\title{
Psychological therapies for the treatment of mental disorders in low- and middle-income countries affected by humanitarian crises
} (Review)

Purgato M, Gastaldon C, Papola D, van Ommeren M, Barbui C, Tol WA

Purgato M, Gastaldon C, Papola D, van Ommeren M, Barbui C, Tol WA.

Psychological therapies for the treatment of mental disorders in low- and middle-income countries affected by humanitarian crises.

Cochrane Database of Systematic Reviews 2018, Issue 7. Art. No.: CD011849.

DOI: 10.1002/14651858.CD011849.pub2.

www.cochranelibrary.com

Psychological therapies for the treatment of mental disorders in low- and middle-income countries affected by humanitarian crises (Review) 
TABLE OF CONTENTS

PLAIN LANGUAGE SUMMARY

SUMMARY OF FINDINGS

BACKGROUND

OBJECTIVES

METHODS

RESULTS

Figure 1.

Figure 2.

Figure 3.

DISCUSSION

AUTHORS' CONCLUSIONS

ACKNOWLEDGEMENTS

REFERENCES

CHARACTERISTICS OF STUDIES

DATA AND ANALYSES

Analysis 1.1. Comparison 1 Psychological therapy vs control comparator - PTSD symptoms - adults, Outcome 1 PTSD at endpoint.

Analysis 1.2. Comparison 1 Psychological therapy vs control comparator - PTSD symptoms - adults, Outcome 2 PTSD at 1 to 4 months.

Analysis 1.3. Comparison 1 Psychological therapy vs control comparator - PTSD symptoms - adults, Outcome 3 PTSD $\geq 6$ months.

Analysis 2.1. Comparison 2 Psychological therapy vs control comparator - PTSD symptoms - children, Outcome 1 PTSD at endpoint.

Analysis 2.2. Comparison 2 Psychological therapy vs control comparator - PTSD symptoms - children, Outcome 2 PTSD at 1 to 4 months.

Analysis 3.1. Comparison 3 Psychological therapy vs control comparator - anxiety symptoms - adults, Outcome 1 Anxiety at endpoint.

Analysis 3.2. Comparison 3 Psychological therapy vs control comparator - anxiety symptoms - adults, Outcome 2 Anxiety at 1 to 4 months.

Analysis 3.3. Comparison 3 Psychological therapy vs control comparator - anxiety symptoms - adults, Outcome 3 Anxiety $\geq 6$ months.

Analysis 4.1. Comparison 4 Psychological therapy vs control comparator - depressive symptoms - adults, Outcome 1 Depression at endpoint.

Analysis 4.2. Comparison 4 Psychological therapy vs control comparator - depressive symptoms - adults, Outcome 2 Depression at 1 to 4 months.

Analysis 4.3. Comparison 4 Psychological therapy vs control comparator - depressive symptoms - adults, Outcome 3 Depression $\geq 6$ months.

Analysis 5.1. Comparison 5 Psychological therapy vs control comparator - dropout -adults, Outcome 1 Dropout.

Analysis 6.1. Comparison 6 Psychological therapy vs control comparator - dropout - children, Outcome 1 Dropout.

Analysis 7.1. Comparison 7 Psychological therapy vs control comparator - functional impairment - adults, Outcome 1 Functional impairment at endpoint.

Analysis 7.2. Comparison 7 Psychological therapy vs control comparator - functional impairment - adults, Outcome 2 Functional impairment at 1 to 4 months.

Analysis 7.3. Comparison 7 Psychological therapy vs control comparator - functional impairment - adults, Outcome 3 Functional impairment $\geq 6$ months.

Analysis 8.1. Comparison 8 Psychological therapy vs control comparator - quality of life - adults, Outcome 1 Quality of life at endpoint.

Analysis 9.1. Comparison 9 Psychological therapy vs control comparator - diagnosis of PTSD - adults, Outcome 1 Diagnosis of PTSD.

Analysis 10.1. Comparison 10 Psychological therapy vs control comparator - diagnosis of PTSD - children, Outcome 1 Diagnosis of PTSD.

Analysis 11.1. Comparison 11 Psychological therapy vs control comparator - diagnosis of depression - adults, Outcome 1 Diagnosis of depression.

Psychological therapies for the treatment of mental disorders in low- and middle-income countries affected by humanitarian crises 
Analysis 12.1. Comparison 12 Subgroup analysis: type of traumatic events - adults, Outcome 1 PTSD symptoms at endpoint. .. Analysis 12.2. Comparison 12 Subgroup analysis: type of traumatic events - adults, Outcome 2 PTSD symptoms at 1 to 4 months.

Analysis 12.3. Comparison 12 Subgroup analysis: type of traumatic events - adults, Outcome 3 PTSD symptoms $\geq 6$ months. ... Analysis 12.4. Comparison 12 Subgroup analysis: type of traumatic events - adults, Outcome 4 Anxiety symptoms at endpoint. Analysis 12.5. Comparison 12 Subgroup analysis: type of traumatic events - adults, Outcome 5 Anxiety symptoms at 1 to 4 months.

Analysis 12.6. Comparison 12 Subgroup analysis: type of traumatic events - adults, Outcome 6 Depressive symptoms at endpoint.

Analysis 12.7. Comparison 12 Subgroup analysis: type of traumatic events - adults, Outcome 7 Depressive symptoms at 1 to 4 months.

Analysis 12.8. Comparison 12 Subgroup analysis: type of traumatic events - adults, Outcome 8 Depressive symptoms $\geq 6$ months.

Analysis 12.9. Comparison 12 Subgroup analysis: type of traumatic events - adults, Outcome 9 Dropout.

Analysis 12.10. Comparison 12 Subgroup analysis: type of traumatic events - adults, Outcome 10 Functional impairment at endpoint.

Analysis 12.11. Comparison 12 Subgroup analysis: type of traumatic events - adults, Outcome 11 Functional impairment at 1 to 4 months.

Analysis 12.12. Comparison 12 Subgroup analysis: type of traumatic events - adults, Outcome 12 Quality of life at endpoint. ... Analysis 12.13. Comparison 12 Subgroup analysis: type of traumatic events - adults, Outcome 13 Diagnosis of PTSD. ............. Analysis 13.1. Comparison 13 Subgroup analysis: type of humanitarian crisis - adults, Outcome 1 PTSD symptoms at endpoint. Analysis 13.2. Comparison 13 Subgroup analysis: type of humanitarian crisis - adults, Outcome 2 PTSD symptoms at 1 to 4 months.

Analysis 13.3. Comparison 13 Subgroup analysis: type of humanitarian crisis - adults, Outcome 3 PTSD symptoms $\geq 6$ months. Analysis 13.4. Comparison 13 Subgroup analysis: type of humanitarian crisis - adults, Outcome 4 Anxiety symptoms at endpoint.

Analysis 13.5. Comparison 13 Subgroup analysis: type of humanitarian crisis - adults, Outcome 5 Anxiety symptoms at 1 to 4 months.

Analysis 13.6. Comparison 13 Subgroup analysis: type of humanitarian crisis - adults, Outcome 6 Depressive symptoms at endpoint.

Analysis 13.7. Comparison 13 Subgroup analysis: type of humanitarian crisis - adults, Outcome 7 Depressive symptoms at 1 to 4 months.

Analysis 13.8. Comparison 13 Subgroup analysis: type of humanitarian crisis - adults, Outcome 8 Depressive symptoms $\geq 6$ months.

Analysis 13.9. Comparison 13 Subgroup analysis: type of humanitarian crisis - adults, Outcome 9 Dropout.

Analysis 13.10. Comparison 13 Subgroup analysis: type of humanitarian crisis - adults, Outcome 10 Functional impairment at endpoint.

Analysis 13.11. Comparison 13 Subgroup analysis: type of humanitarian crisis - adults, Outcome 11 Functional impairment at 1 to 4 months.

Analysis 13.12. Comparison 13 Subgroup analysis: type of humanitarian crisis - adults, Outcome 12 Quality of life at endpoint. Analysis 13.13. Comparison 13 Subgroup analysis: type of humanitarian crisis - adults, Outcome 13 Diagnosis of PTSD. ......... Analysis 14.1. Comparison 14 Subgroup analysis: type of interventionists - adults, Outcome 1 PTSD symptoms at endpoint. ... Analysis 14.2. Comparison 14 Subgroup analysis: type of interventionists - adults, Outcome 2 PTSD symptoms at 1 to 4 months.

Analysis 14.3. Comparison 14 Subgroup analysis: type of interventionists - adults, Outcome 3 PTSD symptoms $\geq 6$ months. .... Analysis 14.4. Comparison 14 Subgroup analysis: type of interventionists - adults, Outcome 4 Anxiety symptoms at endpoint. Analysis 14.5. Comparison 14 Subgroup analysis: type of interventionists - adults, Outcome 5 Anxiety symptoms at 1 to 4 months.

Analysis 14.6. Comparison 14 Subgroup analysis: type of interventionists - adults, Outcome 6 Depressive symptoms at endpoint.

Analysis 14.7. Comparison 14 Subgroup analysis: type of interventionists - adults, Outcome 7 Depressive symptoms at 1 to 4 months.

Analysis 14.8. Comparison 14 Subgroup analysis: type of interventionists - adults, Outcome 8 Depressive symptoms $\geq 6$ months.

Analysis 14.9. Comparison 14 Subgroup analysis: type of interventionists - adults, Outcome 9 Dropout. 
Analysis 14.10. Comparison 14 Subgroup analysis: type of interventionists - adults, Outcome 10 Functional impairment at endpoint.

Analysis 14.11. Comparison 14 Subgroup analysis: type of interventionists - adults, Outcome 11 Functional impairment at 1 to 4 months.

Analysis 14.12. Comparison 14 Subgroup analysis: type of interventionists - adults, Outcome 12 Quality of life at endpoint. .... Analysis 14.13. Comparison 14 Subgroup analysis: type of interventionists - adults, Outcome 13 Diagnosis of PTSD.

Analysis 15.1. Comparison 15 Subgroup analysis: type of control - adults, Outcome 1 PTSD symptoms at endpoint. ................ Analysis 15.2. Comparison 15 Subgroup analysis: type of control - adults, Outcome 2 PTSD symptoms at 1 to 4 months. ........ Analysis 15.3. Comparison 15 Subgroup analysis: type of control - adults, Outcome 3 PTSD symptoms $\geq 6$ months. ................. Analysis 15.4. Comparison 15 Subgroup analysis: type of control - adults, Outcome 4 Anxiety symptoms at endpoint. ............ Analysis 15.5. Comparison 15 Subgroup analysis: type of control - adults, Outcome 5 Anxiety symptoms at 1 to 4 months. ..... Analysis 15.6. Comparison 15 Subgroup analysis: type of control - adults, Outcome 6 Anxiety symptoms $\geq 6$ months. ............. Analysis 15.7. Comparison 15 Subgroup analysis: type of control - adults, Outcome 7 Depressive symptoms at endpoint. ...... Analysis 15.8. Comparison 15 Subgroup analysis: type of control - adults, Outcome 8 Depressive symptoms at 1 to 4 months. .. Analysis 15.9. Comparison 15 Subgroup analysis: type of control - adults, Outcome 9 Depressive symptoms $\geq 6$ months. ....... Analysis 15.10. Comparison 15 Subgroup analysis: type of control - adults, Outcome 10 Dropout.

Analysis 15.11. Comparison 15 Subgroup analysis: type of control - adults, Outcome 11 Functional impairment at endpoint. .. Analysis 15.12. Comparison 15 Subgroup analysis: type of control - adults, Outcome 12 Functional impairment at 1 to 4 months.

Analysis 15.13. Comparison 15 Subgroup analysis: type of control - adults, Outcome 13 Functional impairment $\geq 6$ months. ... Analysis 15.14. Comparison 15 Subgroup analysis: type of control - adults, Outcome 14 Quality of life at endpoint. ................ Analysis 15.15. Comparison 15 Subgroup analysis: type of control - adults, Outcome 15 Diagnosis of PTSD. ........................... Analysis 15.16. Comparison 15 Subgroup analysis: type of control - adults, Outcome 16 Diagnosis of depression. .................. Analysis 15.17. Comparison 15 Subgroup analysis: type of control - adults, Outcome 17 Coping at endpoint. ......................... Analysis 15.18. Comparison 15 Subgroup analysis: type of control - adults, Outcome 18 Coping at 1 to 4 months. .................. Analysis 16.1. Comparison 16 Subgroup analysis: phase of humanitarian crisis - adults, Outcome 1 PTSD symptoms at endpoint.

Analysis 16.2. Comparison 16 Subgroup analysis: phase of humanitarian crisis - adults, Outcome 2 PTSD symptoms at 1 to 4 months.

Analysis 16.3. Comparison 16 Subgroup analysis: phase of humanitarian crisis - adults, Outcome 3 PTSD symptoms $\geq 6$ months.

Analysis 16.4. Comparison 16 Subgroup analysis: phase of humanitarian crisis - adults, Outcome 4 Anxiety symptoms at endpoint.

Analysis 16.5. Comparison 16 Subgroup analysis: phase of humanitarian crisis - adults, Outcome 5 Anxiety symptoms at 1 to 4 months.

Analysis 16.6. Comparison 16 Subgroup analysis: phase of humanitarian crisis - adults, Outcome 6 Depressive symptoms at endpoint.

Analysis 16.7. Comparison 16 Subgroup analysis: phase of humanitarian crisis - adults, Outcome 7 Depressive symptoms at 1 to 4 months.

Analysis 16.8. Comparison 16 Subgroup analysis: phase of humanitarian crisis - adults, Outcome 8 Depressive symptoms $\geq 6$ months.

Analysis 16.9. Comparison 16 Subgroup analysis: phase of humanitarian crisis - adults, Outcome 9 Dropout.

Analysis 16.10. Comparison 16 Subgroup analysis: phase of humanitarian crisis - adults, Outcome 10 Functional impairment at endpoint.

Analysis 16.11. Comparison 16 Subgroup analysis: phase of humanitarian crisis - adults, Outcome 11 Functional impairment at 1 to 4 months.

Analysis 16.12. Comparison 16 Subgroup analysis: phase of humanitarian crisis - adults, Outcome 12 Quality of life at endpoint.

Analysis 16.13. Comparison 16 Subgroup analysis: phase of humanitarian crisis - adults, Outcome 13 Diagnosis of PTSD. ....... Analysis 17.1. Comparison 17 Sensitivity analysis: incomplete outcome data - adults, Outcome 1 PTSD symptoms at endpoint. Analysis 17.2. Comparison 17 Sensitivity analysis: incomplete outcome data - adults, Outcome 2 PTSD symptoms at 1 to 4 months.

Analysis 17.3. Comparison 17 Sensitivity analysis: incomplete outcome data - adults, Outcome 3 PTSD symptoms $\geq 6$ months. 
Analysis 17.4. Comparison 17 Sensitivity analysis: incomplete outcome data - adults, Outcome 4 Anxiety symptoms at endpoint.

Analysis 17.5. Comparison 17 Sensitivity analysis: incomplete outcome data - adults, Outcome 5 Anxiety symptoms at 1 to 4 months.

Analysis 17.6. Comparison 17 Sensitivity analysis: incomplete outcome data - adults, Outcome 6 Depressive symptoms $\geq 6$ months.

Analysis 17.7. Comparison 17 Sensitivity analysis: incomplete outcome data - adults, Outcome 7 Depressive symptoms at endpoint.

Analysis 17.8. Comparison 17 Sensitivity analysis: incomplete outcome data - adults, Outcome 8 Depressive symptoms at 1 to 4 months.

Analysis 17.9. Comparison 17 Sensitivity analysis: incomplete outcome data - adults, Outcome 9 Dropout.

Analysis 17.10. Comparison 17 Sensitivity analysis: incomplete outcome data - adults, Outcome 10 Functional impairment at endpoint.

Analysis 17.11. Comparison 17 Sensitivity analysis: incomplete outcome data - adults, Outcome 11 Functional impairment at 1 to 4 months.

Analysis 17.12. Comparison 17 Sensitivity analysis: incomplete outcome data - adults, Outcome 12 Quality of life at endpoint. . Analysis 17.13. Comparison 17 Sensitivity analysis: incomplete outcome data - adults, Outcome 13 Diagnosis of PTSD.

Analysis 18.1. Comparison 18 Sensitivity analysis: selective reporting - adults, Outcome 1 PTSD symptoms at endpoint. ........ Analysis 18.2. Comparison 18 Sensitivity analysis: selective reporting - adults, Outcome 2 PTSD symptoms at 1 to 4 months. ... Analysis 18.3. Comparison 18 Sensitivity analysis: selective reporting - adults, Outcome 3 Dropout.

Analysis 18.4. Comparison 18 Sensitivity analysis: selective reporting - adults, Outcome 4 Functional impairment at endpoint. 
[Intervention Review]

\section{Psychological therapies for the treatment of mental disorders in low- and middle-income countries affected by humanitarian crises}

Marianna Purgato1,2, Chiara Gastaldon1,2, Davide Papola1,2, Mark van Ommeren³, Corrado Barbui1,2, Wietse A Tol4,5

1Department of Neurosciences, Biomedicine and Movement Sciences, Section of Psychiatry, University of Verona, Verona, Italy. ${ }^{2}$ Cochrane Global Mental Health, University of Verona, Verona, Italy. ${ }^{3}$ Department of Mental Health and Substance Abuse, World Health Organization, Geneva, Switzerland. ${ }^{4}$ Department of Mental Health, Johns Hopkins Bloomberg School of Public Health, Baltimore, Maryland, USA. ${ }^{5}$ Peter C. Alderman Program for Global Mental Health, HealthRight International, New York, USA

Contact: Marianna Purgato, Department of Neurosciences, Biomedicine and Movement Sciences, Section of Psychiatry, University of Verona, Piazzale LA Scuro 10, Verona, 37134, Italy. marianna.purgato@univr.it.

Editorial group: Cochrane Common Mental Disorders Group.

Publication status and date: New, published in Issue 7, 2018.

Citation: Purgato M, Gastaldon C, Papola D, van Ommeren M, Barbui C, Tol WA. Psychological therapies for the treatment of mental disorders in low- and middle-income countries affected by humanitarian crises. Cochrane Database of Systematic Reviews 2018 , Issue 7. Art. No.: CD011849. DOI: 10.1002/14651858.CD011849.pub2.

Copyright @ 2018 The Cochrane Collaboration. Published by John Wiley \& Sons, Ltd.

\section{A B S T R A C T}

\section{Background}

People living in humanitarian settings in low- and middle-income countries (LMICs) are exposed to a constellation of stressors that make them vulnerable to developing mental disorders. Mental disorders with a higher prevalence in these settings include post-traumatic stress disorder (PTSD) and major depressive, anxiety, somatoform (e.g. medically unexplained physical symptoms (MUPS)), and related disorders. A range of psychological therapies are used to manage symptoms of mental disorders in this population.

\section{Objectives}

To compare the effectiveness and acceptability of psychological therapies versus control conditions (wait list, treatment as usual, attention placebo, psychological placebo, or no treatment) aimed at treating people with mental disorders (PTSD and major depressive, anxiety, somatoform, and related disorders) living in LMICs affected by humanitarian crises.

\section{Search methods}

We searched the Cochrane Common Mental Disorders Controlled Trials Register (CCMDCTR), the Cochrane Central Register of Controlled Trials (Wiley), MEDLINE (OVID), Embase (OVID), and PsycINFO (OVID), with results incorporated from searches to 3 February 2016. We also searched the World Health Organization (WHO) trials portal (ICTRP) and ClinicalTrials.gov to identify any unpublished or ongoing studies. We checked the reference lists of relevant studies and reviews.

\section{Selection criteria}

All randomised controlled trials (RCTs) comparing psychological therapies versus control conditions (including no treatment, usual care, wait list, attention placebo, and psychological placebo) to treat adults and children with mental disorders living in LMICs affected by humanitarian crises.

\section{Data collection and analysis}

We used standard Cochrane procedures for collecting data and evaluating risk of bias. We calculated standardised mean differences for continuous outcomes and risk ratios for dichotomous data, using a random-effects model. We analysed data at endpoint (zero to four weeks after therapy); at medium term (one to four months after therapy); and at long term (six months or longer). GRADE (Grades of 
Recommendation, Assessment, Development, and Evaluation) was used to assess the quality of evidence for post-traumatic stress disorder (PTSD), depression, anxiety and withdrawal outcomes.

\section{Main results}

We included 36 studies (33 RCTs) with a total of 3523 participants. Included studies were conducted in sub-Saharan Africa, the Middle East and North Africa, and Asia. Studies were implemented in response to armed conflicts; disasters triggered by natural hazards; and other types of humanitarian crises. Together, the 33 RCTs compared eight psychological treatments against a control comparator.

Four studies included children and adolescents between 5 and 18 years of age. Three studies included mixed populations (two studies included participants between 12 and 25 years of age, and one study included participants between 16 and 65 years of age). Remaining studies included adult populations (18 years of age or older).

Included trials compared a psychological therapy versus a control intervention (wait list in most studies; no treatment; treatment as usual). Psychological therapies were categorised mainly as cognitive-behavioural therapy (CBT) in 23 comparisons (including seven comparisons focused on narrative exposure therapy (NET), two focused on common elements treatment approach (CETA), and one focused on brief behavioural activation treatment (BA)); eye movement desensitisation and reprocessing (EMDR) in two comparisons; interpersonal psychotherapy (IPT) in three comparisons; thought field therapy (TFT) in three comparisons; and trauma or general supportive counselling in two comparisons. Although interventions were described under these categories, several psychotherapeutic elements were common to a range of therapies (i.e. psychoeducation, coping skills).

In adults, psychological therapies may substantially reduce endpoint PTSD symptoms compared to control conditions (standardised mean difference (SMD) -1.07, 95\% confidence interval (CI) - 1.34 to -0.79; 1272 participants; 16 studies; low-quality evidence). The effect is smaller at one to four months (SMD $-0.49,95 \% \mathrm{Cl}-0.68$ to $-0.31 ; 1660$ participants; 18 studies) and at six months (SMD $-0.37,95 \% \mathrm{Cl}-0.61$ to -0.14 ; 400 participants; five studies). Psychological therapies may also substantially reduce endpoint depression symptoms compared to control conditions (SMD - $0.86,95 \% \mathrm{Cl}-1.06$ to $-0.67 ; 1254$ participants; 14 studies; low-quality evidence). Similar to PTSD symptoms, follow-up data at one to four months showed a smaller effect on depression (SMD $-0.42,95 \% \mathrm{Cl}-0.63$ to $-0.21 ; 1386$ participants; 16 studies). Psychological therapies may moderately reduce anxiety at endpoint (SMD $-0.74,95 \% \mathrm{Cl}-0.98$ to $-0.49 ; 694$ participants; five studies; low-quality evidence) and at one to four months' follow-up after treatment (SMD $-0.53,95 \% \mathrm{Cl}-0.66$ to $-0.39 ; 969$ participants; seven studies). Dropout rates are probably similar between study conditions ( $19.5 \%$ with control versus $19.1 \%$ with psychological therapy (RR $0.9895 \% \mathrm{Cl} 0.82$ to $1.16 ; 2930$ participants; 23 studies, moderate quality evidence)).

In children and adolescents, we found very low quality evidence for lower endpoint PTSD symptoms scores in psychotherapy conditions (CBT) compared to control conditions, although the confidence interval is wide (SMD $-1.56,95 \% \mathrm{Cl}-3.13$ to $0.01 ; 130$ participants; three studies;). No RCTs provided data on major depression or anxiety in children. The effect on withdrawal was uncertain (RR $1.8795 \% \mathrm{Cl} 0.47$ to $7.47 ; 138$ participants; 3 studies, low quality evidence).

We did not identify any studies that evaluated psychological treatments on (symptoms of) somatoform disorders or MUPS in LMIC humanitarian settings.

\section{Authors' conclusions}

There is low quality evidence that psychological therapies have large or moderate effects in reducing PTSD, depressive, and anxiety symptoms in adults living in humanitarian settings in LMICs. By one to four month and six month follow-up assessments treatment effects were smaller. Fewer trials were focused on children and adolescents and they provide very low quality evidence of a beneficial effect of psychological therapies in reducing PTSD symptoms at endpoint. Confidence in these findings is influenced by the risk of bias in the studies and by substantial levels of heterogeneity. More research evidence is needed, particularly for children and adolescents over longer periods of follow-up.

\section{PLAIN LANGUAGE SUMMARY}

\section{Talking therapy for the management of mental health in low- and middle-income countries affected by mass human tragedy}

\section{Why is this review important?}

Adults and children and adolescents living in humanitarian contexts (such as in the aftermath of a crisis triggered by natural hazards) in low- and middle-income countries (LMICs) are exposed to multifaceted stressors that make them more vulnerable to developing posttraumatic stress disorder (PTSD), major depression, anxiety, and other negative psychological outcomes.

\section{Who will be interested in this review?}

People who are directly exposed to humanitarian crises and their families and caregivers will be interested in this review, as will healthcare professionals and paraprofessionals working both in LMICs and in high-income settings. Moreover, policy makers, humanitarian programming staff, guideline developers, and agencies (such as non-governmental organisations (NGOs)) working in health and nonhealth sectors (e.g. those providing protection to populations living in humanitarian contexts) may be interested in this review.

Psychological therapies for the treatment of mental disorders in low- and middle-income countries affected by humanitarian crises 


\section{What questions does this review aim to answer?}

Are psychological therapies more effective than control comparator conditions (including no treatment, usual care, wait list, attention placebo, and psychological placebo) in reducing (symptoms of) PTSD and major depressive, anxiety, and somatoform and related disorders (conditions in which people present physical symptoms (e.g. pain) that cannot be explained medically) in people of any age, gender, or religion living in LMICs affected by humanitarian crises?

\section{Which studies were included in this review?}

Review authors searched databases up to February 2016 to find and include all relevant published and unpublished trials. Studies had to include children and/or adults living in LMICs affected by humanitarian crises. Studies also had to be randomised controlled trials (RCTs), which means that people were allocated at random (by chance alone) to receive the treatment or comparator condition.

We included 33 trials with a total of 3523 participants that examined a range of psychological therapies.

\section{What does evidence presented in the review tell us?}

In adults, low-quality evidence shows greater benefit from psychological therapies than from control comparators in reducing (symptoms of) PTSD, major depression, and anxiety disorders. This evidence supports the approach of providing psychological therapies to populations affected by humanitarian crises, although we identified no studies that looked at the effectiveness or acceptability of psychological therapies for depressive and anxiety symptoms beyond six months. Only a small proportion of included trials reported data on children and adolescents, which provided very low-quality evidence of greater benefit derived from psychological treatments. With regard to acceptability, moderate- to low-quality evidence suggests no differences in dropout rates among adults and children and adolescents. Reviewers found no studies evaluating psychological treatments for (symptoms of) somatoform disorders or medically unexplained physical symptoms (MUPS) in adults, nor in children or adolescents, respectively.

\section{What should happen next?}

Researchers should conduct higher-quality trials to further evaluate the effectiveness of psychological therapies provided over longer periods to adults and to children and adolescents. Ideally, trials should be randomised, should use culturally appropriate and validated instruments to evaluate outcomes, and should assess correlates of reductions in treatment effects over time; in addition, researchers should make every effort to ensure high rates of follow-up beyond six months after completion of therapy. 


\section{SUMMARY OF FINDINGS}

Summary of findings for the main comparison. Psychological therapy compared with control for treatment of adults with mental disorders in lowand middle-income countries affected by humanitarian crises

Psychological therapy compared with control for treatment of adults with mental disorders in low- and middle-income countries affected by humanitarian crises

Patient or population: adults exposed to traumatic events

Setting: humanitarian settings in LMICs

Intervention: psychological therapy

Comparison: wait list; no treatment; treatment as usual

\begin{tabular}{|c|c|c|c|c|c|c|}
\hline \multirow[t]{2}{*}{ Outcomes } & \multicolumn{2}{|c|}{ Anticipated absolute effects* $(95 \% \mathrm{Cl})$} & \multirow{2}{*}{$\begin{array}{l}\text { Relative effect } \\
(95 \% \mathrm{Cl})\end{array}$} & \multirow{2}{*}{$\begin{array}{l}\text { No. of partici- } \\
\text { pants } \\
\text { (studies) }\end{array}$} & \multirow{2}{*}{$\begin{array}{l}\text { Quality of the } \\
\text { evidence } \\
\text { (GRADE) }\end{array}$} & \multirow[t]{2}{*}{ Comments } \\
\hline & $\begin{array}{l}\text { Risk with con- } \\
\text { trol }\end{array}$ & $\begin{array}{l}\text { Risk with psychological } \\
\text { therapy }\end{array}$ & & & & \\
\hline $\begin{array}{l}\text { Post-traumatic stress disorder at } \\
\text { endpoint } \\
\text { (measured with IES-R; HTQ; CAPS; } \\
\text { UCLA-PTSD-RI; PDS; PCL-5) }\end{array}$ & - & $\begin{array}{l}\text { SMD } 1.07 \text { lower } \\
\text { (1.34 lower to } 0.79 \text { lower) }\end{array}$ & - & $\begin{array}{l}1272 \\
(16 \mathrm{RCTs})\end{array}$ & $\begin{array}{l}\oplus \oplus \ominus \ominus \\
\text { Lowa,b }^{a}\end{array}$ & $\begin{array}{l}\text { This is a large effect ac- } \\
\text { cording to Cohen } 1992\end{array}$ \\
\hline $\begin{array}{l}\text { Depression at endpoint } \\
\text { (measured with BDI-II, HSCL-25, } \\
\text { HADS) }\end{array}$ & - & $\begin{array}{l}\text { SMD } 0.86 \text { SD lower } \\
\text { (1.06 lower to } 0.67 \text { lower) }\end{array}$ & - & $\begin{array}{l}1254 \\
(14 \mathrm{RCTs})\end{array}$ & $\begin{array}{l}\oplus \oplus \ominus \ominus \\
\text { Lowa,c }\end{array}$ & $\begin{array}{l}\text { This is a large effect ac- } \\
\text { cording to Cohen } 1992\end{array}$ \\
\hline $\begin{array}{l}\text { Anxiety at endpoint } \\
\text { (measured with HADS-A; HSCL-25) }\end{array}$ & - & $\begin{array}{l}\text { SMD } 0.74 \text { SD lower } \\
\text { ( } 0.98 \text { lower to } 0.49 \text { lower) }\end{array}$ & - & $\begin{array}{l}694 \\
(5 \mathrm{RCTS})\end{array}$ & $\begin{array}{l}\oplus \oplus \oplus \ominus \\
\text { Lowd,e }\end{array}$ & $\begin{array}{l}\text { This is a moderate ef- } \\
\text { fect according to Co- } \\
\text { hen } 1992\end{array}$ \\
\hline \multirow[t]{2}{*}{ Dropouts for any reason } & \multicolumn{2}{|l|}{ Study population } & RR 0.98 & 2950 & $\oplus \oplus \oplus \ominus$ & \\
\hline & 195 per 1000 & $\begin{array}{l}191 \text { per } 1000 \\
\text { (160 to } 227)\end{array}$ & & & & \\
\hline $\begin{array}{l}\text { Somatic symptoms and related dis- } \\
\text { orders }\end{array}$ & - & - & - & - & - & No data are available \\
\hline
\end{tabular}

*The risk in the intervention group (and its $95 \%$ confidence interval) is based on the assumed risk in the comparison group and the relative effect of the intervention (and its $95 \% \mathrm{Cl}$ )

$\mathrm{Cl}$ : confidence interval; RCT: randomised controlled trial; RR: risk ratio; SMD: standardised mean difference

GRADE Working Group grades of evidence 
High quality: We are very confident that the true effect lies close to that of the estimate of the effect

Moderate quality: We are moderately confident in the effect estimate: The true effect is likely to be close to the estimate of the effect, but there is a possibility that it is sub-

stantially different

Low quality: Our confidence in the effect estimate is limited: The true effect may be substantially different from the estimate of the effect

Very low quality: We have very little confidence in the effect estimate: The true effect is likely to be substantially different from the estimate of effect

BDI: Beck Depression Inventory

CAPS: Clinician Administered Post-traumatic stress disorder Scale

HADS: Hospital Anxiety and Depression Scale

HSCL: Hopkins Symptoms Checklist

IES-R: Impact of Event Scale-Revised

LMIC: low- and middle-income countries

PCL: Post-traumatic stress disorder Check List

PDS: Post-traumatic stress disorder Diagnostic Scale

UCLA-PTSD- RI: University College of Los Angeles Post-traumatic stress disorder Reaction Index

a Downgraded one level owing to study limitations (outcome assessment was not described as masked in all RCTs)

bDowngraded one level owing to inconsistency (12 was higher than $75 \%)$

'Downgraded one level owing to inconsistency ( 12 was $55 \%$ )

$d$ Downgraded one level owing to imprecision (the $\mathrm{Cl}$ includes no effect)

eDowngraded one level owing to study limitations (high risk of bias detected: performance bias, attrition bias, and concerns about therapist/investigator allegiance)

Summary of findings 2. Psychological therapy compared with control for treatment of children with mental disorders in low- and middle-income countries affected by humanitarian crises

Psychological therapy compared with control for treatment of children with mental disorders in low- and middle-income countries affected by humanitarian crises

Patient or population: children exposed to traumatic events

Settings: humanitarian settings in LMICS

Intervention: psychological therapy

Comparison: wait list; no treatment; treatment as usual

\begin{tabular}{|c|c|c|c|c|c|c|}
\hline \multirow[t]{2}{*}{ Outcomes } & \multicolumn{2}{|c|}{ Anticipated absolute effects ${ }^{\star}(95 \% \mathrm{Cl})$} & \multirow{2}{*}{$\begin{array}{l}\text { Relative effect } \\
(95 \% \mathrm{Cl})\end{array}$} & \multirow{2}{*}{$\begin{array}{l}\text { No. of partici- } \\
\text { pants } \\
\text { (studies) }\end{array}$} & \multirow{2}{*}{$\begin{array}{l}\text { Quality of the } \\
\text { evidence } \\
\text { (GRADE) }\end{array}$} & \multirow[t]{2}{*}{ Comments } \\
\hline & $\begin{array}{l}\text { Risk with con- } \\
\text { trol }\end{array}$ & $\begin{array}{l}\text { Risk with psychological } \\
\text { therapy }\end{array}$ & & & & \\
\hline $\begin{array}{l}\text { Post-traumatic stress disorder at } \\
\text { endpoint }\end{array}$ & - & $\begin{array}{l}\text { SMD }-1.56 \\
\text { (-3.13 lower to } 0.01 \text { high- }\end{array}$ & - & $\begin{array}{l}130 \\
\text { (3 RCTs) }\end{array}$ & $\begin{array}{l}\oplus \oplus \ominus \ominus \\
\text { Very lowa,b,c }\end{array}$ & $\begin{array}{l}\text { This is a large effect ac- } \\
\text { cording to Cohen } 1992\end{array}$ \\
\hline
\end{tabular}




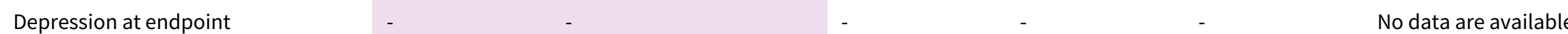

\begin{tabular}{lllllll}
\hline Anxiety at endpoint & - & - & - & No data are available
\end{tabular}

Dropouts for any reason

Study population

352 per 1000

(165 to 1000$)$

Somatic symptoms and related dis

orders

${ }^{*}$ The risk in the intervention group (and its 95\% confidence interval) is based on the assumed risk in the comparison group and the relative effect of the intervention (and its $95 \% \mathrm{Cl}$ )

$\mathrm{Cl}$ : confidence interval; RCT: randomised controlled trial; RR: risk ratio; SMD: standardised mean difference

GRADE Working Group grades of evidence

High quality: Further research is very unlikely to change our confidence in the estimate of effect

Moderate quality: Further research is likely to have an important impact on our confidence in the estimate of effect and may change the estimate

Low quality: Further research is very likely to have an important impact on our confidence in the estimate of effect and is likely to change the estimate

Very low quality: We are very uncertain about the estimate

CRIES: Children Revised Impact of Events Scale

LMIC: low- and middle-income countries

UCLA-PTSD- RI: University College of Los Angeles Post-traumatic stress disorder Reaction Index

a Downgraded one level owing to study limitations (outcome assessment was not described as masked in all RCTs)

$b$ Downgraded one level owing to inconsistency ( ${ }^{2}$ was higher than $75 \%$ )

cDowngraded one level owing to imprecision (outcome based on small number of participants and confidence interval is wide)

${ }^{d}$ Downgraded one level owing to imprecision ( $\mathrm{Cl}$ includes no effect) 


\section{B A C K G R O U N D}

\section{Description of the condition}

The term 'humanitarian crises' is used to refer to a broad group of emergencies, including those triggered by natural, technological, and industrial hazards, as well as armed conflicts (Tol 2011). Humanitarian crises are commonly defined as rapid and serious deteriorations in safety, with numerous victims or numerous people whose lives are in danger or who are in great distress, along with substantial material destruction, forced displacement or population movement, and great difficulty or incapacity of institutional management in handling the situation (Josse 2009). The most commonly affected populations live in lowand middle-income countries (LMICs) (Guha-Sapir 2014; Themner 2014). Humanitarian crises have a wide range of impacts on the mental health of individual survivors. Mental health consequences may include improved mental health (e.g. through post-traumatic growth); maintained mental health and wellbeing despite exposure to adversity (i.e. resilience); transient acute stress reactions and bereavement; and a range of mental disorders (Kane 2017).

Mental health epidemiology in humanitarian settings has most commonly focused on disorders and conditions specifically associated with exposure to stressors, such as post-traumatic stress disorder (PTSD). In classifying outcomes of interest, we followed the Diagnostic and Statistical Manual of Mental Disorders (DSMV) classification. Diagnostic criteria for PTSD include a history of exposure to a traumatic event that meets specific stipulations and symptoms from each of four symptom clusters: intrusion, persistent avoidance, negative alterations in cognition and mood, and alterations in arousal and reactivity associated with the traumatic event (APA 2013; O'Donnell 2014).

Moreover, studies have identified heightened prevalence of disorders that can occur in the absence of exposure to stressors, such as:

1. anxiety disorders, which include disorders that share features of excessive fear and anxiety and related behavioural disturbances;

2. depressive disorders, which are characterised by the presence of sad, empty, or irritable mood, and accompanied by somatic and cognitive changes that affect the individual's capacity to function (APA 2013); and

3. somatic symptom and related disorders (this is an umbrella term introduced in the DSM-V that includes the conditions listed in the DSM-IV as somatoform disorders), including medically unexplained physical symptoms (MUPS) (APA 2013; van Dessel 2014).

Steel et al conducted a meta-analysis of epidemiological studies with adult conflict-affected populations across 181 surveys in 40 countries. In a subset of rigorous studies, prevalence rates were $15.4 \%$ for PTSD (30 studies using representative sampling and diagnostic interviews) and $17.3 \%$ for depression (26 studies using representative sampling and diagnostic interviews). Predictors of PTSD were torture, cumulative exposure to potentially traumatic events (PTEs), time since conflict, and level of political terror in the territory. For depression, predictors were number of PTEs, time since conflict, torture, and residency status (Charlson 2016).

\section{Description of the intervention}

The World Health Organization (WHO) Mental Health Gap Action Program (mhGAP) has developed guidelines specifically focused on the needs of people living in LMICs (WHO 2013; WHO 2016). Pharmacological treatments are available for individuals with PTSD (Hoskins 2015). However, psychological therapies, together with other types of psychosocial interventions, are generally considered the first-line option according to mhGAP guidelines (e.g. for management of acute stress, PTSD, and bereavement) (WHO 2013; WHO 2016).

Psychological therapies are widely used in the management of (symptoms of) PTSD, anxiety, depression, somatoform disorders, MUPS, and related disorders, and are recommended in the mhGAP Intervention Guide (WHO 2016). mhGAP guidelines contain both recommendations on psychological interventions for adults and a specific section dedicated to treatment of children and adolescents. Different types of psychological therapies are available, such as different forms of cognitive-behavioural therapy (CBT), including CBT with a trauma focus (CBT-T), Brief Behavioural Activation treatment (BA), narrative exposure therapy (NET), and the common elements treatment approach (CETA); eye movement desensitisation and reprocessing (EMDR); interpersonal therapy (IPT); thought field therapy (TFT); and psychodynamic therapy.

CBT is often used as an umbrella term that encompasses a wide range of therapeutic approaches, techniques, and systems that share some common elements. CBTs include psychological treatments that combine cognitive components (aimed at thinking differently, for example, by identifying and challenging unrealistic negative thoughts) and behavioural components (aimed at doing things differently, for example, by helping the person to participate in more rewarding activities) (WHO 2016). CBTs assume that psychopathology, or emotional disturbance, is the result of biased cognitions and unhelpful behaviour; these treatments aim to improve symptoms of anxiety by addressing these unhelpful cognitions and behaviours. They are used to treat individuals with depression and somatoform disorders (Allen 2010), and they are used in children and adolescents as well as in adults (James 2015; Olthuis 2015; Watts 2015).

CBT has been applied to all disorders of interest in this review (with various modifications/various emphases for different disorders).

These include the following.

1. CBT-T (cognitive-behavioural therapy with a trauma focus): based on the idea that people who were exposed to a traumatic event have unhelpful thoughts and beliefs related to that event and its consequences. These thoughts and beliefs result in unhelpful avoidance of reminders of the event and a sense of current threat. Treatment usually includes exposure to those reminders and to challenging unhelpful trauma-related thoughts or beliefs (WHO 2016).

a. Among CBT-Ts, NET (narrative exposure therapy) is a standardised short-term approach to trauma-related disorders based on the patient's construction of a narrative about his/her life from birth up to the present situation with focus on detailed exploration of the traumatic experience, while combining testimony therapy and CBT-T exposure elements (Schauer 2011). 
b. CETA (common elements treatment approach): transdiagnostic treatment approach specifically designed to be delivered in low-resource settings, which allows therapists to combine evidence-based treatment elements depending on individual symptom presentation, including psychoeducation, anxiety management, cognitive coping/ restructuring, and elements of exposure (Murray 2014).

c. BA (Brief Behavioural Activation): a manualised type of CBT that is focused on reducing depressive symptoms by helping individuals engage in positive activities on a daily basis, according to the values and goals of that individual in multiple life areas (i.e. relationships, career, and spirituality) (Jakupcak 2010).

2. EMDR (eye movement desensitisation and reprocessing): based on the idea that negative thoughts, feelings, and behaviours result from unprocessed memories of traumatic events. Treatment involves standardised procedures that include focusing simultaneously on:

a. associations of traumatic images, thoughts, emotions, and bodily sensations; and

b. bilateral stimulation that most commonly occurs in the form of repeated eye movements (WHO 2016).

3. IPT (interpersonal psychotherapy): helps people understand their feelings as useful signals of interpersonal encounters (Markowitz 2014). IPT is considered an evidence-based therapy for major depression that focuses specifically on the connection between depressive symptoms and interpersonal problems (Dennis 2007;WHO 2016); it has also been evaluated in the treatment of anxiety disorders, PTSD, and somatoform and related disorders (Markowitz 2015; van Dessel 2014).

4. TFT (thought field trauma): brief trauma intervention that utilises a sequence of self-tapping to stimulate specific acupuncture points while recalling a traumatic event or cue. It facilitates the relaxation response while the person experiences exposure to the problem by simply thinking about the problem (Callahan 2000).

5. Psychodynamic therapy: focused on integration of the traumatic experience into the life experience of the person as a whole, often considering childhood issues as important (Brom 1989).

6. Other interventions, such as generic, problem-solving, or trauma-focused counselling: commonly less structured than psychotherapies and targeting specific needs and problems as expressed by patients. Interventions may also focus on rebuilding skills and coping strategies in social situations while improving communication and social interaction skills, to reduce stress in everyday life (WHO 2016).

\section{How the intervention might work}

These different treatments are based on their own various theoretical models describing putative treatment mechanisms. Previous reviews of psychological treatments for PTSD - Bisson 2013 and Gillies 2016, depression - Cuijpers 2009, Gloaguen 1998, Rigmor 2010, and Watanabe 2007, and anxiety disorders - Abbass 2014 found psychological treatments to be effective. For PTSD in adults, treatments that included specific elements focused on trauma were more effective than treatments that did not include such elements (Bisson 2013). In children and adolescents, CBT interventions appeared to be more effective than control conditions for PTSD (Gillies 2016), depression (Watanabe 2007), and anxiety disorders (James 2015).
For all clinical conditions considered in this review, the mechanism of action of CBT had been explored and categorised as follows.

1. Cognitive mechanisms: increase in adaptive cognitions that may occur through restructuring of maladaptive thought patterns, correction of misinterpretations, changes in attentional focus, and development of adaptive coping thoughts.

2. Behavioural mechanisms: increase in adaptive behavioural responses that may occur through habituation, extinction of maladaptive responses, behavioural activation, associative learning, and reinforcement of adaptive responding.

3. Physiological mechanisms: normalisation of physiological arousal that may occur through habituation, incompatible response training, or changes in autonomic nervous system activity (DePaulo 2014).

CBT for depression is based on the assumption that the person's mood is related to his or her patterns of thought (thoughts tend to be unrealistic or distorted); therefore CBT can help a person learn to recognise negative patterns of thought, to evaluate their validity, and to replace these thoughts with different and functional ways of thinking (Beck 1979; WHO 2016). CBT for anxiety addresses negative patterns/distortions related to the way we look at the world and at ourselves. This involves a cognitive component (focused on how negative thoughts, or cognitions, contribute to anxiety) and a behavioural component (focused on behaviours that trigger anxiety). CBT is based on the premise that fear and anxiety are learnt responses that can be 'unlearnt' (James 2015). CBT has also been used for somatoform-related disorders (van Dessel 2014).

CBT-T is based on the idea that people with PTSD have unhelpful thoughts and beliefs related to a traumatic event and its consequences, and that these beliefs result in unhelpful avoidance of reminders of the event with a sense of current threat. Cognitivebehavioural interventions with a trauma focus usually work with imaginal and/or in vivo (real life) exposure treatment and/or direct challenging of unhelpful trauma-related thoughts and beliefs (WHO 2013). CBT-T protocols usually involve different components such as psychoeducation, anxiety management, exposure, and cognitive restructuring (Bisson 2013). NET is a type of CBT-T that is thought to contextualise the particular associative elements of the fear network - the sensory, affective, and cognitive memories of trauma - to understand and process the memory of a traumatic event in the course of the patient's life. In NET, the patient (with the assistance of the therapist) constructs a chronological narrative of his life story with a focus on traumatic experiences. Fragmented reports of these traumatic experiences will be transformed into a coherent narrative. Empathic understanding, active listening, congruency, and unconditional positive regard are key components of the therapist's behaviour (Schauer 2011). CETA is a transdiagnostic approach that tailors the selection of elements that are common to evidence-based psychotherapies to each individual's symptom profile. It consists of delivering specific components tailored to the individual's needs and culture. Components are engagement, psychoeducation, anxiety management, cognitive restructuring, imaginal gradual exposure, in vivo exposure, safety, and alcohol use assessment (Murray 2014).

$B A$ is a structured CBT program for depression that reinforces positive activities in different areas of an individual's life (e.g. talking and exchanging ideas with others; interacting with and helping other; working). Engagement in these activities is initially

Psychological therapies for the treatment of mental disorders in low- and middle-income countries affected by humanitarian crises 
supported by the therapist (actively) and is intended to become more intrinsic as activities lead to more positive experiences and the satisfaction that comes from living according to one's own goals and values.

TFT is a brief treatment that identifies feelings elicited by thinking about the problem and asking the patient to rate the emotional intensity that he/she feels when thinking about the problem by stimulating selected acupoints on the surface of the skin in a sequence that is specific to the identified emotions. The theory behind TFT is that precisely encoded information becomes activated when an individual thinks about a problem, either subconsciously or consciously (Callahan 2000).

EMDR is based on the idea that negative thoughts, feelings, and behaviours are the result of unprocessed memories. Treatment involves standardised procedures that include simultaneous focus on spontaneous associations of traumatic images, thoughts, emotions, and bodily sensations; and bilateral stimulation, most commonly in the form of repeated eye movements (WHO 2013).

IPT is an evidence-based treatment for individuals with major depression. It is designed to help a person identify and address problems in relationships with family, friends, partners, and other significant people (WHO 2016). IPT addresses the person's ability to assert his/her needs and wishes in interpersonal encounters, to validate the person's anger as a normal interpersonal signal and to encourage its efficient expression, and to take appropriate social risks. Reviewing the person's accomplishments during treatment helps him/her feel more capable and independent (Markowitz 2004).

Psychodynamic therapies aim to resolve inner conflicts arising from the traumatic event by placing emphasis on the unconscious mind. These therapies have also been used in depression, anxiety disorders, and somatic symptom and related disorders.

\section{Why it is important to do this review}

Humanitarian crises impact a large part of the world's population, often affecting populations already beset by adversity (e.g. poverty, gender-based violence, social marginalisation). For example, the Machel report states that just over 1 billion children globally are affected by armed conflicts (UN General Assembly 1996; UNICEF 2009). It is important to note that given the known high burden associated with mental disorders and conditions in these populations, application of treatments with known efficacy has the potential to improve individual functioning while widening wellbeing and economic productivity.

Mental health and psychosocial support interventions are becoming a standard part of humanitarian programmes. Although this was an ideologically divided field, agreement on best practices appears to be growing, as evidenced by international consensusbased documents (IASC 2007; The Sphere Project 2011). These documents advocate for multi-layered systems of care intended to address the diversity of mental health and psychosocial needs in humanitarian settings. As part of such systems of care, pharmacological and psychological treatments are intended to target more severe mental health problems. This review focuses on psychological therapies, given conflicting views in current guidelines on the benefits of pharmacological approaches for conditions specifically related to stress, such as PTSD (Forbes
2010; WHO 2013). In two parallel reviews, we will evaluate the effectiveness of psychosocial approaches in preventing mental disorders and promoting (positive aspects of) mental health and psychosocial well-being.

Bisson et al conducted a systematic review of randomised controlled trials (RCTs) of individual CBT-T, EMDR, CBT without a specific trauma focus, and other therapies (supportive therapy, non-directive counselling, psychodynamic therapy, and presentcentred therapy), as well as group CBT-T and group CBT for PTSD (70 studies; $n=4761$ ) (Bisson 2013). Researchers found that CBT-T and EMDR were effective in reducing clinician-rated PTSD. Individual CBT-T, EMDR, and CBT appeared to be equally effective immediately post treatment, and some evidence shows that CBT-T and EMDR were superior to CBT at follow-up. Individual CBT-T, EMDR, and CBT are more effective than other therapies. $A$ recent Cochrane review on PTSD in children and adolescents included 51 studies $(n=6201)$ with participants exposed to various kinds of traumatic events. Trial authors found evidence to support the effectiveness of psychological therapies for reducing PTSD in children and adolescents (Gillies 2016). However, even though informative, these reviews were not specifically focused on humanitarian settings in LMICs.

In summary, given the broad impact of humanitarian settings on mental health, this review aims to provide a comprehensive evaluation of the effectiveness and acceptability of psychological treatments, across a range of disorders in children and adolescents as well as adults. In conducting this systematic review, we will follow the protocol that we published in the Cochrane Database of Systematic Reviews (Purgato 2015).

\section{O B JECT IVES}

To compare the effectiveness and acceptability of psychological therapies versus control conditions (wait list, treatment as usual, attention placebo, psychological placebo, or no treatment) aimed at treating people with mental disorders (PTSD and major depressive, anxiety, somatoform, and related disorders) living in LMICs affected by humanitarian crises.

\section{METHODS}

\section{Criteria for considering studies for this review \\ Types of studies}

We included RCTs. We also included in the review trials employing a cross-over design - whilst acknowledging that this design is rarely used in psychological treatment studies - using data from the first randomised stage only. We excluded quasi-randomised trials, such as those allocating treatments on alternate days of the week. We considered cluster-randomised trials as eligible for inclusion.

\section{Types of participants}

\section{Participant characteristics}

We included participants of any age, gender, ethnicity, or religion. We conducted separate meta-analyses for studies with children and adolescents (younger than 18 years) and for adults (18 years of age or older) on different trial outcomes. We categorised studies including mixed populations of children and adults according to the mean age of participants. 
We have decided for the first version of this review to consider children and adolescents, as well as adults, according to the methods followed by Tol 2011.

\section{Setting}

We considered studies conducted in LMICs in humanitarian settings, that is, in contexts affected by armed conflicts or by disasters associated with natural, technological, or industrial hazards. We used World Bank criteria for categorising a country as low- or middle-income (World Bank 2013). For 2016, low-income economies were defined as those with a gross national income (GNI) per capita, as calculated using the World Bank Atlas method, of $\$ 1,025$ or less in 2015; middle-income economies as those with a GNI per capita between $\$ 1,026$ and $\$ 12,475$; and highincome economies as those with a GNI per capita of $\$ 12,476$ or more (www.worldbank.org/). We excluded studies undertaken in high-income countries or focused on refugees currently living in high-income countries. Therapies may be delivered in healthcare clinics or in other healthcare facilities, refugee camps, schools, communities, survivors' homes, and detention facilities. We included studies recruiting inpatients and outpatients. We included studies with populations during humanitarian crises, as well as during the period after acute humanitarian crises (e.g. post-conflict settings).

\section{Diagnosis}

We included studies that applied any standardised diagnostic criteria, including Diagnostic and Statistical Manual of Mental Disorders (DSM) III (APA 1980), DSM-III-R (APA 1987), DSM-IV (APA 1994), DSM-IV-TR (APA 2000), DSM-V (APA 2013), or International Classification of Diseases (ICD-10) criteria (WHO 1992), for the following disorders.

1. PTSD.

2. Anxiety disorders (e.g. separation anxiety disorder, selective mutism, specific phobia, social anxiety disorder (social phobia), panic disorder, agoraphobia, substance/medication-induced anxiety disorder).

3. Depressive disorders (e.g. major depressive disorder).

4. Somatoform symptom and related disorders, including medically unexplained physical symptoms (MUPS).

We included studies that assessed the presence of a mental disorder using a structured psychiatric diagnostic interview (e.g. the Mini International Neuropsychiatric Interview (Sheehan 1998)) or scoring above established cutoffs on commonly used rating scales (e.g. the Impact of Events Scale - Revised, for PTSD (Weiss 1997); the Hamilton Depression Rating Scale (HDRS) (Hamilton 1960); or the Beck Depression Inventory for Depression (Beck 1961). Earlier studies may have used ICD-9 (WHO 1978), but ICD-9 is not based on operationalised criteria, so we excluded from this review studies that used ICD-9.

We excluded studies that had a primary focus on the following disorders: substance misuse; dissociative disorders; obsessivecompulsive disorders; and child behavioural disorders. We recognise the importance of these disorders, but we found scant epidemiological data on their prevalence in LMIC humanitarian settings, and we assumed that few studies have evaluated psychological treatments for these disorders in humanitarian settings (Charlson 2016; WHO 2012).
WHO estimates that humanitarian crises may be associated with an increase of $1 \%$ to $2 \%$ in prevalence of (pre-existing) severe neuropsychiatric disorders such as psychosis and epilepsy (WHO 2012). Although it is critical to recognise the importance of these severe neuropsychiatric disorders for humanitarian programming (Jones 2012), we do not discuss them as part of the current review.

Researchers have described the importance of culturally patterned descriptions of symptoms in humanitarian settings that do not fit current psychiatric classification systems. Such cultural concepts of distress have been the topic of epidemiological studies but have not commonly been part of outcome evaluation studies (Kohrt 2013); therefore we did not review them here.

\section{Comorbidity}

We included studies recruiting participants with mental disorder comorbidities (i.e. various combinations of the disorders listed above) and physical comorbidities. We conducted a subgroup analysis to investigate whether the presence of comorbidities affected trial results.

\section{Types of interventions}

\section{Experimental interventions}

Any psychological therapies aimed at treating patients with (symptoms of) PTSD or major depressive, anxiety, somatoform, or related disorders in humanitarian settings in LMICs.
1. CBT (BA and CBT-T: NET, CETA, other CBT).
2. EMDR.
3. IPT.
4. TFT.
5. Psychodynamic therapy.
6. Other psychological therapies.

\section{Comparators}

Control comparators included the following.

1. No treatment.

2. Treatment as usual (TAU) (also called standard/usual care): Participants could receive any appropriate medical care during the course of the study on a naturalistic basis, as deemed necessary by the clinician.

3. Wait list (WL): delayed delivery of the intervention to the control group until after participants in the intervention group have completed treatment. As in TAU, participants in the WL condition could receive any appropriate medical care during the course of the study on a naturalistic basis.

4. Attention placebo: defined as a control condition that is regarded as inactive by both researchers and participants in a trial.

5. Psychological placebo: defined as a control condition that is regarded by researchers as inactive but is regarded by participants as active.

Participants may receive any appropriate medical care during the course of the study on a naturalistic basis, including pharmacotherapy, as deemed necessary by the healthcare staff. We documented any additional intervention(s) received naturalistically by participants allocated to both control and active 
arms. In the present review, we assessed the effectiveness of psychological therapies as delivered in typical clinical settings (not necessarily under ideal experimental conditions).

\section{Format of psychological therapies}

Psychological treatment may be delivered through any means, including, for example, face-to-face meetings, Internet, telephone, or self-help booklets between participant(s) and trained professional(s) or para-professional(s). Both individual and group psychological treatments were eligible for inclusion, with no limit applied to the number of sessions.

\section{Excluded interventions}

We excluded from this review pharmacological treatments, as well psychosocial interventions aimed at preventing mental disorders or promoting (positive aspects of) mental health and psychosocial well-being. Separate parallel reviews have covered the latter two.

\section{Types of outcome measures}

We included studies that met the above inclusion criteria regardless of whether they reported on the following outcomes.

\section{Primary outcomes}

1. Efficacy outcome (symptom severity)

a. PTSD: mean change from baseline to study endpoint on the Harvard Trauma Questionnaire (HTQ) (Mollica 1992), the Posttraumatic Stress Disorder Checklist - Civilian version (PCL-C) (Weathers 1993), the Clinician-Administered PTSD Scale for adults (CAPS) (Blake 1995), the ClinicianAdministered PTSD Scale for Children and Adolescents (CAPS-CA) (Nader 1996), or other rating scales

b. Anxiety disorders: mean change from baseline to study endpoint on the Hospital Anxiety and Depression Scale Anxiety Subscale (HAD-A) for adults (Zigmond 1983), the Screen for Anxiety Related Emotional Disorders (SCARED-5) for children and adolescents (Birmaher 1997), or any other commonly used rating scale

c. Major depressive disorder: mean change scores from baseline to study endpoint on the Depression Self-Rating Scale (Birleson 1987), the Beck Depression Inventory (Beck 1961), the Hamilton Depression Rating Scale (HDRS) (Hamilton 1960), the Montgomery-Asberg Depression Rating Scale (MADRS) (Montgomery 1979), or any other commonly used rating scale

d. Somatic symptom and related disorders: mean change scores from baseline to study endpoint on Somatic Symptom Scale-8 (Gierk 2014), Patient Health Questionnaire-15 (Kroenke 2002), or any other commonly used rating scale

2. Acceptability outcome

a. Number of participants who dropped out of psychological treatment for any reason

\section{Secondary outcomes}

1. Functional impairment: mean change scores from baseline to study endpoint on the Function Impairment Measure (Tol 2011a), the WHO Disability Assessment Schedule 2.0 (WHO 2010), the Global Assessment of Functioning (APA 2000), or other commonly used rating scales
2. Quality of life: mean change scores from baseline to study endpoint on the WHO Quality of Life Scale (WHO 1997), or on other commonly used rating scales

3. Presence or absence of a formal or clinical diagnosis of PTSD, anxiety disorders, depression, or somatic symptom and related disorders evaluated by psychiatric diagnostic interviews. If a psychiatric diagnostic interview was not used, we included studies that applied commonly used symptom checklists

\section{Timing of outcome assessment}

Our primary endpoint was assessed immediately after treatment (zero to four weeks after intervention). We also collected information on every other available follow-up assessment. We categorised follow-up data as follows: follow-up immediately after treatment (at endpoint: zero to four weeks); follow-up at one to four months; and follow-up at six or more months.

\section{Hierarchy of outcome measures}

When more than one outcome measure was available in the domain of interest, as defined in outcomes, and both described the domain adequately, we chose the measure with the most detailed psychometric evaluation or that was used by other trials in the analysis. Secondarily, we chose any measure that trial authors stated was tested for suitability in the population of interest. For primary outcomes, if data from several commonly used rating scales were available, we used the following: for PTSD - HTQ, PCL-C, CAPS, and CAPS-CA; for anxiety - HAD-A for adults (Zigmond 1983) and SCARED-5 for children and adolescents (Birmaher 1997); for depression - HDRS (Hamilton 1960); and for somatic symptom and related disorders - Somatic Symptom Scale-8 (Gierk 2014).

\section{Search methods for identification of studies}

\section{Cochrane Common Mental Disorders Controlled Trials Register (CCMDCTR)}

The Cochrane Common Mental Disorders Group (CCMD) maintains two archived clinical trials registers at its editorial base in York, UK: a references register and a studies-based register. The CCMDCTRReferences Register contains over 40,000 reports of RCTs in depression, anxiety, and neurosis. Approximately $50 \%$ of these references have been tagged to individual, coded trials. The coded trials are held in the CCMDCTR-Studies Register, and records are linked between the two registers through the use of unique Study ID tags. Coding of trials is based on the EU-Psi coding manual, using a controlled vocabulary (please contact the CCMD Information Specialists for further details). Reports of trials for inclusion in the Group's registers are collated from routine (weekly), generic searches of MEDLINE (1950 to 2016), Embase (1974 to 2016), and PsycINFO (1967 to 2016); quarterly searches of the Cochrane Central Register of Controlled Trials (CENTRAL); and review-specific searches of additional databases. Reports of trials are also sourced from international trial registers via the WHO trials portal (the International Clinical Trials Registry Platform (ICTRP)), pharmaceutical company websites, and handsearching of key journals, conference proceedings, and other (non-Cochrane) systematic reviews and meta-analyses.

Details of CCMD generic search strategies (used to identify RCTs) can be found on the Group's website, with an example of the core MEDLINE search used to inform the register displayed in Appendix 1. 


\section{Electronic searches}

\section{Cochrane Specialised Register}

We cross-searched the CCMDCTR-Studies and References Register using terms to represent humanitarian crises in LMICs (only), as this is a specialist mental health database, so already indicative of the diagnosis (3 February 2016).

\#1. (altruis* or humanitarian or human right $\left.^{\star}\right): t i, a b, k w, k y, e m t, m h, m c$

\#2. (catastrophe* or disaster ${ }^{\star}$ or drought* or earthquake* or evacuation* or famine ${ }^{\star}$ or flood or floods or hurricane or cyclone* or landslide* or "land slide" or "mass casualt" or tsunami* or tidal wave* or volcano*):ti,ab,kw, ky,emt, $m h, m c$

\#3. (genocide or "armed conflict" or "mass execution" or "mass violence"):ti, $a b, k w, k y, e m t, m h, m c$

\#4. ((war or conflict) NEAR2 (affect ${ }^{\star}$ or effect ${ }^{\star}$ or expos ${ }^{\star}$ or related or victim* or survivor $\left.{ }^{\star}\right)$ ):ti, $a b, k w, k y, e m t, m h, m c$

\#5. (displac ${ }^{\star}$ NEAR (internal or forced or mass or person* or people* or population $\left.{ }^{\star}\right)$ : ti, $a b, k w, k y, e m t, m h, m c$

\#6. ("forced migration" or refugee ):ti, $a b, k w, k y, e m t, m h, m c$

\#7. (politic* NEAR (persecut* or prison* ${ }^{\star}$ or imprison* ${ }^{*}$ or violen $\left.\left.{ }^{\star}\right)\right): t i, a b, k w, k y, e m t, m h, m c$

$\# 8$. (\#1 or \#2 or \#3 or \#4 or \#5 or \#6 or \#7)

\#9. (bereav $^{\star}$ or orphan ${ }^{\star}$ or widow $\left.{ }^{\star}\right): t i, a b, k w, k y, e m t, m h, m c$

$\# 10$. (abuse* or conflict or persecut ${ }^{\star}$ or rape or torture or violen ${ }^{\star}$ or

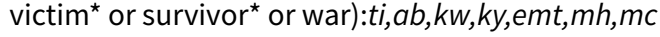

$\# 11$. (aid or relief or rescue or peace ${ }^{\star}$ ):ti, $a b, k w, k y, e m t, m h, m c$

\#12. emergenc ${ }^{*}$ :ti or (emergency NEXT (service* or setting)):ti, $a b, k w, k y, e m t, m h, m c$

\#13. ("critical incident" or "crisis intervention" or

CISD):ti, $a b, k w, k y, e m t, m h, m c$

Lines \#9 to \#13 will be limited to LMIC countries, using a search filter developed by the Norwegian satellite of the Cochrane Effective Practice and Organisation of Care Group (Appendix 2).

\section{Other database searches}

We conducted complementary searches on the following bibliographic databases in February 2015/2016 and September 2017, using relevant subject headings (controlled vocabularies) and search syntax, appropriate to each resource.

a. OVID PsycINFO (all years to $1 / 9 / 17$ ).

b. ProQuest PILOTS database (Published International Literature on Traumatic Stress) (all years to $3 / 2 / 16$ ).

c. Cochrane Central Register of Controlled Trials (CENTRAL) (all years to 2017 , Issue 8).

\section{d. OVID MEDLINE (1946 to $1 / 9 / 17$ ).}

e. OVID Embase (1974 to 1/9/17).

f. International Trial Registries (ClinicalTrials.gov and the WHO ICTRP) (1/9/17).

We applied no restrictions on date, language, or publication status to the searches, however only studies identified from search results to 3 February 2016 have been fully incorporated into the qualitative and quantitative analysis. Studies identified in the 2017 update search have been placed in awaiting classification and will be incorporated into the next version of the revew as appropriate
In the update search (1 September 2017), we made a series of amendments and back-dated where necessary. We added a list of demonyms to the LMIC search strategy (PsycINFO, CENTRAL, MEDLINE, and Embase) to denote the natives or inhabitants of a particular country, and appended terms for warfare to the searches. We also added keywords to identify resource-poor settings (developing nations) (PsycINFO only). At this time we did not repeat the search of the PILOTS database or the Cochrane Common Mental Disorders Controlled Trials Register (CCMDCTR), because the former search did not yield any unique studies in previous searches (to February 2016), and the latter had fallen out of date in the summer of 2016, with the move of the Editorial Group from Bristol to York.

We have reported the search strategies in Appendix 3.

4. We searched international trial registries via the WHO trials portal (ICTRP) and ClinicalTrials.gov to identify unpublished and ongoing studies.

\section{Searching other resources}

\section{Grey literature}

We searched sources of grey literature, including dissertations and theses, humanitarian reports, evaluations published on websites, and clinical guidelines and reports from regulatory agencies (when appropriate). In addition, we searched key agencies and initiatives in this field for relevant reports.

\section{Handsearching}

We handsearched relevant conference proceedings and academic literature (titles not already indexed in Embase or PsycINFO, or already handsearched within the Cochrane Collaboration).

\section{Reference lists}

We checked the reference lists of all included studies and relevant systematic reviews (both Cochrane and non-Cochrane) to identify additional studies missed by the original electronic searches (e.g. unpublished or in-press citations). We also conducted a cited reference search on the Web of Science.

\section{Correspondence}

We contacted trialists and subject experts for information on unpublished and ongoing studies or to request additional trial data.

\section{Data collection and analysis}

\section{Selection of studies}

Two review authors (MP, DP) independently screened titles and abstracts against the inclusion criteria listed above for all studies identified by the search strategy for possible inclusion in the review. We added to a preliminary list all studies rated as possible candidates by either of the two review authors, and we retrieved their full texts. Moreover, we identified and recorded reasons for exclusion of ineligible studies.

We resolved any disagreements through discussion or, if required, by consultation with a third review author (CB). We identified and excluded duplicate records, and we collated multiple reports that related to the same study, so that each study rather than each report is the unit of interest in the review. We recorded the selection

Psychological therapies for the treatment of mental disorders in low- and middle-income countries affected by humanitarian crises 
process in sufficient detail to complete a PRISMA flow diagram and Characteristics of excluded studies table.

\section{Data extraction and management}

We used a data collection form that had been piloted on at least one study in the review to extract study characteristics and outcome data. Two review authors (MP, CG) independently extracted study characteristics and outcome data from included studies. We discussed any disagreements with an additional review author (CB), and when necessary, we contacted study authors to collect further information.

We extracted the following study characteristics.

1. Methods: phase of humanitarian crisis (ongoing, post-conflict, etc.), type of humanitarian crisis, duration of psychological treatment, number of study centres and locations, study setting and dates of study, inclusion criteria, and exclusion criteria.

2. Participants: $\mathrm{N}$, mean age, age range, gender, baseline scores on commonly used rating scales, and type of psychological disorder.

3. Psychological therapies and comparisons (type of therapy administered, who administered therapy, etc.).

4. Outcomes: primary and secondary outcomes specified and collected, and time points reported.

5. Notes: funding for trial and notable conflicts of interest of trial authors.

We extracted data for the following planned comparisons.

1. Psychological treatments versus control for adults.

2. Psychological treatments versus control for children/ adolescents.

We analysed each comparison listed above separately for children and adolescents (younger than 18 years) and for adults (18 years or older) on the different outcomes. Moreover, we looked at whether the type of psychological therapy has an impact on the overall treatment effect by performing a subgroup analysis within each of the main comparisons for each disorder type.

We noted in the Characteristics of included studies table if outcome data were not reported in a usable way. We resolved disagreements by reaching consensus or by involving a third person (CB). Two review authors (CG, DP) working independently transferred data into the Review Manager 5.3 file. We double-checked that data had been entered correctly by comparing data presented in the systematic review against the study reports. A third review author (MP) spot-checked study characteristics and outcomes that had been extracted.

\section{Assessment of risk of bias in included studies}

Two review authors (MP, CG) independently assessed risk of bias for each study using the criteria outlined in the Cochrane Handbook for Systematic Reviews of Interventions (Higgins 2011). We resolved any disagreements by discussion or by involving another review author (CB). We assessed risk of bias according to the following domains.

1. Random sequence generation.

2. Allocation concealment.

3. Blinding of participants and personnel.
4. Blinding of outcome assessment.

5. Incomplete outcome data.

6. Selective outcome reporting.

7. Other bias.

We also included a set of cluster-randomised trials, which we evaluated according to Section 16.3.2 of the Cochrane Handbook for Systematic Reviews of Interventions (Higgins 2011). In particular, we considered:

1. recruitment bias;

2. baseline imbalance;

3. loss of clusters;

4. incorrect analysis; and

5. comparability with individually randomised trials.

In particular for each cluster-RCT, we verified, when possible, whether:

1. all clusters were randomised at the same time;

2. samples were stratified on variables likely to influence outcomes;

3. clusters were pair-matched;

4. baseline comparability between interventions and control groups was evident.

Moreover, we included the following additional items in the 'Risk of bias' assessment (according to the review carried out by Patel 2014): therapist qualifications; treatment fidelity; therapist allegiance.

We judged each potential source of bias as high, low, or unclear, and we provided a supporting quotation from the study report together with a justification for our judgement in the 'Risk of bias' table. We summarised 'Risk of bias' judgements across different studies for each of the domains listed. When information on risk of bias was related to unpublished data or correspondence with a trialist, we noted this in the 'Risk of bias' table.

\section{Measures of treatment effect}

We performed all comparisons between psychological therapy and no treatment, treatment as usual, attention placebo, and wait list.

\section{Dichotomous data}

For dichotomous data, we calculated risk ratios (RRs) with a 95\% confidence interval $(\mathrm{Cl})$. For statistically significant results, we calculated the number needed to treat for an additional beneficial outcome and the number needed to treat for an additional harmful outcome.

\section{Continuous data}

We analysed continuous data as mean differences (MDs) when studies reported outcomes using the same rating scale. We used standardised mean differences (SMDs) when studies assessing the same outcome measured it by using different rating scales (Higgins 2011). We entered data presented as a scale with a consistent direction of effect. We narratively described skewed data reported as medians and interquartile ranges.

Psychological therapies for the treatment of mental disorders in low- and middle-income countries affected by humanitarian crises 


\section{Unit of analysis issues}

\section{Cluster-RCTs}

We included cluster-RCTs when healthcare facilities, schools, or classes within schools rather than single individuals were the unit of allocation (Barbui 2011). Given that variation in response to psychological treatment between clusters may be influenced by cluster membership, we included, when possible, data adjusted with an intracluster correlation coefficient (ICC). When the ICC was not reported or was not available from trialists, we assumed that it was 0.1 (Higgins 2011; Ukoumunne 1999).

\section{Cross-over trials}

We included trials employing a cross-over design. With cross-over trials, there is the possibility of 'carry-over' treatment effect from one period to the next. This means that the observed difference between treatments depends upon the order in which treatments were received; hence the estimated overall treatment effect will be affected (Higgins 2011). Whilst acknowledging that this design is rarely used in psychological treatment studies, we used data from the first randomised stage only.

\section{Studies with multiple treatment groups}

We included studies with two or more formats of the same therapy in meta-analysis by combining group arms into a single group, as recommended in Section 16.5 of the Cochrane Handbook for Systematic Reviews of Interventions (Higgins 2011). Conversely, we included studies with two or more different therapies in meta-analysis without combining group arms of the study into a single group but while considering each intervention and each control group separately. To avoid inclusion of the same group of participants in the same meta-analysis, we followed Section 16.5.4 of the Cochrane Handbook for Systematic Reviews of Interventions (Higgins 2011).

\section{Dealing with missing data}

We contacted investigators or study sponsors to verify key study characteristics and to obtain missing numerical outcome data when possible. We documented all correspondence with trialists and reported in the full review which trialists responded. For not adjusted and could not be identified from the trial report. When ICCs were not available from trial reports nor from trialists directly, we assumed the ICC to be 0.1 (Higgins 2011; Ukoumunne 1999).

For continuous data: We applied an intention-to-treat analysis, whereby all participants with at least one post-baseline measurement are represented by their last observations carried forward. When only the standard error, t-statistics, or $P$ values were reported, we calculated standard deviations according to Altman (Altman 1996).

For dichotomous data: We applied an intention-to-treat analysis, whereby we considered all dropouts not included in the analyses as negative outcomes (i.e. it was assumed they would have experienced the negative outcome by the end of the trial).

\section{Assessment of heterogeneity}

We quantified heterogeneity using the $\mathrm{I}^{2}$ statistic, which calculates the percentage of variability due to heterogeneity rather than to chance. cluster-RCTs, we contacted study authors for an ICC when data were

According to the Cochrane Handbook for Systematic Reviews of Interventions (Higgins 2011), we used the following thresholds for interpretation of 12

1. $0 \%$ to $40 \%$ : might not be important.

2. $30 \%$ to $60 \%$ : may represent moderate heterogeneity.

3. $50 \%$ to $90 \%$ : may represent substantial heterogeneity.

4. $75 \%$ to $100 \%$ : may represent considerable heterogeneity.

The importance of the observed 12 depends on the magnitude and direction of intervention effects and the strength of evidence for heterogeneity (Higgins 2011; Purgato 2012).

\section{Assessment of reporting biases}

To the greatest degree possible, we minimised the impact of reporting biases by undertaking comprehensive searches of multiple sources and increasing efforts to identify unpublished material including protocols of randomised trials without language restrictions.

We used visual inspection of funnel plots to identify asymmetry in any of the comparisons between psychological treatments and comparators. We are aware that funnel plots are of limited power to detect small-study effects. We did not use funnel plots for outcomes when we included 10 or fewer studies, or when all studies were of similar size. In other cases, when funnel plots were possible, we asked for statistical advice regarding their interpretation.

\section{Data synthesis}

We used a random-effects model meta-analysis, given the potential heterogeneity of psychological therapies. A random-effects model has the highest generalisability in empirical examination of summary effect measures for meta-analyses (Furukawa 2002), and this model is based on the assumption that different studies are estimating different, yet related, intervention effects (this deserves particular attention as we included different types of psychological therapies) (der Simonian 1986). We examined the robustness of this summary measure by checking the results under a fixed-effect model. We reported material differences between the models.

For dichotomous data, we calculated risk ratios with $95 \% \mathrm{Cl}$. We analysed continuous scores from different rating scales using SMDs (with 95\% Cl).

\section{Subgroup analysis and investigation of heterogeneity}

We carried out the following subgroup analyses for primary outcomes.

1. Types of psychological therapies.

2. Diagnosis: PTSD; major depressive, anxiety, somatoform, and related disorders. Participants with different diagnoses might respond differently to trial interventions. When possible, we conducted separate analyses for participants with PTSD, major depressive, and anxiety disorders.

3. Presence of comorbidities, as symptoms related to other clinical conditions might influence the response to psychological therapies.

4. Type of traumatic event: We considered the following categories: bereavement, displacement, sexual and other forms of gender-based violence, torture, witness of violence/atrocities, other traumatic events (IASC 2007). Different types of traumatic

Psychological therapies for the treatment of mental disorders in low- and middle-income countries affected by humanitarian crises 14 
events might influence the effectiveness of therapies, as authors have identified different strength of association with negative psychological consequences (US Department of Health and Human Services 2014).

5. Type of humanitarian crisis: We considered the following categories: protracted emergencies such as wars and armed conflicts; communal violence; food shortages; disasters triggered by natural hazards such as geophysical (earthquakes, tsunamis, volcanic eruptions), hydrological (floods, avalanches), climatological (droughts), meteorological (storms, cyclones), or biological hazards (epidemics, plagues); disasters triggered by technological and industrial hazards (e.g. nuclear accidents; oil spills) (reliefweb.int/). We hypothesised that the type of humanitarian crisis may differentially impact mental health outcomes as people's needs, vulnerabilities, and capacities (including their capacity to respond to psychological therapies) may vary according to the different humanitarian contexts in which they live (The Sphere Project 2011).

6. Phase of humanitarian crisis: We hypothesised that the phase of the humanitarian crisis may impact outcomes, as it influences individual vulnerability, capacity to use resources, and psychological reactions (Colliard 2014).

7. Type of interventionist (professional vs paraprofessional): We expected the types of interventionists delivering treatment to have an impact on outcomes. We noted debate in the literature regarding the role of professionals and paraprofessionals in delivering psychological interventions (Montgomery 2010). Given the cost of healthcare interventions and the minimal resources available in humanitarian settings in LMIC, it is important for researchers to investigate potential differences.

8. Type of control: no treatment, treatment as usual, wait list, attention placebo, and psychological placebo.

\section{Sensitivity analysis}

We carried out the following sensitivity analyses.

1. Excluding trials with high risk of bias in the following domains: incomplete outcome data and selective reporting. These biases might impact trial results and interpretation in terms of an intervention's effectiveness estimate, in accordance with availability and completeness of outcome data from study participants,

2. Excluding trials with follow-up performed immediately at the end of the psychological treatment. We kept only studies with at least one follow-up after the first evaluation to assess the longterm outcomes of psychological therapies.

\section{'Summary of findings' tables}

We employed the GRADE approach to interpret findings (Langendam 2013), and use of GRADEpro allowed us to import data from Review Manager 5.3 to create 'Summary of findings' tables. These tables provide outcome-specific information concerning the overall quality of evidence from studies included in the comparison, the magnitude of effect of the psychological therapies examined, and the sum of available data on the outcomes considered. We adhered to standard methods for preparation and presentation of results as outlined in the Cochrane Handbook for Systematic Reviews of Interventions (Higgins 2011).

We included the following outcomes in the 'Summary of findings' tables.

1. PTSD.

2. Major depressive disorder.

3. Anxiety disorders.

4. Somatoform and related disorders (including MUPS).

5. Acceptability (dropout rate).

\section{RE S U LTS}

\section{Description of studies}

\section{Results of the search}

From 3655 records (identified from searches to February 2016), we identified 68 studies (33 RCTs for inclusion, 31 excluded studies, 2 awaiting classification and 2 ongoing) (see Figure 1 for the search flow diagram). 
Figure 1. Study flow diagram.

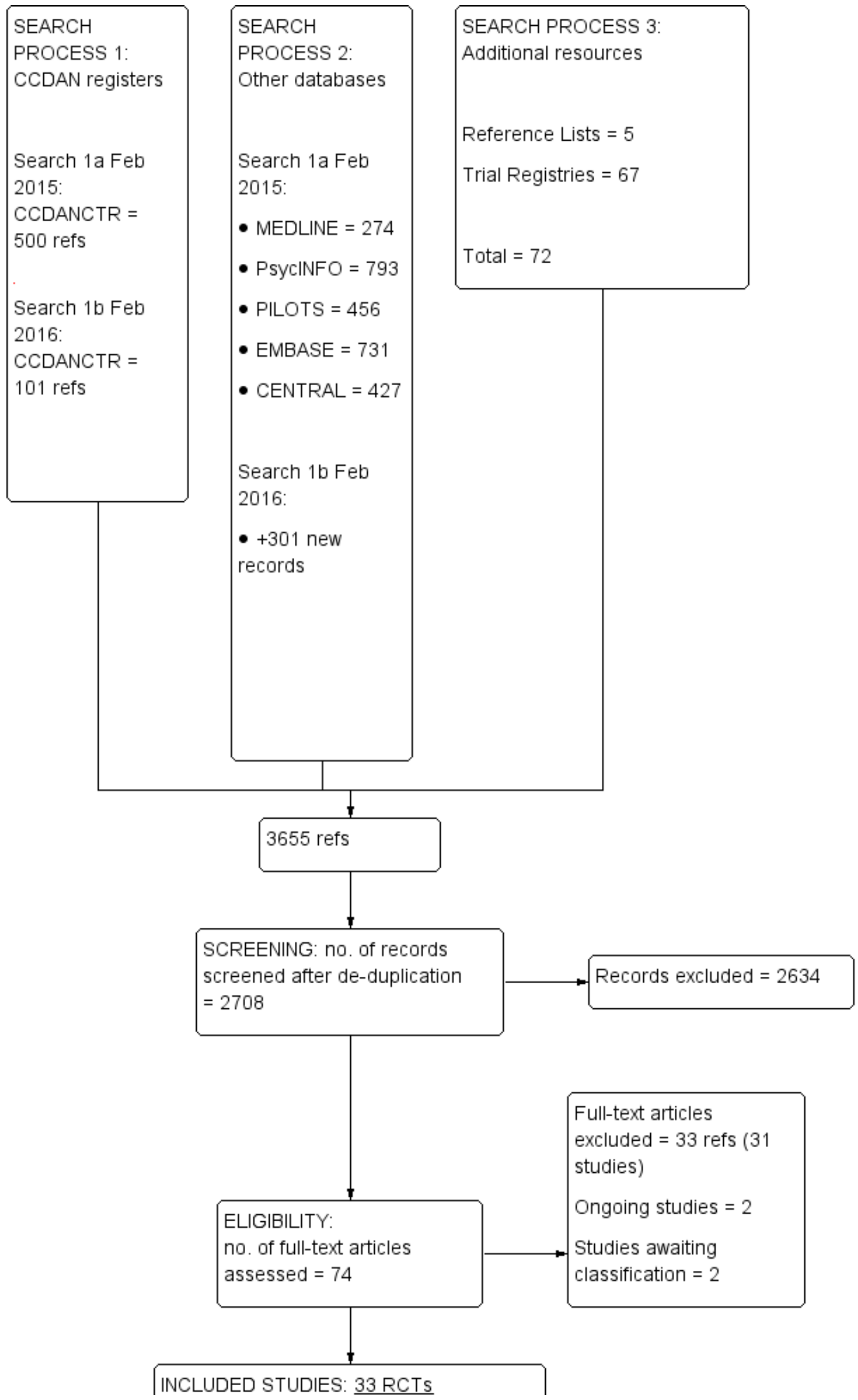

Psychological therapies for the treatment of mental disorders in low- and middle-income countries affected by humanitarian crises 
Figure 1. (Continued)

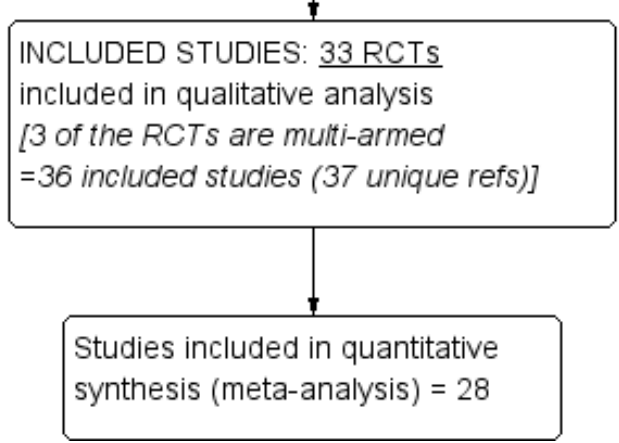

The update search in September 2017 retrieved 574 new records and after screening these we identified a further 8 studies which we've added to awating classification and 1 additional ongoing study. Only results of searches to 3 February 2016 have been formally incorporated into the analysis at this stage (and are reported in the PRISMA flow diagram).

We included 33 RCTs (36 studies, as 3 of the RCTs were multiarmed trials) with a total of 3523 participants (see Characteristics of included studies) and excluded 31 studies (see Characteristics of excluded studies). All included studies were RCTs. We identified no cluster-randomised trials. We have not yet assessed 10 studies - one because it requires translation, another because the fulltext was not available to determine eligibility for this review and the remaining studies are from the 2017 update search, which will be assessed for eligibility in the next version of the revew (Characteristics of studies awaiting classification). We also identified a total of three ongoing studies (Characteristics of ongoing studies).

\section{Included studies}

See Characteristics of included studies.

\section{Settings}

Four of the included studies were done in Turkey (Acarturk 2015; Acaturk 2016; Basoglu 2005; Basoglu 2007); two in Iran (Ahmadizadeh 2013; Azad Marzabadi 2014); two in Kurdistan (Bass 2016; Bolton 2014a); two in Pakistan (Rahman 2016a; Rahman 2016b); one in Kosovo (Wang 2016); three in Thailand (Bolton 2014b; Bryant 2011; Pityaratstian 2015); one in Sri Lanka (Puvimanasinghe 2016); five in China (Chen 2014; Jiang 2014; Wang 2013a; Zang 2013; Zang 2014), and two in Iraq (Knaevelsrud 2015; Weiss 2015a). The remaining 11 studies were undertaken in Africa: three in Uganda (Bolton 2007; Ertl 2011; Neuner 2008a); three in Rwanda (Connolly 2011; Connolly 2013; Jacob 2014); three in the Democratic Republic of the Congo (Hermenau 2013; McMullen 2013; O' Callaghan 2013); one in Mozambique (Igreja 2004); and one in Egypt (Meffert 2014).

We categorised humanitarian crises into five main categories: war or armed conflict (Acarturk 2015; Acaturk 2016; Ahmadizadeh 2013; Azad Marzabadi 2014; Bass 2016; Ertl 2011; Hermenau 2013; Igreja 2004; Jacob 2014; Knaevelsrud 2015; McMullen 2013; Meffert 2014; Neuner 2008a; O' Callaghan 2013; Rahman 2016a; Rahman 2016b; Weiss 2015a); disasters triggered by natural events (Basoglu 2005; Basoglu 2007; Chen 2014; Pityaratstian 2015; Zang 2013; Zang 2014); communal violence (Bolton 2014a; Bolton 2014b; Connolly 2011; Connolly 2013); food shortages (Bryant 2011); and other types (Puvimanasinghe 2016; Wang 2013a). The context of treatment varied across studies: 13 of the included studies delivered interventions in healthcare clinics or facilities (Ahmadizadeh 2013; Azad Marzabadi 2014; Bass 2016; Bolton 2014a; Bryant 2011; Jiang 2014; Meffert 2014; Rahman 2016a; Rahman 2016b; Wang 2016; Weiss 2015a; Zang 2013; Zang 2014); five in community settings (Basoglu 2005; Bolton 2014b; Ertl 2011; Hermenau 2013; Puvimanasinghe 2016); four in refugee camps (Acarturk 2015; Acaturk 2016; Bolton 2007; Neuner 2008a); three at school (Chen 2014; McMullen 2013; Pityaratstian 2015); two at home (Igreja 2004; Jacob 2014); and the remaining six in other settings (Basoglu 2007; Connolly 2011; Connolly 2013; Knaevelsrud 2015; O' Callaghan 2013; Wang 2013a). Most included studies delivered psychological treatments after the acute crisis period had ended.

\section{Sample sizes}

Included studies consisted of 3523 participants, and the number of participants in each trial ranged from 22 in Meffert 2014 to 347 in Bolton 2014b.

\section{Participants}

Included participants in four studies were children and adolescents between 5 and 18 years of age (Chen 2014; McMullen 2013; O' Callaghan 2013; Pityaratstian 2015). Three studies included mixed populations (two studies included participants between 12 and 25 years of age (Ertl 2011; mean age 18.66 years, standard deviation (SD) 3.77 in the CBT group; mean age 18.06 years, SD 3.55 years in the control group; Hermenau 2013; mean age of the whole sample 19 years, SD 2.02), and one study included participants between 16 and 65 years of age (Basoglu 2005; mean age of the whole sample 36.3 years, SD 11.5 years in the control group). Remaining studies included adult populations (18 years or older). In studies with mixed populations, we considered mean age and SD reported in the papers to categorise populations into children or adults for meta-analysis.

In 24 studies, most (more than 50\%) participants were female. In the remaining nine studies, most (more than $50 \%$ ) participants were male.

Regarding types of traumatic events, participants were exposed to bereavement in four studies (Basoglu 2005; Basoglu 2007; Bryant 2011; Chen 2014); displacement in seven studies (Acarturk 2015; Acaturk 2016; Ertl 2011; Meffert 2014; Neuner 2008a; Zang 2013; Zang 2014); sexual and other forms of gender-based violence in

Psychological therapies for the treatment of mental disorders in low- and middle-income countries affected by humanitarian crises 
one study (O' Callaghan 2013); torture and witness to violence or atrocities in 11 studies (Ahmadizadeh 2013; Azad Marzabadi 2014; Bass 2016; Bolton 2014a; Connolly 2011; Connolly 2013; Hermenau 2013; Jacob 2014; McMullen 2013; Wang 2013a; Weiss 2015a); and compound stressors or other types of stressors in the remaining studies.

Regarding types of mental disorders, 25 studies included participants with PTSD, three with PTSD and/or depression, two with anxiety and/or depression and/or emotional distress, and three with depression.

\section{Interventions and comparators}

Included trials compared a psychological therapy versus a control intervention (wait list in most studies; no treatment; treatment as usual). The psychological therapy was categorised as belonging to a type of group CBT in 27 studies, that is, CBT in 16 studies (Ahmadizadeh 2013; Azad Marzabadi 2014; Basoglu 2005; Basoglu 2007; Bolton 2014a; Bryant 2011; Chen 2014; McMullen 2013; O Callaghan 2013; Pityaratstian 2015; Rahman 2016a; Rahman 2016b; Wang 2013a; Wang 2013b; Wang 2016; Weiss 2015a), NET in eight studies (Ertl 2011; Hermenau 2013; Igreja 2004; Jacob 2014; Neuner 2008a; Puvimanasinghe 2016; Zang 2013; Zang 2014), BA in one study (Bolton 2014a), and CETA in two studies (Bolton 2014b; Weiss 2015b).

IPT was studied in three studies (Bolton 2007; Jiang 2014; Meffert 2014), TFT in two studies (Connolly 2011; Connolly 2013), EMDR in two comparisons (Acarturk 2015; Acaturk 2016), and trauma/ supportive counselling in two comparisons (Bass 2016; Neuner 2008b). Although psychological therapies were grouped into these categories, several psychotherapeutic elements were common to a range of therapies. In particular, a focus on psychoeducation and/or coping skills was common to most interventions. Most included trials delivered psychological therapy at the individual level (24 studies); six compared group delivery or mixed delivery (individual and group) of psychological therapies versus control; and the remaining three studies were unclear about the type of delivery provided.

Interventionists were professionals (i.e. trained psychologists or psychiatrists) in 14 comparisons, and paraprofessionals (i.e. trained lay counsellors; community health workers) in the remaining trials.

\section{Outcomes}

At the end of the reviewing process, 28 RCTs provided data for meta-analysis. For primary outcomes, 19 RCTs provided continuous data on PTSD at endpoint, 14 RCTs provided data on depression at endpoint, and five RCTs provided data on anxiety at endpoint. For the primary outcome of acceptability, 26 RCTs provided data on total dropouts for any cause.

Overall, 1402 participants were included in the efficacy analysis for the continuous outcome PTSD at endpoint (1272 adults and 130 children); 1254 were included in the efficacy analysis for the continuous outcome depression at endpoint (all adults: 651 participants randomised to psychological therapy and 603 randomised to control); and 694 participants were included in the continuous outcome anxiety at endpoint (all adults: 370 participants randomised to psychological therapy and 324 randomised to control). A total of 3098 participants were included in the primary outcome of acceptability (2960 adults and 138 children).

For secondary outcomes, 686 participants were included in the efficacy analysis for the continuous outcome function impairment at endpoint (359 participants randomised to psychological therapy and 327 randomised to control); 325 participants were included in the efficacy analysis for the continuous outcome quality of life at endpoint (187 participants randomised to psychological therapy and 138 randomised to control); and 440 participants were included in the efficacy analysis for the dichotomous outcome diagnosis of PTSD at endpoint (402 adults and 36 children).

\section{Excluded studies}

See Characteristics of excluded studies.

Thirty-one studies initially selected did not meet our inclusion criteria and were excluded because of wrong setting (no humanitarian crisis in LMICs) for eight studies; wrong design (no RCT or incorrect randomisation procedure) for nine studies; and wrong comparison (no psychological therapy compared with control) for three studies. Moreover, we excluded 11 RCTs assessing the effectiveness of preventive psychosocial interventions. We will include these studies in the Cochrane review specifically focused on preventive psychosocial interventions in humanitarian settings in LMICs (Purgato 2016a).

\section{Studies awaiting classification}

We classified 10 records as awaiting classification, as we did not have the elements needed to evaluate their eligibility.

See Characteristics of studies awaiting classification.

\section{Ongoing studies}

We classified three studies as ongoing: One is just finished, and results are planned to be published in 2018 (ISRCTN65771265), one is estimated to be completed in 2018 (NCT03012451), and one is at the beginning of the recruitment phase (NCT031090028). See Characteristics of ongoing studies.

\section{Risk of bias in included studies}

See Characteristics of included studies. For graphical representations of overall risk of bias in included studies, see Figure 2 and Figure 3. 
Figure 2. Risk of bias graph: review authors' judgements about each risk of bias item presented as percentages across all included studies.

Random sequence generation (selection bias)

Allocation concealment (selection bias)

Blinding of participants and personnel (performance bias)

Blinding of outcome assessment (detection bias)

Incomplete outcome data (attrition bias)

Selective reporting (reporting bias)

Therapist qualification

Treatment fidelity

Therapistlinvestigator allegiance

Other bias
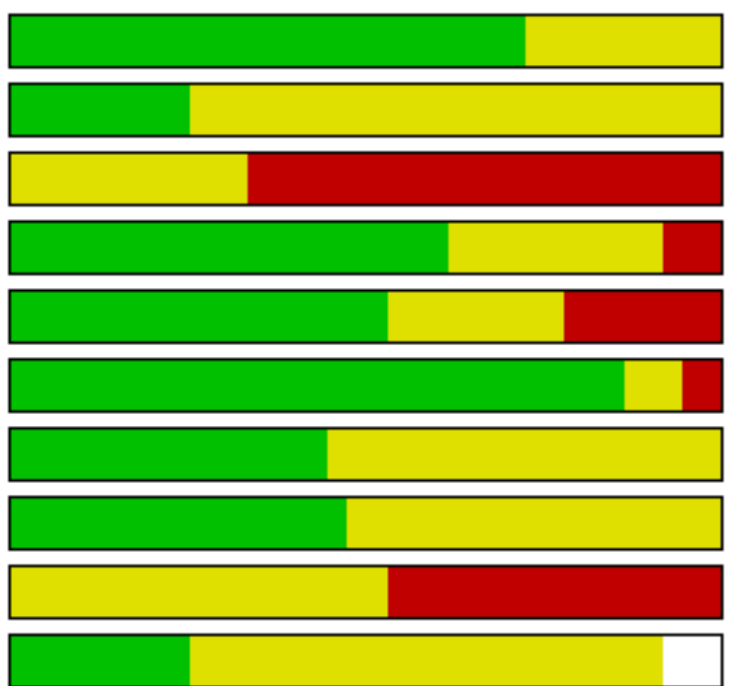

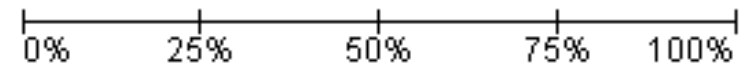


Figure 3. Risk of bias summary: review authors' judgements about each risk of bias item for each included study.

\begin{tabular}{|c|c|c|c|c|c|c|c|c|c|c|}
\hline & 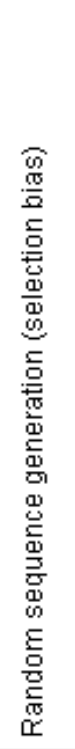 & 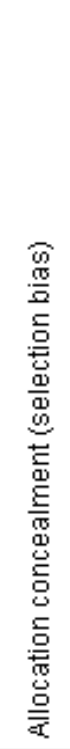 & 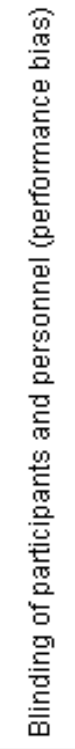 & 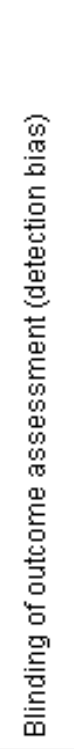 & 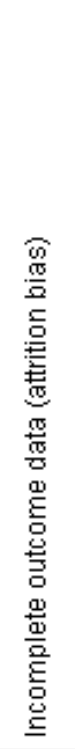 & 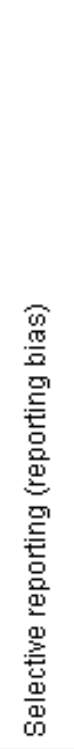 & 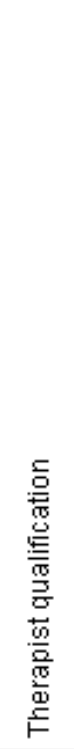 & 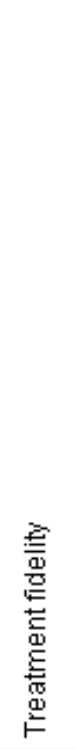 & 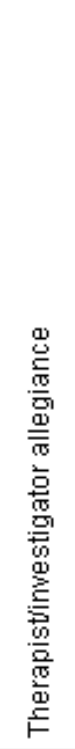 & 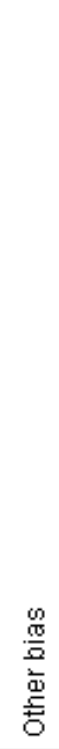 \\
\hline Acarturk 2015 & + & $?$ & $\odot$ & + & + & $\odot$ & + & $\odot$ & $\odot$ & $?$ \\
\hline Acaturk 2016 & $\odot$ & $?$ & $\odot$ & + & 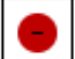 & $\odot$ & + & $\odot$ & - & + \\
\hline Ahmadizadeh 2013 & $?$ & $?$ & $?$ & $?$ & $?$ & $\odot$ & + & $?$ & $?$ & $?$ \\
\hline Azad Marzabadi 2014 & + & $?$ & $?$ & $?$ & $\odot$ & + & $?$ & $?$ & $?$ & $?$ \\
\hline Basoglu 2005 & $?$ & + & $\odot$ & $?$ & $?$ & + & + & $?$ & $?$ & $?$ \\
\hline Basoglu 2007 & $?$ & $?$ & $\odot$ & 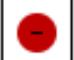 & + & + & $\odot$ & $?$ & $?$ & $?$ \\
\hline Bass 2016 & + & $?$ & $\Theta$ & + & + & $\odot$ & $?$ & $\odot$ & $\odot$ & + \\
\hline Bolton 2007 & + & $?$ & $\odot$ & + & + & + & $?$ & $?$ & $\odot$ & $?$ \\
\hline Bolton 2014a & + & $\odot$ & $?$ & $?$ & $?$ & 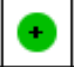 & $?$ & $?$ & $?$ & $\odot$ \\
\hline Bolton 2014b & + & $\odot$ & $\odot$ & + & $\theta$ & + & $?$ & $?$ & $\odot$ & $?$ \\
\hline Bryant 2011 & $\odot$ & $?$ & $?$ & $\odot$ & + & + & + & $\odot$ & $?$ & $?$ \\
\hline Chen 2014 & $?$ & $?$ & $\odot$ & $?$ & $\odot$ & + & $?$ & $?$ & $?$ & $?$ \\
\hline Connolly 2011 & + & $?$ & $?$ & $?$ & $?$ & + & $?$ & $\odot$ & $\odot$ & $?$ \\
\hline Connolly 2013 & + & + & $?$ & $?$ & $?$ & $?$ & $?$ & + & $\Theta$ & $?$ \\
\hline Ertl 2011 & $?$ & $?$ & $\Theta$ & + & $\odot$ & + & $?$ & $\odot$ & $\theta$ & $?$ \\
\hline Hermenau 2013 & + & $?$ & $?$ & $?$ & $?$ & 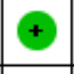 & + & $\odot$ & $\odot$ & $?$ \\
\hline Igreja 2004 & $?$ & $?$ & $?$ & $?$ & $?$ & $\odot$ & + & $?$ & $?$ & $?$ \\
\hline Jacob 2014 & $\odot$ & $?$ & $?$ & + & + & + & + & + & - & $?$ \\
\hline Jiang 2014 & + & $?$ & $?$ & 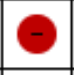 & $?$ & $\odot$ & $\odot$ & $\bullet$ & $?$ & $?$ \\
\hline Knaevelsrud 2015 & + & $?$ & $\odot$ & $?$ & $\odot$ & $\odot$ & $\odot$ & $?$ & $?$ & \\
\hline
\end{tabular}


Figure 3. (Continued)

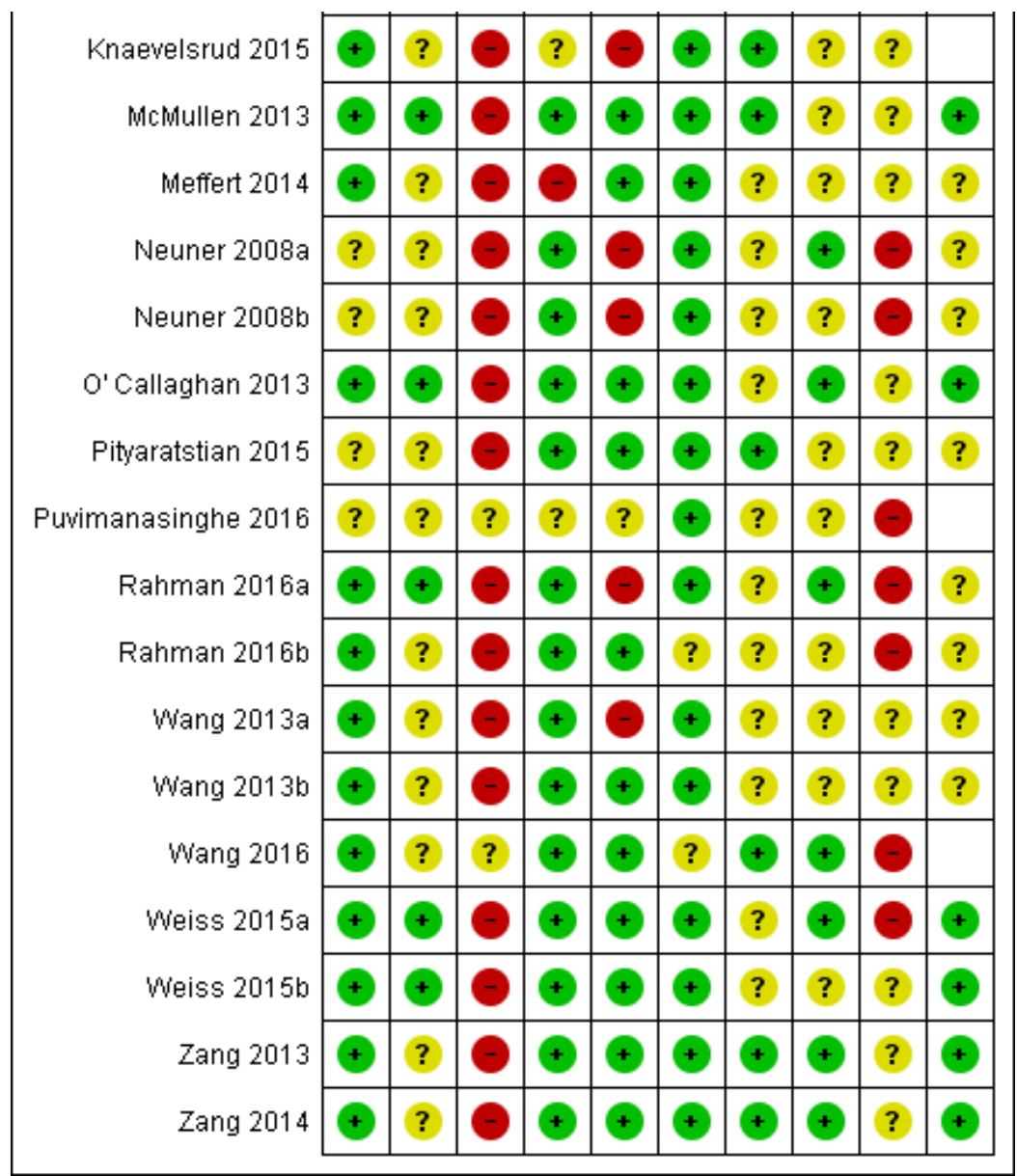

\section{Allocation}

Researchers described generation of a random sequence that we considered to lead to low risk of bias in 26 comparisons (Acarturk 2015; Acaturk 2016; Azad Marzabadi 2014; Bass 2016; Bolton 2007; Bolton 2014a; Bolton 2014b; Bryant 2011; Connolly 2011; Connolly 2013; Hermenau 2013; Jacob 2014; Jiang 2014; Knaevelsrud 2015; McMullen 2013; Meffert 2014; O' Callaghan 2013; Rahman 2016a; Rahman 2016b; Wang 2013a; Wang 2013b; Wang 2016; Weiss 2015a; Weiss 2015b; Zang 2013; Zang 2014) and to unclear risk of bias in the remaining studies. Regarding allocation concealment, we considered nine of the included trials to be at low risk (Basoglu 2005; Bolton 2014a; Bolton 2014b; Connolly 2013; McMullen 2013; O' Callaghan 2013; Rahman 2016a; Weiss 2015a; Weiss 2015b). The 24 remaining RCTs did not describe allocation concealment; we therefore rated them as having unclear risk.

\section{Blinding}

\section{Performance bias}

Participants (both personnel and study participants) would have been aware of whether they had been assigned to an intervention group or a control group in 24 trials (Acarturk 2015; Acaturk 2016; Basoglu 2005; Basoglu 2007; Bass 2016; Bolton 2007; Bolton 2014b; Chen 2014; Ertl 2011; Knaevelsrud 2015; McMullen 2013; Meffert 2014; Neuner 2008a; Neuner 2008b; O' Callaghan 2013; Pityaratstian 2015; Rahman 2016a; Rahman 2016b; Wang 2013a;
Wang 2013b; Weiss 2015a; Weiss 2015b; Zang 2013; Zang 2014); therefore, we rated these studies as having high risk of performance bias. We rated the remaining trials as having unclear risk of performance bias.

\section{Detection bias}

We rated trials as having low risk of bias when researchers described blinded assessment of outcomes (Acarturk 2015; Acaturk 2016; Bass 2016; Bolton 2007; Bolton 2014b; Bryant 2011; Ertl 2011; Jacob 2014; McMullen 2013; Neuner 2008a; Neuner 2008b; O' Callaghan 2013; Pityaratstian 2015; Rahman 2016a; Rahman 2016b; Wang 2013a; Wang 2013b; Wang 2016; Weiss 2015a; Weiss 2015b; Zang 2013; Zang 2014). We rated three trials as having high risk of bias, as the assessors were likely to be aware of participant allocation (Basoglu 2007; Jiang 2014; Meffert 2014); we rated risk in the remaining trials as unclear (Ahmadizadeh 2013; Azad Marzabadi 2014; Basoglu 2005; Bolton 2014a; Chen 2014; Connolly 2011; Connolly 2013; Hermenau 2013; Igreja 2004; Knaevelsrud 2015; Puvimanasinghe 2016).

\section{Incomplete outcome data}

Risk of attrition bias was low in 19 studies, as researchers clearly reported low dropout rates (Acarturk 2015; Azad Marzabadi 2014; Basoglu 2007; Bass 2016; Bolton 2007; Bryant 2011; Ertl 2011; Jacob 2014; McMullen 2013; Meffert 2014; O' Callaghan 2013; Pityaratstian 2015; Rahman 2016b; Wang 2013b; Wang 2016; Weiss 2015a; Weiss 
2015b; Zang 2013; Zang 2014). We considered eight trials to have high risk of attrition bias because researchers reported incomplete outcome data. We rated the remaining trials as having unclear risk.

\section{Selective reporting}

Even though the study protocol was not available for many of the included studies, most included studies showed consistency between Results and Methods sections (31 comparisons).

\section{Other potential sources of bias}

We rated risk of other bias as low in nine trials and as unclear in the remaining trials.

We visually inspected funnel plots to identify asymmetry in any of the comparisons between psychological treatments and comparators when we identified 10 or more studies. We did not identify any asymmetry in the distribution of studies.

We included in our risk of bias evaluation the following additional items.

1. Therapist qualification: We considered 16 trials as having low risk of bias with regard to the qualifications of therapists, as trained professionals in mental health (mainly psychologists or psychiatrists) delivered psychological therapy (Acarturk 2015; Acaturk 2016; Ahmadizadeh 2013; Basoglu 2005; Basoglu 2007; Bryant 2011; Hermenau 2013; Igreja 2004; Jacob 2014; Jiang 2014; Knaevelsrud 2015; McMullen 2013; Pityaratstian 2015; Wang 2016; Zang 2013; Zang 2014). For the remaining trials, we evaluated the risk as unclear.

2. Treatment fidelity: Sixteen trials described the system used to monitor treatment fidelity, and we rated their risk of bias as low (Acarturk 2015; Acaturk 2016; Bass 2016; Bryant 2011; Connolly 2011; Connolly 2013; Ertl 2011; Hermenau 2013; Jiang 2014; Neuner 2008a; O' Callaghan 2013; Rahman 2016a; Wang 2016; Weiss 2015a; Zang 2013; Zang 2014). We evaluated risk as unclear for the remaining trials because researchers provided no details about fidelity checks.

3. Therapist/investigator allegiance: We rated the risk of therapist or investigator allegiance as high in 17 trials and as unclear in the remaining trials.

\section{Effects of interventions}

See: Summary of findings for the main comparison Psychological therapy compared with control for treatment of adults with mental disorders in low- and middle-income countries affected by humanitarian crises; Summary of findings 2 Psychological therapy compared with control for treatment of children with mental disorders in low- and middle-income countries affected by humanitarian crises

All results of this systematic review must be interpreted with consideration of the characteristics and risk of bias profile of each included study (see Characteristics of included studies).

\section{Psychological therapy versus control}

\section{Primary outcomes}

\subsection{PTSD symptoms}

Adults: Scores for PTSD symptoms were significantly lower for psychological therapy conditions than for control conditions at study endpoint (standardised mean difference (SMD) -1.07, 95\% confidence interval $(\mathrm{Cl})-1.34$ to $-0.79 ; 1272$ participants; 16 RCTs; Analysis 1.1). We also collected information on PTSD symptoms at one to four months after completion of the intervention and at six months after the intervention or later. Data indicated that the psychological therapy effect was reduced at one to four months after the intervention (SMD $-0.49,95 \% \mathrm{Cl}-0.68$ to -0.31 ; 1660 participants; 18 RCTs; Analysis 1.2) and at six months after the intervention or later (SMD $-0.37,95 \% \mathrm{Cl}-0.61$ to $-0.14 ; 400$ participants; 5 RCTs; Analysis 1.3).

Children and adolescents: We found that PTSD symptoms were lesser for psychological therapy conditions (all CBT) than for control conditions at endpoint (SMD $-1.56,95 \% \mathrm{Cl}-3.13$ to 0.01 ; 130 participants; 3 RCTs; Analysis 2.1). We did not find a significant effect of psychological therapies over control interventions at one to four months after the intervention (Analysis 2.2). Data for six months after completion of the intervention or later were not available.

The most commonly reported rating scales for PTSD were the Clinician Administered PTSD Sscale (CAPS), Children's Revised Impact of Event Scale (CRIES), the Harvard Trauma Questionnaire (HTQ), the Posttraumatic Stress Disorder Checklist (PCL-5), the Impact of Event Scale - Revised (IES-R), the University of California at Los Angeles post-traumatic stress disorder reaction index (UCLAPTSD), and the Posttraumatic Diagnostic Scale (PDS).

\subsection{Anxiety symptoms}

Adults: In general, we observed significant differences between psychological therapies and controls for anxiety symptoms at endpoint (SMD $-0.74,95 \% \mathrm{Cl}-0.98$ to $-0.49 ; 694$ participants; five RCTs; Analysis 3.1) and at one to four months after the intervention (SMD $-0.53,95 \% \mathrm{Cl}-0.66$ to $-0.39 ; 969$ participants; seven RCTs; Analysis 3.2). We did not identify any significant differences between psychological therapies and controls at six months after the intervention or later (Analysis 3.3).

Children and adolescents: No study provided data on this outcome for children.

The most commonly reported rating scales for anxiety were the Hospital and Anxiety Depression Scale (HADS) and the Hopkins Symptoms Checklist for anxiety (HSCL-25).

\subsection{Depressive symptoms}

Adults: Scores for depressive symptoms were significantly lower for psychological therapy conditions than for control conditions at endpoint (SMD $-0.86,95 \% \mathrm{Cl}-1.06$ to $-0.67 ; 1254$ participants; 14 RCTs; Analysis 4.1). All types of psychological therapy were significantly effective for this outcome. Data on depressive symptoms at one to four months after therapy showed reduced effects of psychological therapies over controls (SMD $-0.42,95 \% \mathrm{Cl}$ -0.63 to $-0.21 ; 1386$ participants; 16 RCTs; Analysis 4.2). However, data at six months or later failed to show a significant difference between psychological therapies and controls (Analysis 4.3).

Children and adolescents: No study provided data on this outcome for children.

The most commonly reported rating scales for depression were the Beck Depression Inventory (BDI-II), HADS, and HSCL-25.

Psychological therapies for the treatment of mental disorders in low- and middle-income countries affected by humanitarian crises 
1.4 Somatoform symptoms and related disorders, including medically unexplained physical symptoms (MUPS)

No data were available for this outcome.

\subsection{Dropouts for any reason}

Adults: We found no difference in the proportions of participants lost to follow-up (RR 0.98, 95\% Cl 0.82 to 1.16; 2960 participants; 23 RCTs; Analysis 5.1).

Children and adolescents: We found no difference in the proportions of children and adolescents lost to follow-up (RR 1.87, 95\% Cl 0.47 to $7.47 ; 138$ participants; three RCTs; Analysis 6.1). Only data on CBT were available for this population.

\section{Secondary outcomes}

\subsection{Functional impairment}

Adults: We observed significant differences in favour of psychological therapies over controls for functional impairment at endpoint (SMD $-0.54,95 \% \mathrm{Cl}-0.79$ to -0.29 ; 686 participants; five RCTs; Analysis 7.1). At one to four months after the intervention, we observed a reduced but statistically significant treatment effect (SMD -0.35, 95\% Cl -0.54 to -0.15 ; 1061 participants; seven RCTs; Analysis 7.2). The effect on functional impairment was not maintained at six months after completion of the intervention or later (Analysis 7.3).

No data on this outcome were available for children.

The most commonly reported rating scales for functional impairment were Social Functioning Impairment (SFI) and the Sri Lanka Index of Psychosocial Status (SLIPSS-A).

\subsection{Quality of life}

Adults: Quality of life was significantly improved in the psychological therapy group compared with the control group at endpoint (SMD $-0.73,95 \% \mathrm{Cl}-1.22$ to -0.25 ; 325 participants; four studies; Analysis 8.1). No follow-up data were available for this outcome.

Children: No study provided data on this outcome for children and adolescents.

The most commonly reported rating scales for quality of life were Quality of LIfe and the Quality of Life Index.

\subsection{Formal or clinical diagnosis of PTSD}

Adults: We noted no significant differences between psychological therapies and control interventions for this outcome (Analysis 9.1).

Children and adolescents: We observed significant differences in favour of psychological therapy over control intervention for this outcome (RR $0.59,95 \% \mathrm{Cl} 0.38$ to $0.90 ; 36$ participants; one RCT; Analysis 10.1).

\subsection{Formal or clinical diagnosis of major depressive disorder}

Adults: We identified a significant difference in favour of psychological therapy (IPT) versus control for this outcome (RR $0.30,95 \% \mathrm{Cl} 0.11$ to 0.80 ; 49 participants; one RCT; Analysis 11.1 ).

\subsection{Formal or clinical diagnosis of anxiety disorders}

Adults: No study provided data for this outcome.

Children: No study provided data for this outcome.

\subsection{Formal or clinical diagnosis of somatoform and related disorders}

Adults: Researchers provided no data for this outcome.

Children and adolescents: Researchers provided no data for this outcome.

\section{Subgroup analyses}

According to availability of data, we were able to undertake most planned subgroup analyses in adult populations; the small number of RCTs that focused on children and adolescents did not allow us to undertake subgroup analyses in child and adolescent populations.

\section{Types of psychotherapies}

\subsection{PTSD symptoms}

At endpoint and at one to four months' follow-up, we identified a difference between subgroups when comparing the effects of psychological therapy versus control for type of psychological therapy ( $P=0.0007$; Analysis 1.1; and $P<0.0001$; Analysis 1.2$)$, but at six months, we identified no differences between subgroups ( $P=$ 0.81 ; Analysis 1.3). For children and adolescents, population testing for subgroup analysis was not applicable.

\subsection{Anxiety symptoms}

For this outcome, subgroup analyses were not possible.

\subsection{Depressive symptoms}

We found evidence of a difference between subgroups at endpoint and at one to four months' follow-up when comparing effects of psychological therapy versus control interventions for type of psychological therapy $(P=0.04$; Analysis 4.1; and $P=0.010$; Analysis 4.2). We detected no differences between subgroups at six months' follow-up ( $P=0.94$; Analysis 4.3).

\subsection{Dropouts for any cause}

We did not identify any differences between subgroups for this outcome in adults $(P=0.32$; Analysis 5.1). For children and adolescents, population subgroup analyses were not possible.

\subsection{Functional impairment}

For this outcome, subgroup analyses were not possible.

\subsection{Quality of life}

We did not identify any differences between subgroups for this outcome in adults $(P=0.72)$.

\subsection{Diagnosis of PTSD}

We found evidence of a difference between subgroups when comparing psychological therapy versus control for this outcome $(P=0.04$; Analysis 9.1). For children and adolescents, population testing for subgroup analysis was not applicable.

Children and adolescents: No study provided data for this outcome.

Psychological therapies for the treatment of mental disorders in low- and middle-income countries affected by humanitarian crises 


\section{Types of traumatic events}

\subsection{PTSD symptoms}

In subgroup analyses according to the type of traumatic event, we found significant differences between subgroups in terms of PTSD symptoms ( $P=0.004$; Analysis 12.1). At one to four months after the intervention, we identified a significant difference between subgroups ( $P=0.02$; Analysis 12.2). At six months after therapy (or later), only data for displaced populations and torture and witnessing violence or atrocities were available. We found no evidence of a difference between subgroups $(P=0.71)$ when comparing effects of psychological therapy over control for displacement, and effects of psychological therapy over control for torture and witnessing of violence or atrocities (see Analysis 12.3).

\subsection{Anxiety symptoms}

We noted that the effect of psychotherapy over control was significant for displacement (SMD $-1.30,95 \% \mathrm{Cl}-1.92$ to -0.67 ; 52 participants; two RCTs). We found no evidence of a difference for the subgroup other types of traumatic events at endpoint $(P=0.07$; Analysis 12.4). At one to four months, we found significant differences between subgroups for anxiety symptoms ( $P$ $=0.009$; Analysis 12.5). No data were available for six months after intervention or later, and no data were available for survivors of sexual and other forms of gender-based violence.

\subsection{Depressive symptoms}

At endpoint, we found no evidence of a difference between subgroups ( $P=0.72$; Analysis 12.6). We found no evidence of a difference between subgroups $(P=0.55)$ when comparing effects of psychological therapy over control for displacement (see Analysis 12.7). At six months, we noted no significant differences between the two traumatic event subgroups for which data were available (displaced populations and those surviving torture and witnessing violence or atrocities) (see Analysis 12.8). Available data were not sufficient for analysis of impacts on the subgroup sexual and other forms of gender-based violence for any time points.

\subsection{Dropouts for any reason}

We detected significant differences between subgroups in the outcome dropouts for any reason ( $P=0.009$; Analysis 12.9$)$.

\subsection{Functional impairment}

We found no evidence of differences between subgroups for the outcome functional impairment $(P=0.74$; Analysis 12.10$)$. At one to four months' follow-up, we identified significant differences between subgroups $(P=0.007$; Analysis 12.11$)$.

\subsection{Quality of life}

We found no evidence of a difference between subgroups $(P=0.72)$ when comparing effects of psychological therapy over control for the outcome quality of life (Analysis 12.12). Follow-up data were not available for this outcome.

\subsection{Diagnosis of PTSD}

We identified significant differences between subgroups for this outcome ( $P=0.004$; Analysis 12.13).

\section{Types of humanitarian crisis}

\subsection{PTSD symptoms}

In subgroup analyses according to types of humanitarian crisis, we found no evidence of a difference between subgroups $(P=$ 0.28 ) when comparing effects of psychological therapy over control interventions (see Analysis 13.1). At one to four months, we did not find evidence of a difference between subgroups $(P=0.57$; Analysis 13.2). At six months or later, only data on war/armed conflict were available; they showed maintained benefit (see Analysis 13.3).

\subsection{Anxiety symptoms}

We found evidence of differences between subgroups for this outcome $(P=0.04$; Analysis 13.4). Data at one to four months showed differences between subgroups $(P=0.03$; Analysis 13.5$)$. No data were available for this outcome at six months.

\subsection{Depressive symptoms}

For this outcome, we found no evidence of differences between subgroups ( $P=0.72$; Analysis 13.6). After one to four months, we identified significant differences between subgroups $(P<0.00001$; Analysis 13.7). At six months' follow-up or later, only data for populations affected by armed conflict were available (see Analysis 13.8).

\subsection{Dropouts for any reason}

We did not detect significant differences between subgroups for this outcome (Analysis 13.9).

\subsection{Functional impairment}

Psychological therapy was more effective than control for this outcome for the subgroups war/armed conflict (SMD $-0.68,95 \% \mathrm{Cl}$ -0.93 to $-0.43 ; 261$ participants; two RCTs). We found no evidence of differences between subgroups $(P=0.74)$ when comparing effects of psychological therapy over control intervention for communal violence; Analysis 13.10). At one to four months' follow-up, we found significant effects for the subgroup communal violence (SMD -0.36, 95\% Cl -0.63 to $-0.08 ; 281$ participants; two RCTs). We found no evidence of a difference between subgroups ( $P=$ 0.29 ) when comparing effects of psychological therapy over control intervention for war/armed conflict (SMD $-0.44,95 \% \mathrm{Cl}-0.75$ to -0.14; 659 participants; four RCTs; Analysis 13.11). No data were reported at six months after completion of the intervention or later.

\subsection{Quality of life}

We found no evidence of differences between subgroups $(P=$ 0.72 ) when comparing psychological therapy over control for the outcome quality of life (see Analysis 13.12). No data were reported for this outcome at follow-up.

\subsection{Diagnosis of PTSD}

We found no evidence of differences between subgroups when comparing effects of psychological therapy over control for this outcome ( $P=0.27$; Analysis 13.13).

\section{Types of interventionists}

\subsection{PTSD symptoms}

We found no differences between subgroups $(P=0.14)$ for this outcome (see Analysis 14.1). Research data confirmed no differences $(P=0.34)$ between subgroups at one to four months' 
follow-up (see Analysis 14.2) and no differences at six months' follow-up ( $P=0.34$; Analysis 14.3).

\subsection{Anxiety symptoms}

We observed significant differences between subgroups for the outcome anxiety symptoms at endpoint $(P=0.04$; Analysis 14.4). Data at one to four months highlighted no differences between subgroups $(P=0.07$; Analysis 14.5$)$. No data were reported at six months' follow-up.

\subsection{Depressive symptoms}

We noted significant differences between subgroups for the outcome depressive symptoms at endpoint ( $P=0.02$; Analysis 14.6). Data at one to four months after completion of the intervention showed no differences between subgroups ( $P=0.92$; Analysis 14.7 ); at six months, it was not possible to compare subgroups, as data were reported for paraprofessionals only (see Analysis 14.8).

\subsection{Dropouts for any cause}

We did not detect any significant differences between subgroups for this outcome (Analysis 14.9).

\subsection{Functional impairment}

Only data for the subgroup paraprofessionals were available for this outcome (see Analysis 14.10 and Analysis 14.11).

\subsection{Quality of life}

Only data for the subgroup professionals were available for this outcome (see Analysis 14.12).

\subsection{Diagnosis of PTSD}

We detected differences between subgroups for this outcome $(P=$ 0.03; Analysis 14.13).

\section{Types of controls}

\subsection{PTSD symptoms}

At endpoint and at 1-4 months, we found differences between subgroups for the outcome PTSD symptoms $(P<0.00001$; Analysis 15.1; Analysis 15.2). After 6 months, we found no evidence of differences between subgroups $(P=0.39)$ for this outcome (see Analysis 15.3).

\subsection{Anxiety symptoms}

At endpoint, we found no evidence of a difference between subgroups $(P=0.63)$ when comparing effects of psychological therapy over control for type of control (see Analysis 15.4). At 1-4 months, we found no evidence of differences between subgroups $(P=0.06$; Analysis 15.5). It was not possible to make comparisons between subgroups according to the type of control for this outcome after 6 months (Analysis 15.6).

\subsection{Depressive symptoms}

We found a difference between subgroups for the outcome depressive symptoms at endpoint ( $P=0.0001$; Analysis 15.7) and at 1-4 months ( $P=0.0001$; Analysis 15.8$)$. At six months, only a small proportion of RCTs provided data, showing no differences between subgroups ( $P=0.49$; Analysis 15.9$)$.

\subsection{Dropouts for any cause}

We found evidence of a difference between subgroups $(P=0.04)$ in the comparison of psychological therapy versus control for the outcome dropout rate (see Analysis 15.10).

\subsection{Functional impairment}

We found no differences between subgroups $(P=0.42)$ when comparing the effect of psychological therapy over control for type of control (see Analysis 15.11). A difference between subgroups was present at one to four months' follow-up ( $P=0.007$; Analysis 15.12). It was not possible to make comparisons between subgroups according to the type of control for this outcome at six months (Analysis 15.13).

\subsection{Quality of life}

It was not possible to make comparisons between subgroups according to the type of control for this outcome.

\subsection{Diagnosis of PTSD}

We found no significant differences between subgroups according to the type of control for this outcome.

\subsection{Diagnosis of depression}

It was not possible to make comparisons between subgroups according to the type of control for this outcome.

\subsection{Coping}

It was not possible to make comparisons between subgroups according to the type of control for this outcome.

\section{Phases of humanitarian crisis}

\subsection{PTSD symptoms}

We did not identify any differences between subgroups $(P=0.07)$ when comparing the effect of psychological therapy over control for the phase of humanitarian crisis at endpoint (see Analysis 16.1); at one to four months after the intervention ( $P=0.07$; Analysis 16.2); and after six months $(P=0.23$; Analysis 16.3$)$.

\subsection{Anxiety symptoms}

At endpoint, we did not detect any differences between subgroups for the outcome anxiety symptoms $(P=0.63$; Analysis 16.4). At one to four months, we found evidence of a difference between subgroups ( $P=0.01$; Analysis 16.5).

\subsection{Depressive symptoms}

For this outcome at endpoint, we identified no differences between subgroups ( $P=0.15$; Analysis 16.6). Data at one to four months showed a difference between subgroups $(P=0.003$; Analysis 16.7). At six months, we did not detect any differences between subgroups ( $P=0.49$; Analysis 16.8).

\subsection{Dropouts for any cause}

We did not identify any differences between subgroups for this outcome (see Analysis 16.9).

\subsection{Functional impairment}

We found no evidence of differences between subgroups $(P=0.69)$ when comparing the effect of psychological therapy over control for the phase of humanitarian crisis (see Analysis 16.10). We found 
no differences between subgroups after one to four months $(P=$ 0.28 ; Analysis 16.11). We did not have data on this outcome for six months' follow-up or later.

\subsection{Quality of life}

We found no differences between subgroups $(P=0.81$; Analysis 16.12).

\subsection{Diagnosis of PTSD}

It was not possible to compare different subgroups for this outcome (see Analysis 16.13).

\section{Sensitivity analyses}

Owing to the small number of RCTs that focused on child and adolescent populations, it was possible to carry out sensitivity analyses only for RCTs including adult populations.

\section{Incomplete outcome data}

\subsection{PTSD symptoms}

Upon performing this sensitivity analysis, we did not identify any material differences in comparison with standard meta-analysis including all RCTs (see Analysis 17.1; Analysis 17.2; Analysis 17.3).

\subsection{Anxiety symptoms}

By performing this sensitivity analysis, we did not identify any material differences compared with standard meta-analysis results (see Analysis 17.4; Analysis 17.5).

\subsection{Depressive symptoms}

Upon performing this sensitivity analysis, we did not identify any material differences in comparison with standard meta-analysis including all RCTs (see Analysis 17.6); Analysis 17.7; Analysis 17.8).

\subsection{Dropouts for any cause}

By performing this sensitivity analysis, we did not identify any significant differences compared with standard meta-analysis results (see Analysis 17.9).

\subsection{Functional impairment}

By performing this sensitivity analysis, we found that the effect of psychological treatment was not significant at endpoint (SMD - 0.25 , $95 \% \mathrm{Cl}-0.74$ to $0.24 ; 141$ participants; two RCTs). At one to four months after completion of the intervention, we did not identify any differences compared with standard meta-analysis results (see Analysis 17.10; Analysis 17.11).

\subsection{Quality of life}

Although meta-analysis including all RCTs highlighted a significant effect of psychological therapy over control, we found no significant effects of psychotherapy over control by performing this sensitivity analysis (see Analysis 17.12).

\subsection{Diagnosis of PTSD}

Results of meta-analysis did not significantly change after we performed this sensitivity analysis. However, IPT psychotherapy was no longer more effective than control, and NET was more effective than control for this outcome (see Analysis 17.13).

\section{Selective reporting}

\subsection{PTSD symptoms}

We did not identify any material change by performing this sensitivity analysis (see Analysis 18.1; Analysis 18.2).

\subsection{Dropouts for any cause}

We did not identify any material change by performing this sensitivity analysis (see Analysis 18.3).

\subsection{Functional impairment}

By performing this sensitivity analysis, we did not identify any significant difference compared with results of standard metaanalysis for this outcome (see Analysis 18.4).

\subsection{Quality of life}

For this outcome, we did not identify any material difference in comparison with results of standard meta-analysis including all RCTs (see Analysis 18.5).

\section{DISCUSSION}

\section{Summary of main results}

With regard to primary outcomes, we observed that symptoms of post-traumatic stress disorder (PTSD) decreased substantially among adults who received psychological therapies compared with those who received treatment as usual, received no treatment, or were on a wait list (low-quality evidence). This beneficial effect was observed immediately after completion of therapy, as well as at medium-term (one to four months after therapy) and long-term follow-up (six months or more after completion of psychological therapy). For children and adolescents, a beneficial effect of psychological therapy was observed immediately after the intervention (very low-quality evidence). However, this effect was not maintained at follow-up (one to four months after the intervention), and long term data were not available.

For depression and anxiety symptom outcomes, only data on adults were available. Low-quality evidence highlighted substantial improvement in depressive and anxiety symptoms in the psychological therapy group compared with the control group both at endpoint and at medium-term follow-up (one to four months). At six months, we did not find substantial treatment benefits and noted reduced strength of effect at each follow-up. Data on depression at six months were derived from just a few studies (with few participants).

The likelihood of leaving the study prematurely for any reason was similar in psychological therapy and control groups for both adults (moderate-quality evidence) and children (low-quality evidence). Data from studies on children and adolescents are based on small numbers of studies and participants.

With regard to secondary outcomes, results show a difference in favour of psychological therapy over control in reducing functional impairment at endpoint for adults and children and adolescents. Follow-up data were available only for adults, and (similar to depression and anxiety symptoms) showed maintained (but reduced) treatment benefit over the medium term but not over the long term. Long-term data on functional impairment are based on a few participants enrolled in one randomised controlled 
trial (RCT). The outcome quality of life was available only for adults at endpoint, and we identified a difference between intervention and control conditions in favour of psychological intervention. The likelihood of receiving a diagnosis of PTSD was reduced at endpoint for children and adolescents who received psychological therapy compared with those allocated to the control condition. However, only data from one small study were available for this outcome. For adults, we did not identify any substantial differences for this outcome at endpoint. No follow-up data were available for this outcome. Regarding a diagnosis of depression, only endpoint data for adults were available from a small study, indicating a positive effect of psychological therapy over control. No data were available on benefits regarding anxiety and somatoform and related diagnoses.

Overall, these findings indicate the benefit of psychological therapies for PTSD symptoms for adults in low- and middleincome country (LMIC) humanitarian settings but show lack of maintained treatment benefit in these settings over the longer term for depression and anxiety symptoms and functional impairment. For children and adolescents, we found that treatment benefits for PTSD symptoms were not maintained at medium term, and these were the only data available regarding maintenance. For children and adolescents, data from one small study highlighted an effect of psychological treatment in reducing PTSD diagnoses. Collectively, these results appear to present a more modest picture of what can be achieved through psychological therapies as opposed to what may be expected from results of meta-analyses of psychological treatments for selected outcomes in other settings. In general, little evidence of low quality was available for outcomes among children and adolescents. Future researchers should aim to understand the reasons for consistent reduction in treatment effects over time in low-resource humanitarian settings, but it is possible that this reduction in benefit of psychological treatments is related to continued exposure to stressors in LMIC humanitarian settings, including chronic poverty, continued (gender-based) violence, and negative impacts of humanitarian crises on social relationships that may protect mental health.

Even though the beneficial effects of psychological therapies have decreased over time, relief of psychological symptoms is an important goal for populations affected by humanitarian crises. Access to mental healthcare services is a right for populations living in humanitarian settings, as elsewhere. Humanitarian crises are often associated with denial of health as a human right both for adults and for children and adolescents. It is clear that the fundamental right to health should be an urgent priority for the humanitarian community, and that provision of psychological therapies is an important part of securing this right (The Lancet 2016).

We were able to conduct most of our planned subgroup analyses for adults, but not for children and adolescents. Firm conclusions based on subgroup analyses are challenging because subgroup analyses could rely on fewer RCTs and study participants. With regard to subgroup analyses for types of traumatic events, we found evidence of a difference between subgroups for PTSD outcome both at endpoint and at medium term. However, such maintained benefits were not found at medium term for displaced populations (PTSD, depression, and anxiety symptoms). For subgroup analyses looking at different types of humanitarian crises, we could not discern clear patterns, and we found no evidence of differences between subgroups for PTSD. Similarly, subgroup analyses by phase of humanitarian crisis and by type of therapist did not appear to result in obvious relationships. Overall, these results indicate that the benefit of psychological treatment for populations exposed to humanitarian crises in low-resource settings is moderated by contextual variables such as types of traumatic events experienced and type of crisis. A larger body of high-quality RCTs would provide a better understanding of how these and other variables can influence treatment benefits.

In addition, an important focus for future research, in our opinion, is to improve understanding of how initial benefits of psychological treatments may be maintained over time. This could be achieved, for example, through (1) booster sessions (e.g. at six months after treatment); (2) more systematic incorporation of problemsolving skills to address ongoing stressors in humanitarian settings; and/or (3) integration of psychological treatments with social interventions that address critical ongoing stressors in humanitarian settings (e.g. poverty alleviation, violence protection interventions).

\section{Overall completeness and applicability of evidence}

Psychological therapies included a relatively wide range of interventions, and we identified no psychodynamic interventions. With regard to the psychological therapies included, many were trauma focused and cognitive-behavioural therapy (CBT) based and/or derived; a small proportion of interventions were based on more generic counselling. Most expected outcomes were reported in these studies, including dropouts for any reason and functioning outcomes. However, data on anxiety and somatoform and related disorder diagnoses were not reported in the included RCTs. Data on children and adolescents were not available for some important outcomes, or were not available at mediumand long-term follow-up. For children, it was not possible to undertake the planned subgroup and sensitivity analyses - owing to the small number of RCTs - leaving a gap in evidence related to the role of specific variables influencing intervention effects. Regarding control conditions, we found that the wait list was the most reported control compared with no treatment and treatment as usual, and psychological placebo was not used as a control condition (in RCTs with adults and in RCTs with children and adolescents). Having said this, however, we acknowledge a level of complexity in defining specific ingredients that compose psychological placebo. For example, studies using control conditions defined as "individual counselling" could potentially be considered as using psychological placebo controls (Bass 2013). We will consider these distinctions/definitions in the next update of this review, depending on the quantity of randomised evidence available on this topic at that time.

Additionally, and for the same reason, we will consider the comparison of psychosocial interventions with active controls in the next update of this review. Comparing active psychosocial interventions will lead to a better understanding of the mechanisms of action of psychosocial interventions.

\section{Quality of the evidence}

We have included risk of bias assessment of included RCTs in Figure 2 and in Figure 3. We added into the risk of bias evaluation items related to psychological therapy and 
interventionist characteristics, according to the Cochrane review published by Patel (Patel 2014).

The risk of bias assessment is crucial in influencing interpretation of trial results and therefore deserves due attention. All included studies were RCTs, but their quality is not easy to assess, especially given the complexity of psychological therapies. Even though a RCT is the design of choice for evaluating the efficacy and acceptability of healthcare interventions (Jüni 2001; Purgato 2010), the evidence upon which the findings of this review are based is relatively poor as evaluated with the Cochrane risk of bias tool, and this is consistent with our grading within the 'Summary of findings' tables (from very low to moderate). Overall, we defined risk of allocation bias as low or unclear because some study authors provided insufficient details to permit a judgement. We considered most of the included RCTs to have high risk of performance bias, as participants were probably aware of whether they were receiving the psychological therapy or the control condition. Having said this, however, we have to acknowledge that in studies focused on psychological interventions, it is very challenging or even impossible to maintain participants' and therapists' blinding to treatment allocation. On the contrary, we evaluated most trials as having low risk of attrition and reporting bias. Regarding the specific items on psychological therapies, most studies failed to describe in detail therapist qualifications, and we evaluated a small proportion of RCTs as having low risk of bias for this item (e.g. RCTs in which the therapist was clearly described as a trained clinical psychologist). Many included RCTs described methods to check treatment fidelity in sufficient detail, but for others, we judged the risk of bias as unclear owing to insufficient information provided. Most included trials did not report details on therapist/investigator allegiance; we evaluated them as having unclear risk of bias. Remaining studies provided information on this item, and we evaluated them as having high risk of bias because investigators who developed psychological interventions were involved in conducting the RCT and in training therapists. Additional possible sources of bias not included in the risk of bias assessment are those specifically related to the topic of this review: socio-cultural differences in relation to psychological suffering; transposition of mental health concepts and therapies from Western to non-Western cultures (Kaiser 2015), with very different understandings and ways of dealing with psychological distress; and social norms and ways of discussing distress with strangers (such as therapists and interpreters) (Barbui 2017). Moreover, even though we were able to collect information about some basic characteristics of therapists (i.e. whether a professional or a paraprofessional), researchers did not always report details on therapists' language and nationality, social/economic class, education, geography, age, and background. These characteristics might have an influence on the establishment of relationship and trust, and thus on study outcomes.

For adults, we judged the quality of evidence on PTSD, depression, and anxiety at end point as low, indicating that additional data from further studies may very likely have an important impact on our confidence in the estimate of effect. The low-quality judgement was due to high levels of heterogeneity across studies, as well as the high risk of performance bias and attrition bias and concerns about the therapist/investigator allegiance. The quality of evidence for the outcome dropouts was moderate, indicating that further research may likely have an important impact on our confidence in the estimate of the effect. We made this judgement as researchers in all RCTs did not describe the outcome assessment as masked. For children, we rated the quality of evidence on the outcome PTSD and dropouts, respectively, as very low and low, indicating for the first outcome uncertainty regarding the estimate of the effect, and for the second outcome, that additional data from further studies may very likely have an important impact on our confidence in the estimate of effect. No data were available on the other outcomes. We based this judgement on high levels of heterogeneity detected between studies, description of the outcome assessment as not masked, and a wide confidence interval that included no effect of the intervention.

In general, we found high levels of heterogeneity between studies, which means that studies had different trends in outcomes. Even though some levels of heterogeneity are expected in studies focused on complex clinical interventions (such as psychological therapies), one should consider this issue when interpreting results and drawing conclusions.

\section{Potential biases in the review process}

Although we attempted to access studies through an extensive search of the literature (including grey literature), it is still possible that we have missed one or more (unpublished) studies. Although it is unlikely that RCTs would be conducted and would not be publicly accessible, not all those conducting research may necessarily value academic publications, so work may be disseminated through other channels.

Data generated from these included RCTs had some limitations. Some RCTs were very small, often including fewer than 100 participants, and in some cases fewer than 20. This lack of statistical power may have affected the findings. Another limitation of this review is that included studies did not report information on some outcomes, such as somatic symptom and related disorders, and broader wellbeing outcomes, such as quality of life and functioning (especially in child and adolescent populations). Moreover, only a small proportion of studies reported data on child and adolescent populations, even though it is known that psychological suffering during childhood and adolescence can negatively impact future achievements (also at academic and work levels) and raise serious risks for health, such as substance abuse and suicidal ideation (Fergusson 2005). In addition, most RCTs reported only short-term or medium-term data, and longer-term follow-up data were available in only a small proportion of studies. This limits conclusions that can be drawn from this review.

To facilitate data reading and interpretation, we decided to pool together data on different types of psychological therapies. We also tested for differences between different types of therapies as part of subgroup analyses.

\section{Agreements and disagreements with other studies or reviews}

Findings of this review are consistent with those of Cochrane reviews that found good evidence for the effectiveness of some types of psychological therapies for children and adolescents exposed to trauma (in particular, CBT) (Gillies 2016); and for adults with PTSD (Bisson 2013). The Cochrane review published by Patel et al was focused on torture survivors and did not identify substantial differences between psychological therapies and controls in terms of immediate effects on PTSD symptoms, 
distress, or quality of life. Moreover, we rated the quality of included RCTs as very low according to the GRADE system (Patel 2014). Results of this review are also consistent with those of the Tol et al review published in 2011 (Tol 2011), which identified substantial beneficial effects of psychological interventions versus control conditions for adults with symptoms of PTSD. For children and adolescents, the Tol review detected a non-substantial trend in favour of interventions versus control conditions for PTSD symptoms, along with a substantial effect for internalising symptoms. Other recent systematic reviews and meta-analyses of psychological interventions for children and adolescents in low- and middle-income countries affected by mass violence (Jordans 2016; Morina 2017; Purgato 2018) have analysed effects of a wider range of interventions compared with our separate analysis of treatment and (in parallel reviews), prevention, and promotion interventions. The systematic review conducted by Morina and colleagues included 21 RCTs on any type of psychological interventions for treating war-related PTSD and depressive symptoms as compared with control conditions (wait list) for survivors of mass violence 19 years of age or younger; and the review conducted by Jordans and colleagues examined the type and effectiveness of psychosocial and mental health interventions in conflict-affected children as reported in 24 articles. These systematic reviews and meta-analyses identified an overall effect for this broad group of interventions. Morina et al found small and medium effects after correcting for publication bias, and Jordans et al noted benefits commonly limited to subgroups of children. These trial authors highlight the need for additional highquality studies with this population.

\section{AUTHORS' CONCLUSIONS}

\section{Implications for practice}

Currently low-quality evidence suggests the effectiveness of psychological therapies over control comparators in decreasing PTSD and depressive and anxiety symptoms, and moderate-quality evidence shows the acceptability of these treatments for adults. Very low-quality evidence suggests that psychological therapies may decrease PTSD symptoms in children and adolescents (with no evidence available with regard to depression and anxiety in children/adolescents) immediately after treatment, and low-quality evidence suggests that these treatments may be acceptable. Heterogeneity between studies was high. We found large treatment benefits at short-term follow-up.

\section{Implications for research}

Results from this review show that psychological therapies are effective in decreasing PTSD and depressive and anxiety symptoms in adults and in children and adolescents. Identification of a shortterm benefit is important for populations living in humanitarian settings in LMICs. Having said this, however, this review revealed a gap in the knowledge base at longer term and in the child and adolescent population. More evidence is needed to evaluate the effectiveness of psychological therapies for longer than six months after the intervention, and for children and adolescents living in humanitarian settings in LMICs. Future trials should be randomised, should use socioculturally appropriate and validated instruments to measure outcomes, and should provide higher rates of follow-up over the longer term. Moreover, the following would be important.

1. To describe in greater detail the types of trauma and the sociocultural and family contexts in which participants live, including, for example, details on socioeconomic status, living arrangements, ethnicity, and healthcare preferences. A full description of humanitarian conditions is particularly important, as factors such as poverty, discrimination, stigma, and lack of social networks may negatively impact psychological and physical health, and thus outcomes of treatment (Miller 2016; Purgato 2016b). Even though we were able to collect a good quantity of information on traumatic events and settings (and to perform subgroup analyses accordingly), this goal would best be achieved by making individual participant data available for meta-analysis.

2. To (continue to) pay attention to cultural applicability of research design, measurement, and interpretation across socioculturally diverse populations, given variation in mental health experiences, prioritisation, and care preferences (Haroz 2016; Kaiser 2015). (Continued) involvement of people from the affected population throughout the research process will prove helpful in gaining an appropriate understanding and interpretation of clinical symptoms, and in assessing and delivering interventions.

3. To choose pragmatic, meaningful, and easily to assess outcomes.

4. To receive due attention to some methodological key issues such as allocation concealment, blinding, and availability of longer-term follow-ups. Moreover, with summary statistics, quality and completeness of information are essential. Metaanalyses of poor quality studies may be seriously misleading because the bias associated with defects in the conduct of primary studies (RCTs) can seriously affect the overall estimate of intervention (Savović 2012). Systematic review authors (not only within Cochrane) should routinely assess risk of bias in trial results.

\section{ACKNOWLEDGEMENTS}

We would like to thank all trial authors who provided additional data for inclusion in the present report, especially Drs. Esfandiar Azad Marzabadi and Suzanne Connolly.

Dr. Marianna Purgato was supported by a Marie Curie International Outgoing Fellowship within the 7th European Community Framework Programme.

CRG Funding Acknowledgement: The National Institute for Health Research (NIHR) is the largest single funder of the Cochrane Common Mental Disorders Group.

Disclaimer: The views and opinions expressed herein are those of the review authors and do not necessarily reflect those of the NIHR, the NHS, or the Department of Health. 


\section{R E F E R E N C E S}

\section{References to studies included in this review}

Acarturk 2015 \{published data only\}

Acarturk C, Konuk E, Cetinkaya M, Senay I, Sijbrandij M, Cuijpers $P$, et al. EMDR for Syrian refugees with post-traumatic stress disorder symptoms: results of a pilot randomized controlled trial. European Journal of Psychotraumatology 2015;6:27414.

\section{Acaturk 2016 \{published data only\}}

Acarturk C, Konuk E, Cetinkaya M, Senay I, Sijbrandij M, Gulen B, et al. The efficacy of eye movement desensitization and reprocessing for post-traumatic stress disorder and depression among Syrian refugees: results of a randomized controlled trial. Psychological Medicine 2016;46:2583-93.

\section{Ahmadizadeh 2013 \{published data only\}}

Ahmadizadeh M, Ahmadi K, Anisi J, Ahmadi AB. Assessment of cognitive behavioral therapy on quality of life of patients with chronic war-related post-traumatic stress disorder. Indian Journal of Psychological Medicine 2013;35(4):341-5.

Azad Marzabadi 2014 \{published data only\}

Azad Marzabadi E, Hashemi Zadeh SM. The effectiveness of mindfulness training in improving the quality of life of the war victims with post traumatic stress disorder (PTSD). Iran Journal of Psychiatry 2014;9(4):228-36.

\section{Basoglu 2005 \{published data only\}}

Başoğlu M, Salcioğlu E, Livanou M, Kalender D, Acar G. Singlesession behavioral treatment of earthquake-related posttraumatic stress disorder: a randomized waiting list controlled trial. Journal of Traumatic Stress 2005;18(1):1-11.

\section{Basoglu 2007 \{published data only\}}

Başoglu M, Salcioglu E, Livanou M. A randomized controlled study of single-session behavioural treatment of earthquakerelated post-traumatic stress disorder using an earthquake simulator. Psychological Medicine 2007;37(2):203-13.

\section{Bass 2016 \{published data only\}}

Bass J, Murray SM, Mohammed TA, Bunn M, Gorman W, Ahmed AM, et al. A randomized controlled trial of a traumainformed support, skills, and psychoeducation intervention for survivors of torture and related trauma in Kurdistan, Northern Iraq. Global Health Science and Practice 2016;4(3):452-66.

\section{Bolton 2007 \{published data only\}}

Bolton P, Bass J, Betancourt T, Speelman L, Onyango G, Clougherty KF, et al. Interventions for depression symptoms among adolescent survivors of war and displacement in Northern Uganda. A randomized controlled trial. Journal of the American Medical Association 2007;298(5):519-27.

\section{Bolton 2014a \{published data only\}}

Bolton P. Controlled trial of mental health interventions for torture survivors in Kurdistan. clinicaltrialsgov/ct2/show/ NCT00925262.
* Bolton P, Bass JK, Zangana GA, Kamal T, Murray SM, Kaysen $D$, et al. A randomized controlled trial of mental health interventions for survivors of systematic violence in Kurdistan, Northern Iraq. BMC Psychiatry 2014;14:360.

Bolton 2014b \{published data only\}

Bolton P, Lee C, Haroz EE, Murray L, Dorsey S, Robinson C, et al. A transdiagnostic community-based mental health treatment for comorbid disorders: development and outcomes of a randomized controlled trial among Burmese refugees in Thailand. PLoS Medicine 2014; Vol. 11, issue 11:e1001757.

Bryant 2011 \{published data only\}

Bryant RA, Ekasawin S, Chakrabhand S, Suwanmitri S, Duangchun $\mathrm{O}$, Chantaluckwong T. A randomized controlled effectiveness trial of cognitive behavior therapy for posttraumatic stress disorder in terrorist-affected people in Thailand. World Psychiatry 2011;10(3):205-9.

\section{Chen 2014 \{published data only\}}

Chen Y, Shen WW, Gao K, Lam CS, Chang WC, Deng H. Effectiveness RCT of a CBT intervention for youths who lost parents in the Sichuan, China, earthquake. Psychiatric Services 2014;65(2):259-62.

\section{Connolly 2011 \{published data only\}}

Connolly S, Sakai C. Brief trauma intervention with Rwandan genocide-survivors using thought field therapy. International Journal of Emergency in Mental Health 2011;13(3):161-72.

Connolly 2013 \{published data only\}

Connolly SM, Roe-Sepowitz D, Sakai C, Edwards J. Utilizing community resources to treat PTSD: a randomized controlled study using thought field therapy. Journal of Traumatic Stress 2013;3:24-32.

\section{Ertl 2011 \{published data only\}}

Ertl V, Pfeiffer A, Schauer E, Elbert T, Neuner F. Communityimplemented trauma therapy for former child soldiers in Northern Uganda: a randomized controlled trial. Journal of the American Medical Association 2011;306(5):503-12.

\section{Hermenau 2013 \{published data only\}}

Hermenau K, Hecker T, Schaal S, Maedl A, Elbert T. Addressing post-traumatic stress and aggression by means of narrative exposure: a randomized controlled trial with ex-combatants in the eastern DRC. Journal of Aggression Maltreatment \& Trauma 2013;22:916-34.

\section{Igreja 2004 \{published data only\}}

Igreja V, Kleijn WC, Schreuder BJ, Van Dijk JA, Verschuur M. Testimony method to ameliorate post-traumatic stress symptoms. Community-based intervention study with Mozambican civil war survivors. British Journal of Psychiatry 2004;184:251-7.

\section{Jacob 2014 \{published data only\}}

Jacob N, Neuner F, Maedl A, Schaal S, Elbert T. Dissemination of psychotherapy for trauma spectrum disorders in

Psychological therapies for the treatment of mental disorders in low- and middle-income countries affected by humanitarian crises 
postconflict settings: a randomized controlled trial in Rwanda. Psychotherapy Psychosomatics 2014;83(6):354-63.

\section{Jiang 2014 \{published data only\}}

Jiang RF, Tong HQ, Delucchi KL, Neylan TC, Shi Q, Meffert SM. Interpersonal psychotherapy versus treatment as usual for PTSD and depression among Sichuan earthquake survivors: a randomized clinical trial. Conflict and Health 2014;8:14.

\section{Knaevelsrud 2015 \{published data only\}}

Knaevelsrud C, Brand J, Lange A, Ruwaard J, Wagner B. Webbased psychotherapy for post-traumatic stress disorder in wartraumatized Arab patients: randomized controlled trial. Journal of Medical Internet Research 2015;17(3):e71.

Wagner B, Brand J, Schulz W, Knaevelsrud C. Online working alliance predicts treatment outcome for post-traumatic stress symptoms in Arab war-traumatized patients. Depression and Anxiety 2012;29:646-51.

\section{McMullen 2013 \{published data only\}}

McMullen J. Randomized control trial of group intervention with former war-affected boys in the Democratic Republic of Congo. clinicaltrialsgov/show/NCT01494831.

\section{* McMullen J, O'Callaghan P, Shannon C, Black A, Eakin J. Group trauma-focused cognitive-behavioural therapy with former child soldiers and other war-affected boys in the DR Congo: a randomised controlled trial. Journal of Child Psychology and Psychiatry 2013;54(11):1231-41.}

\section{Meffert 2014 \{published data only\}}

* Meffert SM, Abdo AO, Alla OAA, Elmakki YOM, Metzler TJ, Marmar CR. A pilot randomized controlled trial of interpersonal psychotherapy for Sudanese refugees in Cairo, Egypt. Psychological Trauma: Theory Research Practice and Policy 2014;6(3):240-9.

\section{Neuner 2008a \{published data only\}}

Neuner F, Onyut PL, Ertl V, Odenwald M, Schauer E, Elbert T. Treatment of post-traumatic stress disorder by trained lay counselors in an African refugee settlement: a randomized controlled trial. Journal of Consulting and Clinical Psychology 2008;76(4):686-94.

\section{Neuner 2008b \{published data only\}}

Neuner F, Onyut PL, Ertl V, Odenwald M, Schauer E, Elbert T. Treatment of post-traumatic stress disorder by trained lay counselors in an African refugee settlement: a randomized controlled trial. Journal of Consulting and Clinical Psychology 2008;76(4):686-94.

\section{O' Callaghan 2013 \{published data only\}}

O'Callaghan P. Is trauma-focused cognitive behavioral therapy effective in reducing post-traumatic stress and psychosocial distress among sexually exploited, war-affected girls in the Democratic Republic of Congo. clinicaltrialsgov [wwwclinicaltrialsgov] NCT01483261.

* O'Callaghan P, McMullen J, Shannon C, Rafferty H, Black A. A randomized controlled trial of trauma-focused cognitive behavioral therapy for sexually exploited, war-affected
Congolese girls. Journal of American Academy of Child \& Adolescent Psychiatry 2013;52(4):359-69.

Pityaratstian 2015 \{published data only\}

Pityaratstian N, Piyasil V, Ketumarn P, Sitdhiraksa N Ularntinon S, Pariwatcharakul P. Randomized controlled trial of group cognitive behavioural therapy for post-traumatic stress disorder in children and adolescents exposed to tsunami in Thailand. Behavioural and Cognitive Psychotherapy 2015;43:549-61.

Puvimanasinghe 2016 \{published data only\}

Puvimanasinghe TS, Price IR. Healing through giving testimony: an empirical study with Sri Lankan torture survivors. Transcultural Psychiatry 2016;53(5):531-50.

\section{Rahman 2016a \{published data only\}}

Rahman A, Hamdani SU, Awan NR, Bryant RA, Dawson KS, Khan MF, et al. Effect of a multicomponent behavioral intervention in adults impaired by psychological distress in a conflict-affected area of Pakistan: a randomized clinical trial. Journal of the American Medical Association 2016;316(24):doi:10.1001/jama.2016.17165.

\section{Rahman 2016b \{published data only\}}

Rahman A, Riaz R, Dawson KS, Hamdani SU, Chiumento A, Sijbrandij M, et al. Problem Management Plus (PM1): pilot trial of a WHO transdiagnostic psychological intervention in conflictaffected Pakistan. World Psychiatry 2016;15(2):182-3.

\section{Wang 2013a \{published data only\}}

Wang Z, Wang J, Maercker A. Chinese My Trauma recovery, a web-based intervention for traumatized persons in two parallel samples: randomized controlled trial. Journal of Medical Internet Research 2013;15(9):e213.

\section{Wang 2013b \{published data only\}}

Wang Z, Wang J, Maercker A. Chinese My Trauma recovery, a web-based intervention for traumatized persons in two parallel samples: randomized controlled trial. Journal of Medical Internet Research 2013;15(9):e213.

\section{Wang 2016 \{published data only\}}

Wang SJ, Bytyci A, Izeti S, Kallaba M, Rushiti F, Montgomery E, et al. A novel bio-psycho-social approach for rehabilitation of traumatized victims of torture and war in the post-conflict context: a pilot randomized controlled trial in Kosovo. Conflict and Health 2016;10(34):1-17.

\section{Weiss 2015a \{published data only\}}

Weiss WM, Murray LK, Zangana GAS, Mahmooth Z, Kaysen D, Dorsey $S$, et al. Community-based mental health treatments for survivors of torture and militant attacks in Southern Iraq: a randomized control trial. BMC Psychiatry 2015;15:249.

\section{Weiss 2015b \{published data only\}}

Weiss WM, Murray LK, Zangana GAS, Mahmooth Z, Kaysen D, Dorsey S, et al. Community-based mental health treatments for survivors of torture and militant attacks in Southern Iraq: a randomized control trial. BMC Psychiatry 2015;15:249.

Psychological therapies for the treatment of mental disorders in low- and middle-income countries affected by humanitarian crises 
Zang 2013 \{published data only\}

Zang Y, Hunt N, Cox T. A randomised controlled pilot study: the effectiveness of narrative exposure therapy with adult survivors of the Sichuan earthquake. BMC Psychiatry 2013;13:41.

\section{Zang 2014 \{published data only\}}

Zang Y, Hunt N, Cox T. Adapting narrative exposure therapy for Chinese earthquake survivors: a pilot randomised controlled feasibility study. BMC Psychiatry 2014;14:262.

\section{References to studies excluded from this review}

\section{Adenauer 2011 \{published data only\}}

Adenauer H, Catani C, Gola H, Keil J, Ruf M, Schauer M, et al. Narrative exposure therapy for PTSD increases top-down processing of aversive stimuli-evidence from a randomized controlled treatment trial. BMC Neuroscience 2011;12:127.

\section{Ager 2011 \{published data only\}}

Ager A, Akesson B, Stark L, Flouri E, Okot B, McCollister F, et al. The impact of the school-based Psychosocial Structured Activities (PSSA) program on conflict-affected children in Northern Uganda. Journal of Child Psychology and Psychiatry 2011;52(11):1124-33.

\section{Akhtar 1994 \{published data only\}}

Akhtar P. Project report: therapeutic effects of music on torture survivors and refugees. Torture 1994;4:121-3.

\section{Ayoughi 2012 \{published data only\}}

Ayoughi S, Missmahl I, Weierstall R, Elbert T. Provision of mental health services in resource-poor settings: a randomised trial comparing counselling with routine medical treatment in North Afghanistan (Mazar-e-Sharif). BMC Psychiatry 2012;12:14.

\section{Barron 2013 \{published data only\}}

Barron IG, Abdallah G, Smith P. Randomized control trial of a CBT trauma recovery program in Palestinian schools. Journal of Loss \& Trauma 2013;18:306-21.

\section{Başoğlu 2003 \{published data only\}}

Başoğlu M, Livanou M, Salcioğlu E, Kalender D. A brief behavioural treatment of chronic post-traumatic stress disorder in earthquake survivors: results from an open clinical trial. Psychological Medicine 2003;33(4):647-54.

\section{Bass 2012 \{published data only\}}

Bass J, Poudyal B, Tol W, Murray L, Nadison M, Bolton P. A controlled trial of problem-solving counseling for war-affected adults in Aceh, Indonesia. Social Psychiatry and Psychiatric Epidemiology 2012;47(2):279-91.

\section{Bass 2013 \{published data only\}}

Bass JK, Annan J, Mclvor Murray S, Kaysen D, Griffiths S, Cetinoglu T, et al. Controlled trial of psychotherapy for Congolese survivors of sexual violence. New England Journal of Medicine 2013;368(23):2182-91.
Beck 2009 \{published data only\}

Beck J, Coffey SF, Foy DW, Keane TM, Blanchard EB. Group cognitive behavior therapy for chronic post-traumatic stress disorder: an initial randomized pilot study. Behavioral Therapy 2009;40:82-92.

\section{Becker 2009 \{published data only\}}

Becker SM. Psychosocial care for women survivors of the tsunami disaster in India. American Journal of Public Health 2009;99(4):654-8.

\section{Berger 2015 \{published data only\}}

Berger R, Gelkopf M, Heineberg Y, Zimbardo P. A school-based intervention for reducing post-traumatic symptomatology and intolerance during political violence. Journal of Educational Psychology 2015;108(6):1-10.

\section{Betancourt 2014 \{published data only\}}

Betancourt TS, McBain R, Newham EA, Akinsulure-Smith A, Brennan R, Weisz JR, Hansen NB. A behavioural intervention for war-affected youth in Sierra Leone: a randomized controlled trial. Journal of the American Academy of Child \& Adolescent Psychiatry 53;12:1288-97.

\section{Bichescu 2007 \{published data only\}}

Bichescu D, Neuner F, Schauer M, Elbert T. Narrative exposure therapy for political imprisonment-related chronic posttraumatic stress disorder and depression. Behavioral Research Therapy 2007;45(9):2212-20.

\section{Bolton 2003 \{published data only\}}

Bolton P, Bass J, Neugebauer R, Verdeli H, Clougherty KF, Wickramaratne $\mathrm{P}$, et al. Group interpersonal psychotherapy for depression in rural Uganda: a randomized controlled trial. JAMA 2003;289(23):3117-24.

\section{Bolton 2009 \{published data only\}}

Bolton P, Murray L, Bass J. School based intervention improves PTSD symptoms in children affected by political violence. Evidence Based Mental Health 2009;12(2):47.

\section{Chibanda 2016 \{published data only\}}

Chibanda D, Weiss H, Verhey R, Simms V, Munjoma R, Rusakaniko $S$, et al. Effect of a primary care-based psychological intervention on symptoms of common mental disorders in Zimbabwe. A randomized clinical trial. Journal of the American Medical Association 2016;316(24):2618-26.

\section{Gordon 2008 \{published data only\}}

Gordon J, Staples J, Blyta A, Bytyqi M, Wilson A. Treatment of post-traumatic stress disorder in postwar Kosovar adolescents using mind-body skills groups: a randomized controlled trial. Journal of Clinical Psychiatry 2008;69:1469-76.

\section{Jordans 2010 \{published data only\}}

Jordans MJ, Komproe IH, Tol WA, Kohrt BA, Luitel NP, Macy RD, et al. Evaluation of a classroom-based psychosocial intervention in conflict-affected nepal: a cluster randomized controlled trial. Journal of Child Psychology and Psychiatry 2010;51(7):818-26. 
Mughal 2015 \{published data only\}

Mughal U, Carrasco D, Brown R, Ayers S. Rehabilitating civilian victims of war through psychosocial intervention in Sierra Leone. Journal of Applied Social Psychology 2015;45:593-601.

\section{Murray 2015 \{published data only\}}

Murray LK, Skavenski S, Kane JC, Mayeya J, Dorsey S, Cohen JA, et al. Effectiveness of trauma-focused cognitive behavioral therapy among trauma-affected children in Lusaka, Zambia; a randomized clinical trial. JAMA Pedriatic 2015;169(8):761-9.

\section{Newnham 2015 \{published data only\}}

Newnham EA, McBain RK, Hann K, Akinsulure-Smith AM, Weisz J, Lilienthal GM, et al. The Youth Readiness Intervention for war-affected youth. Journal of Adolescent Health 2015;56(6):606-11.

\section{O'Callaghan 2014 \{published data only\}}

O'Callaghan P, Branham L, Shannon C, Betancourt T, Dempster M, McMullen J. A pilot study of a family focused, psychosocial intervention with war-exposed youth at risk of attack and abduction in north-eastern Democratic Republic of Congo. Child Abuse \& Neglect 2014;38:1197-207.

Pokharial 2012 \{published data only\}

Pokhariyal GP, Rono R, Munywoki S. Analysis of treatment methods for victims of torture in Kenya and East Africa region. Traumatology 2012;19(2):107-17.

\section{Punamaki 2014 \{published data only\}}

Diab M, Peltonen K, Qouta S, Palosaari E, Punamaki RA. Effectiveness of psychosocial intervention enhancing resilience among war-affected children and the moderating role of family factors. Child Abuse \& Neglect 2015;40:24-35.

Diab M, Punamaki RL, Palosaari E, Qouta S. Can psychosocial intervention improve peer and sibling relations among war-affected children? Impact and mediating analyses in a randomized controlled trial. Social Development 2013;23(2):215-31.

* Punamaki RL, Peltonen K, Diab M, Qouta S. Psychosocial interventions and emotion regulation among war-affected children: randomized controlled trial effects. Traumatology 2014;20(4):241-52.

\section{Richards 2014 \{published data only\}}

Richards J, Foster C, Townsend N, Bauman A. Physical fitness and mental health impact of a sport-for-development intervention in a post-conflict setting: randomized controlled trial nested within an observational study of adolescents in Gulu, Uganda. BMC Public Health 2014;18(14):619.

\section{Robson 2016 \{published data only\}}

Robson RH, Robson PM, Ludwig R, Mitabu C, Philips C. Effectiveness of thought field therapy provided by newly instructed community workers to a traumatized population in Uganda: a randomized trial. Current Research in Psychology 2016;7(1):1-11.

\section{Tiwari 2010 \{published data only\}}

Tiwari A, Fong DY, Yuen KH, Yuk H, Pang P, Humphreys J, et al. Effect of an advocacy intervention on mental health in Chinese women survivors of intimate partner violence: a randomized controlled trial. Journal of the American Medical Association 2010;304(5):536-43.

\section{Tol 2008 \{published data only\}}

Tol WA, Komproe IH, Susanty D, Jordans MJ, Macy RD, de Jong JT. School-based mental health intervention for children affected by political violence in Indonesia: a cluster randomized trial. Journal of the American Medical Association 2008;300(6):544.

\section{Tol 2012 \{published data only\}}

Tol WA, Komproe IH, Jordans MJ, Vallipuram A, Sipsma H, Macy RD, et al. Outcomes and moderators of a preventive school-based mental health intervention for children affected by war in Sri Lanka: a cluster randomized trial. World Psychiatry 2012;11(2):114-22.

\section{Tol 2014 \{published data only\}}

Tol WA, Komproe IH, Jordans MJ, Ndayisaba A, Ntamutumba P, Sipsma $\mathrm{H}$, et al. School based mental health intervention for children in war-affected Burundi: a cluster randomized trial. BMC Medicine 2014;12(56):1-12.

\section{Unterhitzenberger 2014 \{published data only\}}

Unterhitzenberger J, Rosner R. Lessons from writing sessions: a school-based randomized trial with adolescent orphans in Rwanda. European Journal of Psychotraumatology 2014;5:1-10.

\section{References to studies awaiting assessment}

\section{Abbasnehzad 2007 \{published data only\}}

Abbasnezhad M, Nemat Elah Zadeh Mahani K, Zamyad A. Efficacy of "eye movement desensitization and reprocessing" in reducing anxiety and unpleasant feelings due to earthquake experience. Psychological Research 2007;9(3-4):104-17.

\section{Barron 2016 \{published data only\}}

Barron I, Abdallah G, Heltne U. Randomized control trial of teaching recovery techniques in rural occupied Palestine: effect on adolescent dissociation. Journal of Aggression Maltreatment and Trauma 2016;25(9):995-73.

Dawson 2016 \{published data only\}

Dawson KS, Schafer A, Anjuri D, Ndogoni L, Musyoki C, Sijbrandij M, et al. Feasibility trial of a scalable psychological intervention for women affected by urban adversity and genderbased violence in Nairobi. BMC Psychiatry 2016;16.

\section{Esala 2017 \{published data only\}}

Esala JJ, Taing S. Testimony therapy with ritual: a pilot randomized controlled trial. Journal of Traumatic Stress 2017;30(1):94-8.

\section{Khan 2017 \{published data only\}}

Khan MN, Hamdani SU, Chiumento A, Dawson K, Bryant RA, Sijbrandij M, et al. Evaluating feasibility and acceptability 
of a group WHO trans-diagnostic intervention for women with common mental disorders in rural Pakistan: a cluster randomised controlled feasibility trial. Epidemiology and Psychiatric Sciences 2017:1-11.

\section{Mahmoudi-Gharaei 2006 \{published data only\}}

Mahmoudi-Gharaei J, Mohammadi M, Bina MYM, Fakour Y. Supportive and cognitive behavioural group interventions on Bam earthquake related PTSD symptoms in adolescents Persian. Tehran University Medical Journal 2006;64:57-67.

NCT02598024 \{unpublished data only\}

NCT02598024. Treating Earthquake in Nepal Trauma (TENT) Trial 2016. ClinicalTrialsgov/show/NCT02598024 2016.

\section{Reger 2016 \{published data only\}}

Reger GM, Koenen-Woods P, Zetocha K, Smolenski DJ, Holloway KM, Rothbaum BO, et al. Randomized controlled trial of prolonged exposure using imaginal exposure vs. virtual reality exposure in active duty soldiers with deployment-related posttraumatic stress disorder (PTSD). Journal of Consulting and Clinical Psychology 2016;84(11):946-59.

\section{Steinert 2017 \{published data only\}}

Steinert C, Bumke P, Hollekamp R, Larisch A, Leichsenring F, et al. Treating post-traumatic stress disorder by resource activation in Cambodia. World Psychiatry 2016;15(2):183-5.

Steinert C, Bumke PJ, Hollekamp RL, Larisch A, Leichsenring F, Matthes $\mathrm{H}$, et al. Resource activation for treating post-traumatic stress disorder, co-morbid symptoms and impaired functioning: a randomized controlled trial in Cambodia. Psychological Medicine 2017;47(3):553-64.

\section{Weinstein 2016 \{published data only\}}

Weinstein N, Khabbaz F, Legate N. Enhancing need satisfaction to reduce psychological distress in Syrian refugees. Journal of Consulting and Clinical Psychology 2016;84(7):645-50.

\section{References to ongoing studies}

\section{ISRCTN65771265 \{published data only\}}

Greene C, Tol W. Nguvu: Evaluating an integrated approach to reduce intimate partner violence and psychological distress in refugees in Tanzania. apps.who.int/trialsearch/Trial2.aspx? TrialID=ISRCTN65771265

\section{NCT03012451 \{unpublished data only\}}

Panter-Brick C. A psychosocial program impact evaluation in Jordan. clinicaltrials.gov/ct2/show/ NCT03012451?term=humanitarian+OR+low+and+middle +income\&type=Intr\&intr=psychological+OR+psychotherapy+OR + psychosocial\&rank=1.

\section{NCT031090028 \{published data only\}}

NCT03109028. Mental health in refugees and asylum seekers. ClinicalTrialsgov/show/NCT03109028.

\section{Additional references}

\section{Abbass 2014}

Abbass AA, Kisely SR, Town JM, Leichsenring F, Driessen E, De Maat S, et al. Short-term psychodynamic psychotherapies for common mental disorders. Cochrane Database of Systematic Reviews 2014, Issue 7. [DOI: 10.1002/14651858.CD004687.pub4]

\section{Allen 2010}

Allen LA, Woolfolk RL. Cognitive behavioral therapy for somatoform disorders. Psychiatric Clinics of North America 2010;33(3):579-93.

\section{Altman 1996}

Altman DG, Bland JM. Detecting skewness from summary information. British Medical Journal 1996;313(7066):1200.

\section{APA 1980}

American Psychiatric Association. iagnostic and Statistical Manual of Mental Disorders (DSM-III). Diagnostic and Statistical Manual of Mental Disorders (DSM-III). 3rd Edition. Washington, DC: American Psychiatric Association, 1980.

\section{APA 1987}

American Psychiatric Association. Diagnostic and Statistical Manual of Mental Disorders (DSM-III-R). 3rd Edition. Washington, DC: American Psychiatric Association, 1987.

\section{APA 1994}

American Psychiatric Association. .. Diagnostic and Statistical Manual of Mental Disorders (DSM-IV). 4th Edition. Washington, DC: American Psychiatric Association, 1994.

\section{APA 2000}

American Psychiatric Association. Diagnostic and Statistical Manual of Mental Disorders (DSM-IV-TR). 4th Edition. Washington, DC: American Psychiatric Association, 2000.

\section{APA 2013}

American Psychiatric Association. Diagnostic and Statistical Manual of Mental Disorders (DSM-V). 5th Edition. Washington, DC: American Psychiatric Association, 2103.

\section{Barbui 2011}

Barbui C, Cipriani A. Cluster randomised trials. Epidemiology and Psychiatric Sciences 2011;20(4):307-9.

\section{Barbui 2017}

Barbui C, Purgato M, Churchill R, Adams C, Amato L, Macdonald G, et al. Cochrane for global mental health. Lancet Psychiatry 2017;4:e6.

\section{Beck 1961}

Beck AT, Ward CH, Mendelson M, Mock J, Erbaugh J. An inventory for measuring depression. Archives of General Psychiatry 1961;4:561-71.

\section{Beck 1979}

Beck AT, Rush AJ, Shaw BF, Emery G. Cognitive Therapy for Depression. New York: Guilford Press, 1979. 


\section{Birleson 1987}

Birleson P, Hudson I, Grey-Buchanan D, Wolff S. Clinical evaluation of a self-rating scale for depressive disorder in childhood (Depression Self-Rating Scale). Journal of Child Psychology and Psychiatry 1987;28:43-60.

\section{Birmaher 1997}

Birmaher B, Khetarpal S, Brent D, Cully M, Balach L, Kaufman J, et al. The Screen for Child Anxiety Related Emotional Disorders (SCARED): scale construction and psychometric characteristics. Journal of the American Academy of Child \& Adolescent 1997;36(4):545-53.

\section{Bisson 2013}

Bisson JI, Roberts NP, Andrew M, Cooper R, Lewis C. Psychological therapies for chronic posttraumatic stress disorder (PTSD) in adults. Cochrane Database of Systematic Reviews 2013, Issue 12. [DOI: 10.1002/14651858.CD003388.pub4]

\section{Blake 1995}

Blake DD, Weathers FW, Nagy LM, Kaloupek DG, Gusman FD, Charney DS, et al. The development of a clinician-administered PTSD scale. Journal of Traumatic Stress 1995;8(1):75-90.

\section{Brom 1989}

Brom D, Kleber RJ, Defares PB. Brief psychotherapy for posttraumatic stress disorders. Journal of Consulting and Clinical Psychology 1989;57(5):607-12.

\section{Callahan 2000}

Callahan RJ, Callahan J. Stop the nightmares of trauma. Stop the Nightmares of Trauma. Chapel Hill, NC: Professional Press, 2000.

\section{Charlson 2016}

Charlson FJ, Flaxman A, Ferrari AJ, Vos T, Steel Z, Whiteford HA. Post-traumatic stress disorder and major depression in conflictaffected populations: an epidemiological model and predictor analysis. Global Mental Health 2016;3:e4. [DOI: 10.1017] gmh.2015.26]

\section{Cohen 1992}

Cohen J. A power primer. Psychological Bulletin 1992;112(1):155-9.

\section{Colliard 2014}

Colliard C, Bizouerne C, Corna F, ACF Mental Health and Care Practices Team. The Psychological Impact of Humanitarian Crises: A Better Understanding for Better Interventions. Paris: Action contre la Faim - France, 2014.

\section{Cuijpers 2009}

Cuijpers P, van Straten A, Warmerdam L, Andersson G. Psychotherapy versus the combination of psychotherapy and pharmacotherapy in the treatment of depression: a metaanalysis. Depression and Anxiety 2009;26(3):279-88.

\section{Dennis 2007}

Dennis CL, Hodnett E. Psychosocial and psychological interventions for treating postpartum depression. Cochrane
Database of Systematic Reviews 2007, Issue 4. [DOI:

10.1002/14651858.CD006116.pub2]

\section{DePaulo 2014}

DePaulo JR, Bienvenu OJ (editors). The Johns Hopkins Psychiatry Guide. In: DePaulo JR, Bienvenu OJ editor(s). The Johns Hopkins Psychiatry Guide. Baltimore: Johns Hopkins, 2014.

\section{der Simonian 1986}

der Simonian R, Laird N. Meta-analysis in clinical trials. Controlled Clinical Trials 1986;7(3):177-88.

\section{Fergusson 2005}

Fergusson DM, Horwood LJ, Ridder EM, Beautrais AL. Suicidal behaviour in adolescence and subsequent mental health outcomes in young adulthood. Psychological Medicine 2005;35(7):983-93.

\section{Forbes 2010}

Forbes D, Creamer M, Bisson JI, Cohen JA, Crow BE, Foa EB, et al. A guide to guidelines for the treatment of PTSD and related conditions. Journal of Traumatic Stress 2010;23(5):537-52.

\section{Furukawa 2002}

Furukawa TA, Guyatt GH, Griffith LE. Can we individualize the 'number needed to treat'? An empirical study of summary effect measures in meta-analyses. International Journal of Epidemiology 2002;31(1):72-6.

\section{Gierk 2014}

Gierk B, Kohlmann S, Kroenke K, Spangenberg L, Zenger M, Brähler E, et al. The Somatic Symptom Scale-8 (SSS-8): a brief measure of somatic symptom burden. JAMA Internal Medicine 2014;174(3):399-407.

\section{Gillies 2016}

Gillies D, Maiocchi L, Bhandari AP, Taylor F, Gray C, O'Brien L. Psychological therapies for the treatment of post-traumatic stress disorder in children and adolescents. Cochrane Database of Systematic Reviews 2016, Issue 10. [DOI: 10.1002/14651858.CD006726.pub2]

\section{Gloaguen 1998}

Gloaguen V, Cottraux J, Cucherat M, Blackburn IM. A metaanalysis of the effects of cognitive therapy in depressed patients. Journal of Affective Disorders 1998;49:59-72.

\section{Guha-Sapir 2014}

Guha-Sapir D, Hoyois P, Below R. Annual Disaster Statistical Review 2013: the numbers and trends. reliefweb.int/report/ world/annual-disaster-statistical-review-2013-numbers-andtrends.

\section{Hamilton 1960}

Hamilton M. A rating scale for depression. Journal of Neurology, Neurosurgery and Psychiatry 1960;23:56-62.

\section{Haroz 2016}

Haroz EE, Ritchey M, Bass JK, Kohrt BA, Augustinavicius J, Michalopoulos L, et al. How is depression experienced around

Psychological therapies for the treatment of mental disorders in low- and middle-income countries affected by humanitarian crises 
the world? A systematic review of qualitative literature. Social Science and Medicine 2016;16:1-12.

\section{Higgins 2011}

Higgins JP, Green S, editors. Cochrane Handbook for Systematic Reviews of Interventions. Chichester: John Wiley \& Sons, Ltd, 2011.

\section{Hoskins 2015}

Hoskins M, Pearce J, Bethell A, Dankova L, Barbui C, Tol WA, et al. Pharmacotherapy for post-traumatic stress disorder: systematic review and meta-analysis. British Journal of Psychiatry 2015;206(2):93-100.

\section{IASC 2007}

Inter-Agency Standing Committee (IASC). IASC guidelines on mental health and psychosocial support in emergency settings. www.who.int/hac/network/interagency/news/iasc_guidelines_ mental_health_psychososial.pdf?ua=1 2007.

\section{Jakupcak 2010}

Jakupcak M, Wagner A, Paulson A, Varra A, McFall M. Behavioral activation as a primary care-based treatment for PTSD and depression among returning veterans. Journal of Traumatic Stress 2010;23(4):491-5.

\section{James 2015}

James AC, James G, Cowdrey FA, Soler A, Choke A. Cognitive behavioural therapy for anxiety disorders in children and adolescents. Cochrane Database of Systematic Reviews 2015, Issue 2. [DOI: 10.1002/14651858.CD004690.pub4]

\section{Jones 2012}

Jones L, Bellis MA, Wood S, Hughes K, McCoy E, Eckley L, et al. Prevalence and risk of violence against children with disabilities: a systematic review and meta-analysis of observational studies. The Lancet 2012;380(9845):899-907.

\section{Jordans 2016}

Jordans M, Pigott $\mathrm{H}$, Tol WA. Interventions for children affected by armed conflict: a systematic review of mental health and psychosocial support in low- and middle-income countries. Current Psychiatry Report 2016;18(9):1-15.

\section{Josse 2009}

Josse E, Dubois V. Interventions Humanitaires en Santè Mentale Dans Les Violences de Masse. Bruxelles: Groupe de Boeck, 2009.

\section{Jüni 2001}

Jüni P, Altman DG, Egger M. Systematic reviews in health care: assessing the quality of controlled clinical trials. British Medical Journal 2001;323:42-6.

\section{Kaiser 2015}

Kaiser BN, Haroz EE, Kohrt BA, Bolton PA, Bass JK, Hinton DE. "Thinking too much": a systematic review of a common idiom of distress. Social Science and Medicine 2015;147:170-83.

\section{Kane 2017}

Kane JC, Luitel NP, Jordans MJ, Kohrt BA, Weissbecker J, Tol WA. Mental health and psychosocial problems in the aftermath of Nepal earthquakes: findings from a representative cluster sample survey. Epidemiology and Psychiatric Sciences 2017;9:1-10.

\section{Kohrt 2013}

Kohrt BA, Rasmussen A, Kaiser BN, Haroz EE, Maharjan SM, Mutamba BB, et al. Cultural concepts of distress and psychiatric disorders: literature review and research recommendations for global mental health epidemiology. International Journal of Epidemiology 2013;43(2):365-406.

\section{Kroenke 2002}

Kroenke K, Spitzer RL, Williams JB. The PHQ-15: validity of a new measure for evaluating the severity of somatic symptoms. Psychosomatic Medicine 2002;64(2):258-66.

\section{Langendam 2013}

Langendam MW, Akl EA, Dahm P, Glasziou P, Guyatt G, Schünemann HJ. Assessing and presenting summaries of evidence in Cochrane Reviews. Systematic Reviews 2013;23(2):81.

\section{Markowitz 2004}

Markowitz JC, Weissman MM. Interpersonal psychotherapy: principles and applications. World Psychiatry 2004;3(3):136-9.

\section{Markowitz 2014}

Markowitz JC, Lipsitz J, Milrod B. Critical review of outcome research on interpersonal psychotherapy for anxiety disorders. Depression and Anxiety 2014;31(4):316-25.

\section{Markowitz 2015}

Markowitz JC, Petkova E, Neria Y, Van Meter PE, Zhao Y, Hembree $\mathrm{E}$, et al. Is exposure necessary? A randomized clinical trial of interpersonal psychotherapy for PTSD. American Journal of Psychiatry 2015;172(5):430-40.

\section{Miller 2016}

Miller K, Rasmussen A. The mental health of civilians displaced by armed conflict: an ecological model for refugee distress. Epidemiology and Psychiatric Sciences 2016;4:1-10.

\section{Mollica 1992}

Mollica RF, Caspi-Yavin Y, Bollini P, Truong T, Tor S, Lavelle J. The Harvard Trauma Questionnaire. Validating a cross-cultural instrument for measuring torture, trauma, and posttraumatic stress disorder in Indochinese refugees. Journal of Nervous \& Mental Disease 1992;180(2):111-6.

\section{Montgomery 1979}

Montgomery SA, Asberg M. A new depression scale designed to be sensitive to change. British Journal of Psychiatry 1979;134:382-9.

\section{Montgomery 2010}

Montgomery EC, Kunik ME, Wilson N, Stanley MA, Weiss B. Can paraprofessionals deliver cognitive-behavioral therapy to treat anxiety and depressive symptoms?. Bulletin of the Menninger Clinic 2010;74(1):45-62.

Psychological therapies for the treatment of mental disorders in low- and middle-income countries affected by humanitarian crises 


\section{Morina 2017}

Morina N, Malek M, Nickerson A, Bryant RA. Psychological interventions for post-traumatic stress disorder and depression in young survivors of mass violence in low- and middleincome countries: meta-analysis. British Journal of Psychiatry 2017;210:247-54.

\section{Murray 2014}

Murray LK, Dorsey S, Haroz E, Lee C, Alsiary MM, Haydary A, et al. A common elements treatment approach for adult mental health problems in low- and middle-income countries. Cognitive Behavioral Practice 2014;21(2):111-23.

\section{Nader 1996}

Nader K, Kriegler JA, Blake DD, Pynoos RS, Newman E, Weather FW. Clinician administered PTSD scale, child and adolescent version. White River Junction (VT): National Center for PTSD; 1996.

\section{O'Donnell 2014}

O'Donnell ML, Alkemade N, Nickerson A, Creamer M, McFarlane AC, Silove D, et al. Impact of the diagnostic changes to post-traumatic stress disorder for DSM-5 and the proposed changes to ICD-11. British Journal of Psychiatry 2014;205(3):230-5.

\section{Olthuis 2015}

Olthuis JV, Watt MC, Bailey K, Hayden JA, Stewart SH. Therapistsupported Internet cognitive behavioural therapy for anxiety disorders in adults. Cochrane Database of Systematic Reviews 2015, Issue 3. [DOI: 10.1002/14651858.CD011565]

\section{Patel 2014}

Patel N, Kellezi B, Williams AC. Psychological, social and welfare interventions for psychological health and well-being of torture survivors. Cochrane Database of Systematic Reviews 2014, Issue 11. [DOI: 10.1002/14651858.CD009317.pub2]

\section{Purgato 2010}

Purgato M, Barbui C, Cipriani A. Assessing risk of bias in randomized controlled trials. Epidemiologia e Psichiatria Sociale 2010;19(4):296-7.

\section{Purgato 2012}

Purgato M, Adams CE. Heterogeneity: the issue of apples, oranges and fruit pie. Epidemiology and Psychiatric Sciences 2012;21(1):27-9.

\section{Purgato 2015}

Purgato M, Gastaldon C, Papola D, van Ommeren M, Barbui C, Tol WA. Psychological therapies for the treatment of mental disorders in low- and middle-income countries affected by humanitarian crises. Cochrane Database of Systematic Reviews 2015, Issue 10. [DOI: 10.1002/14651858.CD011849]

\section{Purgato 2016a}

Purgato M, Gastaldon C, Papola, van Ommeren M, Barbui C, Tol WA. Psychological and social interventions for the prevention of mental disorders in people living in low- and middle-income countries affected by humanitarian crises.
Cochrane Database of Systematic Reviews 2016, Issue 11. [DOI: 10.1002/14651858.CD012417]

\section{Purgato 2016b}

Purgato M, Tol WA, Bass JK. An ecological model for refugee mental health: implications for research. Epidemiology and Psychiatric Sciences 2016;19:1-3.

\section{Purgato 2018}

Purgato M, Gross AL, Betancourt T, Bolton P, Bonetto C, Gastaldon C, et al. Focused psychosocial interventions for children in low-resource humanitarian settings: a systematic review and individual participant data meta-analysis. Lancet Global Health 2018;6(4):e390-e400. [DOI: 10.1016/ S2214-109X(18)30046-9]

\section{Rigmor 2010}

Rigmor CB, Bjørg H. Effectiveness of psychotherapy for adults with depression: a systematic review of the best available evidence. Procedia - Social and Behavioral Sciences 2010;5:2194-200.

\section{Savović 2012}

Savović J, Jones HE, Altman DG, Harris RJ, Jüni P, Pildal J, et al. Influence of reported study design characteristics on intervention effect estimates from randomized, controlled trials. Annals of Internal Medicine 2012;157(6):429-38.

\section{Schauer 2011}

Schauer M, Neuner F, Elbert T. Narrative Exposure Therapy: A short Term Treatment For Traumatic Stress Disorders (2nd edition). Narrative Exposure Therapy: A Short Term Treatment for Traumatic Stress Disorders. 2nd edition. Cambridge, MA: Hogrefe Publishing, 2011.

\section{Sheehan 1998}

Sheehan DV, Lecrubier Y, Sheehan KH, Amorim P, Janavs J, Weiller E, et al. The Mini-International Neuropsychiatric Interview (M.I.N.I.): the development and validation of a structured diagnostic psychiatric interview for DSM-IV and ICD-10. Journal of Clinical Psychiatry 1998;59(20):22-33.

\section{The Lancet 2016}

The Lancet. Health - an explicit human right. The Lancet 2016;387:917.

\section{The Sphere Project 2011}

The Sphere Project. Humanitarian charter and minimum standards in humanitarian response. www.sphereproject.org/. Accessed 3 August 2015

\section{Themner 2014}

Themner L, Wallensteen P. Armed conflicts, 1946-2013. Journal of Peace Research 2014;51(4):541-54.

\section{Tol 2011}

Tol WA, Barbui C, Galappatti A, Silove D, Betancourt TS, Souza R, et al. Mental health and psychosocial support in humanitarian settings: linking practice and research. The Lancet 2011;378(9802):1581-91. 


\section{Tol 2011a}

Tol WA, Komproe IH, Jordans MJ, Susanty D, de Jong JT. Developing a function impairment measure for children affected by political violence: a mixed methods approach in Indonesia. International Journal for Quality in Health Care 2011;23(4):375-83.

\section{Ukoumunne 1999}

Ukoumunne OC, Gulliford MC, Chinn S, Sterne JA, Burney PG. Methods for evaluating area-wide and organisation-based interventions in health and health care: a systematic review. Health Technology Assessment 1999;3(5):92.

\section{UN General Assembly 1996}

UN General Assembly. Impact of armed conflict on children. www.refworld.org/docid/3b00f2d30.html. Accessed 23 March 2017.

\section{UNICEF 2009}

Office of the Special Representative of the Secretary-General for Children and Armed Conflict in collaboration with UNICEF. Machel Study 10-Year Strategic Review: Children and Conflict in a Changing World. www.unicef.org/publications/ index_49985.html. Accessed 3 August 2015.

\section{US Department of Health and Human Services 2014}

US Department of Health and Human Services. TraumaInformed Care in Behavioral Health Services. Rockville, MD: SAMHSA Publications, 2014.

\section{van Dessel 2014}

van Dessel N, den Boeft M, van der Wouden JC, Kleinstäuber M, Leone SS, Terluin B, et al. Non-pharmacological interventions for somatoform disorders and medically unexplained physical symptoms (MUPS) in adults. Cochrane Database of Systematic Reviews 2014, Issue 11. [DOI: 10.1002/14651858.CD011142.pub2]

\section{Watanabe 2007}

Watanabe N, Hunot V, Omori IM, Churchill R, Furukawa TA. Psychotherapy for depression among children and adolescents: a systematic review. Acta Psychiatrica Scandinavica 2007;116(2):84-95.

\section{Watts 2015}

Watts SE, Turnell A, Kladnitski N, Newby JM, Andrews G. Treatment-as-usual (TAU) is anything but usual: a meta-analysis of CBT versus TAU for anxiety and depression. Journal of Affective Disorders 2015;175:152-67.

\section{Weathers 1993}

Weathers F, Litz B, Herman D, Huska J, Keane T. The PTSD Checklist (PCL): reliability, validity, and diagnostic utility. Annual Convention of the International Society for Traumatic Stress Studies; San Antonio, TX, October 1993. International Society for Traumatic Stress Studies.

\section{Weiss 1997}

Weiss D, Marmar C. The impact of event scale-revised. In: Wilson, JP editor(s). Assessing Psychological Trauma and PTSD. New York: Guilford Press, 1997:399-411.

\section{WHO 1978}

World Health Organization (WHO). The Ninth Revision of the International Classification of Diseases and Related Health Problems (ICD-9). Geneva: WHO, 1978.

\section{WHO 1992}

World Health Organization (WHO). The ICD-10 Classification of Mental and Behavioural Disorders. Geneva: WHO, 1992.

\section{WHO 1997}

Division of mental health and prevention of substance abuse World Health Organization (WHO). WHOQOL. Measuring Quality of Life. Geneva: WHO, 1997.

\section{WHO 2010}

World Health Organization (WHO). Measuring Health and Disability: Manual for WHO Disability Assessment Schedule 2.0 (WHODAS 2.0). In: Ustun TB, Kostanjsek N, Chatterji S, Rehm J editor(s). Measuring Health and Disability: Manual for WHO Disability Assessment Schedule 2.0 (WHODAS 2.0). Geneva: WHO, 2010.

\section{WHO 2012}

World Health Organization (WHO). Assessing Mental Health and Psychosocial Needs and Resources: Assessment Toolkit for Humanitarian Settings. Geneva: WHO, 2012.

\section{WHO 2013}

World Health Organization (WHO). Guidelines for the Management of Conditions Specifically Related to Stress. Geneva: WHO, 2013.

\section{WHO 2016}

World Health Organization (WHO). mhGAP Intervention Guide for Mental, Neurological and Substance Use Disorders in Non-specialized Health Settings. Vol. Version 2.0, Geneva: Department of Mental Health and Substance Abuse, WHO, 2016.

\section{World Bank 2013}

World Bank. Country and leading groups. data.worldbank.org/ about/country-classifications/country-and-lending-groups. Accessed 6 May 2014.

\section{Zigmond 1983}

Zigmond AS, Snaith RP. The hospital anxiety and depression scale. Acta Psychiatrica Scandinavica 1983;67(6):361-70.

* Indicates the major publication for the study 
CHARACTERISTICS OF STUDIES

Characteristics of included studies [ordered by study ID]

Acarturk 2015

\begin{tabular}{|c|c|}
\hline Methods & Single-blind, parallel group, open-label RCT \\
\hline Participants & $\begin{array}{l}\text { Refugees with PTSD symptoms forced to leave Syria owing to the war that started in } 2011 \text {; living in Kilis } \\
\text { Refugee Camp. The camp is located at the border between Turkey and Syria. Refugees, in addition to } \\
\text { displacement and lack of shelter, have been exposed to traumatic events such as torture, serious injury } \\
\text { to self or loved ones, death of family members, husband at war, and arrested family members. This pi- } \\
\text { lot RCT was conducted between April } 2013 \text { and July } 2013\end{array}$ \\
\hline
\end{tabular}

Age range: $19-63$ years

Inclusion criteria: 18 years and older, PTSD symptoms (Impact of Event Scale-Revised - IES-R score 33)

Exclusion criteria: mental retardation, pregnant, using psychiatric medication

Interventions

Eye movement desensitisation and reprocessing (EMDR) intervention: 15 participants

EMDR given in different phases, including history taking, case formulation, and treatment planning; EMDR explanation; trauma memory selection; desensitisation; reporting of current emotions, sensations, and cognitions; and instillation of positive cognition. The closure phase in focused on relaxation

Wait list control group: 14 participants

Wait-list participants did not receive any psychological or pharmacological treatment. They were informed that at the end of the study, they could receive psychological help from the research team

Outcomes

IES-R scores for the EMDR group and the wait list control group. Symptoms of depression as measured with the Beck Depression Inventory-II (BDI-II)

\section{Notes}

\section{Risk of bias}

\begin{tabular}{|c|c|c|}
\hline Bias & Authors' judgement & Support for judgement \\
\hline $\begin{array}{l}\text { Random sequence genera- } \\
\text { tion (selection bias) }\end{array}$ & Low risk & $\begin{array}{l}\text { Quote: "For the allocation of participants to different treatment groups, a com- } \\
\text { puter-generated random number list was used. Participants were randomly } \\
\text { assigned on a 1:1 basis to the EMDR or wait-list group" }\end{array}$ \\
\hline $\begin{array}{l}\text { Allocation concealment } \\
\text { (selection bias) }\end{array}$ & Unclear risk & No information provided \\
\hline $\begin{array}{l}\text { Blinding of participants } \\
\text { and personnel (perfor- } \\
\text { mance bias) } \\
\text { All outcomes }\end{array}$ & High risk & Quote: "The participants and the therapists were aware of the allocated arm" \\
\hline $\begin{array}{l}\text { Blinding of outcome as- } \\
\text { sessment (detection bias) } \\
\text { All outcomes }\end{array}$ & Low risk & Quote: "The outcome assessors were kept blind to the allocation" \\
\hline $\begin{array}{l}\text { Incomplete outcome data } \\
\text { (attrition bias) } \\
\text { All outcomes }\end{array}$ & Low risk & No one withdrew from the study \\
\hline
\end{tabular}


Acarturk 2015 (Continued)

Selective reporting (re- Low risk Rating scales listed in the "Methods" section were reported in the "Results" porting bias) section (IER-R and BDI-II)

\begin{tabular}{|c|c|c|}
\hline Therapist qualification & Low risk & Trained psychologists \\
\hline Treatment fidelity & Low risk & $\begin{array}{l}\text { Quote: "The supervisor personally observed a minimum of one session } \\
\text { with each therapist (with the permission of the participant). The supervisor } \\
\text { checked during live and normal one-on-one and group supervision sessions } \\
\text { whether the therapists were complying with the } 8 \text { Phase EMDR standard pro- } \\
\text { tocol. Treatment fidelity was supported by the supervisor, who attended at } \\
\text { least one session of each therapist" }\end{array}$ \\
\hline
\end{tabular}

\begin{tabular}{lll}
\hline $\begin{array}{l}\text { Therapist/investigator al- } \\
\text { legiance }\end{array}$ & High risk & $\begin{array}{l}\text { Psychologists were trained at EMDR level I by the second trial author, who is } \\
\text { an EMDR Institute-accredited trainer. No further information provided }\end{array}$ \\
\hline Other bias & Unclear risk & Sponsorship bias cannot be ruled out \\
\hline
\end{tabular}

\section{Acaturk 2016}

\begin{tabular}{ll}
\hline Methods & Single-blind, parallel-group, open-label RCT \\
\hline Participants & $\begin{array}{l}\text { Refugees with PTSD symptoms forced to leave Syria owing to the war that started in 2011; living in Kilis } \\
\text { Refugee Camp. The camp is located at the border between Turkey and Syria. Refugees, in addition to } \\
\text { displacement and lack of shelter, have been exposed to traumatic events such as torture, serious in- } \\
\text { jury to self or loved ones, death of family members, husband at war, and arrested family members The } \\
\text { study was conducted between September } 2013 \text { and June } 2014\end{array}$
\end{tabular}

Age range: $19-63$ years

Inclusion criteria: diagnosis of PTSD according to the Diagnostic and Statistical Manual of Mental Disorders, 4th edition (DSM-IV); 18 years of age and older

Exclusion criteria: diagnosis of psychotic disorder or substance abuse according to DSM-IV; pregnant; any psychotherapy during the trial; concurrent use of any psychotropic medication during the trial

Interventions Eye movement desensitisation and reprocessing (EMDR) intervention: 49 participants

EMDR given in different phases, including history taking, case formulation, and treatment planning; EMDR explanation; trauma memory selection; desensitisation; reporting of current emotions, sensations, and cognitions; and instillation of positive cognition. The closure phase is focused on relaxation

Wait list control group: 49 participants

Wait list participants did not receive any psychological or pharmacological treatment. They were informed that at the end of the study, they could receive psychological help from the research team

Exposure to traumatic events and PTSD symptoms measured with the Harvard Trauma Questionnaire
(HTQ) and the Impact of Event Scale - Revised (IES-R); symptoms of depression were measured with the
Beck Depression Inventory (BDI-II) and the Hopkins Symptoms Checklist (HSCL-25)

Notes

\section{Risk of bias}

Bias Authors' judgement Support for judgement


Acaturk 2016 (Continued)

Random sequence genera- Low risk Quote: "After including the participants, another researcher, not involved in tion (selection bias) the current study, used a computer generated random-number list for the allocation of participants to different treatment groups"

\begin{tabular}{lll}
\hline $\begin{array}{l}\text { Allocation concealment } \\
\text { (selection bias) }\end{array}$ & Unclear risk & No information provided \\
\hline $\begin{array}{l}\text { Blinding of participants } \\
\text { and personnel (perfor- } \\
\text { mance bias) }\end{array}$ & High risk & $\begin{array}{l}\text { Quote: "The participants and the therapists were necessarily aware of the allo- } \\
\text { cated arm" }\end{array}$ \\
\begin{tabular}{l} 
All outcomes \\
\hline
\end{tabular} &
\end{tabular}

\begin{tabular}{lll}
\hline $\begin{array}{l}\text { Blinding of outcome as- } \\
\text { sessment (detection bias) } \\
\text { All outcomes }\end{array}$ & Low risk & Quote: "The outcome assessors were kept blind to the allocation" \\
\hline $\begin{array}{l}\text { Incomplete outcome data } \\
\text { (attrition bias) } \\
\text { All outcomes }\end{array}$ & High risk & $\begin{array}{l}\text { The dropout rate was higher than 20\%. Dropouts in intervention group: 12/49; } \\
\text { in control group: 16/49 }\end{array}$ \\
\hline $\begin{array}{l}\text { Selective reporting (re- } \\
\text { porting bias) }\end{array}$ & Low risk & All outcomes are reported \\
\hline $\begin{array}{l}\text { Therapist qualification } \\
\text { Treatment fidelity }\end{array}$ & Low risk & Low risk \\
$\begin{array}{ll}\text { Therapist/investigator al- } \\
\text { legiance }\end{array}$ & High risk & $\begin{array}{l}\text { Trained psychologists } \\
\text { Quote: "The supervisor checked whether the therapists were complying with } \\
\text { the protocol during at least one live session of each therapist and conducted } \\
\text { one-to-one and group supervision sessions" }\end{array}$ \\
\hline $\begin{array}{l}\text { Other bias } \\
\text { Psychologists were trained in EMDR (level II) and R-TEP (Recent Traumatic }\end{array}$ \\
\hline $\begin{array}{l}\text { Episode Protocol) by the second trial author, who is an EMDR Institute-accred- } \\
\text { ited trainer. No further information provided }\end{array}$ \\
\hline \begin{tabular}{l} 
The study appears to be free from other sources of bias \\
\hline
\end{tabular}
\end{tabular}

Ahmadizadeh 2013

\begin{tabular}{ll}
\hline Methods & RCT \\
\hline Participants & $\begin{array}{l}\text { Iranian veterans in } 8 \text { years of consecutive Iraq-Iran War combat (1982-1989), 25-50 years of age, with di- } \\
\text { agnosis of PTSD merely or PTSD with concomitant depression (diagnosed with DSM-IV-TR). They pre- } \\
\text { sented a variety of concerns to the Deployment Health Clinic of Bonyad-e-Shahid and Sepah Pasdaran } \\
\text { between } 2005 \text { and } 2006 \text { in Tehran }\end{array}$ \\
\hline
\end{tabular}

Problem-solving therapy: 25 participants
Exposure therapy: 25 participants
Combined therapy (exposure therapy plus problem solving): 25 participants
Control group: 25 participants

Outcomes

Quality of life using Quality of Life (QoL) questionnaire; psychiatric problems using Symptom Checklist 90-Revised (SCL90-R)

\section{Notes}

Psychological therapies for the treatment of mental disorders in low- and middle-income countries affected by humanitarian crises 
Ahmadizadeh 2013 (Continued)

\section{Risk of bias}

\begin{tabular}{|c|c|c|}
\hline Bias & Authors' judgement & Support for judgement \\
\hline $\begin{array}{l}\text { Random sequence genera- } \\
\text { tion (selection bias) }\end{array}$ & Unclear risk & Quote: "Were randomly allocated to one of four equal interventional groups" \\
\hline $\begin{array}{l}\text { Allocation concealment } \\
\text { (selection bias) }\end{array}$ & Unclear risk & No information provided \\
\hline $\begin{array}{l}\text { Blinding of participants } \\
\text { and personnel (perfor- } \\
\text { mance bias) } \\
\text { All outcomes }\end{array}$ & Unclear risk & No information provided \\
\hline $\begin{array}{l}\text { Blinding of outcome as- } \\
\text { sessment (detection bias) } \\
\text { All outcomes }\end{array}$ & Unclear risk & No information provided \\
\hline $\begin{array}{l}\text { Incomplete outcome data } \\
\text { (attrition bias) } \\
\text { All outcomes }\end{array}$ & Unclear risk & No information provided \\
\hline $\begin{array}{l}\text { Selective reporting (re- } \\
\text { porting bias) }\end{array}$ & High risk & $\begin{array}{l}\text { Study report declares that } 120 \text { patients were included in the study but later } \\
\text { adds that each of } 4 \text { study groups was composed of } 25 \text { individuals }\end{array}$ \\
\hline Therapist qualification & Low risk & Intervention was delivered by "expert therapists" \\
\hline Treatment fidelity & Unclear risk & No information provided \\
\hline $\begin{array}{l}\text { Therapist/investigator al- } \\
\text { legiance }\end{array}$ & Unclear risk & No information provided \\
\hline Other bias & Unclear risk & No information provided \\
\hline
\end{tabular}

Azad Marzabadi 2014

\begin{tabular}{ll}
\hline Methods & RCT \\
\hline Participants & $\begin{array}{l}\text { Warfare victims in Iran-Iraq War, with diagnosis of PTSD and suffering from war-related psychological } \\
\text { disorders. Study was conducted in 2012 at Shahid Rajaee Hospital (Iran) }\end{array}$ \\
\hline Interventions & Mindfulness intervention: mindfulness-based stress reduction training: 14 participants \\
& Wait list control: 14 participants \\
\hline Outcomes & World Health Organization Quality of Life Questionnaire (26-item) \\
\hline Notes & Randomisation procedure and data reporting unclear
\end{tabular}

\section{Risk of bias}

\section{Bias \\ Authors' judgement Support for judgement}


Azad Marzabadi 2014 (Continued)

$\begin{array}{ll}\begin{array}{l}\text { Random sequence genera- } \\ \text { tion (selection bias) }\end{array} & \text { Low risk } \\ \end{array}$

\begin{tabular}{|c|c|c|}
\hline $\begin{array}{l}\text { Allocation concealment } \\
\text { (selection bias) }\end{array}$ & Unclear risk & $\begin{array}{l}\text { Quote: "hidden and confidential assignment." No further information provid- } \\
\text { ed on how allocation was concealed }\end{array}$ \\
\hline
\end{tabular}

\begin{tabular}{|c|c|c|}
\hline $\begin{array}{l}\text { Blinding of participants } \\
\text { and personnel (perfor- }\end{array}$ & Unclear risk & $\begin{array}{l}\text { Quote: "after the intervention [group] and the control group [were] deter- } \\
\text { mined, participants had no more information about the research process" }\end{array}$ \\
\hline
\end{tabular}

mance bias)

All outcomes

\begin{tabular}{lll}
\hline $\begin{array}{l}\text { Blinding of outcome as- } \\
\text { sessment (detection bias) } \\
\text { All outcomes }\end{array}$ & Unclear risk & No information provided \\
\hline $\begin{array}{l}\text { Incomplete outcome data } \\
\text { (attrition bias) }\end{array}$ & Low risk & $\begin{array}{l}\text { Dropouts were reported (2/16 in the intervention group; } 2 / 16 \text { in the wait list } \\
\text { control group) }\end{array}$
\end{tabular}

\begin{tabular}{|c|c|c|}
\hline $\begin{array}{l}\text { Selective reporting (re- } \\
\text { porting bias) }\end{array}$ & Low risk & Outcomes listed in the "Methods" were also reported in the "Results" \\
\hline Therapist qualification & Unclear risk & No information provided on therapist qualification and background \\
\hline Treatment fidelity & Unclear risk & No information on how fidelity to treatment was recorded/checked \\
\hline $\begin{array}{l}\text { Therapist/investigator al- } \\
\text { legiance }\end{array}$ & Unclear risk & No information provided \\
\hline Other bias & Unclear risk & No information provided \\
\hline
\end{tabular}

\section{Basoglu 2005}

\begin{tabular}{ll}
\hline Methods & Six-week, single-blind RCT \\
\hline Participants & $\begin{array}{l}\text { Earthquake survivors in Turkey. In 1999, an earthquake of magnitude } 7.4 \text { on the Richter scale occurred } \\
\text { in the northwestern part of Turkey, causing more than } 17,000 \text { deaths and } 44,000 \text { injuries. This region } \\
\text { was hit by a second earthquake in November } 1999, \text { which caused additional thousands of deaths and } \\
\text { injuries. This study was conducted 3 years after the first major earthquake occurred }\end{array}$ \\
& Age range: $16-65$ years (mean age 36.3, SD 11.5) \\
Inclusion criteria: TSSC score higher than 20, literate, diagnosis of PTSD according to DSM-IV criteria & Exclusion criteria: alcohol or drug dependence, severe depression with suicidal intent, psychotic ill- \\
& ness, predominating grief, use of benzodiazepines, use of a stable dose of antidepressants for less than \\
& 2 months at the time of assessment, and previous CBT for earthquake-related traumatic stress prob- \\
lems & Modified behavioural treatment (SSBT): 31 participants \\
& Treatment employed a shorter version of CBT, which was modified by limiting cognitive interventions \\
to explanation of the treatment rationale only; focusing on reduction of fear and avoidance; and shift- \\
ing focus from habituation to anxiogenic stimuli to enhancement of sense of control over traumatic \\
stressors \\
Wait list condition: 28 participants
\end{tabular}

Psychological therapies for the treatment of mental disorders in low- and middle-income countries affected by humanitarian crises 
Basoglu 2005 (Continued)

Outcomes
Semi-Structured Interview for Survivors of Earthquake; Structured Clinical Interview for DSM-IV (Major Depressive Episode Module; SCID-I/NP, Version 2); Clinician-Administered PTSD Scale (CAPS); and Clinician's Global Impression-Improvement (CGI)

Self-rated measures included TSSC; Fear and Avoidance Questionnaire (FAQ); Beck Depression Inventory (BDI); Work and Social Adjustment Scale (WSA); and Patient's Global Impression/Improvement

Notes

\section{Risk of bias}

\begin{tabular}{|c|c|c|}
\hline Bias & Authors' judgement & Support for judgement \\
\hline \multirow[t]{2}{*}{$\begin{array}{l}\text { Random sequence genera- } \\
\text { tion (selection bias) }\end{array}$} & Unclear risk & $\begin{array}{l}\text { Quote: "Random allocation was conducted according to a computer-generat- } \\
\text { ed randomisation list. Blocking was used to ensure approximately equal cell } \\
\text { sizes" "Whenever a participant was not available for the second assessment } \\
(\mathrm{N}=10) \text {, she or he was replaced by the next eligible individual" }\end{array}$ \\
\hline & & No further information \\
\hline $\begin{array}{l}\text { Allocation concealment } \\
\text { (selection bias) }\end{array}$ & Low risk & $\begin{array}{l}\text { Quote: "The participants were recruited into the study by four independent as- } \\
\text { sessors, who did not have access to the random assignment schedule" }\end{array}$ \\
\hline $\begin{array}{l}\text { Blinding of participants } \\
\text { and personnel (perfor- } \\
\text { mance bias) } \\
\text { All outcomes }\end{array}$ & High risk & $\begin{array}{l}\text { Quote: "Blindness could not be maintained in } 11(19 \%) \text { cases ( } 8 \text { SSBT and } 3 \\
\text { WL), often because the participants unintentionally revealed their experimen- } \\
\text { tal condition. These cases did not significantly differ in assessor-rated treat- } \\
\text { ment outcome from the others, suggesting that unblinding did not affect the } \\
\text { assessors' ratings" }\end{array}$ \\
\hline
\end{tabular}

Blinding of outcome as- Unclear risk sessment (detection bias) All outcomes
Quote: "The relationship between correct guessing and the assessors' ratings of clinical outcome could not be examined because there were too few people in each group whose treatment condition was incorrectly guessed. Although correct guessing may have biased the assessors' ratings, such bias did not seem to lead to a substantial difference between assessor and patient ratings of the same constructs, as evidenced by the magnitude of correlations between the two ratings of global improvement, as well as between the TSSC and CAPS scores (ranging from .69 to .97; all $\mathrm{P}<.001$ ) at all follow-up points"

At baseline, 5 participants refused treatment on grounds of "feeling better"; at week 6 , there were 2 dropouts - 1 prescribed. No further information provided
Incomplete outcome data Unclear risk (attrition bias)

All outcomes
Primary outcomes are reported

Selective reporting (re- Low risk $\quad$ Primary outcomes are reported
porting bias)

\begin{tabular}{lll}
\hline Therapist qualification Low risk & Trained psychologists
\end{tabular}

\begin{tabular}{ll}
\hline Treatment fidelity $\quad$ Unclear risk & $\begin{array}{l}\text { Quote: "Nineteen of the treatment sessions could be audiotaped; all of them } \\
\text { were assessed for compliance with the treatment protocol and rated as satis- } \\
\text { factory" }\end{array}$ \\
\hline
\end{tabular}

Therapist/investigator al- Unclear risk No information provided
legiance

\begin{tabular}{|c|c|c|}
\hline Other bias & Unclear risk & Sponsorship bias cannot be ruled out \\
\hline
\end{tabular}


Basoglu 2007

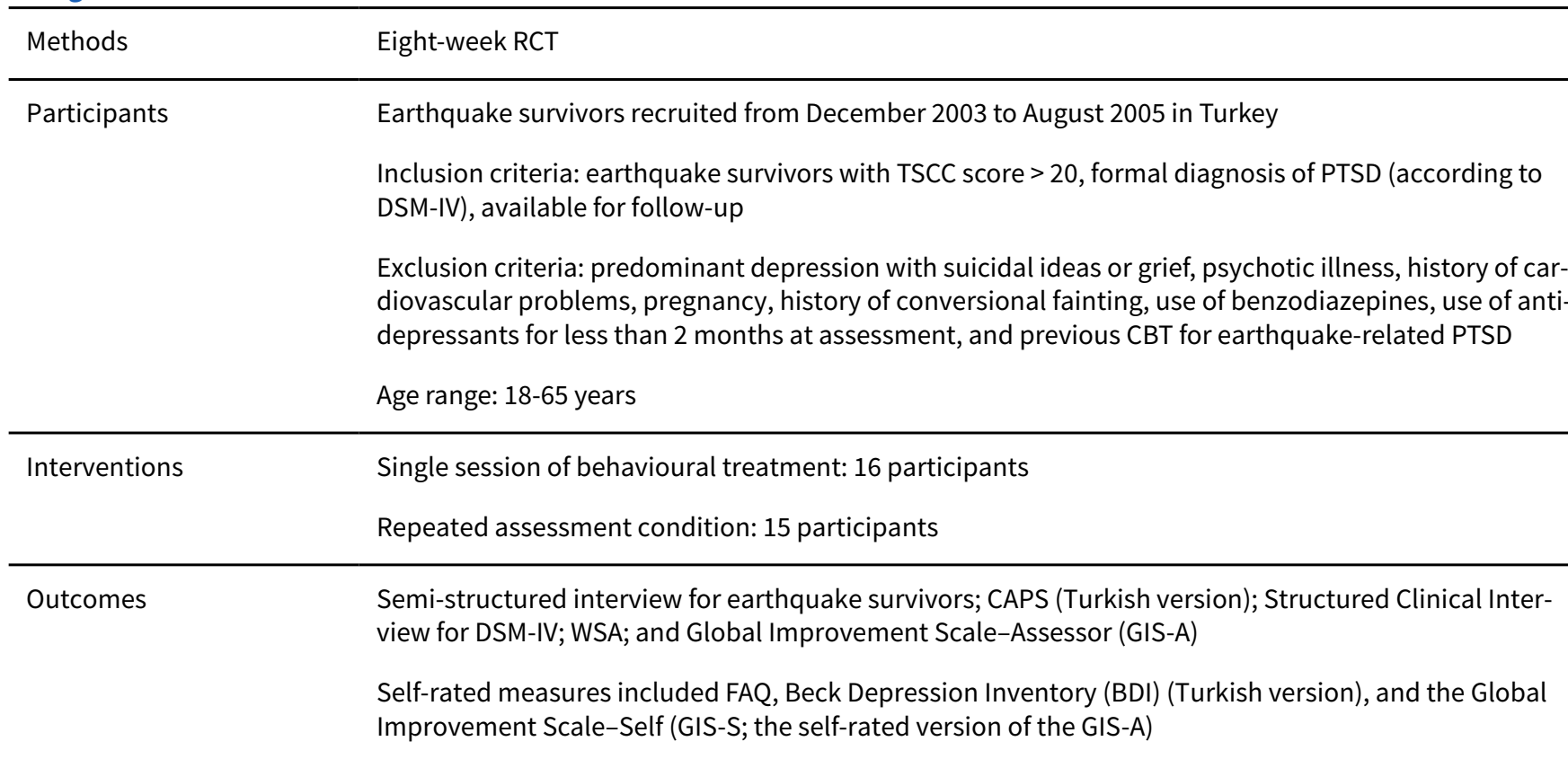

Notes

\section{Risk of bias}

Bias

Authors' judgement Support for judgement

Random sequence genera- Unclear risk tion (selection bias)

Quote: "31 participants were randomised [...]. A computer-generated sequence of random numbers that ensured equal cell sizes and did not lead to allocation of more than two consecutive cases to the same experimental condition was used in the randomisation [...] participants who did not have treatment or at least one follow-up after treatment were replaced so that the random sequence could be preserved"

\begin{tabular}{|c|c|c|}
\hline $\begin{array}{l}\text { Allocation concealment } \\
\text { (selection bias) }\end{array}$ & Unclear risk & $\begin{array}{l}\text { Quote: "Participants were enrolled by two independent assessors (psycholo- } \\
\text { gists) and randomisation was conducted by the second author, who did not } \\
\text { participate in baseline assessments" }\end{array}$ \\
\hline $\begin{array}{l}\text { Blinding of participants } \\
\text { and personnel (perfor- } \\
\text { mance bias) } \\
\text { All outcomes }\end{array}$ & High risk & $\begin{array}{l}\text { Participants were aware of their treatment allocation } \\
\text { Quote: "In six cases, follow-up assessments were conducted by the therapist } \\
\text { because the assessors had to leave the study due to an unexpected shortage } \\
\text { of funding. Four participants in the treatment group unintentionally revealed } \\
\text { their experimental condition during assessment. [...] The rate of correct guess- } \\
\text { ing was higher than expected by chance" }\end{array}$ \\
\hline $\begin{array}{l}\text { Blinding of outcome as- } \\
\text { sessment (detection bias) } \\
\text { All outcomes }\end{array}$ & High risk & $\begin{array}{l}\text { Quote: "In six cases, follow-up assessments were conducted by the therapist } \\
\text { because the assessors had to leave the study due to an unexpected shortage } \\
\text { of funding. Four participants in the treatment group unintentionally revealed } \\
\text { their experimental condition during assessment. [...] The rate of correct guess- } \\
\text { ing was higher than expected by chance" }\end{array}$ \\
\hline
\end{tabular}

Incomplete outcome data Low risk Only 1 participant missed assessment at week 4

(attrition bias)

All outcomes 
Basoglu 2007 (Continued)

Selective reporting (re- Low risk Primary outcomes were reported porting bias)

Therapist qualification Low risk

Quote: "The treatment was conducted by E.S. in accordance with a protocol. The therapist had extensive experience in treatment delivery from previous studies" E.S. is a trained psychologist

Treatment fidelity Unclear risk

Quote: "Treatment integrity checks were not conducted because the treatment protocol closely reflected the way treatment was delivered in routine fieldwork. Audiotaping of the entire session was not possible because of the loud noise generated by the earthquake simulator"

\begin{tabular}{lll}
\hline $\begin{array}{l}\text { Therapist/investigator al- } \\
\text { legiance }\end{array}$ & Unclear risk & No information provided \\
\hline Other bias & Unclear risk & Sponsorship bias cannot be ruled out \\
\hline
\end{tabular}

\section{Bass 2016}

\begin{tabular}{ll}
\hline Methods & RCT \\
\hline Participants & $\begin{array}{l}\text { The study was undertaken in the Dohuk region, which was characterised by violence since } 1980 \text { (Sad- } \\
\text { dam Hussein violent campaigns). Recently, the brutal advance of ISIS (the Islamic State of Iraq and } \\
\text { Syria) exacerbated this situation and made Dohuk a major site for refuge for displaced Iraquis and } \\
\text { refugees. Trial eligibility criteria comprised being age } 18 \text { or older; residing in the Dohuk governorate } \\
\text { (Northern Iraq); reporting experiences of torture; presenting with significant depressive symptoms; not } \\
\text { currently being psychotic or actively suicidal; and being mentally competent to give consent }\end{array}$ \\
& Trial recruitment ran from June 2009 through June 2010 \\
\hline Interventions & Counselling sessions (mean number of sessions attended was 11.29): 159 participants \\
& Wait list control: 50 participants \\
\hline Outcomes & $\begin{array}{l}\text { Study aimed to assess the impact of the intervention on primary outcomes of depressive symptoms } \\
\text { and dysfunction (using Hopkins Symptoms Checklist - HSCL-25) and on secondary outcomes of post- } \\
\text { traumatic stress (using Harvard Trauma Questionnaire - HTQ), traumatic grief, and anxiety symptoms } \\
\text { (using HSCL-25) }\end{array}$
\end{tabular}

Notes

\section{Risk of bias}

Bias Authors' judgement Support for judgement

Random sequence genera- Low risk Quote: "Study CMHWs were provided with a set of pre-numbered consent tion (selection bias) forms with the designation of intervention or wait list control status on a piece of paper that was folded and stapled to the back. ID numbers were randomly allocated to study condition by study author using Stata's randomisation function"

$\begin{aligned} & \text { Allocation concealment } \\ & \text { (selection bias) }\end{aligned}$
Unclear risk $\quad$ No information provided


Bass 2016 (Continued)

Blinding of participants and personnel (perfor-

High risk Participants were aware of their treatment allocation mance bias)

All outcomes

\section{Blinding of outcome as- Low risk} sessment (detection bias) All outcomes
Quote: "The majority ( $82 \%, n=154)$ of the follow-up interviews were implemented by CMHWs who were blinded to the participant's treatment status, whereas $18 \%(n=34)$ were implemented by CMHWs or study supervisors who were unblinded. [...] Analyses were done with and without the 34 participants who were assessed unblinded to evaluate the impact of the unblinded subjects"

Incomplete outcome data $\quad$ Low risk $\quad$ Dropout rate below 20\%
(attrition bias)

\begin{tabular}{lll}
\hline $\begin{array}{l}\text { Selective reporting (re- } \\
\text { porting bias) }\end{array}$ & Low risk & All outcomes are reported \\
\hline Therapist qualification & Unclear risk & $\begin{array}{l}\text { The intervention was delivered by trained community mental health workers } \\
\text { (CMHWs included pharmacists, nurses, and physician assistants) }\end{array}$ \\
\hline
\end{tabular}

Treatment fidelity Low risk

\begin{abstract}
Quote: "Fidelity to the treatment model was promoted by monthly on-site group supervision by a psychiatrist as well as weekly check-ins via mobile phone. (...) To monitor adherence to the counselling protocol during the onsite meetings, the psychiatrist reviewed clinical notes, which included how CMHWs responded to the client's needs and checklists of the different activities the CMHW could have provided. The client monitoring form also included a brief checklist of common mental health symptoms that was used to review client progress and help CMHW and supervisor decide, together with the client, when treatment would be completed"
\end{abstract}

Therapist/investigator al- High risk A study coauthor was part of the NGO that developed the intervention prolegiance

Other bias Low risk The study appears to be free of other sources of bias

\section{Bolton 2007}

\begin{tabular}{|c|c|}
\hline Methods & $\mathrm{RCT}$ \\
\hline Participants & $\begin{array}{l}\text { Acholi adolescents } 14 \text { to } 17 \text { years of age, living in } 2 \text { camps for internally displaced persons near Gulu, } \\
\text { Uganda. The war in Northern Uganda represents one of the most violent and persistent complex hu- } \\
\text { manitarian emergencies in the world. Over } 1.8 \text { million individuals, mainly ethnic Acholi, have been in- } \\
\text { ternally displaced during } 20 \text { years of conflict between the government of Uganda and the Lord's Resis- } \\
\text { tance Army. Eligible participants scored greater than } 32 \text { on the depression symptom scale and greater } \\
\text { than } 0 \text { on the function scale; had symptoms for at least } 1 \text { month; and resided in the camps during the } \\
\text { preceding month. Exclusion criteria were inability to be interviewed due to a cognitive or physical dis- } \\
\text { ability and severe suicidal ideation or behaviour }\end{array}$ \\
\hline
\end{tabular}

Interventions

Wait list: 104 participants

Creative play $(\mathrm{CP}): 105$ participants 
Bolton 2007 (Continued)

Outcomes

Depressive symptoms; anxiety symptoms; conduct problems; functioning. Recovery, defined as a reduction of $50 \%$ or more of an individual's baseline symptom severity score; remission, defined as at or less than a predefined cutoff score of 15.6 points (mean score of non-cases in the validation study)

Notes

\section{Risk of bias}

\section{Bias Authors' judgement Support for judgement}

Random sequence genera- Low risk tion (selection bias)
Quote: "Random allocation was done by computerized generation of a random number between 1 and 400 for each eligible participant, ordering them by number and assigning the first third to IPT-G, the second third to CP and the final third to the wait-control group"

\begin{tabular}{|c|c|c|}
\hline $\begin{array}{l}\text { Allocation concealment } \\
\text { (selection bias) }\end{array}$ & Unclear risk & No information provided \\
\hline $\begin{array}{l}\text { Blinding of participants } \\
\text { and personnel (perfor- } \\
\text { mance bias) } \\
\text { All outcomes }\end{array}$ & High risk & $\begin{array}{l}\text { Quote: "Informed consent included advising each youth of the study group to } \\
\text { which he or she had been allocated" }\end{array}$ \\
\hline $\begin{array}{l}\text { Blinding of outcome as- } \\
\text { sessment (detection bias) } \\
\text { All outcomes }\end{array}$ & Low risk & Quote: "Interviewers were blinded to interviewees' intervention status" \\
\hline $\begin{array}{l}\text { Incomplete outcome data } \\
\text { (attrition bias) } \\
\text { All outcomes }\end{array}$ & Low risk & $\begin{array}{l}\text { Dropouts reported at study endpoint ( } 17 / 105 \text { missing from creative play group; } \\
9 / 105 \text { missing from interpersonal psychotherapy group; } 16 / 104 \text { missing from } \\
\text { control group) }\end{array}$ \\
\hline $\begin{array}{l}\text { Selective reporting (re- } \\
\text { porting bias) }\end{array}$ & Low risk & All outcomes are correctly reported \\
\hline Therapist qualification & Unclear risk & Interventions were delivered by trained facilitators \\
\hline Treatment fidelity & Unclear risk & $\begin{array}{l}\text { Quote: "IPT supervisors also provided weekly written reports that were re- } \\
\text { viewed and discussed with study staff during the phone meetings for adher- } \\
\text { ence to the treatment model and to monitor human subject protection" }\end{array}$ \\
\hline
\end{tabular}

Therapist/investigator al- High risk Manualised IPT was developed in the United States, and study authors were inlegiance volved in training facilitators

\begin{tabular}{lll}
\hline Other bias & Unclear risk & No information provided \\
\hline
\end{tabular}

\section{Bolton 2014a}

\begin{tabular}{ll}
\hline Methods & RCT \\
\hline Participants & $\begin{array}{l}\text { Kurdistan, Iraq. The region was exposed to conflict and violence. At the time of the study, Kurdistan } \\
\text { was experiencing relatively little violence, and this period was alternated with substantial conflict in } \\
\text { nearby areas under control of the ISIL (Islamic State of Iraq and the Levant) }\end{array}$ \\
& $\begin{array}{l}\text { Inclusion criteria: survivors of systematic violence living in the governor rates of Erbil or Sulaimaniyah, } \\
18 \text { years of age or over, fluent in Sorani Kurdish, reported significant depression symptoms on the }\end{array}$
\end{tabular}


Bolton 2014a (Continued)

adapted HSCL-25, had no current psychotic symptoms or active suicidality, and appeared mentally competent to consent. Recruitment occurred between May 2009 and June 2010

Exclusion criteria: inability to be interviewed due to a cognitive or physical disability, severe suicidal ideation or behaviour

\begin{tabular}{ll} 
Interventions & $\begin{array}{l}\text { Intervention status: behavioural activation (BA) treatment for depression: } 114 \text { participants; cognitive } \\
\text { processing therapy: (CPT) } 101 \text { participants }\end{array}$ \\
& Wait list control status: 66 participants \\
\hline Outcomes & $\begin{array}{l}\text { Primary outcomes: depression and dysfunction severity scores on scales adapted and validated for lo- } \\
\text { cal use } \\
\text { Secondary outcomes: post-traumatic stress; anxiety; traumatic grief }\end{array}$
\end{tabular}

Notes

\section{Risk of bias}

Bias

Authors' judgement Support for judgement
Random sequence genera- Low risk tion (selection bias)

Quote: "We used a two-tier randomisation process. First, $20 \mathrm{CMHWs}$ who worked at primary clinics throughout rural Erbil and Sulaimaniyag governorates were randomised to receive training either $\mathrm{BA}(\mathrm{N}=11)$ or $\mathrm{CPT}(\mathrm{N}=9)(\ldots)$. The second-tier randomisation happened at the level of the study participant. Study participants were randomised to study condition (treatment or wait list control) by the CMHW they saw at their local primary care center where they went for treatment. The CMHWs received 20 participant IDs randomly assigned to intervention or control in the ratio of 3:1 of treatment to wait controls. Randomisation of CMHWs and participant IDs was done by JB using Stata's randomisation function"

$\begin{array}{ll}\begin{array}{l}\text { Allocation concealment } \\ \text { (selection bias) }\end{array} & \begin{array}{l}\text { Quote: "if a person consented, the CMHW opened a sealed envelope attached } \\ \text { to the consent form containing the participant's assignment" }\end{array}\end{array}$

Blinding of participants Unclear risk No information provided on blinding of participants
and personnel (performance bias)

All outcomes
Quote: "CMHWs or supervisors blind to participants' treatment status did 197 (85\%) of the interviews; 35 (15\%) were implemented by unblinded CMHWs. The latter group included participants who terminated treatment and refused further contact. Rather than forgo assessment, the treating CMHW did the interview" sessment (detection bias)

All outcomes
Unclear risk

\begin{tabular}{|c|c|c|}
\hline $\begin{array}{l}\text { Incomplete outcome data } \\
\text { (attrition bias) } \\
\text { All outcomes }\end{array}$ & Unclear risk & $\begin{array}{l}\text { Dropouts were reported: 32/114 BA group; } 34 / 101 \text { CPT group; } 13 / 66 \text { wait list } \\
\text { group }\end{array}$ \\
\hline
\end{tabular}

All outcomes

Dropouts were reported: 32/114 BA group; 34/101 CPT group; 13/66 wait list Outcomes listed in the "Methods" were also reported in the "Results" porting bias)

Low risk

\begin{tabular}{lll}
\hline Therapist qualification & Unclear risk & Interventions were delivered by community mental health workers (CMHWs) \\
\hline Treatment fidelity & Unclear risk & No information provided \\
\hline
\end{tabular}


Bolton 2014a (Continued)

Therapist/investigator al- Unclear risk No information provided
legiance

Other bias Low risk The study seems to be free of other bias

\section{Bolton 2014b}

\begin{tabular}{|c|c|}
\hline Methods & Single-blind, wait list RCT \\
\hline \multirow[t]{3}{*}{ Participants } & $\begin{array}{l}\text { Burmese adults displaced into Thailand owing to severe life conditions under decades of military rule } \\
\text { (since 1984), imprisonment of political prisoners, attacks to ethnic minority groups, forced labor, and } \\
\text { forced displacement }\end{array}$ \\
\hline & Age $>18$ years \\
\hline & $\begin{array}{l}\text { Eligibility criteria: witnessed or experienced a traumatic event; moderate to severe depression and/ } \\
\text { or PTSS based on locally validated measures (by applying modified versions of previously developed } \\
\text { DSM-IV-based algorithms to baseline interviews with the Hopkins Symptom Checklist } 25 \text { (HSCL-25) and } \\
\text { the Harvard Trauma Questionnaire (HTQ) for depression and PTSS) }\end{array}$ \\
\hline
\end{tabular}

Interventions

CETA (Common Elements Treatment Approach): 182 participants

Wait list: 165 participants

\section{Outcomes}

Primary outcomes: 15-item Hopkins Symptoms Checklist (HSCL-25) depression subscale for depressive symptoms; 30-item Harvard Trauma Questionnaire (HTQ) for post-traumatic stress symptoms

Secondary outcomes: functional impairment, measured using locally developed sex-specific scales (not described); anxiety symptoms, measured using the 10-item HSCL-25 anxiety subscale; aggression, measured with the 12-item Aggression Questionnaire, adapted for local use; alcohol use, measured with the Alcohol Use Disorders Identification Test (AUDIT)

\section{Notes}

\section{Risk of bias}

\begin{tabular}{|c|c|c|}
\hline Bias & Authors' judgement & Support for judgement \\
\hline $\begin{array}{l}\text { Random sequence genera- } \\
\text { tion (selection bias) }\end{array}$ & Low risk & $\begin{array}{l}\text { Quote: "Each counsellor assigned participants the next available ID number } \\
\text { from a block of } 20 \text { sequential participant ID numbers per counsellor random- } \\
\text { ly allocated to intervention or wait-list control (WLC) status. The project site di- } \\
\text { rector generated these random numbers using STATA" }\end{array}$ \\
\hline $\begin{array}{l}\text { Allocation concealment } \\
\text { (selection bias) }\end{array}$ & Low risk & $\begin{array}{l}\text { Quote: "Counselors opened a pre-sealed envelope (corresponding to the ID } \\
\text { number) containing assignment to immediate treatment or wait-list" }\end{array}$ \\
\hline $\begin{array}{l}\text { Blinding of participants } \\
\text { and personnel (perfor- } \\
\text { mance bias) } \\
\text { All outcomes }\end{array}$ & High risk & Single-blinded study; participants were aware of allocation \\
\hline $\begin{array}{l}\text { Blinding of outcome as- } \\
\text { sessment (detection bias) } \\
\text { All outcomes }\end{array}$ & Low risk & $\begin{array}{l}\text { Quote: "Interviewers at baseline and follow-up did not know to which study } \\
\text { arm the interviewees belonged [...]. After treatment or the wait-list period, in- } \\
\text { terviewers not otherwise involved in the study conducted post-intervention } \\
\text { assessments while masked to treatment/control status and baseline scores" }\end{array}$ \\
\hline
\end{tabular}


Bolton 2014b (Continued)

Incomplete outcome data High risk_ Dropout of intervention group: 34/182; dropout of control group: $39 / 165$ (attrition bias)

All outcomes

Selective reporting (re- Low risk $\quad$ All outcomes were reported
porting bias)

\begin{tabular}{ll}
\hline Therapist qualification $\quad$ Unclear risk & $\begin{array}{l}\text { Quote: "Qualifications were literacy in Burmese and demonstrated interest in } \\
\text { mental health and counselling" }\end{array}$
\end{tabular}

Treatment fidelity Unclear risk

\begin{abstract}
Quote: "Fidelity tracking was done through a multi-tier review approach. Specifically, counsellors tracked their own fidelity by following their step sheets and checking off each step on their own step sheets. They also completed a monitoring form for each session, which included documentation on the component delivered and some steps for each component. Supervisors reviewed fidelity during the supervision groups by reviewing the monitoring forms and requiring in-person objective reporting (...). The final and third layer of fidelity checking was completed during weekly Internet calls between supervisors and US-based CETA trainers"
\end{abstract}

Therapist/investigator al- High risk Intervention was developed by 2 of the trial authors legiance

Other bias Unclear risk No information provided

Bryant 2011

\begin{tabular}{|c|c|}
\hline Methods & $\mathrm{RCT}$ \\
\hline \multirow[t]{3}{*}{ Participants } & $\begin{array}{l}\text { People who were directly exposed to terrorist attacks in southern Thailand, where over } 3000 \text { people } \\
\text { were killed since 2004. This terrorist activity has arisen from extremist Muslim separatists operating } \\
\text { against the Thai government. Many terrorist activities in the } 3 \text { southern provinces of Thailand have tar- } \\
\text { geted schools, health workers, and other non-combatant people. People were identified between May } \\
2007 \text { and February } 2009\end{array}$ \\
\hline & $\begin{array}{l}\text { Inclusion criteria: directly exposed to a terrorist attack, primary diagnosis of PTSD based on DSM-IV cri- } \\
\text { teria }\end{array}$ \\
\hline & $\begin{array}{l}\text { Exclusion criteria: severe suicidal risk, psychosis, substance dependence, younger than } 17 \text { years or old- } \\
\text { er than } 70 \text { years }\end{array}$ \\
\hline
\end{tabular}

Interventions Cognitive-behavioural therapy (CBT): 16 participants

Treatment as usual (TAU): 12 participants

Outcomes PTSD diagnosis was determined by the PTSD Symptom Scale - Interview (PSS-I). Depression was assessed using the Beck Depression Inventory-II. Complicated grief was assessed using the Inventory of Complicated Grief

\title{
Notes
}

\section{Risk of bias}

Bias Authors' judgement Support for judgement


Bryant 2011 (Continued)

Random sequence genera- Low risk tion (selection bias)

Quote: "Patients were randomised according to a random numbers system ad ministered by health officials in Bangkok (fully independent of counsellors and the study co-ordinator)"

\begin{tabular}{lll}
\hline $\begin{array}{l}\text { Allocation concealment } \\
\text { (selection bias) }\end{array}$ & Unclear risk & No information provided
\end{tabular}

Blinding of participants Unclear risk and personnel (perfor-

clear risk

mance bias)

All outcomes

Quote: "TAU comprised the equivalent number of sessions of supportive counselling being provided by psychiatrists who were not trained in CBT"

No further information

\begin{tabular}{|c|c|c|}
\hline $\begin{array}{l}\text { Blinding of outcome as- } \\
\text { sessment (detection bias) } \\
\text { All outcomes }\end{array}$ & Low risk & $\begin{array}{l}\text { Quote: "Assessments conducted at post-treatment and } 3 \text { months following } \\
\text { treatment were conducted by independent personnel unaware of patients' } \\
\text { treatment condition" }\end{array}$ \\
\hline
\end{tabular}

Quote: "There were no treatment dropouts"

Incomplete outcome data Low risk

(attrition bias)

All outcomes

\begin{tabular}{lll}
\hline $\begin{array}{l}\text { Selective reporting (re- } \\
\text { porting bias) }\end{array}$ & Low risk & Primary outcomes are reported \\
\hline Therapist qualification & Low risk & Trained psychologists or psychiatric nurses \\
\hline Treatment fidelity & Low risk & $\begin{array}{l}\text { Quote: "At the completion of treatment sessions, therapists compiled check- } \\
\text { lists that itemized each of the therapy components. Therapists providing CBT } \\
\text { indicated on their checklists that all patients in CBT received education, anxi- } \\
\text { ety management, imaginal exposure, and cognitive restructuring" }\end{array}$
\end{tabular}

\begin{tabular}{lll}
\hline $\begin{array}{l}\text { Therapist/investigator al- } \\
\text { legiance }\end{array}$ & Unclear risk & No information provided \\
\hline Other bias & Unclear risk & No information provided \\
\hline
\end{tabular}

Chen 2014

\begin{tabular}{ll}
\hline Methods & 3-Arm RCT \\
\hline Participants & $\begin{array}{l}\text { Two years after Sichuan earthquake (2010-2011), in which survivors experienced horrific sights and } \\
\text { sounds, as their school buildings collapsed with students inside. A great number of survivors' friends } \\
\text { and classmates were injured or died }\end{array}$ \\
& $\begin{array}{l}\text { Adolescents who lost at least } 1 \text { parent in the earthquake; scored }>18 \text { points on the CRIES-13 and thus } \\
\text { were considered to have PTSD symptoms }\end{array}$ \\
\hline
\end{tabular}

Cognitive-behavioural therapy (CBT): 16 participants
General support group: 12 participants
Non-treatment group: 12 participants

Outcomes

Chinese versions of 3 psychological instruments were used to evaluate outcomes of the interventions: The CRIES-13 was used to assess PTSD symptoms, the Center for Epidemiologic Studies Depression Scale (CES-D) to assess depressive symptoms, and the Connor-Davidson Resilience Scale (CD-RISC) to measure psychological resilience

Psychological therapies for the treatment of mental disorders in low- and middle-income countries affected by humanitarian crises 
Chen 2014 (Continued)

Notes

\section{Risk of bias}

\begin{tabular}{|c|c|c|}
\hline Bias & Authors' judgement & Support for judgement \\
\hline $\begin{array}{l}\text { Random sequence genera- } \\
\text { tion (selection bias) }\end{array}$ & Unclear risk & $\begin{array}{l}\text { Quote: "The } 40 \text { adolescents were randomly divided into three groups" } \\
\text { No further information }\end{array}$ \\
\hline $\begin{array}{l}\text { Allocation concealment } \\
\text { (selection bias) }\end{array}$ & Unclear risk & No information provided \\
\hline $\begin{array}{l}\text { Blinding of participants } \\
\text { and personnel (perfor- } \\
\text { mance bias) } \\
\text { All outcomes }\end{array}$ & High risk & $\begin{array}{l}\text { The non-treatment group did not receive any psychological placebo } \\
\text { Quote: "The volunteers visited the ten adolescents' homes weekly for six } \\
\text { weeks to provide support and assistance to them in dealing with problems at } \\
\text { home and at school. No intervention or services were provided to the } 12 \text { stu- } \\
\text { dents in the control group" }\end{array}$ \\
\hline $\begin{array}{l}\text { Blinding of outcome as- } \\
\text { sessment (detection bias) } \\
\text { All outcomes }\end{array}$ & Unclear risk & No information provided \\
\hline $\begin{array}{l}\text { Incomplete outcome data } \\
\text { (attrition bias) } \\
\text { All outcomes }\end{array}$ & High risk & $\begin{array}{l}\text { Dropout of intervention sample 6/16; dropout of control group } 2 / 12 \\
\text { Quote: "Only } 32 \text { adolescents completed the entire study. Six students were } \\
\text { dropped from the CBT group, and two students were dropped from the gener- } \\
\text { al support group" }\end{array}$ \\
\hline $\begin{array}{l}\text { Selective reporting (re- } \\
\text { porting bias) }\end{array}$ & Low risk & Primary outcomes are reported \\
\hline Therapist qualification & Unclear risk & Intervention was delivered by school staff \\
\hline Treatment fidelity & Unclear risk & No information provided \\
\hline $\begin{array}{l}\text { Therapist/investigator al- } \\
\text { legiance }\end{array}$ & Unclear risk & No information provided \\
\hline Other bias & Unclear risk & No information provided \\
\hline
\end{tabular}

\section{Connolly 2011}

\begin{tabular}{ll}
\hline Methods & RCT \\
\hline Participants & $\begin{array}{l}145 \text { adult survivors of the } 1994 \text { genocide in Rwanda, in which during a 100-day period an estimated } \\
800,000 \text { members of the minority ethnic population and many moderates belonging to the majority } \\
\text { ethnic population were massacred by a group of radicals of the majority population. Trial participants } \\
\text { volunteered to receive brief treatment for symptoms of trauma. All participants met the DSM-IV criteri- } \\
\text { on A1 for Post-Traumatic Stress Disorder symptoms }\end{array}$
\end{tabular}

Age range: $18-73$ years

Interventions

Thought field therapy (TFT) is a brief treatment that includes exposure to the problem and identification of feelings elicited by thinking about the problem and stimulation of selected acupoints on the 
Connolly 2011 (Continued)

surface of the skin in a sequence that is specific to identified emotions. Elements of PTSD are targeted via a trauma treatment protocol

\begin{tabular}{ll}
\hline Outcomes & PTSD symptoms, as measured with the Modified PTSD Symptom Scale (MPSS); other symptoms that \\
trauma victims experience (i.e. anxiety, depression, anger/irritability, intrusive experience, etc.), as \\
measured with the Trauma Symptom Inventory (TSI)
\end{tabular}

Notes

\section{Risk of bias}

\section{Bias}

Random sequence genera- Low risk tion (selection bias)

\section{Authors' judgement Support for judgement}

Quote: "A randomised wait list control group design was used (...) participants were randomly assigned to an immediate treatment group or the wait list control group. Blank surveys were in file folders delineated as treatment (blue folders) or wait list group (red folders) and were stacked alternately. The intake person removed the top file from the stack and assigned the participant to that group, continuing with alternating group assignments"

\begin{tabular}{lll}
\hline $\begin{array}{l}\text { Allocation concealment } \\
\text { (selection bias) }\end{array}$ & Unclear risk & No information provided \\
\hline $\begin{array}{l}\text { Blinding of participants } \\
\text { and personnel (perfor- }\end{array}$ & Unclear risk & No information provided \\
mance bias) & \\
All outcomes & \\
\hline
\end{tabular}

\section{Connolly 2013}

\begin{tabular}{ll}
\hline Methods & RCT \\
\hline
\end{tabular}

Psychological therapies for the treatment of mental disorders in low- and middle-income countries affected by humanitarian crises 
Connolly 2013 (Continued)

Participants

164 adult survivors of the 1994 genocide in Rwanda, who volunteered to receive brief treatment for symptoms of trauma. All participants met the DSM-IV criterion A1 for Post- Traumatic Stress Disorder symptoms

Age range: $18-100$ years

Thought field therapy (TFT) is a brief treatment that includes exposure to the problem and identifica-
tion of feelings elicited by thinking about the problem and stimulation of selected acupoints on the
surface of the skin in a sequence that is specific to identified emotions. Elements of PTSD are targeted
via a trauma treatment protocol

Outcomes

PTSD symptoms, as measured with the Modified PTSD Symptom Scale (MPSS); other symptoms that trauma victims experience (i.e. anxiety, depression, anger/irritability, intrusive experience, etc.), as measured with the Trauma Symptom Investory (TSI)

\section{Notes}

\section{Risk of bias}

\begin{tabular}{lll}
\hline Bias & Authors' judgement & Support for judgement \\
\hline $\begin{array}{l}\text { Random sequence genera- } \\
\text { tion (selection bias) }\end{array}$ & Low risk & $\begin{array}{l}\text { Quote: "A randomised controlled study (...) participants were randomly as- } \\
\text { signed to an immediate treatment group or the wait list control group" }\end{array}$ \\
\hline $\begin{array}{l}\text { Allocation concealment } \\
\text { (selection bias) }\end{array}$ & Low risk & $\begin{array}{l}\text { Quote: "Clank surveys were in file folders delineated as treatment (blue fold- } \\
\text { ers) or wait list control group (red folders) and were stacked alternately. The } \\
\text { intake person took the top file from the stack and assigned the participant to } \\
\text { that group and continued with the alternating group assignments. The person } \\
\text { handing out the folders was not aware of the implications of the different col- } \\
\text { ored folders" }\end{array}$ \\
\hline
\end{tabular}

Blinding of participants $\quad$ Unclear risk No information provided
and personnel (perfor-
mance bias)
All outcomes

\begin{tabular}{lll}
\hline $\begin{array}{l}\text { Blinding of outcome as- } \\
\text { sessment (detection bias) } \\
\text { All outcomes }\end{array}$ & Unclear risk & No information provided \\
\hline $\begin{array}{l}\text { Incomplete outcome data } \\
\text { (attrition bias) } \\
\text { All outcomes }\end{array}$ & Unclear risk & The number of participants evaluated at the study endpoint is unclear \\
\hline $\begin{array}{l}\text { Selective reporting (re- } \\
\text { porting bias) }\end{array}$ & Unclear risk & $\begin{array}{l}\text { All outcomes listed in the "Methods" section are reported in the "Results" sec- } \\
\text { tion, but only effect sizes were reported for study endpoint (and the number of } \\
\text { participants evaluated at endpoint is not clear) }\end{array}$ \\
\hline
\end{tabular}

\begin{tabular}{|c|c|c|}
\hline Therapist qualification & Unclear risk & Quote: "The selected therapists included respected community leaders" \\
\hline Treatment fidelity & Low risk & $\begin{array}{l}\text { Quote: "The trainers were available for supervision throughout the entire } \\
\text { study, ensuring that the newly trained Rwandan therapists adhered to the } \\
\text { standard Thought Field Therapy algorithm protocols throughout the treat- } \\
\text { ment phases of the study" }\end{array}$ \\
\hline
\end{tabular}

Therapist/investigator al- High risk Study authors were involved in treatment development
legiance


Connolly 2013 (Continued)

Other bias Unclear risk No information provided

Ertl 2011

\begin{tabular}{ll}
\hline Methods & RCT \\
\hline Participants & $\begin{array}{l}\text { The trial was conducted in Northern Uganda, where children were forced to be actively involved as } \\
\text { child soldiers, helpers, or sexual slaves in the civil war }\end{array}$ \\
& $\begin{array}{l}\text { Age: } 12-25 \text { years; former child soldiers with PTSD, confirmed by clinical experts according to the CAPS; } \\
\text { patients with suicidal ideation, substance abuse, or depression (2 former child soldiers were not en- } \\
\text { rolled because of the presence of psychotic symptoms) }\end{array}$ \\
\hline Interventions & $\begin{array}{l}\text { Narrative exposure therapy (NET): } 29 \text { participants } \\
\text { Supportive counselling: } 28 \text { participants } \\
\text { Wait list: } 28 \text { participants }\end{array}$ \\
\hline Outcomes & $\begin{array}{l}\text { Primary outcome measure was change in PTSD severity, assessed over a 1-year period after treat- } \\
\text { ment (3, } 6,12 \text { months). Secondary outcome measures were depression symptoms, severity of suicidal } \\
\text { ideation, feelings of guilt, and perceived stigmatisation }\end{array}$ \\
\hline
\end{tabular}

Notes

\section{Risk of bias}

\begin{tabular}{lll}
\hline Bias & Authors' judgement & Support for judgement \\
\hline $\begin{array}{l}\text { Random sequence genera- } \\
\text { tion (selection bias) }\end{array}$ & Unclear risk & Quote: "Participants were randomised into 3 treatment[s]" \\
\hline $\begin{array}{l}\text { Allocation concealment } \\
\text { (selection bias) }\end{array}$ & Unclear risk & No information provided \\
\hline $\begin{array}{l}\text { Blinding of participants } \\
\text { and personnel (perfor- } \\
\text { mance bias) }\end{array}$ & High risk & Participants were aware of the allocation \\
All outcomes & \\
\hline
\end{tabular}

\begin{tabular}{|c|c|c|}
\hline $\begin{array}{l}\text { Blinding of outcome as- } \\
\text { sessment (detection bias) } \\
\text { All outcomes }\end{array}$ & Low risk & $\begin{array}{l}\text { Quote: "Pretreatment assessments as well as follow-up assessments at } 3 \\
\text { months, } 6 \text { months, and } 12 \text { months after treatment were conducted by } 13 \text { clini- } \\
\text { cal psychologists blinded to treatment conditions" }\end{array}$ \\
\hline
\end{tabular}

\begin{tabular}{lll}
\hline $\begin{array}{l}\text { Incomplete outcome data } \\
\text { (attrition bias) } \\
\text { All outcomes }\end{array}$ & Low risk & $3 / 29 ; 4 / 28 ; 0 / 28$ \\
\hline $\begin{array}{l}\text { Selective reporting (re- } \\
\text { porting bias) }\end{array}$ & Low risk & All outcomes are reported \\
\hline Therapist qualification & Unclear risk & $\begin{array}{l}\text { Quote: "Treatments were carried out by 14 intensively trained local lay coun- } \\
\text { sellors" }\end{array}$ \\
\hline Treatment fidelity & Low risk & $\begin{array}{l}\text { Quote: "Treatment fidelity and therapeutic competence were monitored by } \\
\text { case discussions in supervision meetings, observation and evaluation of treat- }\end{array}$
\end{tabular}


Ertl 2011 (Continued)

ment sessions via video recordings, and review of the obligatory treatment process notes for each session. In the case of Narrative Exposure Therapy, testimonies were additionally reviewed to check for trauma focus and richness of detail. No deviations from the study protocol were noted"

Therapist/investigator al- High risk Study authors were involved in treatment development
legiance

Study authors were involved in treatment development

Other bias Unclear risk No information provided

Hermenau 2013

\begin{tabular}{ll}
\hline Methods & RCT \\
\hline Participants & $\begin{array}{l}\text { The trial was conducted in the Democratic Republic of the Congo, which was affected by war and ongo- } \\
\text { ing conflicts. Adults (mean age } 19 \text { years), 100\% men, who reported combat experience }\end{array}$
\end{tabular}

Interventions Narrative exposure therapy for forensic offender rehabilitation (FORNET): 19 participants

No intervention: 19 participants

\begin{tabular}{ll}
\hline Outcomes & PTSD and appetitive aggression \\
\hline Notes & \\
\hline
\end{tabular}

\section{Risk of bias}

Bias Authors' judgement Support for judgement

Random sequence genera- Low risk Quote: "Randomised controlled clinical trial (...) We included all 38 particition (selection bias) pants who were present at the time of the pretest at the reintegration center and matched them into 19 pairs of ex-combatants. We then randomly assigned one member of each pair to the treatment group and the other one to the control group. The series of random numbers was obtained via www.random.org"

\begin{tabular}{lll}
\hline $\begin{array}{l}\text { Allocation concealment } \\
\text { (selection bias) }\end{array}$ & Unclear risk & No information provided \\
\hline
\end{tabular}

Blinding of participants Unclear risk No information provided
and personnel (performance bias)

All outcomes

\begin{tabular}{lll}
\hline $\begin{array}{l}\text { Blinding of outcome as- } \\
\text { sessment (detection bias) } \\
\text { All outcomes }\end{array}$ & Unclear risk & It is unclear how blinding was maintained and whether it was successful \\
\hline $\begin{array}{l}\text { Incomplete outcome data } \\
\begin{array}{l}\text { (attrition bias) } \\
\text { All outcomes }\end{array}\end{array}$ & Unclear risk & $\begin{array}{l}\text { Dropouts were reported (4/19 in the intervention group; and consequently } 4 \\
\text { matched participants excluded from analyses by study authors) }\end{array}$ \\
\hline $\begin{array}{l}\text { Selective reporting (re- } \\
\text { porting bias) }\end{array}$ & Low risk & $\begin{array}{l}\text { All outcomes listed in the "Methods" section were reported in the "Results" } \\
\text { section }\end{array}$ \\
\hline
\end{tabular}


Hermenau 2013 (Continued)

Therapist qualification Low risk Intervews and interventions were implemented by trial authors and 2 additional interviewers; all were psychologists from the University of Konstanz, with extensive work experience in East Africa

\begin{tabular}{lll}
\hline Treatment fidelity & Low risk & The intervention was delivered according to the FORNET Manual \\
\hline $\begin{array}{l}\text { Therapist/investigator al- } \\
\text { legiance }\end{array}$ & High risk & $\begin{array}{l}\text { Trial authors implemented the intervention and contributed to intervention } \\
\text { development }\end{array}$ \\
\hline Other bias & Unclear risk & No information provided \\
\hline
\end{tabular}

Igreja 2004

\begin{tabular}{ll}
\hline Methods & RCT \\
\hline Participants & $\begin{array}{l}\text { Adult men or women who were Mozambican civil war survivors (Mozambique is characterised by al- } \\
\text { most 3 decades of war); born in the region; having survived the war while living within regional war } \\
\text { zones. Former refugees or displaced persons from other regions were excluded }\end{array}$ \\
& Age: mean 40 (SD 14) years \\
\hline Interventions & Testimony method: 66 participants \\
& Control group: 71 participants \\
\hline Outcomes & $\begin{array}{l}\text { Psychiatric morbidity was measured with the Self-Report Questionnaire (SRQ); shocking experiences } \\
\text { by a section of the Harvard Trauma Questionnaire (HTQ); prevalence of nightmares with the Nocturnal } \\
\text { Intrusions after Traumatic Experiences questionnaire (NITE); and post-traumatic stress symptoms with } \\
\text { the Self-Inventory for PTSD (SIFP) }\end{array}$
\end{tabular}

Notes

\section{Risk of bias}

\section{Bias Authors' judgement Support for judgement}

Random sequence genera- Unclear risk Quote: "During the baseline measurements, every participant was given a contion (selection bias) secutive number. By using a cut-off point in the measurements of post-traumatic stress symptoms, the group was divided into a case $(\mathrm{N}=137)$ and a noncase group ( $\mathrm{N}=69)$. Depending on whether their number was an even or odd one, participants belonging to the case group were respectively assigned to an intervention group $(\mathrm{N}=66)$ or a control group $(\mathrm{N}=71) "$

\begin{tabular}{lll}
\hline $\begin{array}{l}\text { Allocation concealment } \\
\text { (selection bias) }\end{array}$ & Unclear risk & No information provided \\
\hline $\begin{array}{l}\text { Blinding of participants } \\
\text { and personnel (perfor- }\end{array}$ & Unclear risk & No information provided \\
mance bias) & \\
All outcomes & \\
\hline
\end{tabular}

Blinding of outcome as- Unclear risk Unclear who conducted assessments - all by interview sessment (detection bias)

All outcomes 
Igreja 2004 (Continued)

Incomplete outcome data Unclear risk No information provided
(attrition bias)

All outcomes

\begin{tabular}{lll}
\hline $\begin{array}{l}\text { Selective reporting (re- } \\
\text { porting bias) }\end{array}$ & High risk & $\begin{array}{l}\text { Not all of the study's prespecified outcomes have been reported. No protocol } \\
\text { available }\end{array}$ \\
\hline Therapist qualification & Low risk & $\begin{array}{l}\text { Intervention was delivered by a professional, in collaboration with 3 native } \\
\text { speakers acting as interpreters }\end{array}$ \\
\hline Treatment fidelity & Unclear risk & No information provided \\
\hline $\begin{array}{l}\text { Therapist/investigator al- } \\
\text { legiance }\end{array}$ & Unclear risk & No information provided \\
\hline Other bias & Unclear risk & No information provided \\
\hline
\end{tabular}

Jacob 2014

\begin{tabular}{ll}
\hline Methods & RCT \\
\hline Participants & $\begin{array}{l}\text { Widowed or orphaned survivors of the } 1994 \text { Rwandan genocide with a PTSD diagnosis based on DSM- } \\
\text { IV-TR criteria }\end{array}$ \\
\hline
\end{tabular}

Interventions Narrative exposure therapy (NET): 38 participants

Therapy consisted of 8 weekly sessions lasting from 90 minutes to a maximum of 150 minutes. Sessions include psychoeducation about PTSD (first session); narration of the major emotionally arousing events of the entire life (the following 5 sessions); social interventions to explicitly target symptoms of depression and grief related to losses and to raise awareness about major current problems (final 2 sessions)

Wait list: 38 participants

\begin{tabular}{ll}
\hline Outcomes & Clinician Administered PTSD scale (CAPS) total score \\
\hline Notes & \\
\hline
\end{tabular}

\section{Risk of bias}

\begin{tabular}{|c|c|c|}
\hline Bias & Authors' judgement & Support for judgement \\
\hline $\begin{array}{l}\text { Random sequence genera- } \\
\text { tion (selection bias) }\end{array}$ & Low risk & $\begin{array}{l}\text { Quote: "We used simple randomisation by drawing lots to assign participants } \\
\text { to the two different groups (NET-1 and WL/NET-2)" }\end{array}$ \\
\hline $\begin{array}{l}\text { Allocation concealment } \\
\text { (selection bias) }\end{array}$ & Unclear risk & No information provided \\
\hline $\begin{array}{l}\text { Blinding of participants } \\
\text { and personnel (perfor- } \\
\text { mance bias) } \\
\text { All outcomes }\end{array}$ & Unclear risk & $\begin{array}{l}\text { Quote: "We did not inform participants about their group assignment" } \\
\text { No details on how blinding was maintained and whether it was successful }\end{array}$ \\
\hline $\begin{array}{l}\text { Blinding of outcome as- } \\
\text { sessment (detection bias) } \\
\text { All outcomes }\end{array}$ & Low risk & Quote: "Expert interviewers were blind to the allocation" \\
\hline
\end{tabular}

Psychological therapies for the treatment of mental disorders in low- and middle-income countries affected by humanitarian crises 
Jacob 2014 (Continued)

Incomplete outcome data Low risk $\quad$ Dropouts were reported (NET group 1/38; WL group 2/38)
(attrition bias)

All outcomes

\begin{tabular}{lll}
\hline $\begin{array}{l}\text { Selective reporting (re- } \\
\text { porting bias) }\end{array}$ & Low risk & $\begin{array}{l}\text { Outcomes listed in the "Methods" section were reported in the "Results" sec- } \\
\text { tion }\end{array}$ \\
\hline Therapist qualification & Low risk & Trained clinical psychologists \\
\hline $\begin{array}{l}\text { Treatment fidelity } \\
\text { Low risk }\end{array}$ & $\begin{array}{l}\text { Quote: "To measure adherence to treatment, therapists completed protocol } \\
\text { sheets that reviewed the main aspects of each therapy session, including dura- } \\
\text { tion, reported problems, and medications taken" }\end{array}$ \\
\hline $\begin{array}{l}\text { Therapist/investigator al- } \\
\text { legiance }\end{array}$ & High risk & Study authors were involved in development of treatment \\
\hline Other bias & Unclear risk & No information provided \\
\hline
\end{tabular}

Jiang 2014

\begin{tabular}{ll}
\hline Methods & Two-group, parallel RCT \\
\hline Participants & $\begin{array}{l}\text { Chinese adults meeting criteria for PTSD as assessed by the CAPS. Participants survived the 2008 earth- } \\
\text { quake in Sichuan, which caused more than } 69,000 \text { deaths, including more than } 5000 \text { students in school } \\
\text { at the time of the event }\end{array}$
\end{tabular}

Interventions

Interpersonal psychotherapy (IPT) + treatment as usual (TAU): 27 participants

IPT addressed trauma-related mental disorders. It was focused on 4 areas, depending on the cause of the patient's distress: interpersonal disputes, role transitions, grief/loss, and interpersonal sensitivity/deficit

\section{TAU: 22 participants}

TAU included continuation of selective serotonin reuptake inhibitors (SSRIs), serotonin-norepinephrine reuptake inhibitors (SNRIs), benzodiazepines, and crisis counselling services. Those receiving medications met weekly with psychiatrists for medication management and had access to mental health professionals for interim crisis care. After 12 weeks, those assigned to TAU were offered IPT treatment

$\begin{array}{ll}\text { Outcomes } & \text { Primary outcomes: diagnosis of PTSD on CAPS and/or diagnosis of MDD on SCID } \\ & \text { Secondary outcomes: Beck Depression Inventory (BDI-II); Generalized Self Efficacy (GSE); State Trait } \\ & \text { Anger (STAXI); Conflict Tactics Scale (CTS); Social Adjustment Scale (SAS); Quality of Life Index (QLI) }\end{array}$

Notes

\section{Risk of bias}

\begin{tabular}{lll}
\hline Bias & Authors' judgement & Support for judgement \\
\hline $\begin{array}{l}\text { Random sequence genera- } \\
\text { tion (selection bias) }\end{array}$ & Low risk & $\begin{array}{l}\text { Quote: "Individuals were randomised" } \\
\text { Probably done }\end{array}$ \\
\hline $\begin{array}{l}\text { Allocation concealment } \\
\text { (selection bias) }\end{array}$ & Unclear risk & No information provided \\
\hline
\end{tabular}

Psychological therapies for the treatment of mental disorders in low- and middle-income countries affected by humanitarian crises 
Jiang 2014 (Continued)

Blinding of participants Unclear risk No information provided and personnel (perfor-

mance bias)

All outcomes

\begin{tabular}{|c|c|c|}
\hline $\begin{array}{l}\text { Blinding of outcome as- } \\
\text { essment (detection bias) } \\
\text { All outcomes }\end{array}$ & High risk & $\begin{array}{l}\text { Quote: "Unexpected personnel and budget constraints required that the un- } \\
\text { blinded study coordinator and unblinded assistant carry out these assess- } \\
\text { ments" }\end{array}$ \\
\hline
\end{tabular}

Unclear risk

Dropouts were reported (IPT group 8/27; TAU group 3/22)

Incomplete outcome data

(attrition bias)

All outcomes

\begin{tabular}{|c|c|c|}
\hline $\begin{array}{l}\text { Selective reporting (re- } \\
\text { porting bias) }\end{array}$ & Low risk & $\begin{array}{l}\text { Primary and secondary outcomes listed in the "Methods" section were report- } \\
\text { ed in the "Results" section }\end{array}$ \\
\hline
\end{tabular}

\begin{tabular}{ll}
\hline Therapist qualification $\quad$ Low risk & $\begin{array}{l}\text { Therapists were } 5 \text { psychologists, } 4 \text { psychiatrists, and } 1 \text { teacher experienced in } \\
\text { processing emotional trauma secondary to earthquake }\end{array}$
\end{tabular}

Treatment fidelity Low risk

Quote: "Treatment adherence was assessed for each session by the therapist supervisor. Supervisors rated therapists' adherence to IPT protocol using a ten-point scale assessing overall quality of the session (three items) and quality of key components of each of four phases (2-5 items), and two reverse coded items for off-protocol treatments, such as CBT"

\begin{tabular}{lll}
\hline $\begin{array}{l}\text { Therapist/investigator al- } \\
\text { legiance }\end{array}$ & Unclear risk & No information provided \\
\hline Other bias & Unclear risk & No information provided \\
\hline
\end{tabular}

\section{Knaevelsrud 2015}

\begin{tabular}{|c|c|}
\hline Methods & $\mathrm{RCT}$ \\
\hline \multirow[t]{2}{*}{ Participants } & $\begin{array}{l}\text { Arabic-speaking adults between } 18 \text { and } 65 \text { years of age, with a history of trauma according to DSM-IV } \\
\text { criteria accompanied by post-traumatic stress symptoms. Participants lived in areas of ongoing war, } \\
\text { conflict, and violence. They were exposed to traumatic events such as torture, killings, severe human } \\
\text { rights abuses, and rape. Between } 2004 \text { and } 2007 \text {, Iraqis were at the highest risk worldwide of dying in a } \\
\text { violent conflict. The study was conducted between January } 2009 \text { and November } 2011\end{array}$ \\
\hline & $\begin{array}{l}\text { The Posttraumatic Stress Diagnostic Scale (PDS) was used to identify whether patients reported the } \\
\text { minimum number of symptoms required by DSM-IV for each of the symptom clusters (at least } 1 \text { intru- } \\
\text { sion, } 3 \text { avoidance, and } 2 \text { hyperarousal symptoms). Additionally, the minimum score on the PDS to be } \\
\text { included in the trial was } 11 \text {, indicating moderate symptom severity. Exclusion criteria were currently } \\
\text { receiving treatment elsewhere, substance abuse or dependence, high risk of suicide, psychotic symp- } \\
\text { toms, and low symptom severity }\end{array}$ \\
\hline
\end{tabular}

Interventions Web-based CBT intervention: 79 participants

Wait list: 80 participants

\section{Outcomes}

Post-traumatic stress symptoms (measured with PDS); depression and anxiety (measured with HSCL-25); somatisation (measured with the somatisation subscale of the Symptom Checklist 90- Revised (SCL-90)); quality of life (measured with the EUROHIS-QOL)

Notes

Psychological therapies for the treatment of mental disorders in low- and middle-income countries affected by humanitarian crises 
Knaevelsrud 2015 (Continued)

\section{Risk of bias}

\begin{tabular}{|c|c|c|}
\hline Bias & Authors' judgement & Support for judgement \\
\hline $\begin{array}{l}\text { Random sequence genera- } \\
\text { tion (selection bias) }\end{array}$ & Low risk & $\begin{array}{l}\text { Quote: "Participants (...) were randomly assigned to either the Internet-based } \\
\text { treatment or a waiting list control condition. Randomisation was based on a } \\
\text { computer-generated randomisation list" }\end{array}$ \\
\hline $\begin{array}{l}\text { Allocation concealment } \\
\text { (selection bias) }\end{array}$ & Unclear risk & No information provided \\
\hline $\begin{array}{l}\text { Blinding of participants } \\
\text { and personnel (perfor- } \\
\text { mance bias) } \\
\text { All outcomes }\end{array}$ & High risk & $\begin{array}{l}\text { Quote: "Researchers and psychotherapists were not masked to the interven- } \\
\text { tion" }\end{array}$ \\
\hline $\begin{array}{l}\text { Blinding of outcome as- } \\
\text { sessment (detection bias) } \\
\text { All outcomes }\end{array}$ & Unclear risk & No information provided \\
\hline $\begin{array}{l}\text { Incomplete outcome data } \\
\text { (attrition bias) } \\
\text { All outcomes }\end{array}$ & High risk & $\begin{array}{l}\text { Dropouts were reported and were higher than } 20 \% \text { (32/79 in the intervention } \\
\text { group; } 33 / 80 \text { in the wait list group) }\end{array}$ \\
\hline $\begin{array}{l}\text { Selective reporting (re- } \\
\text { porting bias) }\end{array}$ & Low risk & $\begin{array}{l}\text { Outcomes listed in the "Methods" section were consistently reported in the } \\
\text { "Results" section }\end{array}$ \\
\hline Therapist qualification & Low risk & Trained psychotherapists or psychiatrists \\
\hline Treatment fidelity & Unclear risk & Intervention was manualised and Web-based \\
\hline $\begin{array}{l}\text { Therapist/investigator al- } \\
\text { legiance }\end{array}$ & Unclear risk & No information provided \\
\hline
\end{tabular}

\section{McMullen 2013}

Methods Parallel-group study with a wait list control group and equal randomisation (1:1)

Participants The trial was conducted in the Democratic Republic of the Congo, which is thought to have approximately 30,000 children fighting or living with armed group

Inclusion criteria: male, younger than 18, either a former child soldier (abducted or recruited by an armed group) or a witness to a violent event involving a real or perceived direct threat to life. To keep the trial naturalistic, adolescents with suicidal ideation, substance abuse, or other mental health difficulties were not excluded

Interventions intervention took place during May to July 2011 in Beni, a town in the North Kivu province. The city
was affected by years of war, atrocities, and human rights violations against civilians
Trauma-focused cognitive-behavioural therapy (TF-CBT): 25 participants
Wait list: 25 participants
Outcomes
$\begin{aligned} & \text { The UCLA-PTSD-RI was used to assess levels of post-traumatic stress symptoms. The African Youth Psy- } \\ & \text { chosocial Assessment was used to measure levels of psychosocial distress }\end{aligned}$

Psychological therapies for the treatment of mental disorders in low- and middle-income countries affected by humanitarian crises 
McMullen 2013 (Continued)

Notes

\section{Risk of bias}

Bias Authors' judgement Support for judgement

Random sequence genera- Low risk

tion (selection bias)

Quote: "They were then randomly allocated, by the first author, to either TFCBT intervention group or wait-list control group using a matched dyad sequence from a computer randomisation program (www.random.org) generated by the third author (off site)"

\begin{tabular}{ll}
\hline $\begin{array}{l}\text { Allocation concealment } \\
\text { (selection bias) }\end{array}$ & Low risk
\end{tabular}$\quad \begin{aligned} & \text { Quote: "To prevent selection bias, the first author was not present during as- } \\
& \text { sessment and did not meet the participants prior to the group allocation" }\end{aligned}$

\begin{tabular}{ll}
\hline $\begin{array}{l}\text { Blinding of participants } \\
\text { and personnel (perfor- }\end{array}$ & High risk
\end{tabular}$\quad \begin{aligned} & \text { No information was provided, but it's hard to believe that blinding of partici- } \\
& \text { pants could have been respected in such a study design }\end{aligned}$
mance bias)

All outcomes

\begin{tabular}{|c|c|c|}
\hline $\begin{array}{l}\text { Blinding of outcome as- } \\
\text { sessment (detection bias) }\end{array}$ & Low risk & $\begin{array}{l}\text { Quote: "None of the interviewers participated in the intervention to ensure } \\
\text { blinding of treatment allocation" }\end{array}$ \\
\hline
\end{tabular}

Alloutcomes

Incomplete outcome data Low risk
(attrition bias)

(attrition bias)

Less than $20 \%$ of patients abandoned the study prematurely

All outcomes

Study endpoint: 1/25 missing from TF-CBT group; $1 / 25$ missing from WL group

\begin{tabular}{lll}
\hline $\begin{array}{l}\text { Selective reporting (re- } \\
\text { porting bias) }\end{array}$ & Low risk & All outcomes are reported \\
\hline Therapist qualification & Low risk & $\begin{array}{l}\text { Interventon was delivered by } 2 \text { of the study authors (with PhD degree) and by } 2 \\
\text { experienced Congolese counsellors through a local interpreter }\end{array}$
\end{tabular}

\begin{tabular}{|c|c|c|}
\hline \multirow[t]{2}{*}{ Treatment fidelity } & Unclear risk & $\begin{array}{l}\text { Quote: "Daily training and evaluation sessions were held with these facilitators } \\
\text { to ensure fidelity to the original intervention and to maximise cultural appro- } \\
\text { priateness" }\end{array}$ \\
\hline & & No further information provided \\
\hline $\begin{array}{l}\text { Therapist/investigator al- } \\
\text { legiance }\end{array}$ & Unclear risk & $\begin{array}{l}\text { Study authors were involved in translation/cultural adaptation of the interven- } \\
\text { tion; in training staff in administering pre-intervention interviews }\end{array}$ \\
\hline Other bias & Low risk & $\begin{array}{l}\text { Quote: "No external funding was provided for this study. This project was } \\
\text { made possible through generous support from Queens University, colleagues, } \\
\text { family and friends" }\end{array}$ \\
\hline
\end{tabular}

Meffert 2014

\begin{tabular}{ll}
\hline Methods & Pilot RCT \\
\hline Participants & $\begin{array}{l}\text { Sudanese refugees living in Cairo, Egypt. Darfurians have been attacked by government-backed mili- } \\
\text { tias and have fled to Egypt. They have undergone intense traumatic exposure, including rape, murder } \\
\text { of family members, and narrow escape from death. The trial was conducted from April to August } 2008 \\
\text { Patients were screened according to the following selection criteria: absence of cognitive dysfunction } \\
\text { that required a higher level of care or interfered with the ability to participate in IPT, absence of severe }\end{array}$
\end{tabular}

Psychological therapies for the treatment of mental disorders in low- and middle-income countries affected by humanitarian crises 
Meffert 2014 (Continued)

thought or mood disorder symptoms that required a higher level of care or interfered with the ability to participate in IPT, absence of drug and alcohol dependence, HTQ score of 2.3 or greater, ability to attend twice-weekly therapy sessions for 3 weeks and return for regular screening, and ability to give verbal informed consent

Age: 21-42 years

\begin{tabular}{ll}
\hline Interventions & Interpersonal psychotherapy (IPT): 13 participants \\
& Wait list control: 9 participants \\
\hline Outcomes & $\begin{array}{l}\text { PTSD (Harvard Trauma Questionnaire - HTQ); depression (Beck Depression Inventory-II - BDI-II); family } \\
\text { violence (Conflict Tactics Scale - CTS); anger expression (State-Trait Anger Expression Inventory - STAXI) }\end{array}$ \\
\hline Notes &
\end{tabular}

\section{Risk of bias}

Bias Authors' judgement Support for judgement

Random sequence genera- Low risk tion (selection bias)

Quote: "Eligible participants were randomly assigned using a computer-generated random allocation sequence"

Allocation concealment Unclear risk No information provided

(selection bias)

\begin{tabular}{|c|c|c|}
\hline $\begin{array}{l}\text { Blinding of participants } \\
\text { and personnel (perfor- }\end{array}$ & High risk & Quote: "Participants were not blinded to group status" \\
\hline
\end{tabular}

mance bias)

All outcomes

\begin{tabular}{|c|c|c|}
\hline $\begin{array}{l}\text { Blinding of outcome as- } \\
\text { sessment (detection bias) } \\
\text { All outcomes }\end{array}$ & High risk & Quote: "Therapists were not blind to group status" \\
\hline $\begin{array}{l}\text { Incomplete outcome data } \\
\text { (attrition bias) }\end{array}$ & Low risk & Less than $20 \%$ of participants abandoned the study prematurely \\
\hline All outcomes & & Study endpoint: 2/13 missing from IPT group; $1 / 9$ missing from WL group \\
\hline $\begin{array}{l}\text { Selective reporting (re- } \\
\text { porting bias) }\end{array}$ & Low risk & $\begin{array}{l}\text { The study protocol is not available, but it is clear that published reports in- } \\
\text { clude all expected outcomes }\end{array}$ \\
\hline Therapist qualification & Unclear risk & $\begin{array}{l}\text { Quote: "Sudanese mental health professionals were not available in Cairo, so } \\
\text { community members without previous mental health education were trained } \\
\text { to deliver the care" }\end{array}$ \\
\hline Treatment fidelity & Unclear risk & No information provided \\
\hline $\begin{array}{l}\text { Therapist/investigator al- } \\
\text { legiance }\end{array}$ & Unclear risk & No information provided \\
\hline Other bias & Unclear risk & Sponsorship bias cannot be ruled out \\
\hline
\end{tabular}

\section{Neuner 2008a}

Methods RCT

Psychological therapies for the treatment of mental disorders in low- and middle-income countries affected by humanitarian crises 
Neuner 2008a (Continued)

Participants

Rwandan and Somalian refugees living in the Navikale refugee settlement in Uganda. Participants who fulfilled DSM-IV criteria of PTSD (assessed with the PDS) and lived in 2 villages closest to the research base in the settlement were included

Interventions Narrative exposure therapy (NET): 111 participants

Monitoring group: 55 participants

Notes

\section{Risk of bias}

Bias Authors' judgement Support for judgement

Random sequence genera- Unclear risk tion (selection bias)
Quote: "The list of participants was ordered randomly; the first 4 were consecutively assigned to the NET, TC, NET, and TC groups; and the fifth was assigned to the MG (monitoring) group. This procedure was repeated until all 277 participants were assigned"

\begin{tabular}{|c|c|c|}
\hline $\begin{array}{l}\text { Allocation concealment } \\
\text { (selection bias) }\end{array}$ & Unclear risk & No information provided \\
\hline $\begin{array}{l}\text { Blinding of participants } \\
\text { and personnel (perfor- } \\
\text { mance bias) } \\
\text { All outcomes }\end{array}$ & High risk & Participants were aware of their treatment allocation \\
\hline $\begin{array}{l}\text { Blinding of outcome as- } \\
\text { sessment (detection bias) } \\
\text { All outcomes }\end{array}$ & Low risk & Interviewers were blind with respect to the particular treatment condition \\
\hline $\begin{array}{l}\text { Incomplete outcome data } \\
\text { (attrition bias) } \\
\text { All outcomes }\end{array}$ & High risk & $\begin{array}{l}\text { More than } 20 \% \text { of participants abandoned the study prematurely } \\
\text { Study endpoint: } 50 / 111 \text { missing from NET group; } 52 / 111 \text { missing from TC } \\
\text { group }\end{array}$ \\
\hline $\begin{array}{l}\text { Selective reporting (re- } \\
\text { porting bias) }\end{array}$ & Low risk & Only 1 outcome measure tested \\
\hline Therapist qualification & Unclear risk & Quote: "Nine refugees were trained as counsellors" \\
\hline Treatment fidelity & Low risk & $\begin{array}{l}\text { Quote: "Treatment adherence was monitored by case discussions in supervi- } \\
\text { sion meetings, by the direct observation of treatment sessions, and by a re- } \\
\text { view of the testimonies and treatment protocols. No major deviation from the } \\
\text { study protocol was noticed" }\end{array}$ \\
\hline $\begin{array}{l}\text { Therapist/investigator al- } \\
\text { legiance }\end{array}$ & High risk & Study authors were involved in treatment development \\
\hline Other bias & Unclear risk & Sponsorship bias cannot be ruled out \\
\hline
\end{tabular}

Methods RCT

Psychological therapies for the treatment of mental disorders in low- and middle-income countries affected by humanitarian crises 
Neuner 2008b (Continued)

Participants

Refugees who fulfilled DSM-IV criteria for PTSD (assessed with the PDS) who lived in 2 villages closest to the research base in the settlement

\begin{tabular}{ll}
\hline Interventions & Trauma counselling (TC): 111 participants \\
& Monitoring group (MG): 55 participants \\
\hline Outcomes & PTSD symptoms
\end{tabular}

Notes

\section{Risk of bias}

\begin{tabular}{|c|c|c|}
\hline Bias & Authors' judgement & Support for judgement \\
\hline $\begin{array}{l}\text { Random sequence genera- } \\
\text { tion (selection bias) }\end{array}$ & Unclear risk & $\begin{array}{l}\text { Quote: "The list of participants was ordered randomly; the first } 4 \text { were consec- } \\
\text { utively assigned to the NET, TC, NET, and TC groups; and the fifth was assigned } \\
\text { to the MG group. This procedure was repeated until all } 277 \text { participants were } \\
\text { assigned" }\end{array}$ \\
\hline $\begin{array}{l}\text { Allocation concealment } \\
\text { (selection bias) }\end{array}$ & Unclear risk & No information provided \\
\hline $\begin{array}{l}\text { Blinding of participants } \\
\text { and personnel (perfor- } \\
\text { mance bias) } \\
\text { All outcomes }\end{array}$ & High risk & Participants were aware of their treatment allocation \\
\hline $\begin{array}{l}\text { Blinding of outcome as- } \\
\text { sessment (detection bias) } \\
\text { All outcomes }\end{array}$ & Low risk & Interviewers were blind with respect to the particular treatment condition \\
\hline $\begin{array}{l}\text { Incomplete outcome data } \\
\text { (attrition bias) } \\
\text { All outcomes }\end{array}$ & High risk & $\begin{array}{l}\text { More than } 20 \% \text { of participants abandoned the study prematurely } \\
\text { Study endpoint: 50/111 missing from NET group; 52/111 missing from TC } \\
\text { group }\end{array}$ \\
\hline $\begin{array}{l}\text { Selective reporting (re- } \\
\text { porting bias) }\end{array}$ & Low risk & Only 1 outcome measure tested \\
\hline Therapist qualification & Unclear risk & Trained counsellors \\
\hline Treatment fidelity & Unclear risk & No information provided \\
\hline $\begin{array}{l}\text { Therapist/investigator al- } \\
\text { legiance }\end{array}$ & High risk & Study authors were involved in treatment development \\
\hline Other bias & Unclear risk & Sponsorship bias cannot be ruled out \\
\hline
\end{tabular}

\section{O' Callaghan 2013}

\begin{tabular}{ll}
\hline Methods & $\begin{array}{l}\text { Single-centred, equal randomisation, single-blind (outcome assessors), parallel-group (active and wait } \\
\text { list control) study }\end{array}$ \\
\hline Participants & $\begin{array}{l}\text { The study was conducted in the Democratic Republic of the Congo, which was affected by the highest } \\
\text { rate of gender-based violence in the world. The consequences of war and sexual violence for girls in- }\end{array}$
\end{tabular}

Psychological therapies for the treatment of mental disorders in low- and middle-income countries affected by humanitarian crises 
clude physical injury, sexually transmitted infection and pregnancy, psychological distress, stigmatisation, rejection by family, and poverty

Fifty-two war-affected girls who had witnessed or had personal experience of rape or sexual abuse (described in the questionnaire as inappropriate sexual touch)

Age range: $12-17$ years

Interventions $\quad$ Trauma-focused cognitive-behavioural therapy (TF-CBT): 24 participants
Wait list (WL): 28 participants

Outcomes

The UCLA-PTSD-RI was used to assess the severity of post-traumatic stress symptoms. AYPA (African Youth Psychosocial Assessment Instrument) was used to assess the psychosocial functioning

\section{Notes}

\section{Risk of bias}

\begin{tabular}{lll}
\hline Bias & Authors' judgement & Support for judgement \\
\hline $\begin{array}{l}\text { Random sequence genera- } \\
\text { tion (selection bias) }\end{array}$ & Low risk & $\begin{array}{l}\text { Quote: "Participants were randomly assigned using a computer-generated } \\
\text { random sequence of numbers" }\end{array}$ \\
\hline $\begin{array}{l}\text { Allocation concealment } \\
\text { (selection bias) }\end{array}$ & Low risk & $\begin{array}{l}\text { Quote: "Selection bias was reduced by ensuring that treatment allocation was } \\
\text { concealed from those responsible for participant enrolment and by ensuring } \\
\text { that the person responsible for assigned participants had met none of the par- } \\
\text { ticipants before the group allocation" }\end{array}$
\end{tabular}

Blinding of participants High risk Participants were aware of their treatment allocation
and personnel (perfor-
mance bias)
All outcomes

\begin{tabular}{|c|c|c|}
\hline $\begin{array}{l}\text { Blinding of outcome as- } \\
\text { sessment (detection bias) } \\
\text { All outcomes }\end{array}$ & Low risk & $\begin{array}{l}\text { Quote: "The interviewers (outcome assessors) were blinded to the intervention } \\
\text { allocation" }\end{array}$ \\
\hline
\end{tabular}

\begin{tabular}{|c|c|c|}
\hline $\begin{array}{l}\text { Incomplete outcome data } \\
\text { (attrition bias) }\end{array}$ & Low risk & $\begin{array}{l}\text { Study endpoint: } 4 / 24 \text { missing from TF-CBT group; } 2 / 28 \text { missing from WL con- } \\
\text { trol group }\end{array}$ \\
\hline
\end{tabular}

All outcomes

\begin{tabular}{|c|c|c|}
\hline $\begin{array}{l}\text { Selective reporting (re- } \\
\text { porting bias) }\end{array}$ & Low risk & All outcomes are reported \\
\hline Therapist qualification & Unclear risk & Intervention was delivered by non-clinical facilitators \\
\hline Treatment fidelity & Low risk & $\begin{array}{l}\text { Quote: "The lead researcher (...) monitored each session to ensure treatment } \\
\text { integrity and to check that examples, activities, and teaching points discussed } \\
\text { at the preintervention meeting were addressed" }\end{array}$ \\
\hline $\begin{array}{l}\text { Therapist/investigator al- } \\
\text { legiance }\end{array}$ & Unclear risk & No information provided \\
\hline Other bias & Low risk & The study appears to be free of other sources of bias \\
\hline
\end{tabular}


Pityaratstian 2015

\begin{tabular}{|c|c|c|}
\hline Methods & \multicolumn{2}{|l|}{ Open-label RCT } \\
\hline Participants & \multicolumn{2}{|c|}{$\begin{array}{l}\text { The study was conducted in Thailand, after the devastating Tsunami that struck Asian and African } \\
\text { coastlines in December } 2004\end{array}$} \\
\hline & \multicolumn{2}{|c|}{$\begin{array}{l}\text { Inclusion criterion: primary diagnosis of DSM-IV-TR PTSD. Diagnosis was made from clinical interview } \\
\text { by board-certified child psychiatrists using a checklist according to DSM-IV-TR criteria of PTSD }\end{array}$} \\
\hline & \multicolumn{2}{|l|}{ Age range: $10-15$ years } \\
\hline Interventions & \multicolumn{2}{|c|}{ Cognitive-behavioural therapy (CBT): 18 participants } \\
\hline & \multicolumn{2}{|c|}{ Wait list (WL): 18 participants } \\
\hline Outcomes & \multicolumn{2}{|c|}{$\begin{array}{l}\text { Primary outcome: PTSD assessed with the Thai version of Children Revised Impact of Events Scale } \\
\text { (CRIES) and UCLA-PTSD-RI }\end{array}$} \\
\hline \multicolumn{3}{|l|}{ Notes } \\
\hline \multicolumn{3}{|l|}{ Risk of bias } \\
\hline Bias & Authors' judgement & Support for judgement \\
\hline $\begin{array}{l}\text { Random sequence genera- } \\
\text { tion (selection bias) }\end{array}$ & Unclear risk & Quote: "Block randomisation was carried out" \\
\hline $\begin{array}{l}\text { Allocation concealment } \\
\text { (selection bias) }\end{array}$ & Unclear risk & No information provided \\
\hline $\begin{array}{l}\text { Blinding of participants } \\
\text { and personnel (perfor- } \\
\text { mance bias) } \\
\text { All outcomes }\end{array}$ & High risk & No information provided \\
\hline $\begin{array}{l}\text { Blinding of outcome as- } \\
\text { sessment (detection bias) } \\
\text { All outcomes }\end{array}$ & Low risk & $\begin{array}{l}\text { Quote: "Participants were assessed at pretreatment, posttreatment, and 1- } \\
\text { month follow-up by blind assessors" }\end{array}$ \\
\hline $\begin{array}{l}\text { Incomplete outcome data } \\
\text { (attrition bias) } \\
\text { All outcomes }\end{array}$ & Low risk & No participants dropped out from the programme \\
\hline $\begin{array}{l}\text { Selective reporting (re- } \\
\text { porting bias) }\end{array}$ & Low risk & Two rating scales in "Methods"; both reported in "Results" (CRIES, PTSD-RI) \\
\hline Therapist qualification & Low risk & Intervention was delivered by certified child psychiatrists \\
\hline Treatment fidelity & Unclear risk & No information provided \\
\hline $\begin{array}{l}\text { Therapist/investigator al- } \\
\text { legiance }\end{array}$ & Unclear risk & Study authors were involved in training therapists \\
\hline Other bias & Unclear risk & Sponsorship bias cannot be ruled out \\
\hline
\end{tabular}


Puvimanasinghe 2016

\begin{tabular}{|c|c|c|}
\hline Methods & \multicolumn{2}{|l|}{$\mathrm{RCT}$} \\
\hline Participants & \multicolumn{2}{|c|}{$\begin{array}{l}\text { Sri Lankan survivors of torture and ill treatment, aged } 18 \text { years or over. All had experienced violence } \\
\text { personally or had significant others who experienced violence within the previous } 5 \text { years. Exclusion } \\
\text { criteria were applied to individuals showing signs of severe depression or other severe symptoms, or } \\
\text { who were not motivated to give their testimony }\end{array}$} \\
\hline Interventions & \multicolumn{2}{|c|}{ Testimony therapy: 13 participants } \\
\hline & \multicolumn{2}{|l|}{ Wait list: 13 participants } \\
\hline Outcomes & \multicolumn{2}{|c|}{$\begin{array}{l}\text { Psychosocial functioning (measured with the Sri Lanka Index of Psychosocial Status - SLIPSS-A); social } \\
\text { participation (measured with the Participation Scale - P-Scale); emotional wellbeing (measured with } \\
\text { the WHO Five Wellbeing Index - WHO-5) }\end{array}$} \\
\hline \multicolumn{3}{|l|}{ Notes } \\
\hline \multicolumn{3}{|l|}{ Risk of bias } \\
\hline Bias & Authors' judgement & Support for judgement \\
\hline $\begin{array}{l}\text { Random sequence genera- } \\
\text { tion (selection bias) }\end{array}$ & Unclear risk & $\begin{array}{l}\text { Quote: "Participants were randomly assigned to either the treatment group to } \\
\text { receive Testimony therapy, or to the waiting list type control condition" }\end{array}$ \\
\hline $\begin{array}{l}\text { Allocation concealment } \\
\text { (selection bias) }\end{array}$ & Unclear risk & No information provided \\
\hline $\begin{array}{l}\text { Blinding of participants } \\
\text { and personnel (perfor- } \\
\text { mance bias) } \\
\text { All outcomes }\end{array}$ & Unclear risk & No information provided \\
\hline $\begin{array}{l}\text { Blinding of outcome as- } \\
\text { sessment (detection bias) } \\
\text { All outcomes }\end{array}$ & Unclear risk & No information provided \\
\hline $\begin{array}{l}\text { Incomplete outcome data } \\
\text { (attrition bias) } \\
\text { All outcomes }\end{array}$ & Unclear risk & No information provided \\
\hline $\begin{array}{l}\text { Selective reporting (re- } \\
\text { porting bias) }\end{array}$ & Low risk & Outcomes listed in the "Methods" were also reported in the "Results" \\
\hline Therapist qualification & Unclear risk & Intervention was delivered by paraprofessionals \\
\hline Treatment fidelity & Unclear risk & No information provided \\
\hline $\begin{array}{l}\text { Therapist/investigator al- } \\
\text { legiance }\end{array}$ & High risk & $\begin{array}{l}\text { Quote: "The first author contributed to the development of the manual for this } \\
\text { version" }\end{array}$ \\
\hline
\end{tabular}

Rahman 2016a

\begin{tabular}{ll}
\hline Methods & Single-blind, individual RCT \\
\hline Participants & $\begin{array}{l}\text { Adults from Peshawar (a conflict-affected area of Pakistan) currently experiencing emotional distress. } \\
\text { The study was conducted between November } 2014 \text { and Juanuary } 2016\end{array}$ \\
\hline
\end{tabular}

Psychological therapies for the treatment of mental disorders in low- and middle-income countries affected by humanitarian crises 
To be included, participants need to score both 3 or higher on a screening questionnaire for common mental disorders (12-item General Health Questionnaire (GHQ-12)) and 17 or higher on a questionnaire for functional impairments (WHO Disability Assessment Schedule 2.0 (WHODAS 2.0))

Age range: $18-65$ years

\begin{tabular}{ll} 
Interventions & Problem management plus (based on problem-solving and behavioural techniques): 172 participants \\
& Enhanced usual care: 174 participants \\
\hline Outcomes & $\begin{array}{l}\text { Primary outcomes: anxiety and depressive symptoms measured by the Hospital Anxiety and Depres- } \\
\text { sion Scale (HADS-A; HADS-D; HADS total score) } \\
\text { Secondary outcomes: Post-traumatic stress disorder symptoms were measured by the 20-item Post- } \\
\text { traumatic Stress Disorder Checklist for DSM-5 (PCL-5). Functional impairment was measured by the } \\
\text { WHODAS 2.0. The Psychological Outcome Profiles (PSYCHLOPS) instrument was used to measure } \\
\text { progress on problems for which the person sought help. Participants were also assessed for symptoms } \\
\text { of depressive disorder with the 9-item Patient Health Questionnaire (PHQ-9) }\end{array}$
\end{tabular}

Notes

\section{Risk of bias}

\begin{tabular}{lll}
\hline Bias & Authors' judgement & Support for judgement \\
\hline $\begin{array}{l}\text { Random sequence genera- } \\
\text { tion (selection bias) }\end{array}$ & Low risk & $\begin{array}{l}\text { Quote: "Randomization was performed using computerized software on 1:1 } \\
\text { basis" }\end{array}$ \\
\hline $\begin{array}{l}\text { Allocation concealment } \\
\text { (selection bias) }\end{array}$ & Low risk & $\begin{array}{l}\text { Quote: "Randomization was conducted after baseline assessment by an inde- } \\
\text { pendent research assistant located off site and not involved in any other as- } \\
\text { pect of the study (...). Allocation concealment was ensured by keeping the ran- } \\
\text { dom assignments in sequentially numbered, opaque, sealed envelopes at the } \\
\text { off-site center" }\end{array}$
\end{tabular}

Blinding of participants $\quad$ High risk
and personnel (perfor-
mance bias)
All outcomes

\begin{tabular}{|c|c|c|}
\hline $\begin{array}{l}\text { Blinding of outcome as- } \\
\text { sessment (detection bias) } \\
\text { All outcomes }\end{array}$ & Low risk & $\begin{array}{l}\text { Quote: "All assessments were performed by trained research assistants blind } \\
\text { to allocation status of participants who received 2-day training covering ad- } \\
\text { ministration" }\end{array}$ \\
\hline
\end{tabular}

\begin{tabular}{lll}
\hline $\begin{array}{l}\text { Incomplete outcome data } \\
\text { (attrition bias) } \\
\text { All outcomes }\end{array}$ & High risk & $\begin{array}{l}\text { More than 20\% of participants abandoned the study prematurely } \\
\text { Study endpoint: 112/172 missing from intervention group; } 97 / 174 \text { missing } \\
\text { from enhanced usual care group }\end{array}$ \\
\hline $\begin{array}{l}\text { Selective reporting (re- } \\
\text { porting bias) }\end{array}$ & Low risk & $\begin{array}{l}\text { All outcomes stated in the "Methods" section were correctly reported. The } \\
\text { study protocol was publicly accessible }\end{array}$ \\
\hline
\end{tabular}

\begin{tabular}{|c|c|c|}
\hline Therapist qualification & Unclear risk & Intervention was delivered by lay health workers \\
\hline Treatment fidelity & Low risk & $\begin{array}{l}\text { Quote: "One in-country supervisor directly observed a randomly selected sam- } \\
\text { ple of } 10 \% \text { of health workers' sessions (...) and used a checklist to systemati- } \\
\text { cally assess fidelity to the intervention. The session-wise checklist consisted } \\
\text { of items capturing key intervention strategies for each session. The responses } \\
\text { were recorded as yes or no for each given strategy for the particular session. } \\
\text { Based on this evaluation, the supervisor rated each session overall as satisfac- }\end{array}$ \\
\hline
\end{tabular}


Rahman 2016a (Continued)

tory or unsatisfactory in terms of fidelity achieved. Identified weak areas were reinforced during supervision"

\begin{tabular}{lll}
\hline $\begin{array}{l}\text { Therapist/investigator al- } \\
\text { legiance }\end{array}$ & High risk & Study authors were involved in treatment development \\
\hline Other bias & Unclear risk & The study appears to be free of other sources of bias \\
\hline
\end{tabular}

\section{Rahman 2016b}

\begin{tabular}{ll}
\hline Methods & Single-blind, individual RCT \\
\hline Participants & $\begin{array}{l}\text { Adults from Peshawar (Pakistan) currently experiencing emotional distress. To be included, partici- } \\
\text { pants need to score both } 2 \text { or above on a screening questionnaire for common mental disorders (12- } \\
\text { item General Health Questionnaire (GHQ-12)) and 17 or higher on a questionnaire for functional impair- } \\
\text { ments (WHO Disability Assessment Schedule 2.0 (WHODAS 2.0)) }\end{array}$ \\
& Age range: 18-65 years \\
\hline Interventions & Problem management (PM) plus (based on problem-solving and behavioural techniques): 30 partici- \\
& Treatment as usual (TAU): 30 participants \\
\hline Outcomes & $\begin{array}{l}\text { Primary outcome: psychological distress, measured by GHQ-12 } \\
\text { Other outcomes included functioning, measured by the 12-item interviewer-administered screener ver- } \\
\text { sion of the WHODAS 2.0; and post-traumatic stress symptoms, measured with the PTSD Checklist for } \\
\text { DSM-5 (PCL-5) }\end{array}$ \\
\hline
\end{tabular}

Notes

\section{Risk of bias}

\begin{tabular}{|c|c|c|}
\hline Bias & Authors' judgement & Support for judgement \\
\hline $\begin{array}{l}\text { Random sequence genera- } \\
\text { tion (selection bias) }\end{array}$ & Low risk & $\begin{array}{l}\text { Quote: "Randomization to the PM+ intervention or treatment as usual was per- } \\
\text { formed by an independent researcher not involved in the project using com- } \\
\text { puterized software on a 1:1 basis, stratified for gender" }\end{array}$ \\
\hline
\end{tabular}

\begin{tabular}{lll}
\hline $\begin{array}{l}\text { Allocation concealment } \\
\text { (selection bias) }\end{array}$ & Unclear risk & No information provided \\
\hline $\begin{array}{l}\text { Blinding of participants } \\
\text { and personnel (perfor- } \\
\text { mance bias) }\end{array}$ & High risk & The trial was single blind \\
All outcomes & \\
\hline
\end{tabular}

\begin{tabular}{lll}
\hline $\begin{array}{l}\text { Blinding of outcome as- } \\
\text { sessment (detection bias) } \\
\text { All outcomes }\end{array}$ & Low risk & Assessed by independent raters \\
\hline $\begin{array}{l}\text { Incomplete outcome data } \\
\text { (attrition bias) } \\
\text { All outcomes }\end{array}$ & Low risk & Less than $20 \%$ of participants abandoned the study prematurely \\
& $\begin{array}{l}\text { Study endpoint: } 5 / 30 \text { missing from intervention group; } 4 / 30 \text { missing from en- } \\
\text { hanced usual care group }\end{array}$
\end{tabular}

Psychological therapies for the treatment of mental disorders in low- and middle-income countries affected by humanitarian crises 
Rahman 2016b (Continued)

Selective reporting (re- Unclear risk $\quad$ This was a pilot study (see Rahman 2016). A flow diagram of progress through porting bias) phases is missing. OR for the primary outcome missing

Quote: "There was no significant change in GHQ-12 scores"

\begin{tabular}{lll}
\hline Therapist qualification & Unclear risk & Intervention was delivered by trained "non-specialists" \\
\hline Treatment fidelity & Unclear risk & No information provided \\
\hline $\begin{array}{l}\text { Therapist/investigator al- } \\
\text { legiance }\end{array}$ & High risk & Study authors were involved in treatment development \\
\hline Other bias & Unclear risk & The study appears to be free of other sources of bias \\
\hline
\end{tabular}

Wang 2013a

\begin{tabular}{ll}
\hline Methods & RCT \\
\hline Participants & $\begin{array}{l}\text { People experienced at least } 1 \text { traumatic event (most participants experienced natural disaster - the } \\
2008 \text { Sichuan earthquake) according to DSM-IV trauma criteria, with the latest traumatic event happen- } \\
\text { ing } 3 \text { to } 60 \text { months before, and most reported at least } 2 \text { PTSD symptoms on the trauma screening ques- } \\
\text { tionnaire. Respondents were excluded if they had insufficient reading or auditory comprehension com- } \\
\text { petency in the Chinese language, insufficient Internet access time (<360 min in } 4 \text { weeks), or acute psy- } \\
\text { chotic symptoms, or if they were receiving other mental health intervention }\end{array}$
\end{tabular}

Interventions

Chinese My Trauma Recovery (CMTR) programme (urban/unsupported sample): 50 participants

Wait list (WL) (urban/unsupported sample): 44 participants

Outcomes Primary outcome: PTSD symptoms measured with the Post-Traumatic Diagnostic Scale (PDS)

Secondary outcomes: depressive symptoms measured with Symptom Checklist 90-Depression (SCL-D); cognitive changes after traumatic experiences measured with Post-Traumatic Cognitive Changes (PCC); functional impairment after trauma experiences measured by Social Functioning Impairment (SFI); and Trauma Coping Self-Efficacy Scale (CSE) used to determine to what extent participants felt capable of coping with PTSD reactions at different assessment points

Notes

\section{Risk of bias}

\begin{tabular}{|c|c|c|}
\hline Bias & Authors' judgement & Support for judgement \\
\hline $\begin{array}{l}\text { Random sequence genera- } \\
\text { tion (selection bias) }\end{array}$ & Low risk & $\begin{array}{l}\text { Quote: "The participants were randomly assigned to the treatment or waiting } \\
\text { list condition based on a computer-generated randomisation list" }\end{array}$ \\
\hline $\begin{array}{l}\text { Allocation concealment } \\
\text { (selection bias) }\end{array}$ & Unclear risk & No information provided \\
\hline $\begin{array}{l}\text { Blinding of participants } \\
\text { and personnel (perfor- } \\
\text { mance bias) } \\
\text { All outcomes }\end{array}$ & High risk & $\begin{array}{l}\text { No information is provided, but it's hard to believe that blinding of partici- } \\
\text { pants could have been respected in such a study design }\end{array}$ \\
\hline $\begin{array}{l}\text { Blinding of outcome as- } \\
\text { sessment (detection bias) }\end{array}$ & Low risk & $\begin{array}{l}\text { Quote: "Assessment had been computer-generated on a professional Chinese } \\
\text { survey website (equals a blinded assessment)" }\end{array}$ \\
\hline
\end{tabular}

Psychological therapies for the treatment of mental disorders in low- and middle-income countries affected by humanitarian crises 
Wang 2013a (Continued)

All outcomes

\begin{tabular}{lll}
\hline $\begin{array}{l}\text { Incomplete outcome data } \\
\text { (attrition bias) } \\
\text { All outcomes }\end{array}$ & High risk & More than 20\% of participants abandoned the study prematurely \\
\hline $\begin{array}{l}\text { Selective reporting (re- } \\
\text { porting bias) }\end{array}$ & Low risk & All outcomes are correctly reported \\
\hline $\begin{array}{l}\text { Therapist qualification } \\
\text { Ureatment fidelity }\end{array}$ & Unclear risk & $\begin{array}{l}\text { Self-help trauma intervention programme available on Internet and translated } \\
\text { by study authors }\end{array}$ \\
\hline $\begin{array}{l}\text { Therapist/investigator al- } \\
\text { legiance }\end{array}$ & Unclear risk & No information provided \\
\hline Other bias & Unclear risk & The programme was translated by study authors \\
\hline
\end{tabular}

Wang 2013b

\begin{tabular}{ll}
\hline Methods & RCT \\
\hline Participants & $\begin{array}{l}\text { People experienced at least } 1 \text { traumatic event according to DSM-IV trauma criteria, with the latest } \\
\text { traumatic event happening } 3 \text { to } 60 \text { months before; at least } 2 \text { reported PTSD symptoms on the trau- } \\
\text { ma screening questionnaire. Respondents were excluded if they had insufficient reading or auditory } \\
\text { comprehension competency in the Chinese language, insufficient Internet access time (<360 min in } 4 \\
\text { weeks), or acute psychotic symptoms, or were receiving other mental health intervention }\end{array}$ \\
\hline Interventions & Chinese My Trauma Recovery (CMTR) programme (rural/supported sample): 49 participants \\
\hline Outcomes & Primary outcome: PTSD symptoms measured with the Post-Traumatic Diagnostic Scale (PDS) \\
& $\begin{array}{l}\text { Secondary outcomes: depressive symptoms measured with Symptom Checklist 90-Depression (SCL-D); } \\
\text { cognitive changes after traumatic experiences measured with Post-Traumatic Cognitive Changes (PCC); } \\
\text { functional impairment after trauma experiences measured by Social Functioning Impairment (SFI); and } \\
\text { Trauma Coping Self-Efficacy Scale (CSE) used to measure to what extent participants felt capable of } \\
\text { coping with PTSD reactions at different assessment points }\end{array}$
\end{tabular}

Notes

\section{Risk of bias}

\begin{tabular}{lll}
\hline Bias & Authors' judgement & Support for judgement \\
\hline $\begin{array}{l}\text { Random sequence genera- } \\
\text { tion (selection bias) }\end{array}$ & Low risk & $\begin{array}{l}\text { Quote: "The participants were randomly assigned to the treatment or waiting } \\
\text { list condition based on a computer-generated randomisation list" }\end{array}$ \\
\hline $\begin{array}{l}\text { Allocation concealment } \\
\text { (selection bias) }\end{array}$ & Unclear risk & No information provided \\
\hline $\begin{array}{l}\text { Blinding of participants } \\
\text { and personnel (perfor- } \\
\text { mance bias) }\end{array}$ & High risk & $\begin{array}{l}\text { No information is provided, but it's hard to believe that blinding of partici- } \\
\text { pants could have been respected in such a study design }\end{array}$ \\
\end{tabular}

Psychological therapies for the treatment of mental disorders in low- and middle-income countries affected by humanitarian crises 
Wang 2013b (Continued)

All outcomes

\begin{tabular}{|c|c|c|}
\hline $\begin{array}{l}\text { Blinding of outcome as- } \\
\text { sessment (detection bias) }\end{array}$ & Low risk & $\begin{array}{l}\text { Quote: "Assessment had been computer-generated on a professional Chinese } \\
\text { survey website (equals a blinded assessment)" }\end{array}$ \\
\hline
\end{tabular}

All outcomes

$\begin{array}{ll}\begin{array}{l}\text { Incomplete outcome data } \\ \text { (attrition bias) }\end{array} & \text { Low risk } \\ \text { All outcomes } & \text { Less than } 20 \% \text { of participants abandoned the study prematurely } \\ \end{array}$

All outcomes

All outcomes are correctly reported

Selective reporting (re- Low risk All outcomes are correctly reported
porting bias)

\begin{tabular}{lll}
\hline Therapist qualification & Unclear risk & $\begin{array}{l}\text { Self-help trauma intervention programme available on Internet and translated } \\
\text { by study authors }\end{array}$ \\
\hline Treatment fidelity & Unclear risk & No information provided \\
\hline $\begin{array}{l}\text { Therapist/investigator al- } \\
\text { legiance }\end{array}$ & Unclear risk & The programme was translated by study authors \\
\hline Other bias & Unclear risk & Sponsorship bias cannot be ruled out \\
\hline
\end{tabular}

Wang 2016

\begin{tabular}{ll}
\hline Methods & RCT \\
\hline Participants & The study was conducted with war-affected population in Kosovo, between 2012 and 2013 \\
& $\begin{array}{l}\text { Participants } 18 \text { to } 65 \text { years old reporting } 1 \text { or more of the following experiences: torture and other cruel, } \\
\text { inhuman or degrading treatment or punishment, using UN definition; sexual harassment, molestation, } \\
\text { rape, or insertion of a blunt object into a genital organ and/or the rectum; arrest or detention without } \\
\text { warrant or order; or extrajudicial execution of family members, perpetrated by members of law en- } \\
\text { forcement agency. All participants should meet DSM-IV criteria for comorbid chronic pain and } 1 \text { of the } \\
\text { affective disorders: PTSD, depression, or anxiety }\end{array}$
\end{tabular}

Interventions Multi-disciplinary intervention (including 10 individual therapy sessions, using biofeedback-supported CBT; and 10 group sessions based on physiotherapy and exercise on a weekly basis over a 3-month period: 17 participants

Wait list: 17 participants

Participants were provided with 2 bottles of multi-vitamin for daily intake

$\begin{array}{ll}\text { Outcomes } & \text { Outcomes comprised } 4 \text { subtypes: mental health (PTSD, depression, and anxiety); emotional well-be- } \\ \text { ing (anger, aggressiveness, inferiority complex, social isolation, and police or military phobia); physical } \\ \text { health (chronic pain symptoms, body mass index, handgrip strength, and standing balance); function- } \\ \text { ing and social outcomes (income, employment rate, and disability score) }\end{array}$

Notes

\section{Risk of bias}

\begin{tabular}{lll}
\hline Bias & Authors' judgement & Support for judgement \\
\hline $\begin{array}{ll}\text { Random sequence genera- } \\
\text { tion (selection bias) }\end{array}$ & Low risk & $\begin{array}{l}\text { Quote: "The } 34 \text { participants were randomly allocated to the intervention group } \\
\text { or the waiting list group by a block randomisation procedure using a comput- }\end{array}$
\end{tabular}

Psychological therapies for the treatment of mental disorders in low- and middle-income countries affected by humanitarian crises 
Wang 2016 (Continued)

erised random number generator by two blocks of size 17 , created by DIGNITY staff not involved in the trial"

\begin{tabular}{|c|c|c|}
\hline $\begin{array}{l}\text { Allocation concealment } \\
\text { (selection bias) }\end{array}$ & Unclear risk & No information provided \\
\hline
\end{tabular}

\begin{tabular}{|c|c|c|}
\hline $\begin{array}{l}\text { Blinding of outcome as- } \\
\text { sessment (detection bias) }\end{array}$ & Low risk & $\begin{array}{l}\text { Quote: "The baseline and outcome assessor [were] blinded to which group } \\
\text { was which, throughout the study" }\end{array}$ \\
\hline
\end{tabular}

All outcomes

\begin{tabular}{|c|c|c|}
\hline $\begin{array}{l}\text { Incomplete outcome data } \\
\text { (attrition bias) }\end{array}$ & Low risk & $\begin{array}{l}\text { Dropouts were reported. At } 3 \text { months' follow-up, dropouts were } 2 / 17 \text { in the in- } \\
\text { tervention group and } 0 / 17 \text { in the wait list group }\end{array}$ \\
\hline
\end{tabular}

All outcomes

\begin{tabular}{lll}
\hline $\begin{array}{l}\text { Selective reporting (re- } \\
\text { porting bias) }\end{array}$ & Unclear risk & $\begin{array}{l}\text { Outcomes are reported. At follow-up, only differences between means are re- } \\
\text { ported instead of rating scale scores }\end{array}$ \\
\hline Therapist qualification & Low risk & $\begin{array}{l}\text { Intervention was delivered by trained psychologists and a medical doctor with } \\
\text { experience in CBT }\end{array}$ \\
\hline Treatment fidelity & Low risk & $\begin{array}{l}\text { Quote: "The supervision also aimed to ensure that the therapists were all fol- } \\
\text { lowing the therapy protocol in the same way. Additionally, to check treatment } \\
\text { integrity and compliance for quality assurance, at least 15\% of the CBT treat- } \\
\text { ment sessions were randomly selected and recorded using a digital audio } \\
\text { recorder. An independent assessor from Israel (...) reviewed these recorded } \\
\text { sessions with the assistance of an experienced interpreter with medical doctor } \\
\text { background" }\end{array}$ \\
\hline
\end{tabular}

\begin{tabular}{l}
$\begin{array}{l}\text { Therapist/investigator al- High risk } \\
\text { legiance }\end{array}$ \\
\hline
\end{tabular}

Weiss 2015a

\begin{tabular}{ll}
\hline Methods & RCT \\
\hline Participants & $\begin{array}{l}\text { The study was conducted in Southern Iraq (recruitment was active between April } 2011 \text { and January } \\
\text { 2012). Participants were survivors of systematic violence referred to the CMHWs by physicians in the } \\
\text { health center where they worked, from local prisoners' associations, and through self-referral after } \\
\text { learning of services through public service announcements or by word of mouth. Survivors were de- } \\
\text { fined as persons having experienced or witnessed physical torture or militant attacks. A survivor who } \\
\text { was } 18 \text { years of age or older and who met the symptom criterion was eligible for the trial }\end{array}$ \\
\hline Interventions & Common Elements Treatment Approach (CETA): 99 participants \\
& Wait list: 50 participants \\
\hline Outcomes & $\begin{array}{l}\text { Primary outcome was trauma symptoms (locally validated Harvard Trauma Questionnaire - HTQ) } \\
\text { Secondary outcomes were dysfunction (assessed by mean item scores for gender-specific items on the } \\
\text { locally developed dysfunction scale), anxiety, and depression (assessed by the mean item score on the } \\
\text { locally validated HSCL-25) }\end{array}$ \\
\hline
\end{tabular}

Psychological therapies for the treatment of mental disorders in low- and middle-income countries affected by humanitarian crises 
Weiss 2015a (Continued)

Notes

\section{Risk of bias}

\begin{tabular}{lll}
\hline Bias & Authors' judgement & Support for judgement \\
\hline $\begin{array}{l}\text { Random sequence genera- } \\
\text { tion (selection bias) }\end{array}$ & Low risk & Quote: "The assignment was generated using a random number generator" \\
\hline $\begin{array}{l}\text { Allocation concealment } \\
\text { (selection bias) }\end{array}$ & Low risk & $\begin{array}{l}\text { Quote: "A piece of paper indicating the treatment assignment (intervention or } \\
\text { wait list) was stapled directly to the back of the study consent forms that were } \\
\text { pre-numbered with the participant identification number. This paper could } \\
\text { only be read if removed from the consent form" }\end{array}$ \\
\hline
\end{tabular}

Blinding of participants High risk Participants were aware of their allocation

and personnel (perfor-

mance bias)

All outcomes

\begin{tabular}{|c|c|c|}
\hline $\begin{array}{l}\text { Blinding of outcome as- } \\
\text { sessment (detection bias) } \\
\text { All outcomes }\end{array}$ & Low risk & $\begin{array}{l}\text { Quote. "To maintain blinding, follow-up interviews were done by a different } \\
\text { community mental health worker than the one who recruited the participant } \\
\text { so they were unaware of the participant's assignment" }\end{array}$ \\
\hline
\end{tabular}

All outcomes

so they were unaware of the participant's assignment"

Incomplete outcome data Low risk

Study endpoint: 2/99 missing from CETA group; 2/50 missing from WL group

(attrition bias)

All outcomes

\begin{tabular}{ll}
\hline $\begin{array}{l}\text { Selective reporting (re- } \\
\text { porting bias) }\end{array}$ & Low risk
\end{tabular}

\begin{tabular}{lll}
\hline $\begin{array}{l}\text { Therapist qualification } \\
\text { Treatment fidelity }\end{array}$ & Low risk & $\begin{array}{l}\text { Intervention was delivered by trained community mental health workers } \\
\text { (CMHWs) }\end{array}$ \\
\hline $\begin{array}{l}\text { Therapist/investigator al- } \\
\text { legiance }\end{array}$ & High risk & $\begin{array}{l}\text { Quote: "Fidelity was tracked by CMHW self report of elements delivered, super- } \\
\text { visor review of notes and CMHW reports, and finally by trainer review" }\end{array}$ \\
\hline Other bias & Low risk & Study authors developed the intervention \\
& $\begin{array}{l}\text { The research and the services programme provided by Heartland Alliance } \\
\text { were solely funded by the USAID Victims of Torture fund (VOT) Award DFD- } \\
\text { A-00-08-00308-00. USAID/VOT was not involved in the research or programme } \\
\text { design or implementation, nor in the management or analysis of data. }\end{array}$ \\
& The study appears to be free of other sources of bias
\end{tabular}

Weiss 2015b

\begin{tabular}{ll}
\hline Methods & RCT \\
\hline Participants & $\begin{array}{l}\text { Participants were survivors of systematic violence referred to CMHWs by physicians in the health center } \\
\text { where they worked, from local prisoners' associations, and through self-referral after learning of ser- } \\
\text { vices through public service announcements or by word of mouth. Survivors were defined as persons } \\
\text { having experienced or witnessed physical torture or militant attacks. A survivor who was } 18 \text { years of } \\
\text { age or older and who met the symptom criterion was eligible for the trial }\end{array}$ \\
\hline
\end{tabular}

Psychological therapies for the treatment of mental disorders in low- and middle-income countries affected by humanitarian crises 
Weiss 2015b (Continued)

Interventions Cognitive processing therapy (CPT): 129 participants

Wait list: 64 participants

Outcomes Primary outcome was trauma symptoms (locally validated Harvard Trauma Questionnaire)

Secondary outcomes were dysfunction (assessed by mean item scores for gender-specific items on the locally developed dysfunction scale), anxiety, and depression (assessed by the mean item score on the locally validated HSCL-25)

Notes

\section{Risk of bias}

\begin{tabular}{lll}
\hline Bias & Authors' judgement & Support for judgement \\
\hline $\begin{array}{l}\text { Random sequence genera- } \\
\text { tion (selection bias) }\end{array}$ & Low risk & Quote: "The assignment was generated using a random number generator" \\
\hline $\begin{array}{l}\text { Allocation concealment } \\
\text { (selection bias) }\end{array}$ & Low risk & $\begin{array}{l}\text { Quote. "A piece of paper indicating the treatment assignment (intervention or } \\
\text { wait list) was stapled directly to the back of the study consent forms that were } \\
\text { pre-numbered with the participant identification number. } \\
\text { only be read if removed from the consent form" }\end{array}$ \\
\hline
\end{tabular}

Blinding of participants High risk Participants were aware of their allocation
and personnel (perfor-

\begin{tabular}{|c|c|c|}
\hline $\begin{array}{l}\text { Blinding of outcome as- } \\
\text { sessment (detection bias) } \\
\text { All outcomes }\end{array}$ & Low risk & $\begin{array}{l}\text { Quote. "To maintain blinding, follow-up interviews were done by a different } \\
\text { community mental health worker than the one who recruited the participant } \\
\text { so they were unaware of the participant's assignment" }\end{array}$ \\
\hline
\end{tabular}

\begin{tabular}{|c|c|c|}
\hline $\begin{array}{l}\text { Incomplete outcome data } \\
\text { (attrition bias) } \\
\text { All outcomes }\end{array}$ & Low risk & Study endpoint: 22/129 missing from CPT group; 3/64 missing from WL group \\
\hline $\begin{array}{l}\text { Selective reporting (re- } \\
\text { porting bias) }\end{array}$ & Low risk & $\begin{array}{l}\text { The study protocol is not available, but it is clear that published reports in- } \\
\text { clude all expected outcomes }\end{array}$ \\
\hline Therapist qualification & Unclear risk & $\begin{array}{l}\text { Intervention was delivered by trained community mental health workers } \\
\text { (CMHWs) }\end{array}$ \\
\hline Treatment fidelity & Unclear risk & No information provided \\
\hline $\begin{array}{l}\text { Therapist/investigator al- } \\
\text { legiance }\end{array}$ & Unclear risk & No information provided \\
\hline \multirow[t]{2}{*}{ Other bias } & Low risk & $\begin{array}{l}\text { The research and the services programme provided by Heartland Alliance } \\
\text { was solely funded by the USAID Victims of Torture fund (VOT) Award DFD- } \\
\text { A-00-08-00308-00. USAID/VOT was not involved in the research or programme } \\
\text { design or implementation, nor in the management or analysis of data. }\end{array}$ \\
\hline & & The study appears to be free of other sources of bias \\
\hline
\end{tabular}


Zang 2013

\begin{tabular}{ll}
\hline Methods & RCT \\
\hline Participants & $\begin{array}{l}\text { The study was conducted after the Sichuan earthquake of 2008. Randomisation took place between } \\
\text { December } 2009 \text { and March } 2010 \text { (19 to } 23 \text { months after the earthquake) } \\
\text { All eligible participants were adults } 18 \text { years of age or over who met DSM-IV criteria for PTSD as mea- } \\
\text { sured by the PDS }\end{array}$ \\
\hline Interventions & Warrative exposure therapy (NET): 11 participants 11 participants \\
\hline Outcomes & $\begin{array}{l}\text { Severity of PTSD symptoms was assessed by the Impact of Event Scale-Revised (IES-R); depression and } \\
\text { anxiety on the Hospital Anxiety and Depression Scale (HADS); general health with the Chinese version } \\
\text { of the General Health Questionnaire-28 (GHQ-28); both positive and negative post-traumatic changes } \\
\text { on the Short Form of the Changes in Outlook Questionnaire (CiQQ-S); social support by the Multidimen- } \\
\text { sional Scale of Perceived Social Support (MSPSS); and coping strategies with the Simplified Coping } \\
\text { Style Questionnaire (SCSQ) }\end{array}$
\end{tabular}

Notes

\section{Risk of bias}

\begin{tabular}{lll}
\hline Bias & Authors' judgement & Support for judgement \\
\hline $\begin{array}{l}\text { Random sequence genera- } \\
\text { tion (selection bias) }\end{array}$ & Low risk & $\begin{array}{l}\text { Quote: "Twenty two participants were randomly allocated to either NET } \\
(\mathrm{N}=11) \text { or a waiting list condition (WL; N=11) by a computer-generated list of } \\
\text { random numbers" }\end{array}$ \\
& &
\end{tabular}

\begin{tabular}{|c|c|c|}
\hline $\begin{array}{l}\text { Allocation concealment } \\
\text { (selection bias) }\end{array}$ & Unclear risk & No information provided \\
\hline
\end{tabular}

\begin{tabular}{|c|c|c|}
\hline $\begin{array}{l}\text { Blinding of participants } \\
\text { and personnel (perfor- }\end{array}$ & High risk & $\begin{array}{l}\text { No information is provided, but it's hard to believe that blinding of partici- } \\
\text { pants could have been respected in such a study design }\end{array}$ \\
\hline
\end{tabular}
mance bias)

All outcomes

Blinding of outcome as- $\quad$ Low risk
sessment (detection bias)

All outcomes

\begin{tabular}{lll}
\hline $\begin{array}{l}\text { Incomplete outcome data } \\
\text { (attrition bias) } \\
\text { All outcomes }\end{array}$ & Low risk & No participants dropped out from the programme \\
\hline $\begin{array}{l}\text { Selective reporting (re- } \\
\text { porting bias) }\end{array}$ & Low risk & $\begin{array}{l}\text { The study protocol is not available, but it is clear that published reports in- } \\
\text { clude all expected outcomes }\end{array}$ \\
\hline Therapist qualification & Low risk & Intervention was delivered by trained psychologists \\
\hline Treatment fidelity & Low risk & $\begin{array}{l}\text { Quote: "Treatment adherence was monitored by the direct observation of } \\
\text { treatment sessions, by case discussions in supervision meetings, and by a re- } \\
\text { view of the records and treatment protocols" }\end{array}$
\end{tabular}

\begin{tabular}{lll}
\hline $\begin{array}{l}\text { Therapist/investigator al- } \\
\text { legiance }\end{array}$ & Unclear risk & No information provided \\
\hline Other bias & Low risk & The study appears to be free of other sources of bias \\
\hline
\end{tabular}

Psychological therapies for the treatment of mental disorders in low- and middle-income countries affected by humanitarian crises Quote: "The pre and post treatment assessments were carried out by a researcher not involved in treatment and blind to the treatment conditions. The details of the condition were unknown to the researcher" 
Zang 2014

\begin{tabular}{ll} 
Methods & Randomised, wait list controlled study. \\
\hline Participants & $\begin{array}{l}\text { The study was conducted after the Sichuan earthquake in 2008. Randomisation took place between } \\
\text { October } 2010 \text { and January } 2011 \text { (30 to } 34 \text { months after the earthquake) } \\
\text { Eligible participants were } 18 \text { years of age or older and met DSM-IV criteria for PTSD as measured by the } \\
\text { PDS. Exclusion criteria included suicidal ideation or substance abuse, participation in another psycho- } \\
\text { logical treatment programme, and an inability to finish treatment }\end{array}$ \\
\hline Narrative exposure therapy (NET): 10 participants \\
NET-R: 10 participants \\
Wait list: 10 participants \\
$\begin{array}{l}\text { Severity of PTSD symptoms was assessed via the Impact of Event Scale-Revised (IES-R); depression and } \\
\text { anxiety with the Hospital Anxiety and Depression Scale (HADS); general health on the Chinese version } \\
\text { of the General Health Questionnaire-28 (GHQ-28); both positive and negative post-traumatic changes } \\
\text { on the Short Form of the Changes in Outlook Questionnaire (CiQQ-S); social support by the Multidimen- } \\
\text { sional Scale of Perceived Social Support (MSPSS); and coping strategies by the 28-item Brief COPE }\end{array}$ \\
\hline Outcomes
\end{tabular}

Notes

\section{Risk of bias}

\begin{tabular}{|c|c|c|}
\hline Bias & Authors' judgement & Support for judgement \\
\hline $\begin{array}{l}\text { Random sequence genera- } \\
\text { tion (selection bias) }\end{array}$ & Low risk & $\begin{array}{l}\text { "Participants were randomly allocated }[. . .] \text { by a computer-generated list of } \\
\text { random numbers" }\end{array}$ \\
\hline $\begin{array}{l}\text { Allocation concealment } \\
\text { (selection bias) }\end{array}$ & Unclear risk & No information provided \\
\hline $\begin{array}{l}\text { Blinding of participants } \\
\text { and personnel (perfor- } \\
\text { mance bias) } \\
\text { All outcomes }\end{array}$ & High risk & $\begin{array}{l}\text { No information is provided, but it's hard to believe that blinding of partici- } \\
\text { pants could have been respected in such a study design }\end{array}$ \\
\hline $\begin{array}{l}\text { Blinding of outcome as- } \\
\text { sessment (detection bias) } \\
\text { All outcomes }\end{array}$ & Low risk & $\begin{array}{l}\text { Quote: "The pre- and post- treatment assessments were carried out by a } \\
\text { trained assessor not involved in the treatments and blind to the treatment } \\
\text { conditions" }\end{array}$ \\
\hline $\begin{array}{l}\text { Incomplete outcome data } \\
\text { (attrition bias) } \\
\text { All outcomes }\end{array}$ & Low risk & No participants dropped out from the programme \\
\hline $\begin{array}{l}\text { Selective reporting (re- } \\
\text { porting bias) }\end{array}$ & Low risk & $\begin{array}{l}\text { The study protocol is not available, but it is clear that published reports in- } \\
\text { clude all expected outcomes }\end{array}$ \\
\hline Therapist qualification & Low risk & Intervention was delivered by trained psychologists \\
\hline Treatment fidelity & Low risk & $\begin{array}{l}\text { Quote: "Treatment adherence was monitored by the direct observation of } \\
\text { treatment sessions, by case discussions in supervision meetings, and by a re- } \\
\text { view of the records and treatment protocols" }\end{array}$ \\
\hline
\end{tabular}


Zang 2014 (Continued)

Therapist/investigator al- Unclear risk No information provided legiance

Other bias

Low risk

Sponsorship: "The research was also supported and partially sponsored by the Building Experience and Skill Travel Scholarships (BESTS) of the University of Nottingham"

AUDIT: Alcohol Use Disorders Identification Test

AYPA: African Youth Psychosocial Assessment Instrument

$\mathrm{BA}$; behavioural activation

BDI: Beck Depression Inventory

CAPS: Clinician Administered Post-traumatic stress disorder Scale

CBT: cognitive-behavioural therapy

CD-RISC: Connor-Davidson Resilience Scale

CES-D: Center for Epidemiologic Studies Depression Scale

CETA: common elements treatment approach

CGI: Clinician's Global Impression-Improvement

CiQQ-S: Changes in Outlook Questionnaire

CMHW: community mental health worker

CMTR: Chinese My Trauma Recovery

$\mathrm{CP}:$ creative play

CPT: cognitive processing therapy

CRIES: Children Revised Impact of Events Scale

CSE: Trauma Coping Self-Efficacy Scale

CTS: Conflict Tactics Scale

DSM: Diagnostic and Statistical Manual of Mental Disorders

EUROHIS-QOL: European Health Interview Survey-Quality of Life

EMDR: eye movement desensitisation and reprocessing

FAQ: Fear and Avoidance Questionnaire

FORNET: Narrative exposure therapy for forensic offender rehabilitation

GHQ: General Health Questionnaire

GIS: Global Improvement Scale

GSE: Generalized Self Efficacy

HADS: Hospital Anxiety and Depression Scale

HSCL: Hopkins Symptoms Checklist

HTQ: Harvard Trauma Questionnaire

IES-R: Impact of Event Scale-Revised

IPT: interpersonal psychotherapy

IPT-G: group interpersonal psychotherapy

ISIL: Islamic State of Iraq and the Levant

ISIS: Islamic State of Iraq and Syria

MDD: major depressive disorder

MG: monitoring

min: minute

MPSS: Modified Post-traumatic stress disorder Symptom Scale

MSPSS: Multidimensional Scale of Perceived Social Support

$\mathrm{N}$ : number

NET: narrative exposure therapy

NGO: non-governmental organisation

NITE: Nocturnal Intrusions after Traumatic Experiences questionnaire

OR: odds ratio

PCC: Post-Traumatic Cognitive Changes

PCL: Post-traumatic stress disorder Check List

PDS: Post-traumatic stress disorder Diagnostic Scale

PHQ: Patient Health Questionnaire

PM: problem management

PSS-I : Post-traumatic stress disorder Symptom Scale - Interview

PSYCHLOPS: Psychological Outcome Profiles

PTSD; post-traumatic stress disorder

Psychological therapies for the treatment of mental disorders in low- and middle-income countries affected by humanitarian crises

Copyright $\odot 2018$ The Cochrane Collaboration. Published by John Wiley \& Sons, Ltd. 
PTSD-RI: Post-traumatic stress disorder Reaction Index

PTSS: post-traumatic stress syndrome

QoL: quality of life

QLI: Quality of Life Index

$\mathrm{RCT}$ : randomised controlled trial

R-TEP: Recent Traumatic Episode Protocol

SAS: Social Adjustment Scale

SCID: structured clinical interview

SCL-90: Symptom Checklist 90

SCL-D: Symptom Checklist 90-Depression

SCL90-R: Symptom Checklist 90-Revised

SCSQ: Simplified Coping Style Questionnaire

SD: standard deviation

SFI: Social Functioning Impairment

SIFP: Self-Inventory for post-traumatic stress disorder

SLIPSS-A: Sri Lanka Index of Psychosocial Status

SNRI: serotonin-norepinephrine reuptake inhibitor

SRQ: Self-Report Questionnaire

SSBT: school-based support team

SSRI: selective serotonin reuptake inhibitor

STAXI: State Trait Anger Expression Inventory

TC: trauma counselling

TF-CBT: trauma-focused cognitive-behavioural therapy

TFT: thought field therapy

TSSC: Traumatic Stress Symptom Checklist

TSI: Trauma Symptom Inventory

UCLA-PTSD- RI: University College of Los Angeles Post-traumatic stress disorder Reaction Index

UN: United Nations

WHO: World Health Organisation

WHODAS: World Health Organisation Disability Assessment Schedule

WHO-5: World Health Organisation Five Wellbeing Index

WL: wait list

WLC: wait list control

WSA: Work and Social Adjustment Scale

Characteristics of excluded studies [ordered by study ID]

\begin{tabular}{ll}
\hline Study & Reason for exclusion \\
\hline Adenauer 2011 & Wrong setting: The study was carried out with refugees resettled in high-income countries \\
\hline Ager 2011 & Incorrect randomisation process \\
\hline Akhtar 1994 & Wrong study design (no RCT) \\
\hline Ayoughi 2012 & Incorrect randomisation process \\
\hline Barron 2013 & Wrong setting \\
\hline Bass 2012 & Wrong study design (no RCT) \\
\hline Bass 2013 & Wrong comparison \\
\hline Başoğlu 2003 & Wrong study design (no RCT) \\
\hline Beck 2009 & Wrong setting \\
\hline Becker 2009 & Wrong study design (no RCT) \\
\hline
\end{tabular}

Psychological therapies for the treatment of mental disorders in low- and middle-income countries affected by humanitarian crises

Copyright $\odot 2018$ The Cochrane Collaboration. Published by John Wiley \& Sons, Ltd. 


Study Reason for exclusion

Berger $2015 \quad$ Wrong setting

Betancourt 2014

\section{Bichescu 2007}

Bolton 2003

Bolton 2009

Chibanda 2016

Gordon 2008

Wrong intervention and population (This intervention is classified as preventive psychosocial intervention for people suffering from psychological distress; therefore, the study will be included in the Cochrane review focused on preventive psychosocial interventions)

Active treatments compared: narrative exposure therapy vs psychoeducation

Wrong setting (not a humanitarian crisis)

Wrong study design (no RCT)

Wrong intervention and population (This intervention is classified as preventive psychosocial intervention for people suffering from psychological distress; therefore, the study will be included in the Cochrane review focused on preventive psychosocial interventions)

Wrong intervention and population (This intervention is classified as preventive psychosocial intervention for people suffering from psychological distress; therefore, the study will be included in the Cochrane review focused on preventive psychosocial interventions)

\begin{tabular}{|c|c|}
\hline Jordans 2010 & $\begin{array}{l}\text { Wrong intervention and population (This intervention is classified as preventive psychosocial inter- } \\
\text { vention for people suffering from psychological distress; therefore, the study will be included in the } \\
\text { Cochrane review focused on preventive psychosocial interventions) }\end{array}$ \\
\hline Mughal 2015 & Wrong population (not randomised) \\
\hline Murray 2015 & Wrong setting (not a humanitarian crisis) \\
\hline Newnham 2015 & Wrong study design (no RCT) \\
\hline O'Callaghan 2014 & $\begin{array}{l}\text { Wrong intervention and population (This intervention is classified as preventive psychosocial inter- } \\
\text { vention for people suffering from psychological distress; therefore, the study will be included in the } \\
\text { Cochrane review focused on preventive psychosocial interventions) }\end{array}$ \\
\hline Pokharial 2012 & Wrong comparison \\
\hline Punamaki 2014 & $\begin{array}{l}\text { Wrong intervention and population (This intervention is classified as preventive psychosocial inter- } \\
\text { vention for people suffering from psychological distress; therefore, the study will be included in the } \\
\text { Cochrane review focused on preventive psychosocial interventions) }\end{array}$ \\
\hline Richards 2014 & $\begin{array}{l}\text { Wrong intervention and population (This intervention is classified as preventive psychosocial inter- } \\
\text { vention for people suffering from psychological distress; therefore, the study will be included in the } \\
\text { Cochrane review focused on preventive psychosocial interventions) }\end{array}$ \\
\hline Robson 2016 & Wrong setting (not a humanitarian crisis) \\
\hline Tiwari 2010 & Wrong setting \\
\hline Tol 2008 & $\begin{array}{l}\text { Wrong intervention and population (This intervention is classified as preventive psychosocial inter- } \\
\text { vention for people suffering from psychological distress; therefore, the study will be included in the } \\
\text { Cochrane review focused on preventive psychosocial interventions) }\end{array}$ \\
\hline Tol 2012 & $\begin{array}{l}\text { Wrong intervention and population (This intervention is classified as preventive psychosocial inter- } \\
\text { vention for people suffering from psychological distress; therefore, the study will be included in the } \\
\text { Cochrane review focused on preventive psychosocial interventions) }\end{array}$ \\
\hline
\end{tabular}

Psychological therapies for the treatment of mental disorders in low- and middle-income countries affected by humanitarian crises (Review)

Copyright $\odot 2018$ The Cochrane Collaboration. Published by John Wiley \& Sons, Ltd. 


\begin{tabular}{ll}
\hline Study & Reason for exclusion \\
\hline Tol 2014 & $\begin{array}{l}\text { Wrong intervention and population (This intervention is classified as preventive psychosocial inter- } \\
\text { vention for people suffering from psychological distress; therefore, the study will be included in the } \\
\text { Cochrane review focused on preventive psychosocial interventions) }\end{array}$ \\
\hline Unterhitzenberger 2014 & $\begin{array}{l}\text { Wrong intervention and population (This intervention is classified as preventive psychosocial inter- } \\
\text { vention for people suffering from psychological distress; therefore, the study will be included in the } \\
\text { Cochrane review focused on preventive psychosocial interventions) }\end{array}$ \\
\hline
\end{tabular}

RCT: randomised controlled trial

Characteristics of studies awaiting assessment [ordered by study ID]

Abbasnehzad 2007

\begin{tabular}{ll}
\hline Methods & RCT \\
\hline Participants & Adults who underwent the stress of the earthquake in Bam \\
\hline Interventions & Eye movement desensitisation and reprocessing (EMDR) intervention: 21 participants \\
& Wait list control: 20 participants \\
\hline Outcomes & $\begin{array}{l}\text { Anxiety (measured with the Beck Anxiety Inventory), depression (measured with the Back Depres- } \\
\text { sion Inventory), negative emotions }\end{array}$ \\
\hline Notes & The full paper of this study was not available \\
\hline
\end{tabular}

Barron 2016

\begin{tabular}{ll}
\hline Methods & RCT \\
\hline Participants & Adolescents in Palestine with high levels of PTSD \\
\hline Interventions & Teaching recovery techniques vs wait list \\
\hline Outcomes & PTSD symptoms, depression, dissociation \\
\hline Notes & Unclear setting \\
\hline
\end{tabular}

\section{Dawson 2016}

\begin{tabular}{ll}
\hline Methods & RCT \\
\hline Participants & Women exposed to gender-based violence in Nairobi \\
\hline Interventions & Problem management plus vs enhanced TAU \\
\hline Outcomes & General psychological distress, PTSD symptoms \\
\hline Notes & \\
\hline
\end{tabular}

Psychological therapies for the treatment of mental disorders in low- and middle-income countries affected by humanitarian crises 
Esala 2017

\begin{tabular}{ll}
\hline Methods & RCT \\
\hline Participants & Torture survivors across Cambodia \\
\hline Interventions & Testimony therapy vs control \\
\hline Outcomes & PTSD symptoms, anxiety, depressive symptoms
\end{tabular}

\section{Notes}

\section{Khan 2017}

\begin{tabular}{ll}
\hline Methods & RCT \\
\hline Participants & Women in rural Pakistan with common mental disorders \\
\hline Interventions & Problem management plus vs enhanced TAU \\
\hline Outcomes & Depression, anxiety, general psychological profile and functioning, PTSD symptoms \\
\hline Notes & \\
\hline
\end{tabular}

Mahmoudi-Gharaei 2006

\begin{tabular}{ll}
\hline Methods & RCT \\
\hline Participants & Adolescents with PTSD symptoms who survived the Bam earthquake \\
\hline Interventions & Group cognitive-behavioural therapy (CBT) plus art and sport interventions \\
& $\begin{array}{l}\text { Sport interventions without CBT } \\
\text { Control }\end{array}$ \\
\hline Outcomes & PTSD symptoms \\
\hline Notes & The full paper was available only in Arabic \\
\hline PCT02598024 & RCT \\
\hline Methods & No information available \\
\hline Participants & NET vs control \\
\hline Interventions & PTSD symptoms, disability \\
\hline Outcomes & \\
\hline
\end{tabular}


NCT02598024 (Continued)
Notes
No information on study methods and participants

Reger 2016

\begin{tabular}{ll}
\hline Methods & RCT \\
\hline Participants & Military population \\
\hline Interventions & Prolonged exposure vs virtual reality exposure vs wait list \\
\hline Outcomes & PTSD symptoms \\
\hline Notes & Unclear setting \\
\hline
\end{tabular}

Steinert 2017

\begin{tabular}{ll}
\hline Methods & RCT \\
\hline Participants & Outpatients living in Cambodia \\
\hline Interventions & Resource activation vs wait list \\
\hline Outcomes & PTSD, anxiety, depression, impaired functioning \\
\hline
\end{tabular}

Notes

Weinstein 2016

\begin{tabular}{ll}
\hline Methods & RCT \\
\hline Participants & Syrian refugees resettled in Jordan \\
\hline Interventions & Psychological intervention vs control \\
\hline Outcomes & Symptoms of depression and generalised stress, PTSD \\
\hline Notes & \\
\hline
\end{tabular}

NET: narrative exposure therapy

PTSD: post-traumatic stress disorder

RCT: randomised controlled trial

TAU: treatment as usual

Characteristics of ongoing studies [ordered by study ID]

\section{ISRCTN65771265}

Trial name or title

Nguvu: a randomised controlled trial of an integrated intervention to reduce intimate partner violence and psychological distress in adult, female Congolese refugees in Tanzania

Psychological therapies for the treatment of mental disorders in low- and middle-income countries affected by humanitarian crises 
ISRCTN65771265 (Continued)

\begin{tabular}{ll} 
Methods & Randomised controlled trial \\
\hline Participants & Adult (18 years or older) Congolese female refugees (in Tanzania) \\
\hline Interventions & $\begin{array}{l}\text { Nguvu intervention: 8-week programme that integrates advocacy and empowerment counselling } \\
\text { with cognitive processing therapy } \\
\text { Control: TAU (standard mental health and protection services) }\end{array}$
\end{tabular}

Outcomes

Primary outcomes: Domestic Violence Module of the Demographic and Health Survey at baseline and at 9 and 24 weeks post enrolment; psychological distress symptoms measured on the 25-item Hopkins Symptom Checklist (HSCL-25); and PTSD symptom items on the Harvard Trauma Questionnaire (HTQ) at baseline and at 9 and 24 weeks post enrolment

Secondary outcomes: functional impairment measured by 22 items developed from qualitative data at baseline and at 9 and 24 weeks post enrolment

\begin{tabular}{ll}
\hline Starting date & $27 / 06 / 2016$ \\
\hline Contact information & $\begin{array}{l}\text { Claire Greene; Wietse Tol; Johns Hopkins Bloomberg School of Public Health, Johns Hopkins Uni- } \\
\text { versity, Baltimore (USA) }\end{array}$ \\
\hline Notes & \\
\hline
\end{tabular}

\section{NCT03012451}

\begin{tabular}{ll}
\hline Trial name or title & A psychosocial program impact evaluation in Jordan \\
\hline Methods & Randomised controlled trial \\
\hline Participants & $\begin{array}{l}\text { Syrian refugee and Jordanian host-community youth (12 to 18 years) residing in } 4 \text { urban centres in } \\
\text { northern Jordan }\end{array}$ \\
\hline Interventions & $\begin{array}{l}\text { Advancing Adolescents behavioural programme } \\
\text { Control: wait list }\end{array}$ \\
\hline Outcomes & $\begin{array}{l}\text { Psychological stress; mental health difficulties; prosocial behaviour; resilience; biological stress; } \\
\text { cognitive function; PTSD measures }\end{array}$ \\
\hline Starting date & 21/12/2016 \\
\hline Contact information & Catherine Panter-Brick, Yale University, New Haven, USA \\
\hline
\end{tabular}

\section{NCT031090028}

\begin{tabular}{ll}
\hline Trial name or title & Stepped care model supporting mental health in refugees and asylum seekers \\
\hline Methods & Randomised controlled trial \\
\hline Participants & Refugees and asylum seekers \\
\hline
\end{tabular}

Psychological therapies for the treatment of mental disorders in low- and middle-income countries affected by humanitarian crises 
NCT031090028 (Continued)

\begin{tabular}{ll} 
Interventions & Smartphone-based interventions vs TAU \\
\hline Outcomes & Depression severity and traumatic events \\
\hline Starting date & $6 / 04 / 2017$ \\
\hline Contact information & Malek Bajbouj, Germany \\
\hline Notes & Not yet recruiting \\
\hline
\end{tabular}

PTSD: post-traumatic stress disorder

TAU: treatment as usual

\section{DATA AND ANALYSES}

Comparison 1. Psychological therapy vs control comparator - PTSD symptoms - adults

\begin{tabular}{|c|c|c|c|c|}
\hline Outcome or subgroup title & No. of studies & $\begin{array}{l}\text { No. of partici- } \\
\text { pants }\end{array}$ & Statistical method & Effect size \\
\hline 1 PTSD at endpoint & 16 & 1272 & $\begin{array}{l}\text { Std. Mean Difference (IV, Random, } \\
95 \% \mathrm{CI} \text { ) }\end{array}$ & $-1.07[-1.34,-0.79]$ \\
\hline 1.1 EMDR vs control - Adults & 2 & 99 & $\begin{array}{l}\text { Std. Mean Difference (IV, Random, } \\
95 \% \mathrm{Cl} \text { ) }\end{array}$ & $-2.01[-2.50,-1.52]$ \\
\hline 1.2 CBT vs control - Adults & 12 & 1008 & $\begin{array}{l}\text { Std. Mean Difference (IV, Random, } \\
95 \% \mathrm{CI})\end{array}$ & $-0.85[-1.13,-0.58]$ \\
\hline 1.3 IPT vs control - Adults & 1 & 20 & $\begin{array}{l}\text { Std. Mean Difference (IV, Random, } \\
95 \% \mathrm{CI} \text { ) }\end{array}$ & $-1.45[-2.46,-0.43]$ \\
\hline 1.4 TFT vs control - Adults & 1 & 145 & $\begin{array}{l}\text { Std. Mean Difference (IV, Random, } \\
95 \% \mathrm{CI} \text { ) }\end{array}$ & $-1.27[-1.63,-0.91]$ \\
\hline 2 PTSD at 1 to 4 months & 17 & 1590 & $\begin{array}{l}\text { Std. Mean Difference (IV, Random, } \\
95 \% \mathrm{CI} \text { ) }\end{array}$ & $-0.49[-0.68,-0.31]$ \\
\hline 2.1 EMDR vs control - Adults & 1 & 64 & $\begin{array}{l}\text { Std. Mean Difference (IV, Random, } \\
95 \% \mathrm{CI} \text { ) }\end{array}$ & $-1.76[-2.34,-1.18]$ \\
\hline 2.2 CBT vs control - Adults & 15 & 1488 & $\begin{array}{l}\text { Std. Mean Difference (IV, Random, } \\
95 \% \mathrm{CI} \text { ) }\end{array}$ & $-0.40[-0.54,-0.26]$ \\
\hline 2.3 IPT vs control - Adults & 1 & 38 & $\begin{array}{l}\text { Std. Mean Difference (IV, Random, } \\
95 \% \mathrm{CI})\end{array}$ & $-0.99[-1.67,-0.32]$ \\
\hline 3 PTSD $\geq 6$ months & 5 & 400 & $\begin{array}{l}\text { Std. Mean Difference (IV, Random, } \\
95 \% \mathrm{CI} \text { ) }\end{array}$ & $-0.37[-0.61,-0.14]$ \\
\hline 3.1 CBT vs control - Adults & 3 & 150 & $\begin{array}{l}\text { Std. Mean Difference (IV, Random, } \\
95 \% \mathrm{CI})\end{array}$ & $-0.45[-0.82,-0.09]$ \\
\hline
\end{tabular}

Psychological therapies for the treatment of mental disorders in low- and middle-income countries affected by humanitarian crises 


\begin{tabular}{lllll}
\hline Outcome or subgroup title & No. of studies & $\begin{array}{l}\text { No. of partici- } \\
\text { pants }\end{array}$ & Statistical method & Effect size \\
\hline $\begin{array}{l}\text { 3.2 Trauma/supportive } \\
\text { counselling vs control - } \\
\text { Adults }\end{array}$ & 2 & 250 & $\begin{array}{l}\text { Std. Mean Difference (IV, Random, } \\
95 \% \mathrm{Cl})\end{array}$ & $-0.38[-0.84,0.08]$ \\
\hline
\end{tabular}

Analysis 1.1. Comparison 1 Psychological therapy vs control comparator - PTSD symptoms - adults, Outcome 1 PTSD at endpoint.

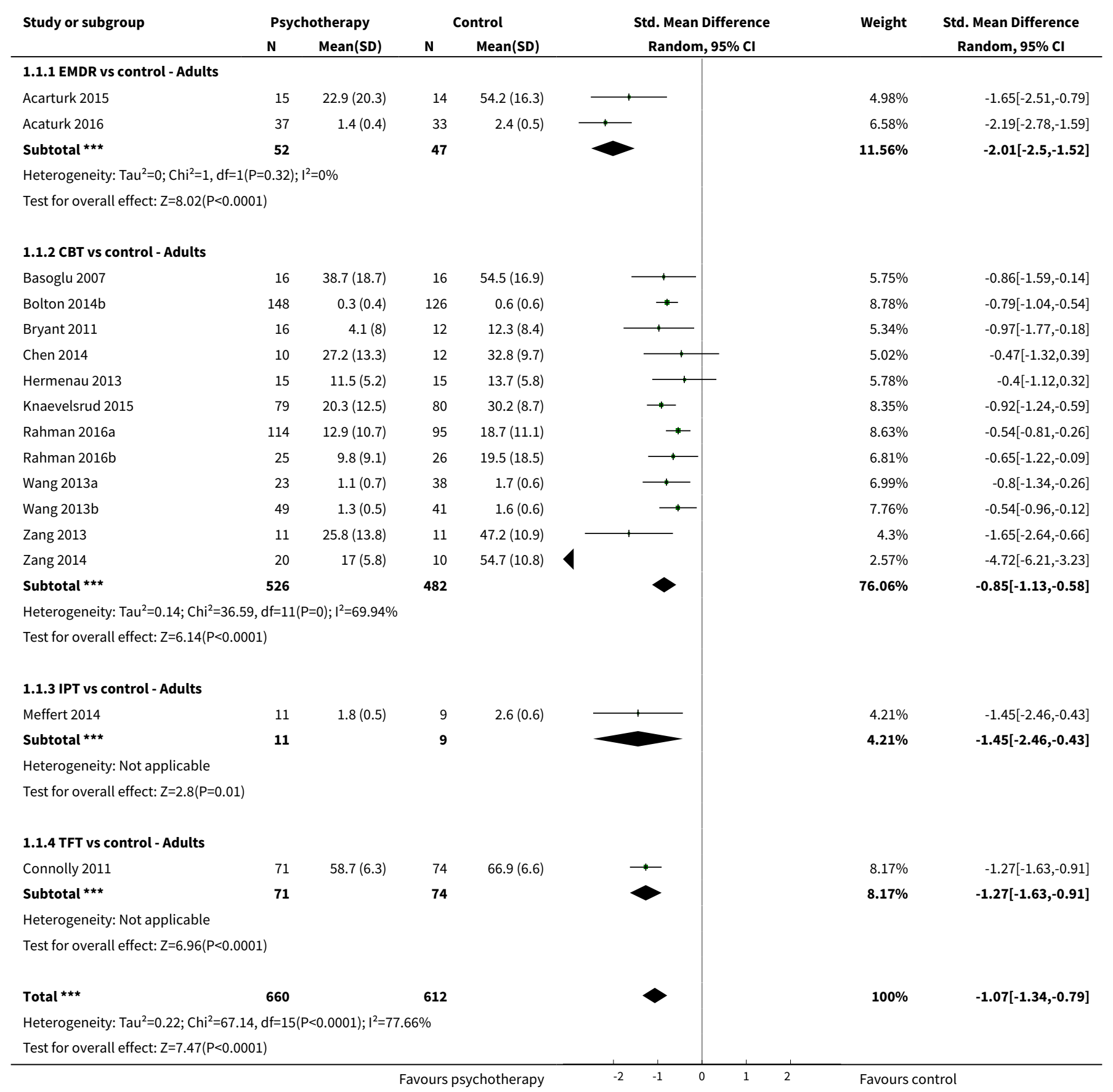

Psychological therapies for the treatment of mental disorders in low- and middle-income countries affected by humanitarian crises 


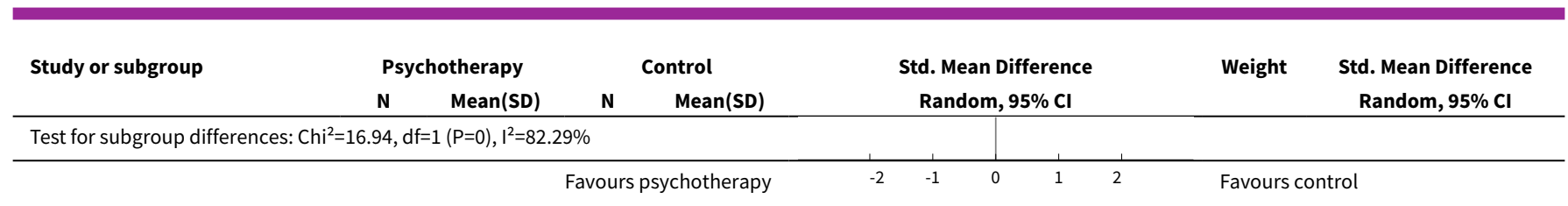

Analysis 1.2. Comparison 1 Psychological therapy vs control comparator - PTSD symptoms - adults, Outcome 2 PTSD at 1 to 4 months.

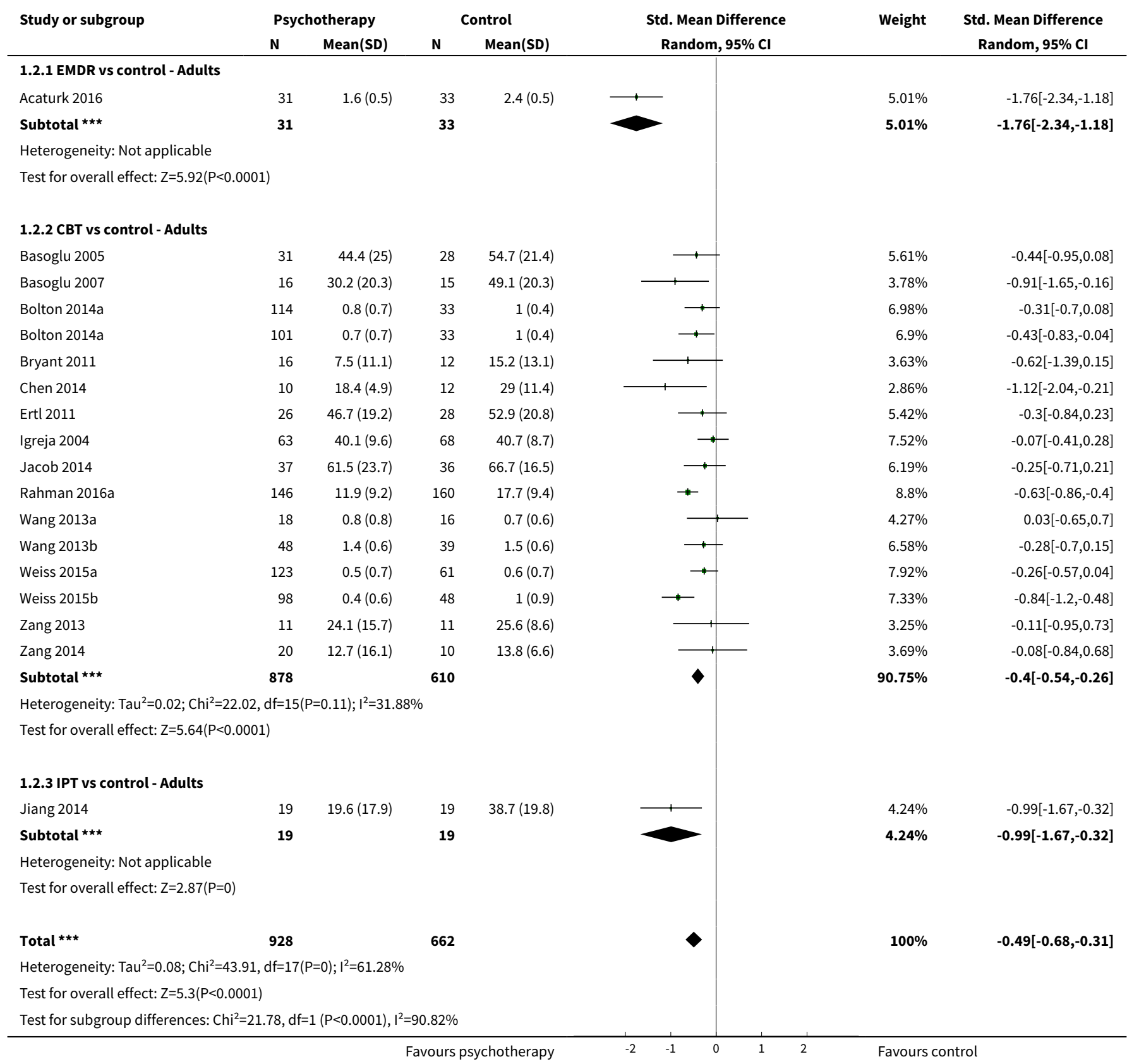

Psychological therapies for the treatment of mental disorders in low- and middle-income countries affected by humanitarian crises 
Analysis 1.3. Comparison 1 Psychological therapy vs control comparator - PTSD symptoms - adults, Outcome 3 PTSD $\geq 6$ months.

\begin{tabular}{|c|c|c|c|c|c|c|c|}
\hline \multirow[t]{2}{*}{ Study or subgroup } & \multicolumn{2}{|c|}{ Psychotherapy } & \multicolumn{2}{|c|}{ Control } & \multirow{2}{*}{$\begin{array}{c}\text { Std. Mean Difference } \\
\text { Random, } 95 \% \mathrm{Cl}\end{array}$} & \multirow[t]{2}{*}{ Weight } & \multirow{2}{*}{$\begin{array}{c}\text { Std. Mean Difference } \\
\text { Random, } 95 \% \mathrm{Cl}\end{array}$} \\
\hline & $\mathbf{N}$ & Mean(SD) & $\mathbf{N}$ & Mean(SD) & & & \\
\hline \multicolumn{8}{|c|}{ 1.3.1 CBT vs control - Adults } \\
\hline Ertl 2011 & 26 & $43(21.5)$ & 28 & $48.6(23.7)$ & $\longrightarrow$ & $19.35 \%$ & $-0.24[-0.78,0.29]$ \\
\hline Hermenau 2013 & 15 & $8.9(7.6)$ & 15 & $16.5(12.5)$ & & $10.11 \%$ & $-0.72[-1.46,0.03]$ \\
\hline Neuner 2008a & 56 & $6.1(6.8)$ & 10 & $10.1(8.1)$ & & $12.02 \%$ & $-0.56[-1.25,0.12]$ \\
\hline Subtotal $\star \star \star$ & 97 & & 53 & & & $41.48 \%$ & $-0.45[-0.82,-0.09]$ \\
\hline \multicolumn{8}{|c|}{ Heterogeneity: $\operatorname{Tau}^{2}=0 ; \mathrm{Chi}^{2}=1.17, \mathrm{df}=2(\mathrm{P}=0.56) ; \mathrm{I}^{2}=0 \%$} \\
\hline \multicolumn{8}{|c|}{ Test for overall effect: $\mathrm{Z}=2.42(\mathrm{P}=0.02)$} \\
\hline \multicolumn{8}{|c|}{ 1.3.2 Trauma/supportive counselling vs control - Adults } \\
\hline Bass 2016 & 145 & $0.7(0.6)$ & 43 & $0.9(0.6)$ & +1 & $47.78 \%$ & $-0.22[-0.57,0.12]$ \\
\hline Neuner 2008b & 53 & $5(6.6)$ & 9 & $10.1(8.1)$ & & $10.75 \%$ & $-0.74[-1.46,-0.02]$ \\
\hline Subtotal $\star \star \star$ & 198 & & 52 & & & $58.52 \%$ & $-0.38[-0.84,0.08]$ \\
\hline \multicolumn{8}{|c|}{ Test for overall effect: $Z=1.61(P=0.11)$} \\
\hline 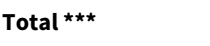 & 295 & & 105 & & & $100 \%$ & $-0.37[-0.61,-0.14]$ \\
\hline \multicolumn{8}{|c|}{ Heterogeneity: $\operatorname{Tau}^{2}=0 ; \mathrm{Chi}^{2}=3.07, \mathrm{df}=4(\mathrm{P}=0.55) ; \mathrm{I}^{2}=0 \%$} \\
\hline \multicolumn{8}{|c|}{ Test for overall effect: $\mathrm{Z}=3.11(\mathrm{P}=0)$} \\
\hline Test for subgroup dif & $06, \mathrm{df}=$ & $\mathrm{P}=0.81), \mathrm{I}^{2}=0^{\mathrm{C}}$ & & & & & \\
\hline
\end{tabular}

\section{Comparison 2. Psychological therapy vs control comparator - PTSD symptoms - children}

\begin{tabular}{lllll}
\hline $\begin{array}{l}\text { Outcome or subgroup ti- } \\
\text { tle }\end{array}$ & No. of studies & $\begin{array}{l}\text { No. of partici- } \\
\text { pants }\end{array}$ & Statistical method & Effect size \\
\hline 1 PTSD at endpoint & 3 & 130 & $\begin{array}{l}\text { Std. Mean Difference (IV, Random, 95\% } \\
\text { Cl) }\end{array}$ & $-1.56[-3.13,0.01]$ \\
\hline $\begin{array}{l}1.1 \text { CBT vs control - Chil- } \\
\text { dren }\end{array}$ & 3 & 130 & $\begin{array}{l}\text { Std. Mean Difference (IV, Random, 95\% } \\
\text { CI) }\end{array}$ & $-1.56[-3.13,0.01]$ \\
\hline 2 PTSD at 1 to 4 months & 1 & 36 & $\begin{array}{l}\text { Std. Mean Difference (IV, Random, 95\% } \\
\text { CI) }\end{array}$ & $-0.57[-1.24,0.10]$ \\
\hline $\begin{array}{l}2.1 \text { CBT vs control - Chil- } \\
\text { dren }\end{array}$ & 1 & 36 & $\begin{array}{l}\text { Std. Mean Difference (IV, Random, 95\% } \\
\text { CI) }\end{array}$ & $-0.57[-1.24,0.10]$ \\
\hline
\end{tabular}

Analysis 2.1. Comparison 2 Psychological therapy vs control comparator - PTSD symptoms - children, Outcome 1 PTSD at endpoint.

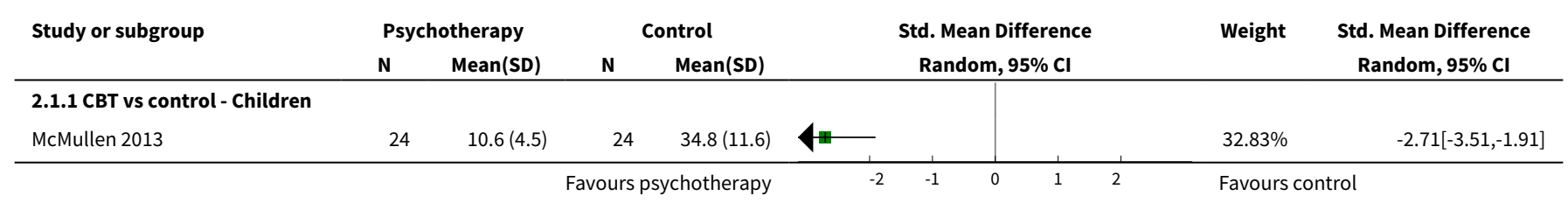




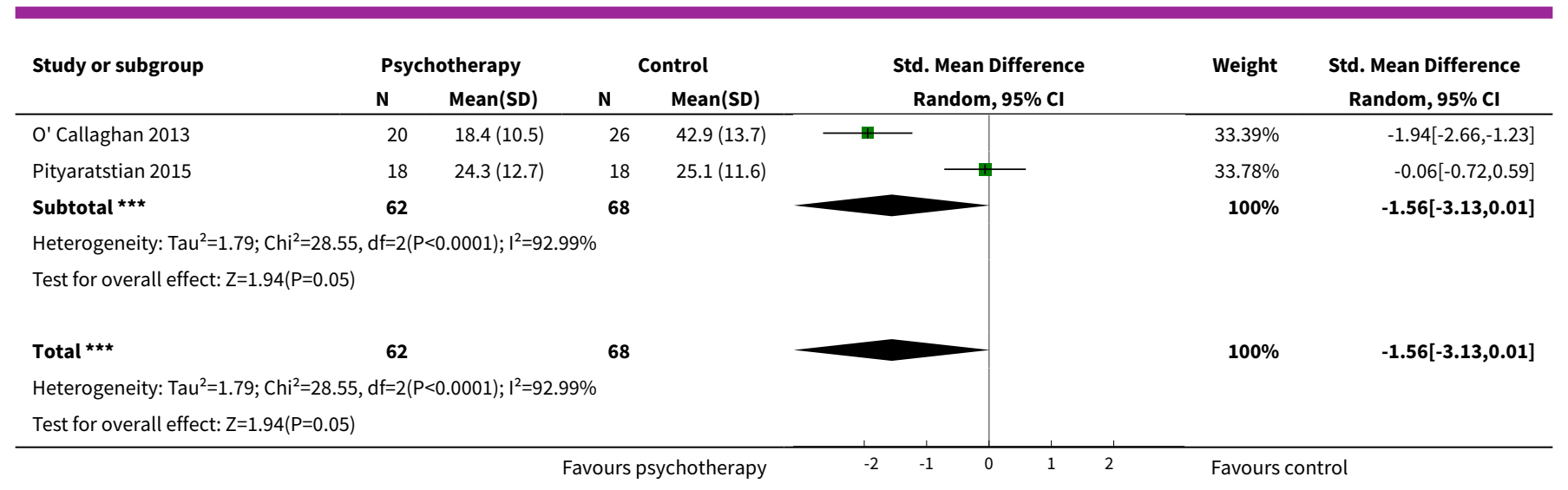

Analysis 2.2. Comparison 2 Psychological therapy vs control comparator - PTSD symptoms - children, Outcome 2 PTSD at 1 to 4 months.

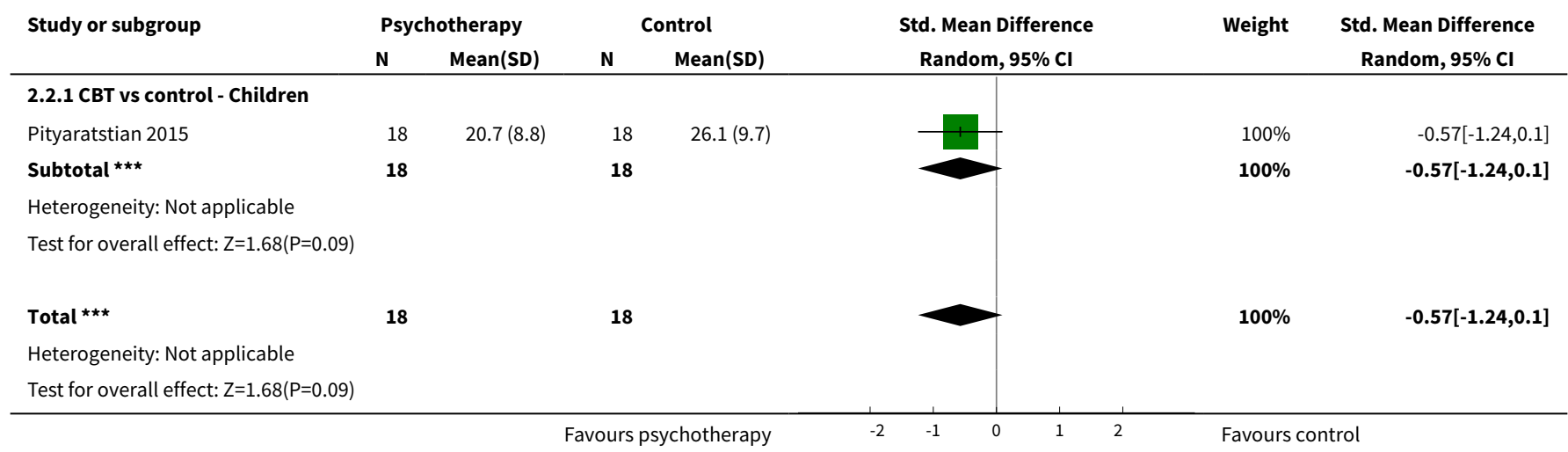

\section{Comparison 3. Psychological therapy vs control comparator - anxiety symptoms - adults}

\begin{tabular}{|c|c|c|c|c|}
\hline Outcome or subgroup title & No. of studies & $\begin{array}{l}\text { No. of partici- } \\
\text { pants }\end{array}$ & Statistical method & Effect size \\
\hline 1 Anxiety at endpoint & 5 & 694 & $\begin{array}{l}\text { Std. Mean Difference (IV, Random, } \\
95 \% \mathrm{CI} \text { ) }\end{array}$ & $-0.74[-0.98,-0.49]$ \\
\hline 1.1 CBT vs control - Adults & 5 & 694 & $\begin{array}{l}\text { Std. Mean Difference (IV, Random, } \\
95 \% \mathrm{CI} \text { ) }\end{array}$ & $-0.74[-0.98,-0.49]$ \\
\hline 2 Anxiety at 1 to 4 months & 6 & 969 & $\begin{array}{l}\text { Std. Mean Difference (IV, Fixed, 95\% } \\
\text { CI) }\end{array}$ & $-0.53[-0.66,-0.39]$ \\
\hline 2.1 CBT vs control - Adults & 6 & 969 & $\begin{array}{l}\text { Std. Mean Difference (IV, Fixed, 95\% } \\
\mathrm{Cl} \text { ) }\end{array}$ & $-0.53[-0.66,-0.39]$ \\
\hline 3 Anxiety $\geq 6$ months & 1 & 188 & Mean Difference (IV, Fixed, 95\% CI) & $-0.15[-0.42,0.12]$ \\
\hline $\begin{array}{l}\text { 3.1 Trauma/Supportive } \\
\text { counselling vs control - } \\
\text { Adults }\end{array}$ & 1 & 188 & Mean Difference (IV, Fixed, 95\% CI) & $-0.15[-0.42,0.12]$ \\
\hline
\end{tabular}

Psychological therapies for the treatment of mental disorders in low- and middle-income countries affected by humanitarian crises 
Analysis 3.1. Comparison 3 Psychological therapy vs control comparator - anxiety symptoms - adults, Outcome 1 Anxiety at endpoint.

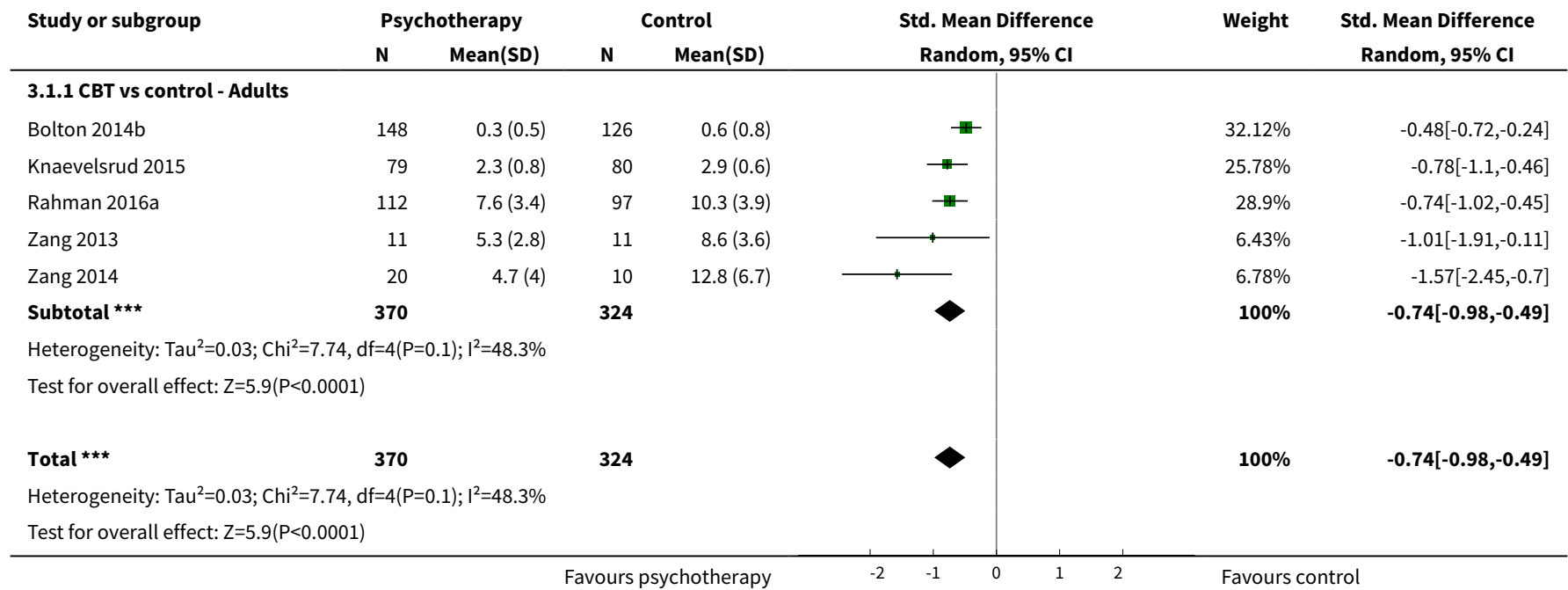

Analysis 3.2. Comparison 3 Psychological therapy vs control comparator - anxiety symptoms - adults, Outcome 2 Anxiety at 1 to 4 months.

\begin{tabular}{|c|c|c|c|c|c|c|c|}
\hline \multirow[t]{2}{*}{ Study or subgroup } & \multicolumn{2}{|c|}{ Psychotherapy } & \multicolumn{2}{|c|}{ Control } & \multirow{2}{*}{$\begin{array}{c}\text { Std. Mean Difference } \\
\text { Fixed, } 95 \% \mathrm{Cl}\end{array}$} & \multirow[t]{2}{*}{ Weight } & \multirow{2}{*}{$\begin{array}{c}\text { Std. Mean Difference } \\
\text { Fixed, } 95 \% \mathrm{Cl}\end{array}$} \\
\hline & $\mathbf{N}$ & Mean(SD) & $\mathbf{N}$ & $\operatorname{Mean}(S D)$ & & & \\
\hline \multicolumn{8}{|c|}{ 3.2.1 CBT vs control - Adults } \\
\hline Bolton 2014a & 114 & $0.8(1.1)$ & 33 & $0.9(0.5)$ & $\longrightarrow$ & $12.57 \%$ & $-0.2[-0.59,0.19]$ \\
\hline Bolton 2014a & 101 & $0.8(1)$ & 33 & $1(0.5)$ & $\longrightarrow$ & $12.19 \%$ & $-0.24[-0.64,0.15]$ \\
\hline Rahman 2016a & 146 & $7.3(3.6)$ & 160 & $10(3.9)$ & \#- & $35.21 \%$ & $-0.74[-0.97,-0.51]$ \\
\hline Weiss 2015a & 123 & $0.5(0.5)$ & 61 & $0.7(0.7)$ & $\rightarrow$ & $19.82 \%$ & $-0.36[-0.67,-0.05]$ \\
\hline Zang 2013 & 11 & $5.5(3)$ & 11 & $4.8(2.5)$ & + & $2.69 \%$ & $0.22[-0.62,1.06]$ \\
\hline Zang 2014 & 20 & $3.4(3.2)$ & 10 & $3.6(2.2)$ & 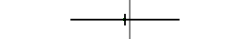 & $3.28 \%$ & $-0.07[-0.83,0.69]$ \\
\hline Subtotal $\star \star \star$ & 613 & & 356 & & 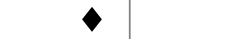 & $100 \%$ & $-0.53[-0.66,-0.39]$ \\
\hline \multicolumn{8}{|c|}{ Heterogeneity: $\mathrm{Tau}^{2}=0 ; \mathrm{Chi}^{2}=20.32, \mathrm{df}=6(\mathrm{P}=0) ; \mathrm{I}^{2}=70.47 \%$} \\
\hline \multicolumn{8}{|c|}{ Test for overall effect: $Z=7.48(P<0.0001)$} \\
\hline \multicolumn{8}{|c|}{ Heterogeneity: $\mathrm{Tau}^{2}=0 ; \mathrm{Chi}^{2}=20.32, \mathrm{df}=6(\mathrm{P}=0) ; \mathrm{I}^{2}=70.47 \%$} \\
\hline Test for overall effect & & & & & & & \\
\hline
\end{tabular}

Psychological therapies for the treatment of mental disorders in low- and middle-income countries affected by humanitarian crises 
Analysis 3.3. Comparison 3 Psychological therapy vs control comparator - anxiety symptoms - adults, Outcome 3 Anxiety $\geq 6$ months.

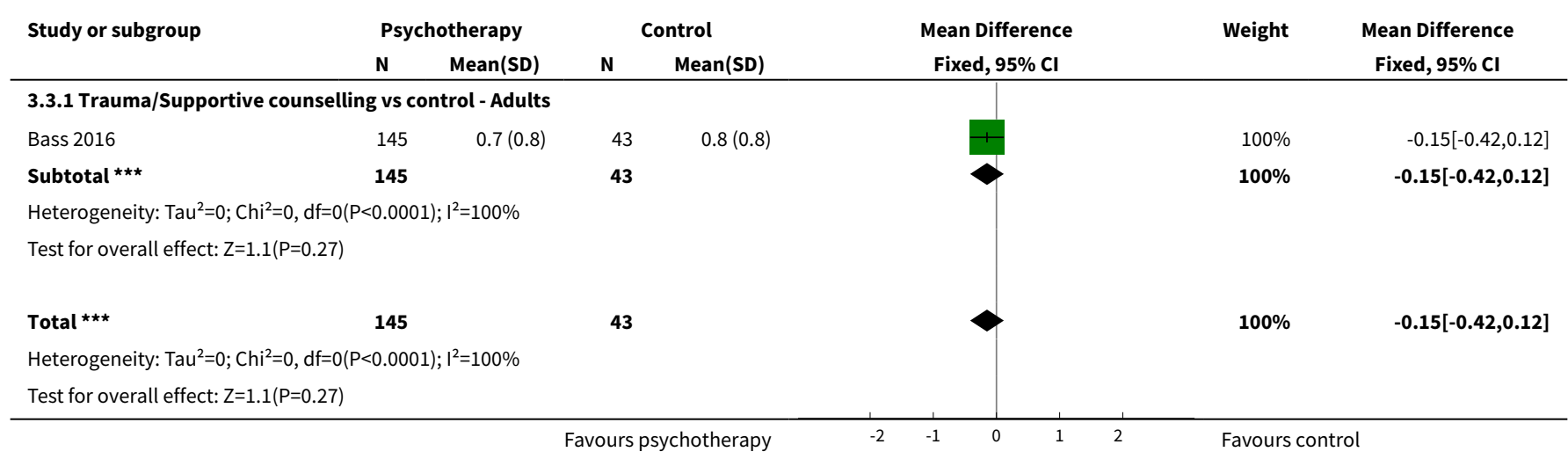

Comparison 4. Psychological therapy vs control comparator - depressive symptoms - adults

\begin{tabular}{|c|c|c|c|c|}
\hline Outcome or subgroup title & No. of studies & $\begin{array}{l}\text { No. of partici- } \\
\text { pants }\end{array}$ & Statistical method & Effect size \\
\hline 1 Depression at endpoint & 14 & 1254 & $\begin{array}{l}\text { Std. Mean Difference (IV, Random, } \\
95 \% \mathrm{CI} \text { ) }\end{array}$ & $-0.86[-1.06,-0.67]$ \\
\hline 1.1 EMDR vs control - Adults & 2 & 99 & $\begin{array}{l}\text { Std. Mean Difference (IV, Random, } \\
95 \% \mathrm{CI} \text { ) }\end{array}$ & $-1.43[-1.88,-0.99]$ \\
\hline 1.2 CBT vs control - Adults & 10 & 926 & $\begin{array}{l}\text { Std. Mean Difference (IV, Random, } \\
95 \% \mathrm{CI} \text { ) }\end{array}$ & $-0.81[-1.02,-0.60]$ \\
\hline 1.3 IPT vs control - Adults & 2 & 229 & $\begin{array}{l}\text { Std. Mean Difference (IV, Random, } \\
95 \% \mathrm{CI} \text { ) }\end{array}$ & $-0.84[-1.60,-0.08]$ \\
\hline $\begin{array}{l}2 \text { Depression at } 1 \text { to } 4 \\
\text { months }\end{array}$ & 15 & 1386 & $\begin{array}{l}\text { Std. Mean Difference (IV, Random, } \\
95 \% \mathrm{CI} \text { ) }\end{array}$ & $-0.42[-0.63,-0.21]$ \\
\hline 2.1 EMDR vs control - Adults & 1 & 64 & $\begin{array}{l}\text { Std. Mean Difference (IV, Random, } \\
95 \% \mathrm{CI} \text { ) }\end{array}$ & $-1.21[-1.74,-0.67]$ \\
\hline 2.2 CBT vs control - Adults & 13 & 1284 & $\begin{array}{l}\text { Std. Mean Difference (IV, Random, } \\
95 \% \mathrm{CI} \text { ) }\end{array}$ & $-0.35[-0.56,-0.13]$ \\
\hline 2.3 IPT vs control - Adults & 1 & 38 & $\begin{array}{l}\text { Std. Mean Difference (IV, Random, } \\
95 \% \mathrm{CI} \text { ) }\end{array}$ & $-0.77[-1.43,-0.11]$ \\
\hline 3 Depression $\geq 6$ months & 2 & 242 & Mean Difference (IV, Fixed, 95\% CI) & $-0.19[-0.44,0.07]$ \\
\hline 3.1 CBT vs control - Adults & 1 & 54 & Mean Difference (IV, Fixed, 95\% CI) & $-0.13[-1.72,1.46]$ \\
\hline $\begin{array}{l}\text { 3.2 Trauma/Supportive } \\
\text { counselling vs control - } \\
\text { Adults }\end{array}$ & 1 & 188 & Mean Difference (IV, Fixed, 95\% Cl) & $-0.19[-0.45,0.07]$ \\
\hline
\end{tabular}


Analysis 4.1. Comparison 4 Psychological therapy vs control comparator - depressive symptoms - adults, Outcome 1 Depression at endpoint.

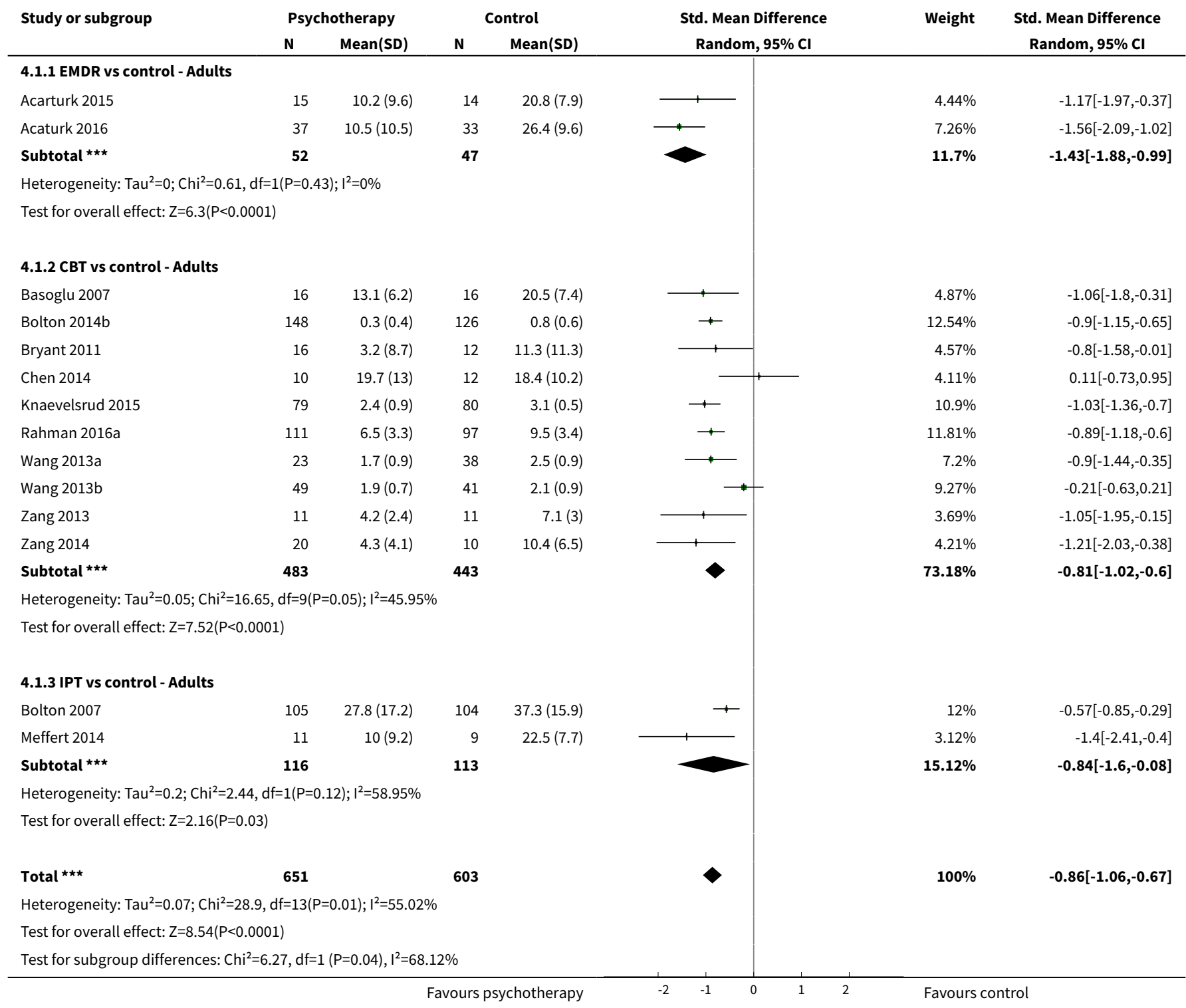

Analysis 4.2. Comparison 4 Psychological therapy vs control comparator - depressive symptoms - adults, Outcome 2 Depression at 1 to 4 months.

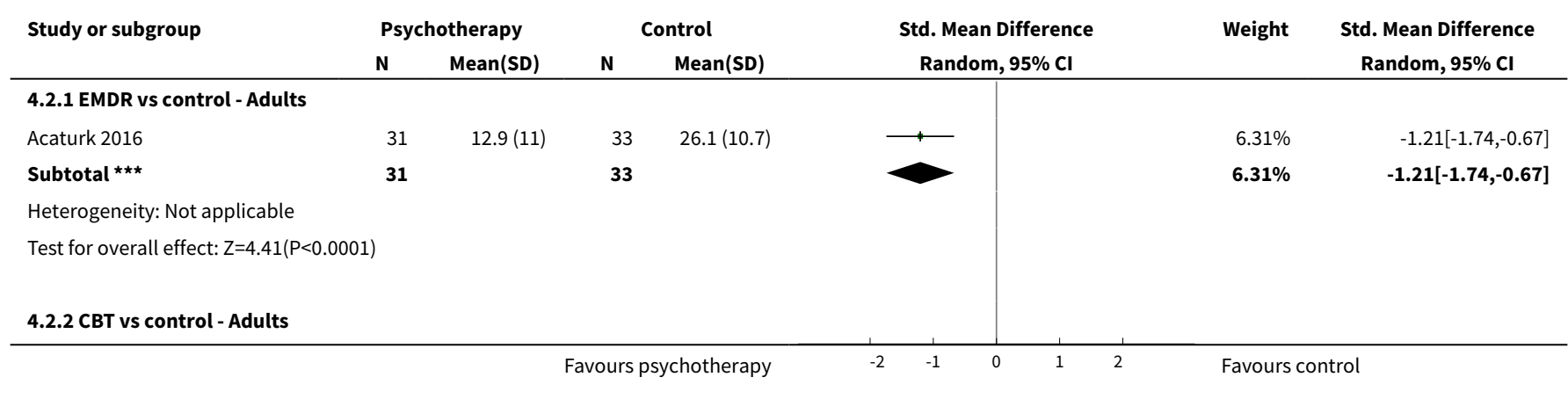

Psychological therapies for the treatment of mental disorders in low- and middle-income countries affected by humanitarian crises 94 (Review)

Copyright ( 2018 The Cochrane Collaboration. Published by John Wiley \& Sons, Ltd. 


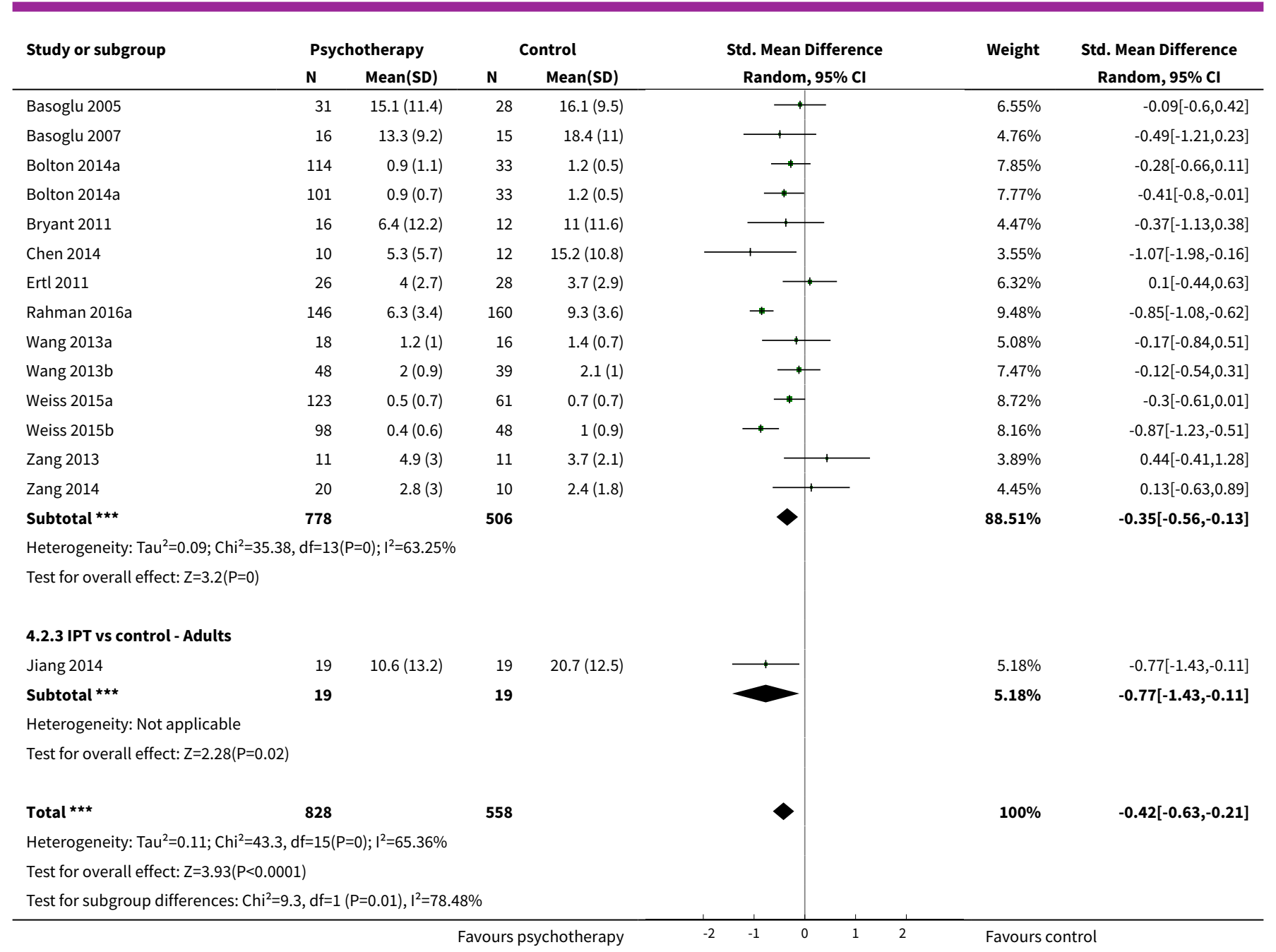

\section{Analysis 4.3. Comparison 4 Psychological therapy vs control comparator - depressive symptoms - adults, Outcome 3 Depression $\geq 6$ months.}

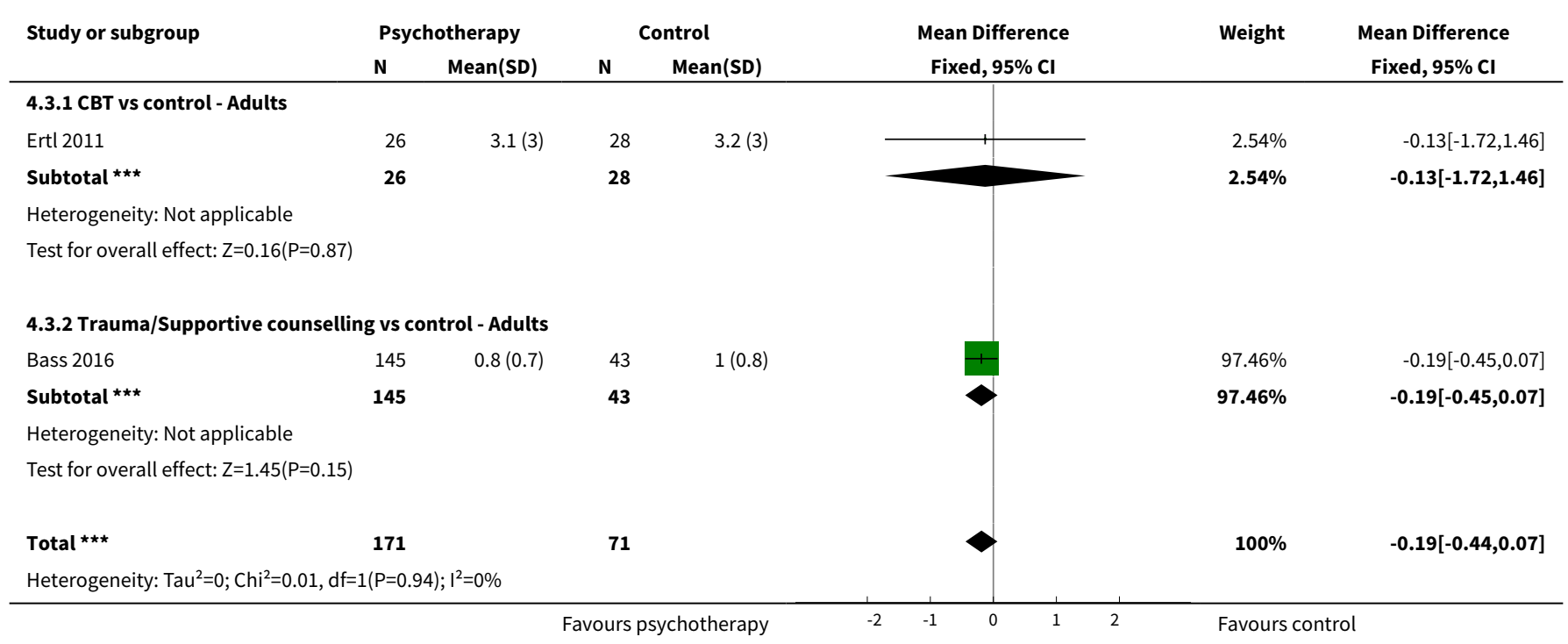

Psychological therapies for the treatment of mental disorders in low- and middle-income countries affected by humanitarian crises 


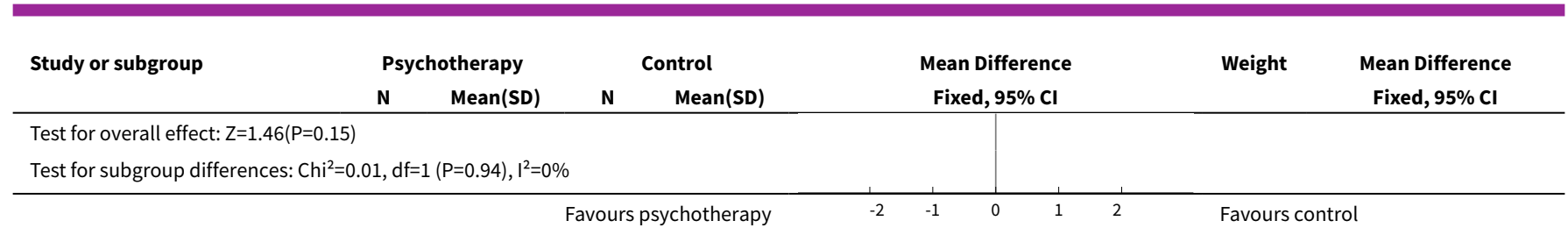

Comparison 5. Psychological therapy vs control comparator - dropout -adults

\begin{tabular}{lllll}
\hline Outcome or subgroup title & No. of studies & $\begin{array}{l}\text { No. of partici- } \\
\text { pants }\end{array}$ & Statistical method & Effect size \\
\hline 1 Dropout & 26 & 2960 & Risk Ratio (M-H, Random, 95\% Cl) & $0.98[0.82,1.16]$ \\
\hline 1.1 EMDR vs control - Adults & 2 & 127 & Risk Ratio (M-H, Random, 95\% Cl) & $0.75[0.40,1.42]$ \\
\hline 1.2 CBT vs control - Adults & 19 & 2206 & Risk Ratio (M-H, Random, 95\% Cl) & $1.06[0.86,1.30]$ \\
\hline 1.3 IPT vs control - Adults & 3 & 280 & Risk Ratio (M-H, Random, 95\% Cl) & $1.03[0.39,2.73]$ \\
\hline $\begin{array}{l}1.4 \text { Trauma/Supportive coun- } \\
\text { selling vs control - Adults }\end{array}$ & 2 & 347 & Risk Ratio (M-H, Random, 95\% Cl) & $0.77[0.57,1.04]$ \\
\hline
\end{tabular}

Analysis 5.1. Comparison 5 Psychological therapy vs control comparator - dropout -adults, Outcome 1 Dropout.

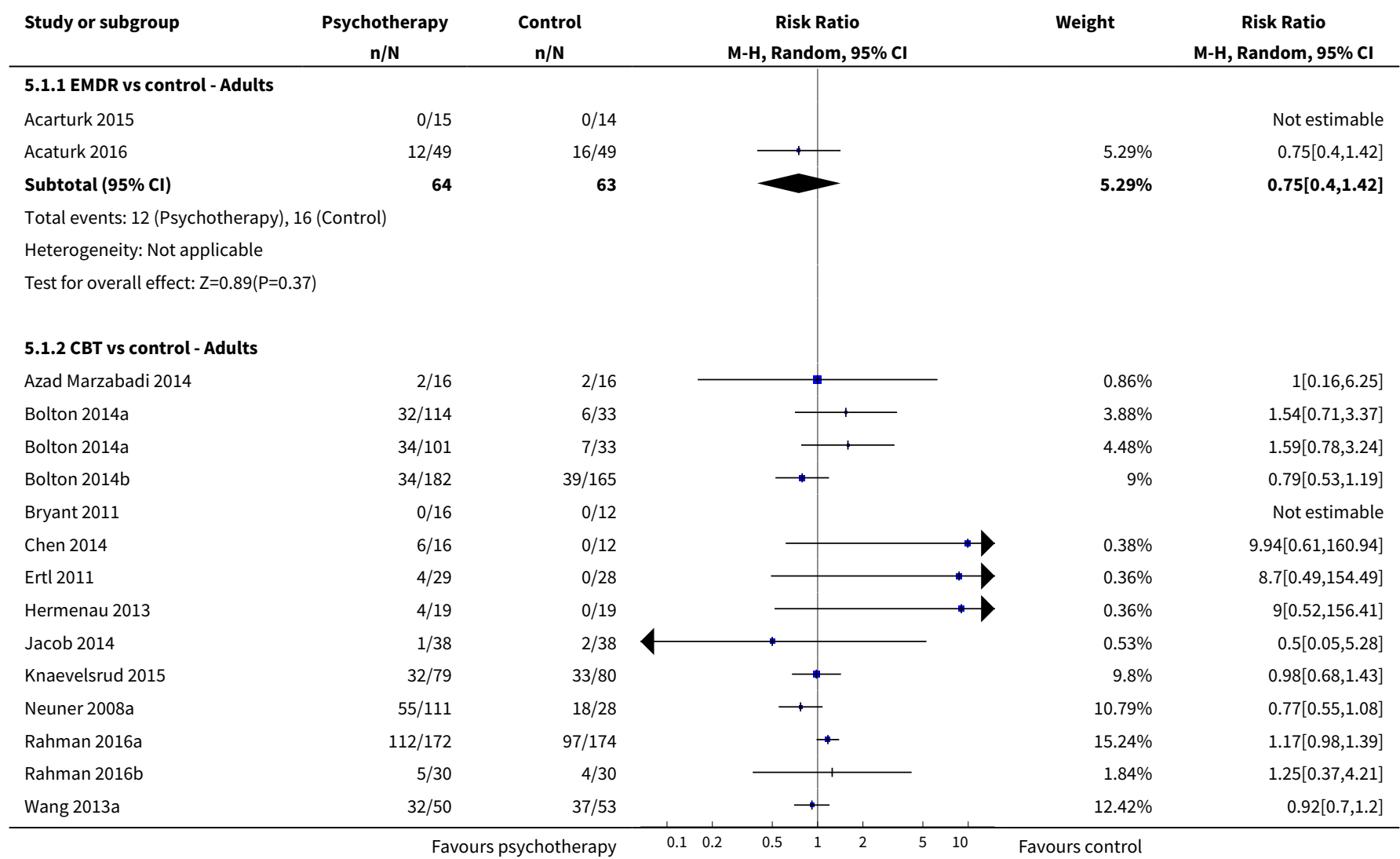




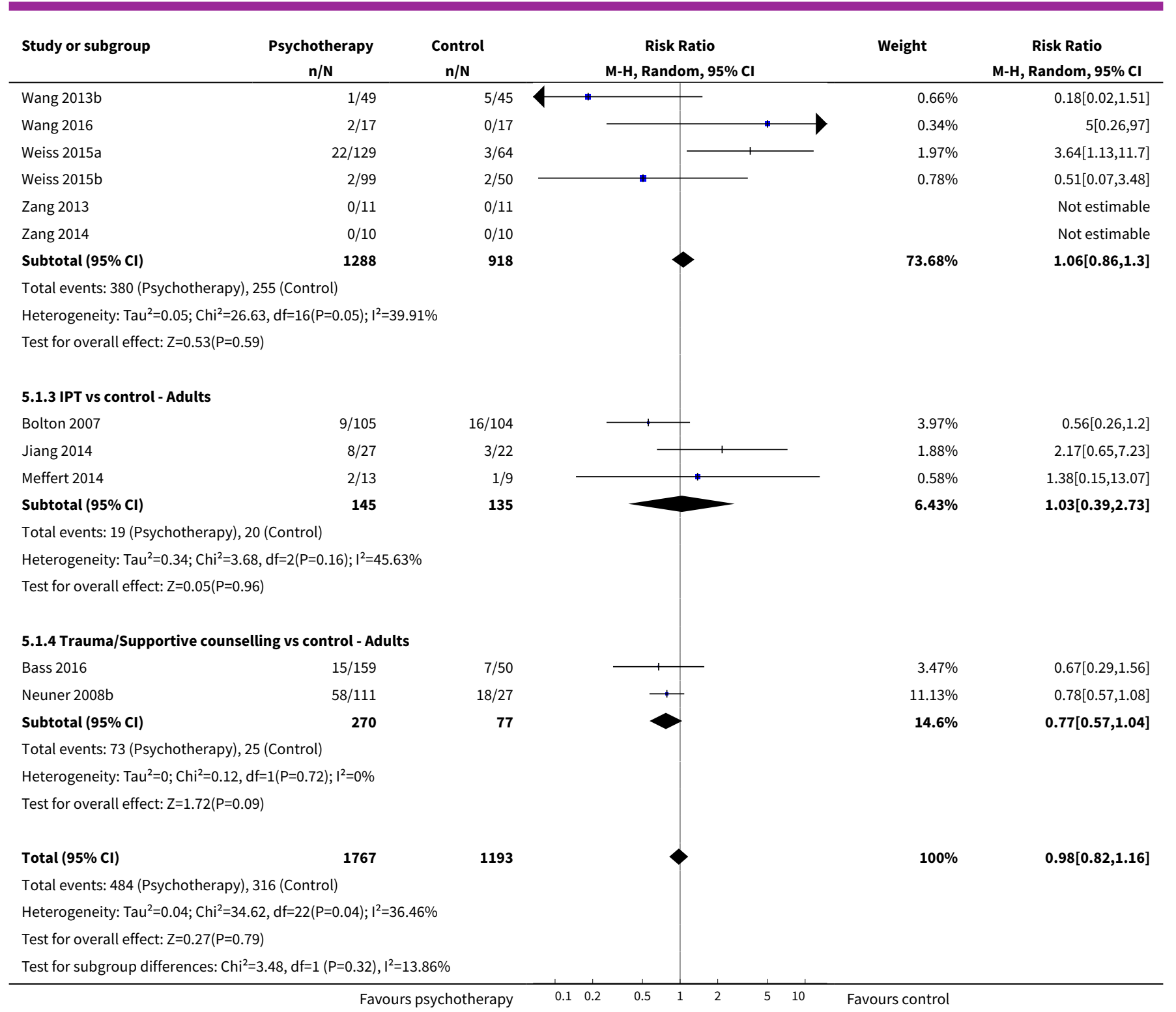

Comparison 6. Psychological therapy vs control comparator - dropout - children

\begin{tabular}{llllll}
\hline Outcome or subgroup title & No. of studies & $\begin{array}{l}\text { No. of partici- } \\
\text { pants }\end{array}$ & Statistical method & Effect size \\
\hline 1 Dropout & 3 & 138 & Risk Ratio (M-H, Random, 95\% Cl) & $1.87[0.47,7.47]$ \\
\hline 1.1 CBT vs control - Children & 3 & 138 & Risk Ratio (M-H, Random, 95\% Cl) & $1.87[0.47,7.47]$ \\
\hline
\end{tabular}


Analysis 6.1. Comparison 6 Psychological therapy vs control comparator - dropout - children, Outcome 1 Dropout.

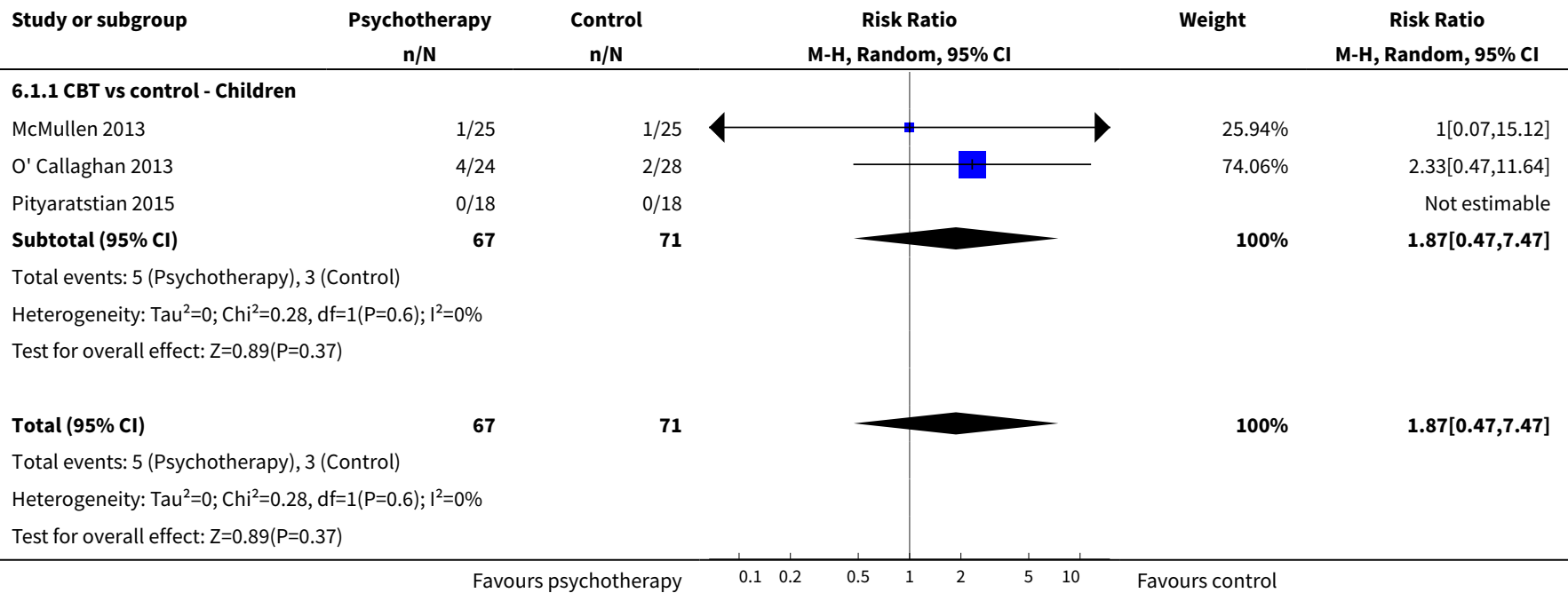

Comparison 7. Psychological therapy vs control comparator - functional impairment - adults

\begin{tabular}{|c|c|c|c|c|}
\hline Outcome or subgroup title & No. of studies & $\begin{array}{l}\text { No. of partici- } \\
\text { pants }\end{array}$ & Statistical method & Effect size \\
\hline $\begin{array}{l}1 \text { Functional impairment at end- } \\
\text { point }\end{array}$ & 5 & 686 & $\begin{array}{l}\text { Std. Mean Difference (IV, Random, } \\
95 \% \mathrm{CI})\end{array}$ & $-0.54[-0.79,-0.29]$ \\
\hline 1.1 CBT vs control - Adults & 5 & 686 & $\begin{array}{l}\text { Std. Mean Difference (IV, Random, } \\
95 \% \mathrm{CI})\end{array}$ & $-0.54[-0.79,-0.29]$ \\
\hline $\begin{array}{l}2 \text { Functional impairment at } 1 \text { to } \\
4 \text { months }\end{array}$ & 7 & 1061 & $\begin{array}{l}\text { Std. Mean Difference (IV, Random, } \\
95 \% \mathrm{CI})\end{array}$ & $-0.35[-0.54,-0.15]$ \\
\hline 2.1 CBT vs control - Adults & 7 & 1061 & $\begin{array}{l}\text { Std. Mean Difference (IV, Random, } \\
95 \% \mathrm{CI} \text { ) }\end{array}$ & $-0.35[-0.54,-0.15]$ \\
\hline $\begin{array}{l}3 \text { Functional impairment } \geq 6 \\
\text { months }\end{array}$ & 1 & 188 & $\begin{array}{l}\text { Std. Mean Difference (IV, Random, } \\
95 \% \mathrm{CI})\end{array}$ & $-0.25[-0.59,0.09]$ \\
\hline $\begin{array}{l}\text { 3.1 Trauma/Supportive coun- } \\
\text { selling vs control - Adults }\end{array}$ & 1 & 188 & $\begin{array}{l}\text { Std. Mean Difference (IV, Random, } \\
95 \% \mathrm{CI})\end{array}$ & $-0.25[-0.59,0.09]$ \\
\hline
\end{tabular}

Analysis 7.1. Comparison 7 Psychological therapy vs control comparator functional impairment - adults, Outcome 1 Functional impairment at endpoint.

\begin{tabular}{|c|c|c|c|c|c|c|c|c|}
\hline \multirow[t]{2}{*}{ Study or subgroup } & \multicolumn{2}{|c|}{ Psychotherapy } & \multicolumn{2}{|c|}{ Control } & \multirow{2}{*}{\multicolumn{2}{|c|}{$\begin{array}{c}\text { Std. Mean Difference } \\
\text { Random, } 95 \% \mathrm{Cl}\end{array}$}} & \multirow[t]{2}{*}{ Weight } & \multirow{2}{*}{$\begin{array}{c}\text { Std. Mean Difference } \\
\text { Random, } 95 \% \mathrm{Cl}\end{array}$} \\
\hline & $\mathbf{N}$ & Mean(SD) & $\mathbf{N}$ & Mean(SD) & & & & \\
\hline \multicolumn{9}{|c|}{ 7.1.1 CBT vs control - Adults } \\
\hline Bolton 2014b & 148 & $0.3(0.6)$ & 126 & $0.7(0.8)$ & 世- & & $28.35 \%$ & $-0.54[-0.79,-0.3]$ \\
\hline Rahman 2016a & 114 & $17.1(5.3)$ & 96 & $22.5(9.6)$ & $\rightarrow$ & & $26.01 \%$ & $-0.72[-1,-0.44]$ \\
\hline
\end{tabular}




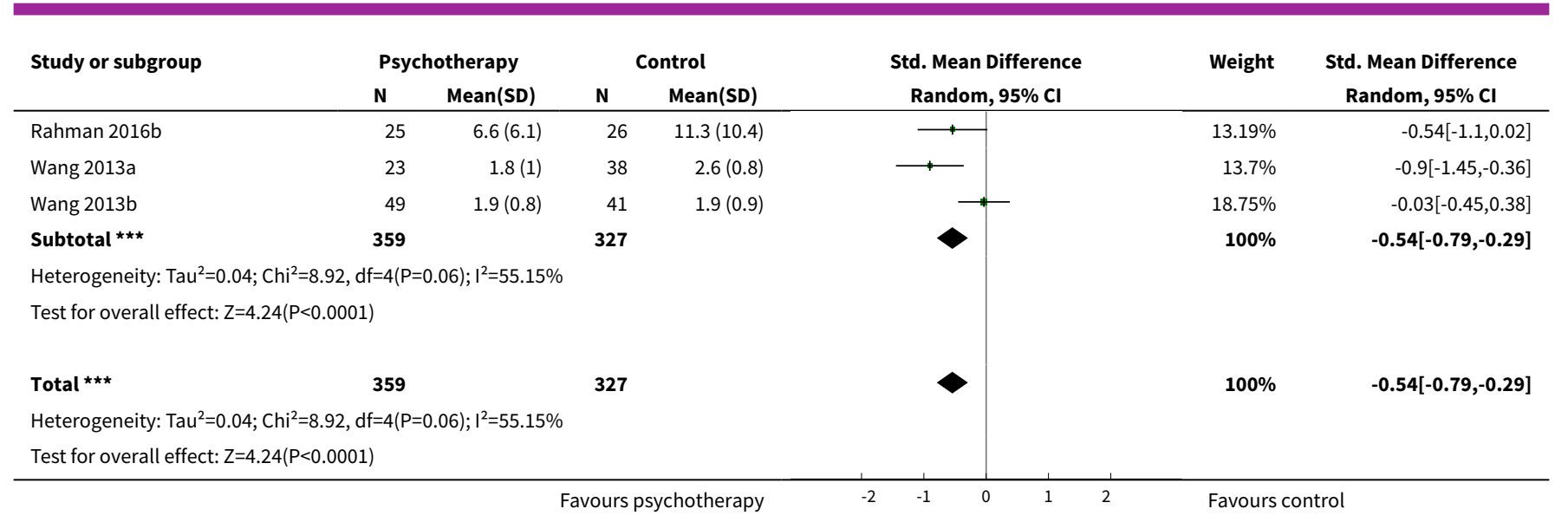

Analysis 7.2. Comparison 7 Psychological therapy vs control comparator -

functional impairment - adults, Outcome 2 Functional impairment at 1 to 4 months.

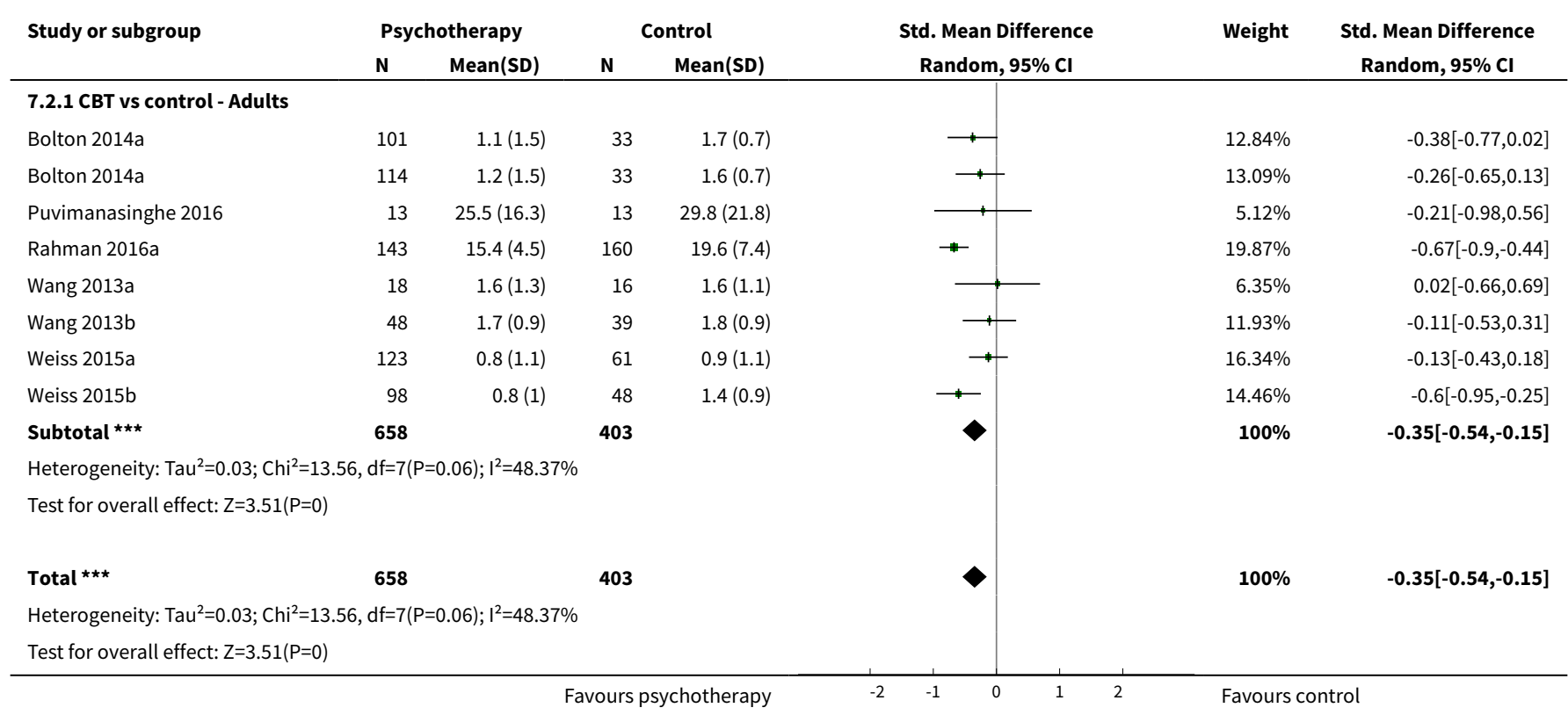

Analysis 7.3. Comparison 7 Psychological therapy vs control comparator functional impairment - adults, Outcome 3 Functional impairment $\geq 6$ months.

\begin{tabular}{|c|c|c|c|c|c|c|c|}
\hline \multirow[t]{2}{*}{ Study or subgroup } & \multicolumn{2}{|c|}{ Psychotherapy } & \multicolumn{2}{|c|}{ Control } & \multirow{2}{*}{$\begin{array}{c}\text { Std. Mean Difference } \\
\text { Random, } 95 \% \mathrm{Cl}\end{array}$} & \multirow[t]{2}{*}{ Weight } & \multirow{2}{*}{$\begin{array}{c}\text { Std. Mean Difference } \\
\text { Random, } 95 \% \mathrm{Cl}\end{array}$} \\
\hline & $\mathbf{N}$ & Mean(SD) & $\mathbf{N}$ & $\operatorname{Mean}(\mathrm{SD})$ & & & \\
\hline \multicolumn{8}{|c|}{ 7.3.1 Trauma/Supportive counselling vs control - Adults } \\
\hline Bass 2016 & 145 & $1.2(1.3)$ & 43 & $1.5(1.2)$ & & $100 \%$ & $-0.25[-0.59,0.09]$ \\
\hline Subtotal $* \star \star$ & 145 & & 43 & & & $100 \%$ & $-0.25[-0.59,0.09]$ \\
\hline \multicolumn{8}{|c|}{ Heterogeneity: Not applicable } \\
\hline \multicolumn{8}{|c|}{ Test for overall effect: $\mathrm{Z}=1.44(\mathrm{P}=0.15)$} \\
\hline Total $\star \star \star ~$ & 145 & & 43 & & & $100 \%$ & $-0.25[-0.59,0.09]$ \\
\hline
\end{tabular}

Psychological therapies for the treatment of mental disorders in low- and middle-income countries affected by humanitarian crises 99 (Review)

Copyright ( 2018 The Cochrane Collaboration. Published by John Wiley \& Sons, Ltd. 


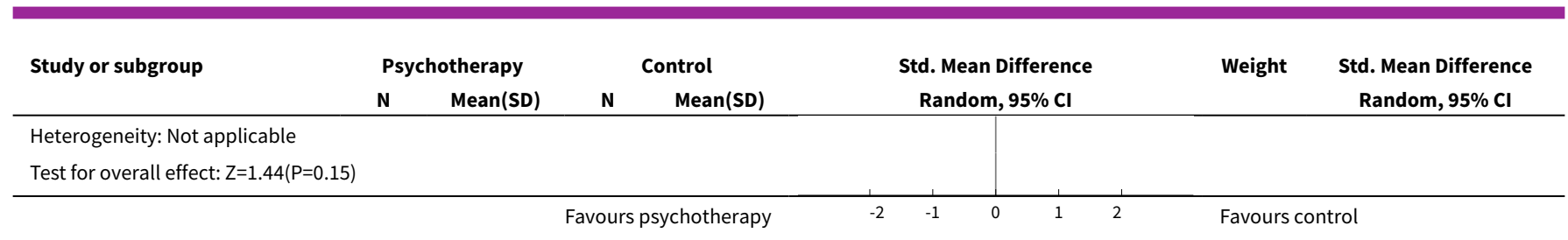

Comparison 8. Psychological therapy vs control comparator - quality of life - adults

\begin{tabular}{lllll}
\hline Outcome or subgroup title & No. of studies & $\begin{array}{l}\text { No. of partici- } \\
\text { pants }\end{array}$ & Statistical method & Effect size \\
\hline 1 Quality of life at endpoint & 4 & 325 & $\begin{array}{l}\text { Std. Mean Difference (IV, Random, } \\
95 \% \text { Cl) }\end{array}$ & -0.73 [-1.22, -0.25] \\
\hline 1.1 IPT vs control - Adults & 1 & 38 & $\begin{array}{l}\text { Std. Mean Difference (IV, Random, } \\
95 \% \text { Cl) }\end{array}$ & -0.88 [-1.55, -0.21] \\
\hline 1.2 CBT vs control - Adults & 3 & 287 & $\begin{array}{l}\text { Std. Mean Difference (IV, Random, } \\
95 \% \text { Cl) }\end{array}$ & $-0.71[-1.35,-0.07]$ \\
\hline
\end{tabular}

Analysis 8.1. Comparison 8 Psychological therapy vs control comparator - quality of life - adults, Outcome 1 Quality of life at endpoint.

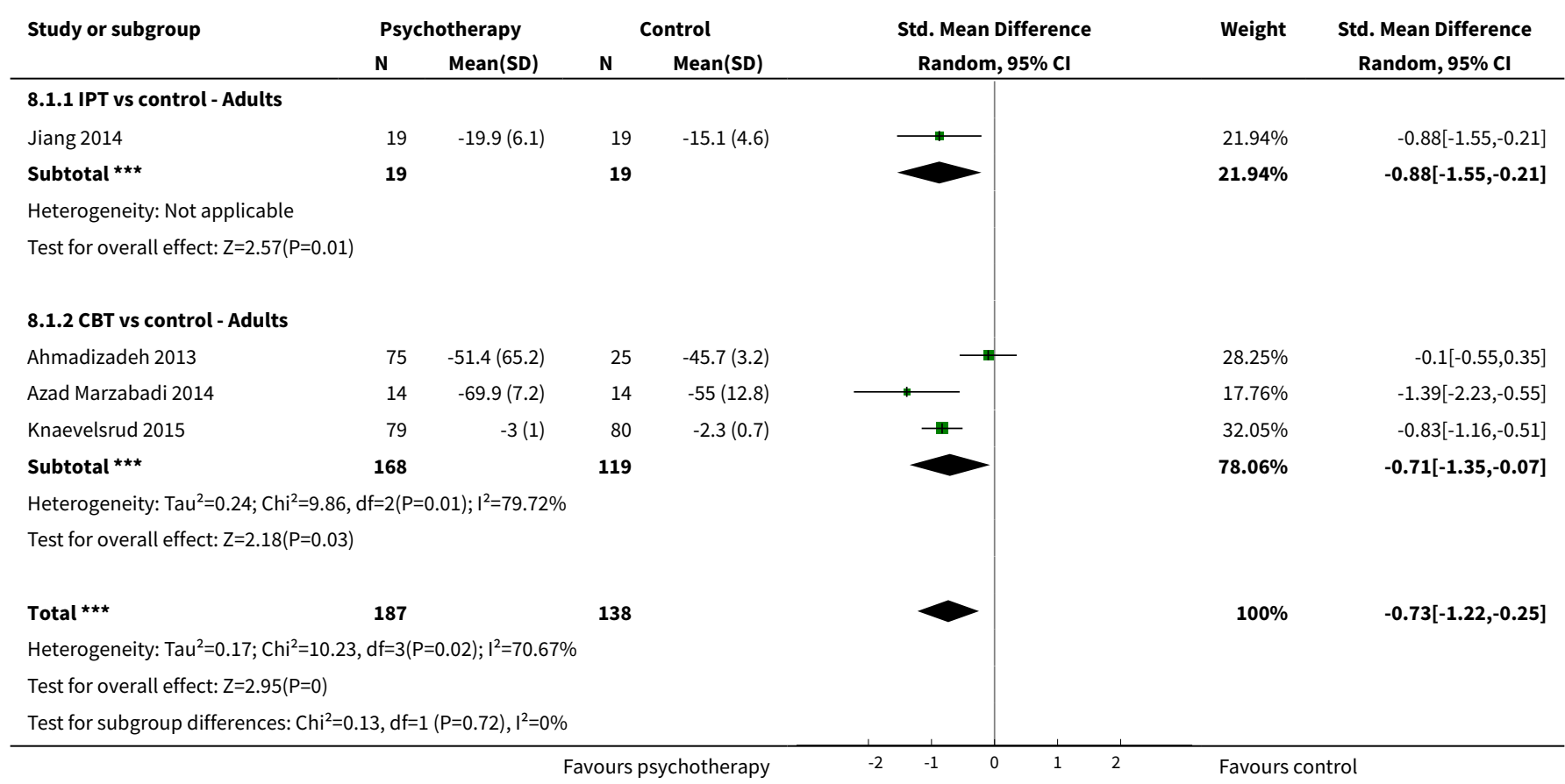


Comparison 9. Psychological therapy vs control comparator - diagnosis of PTSD - adults

\begin{tabular}{|c|c|c|c|c|}
\hline Outcome or subgroup title & No. of studies & $\begin{array}{l}\text { No. of partici- } \\
\text { pants }\end{array}$ & Statistical method & Effect size \\
\hline 1 Diagnosis of PTSD & 4 & 402 & $\begin{array}{l}\text { Risk Ratio (M-H, Random, 95\% } \\
\text { Cl) }\end{array}$ & $1.07[0.43,2.68]$ \\
\hline 1.1 IPT vs control - Adults & 1 & 49 & $\begin{array}{l}\text { Risk Ratio (M-H, Random, 95\% } \\
\mathrm{Cl})\end{array}$ & $0.31[0.09,1.02]$ \\
\hline 1.2 CBT vs control - Adults & 2 & 214 & $\begin{array}{l}\text { Risk Ratio (M-H, Random, 95\% } \\
\mathrm{Cl} \text { ) }\end{array}$ & $1.28[0.28,5.77]$ \\
\hline $\begin{array}{l}\text { 1.3 Trauma/Supportive coun- } \\
\text { selling vs control - Adults }\end{array}$ & 1 & 139 & $\begin{array}{l}\text { Risk Ratio (M-H, Random, 95\% } \\
\text { Cl) }\end{array}$ & $2.27[0.88,5.85]$ \\
\hline
\end{tabular}

Analysis 9.1. Comparison 9 Psychological therapy vs control comparator - diagnosis of PTSD - adults, Outcome 1 Diagnosis of PTSD.

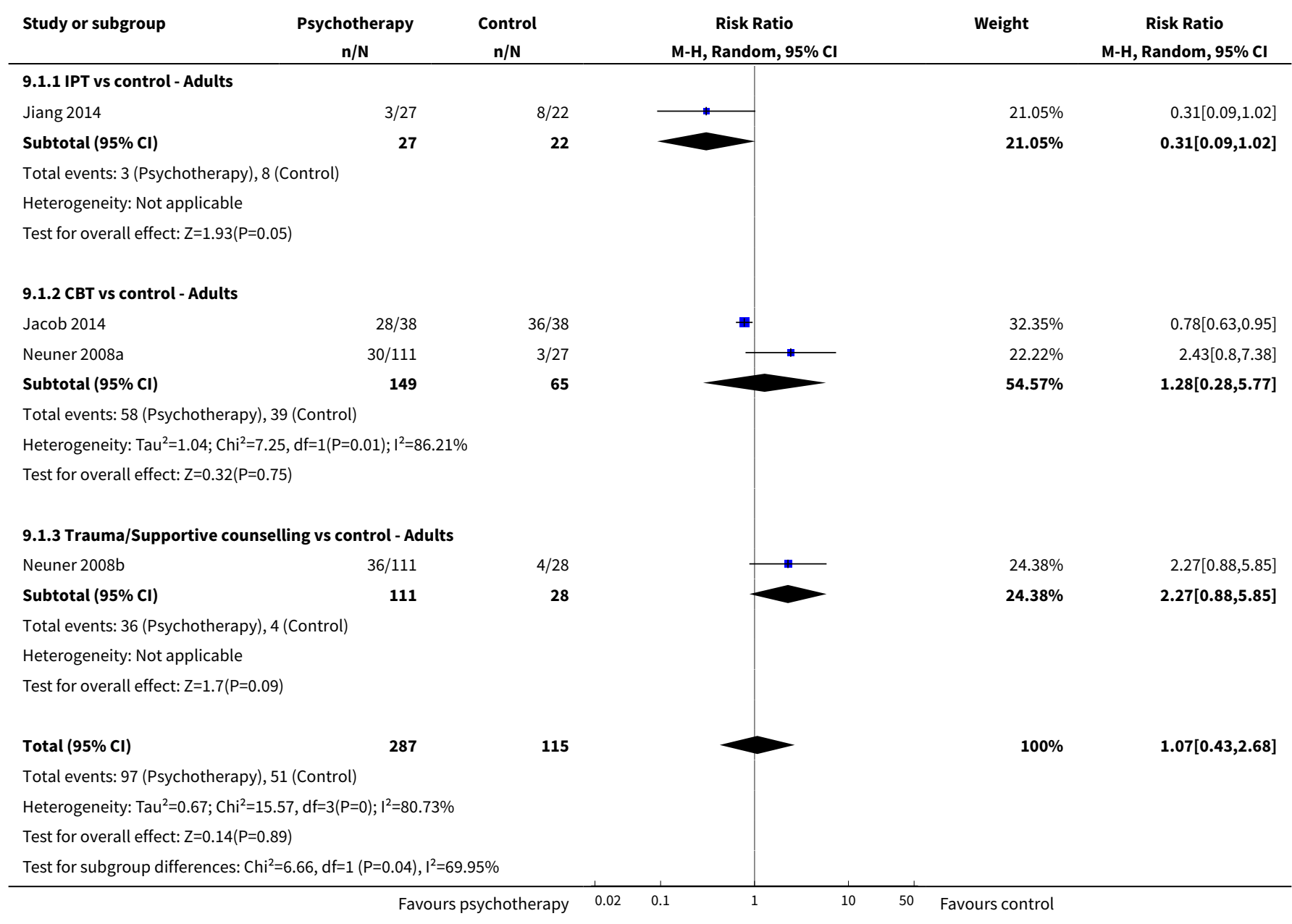


Comparison 10. Psychological therapy vs control comparator - diagnosis of PTSD - children

\begin{tabular}{llllll}
\hline Outcome or subgroup title & No. of studies & $\begin{array}{l}\text { No. of partici- } \\
\text { pants }\end{array}$ & Statistical method & Effect size \\
\hline 1 Diagnosis of PTSD & 1 & 36 & Risk Ratio (M-H, Random, 95\% Cl) & $0.59[0.38,0.90]$ \\
\hline 1.1 CBT vs control - Children & 1 & 36 & Risk Ratio (M-H, Random, 95\% Cl) & $0.59[0.38,0.90]$ \\
\hline
\end{tabular}

Analysis 10.1. Comparison 10 Psychological therapy vs control comparator - diagnosis of PTSD - children, Outcome 1 Diagnosis of PTSD.

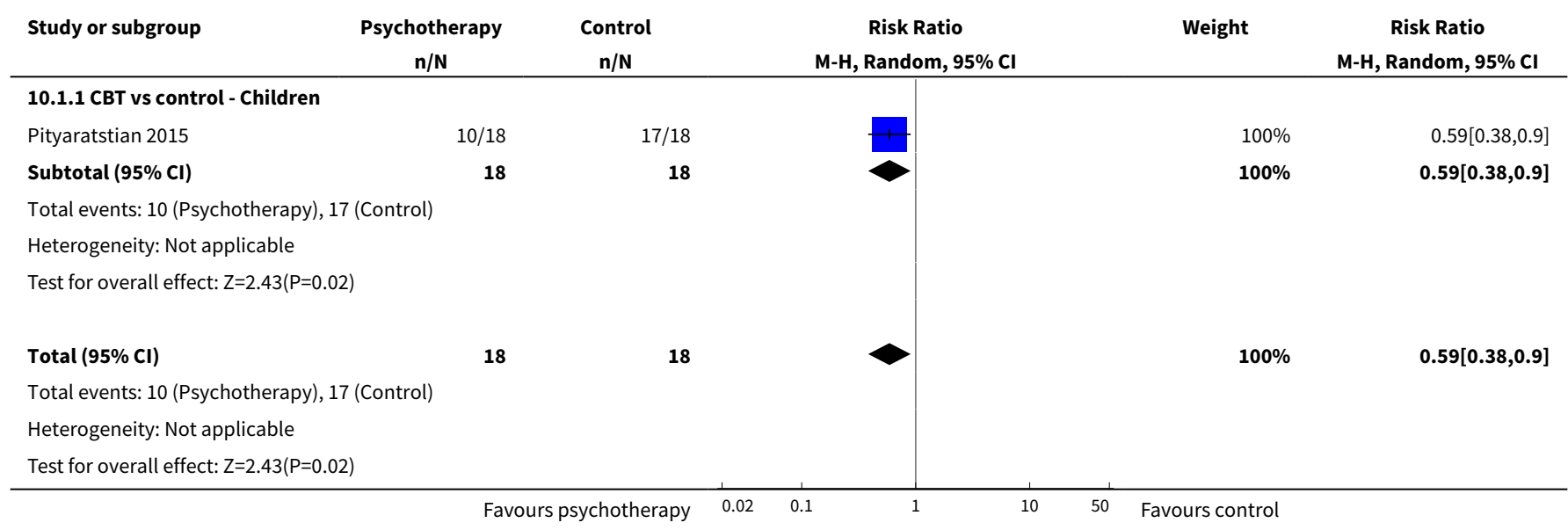

Comparison 11. Psychological therapy vs control comparator - diagnosis of depression - adults

\begin{tabular}{lllll}
\hline Outcome or subgroup title & No. of studies & $\begin{array}{l}\text { No. of partici- } \\
\text { pants }\end{array}$ & Statistical method & Effect size \\
\hline 1 Diagnosis of depression & 1 & 49 & Risk Ratio (M-H, Random, 95\% Cl) & $0.30[0.11,0.80]$ \\
\hline 1.1 IPT vs control - Adults & 1 & 49 & Risk Ratio (M-H, Random, 95\% Cl) & $0.30[0.11,0.80]$ \\
\hline
\end{tabular}

Analysis 11.1. Comparison 11 Psychological therapy vs control comparator - diagnosis of depression - adults, Outcome 1 Diagnosis of depression.

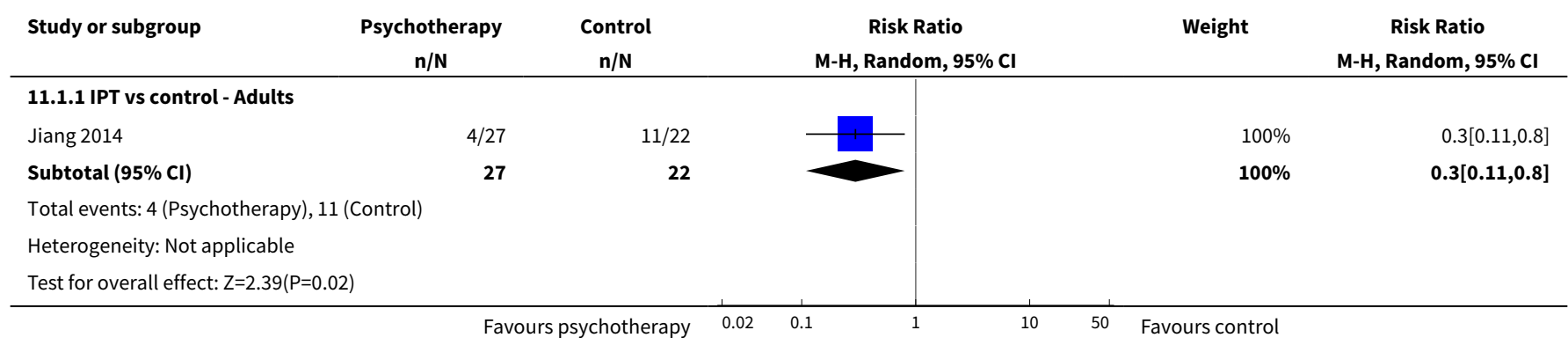




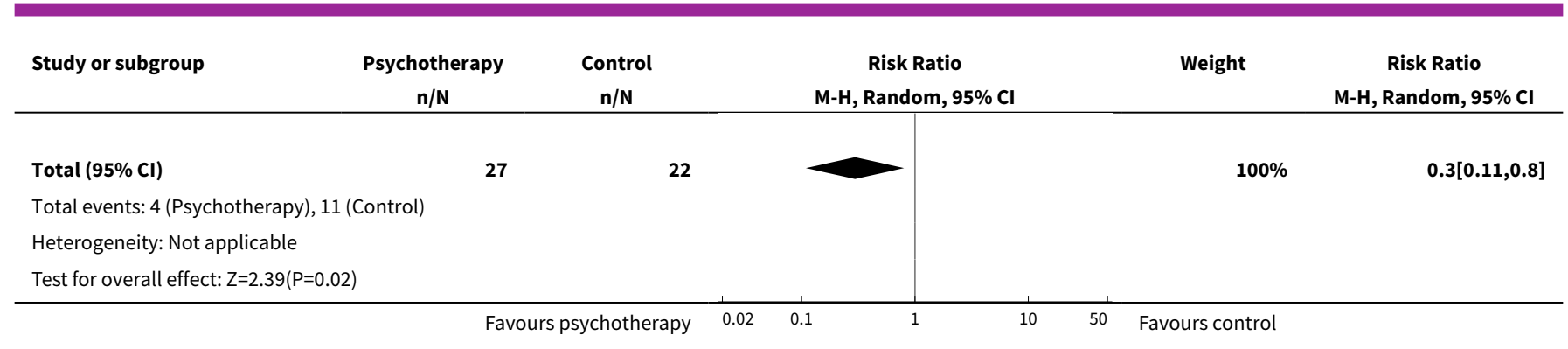

\section{Comparison 12. Subgroup analysis: type of traumatic events - adults}

\begin{tabular}{|c|c|c|c|c|}
\hline Outcome or subgroup title & No. of studies & $\begin{array}{l}\text { No. of partici- } \\
\text { pants }\end{array}$ & Statistical method & Effect size \\
\hline $\begin{array}{l}1 \text { PTSD symptoms at end- } \\
\text { point }\end{array}$ & 15 & 1250 & $\begin{array}{l}\text { Std. Mean Difference (IV, Random, } \\
95 \% \mathrm{Cl} \text { ) }\end{array}$ & $-1.10[-1.39,-0.81]$ \\
\hline 1.1 Bereavement & 4 & 320 & $\begin{array}{l}\text { Std. Mean Difference (IV, Random, } \\
95 \% \mathrm{CI} \text { ) }\end{array}$ & $-0.62[-0.85,-0.40]$ \\
\hline 1.2 Displacement & 5 & 171 & $\begin{array}{l}\text { Std. Mean Difference (IV, Random, } \\
95 \% \mathrm{CI})\end{array}$ & $-2.19[-3.00,-1.37]$ \\
\hline $\begin{array}{l}1.3 \text { Tortures/witnesses of vi- } \\
\text { olence/atrocities }\end{array}$ & 5 & 485 & $\begin{array}{l}\text { Std. Mean Difference (IV, Random, } \\
95 \% \mathrm{CI} \text { ) }\end{array}$ & $-0.84[-1.14,-0.55]$ \\
\hline 1.4 Other traumatic events & 1 & 274 & $\begin{array}{l}\text { Std. Mean Difference (IV, Random, } \\
95 \% \mathrm{Cl} \text { ) }\end{array}$ & $-0.79[-1.04,-0.54]$ \\
\hline $\begin{array}{l}2 \text { PTSD symptoms at } 1 \text { to } 4 \\
\text { months }\end{array}$ & 16 & 1421 & $\begin{array}{l}\text { Std. Mean Difference (IV, Random, } \\
95 \% \mathrm{CI})\end{array}$ & $-0.49[-0.69,-0.29]$ \\
\hline 2.1 Bereavement & 5 & 462 & $\begin{array}{l}\text { Std. Mean Difference (IV, Random, } \\
95 \% \mathrm{CI})\end{array}$ & $-0.65[-0.83,-0.46]$ \\
\hline 2.2 Displacement & 4 & 170 & $\begin{array}{l}\text { Std. Mean Difference (IV, Random, } \\
95 \% \mathrm{CI})\end{array}$ & $-0.59[-1.43,0.25]$ \\
\hline $\begin{array}{l}2.3 \text { Tortures/witnesses of vi- } \\
\text { olence/atrocities }\end{array}$ & 6 & 658 & $\begin{array}{l}\text { Std. Mean Difference (IV, Random, } \\
95 \% \mathrm{CI})\end{array}$ & $-0.38[-0.61,-0.16]$ \\
\hline 2.4 Other traumatic events & 1 & 131 & $\begin{array}{l}\text { Std. Mean Difference (IV, Random, } \\
95 \% \mathrm{CI})\end{array}$ & $-0.07[-0.41,0.28]$ \\
\hline $\begin{array}{l}3 \text { PTSD symptoms } \geq 6 \\
\text { months }\end{array}$ & 5 & & $\begin{array}{l}\text { Std. Mean Difference (IV, Random, } \\
95 \% \mathrm{Cl} \text { ) }\end{array}$ & Subtotals only \\
\hline 3.1 Displacement & 3 & 182 & $\begin{array}{l}\text { Std. Mean Difference (IV, Random, } \\
95 \% \mathrm{Cl} \text { ) }\end{array}$ & $-0.46[-0.82,-0.10]$ \\
\hline $\begin{array}{l}\text { 3.2 Tortures/witnesses of vi- } \\
\text { olence/atrocities }\end{array}$ & 2 & 218 & $\begin{array}{l}\text { Std. Mean Difference (IV, Random, } \\
95 \% \mathrm{CI} \text { ) }\end{array}$ & $-0.36[-0.78,0.07]$ \\
\hline $\begin{array}{l}4 \text { Anxiety symptoms at end- } \\
\text { point }\end{array}$ & 5 & 694 & $\begin{array}{l}\text { Std. Mean Difference (IV, Random, } \\
95 \% \mathrm{CI} \text { ) }\end{array}$ & $-0.74[-0.98,-0.49]$ \\
\hline
\end{tabular}

Psychological therapies for the treatment of mental disorders in low- and middle-income countries affected by humanitarian crises 


\begin{tabular}{|c|c|c|c|c|}
\hline Outcome or subgroup title & No. of studies & $\begin{array}{l}\text { No. of partici- } \\
\text { pants }\end{array}$ & Statistical method & Effect size \\
\hline 4.1 Displacement & 2 & 52 & $\begin{array}{l}\text { Std. Mean Difference (IV, Random, } \\
95 \% \mathrm{CI} \text { ) }\end{array}$ & $-1.30[-1.92,-0.67]$ \\
\hline $\begin{array}{l}4.2 \text { Tortures/witnesses of vi- } \\
\text { olence/atrocities }\end{array}$ & 1 & 159 & $\begin{array}{l}\text { Std. Mean Difference (IV, Random, } \\
95 \% \mathrm{Cl} \text { ) }\end{array}$ & $-0.78[-1.10,-0.46]$ \\
\hline 4.3 Other traumatic events & 1 & 274 & $\begin{array}{l}\text { Std. Mean Difference (IV, Random, } \\
95 \% \mathrm{Cl} \text { ) }\end{array}$ & $-0.48[-0.72,-0.24]$ \\
\hline 4.4 Bereavement & 1 & 209 & $\begin{array}{l}\text { Std. Mean Difference (IV, Random, } \\
95 \% \mathrm{CI} \text { ) }\end{array}$ & $-0.74[-1.02,-0.45]$ \\
\hline $\begin{array}{l}5 \text { Anxiety symptoms at } 1 \text { to } \\
4 \text { months }\end{array}$ & 6 & 969 & $\begin{array}{l}\text { Std. Mean Difference (IV, Random, } \\
95 \% \mathrm{CI})\end{array}$ & $-0.91[-1.45,-0.37]$ \\
\hline 5.1 Displacement & 2 & 52 & $\begin{array}{l}\text { Std. Mean Difference (IV, Random, } \\
95 \% \mathrm{Cl} \text { ) }\end{array}$ & $0.06[-0.50,0.63]$ \\
\hline $\begin{array}{l}5.2 \text { Tortures/witnesses of vi- } \\
\text { olence/atrocities }\end{array}$ & 3 & 611 & $\begin{array}{l}\text { Std. Mean Difference (IV, Random, } \\
95 \% \mathrm{Cl} \text { ) }\end{array}$ & $-1.35[-2.20,-0.51]$ \\
\hline 5.3 Bereavement & 1 & 306 & $\begin{array}{l}\text { Std. Mean Difference (IV, Random, } \\
95 \% \mathrm{Cl} \text { ) }\end{array}$ & $-0.74[-0.97,-0.51]$ \\
\hline $\begin{array}{l}6 \text { Depressive symptoms at } \\
\text { endpoint }\end{array}$ & 13 & 1232 & $\begin{array}{l}\text { Std. Mean Difference (IV, Random, } \\
95 \% \mathrm{Cl} \text { ) }\end{array}$ & $-0.90[-1.09,-0.71]$ \\
\hline 6.1 Bereavement & 3 & 268 & $\begin{array}{l}\text { Std. Mean Difference (IV, Random, } \\
95 \% \mathrm{Cl} \text { ) }\end{array}$ & $-0.90[-1.15,-0.65]$ \\
\hline 6.2 Displacement & 6 & 380 & $\begin{array}{l}\text { Std. Mean Difference (IV, Random, } \\
95 \% \mathrm{Cl} \text { ) }\end{array}$ & $-1.11[-1.54,-0.68]$ \\
\hline $\begin{array}{l}6.3 \text { Tortures/witnesses of vi- } \\
\text { olence/atrocities }\end{array}$ & 3 & 310 & $\begin{array}{l}\text { Std. Mean Difference (IV, Random, } \\
95 \% \mathrm{Cl} \text { ) }\end{array}$ & $-0.71[-1.24,-0.18]$ \\
\hline 6.4 Other traumatic events & 1 & 274 & $\begin{array}{l}\text { Std. Mean Difference (IV, Random, } \\
95 \% \mathrm{CI})\end{array}$ & $-0.90[-1.15,-0.65]$ \\
\hline $\begin{array}{l}7 \text { Depressive symptoms at } 1 \\
\text { to } 4 \text { months }\end{array}$ & 14 & & $\begin{array}{l}\text { Std. Mean Difference (IV, Random, } \\
95 \% \mathrm{CI} \text { ) }\end{array}$ & Subtotals only \\
\hline 7.1 Bereavement & 5 & 462 & $\begin{array}{l}\text { Std. Mean Difference (IV, Random, } \\
95 \% \mathrm{Cl} \text { ) }\end{array}$ & $-0.56[-0.90,-0.23]$ \\
\hline 7.2 Displacement & 4 & 170 & $\begin{array}{l}\text { Std. Mean Difference (IV, Random, } \\
95 \% \mathrm{CI} \text { ) }\end{array}$ & $-0.17[-0.95,0.61]$ \\
\hline $\begin{array}{l}\text { 7.3 Tortures/witnesses of vi- } \\
\text { olence/atrocities }\end{array}$ & 5 & 732 & $\begin{array}{l}\text { Std. Mean Difference (IV, Random, } \\
95 \% \mathrm{CI} \text { ) }\end{array}$ & $-0.38[-0.61,-0.16]$ \\
\hline $\begin{array}{l}8 \text { Depressive symptoms } \geq 6 \\
\text { months }\end{array}$ & 2 & & $\begin{array}{l}\text { Std. Mean Difference (IV, Random, } \\
95 \% \mathrm{CI})\end{array}$ & Subtotals only \\
\hline
\end{tabular}




\begin{tabular}{|c|c|c|c|c|}
\hline Outcome or subgroup title & No. of studies & $\begin{array}{l}\text { No. of partici- } \\
\text { pants }\end{array}$ & Statistical method & Effect size \\
\hline 8.1 Displacement & 1 & 54 & $\begin{array}{l}\text { Std. Mean Difference (IV, Random, } \\
95 \% \mathrm{Cl})\end{array}$ & $-0.04[-0.58,0.49]$ \\
\hline $\begin{array}{l}8.2 \text { Tortures/witnesses of vi- } \\
\text { olence/atrocities }\end{array}$ & 1 & 188 & $\begin{array}{l}\text { Std. Mean Difference (IV, Random, } \\
95 \% \mathrm{Cl})\end{array}$ & $-0.26[-0.61,0.08]$ \\
\hline 9 Dropout & 24 & & Risk Ratio (M-H, Random, 95\% Cl) & Subtotals only \\
\hline 9.1 Bereavement & 4 & 483 & Risk Ratio (M-H, Random, 95\% Cl) & $1.18[1.00,1.40]$ \\
\hline 9.2 Displacement & 9 & 734 & Risk Ratio (M-H, Random, 95\% Cl) & $0.77[0.63,0.95]$ \\
\hline $\begin{array}{l}9.3 \text { Tortures/witnesses of vi- } \\
\text { olence/atrocities }\end{array}$ & 10 & 1209 & Risk Ratio (M-H, Random, 95\% Cl) & $1.16[0.76,1.78]$ \\
\hline 9.4 Other traumatic events & 1 & 347 & Risk Ratio (M-H, Random, 95\% Cl) & $0.79[0.53,1.19]$ \\
\hline $\begin{array}{l}10 \text { Functional impairment } \\
\text { at endpoint }\end{array}$ & 5 & 686 & $\begin{array}{l}\text { Std. Mean Difference (IV, Random, } \\
95 \% \mathrm{Cl})\end{array}$ & $-0.55[-0.80,-0.30]$ \\
\hline $\begin{array}{l}\text { 10.1 Tortures/witnesses of } \\
\text { violence/atrocities }\end{array}$ & 2 & 151 & $\begin{array}{l}\text { Std. Mean Difference (IV, Random, } \\
95 \% \mathrm{Cl})\end{array}$ & $-0.45[-1.30,0.40]$ \\
\hline 10.2 Other traumatic events & 1 & 274 & $\begin{array}{l}\text { Std. Mean Difference (IV, Random, } \\
95 \% \mathrm{Cl})\end{array}$ & $-0.56[-0.80,-0.32]$ \\
\hline 10.3 Bereavement & 2 & 261 & $\begin{array}{l}\text { Std. Mean Difference (IV, Random, } \\
95 \% \mathrm{Cl})\end{array}$ & $-0.68[-0.93,-0.43]$ \\
\hline $\begin{array}{l}11 \text { Functional impairment } \\
\text { at } 1 \text { to } 4 \text { months }\end{array}$ & 7 & 1061 & $\begin{array}{l}\text { Std. Mean Difference (IV, Random, } \\
95 \% \mathrm{Cl})\end{array}$ & $-0.36[-0.55,-0.16]$ \\
\hline $\begin{array}{l}11.1 \text { Tortures/witnesses of } \\
\text { violence/atrocities }\end{array}$ & 6 & 758 & $\begin{array}{l}\text { Std. Mean Difference (IV, Random, } \\
95 \% \mathrm{Cl})\end{array}$ & $-0.29[-0.45,-0.12]$ \\
\hline 11.2 Bereavement & 1 & 303 & $\begin{array}{l}\text { Std. Mean Difference (IV, Random, } \\
95 \% \mathrm{Cl})\end{array}$ & $-0.67[-0.90,-0.44]$ \\
\hline $\begin{array}{l}12 \text { Quality of life at end- } \\
\text { point }\end{array}$ & 4 & 325 & $\begin{array}{l}\text { Std. Mean Difference (IV, Random, } \\
95 \% \mathrm{Cl})\end{array}$ & $-0.73[-1.22,-0.25]$ \\
\hline 12.1 Bereavement & 1 & 38 & $\begin{array}{l}\text { Std. Mean Difference (IV, Random, } \\
95 \% \mathrm{Cl})\end{array}$ & $-0.88[-1.55,-0.21]$ \\
\hline $\begin{array}{l}12.2 \text { Tortures/witnesses of } \\
\text { violence/atrocities }\end{array}$ & 3 & 287 & $\begin{array}{l}\text { Std. Mean Difference (IV, Random, } \\
95 \% \mathrm{Cl})\end{array}$ & $-0.71[-1.35,-0.07]$ \\
\hline 13 Diagnosis of PTSD & 4 & 402 & Risk Ratio (M-H, Random, 95\% Cl) & $1.07[0.43,2.68]$ \\
\hline 13.1 Bereavement & 1 & 49 & Risk Ratio (M-H, Random, 95\% Cl) & $0.31[0.09,1.02]$ \\
\hline 13.2 Displacement & 2 & 277 & Risk Ratio (M-H, Random, 95\% Cl) & $2.34[1.14,4.80]$ \\
\hline $\begin{array}{l}13.3 \text { Tortures/witnesses of } \\
\text { violence/atrocities }\end{array}$ & 1 & 76 & Risk Ratio (M-H, Random, 95\% Cl) & $0.78[0.63,0.95]$ \\
\hline
\end{tabular}

Psychological therapies for the treatment of mental disorders in low- and middle-income countries affected by humanitarian crises 
Analysis 12.1. Comparison 12 Subgroup analysis: type of traumatic events - adults, Outcome 1 PTSD symptoms at endpoint.

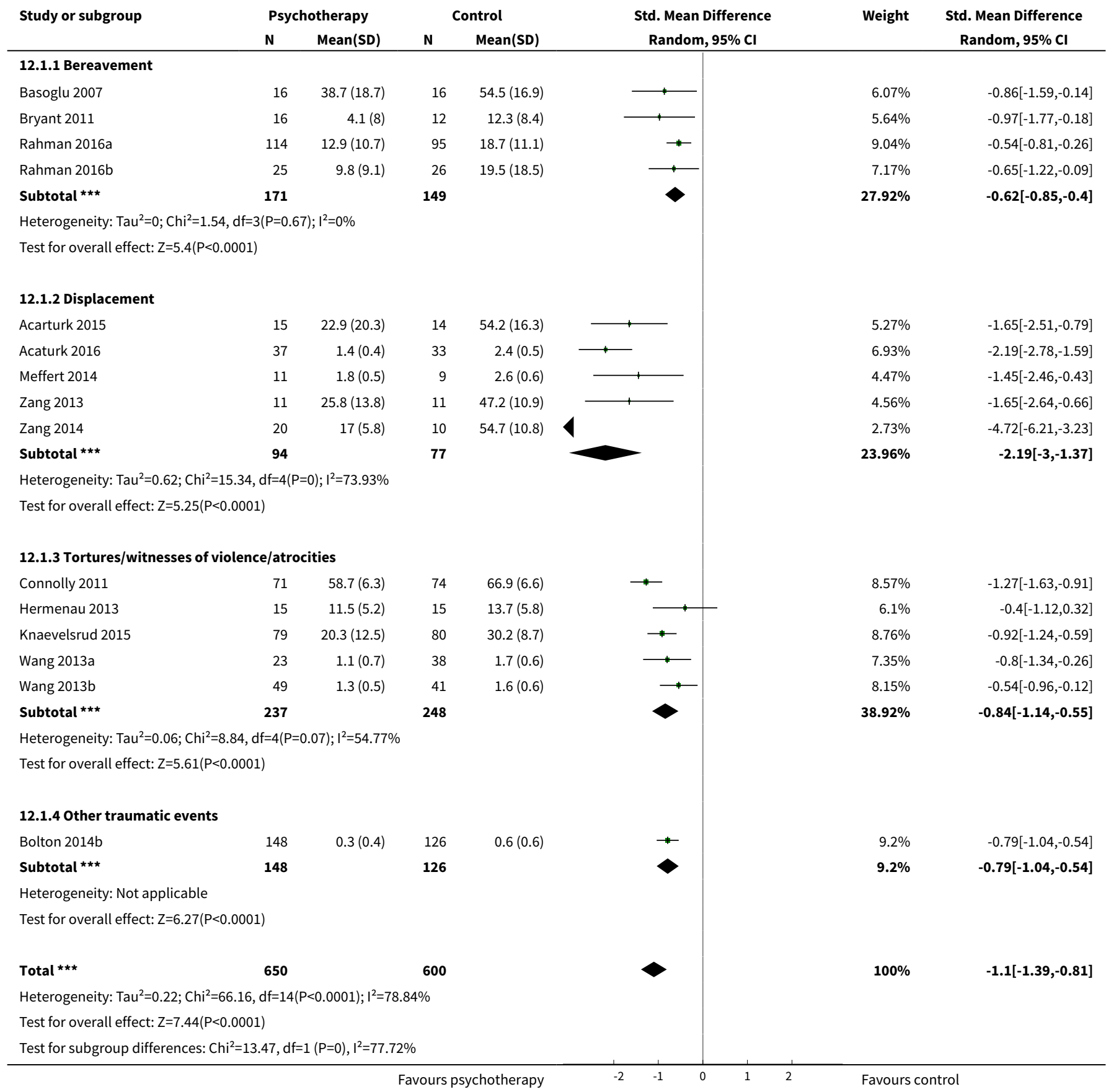

Psychological therapies for the treatment of mental disorders in low- and middle-income countries affected by humanitarian crises 


\section{Analysis 12.2. Comparison 12 Subgroup analysis: type of traumatic} events - adults, Outcome 2 PTSD symptoms at 1 to 4 months.

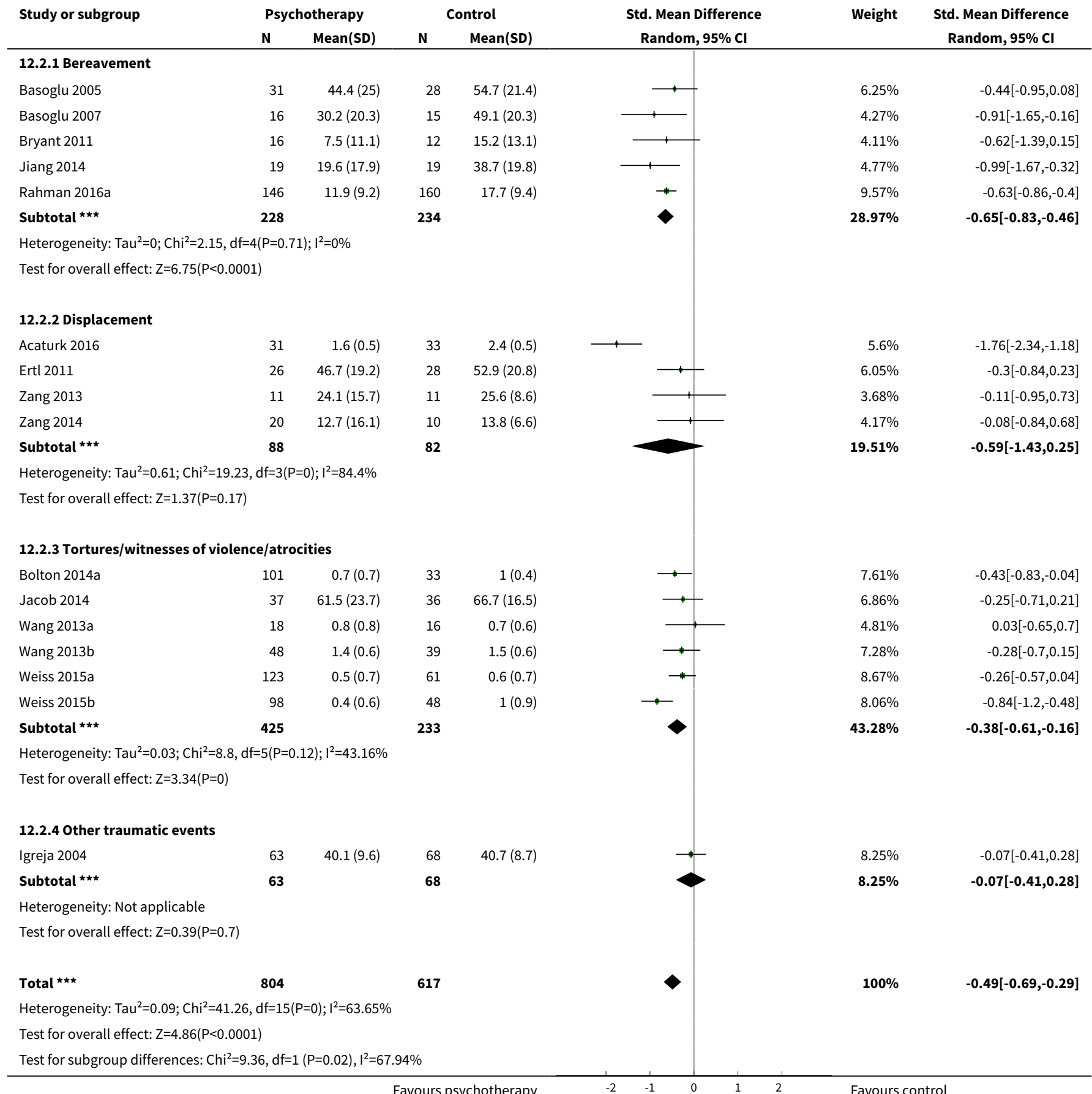

Favours psychotherapy 
Analysis 12.3. Comparison 12 Subgroup analysis: type of traumatic events - adults, Outcome 3 PTSD symptoms $\geq 6$ months.

\begin{tabular}{|c|c|c|c|c|c|c|c|}
\hline \multirow[t]{2}{*}{ Study or subgroup } & \multicolumn{2}{|c|}{ Psychotherapy } & \multicolumn{2}{|c|}{ Control } & \multirow{2}{*}{$\begin{array}{c}\text { Std. Mean Difference } \\
\text { Random, } 95 \% \mathrm{Cl} \\
\end{array}$} & \multirow[t]{2}{*}{ Weight } & \multirow{2}{*}{$\begin{array}{c}\text { Std. Mean Difference } \\
\text { Random, } 95 \% \mathrm{Cl}\end{array}$} \\
\hline & $\mathbf{N}$ & Mean(SD) & $\mathbf{N}$ & Mean(SD) & & & \\
\hline \multicolumn{8}{|l|}{ 12.3.1 Displacement } \\
\hline Ertl 2011 & 26 & $43(21.5)$ & 28 & $48.6(23.7)$ & -1 & $45.96 \%$ & $-0.24[-0.78,0.29]$ \\
\hline Neuner 2008a & 56 & $6.1(6.8)$ & 10 & $10.1(8.1)$ & & $28.53 \%$ & $-0.56[-1.25,0.12]$ \\
\hline Neuner 2008b & 53 & $5(6.6)$ & 9 & $10.1(8.1)$ & & $25.51 \%$ & $-0.74[-1.46,-0.02]$ \\
\hline 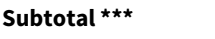 & 135 & & 47 & & & $100 \%$ & $-0.46[-0.82,-0.1]$ \\
\hline \multicolumn{8}{|c|}{ Test for overall effect: $Z=2.49(P=0.01)$} \\
\hline \multicolumn{8}{|c|}{ 12.3.2 Tortures/witnesses of violence/atrocities } \\
\hline Bass 2016 & 145 & $0.7(0.6)$ & 43 & $0.9(0.6)$ & & $73.33 \%$ & $-0.22[-0.57,0.12]$ \\
\hline Hermenau 2013 & 15 & $8.9(7.6)$ & 15 & $16.5(12.5)$ & & $26.67 \%$ & $-0.72[-1.46,0.03]$ \\
\hline 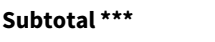 & 160 & & 58 & & & $100 \%$ & $-0.36[-0.78,0.07]$ \\
\hline \multicolumn{8}{|c|}{ Test for overall effect: $Z=1.63(P=0.1)$} \\
\hline \multicolumn{8}{|c|}{ Test for subgroup differences: $\mathrm{Chi}^{2}=0.14, \mathrm{df}=1(\mathrm{P}=0.71), \mathrm{I}^{2}=0 \%$} \\
\hline
\end{tabular}

Analysis 12.4. Comparison 12 Subgroup analysis: type of traumatic events - adults, Outcome 4 Anxiety symptoms at endpoint.

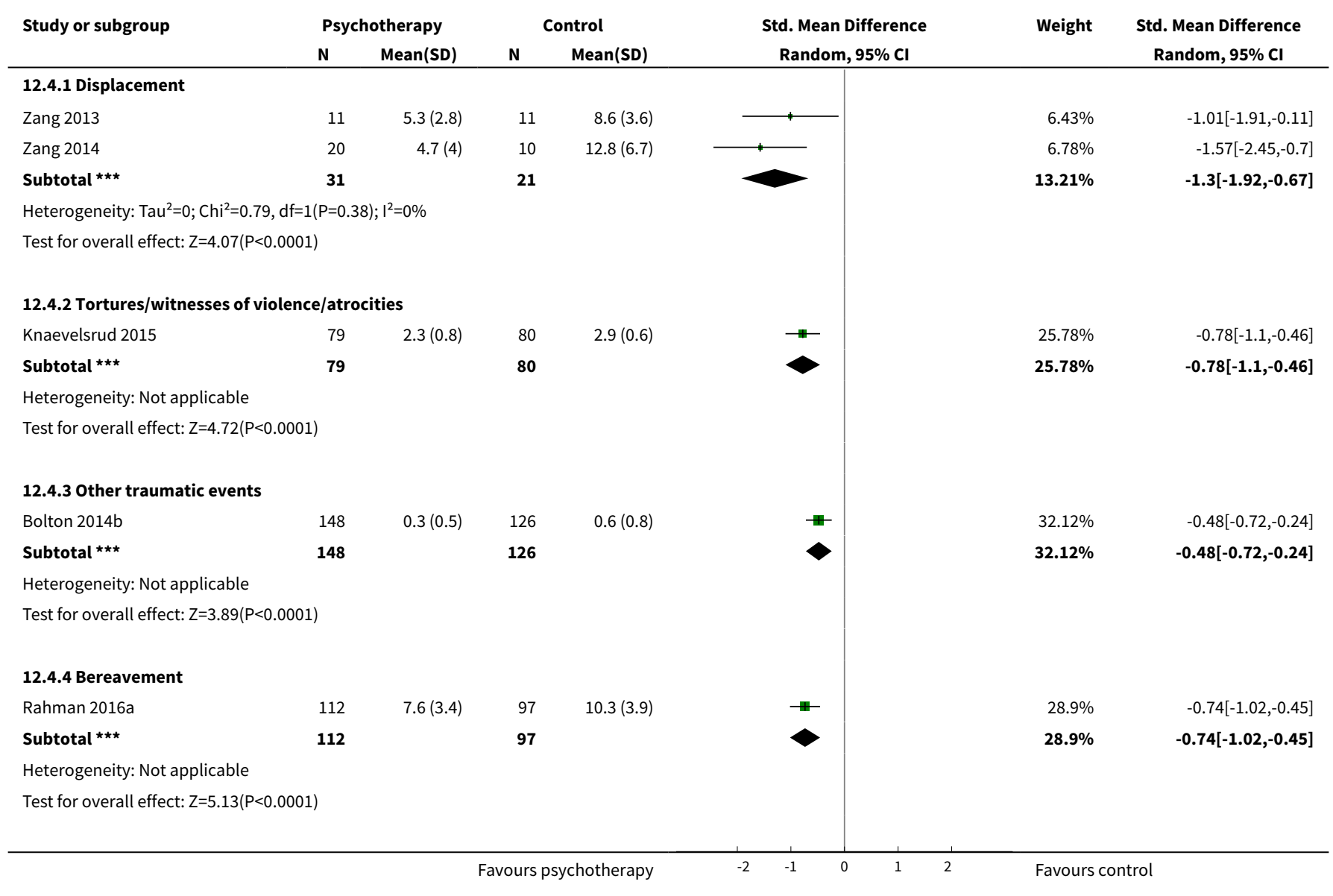

Psychological therapies for the treatment of mental disorders in low- and middle-income countries affected by humanitarian crises 


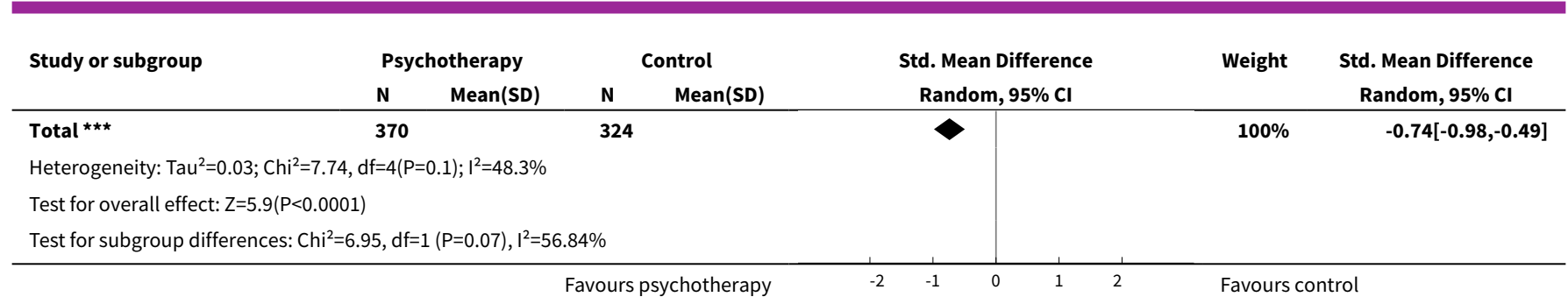

\section{Analysis 12.5. Comparison 12 Subgroup analysis: type of traumatic} events - adults, Outcome 5 Anxiety symptoms at 1 to 4 months.

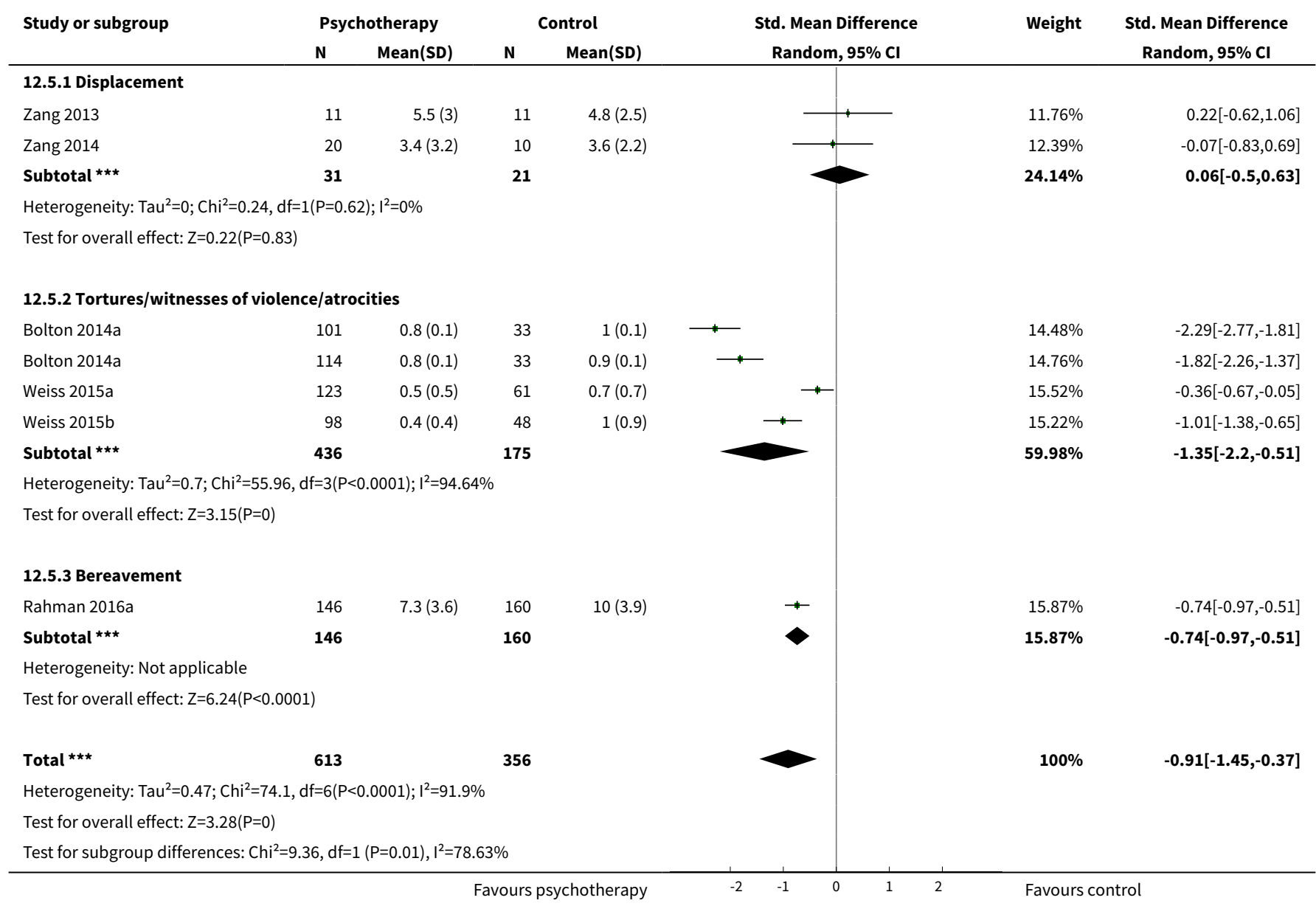

Analysis 12.6. Comparison 12 Subgroup analysis: type of traumatic events - adults, Outcome 6 Depressive symptoms at endpoint.

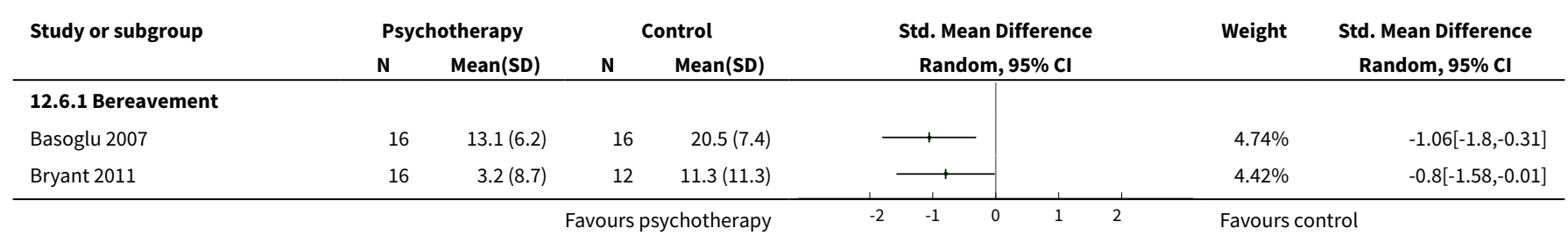

Psychological therapies for the treatment of mental disorders in low- and middle-income countries affected by humanitarian crises 109 (Review)

Copyright (c) 2018 The Cochrane Collaboration. Published by John Wiley \& Sons, Ltd. 


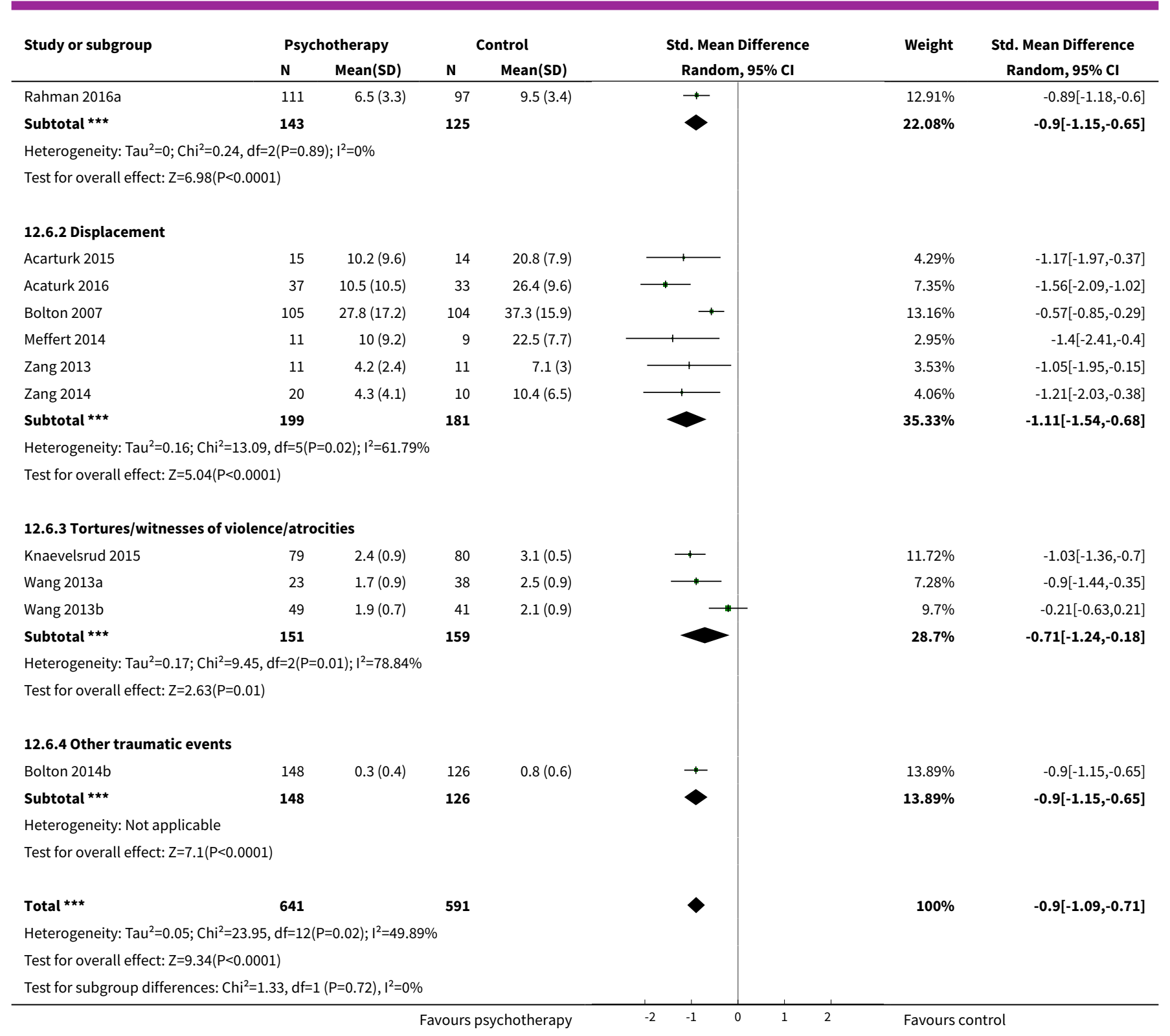

\section{Analysis 12.7. Comparison 12 Subgroup analysis: type of traumatic} events - adults, Outcome 7 Depressive symptoms at 1 to 4 months.

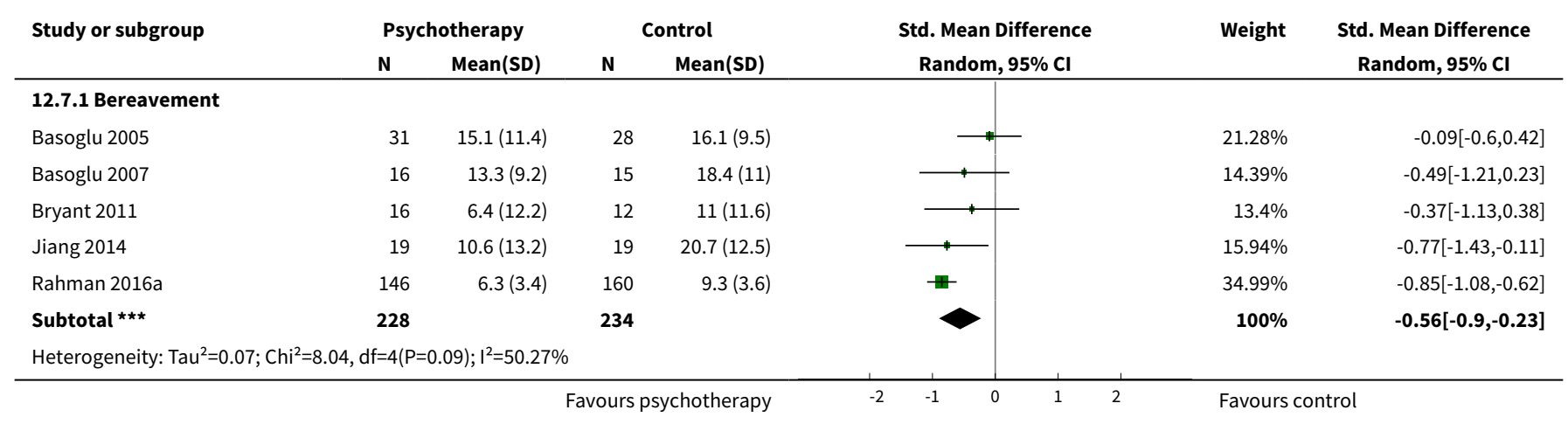

Psychological therapies for the treatment of mental disorders in low- and middle-income countries affected by humanitarian crises 


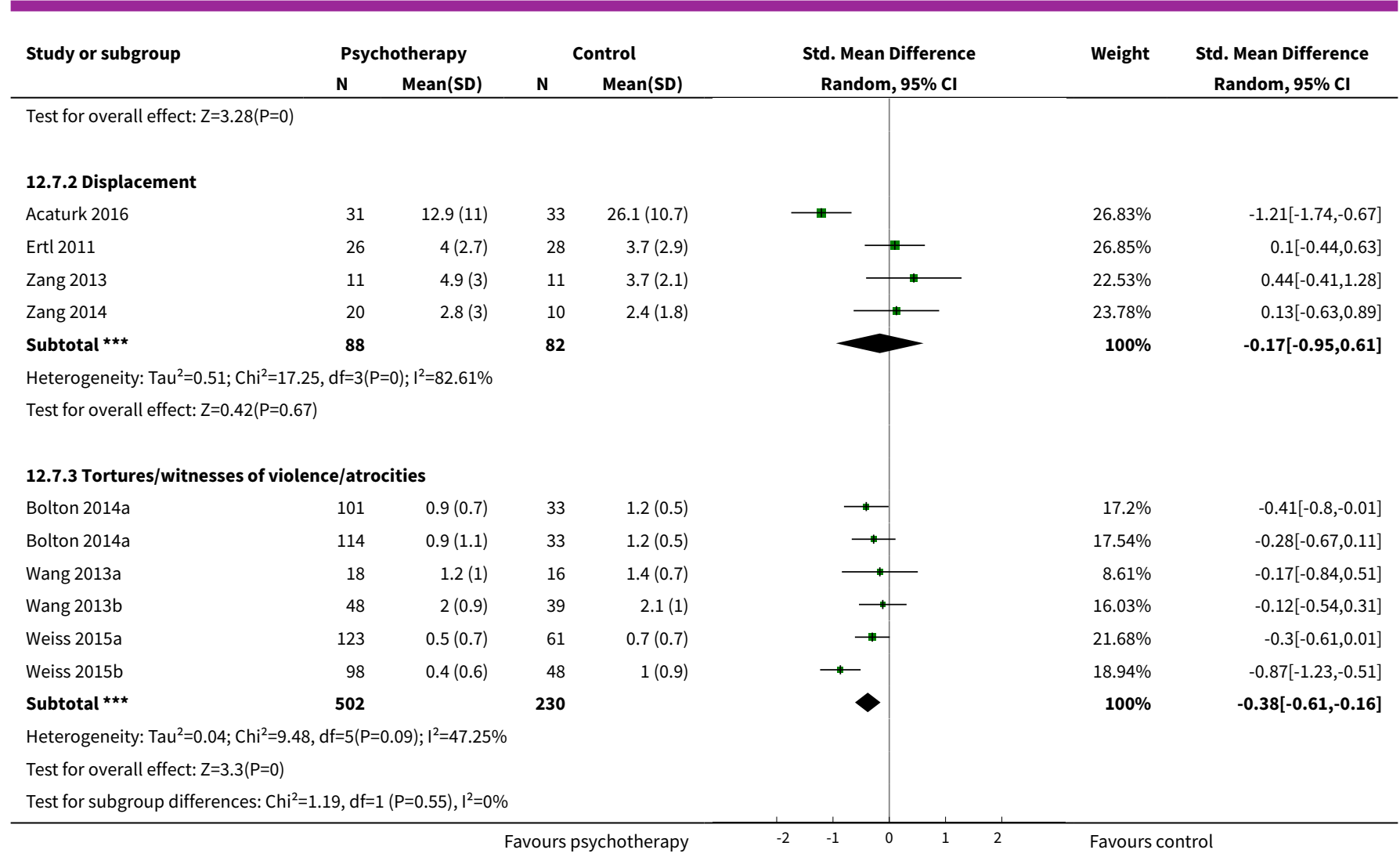

\section{Analysis 12.8. Comparison 12 Subgroup analysis: type of traumatic events - adults, Outcome 8 Depressive symptoms $\geq 6$ months.}

\begin{tabular}{|c|c|c|c|c|c|c|c|}
\hline \multirow[t]{2}{*}{ Study or subgroup } & \multicolumn{2}{|c|}{ Psychotherapy } & \multicolumn{2}{|c|}{ Control } & \multirow{2}{*}{$\begin{array}{c}\text { Std. Mean Difference } \\
\text { Random, } 95 \% \mathrm{Cl} \\
\end{array}$} & \multirow[t]{2}{*}{ Weight } & \multirow{2}{*}{$\begin{array}{c}\text { Std. Mean Difference } \\
\text { Random, } 95 \% \mathrm{CI}\end{array}$} \\
\hline & $\mathbf{N}$ & Mean(SD) & $\mathbf{N}$ & Mean(SD) & & & \\
\hline \multicolumn{8}{|l|}{ 12.8.1 Displacement } \\
\hline Ertl 2011 & 26 & $3.1(3)$ & 28 & $3.2(3)$ & & $100 \%$ & $-0.04[-0.58,0.49]$ \\
\hline Subtotal $\star \star \star ~$ & 26 & & 28 & & & $100 \%$ & $-0.04[-0.58,0.49]$ \\
\hline \multicolumn{8}{|c|}{ Heterogeneity: Not applicable } \\
\hline \multicolumn{8}{|c|}{ Test for overall effect: $Z=0.16(P=0.87)$} \\
\hline \multicolumn{8}{|c|}{ 12.8.2 Tortures/witnesses of violence/atrocities } \\
\hline Bass 2016 & 145 & $0.8(0.7)$ & 43 & $1(0.8)$ & & $100 \%$ & $-0.26[-0.61,0.08]$ \\
\hline Subtotal $\star \star \star$ & 145 & & 43 & & & $100 \%$ & $-0.26[-0.61,0.08]$ \\
\hline \multicolumn{8}{|c|}{ Heterogeneity: Not applicable } \\
\hline \multicolumn{8}{|c|}{ Test for overall effect: $Z=1.52(P=0.13)$} \\
\hline Test for subgroup dif & $47, \mathrm{df}=$ & $P=0.49), I^{2}=09$ & & & & & \\
\hline
\end{tabular}

Psychological therapies for the treatment of mental disorders in low- and middle-income countries affected by humanitarian crises 
Analysis 12.9. Comparison 12 Subgroup analysis: type of traumatic events - adults, Outcome 9 Dropout.

\begin{tabular}{|c|c|c|c|c|c|}
\hline Study or subgroup & $\begin{array}{c}\text { Psychotherapy } \\
\mathrm{n} / \mathrm{N} \\
\end{array}$ & $\begin{array}{c}\text { Control } \\
\mathrm{n} / \mathrm{N}\end{array}$ & $\begin{array}{c}\text { Risk Ratio } \\
\text { M-H, Random, } 95 \% \text { CI }\end{array}$ & Weight & $\begin{array}{c}\text { Risk Ratio } \\
\text { M-H, Random, } 95 \% \mathrm{CI}\end{array}$ \\
\hline \multicolumn{6}{|l|}{ 12.9.1 Bereavement } \\
\hline Bryant 2011 & $0 / 16$ & $0 / 12$ & & & Not estimable \\
\hline Jiang 2014 & $8 / 27$ & $3 / 22$ & -1 & $1.96 \%$ & $2.17[0.65,7.23]$ \\
\hline Rahman 2016a & $112 / 172$ & $97 / 174$ & & $96.11 \%$ & $1.17[0.98,1.39]$ \\
\hline Rahman 2016b & $5 / 30$ & $4 / 30$ & 1 & $1.92 \%$ & $1.25[0.37,4.21]$ \\
\hline \multicolumn{6}{|c|}{ Total events: 125 (Psychotherapy), 104 (Control) } \\
\hline \multicolumn{6}{|c|}{ Heterogeneity: $\operatorname{Tau}^{2}=0 ; \mathrm{Chi}^{2}=1.05, \mathrm{df}=2(\mathrm{P}=0.59) ; \mathrm{I}^{2}=0 \%$} \\
\hline \multicolumn{6}{|c|}{ Test for overall effect: $\mathrm{Z}=1.97(\mathrm{P}=0.05)$} \\
\hline \multicolumn{6}{|l|}{ 12.9.2 Displacement } \\
\hline Acarturk 2015 & $0 / 15$ & $0 / 14$ & & & Not estimable \\
\hline Bolton 2007 & $9 / 105$ & $16 / 104$ & $\longrightarrow$ & $7.27 \%$ & $0.56[0.26,1.2]$ \\
\hline Ertl 2011 & $4 / 29$ & $0 / 28$ & & $0.52 \%$ & $8.7[0.49,154.49]$ \\
\hline Meffert 2014 & $2 / 13$ & $1 / 9$ & & $0.86 \%$ & $1.38[0.15,13.07]$ \\
\hline Neuner 2008a & $55 / 111$ & $18 / 28$ & 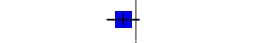 & $38.7 \%$ & $0.77[0.55,1.08]$ \\
\hline Neuner 2008b & $58 / 111$ & $18 / 27$ & \# & $41.97 \%$ & $0.78[0.57,1.08]$ \\
\hline Zang 2013 & $0 / 11$ & $0 / 11$ & & & Not estimable \\
\hline Zang 2014 & $0 / 10$ & $0 / 10$ & & & Not estimable \\
\hline Subtotal $(95 \% \mathrm{Cl})$ & 454 & 280 & $\nabla$ & $100 \%$ & $0.77[0.63,0.95]$ \\
\hline \multicolumn{6}{|c|}{ Total events: 140 (Psychotherapy), 69 (Control) } \\
\hline \multicolumn{6}{|c|}{ Heterogeneity: $\operatorname{Tau}^{2}=0 ; \mathrm{Chi}^{2}=3.73, \mathrm{df}=5(\mathrm{P}=0.59) ; \mathrm{I}^{2}=0 \%$} \\
\hline \multicolumn{6}{|c|}{ Test for overall effect: $Z=2.47(P=0.01)$} \\
\hline \multicolumn{6}{|c|}{ 12.9.3 Tortures/witnesses of violence/atrocities } \\
\hline Bolton 2014a & $34 / 101$ & $7 / 33$ & - & $16.26 \%$ & $1.59[0.78,3.24]$ \\
\hline Bolton 2014a & $32 / 114$ & $6 / 33$ & $\rightarrow$ & $14.89 \%$ & $1.54[0.71,3.37]$ \\
\hline Hermenau 2013 & $4 / 19$ & $0 / 19$ & & $2.07 \%$ & $9[0.52,156.41]$ \\
\hline Jacob 2014 & $1 / 38$ & $2 / 38$ & - & $2.94 \%$ & $0.5[0.05,5.28]$ \\
\hline Wang 2013a & $32 / 50$ & $37 / 53$ & & $26.53 \%$ & $0.92[0.7,1.2]$ \\
\hline Wang 2013b & $1 / 49$ & $5 / 45$ & & $3.59 \%$ & $0.18[0.02,1.51]$ \\
\hline Wang 2016 & $2 / 17$ & $0 / 17$ & 1 & $1.93 \%$ & $5[0.26,97]$ \\
\hline Weiss 2015a & $22 / 129$ & $3 / 64$ & $\longrightarrow$ & $9.2 \%$ & $3.64[1.13,11.7]$ \\
\hline Weiss 2015b & $2 / 99$ & $2 / 50$ & & $4.19 \%$ & $0.51[0.07,3.48]$ \\
\hline Subtotal $(95 \% \mathrm{Cl})$ & 791 & 418 & & $100 \%$ & $1.16[0.76,1.78]$ \\
\hline \multicolumn{6}{|c|}{ Total events: 147 (Psychotherapy), 71 (Control) } \\
\hline \multicolumn{6}{|c|}{ Heterogeneity: $\mathrm{Tau}^{2}=0.16 ; \mathrm{Chi}^{2}=16.77, \mathrm{df}=10(P=0.08) ; \mathrm{I}^{2}=40.37 \%$} \\
\hline \multicolumn{6}{|c|}{ Test for overall effect: $Z=0.68(P=0.5)$} \\
\hline \multicolumn{6}{|c|}{ 12.9.4 Other traumatic events } \\
\hline Bolton 2014b & $34 / 182$ & $39 / 165$ & & $100 \%$ & $0.79[0.53,1.19]$ \\
\hline Subtotal $(95 \% \mathrm{CI})$ & 182 & 165 & & $100 \%$ & $0.79[0.53,1.19]$ \\
\hline \multicolumn{6}{|c|}{ Total events: 34 (Psychotherapy), 39 (Control) } \\
\hline \multicolumn{6}{|c|}{ Heterogeneity: Not applicable } \\
\hline \multicolumn{6}{|c|}{ Test for overall effect: $Z=1.13(P=0.26)$} \\
\hline Test for subgroup diffe & 1.69, $d f=1(P=0.01$ & $.34 \%$ & & & \\
\hline
\end{tabular}

Psychological therapies for the treatment of mental disorders in low- and middle-income countries affected by humanitarian crises 
Analysis 12.10. Comparison 12 Subgroup analysis: type of traumatic events - adults, Outcome 10 Functional impairment at endpoint.

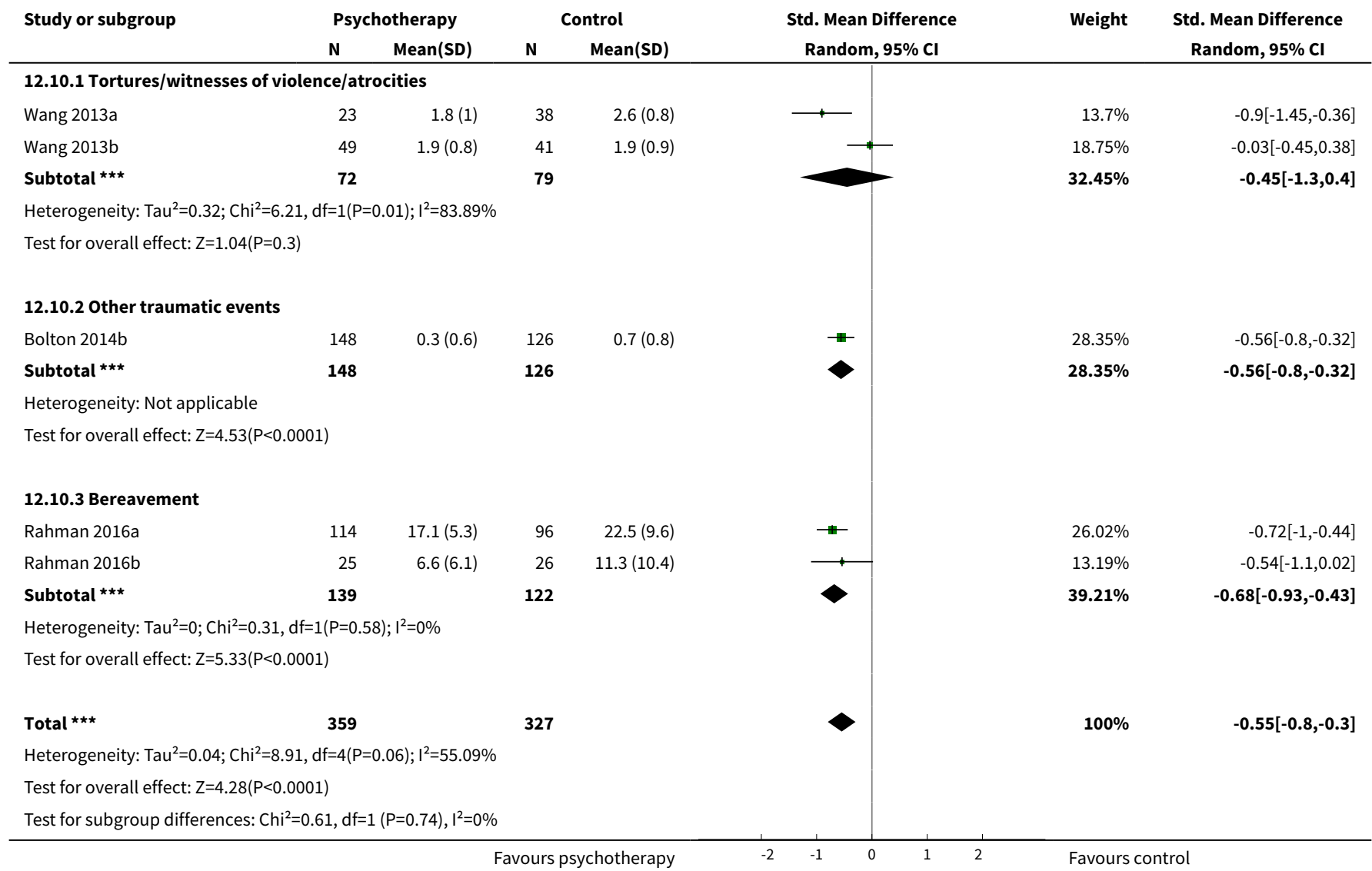

Analysis 12.11. Comparison 12 Subgroup analysis: type of traumatic events - adults, Outcome 11 Functional impairment at 1 to 4 months.

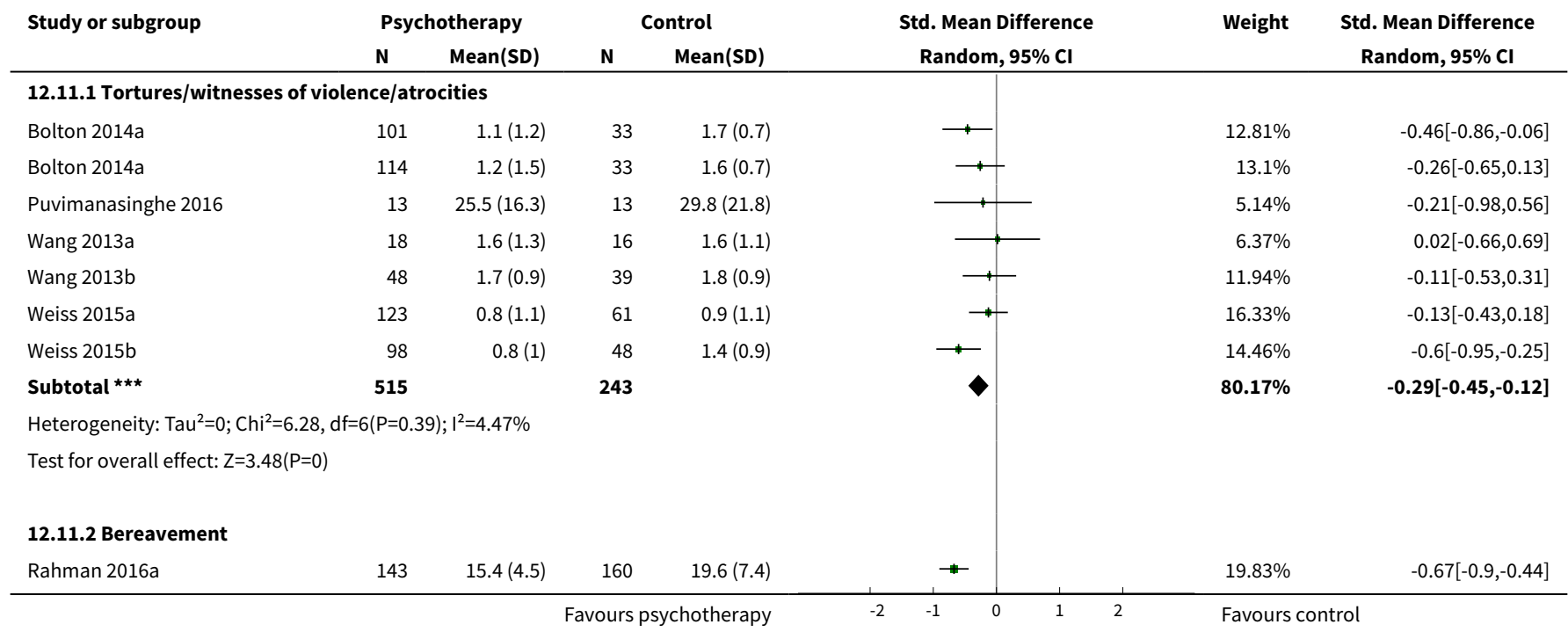

Psychological therapies for the treatment of mental disorders in low- and middle-income countries affected by humanitarian crises 


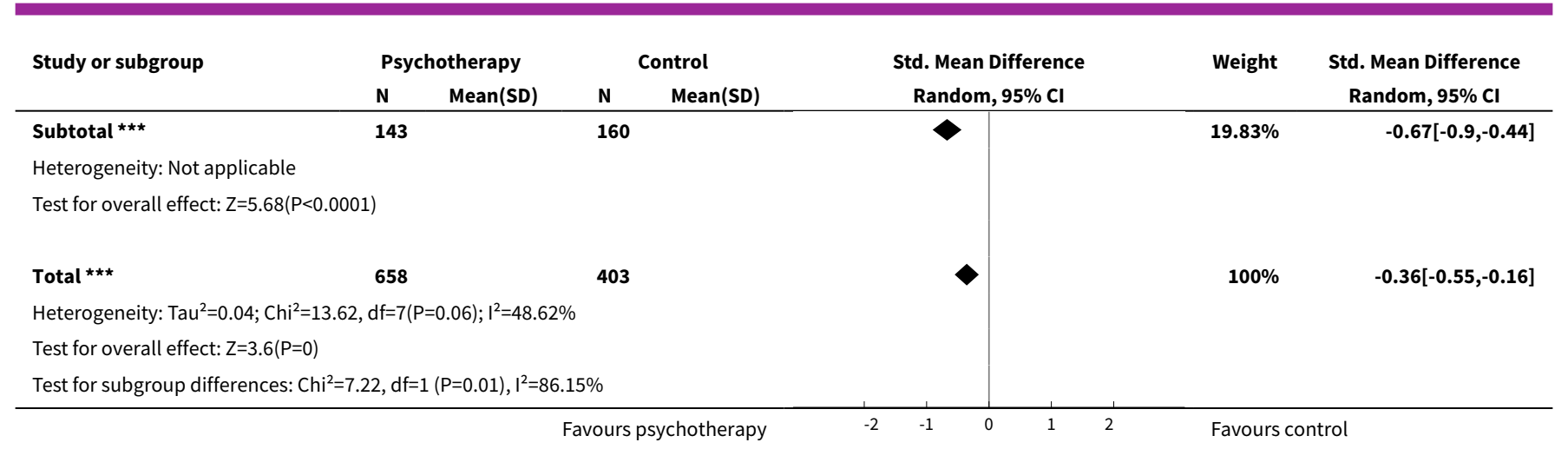

Analysis 12.12. Comparison 12 Subgroup analysis: type of traumatic events - adults, Outcome 12 Quality of life at endpoint.

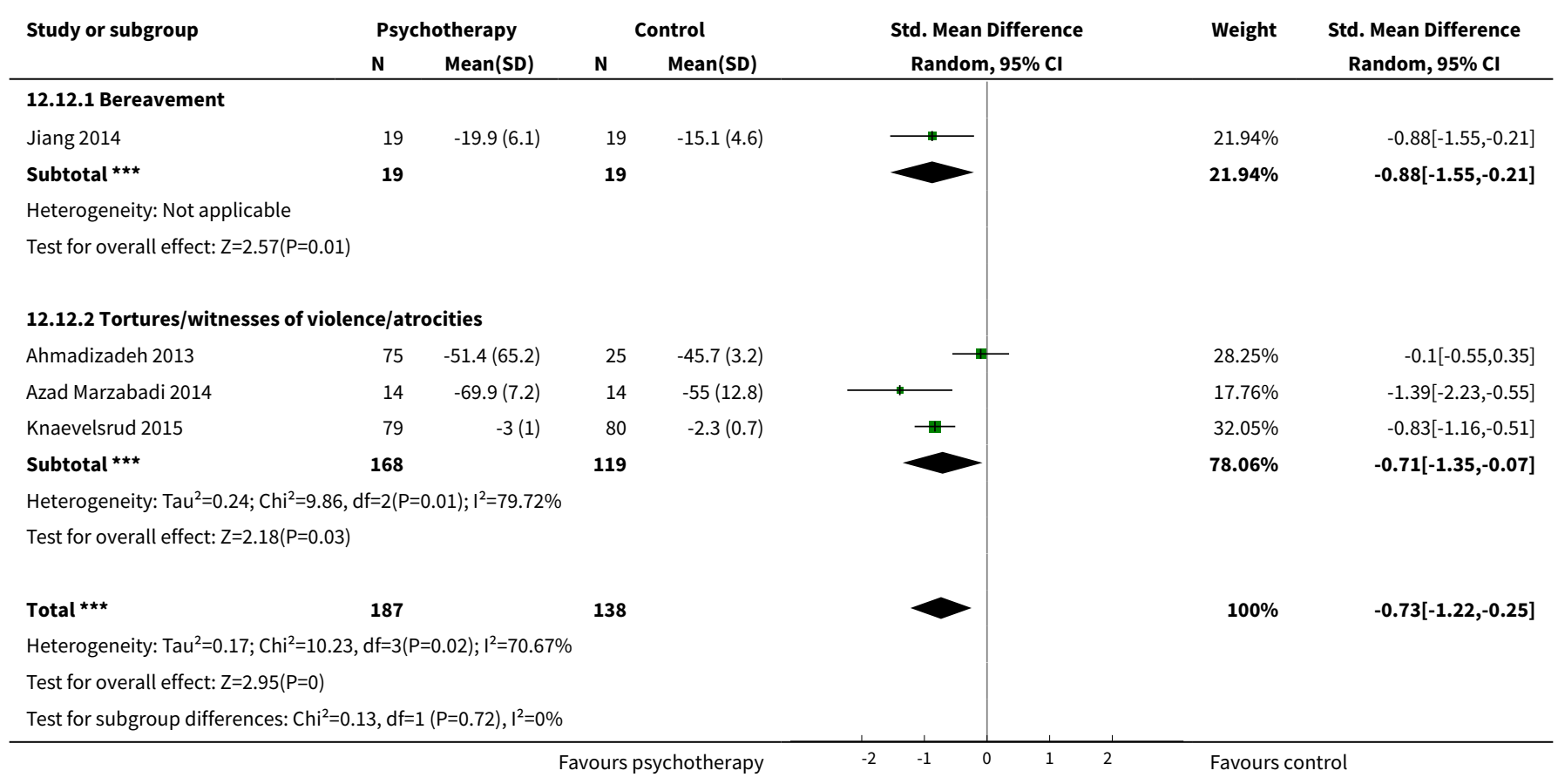

Analysis 12.13. Comparison 12 Subgroup analysis: type of traumatic events - adults, Outcome 13 Diagnosis of PTSD.

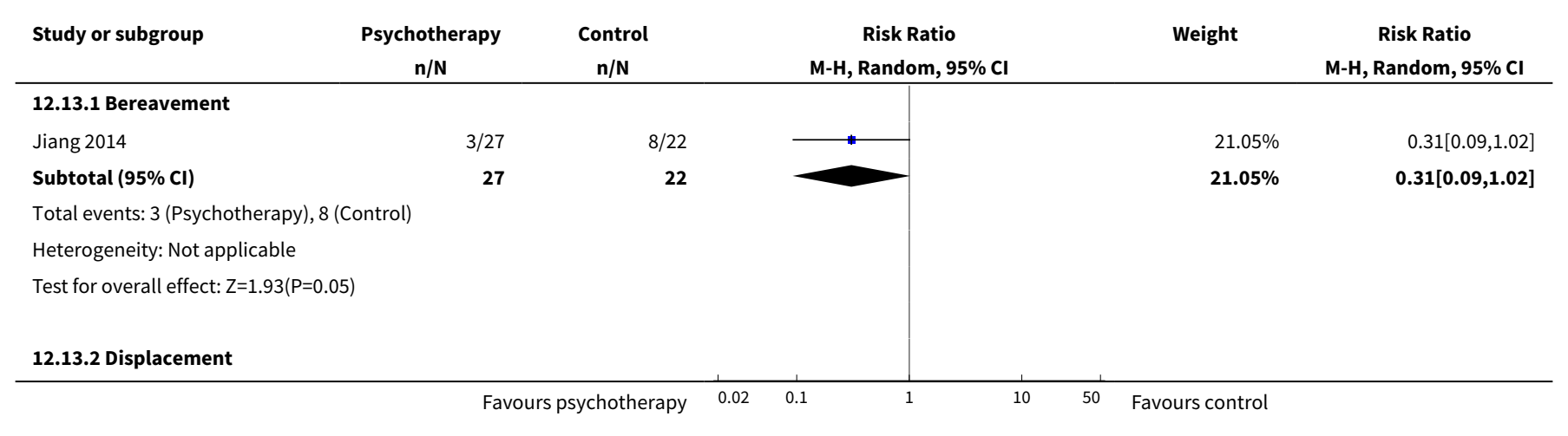

Psychological therapies for the treatment of mental disorders in low- and middle-income countries affected by humanitarian crises 


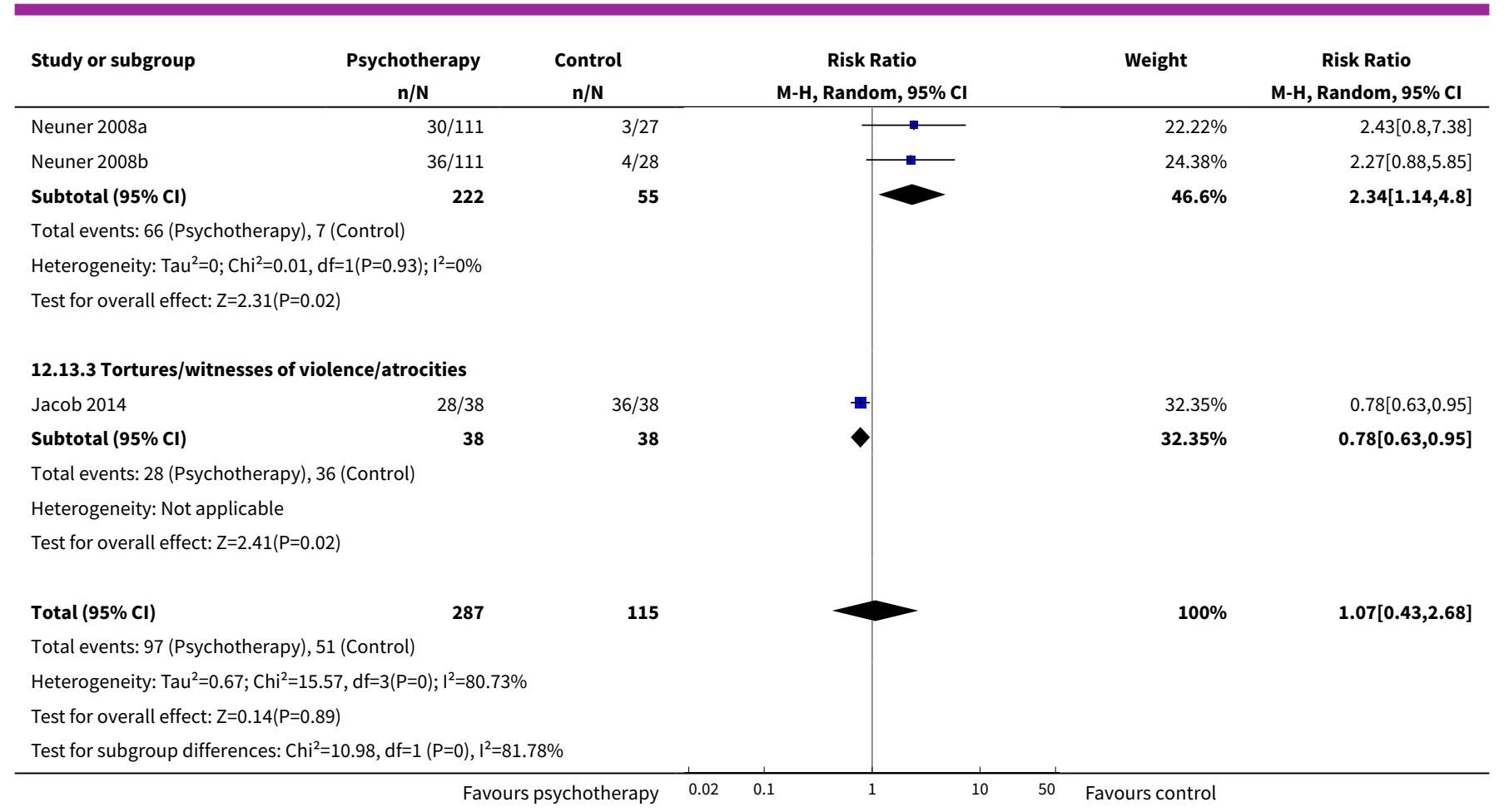

\section{Comparison 13. Subgroup analysis: type of humanitarian crisis - adults}

\begin{tabular}{|c|c|c|c|c|}
\hline Outcome or subgroup title & No. of studies & $\begin{array}{l}\text { No. of partici- } \\
\text { pants }\end{array}$ & Statistical method & Effect size \\
\hline $\begin{array}{l}1 \text { PTSD symptoms at end- } \\
\text { point }\end{array}$ & 15 & 1250 & $\begin{array}{l}\text { Std. Mean Difference (IV, Random, } \\
95 \% \mathrm{Cl})\end{array}$ & $-1.10[-1.39,-0.81]$ \\
\hline 1.1 War/armed conflict & 7 & 568 & $\begin{array}{l}\text { Std. Mean Difference (IV, Random, } \\
95 \% \mathrm{CI})\end{array}$ & $-1.06[-1.51,-0.61]$ \\
\hline $\begin{array}{l}1.2 \text { Natural hazards (geo- } \\
\text { physical: earthquake; vol- } \\
\text { canic eruptions) }\end{array}$ & 3 & 84 & $\begin{array}{l}\text { Std. Mean Difference (IV, Random, } \\
95 \% \mathrm{CI} \text { ) }\end{array}$ & $-2.31[-4.22,-0.39]$ \\
\hline 1.3 Communal violence & 2 & 419 & $\begin{array}{l}\text { Std. Mean Difference (IV, Random, } \\
95 \% \mathrm{Cl})\end{array}$ & $-1.01[-1.48,-0.54]$ \\
\hline 1.4 Food shortages & 1 & 28 & $\begin{array}{l}\text { Std. Mean Difference (IV, Random, } \\
95 \% \mathrm{CI} \text { ) }\end{array}$ & $-0.97[-1.77,-0.18]$ \\
\hline 1.5 Other & 2 & 151 & $\begin{array}{l}\text { Std. Mean Difference (IV, Random, } \\
95 \% \mathrm{Cl} \text { ) }\end{array}$ & $-0.64[-0.97,-0.31]$ \\
\hline $\begin{array}{l}2 \text { PTSD symptoms at } 1 \text { to } 4 \\
\text { months }\end{array}$ & 16 & 1568 & $\begin{array}{l}\text { Std. Mean Difference (IV, Random, } \\
95 \% \mathrm{Cl})\end{array}$ & $-0.47[-0.66,-0.29]$ \\
\hline 2.1 War/armed conflict & 7 & 958 & $\begin{array}{l}\text { Std. Mean Difference (IV, Random, } \\
95 \% \mathrm{CI})\end{array}$ & $-0.56[-0.88,-0.23]$ \\
\hline
\end{tabular}




\begin{tabular}{|c|c|c|c|c|}
\hline Outcome or subgroup title & No. of studies & $\begin{array}{l}\text { No. of partici- } \\
\text { pants }\end{array}$ & Statistical method & Effect size \\
\hline $\begin{array}{l}2.2 \text { Natural hazards (geo- } \\
\text { physical: earthquake; vol- } \\
\text { canic eruptions) }\end{array}$ & 5 & 180 & $\begin{array}{l}\text { Std. Mean Difference (IV, Random, } \\
95 \% \mathrm{Cl})\end{array}$ & $-0.52[-0.88,-0.17]$ \\
\hline 2.3 Communal violence & 1 & 281 & $\begin{array}{l}\text { Std. Mean Difference (IV, Random, } \\
95 \% \mathrm{Cl} \text { ) }\end{array}$ & $-0.37[-0.65,-0.09]$ \\
\hline 2.4 Food shortages & 1 & 28 & $\begin{array}{l}\text { Std. Mean Difference (IV, Random, } \\
95 \% \mathrm{Cl})\end{array}$ & $-0.62[-1.39,0.15]$ \\
\hline 2.5 Other & 2 & 121 & $\begin{array}{l}\text { Std. Mean Difference (IV, Random, } \\
95 \% \mathrm{Cl} \text { ) }\end{array}$ & $-0.19[-0.55,0.17]$ \\
\hline $\begin{array}{l}3 \text { PTSD symptoms } \geq 6 \\
\text { months }\end{array}$ & 5 & & $\begin{array}{l}\text { Std. Mean Difference (IV, Random, } \\
95 \% \mathrm{CI})\end{array}$ & Subtotals only \\
\hline 3.1 War/armed conflict & 5 & 400 & $\begin{array}{l}\text { Std. Mean Difference (IV, Random, } \\
95 \% \mathrm{CI} \text { ) }\end{array}$ & $-0.37[-0.61,-0.14]$ \\
\hline $\begin{array}{l}4 \text { Anxiety symptoms at end- } \\
\text { point }\end{array}$ & 4 & 535 & $\begin{array}{l}\text { Std. Mean Difference (IV, Random, } \\
95 \% \mathrm{Cl} \text { ) }\end{array}$ & $-0.76[-1.10,-0.42]$ \\
\hline $\begin{array}{l}\text { 4.1 Natural hazards (geo- } \\
\text { physical: earthquake; vol- } \\
\text { canic eruptions) }\end{array}$ & 2 & 52 & $\begin{array}{l}\text { Std. Mean Difference (IV, Random, } \\
95 \% \mathrm{CI})\end{array}$ & $-1.30[-1.92,-0.67]$ \\
\hline 4.2 Communal violence & 1 & 274 & $\begin{array}{l}\text { Std. Mean Difference (IV, Random, } \\
95 \% \mathrm{Cl} \text { ) }\end{array}$ & $-0.48[-0.72,-0.24]$ \\
\hline 4.3 War/armed conflict & 1 & 209 & $\begin{array}{l}\text { Std. Mean Difference (IV, Random, } \\
95 \% \mathrm{Cl} \text { ) }\end{array}$ & $-0.74[-1.02,-0.45]$ \\
\hline $\begin{array}{l}5 \text { Anxiety symptoms at } 1 \text { to } \\
4 \text { months }\end{array}$ & 6 & 969 & $\begin{array}{l}\text { Std. Mean Difference (IV, Random, } \\
95 \% \mathrm{CI})\end{array}$ & $-0.42[-0.70,-0.14]$ \\
\hline 5.1 War/armed conflict & 3 & 636 & $\begin{array}{l}\text { Std. Mean Difference (IV, Random, } \\
95 \% \mathrm{Cl} \text { ) }\end{array}$ & $-0.69[-1.03,-0.36]$ \\
\hline $\begin{array}{l}5.2 \text { Natural hazards (geo- } \\
\text { physical: earthquake; vol- } \\
\text { canic eruptions) }\end{array}$ & 2 & 52 & $\begin{array}{l}\text { Std. Mean Difference (IV, Random, } \\
95 \% \mathrm{Cl} \text { ) }\end{array}$ & $0.06[-0.50,0.63]$ \\
\hline 5.3 Communal violence & 1 & 281 & $\begin{array}{l}\text { Std. Mean Difference (IV, Random, } \\
95 \% \mathrm{CI})\end{array}$ & $-0.21[-0.49,0.07]$ \\
\hline $\begin{array}{l}6 \text { Depressive symptoms at } \\
\text { endpoint }\end{array}$ & 14 & 1378 & $\begin{array}{l}\text { Std. Mean Difference (IV, Random, } \\
95 \% \mathrm{CI})\end{array}$ & $-0.89[-1.06,-0.72]$ \\
\hline 6.1 War/armed conflict & 7 & 841 & $\begin{array}{l}\text { Std. Mean Difference (IV, Random, } \\
95 \% \mathrm{Cl})\end{array}$ & $-0.96[-1.20,-0.73]$ \\
\hline $\begin{array}{l}6.2 \text { Natural hazards (geo- } \\
\text { physical: earthquake; vol- } \\
\text { canic eruptions) }\end{array}$ & 3 & 84 & $\begin{array}{l}\text { Std. Mean Difference (IV, Random, } \\
95 \% \mathrm{Cl} \text { ) }\end{array}$ & $-1.10[-1.58,-0.63]$ \\
\hline
\end{tabular}




\begin{tabular}{|c|c|c|c|c|}
\hline Outcome or subgroup title & No. of studies & $\begin{array}{l}\text { No. of partici- } \\
\text { pants }\end{array}$ & Statistical method & Effect size \\
\hline 6.3 Communal violence & 1 & 274 & $\begin{array}{l}\text { Std. Mean Difference (IV, Random, } \\
95 \% \mathrm{CI})\end{array}$ & $-0.90[-1.15,-0.65]$ \\
\hline 6.4 Food shortages & 1 & 28 & $\begin{array}{l}\text { Std. Mean Difference (IV, Random, } \\
95 \% \mathrm{Cl})\end{array}$ & $-0.80[-1.58,-0.01]$ \\
\hline 6.5 Other & 2 & 151 & $\begin{array}{l}\text { Std. Mean Difference (IV, Random, } \\
95 \% \mathrm{Cl})\end{array}$ & $-0.53[-1.20,0.14]$ \\
\hline $\begin{array}{l}7 \text { Depressive symptoms at } 1 \\
\text { to } 4 \text { months }\end{array}$ & 14 & 1093 & $\begin{array}{l}\text { Std. Mean Difference (IV, Random, } \\
95 \% \mathrm{CI})\end{array}$ & $-0.60[-1.05,-0.15]$ \\
\hline 7.1 War/armed conflict & 4 & 608 & $\begin{array}{l}\text { Std. Mean Difference (IV, Random, } \\
95 \% \mathrm{Cl})\end{array}$ & $-0.57[-1.04,-0.10]$ \\
\hline $\begin{array}{l}7.2 \text { Natural hazards (geo- } \\
\text { physical: earthquake; vol- } \\
\text { canic eruptions) }\end{array}$ & 6 & 202 & $\begin{array}{l}\text { Std. Mean Difference (IV, Random, } \\
95 \% \mathrm{Cl})\end{array}$ & $-0.30[-0.71,0.11]$ \\
\hline 7.3 Communal violence & 1 & 134 & $\begin{array}{l}\text { Std. Mean Difference (IV, Random, } \\
95 \% \mathrm{Cl})\end{array}$ & $-3.56[-4.15,-2.98]$ \\
\hline 7.4 Food shortages & 1 & 28 & $\begin{array}{l}\text { Std. Mean Difference (IV, Random, } \\
95 \% \mathrm{CI})\end{array}$ & $-0.37[-1.13,0.38]$ \\
\hline 7.5 Other & 2 & 121 & $\begin{array}{l}\text { Std. Mean Difference (IV, Random, } \\
95 \% \mathrm{Cl})\end{array}$ & $-0.13[-0.49,0.23]$ \\
\hline $\begin{array}{l}8 \text { Depressive symptoms } \geq 6 \\
\text { months }\end{array}$ & 2 & & $\begin{array}{l}\text { Std. Mean Difference (IV, Random, } \\
95 \% \mathrm{Cl})\end{array}$ & Subtotals only \\
\hline 8.1 War/armed conflict & 2 & 242 & $\begin{array}{l}\text { Std. Mean Difference (IV, Random, } \\
95 \% \mathrm{CI})\end{array}$ & $-0.20[-0.49,0.09]$ \\
\hline 9 Dropout & 25 & 2354 & Risk Ratio (M-H, Random, 95\% Cl) & $0.93[0.78,1.11]$ \\
\hline 9.1 War/armed conflict & 16 & 1529 & Risk Ratio (M-H, Random, 95\% Cl) & $0.93[0.74,1.15]$ \\
\hline $\begin{array}{l}9.2 \text { Natural hazards (geo- } \\
\text { physical: earthquake; vol- } \\
\text { canic eruptions) }\end{array}$ & 4 & 106 & Risk Ratio (M-H, Random, 95\% Cl) & $9.94[0.61,160.94]$ \\
\hline 9.3 Communal violence & 2 & 494 & Risk Ratio (M-H, Random, 95\% Cl) & $1.01[0.54,1.91]$ \\
\hline 9.4 Food shortages & 1 & 28 & Risk Ratio (M-H, Random, 95\% Cl) & $0.0[0.0,0.0]$ \\
\hline 9.5 Other & 2 & 197 & Risk Ratio (M-H, Random, 95\% Cl) & $0.56[0.12,2.67]$ \\
\hline $\begin{array}{l}10 \text { Functional impairment } \\
\text { at endpoint }\end{array}$ & 5 & 686 & $\begin{array}{l}\text { Std. Mean Difference (IV, Random, } \\
95 \% \mathrm{Cl})\end{array}$ & $-0.55[-0.80,-0.30]$ \\
\hline 10.1 Communal violence & 1 & 274 & $\begin{array}{l}\text { Std. Mean Difference (IV, Random, } \\
95 \% \mathrm{Cl})\end{array}$ & $-0.56[-0.80,-0.32]$ \\
\hline
\end{tabular}




\begin{tabular}{|c|c|c|c|c|}
\hline Outcome or subgroup title & No. of studies & $\begin{array}{l}\text { No. of partici- } \\
\text { pants }\end{array}$ & Statistical method & Effect size \\
\hline 10.2 Other & 2 & 151 & $\begin{array}{l}\text { Std. Mean Difference (IV, Random, } \\
95 \% \mathrm{CI})\end{array}$ & $-0.45[-1.30,0.40]$ \\
\hline 10.3 War/armed conflict & 2 & 261 & $\begin{array}{l}\text { Std. Mean Difference (IV, Random, } \\
95 \% \mathrm{Cl})\end{array}$ & $-0.68[-0.93,-0.43]$ \\
\hline $\begin{array}{l}11 \text { Functional impairment } \\
\text { at } 1 \text { to } 4 \text { months }\end{array}$ & 7 & 1061 & $\begin{array}{l}\text { Std. Mean Difference (IV, Random, } \\
95 \% \mathrm{CI})\end{array}$ & $-0.36[-0.55,-0.16]$ \\
\hline 11.1 War/armed conflict & 4 & 659 & $\begin{array}{l}\text { Std. Mean Difference (IV, Random, } \\
95 \% \mathrm{CI})\end{array}$ & $-0.44[-0.75,-0.14]$ \\
\hline 11.2 Communal violence & 1 & 281 & $\begin{array}{l}\text { Std. Mean Difference (IV, Random, } \\
95 \% \mathrm{CI})\end{array}$ & $-0.36[-0.63,-0.08]$ \\
\hline 11.3 Other & 2 & 121 & $\begin{array}{l}\text { Std. Mean Difference (IV, Random, } \\
95 \% \mathrm{Cl} \text { ) }\end{array}$ & $-0.08[-0.43,0.28]$ \\
\hline $\begin{array}{l}12 \text { Quality of life at end- } \\
\text { point }\end{array}$ & 4 & 325 & $\begin{array}{l}\text { Std. Mean Difference (IV, Random, } \\
95 \% \mathrm{CI} \text { ) }\end{array}$ & $-0.73[-1.22,-0.25]$ \\
\hline 12.1 War/armed conflict & 3 & 287 & $\begin{array}{l}\text { Std. Mean Difference (IV, Random, } \\
95 \% \mathrm{Cl})\end{array}$ & $-0.71[-1.35,-0.07]$ \\
\hline $\begin{array}{l}12.2 \text { Natural hazards (geo- } \\
\text { physical: earthquake; vol- } \\
\text { canic eruptions) }\end{array}$ & 1 & 38 & $\begin{array}{l}\text { Std. Mean Difference (IV, Random, } \\
95 \% \mathrm{CI})\end{array}$ & $-0.88[-1.55,-0.21]$ \\
\hline 13 Diagnosis of PTSD & 4 & 299 & Risk Ratio (M-H, Random, 95\% Cl) & $0.74[0.46,1.17]$ \\
\hline 13.1 War/armed conflict & 2 & 214 & Risk Ratio (M-H, Random, 95\% Cl) & $1.28[0.28,5.77]$ \\
\hline $\begin{array}{l}13.2 \text { Natural hazards (geo- } \\
\text { physical: earthquake; vol- } \\
\text { canic eruptions) }\end{array}$ & 2 & 85 & Risk Ratio (M-H, Random, 95\% Cl) & $0.52[0.29,0.92]$ \\
\hline
\end{tabular}

Analysis 13.1. Comparison 13 Subgroup analysis: type of humanitarian crisis - adults, Outcome 1 PTSD symptoms at endpoint.

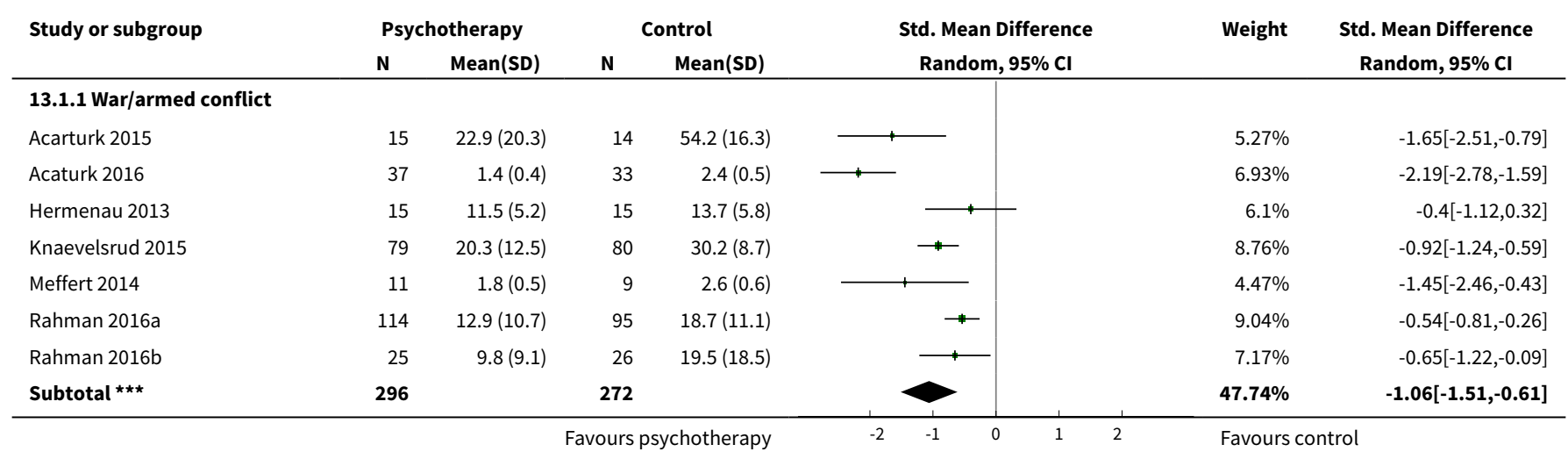




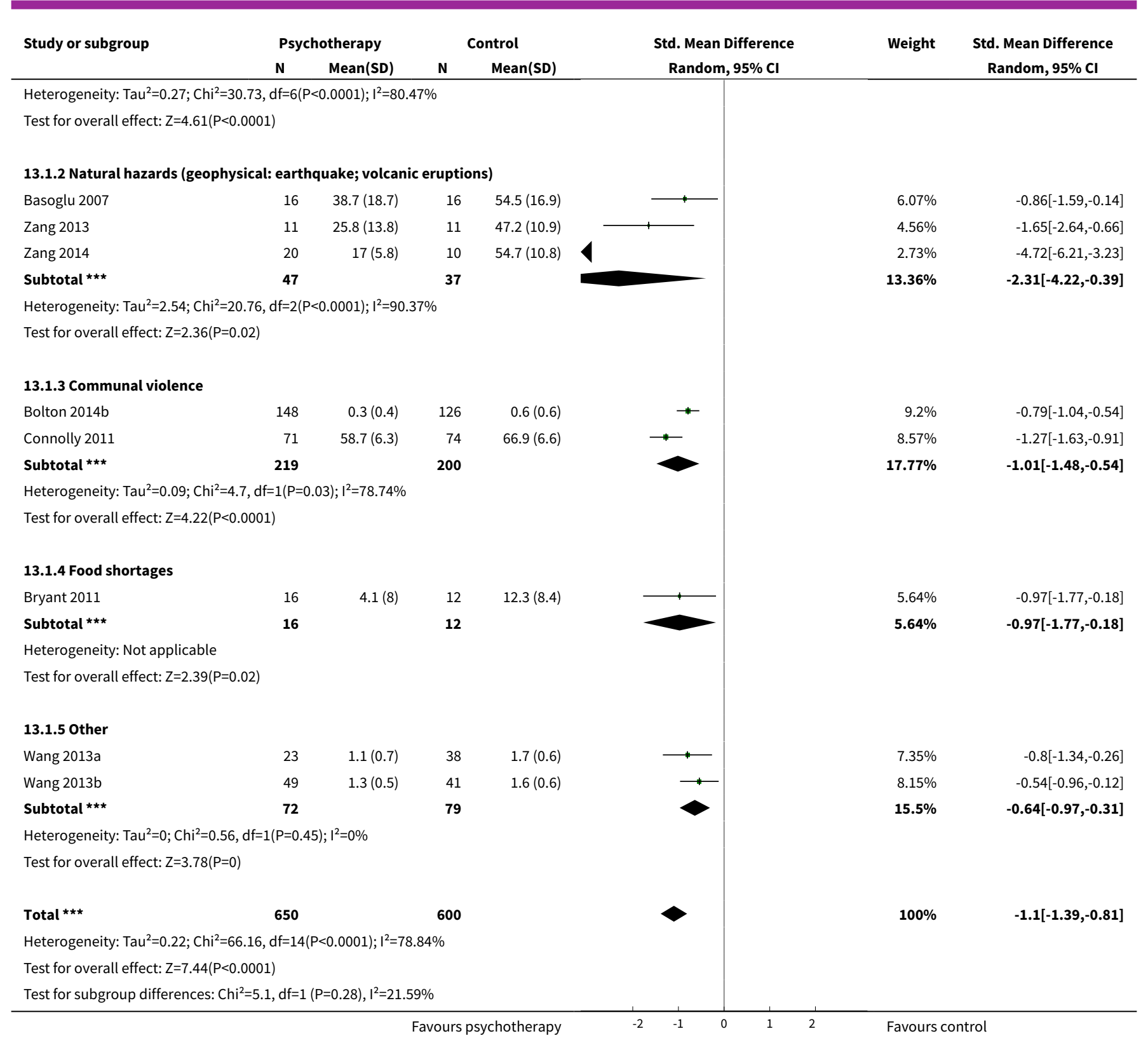

\section{Analysis 13.2. Comparison 13 Subgroup analysis: type of humanitarian crisis - adults, Outcome 2 PTSD symptoms at 1 to 4 months.}

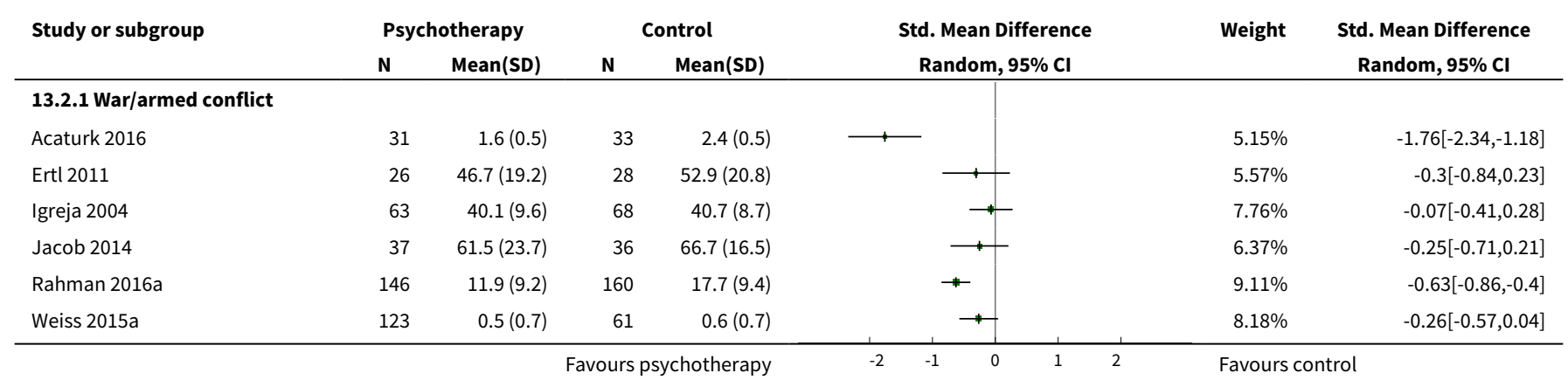

Psychological therapies for the treatment of mental disorders in low- and middle-income countries affected by humanitarian crises 


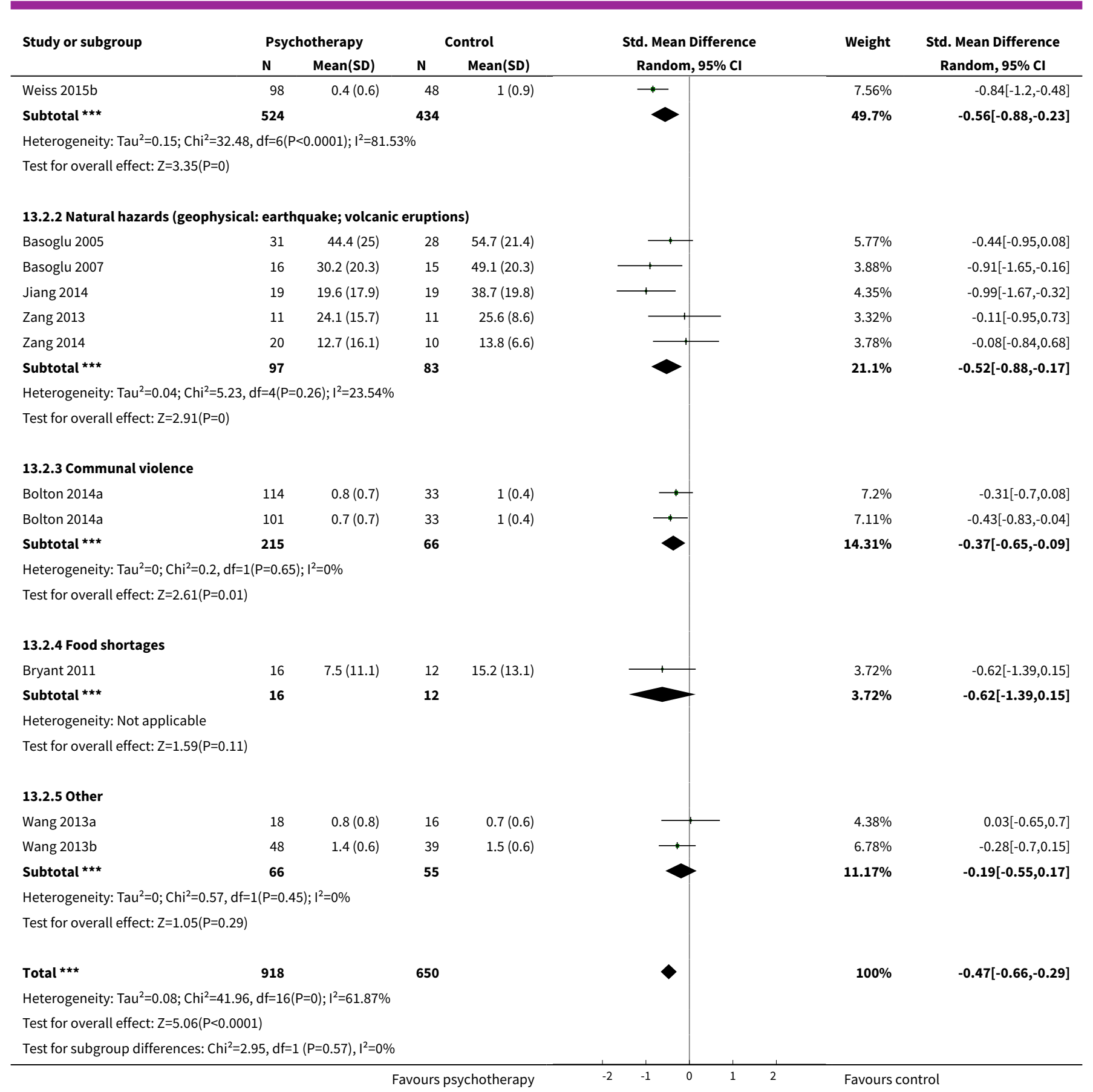

Analysis 13.3. Comparison 13 Subgroup analysis: type of humanitarian crisis - adults, Outcome 3 PTSD symptoms $\geq 6$ months.

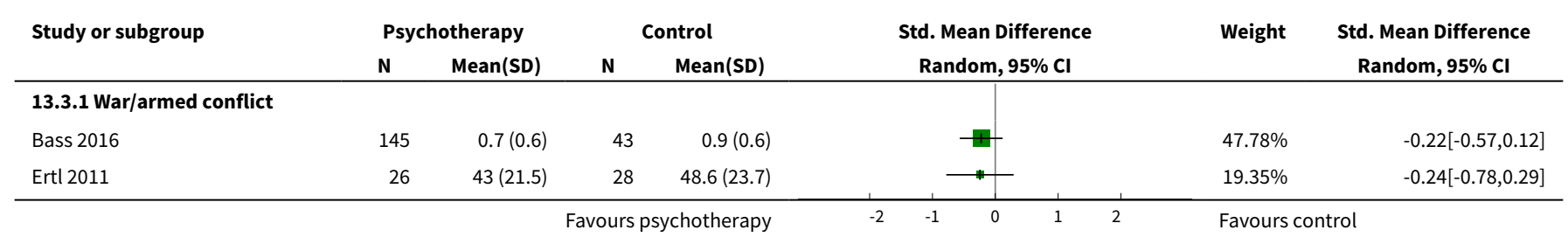

Psychological therapies for the treatment of mental disorders in low- and middle-income countries affected by humanitarian crises 


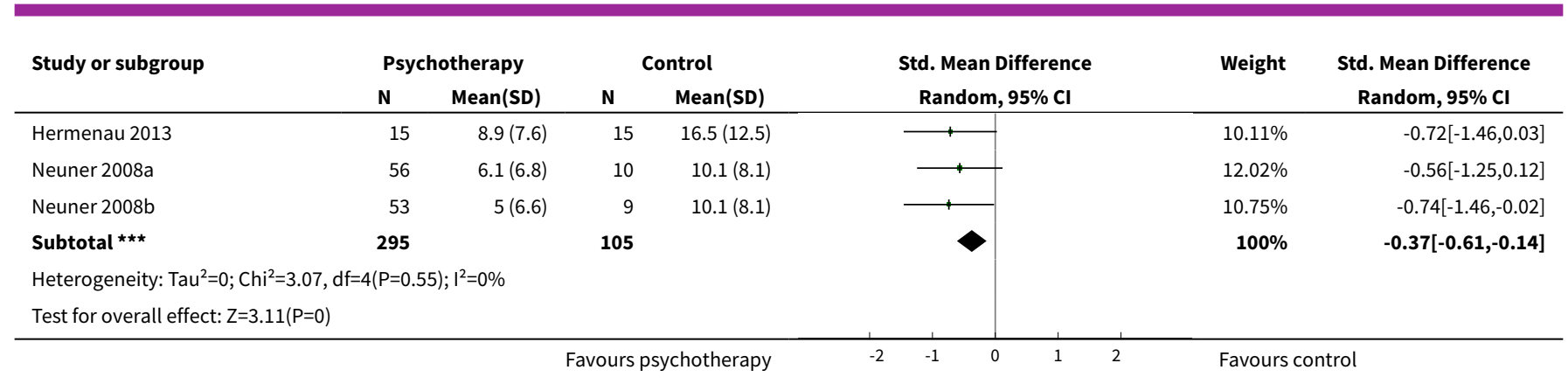

Analysis 13.4. Comparison 13 Subgroup analysis: type of humanitarian crisis - adults, Outcome 4 Anxiety symptoms at endpoint.

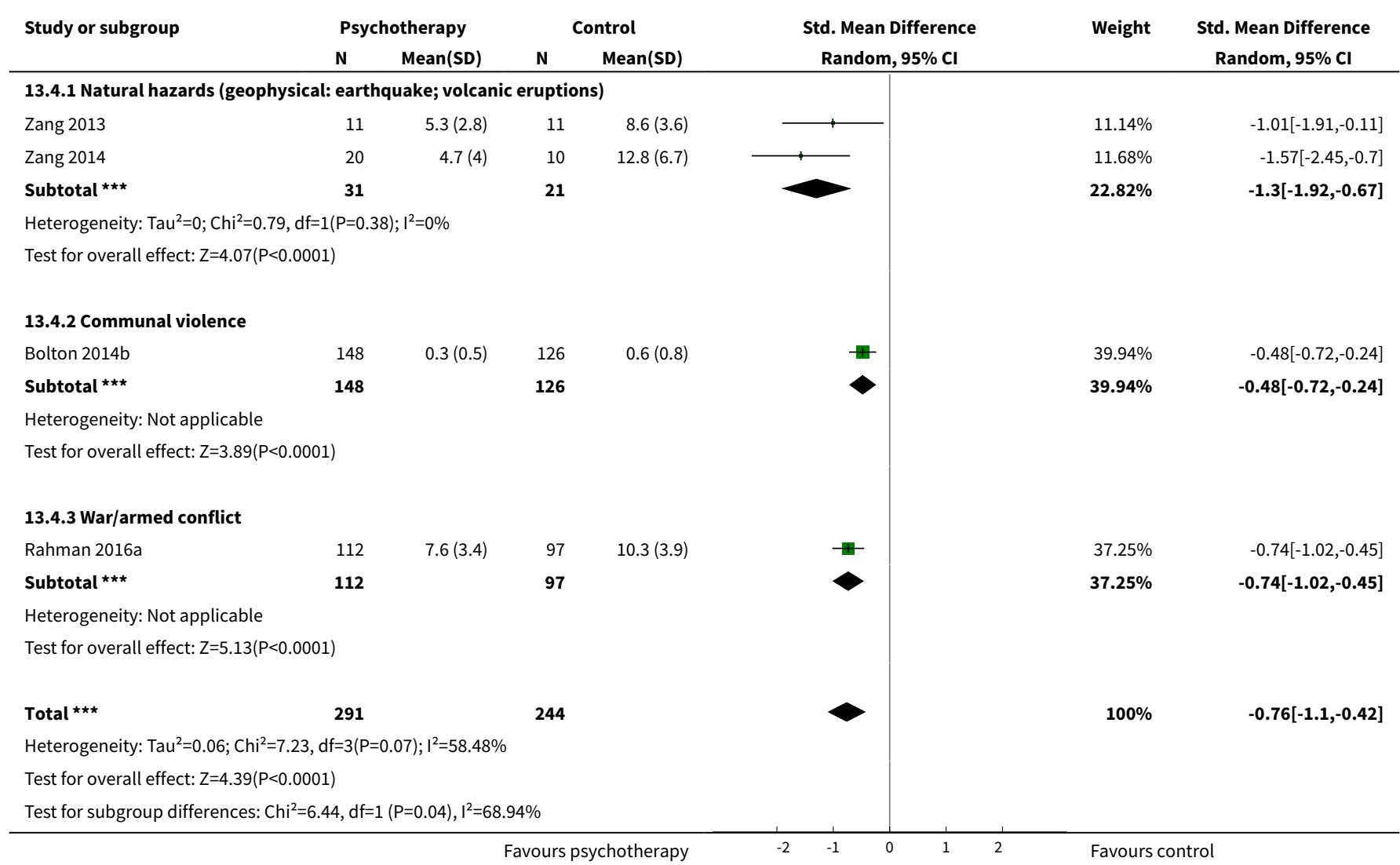

Analysis 13.5. Comparison 13 Subgroup analysis: type of humanitarian crisis - adults, Outcome 5 Anxiety symptoms at 1 to 4 months.

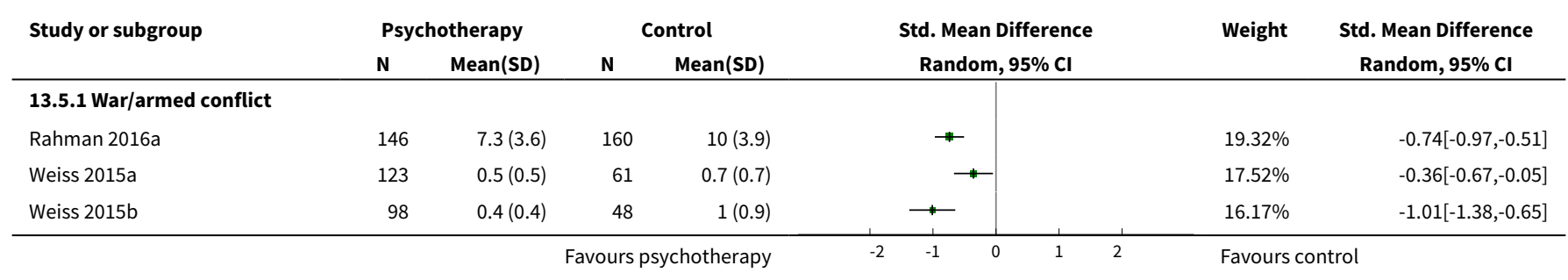




\begin{tabular}{|c|c|c|c|c|c|c|c|}
\hline \multirow{3}{*}{$\begin{array}{l}\text { Study or subgroup } \\
\text { Subtotal } \star \star \star\end{array}$} & \multicolumn{2}{|c|}{ Psychotherapy } & \multicolumn{2}{|c|}{ Control } & \multirow{2}{*}{$\begin{array}{c}\text { Std. Mean Difference } \\
\text { Random, } 95 \% \mathrm{Cl}\end{array}$} & \multirow[t]{2}{*}{ Weight } & \multirow{2}{*}{$\begin{array}{c}\text { Std. Mean Difference } \\
\text { Random, } 95 \% \mathrm{Cl}\end{array}$} \\
\hline & $\mathbf{N}$ & Mean(SD) & $\mathbf{N}$ & Mean(SD) & & & \\
\hline & 367 & & 269 & & & $53.02 \%$ & $-0.69[-1.03,-0.36]$ \\
\hline \multicolumn{8}{|c|}{ Heterogeneity: $\mathrm{Tau}^{2}=0.06 ; \mathrm{Chi}^{2}=7.59, \mathrm{df}=2(\mathrm{P}=0.02) ; \mathrm{I}^{2}=73.63 \%$} \\
\hline \multicolumn{8}{|c|}{ Test for overall effect: $Z=4.05(P<0.0001)$} \\
\hline \multicolumn{8}{|c|}{ 13.5.2 Natural hazards (geophysical: earthquake; volcanic eruptions) } \\
\hline Zang 2013 & 11 & $5.5(3)$ & 11 & $4.8(2.5)$ & + & $7.44 \%$ & $0.22[-0.62,1.06]$ \\
\hline Zang 2014 & 20 & $3.4(3.2)$ & 10 & $3.6(2.2)$ & & $8.46 \%$ & $-0.07[-0.83,0.69]$ \\
\hline Subtotal ${ }^{\star \star \star}$ & 31 & & 21 & & & $15.9 \%$ & $0.06[-0.5,0.63]$ \\
\hline \multicolumn{8}{|c|}{ Heterogeneity: $\operatorname{Tau}^{2}=0 ; \mathrm{Chi}^{2}=0.24, \mathrm{df}=1(\mathrm{P}=0.62) ; \mathrm{I}^{2}=0 \%$} \\
\hline \multicolumn{8}{|c|}{ Test for overall effect: $\mathrm{Z}=0.22(\mathrm{P}=0.83)$} \\
\hline \multicolumn{8}{|c|}{ 13.5.3 Communal violence } \\
\hline Bolton 2014a & 114 & $0.8(1.2)$ & 33 & $0.9(0.5)$ & $\rightarrow$ & $15.61 \%$ & $-0.18[-0.57,0.21]$ \\
\hline Bolton 2014a & 101 & $0.8(1)$ & 33 & $1(0.5)$ & $\rightarrow$ & $15.47 \%$ & $-0.24[-0.64,0.15]$ \\
\hline Subtotal $\star \star \star$ & 215 & & 66 & & & $31.08 \%$ & $-0.21[-0.49,0.07]$ \\
\hline \multicolumn{8}{|c|}{ Heterogeneity: $\mathrm{Tau}^{2}=0 ; \mathrm{Chi}^{2}=0.05, \mathrm{df}=1(\mathrm{P}=0.82) ; \mathrm{I}^{2}=0 \%$} \\
\hline \multicolumn{8}{|c|}{ Test for overall effect: $Z=1.49(P=0.14)$} \\
\hline 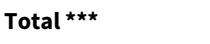 & 613 & & 356 & & & $100 \%$ & $-0.42[-0.7,-0.14]$ \\
\hline \multicolumn{8}{|c|}{ Heterogeneity: $\operatorname{Tau}^{2}=0.09 ; \mathrm{Chi}^{2}=20.63, \mathrm{df}=6(\mathrm{P}=0) ; \mathrm{I}^{2}=70.91 \%$} \\
\hline \multicolumn{8}{|c|}{ Test for overall effect: $Z=2.96(P=0)$} \\
\hline Test for subgroup dif & $5, \mathrm{df}=$ & $P=0.03), I^{2}=71$ & & & & & \\
\hline
\end{tabular}

\section{Analysis 13.6. Comparison 13 Subgroup analysis: type of humanitarian crisis - adults, Outcome 6 Depressive symptoms at endpoint.}

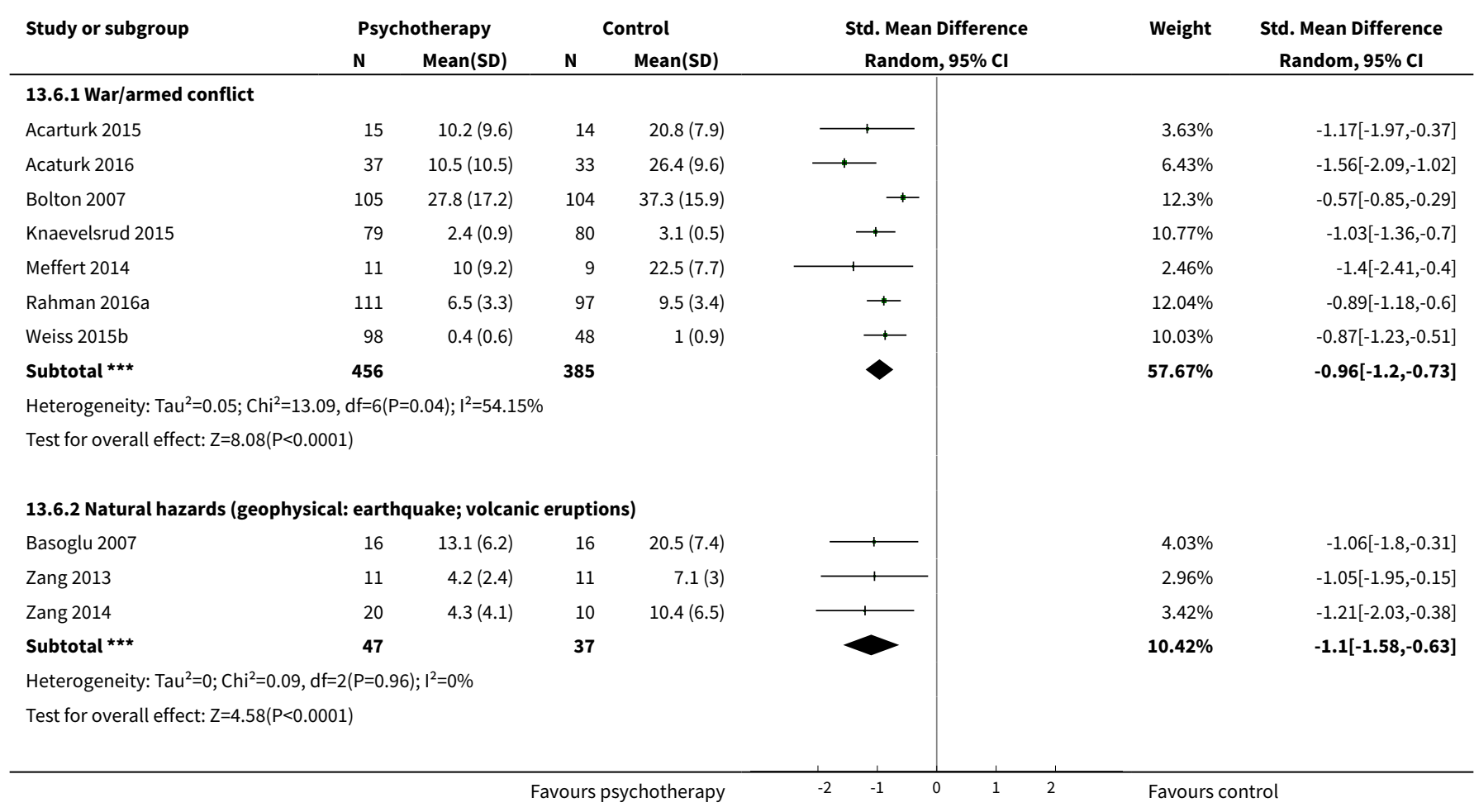

Psychological therapies for the treatment of mental disorders in low- and middle-income countries affected by humanitarian crises 


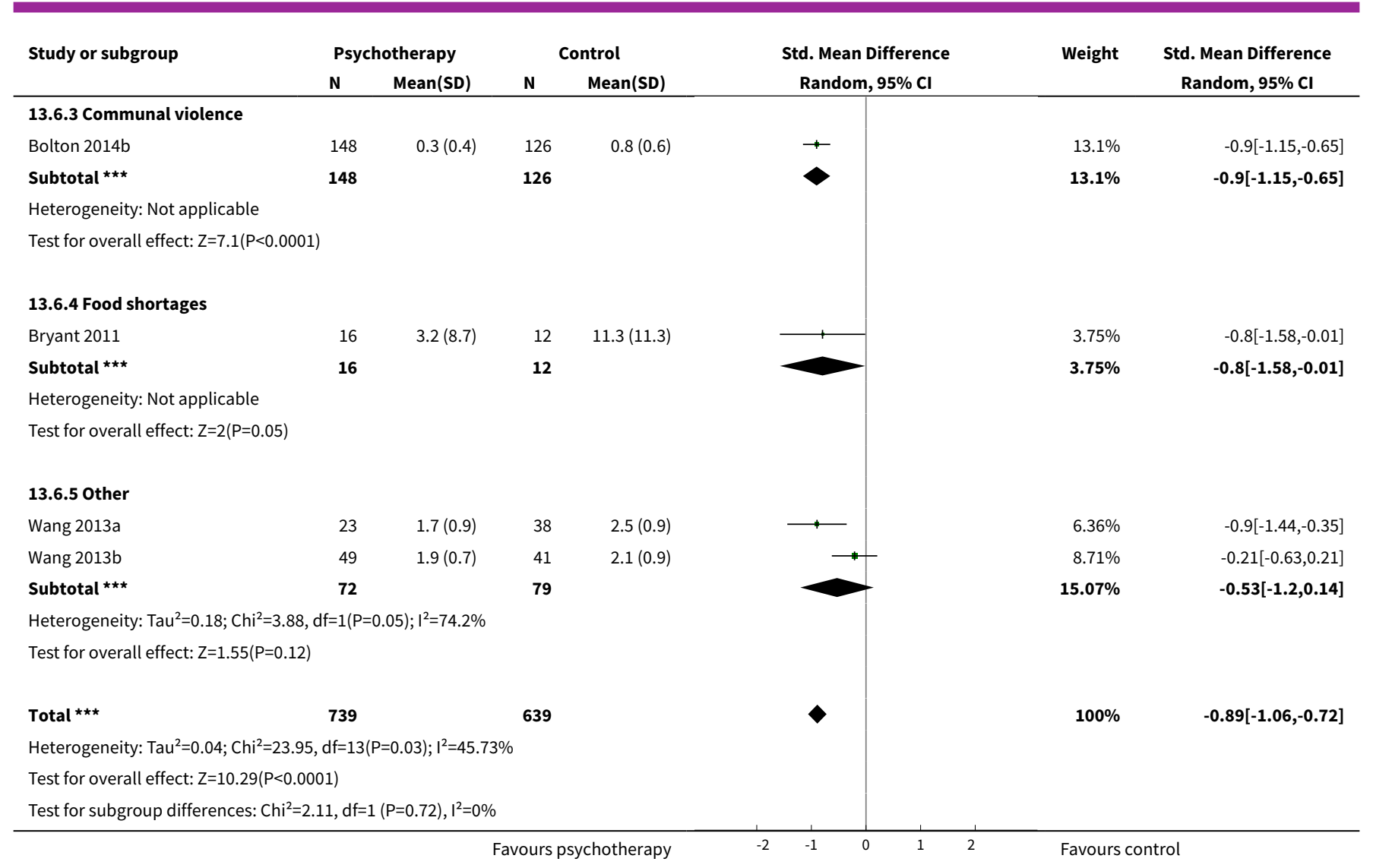

\section{Analysis 13.7. Comparison 13 Subgroup analysis: type of humanitarian crisis - adults, Outcome 7 Depressive symptoms at 1 to 4 months.}

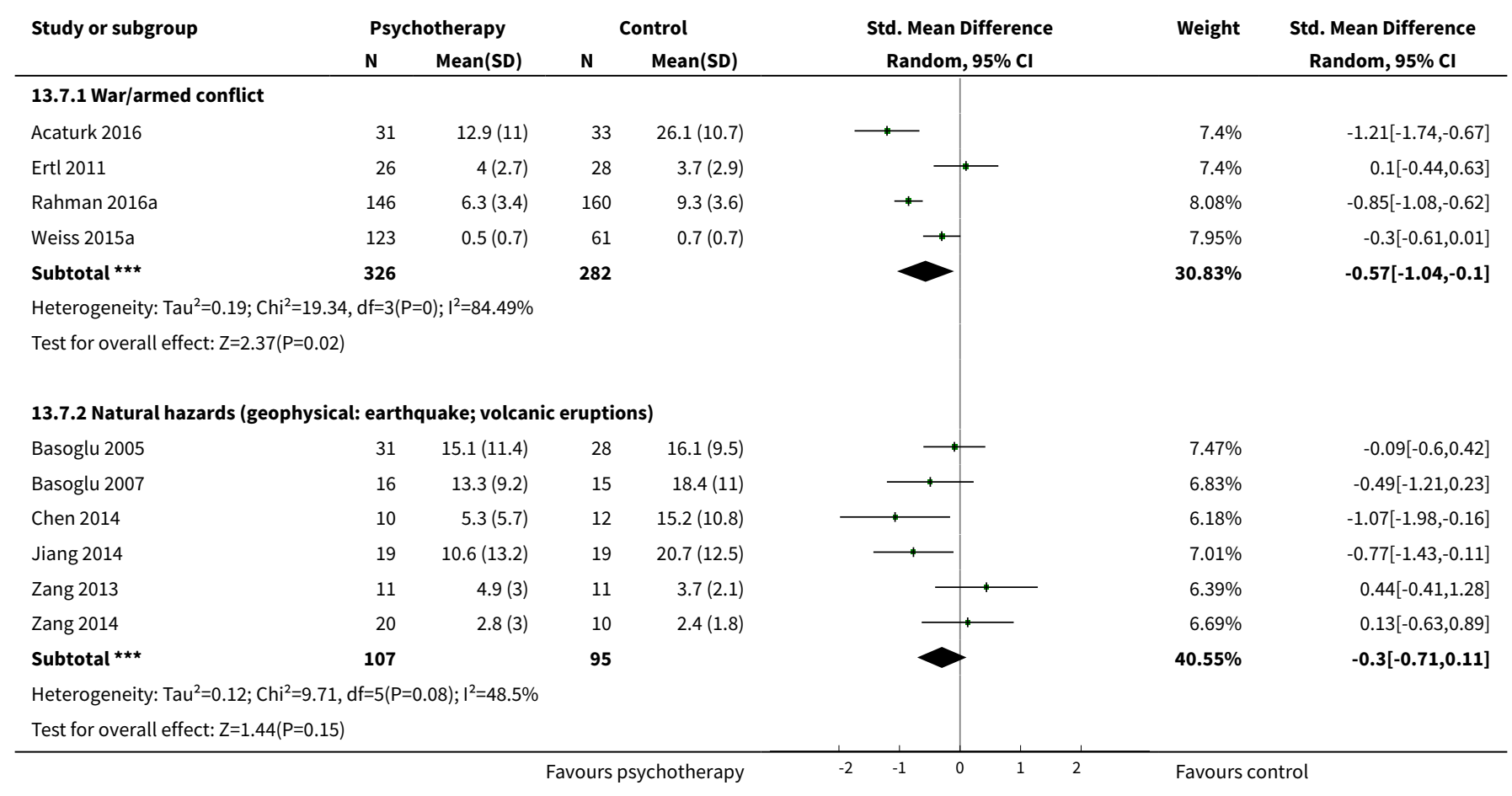

Psychological therapies for the treatment of mental disorders in low- and middle-income countries affected by humanitarian crises 


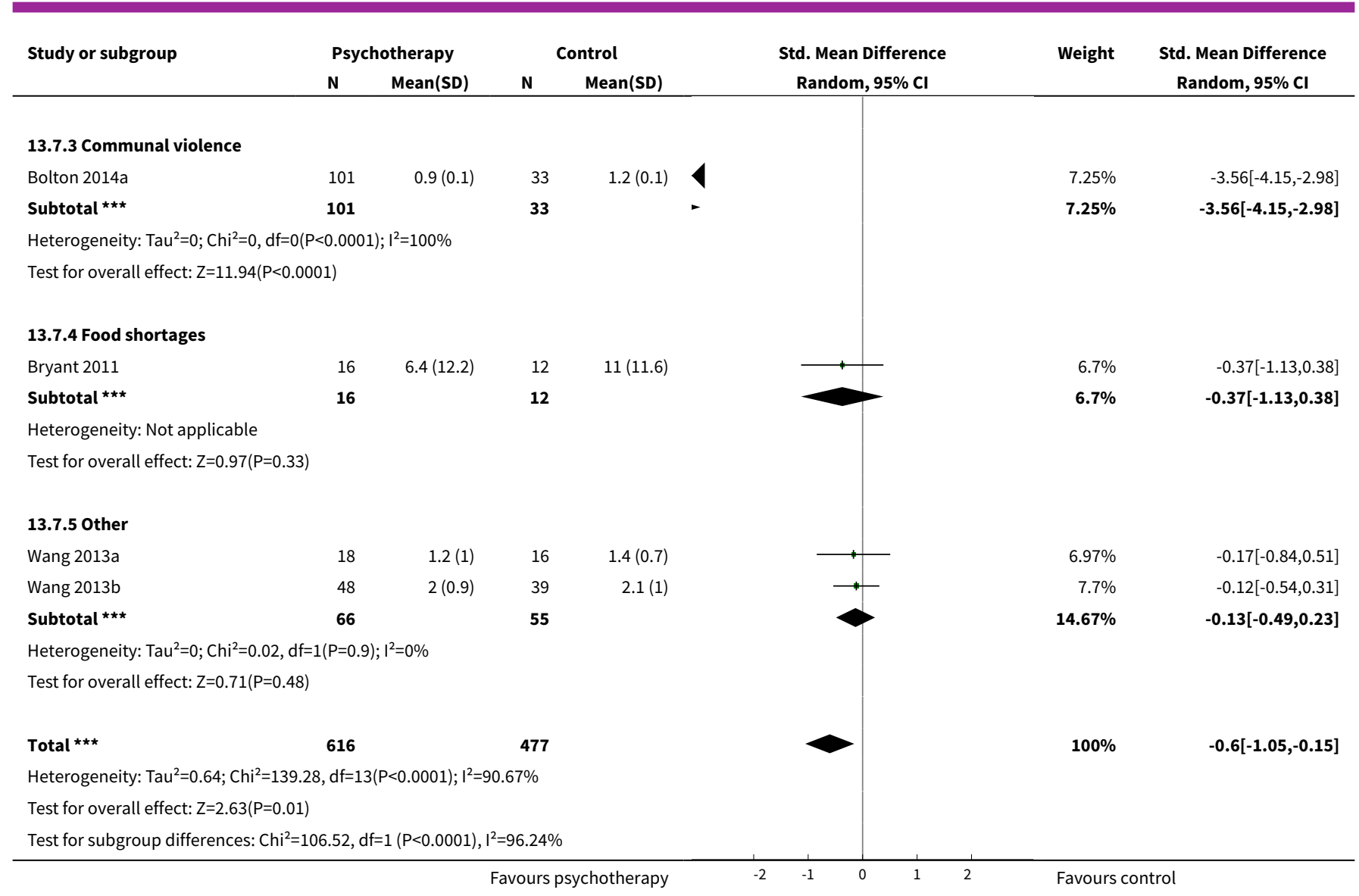

Analysis 13.8. Comparison 13 Subgroup analysis: type of humanitarian crisis - adults, Outcome 8 Depressive symptoms $\geq 6$ months.

\begin{tabular}{|c|c|c|c|c|c|c|c|}
\hline \multirow[t]{2}{*}{ Study or subgroup } & \multicolumn{2}{|c|}{ Psychotherapy } & \multicolumn{2}{|c|}{ Control } & \multirow{2}{*}{$\begin{array}{c}\text { Std. Mean Difference } \\
\text { Random, } 95 \% \mathrm{Cl}\end{array}$} & \multirow[t]{2}{*}{ Weight } & \multirow{2}{*}{$\begin{array}{c}\text { Std. Mean Difference } \\
\text { Random, } 95 \% \mathrm{Cl}\end{array}$} \\
\hline & $\mathbf{N}$ & Mean(SD) & $\mathbf{N}$ & Mean(SD) & & & \\
\hline \multicolumn{8}{|c|}{ 13.8.1 War/armed conflict } \\
\hline Bass 2016 & 145 & $0.8(0.7)$ & 43 & $1(0.8)$ & & $70.97 \%$ & $-0.26[-0.61,0.08]$ \\
\hline Ertl 2011 & 26 & $3.1(3)$ & 28 & $3.2(3)$ & 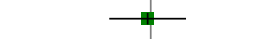 & $29.03 \%$ & $-0.04[-0.58,0.49]$ \\
\hline 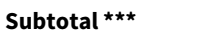 & 171 & & 71 & & & $100 \%$ & $-0.2[-0.49,0.09]$ \\
\hline \multicolumn{8}{|c|}{ Heterogeneity: $\operatorname{Tau}^{2}=0 ; \mathrm{Chi}^{2}=0.47, \mathrm{df}=1(\mathrm{P}=0.49) ; \mathrm{I}^{2}=0 \%$} \\
\hline
\end{tabular}

Analysis 13.9. Comparison 13 Subgroup analysis: type of humanitarian crisis - adults, Outcome 9 Dropout.

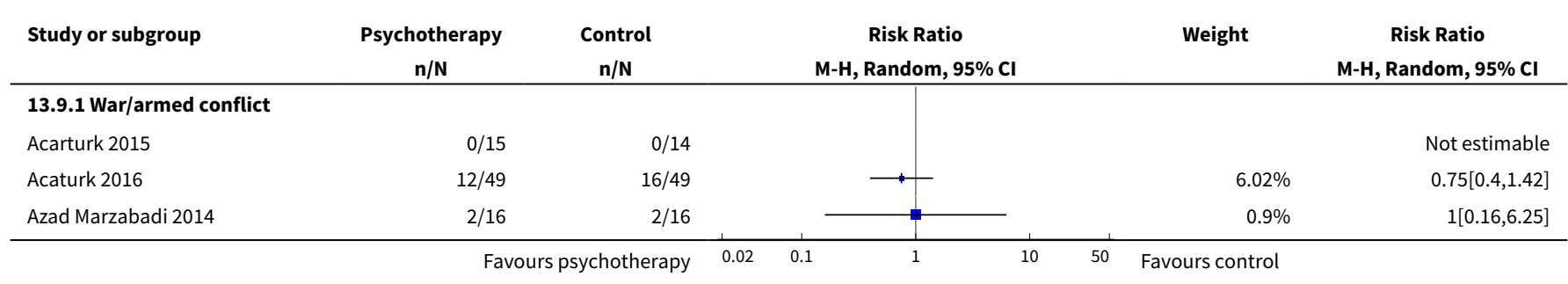

Psychological therapies for the treatment of mental disorders in low- and middle-income countries affected by humanitarian crises 




Psychological therapies for the treatment of mental disorders in low- and middle-income countries affected by humanitarian crises 


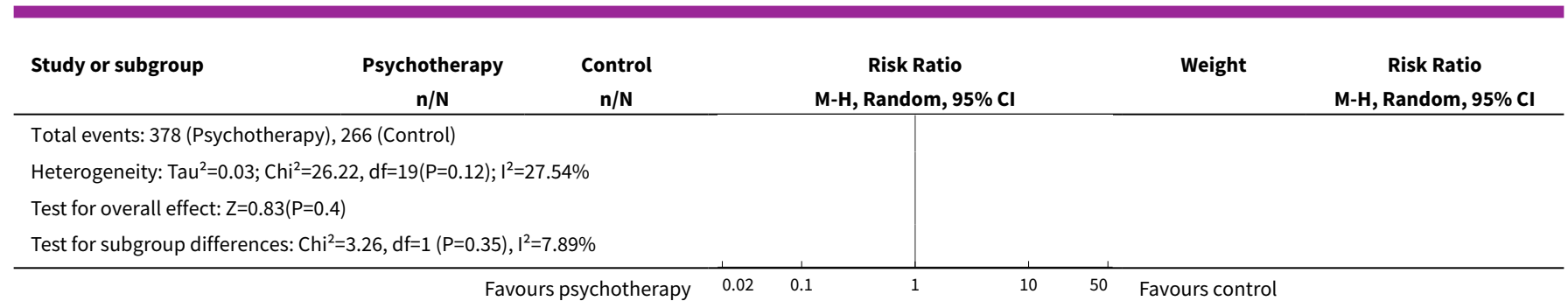

\section{Analysis 13.10. Comparison 13 Subgroup analysis: type of humanitarian} crisis - adults, Outcome 10 Functional impairment at endpoint.

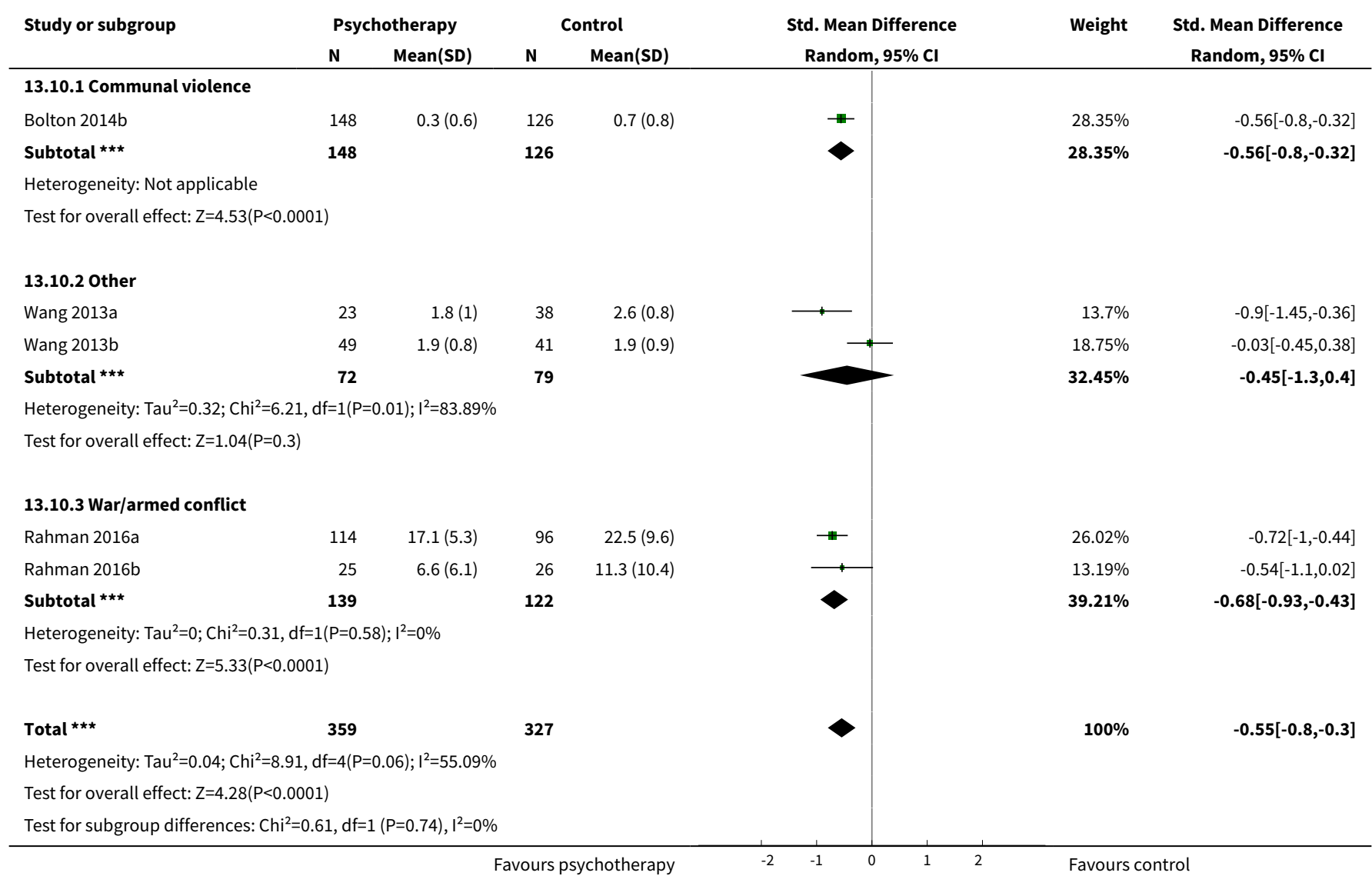

Analysis 13.11. Comparison 13 Subgroup analysis: type of humanitarian crisis - adults, Outcome 11 Functional impairment at 1 to 4 months.

\begin{tabular}{|c|c|c|c|c|c|c|c|c|}
\hline \multirow[t]{2}{*}{ Study or subgroup } & \multicolumn{2}{|c|}{ Psychotherapy } & \multicolumn{2}{|c|}{ Control } & \multirow{2}{*}{\multicolumn{2}{|c|}{$\begin{array}{c}\text { Std. Mean Difference } \\
\text { Random, } 95 \% \mathrm{Cl}\end{array}$}} & \multirow[t]{2}{*}{ Weight } & \multirow{2}{*}{$\begin{array}{c}\text { Std. Mean Difference } \\
\text { Random, } 95 \% \mathrm{Cl}\end{array}$} \\
\hline & $\mathbf{N}$ & Mean(SD) & $\mathbf{N}$ & $\operatorname{Mean}(S D)$ & & & & \\
\hline \multicolumn{9}{|c|}{ 13.11.1 War/armed conflict } \\
\hline Puvimanasinghe 2016 & 13 & $25.5(16.3)$ & 13 & $29.8(21.8)$ & $\longrightarrow$ & & $5.14 \%$ & $-0.21[-0.98,0.56]$ \\
\hline Rahman 2016a & 143 & $15.4(4.5)$ & 160 & $19.6(7.4)$ & $\#$ & & $19.83 \%$ & $-0.67[-0.9,-0.44]$ \\
\hline Weiss 2015a & 123 & $0.8(1.1)$ & 61 & $0.9(1.1)$ & $\rightarrow$ & & $16.33 \%$ & $-0.13[-0.43,0.18]$ \\
\hline Weiss 2015b & 98 & $0.8(1)$ & 48 & $1.4(0.9)$ & $\longrightarrow$ & & $14.46 \%$ & $-0.6[-0.95,-0.25]$ \\
\hline
\end{tabular}




\begin{tabular}{|c|c|c|c|c|c|c|c|}
\hline \multirow{3}{*}{$\begin{array}{l}\text { Study or subgroup } \\
\text { Subtotal } \star \star \star\end{array}$} & \multicolumn{2}{|c|}{ Psychotherapy } & \multicolumn{2}{|c|}{ Control } & \multirow{2}{*}{$\begin{array}{l}\text { Std. Mean Difference } \\
\text { Random, } 95 \% \mathrm{Cl}\end{array}$} & \multirow[t]{2}{*}{ Weight } & \multirow{2}{*}{$\begin{array}{c}\text { Std. Mean Difference } \\
\text { Random, } 95 \% \mathrm{Cl}\end{array}$} \\
\hline & $\mathbf{N}$ & $\operatorname{Mean}(S D)$ & $\mathbf{N}$ & $\operatorname{Mean}(S D)$ & & & \\
\hline & 377 & & 282 & & & $55.77 \%$ & $-0.44[-0.75,-0.14]$ \\
\hline \multicolumn{8}{|c|}{ Heterogeneity: Tau $^{2}=0.06 ; \mathrm{Chi}^{2}=8.59, \mathrm{df}=3(\mathrm{P}=0.04) ; \mathrm{I}^{2}=65.07 \%$} \\
\hline \multicolumn{8}{|c|}{ Test for overall effect: $\mathrm{Z}=2.88(\mathrm{P}=0)$} \\
\hline \multicolumn{8}{|c|}{ 13.11.2 Communal violence } \\
\hline Bolton 2014a & 114 & $1.2(1.5)$ & 33 & $1.6(0.7)$ & $\longrightarrow$ & $13.1 \%$ & $-0.26[-0.65,0.13]$ \\
\hline Bolton 2014a & 101 & $1.1(1.2)$ & 33 & $1.7(0.7)$ & $\longrightarrow$ & $12.81 \%$ & $-0.46[-0.86,-0.06]$ \\
\hline Subtotal ${ }^{\star \star \star}$ & 215 & & 66 & & & $25.91 \%$ & $-0.36[-0.63,-0.08]$ \\
\hline \multicolumn{8}{|c|}{ Heterogeneity: Tau $^{2}=0 ; \mathrm{Chi}^{2}=0.5, \mathrm{df}=1(\mathrm{P}=0.48) ; \mathrm{I}^{2}=0 \%$} \\
\hline \multicolumn{8}{|c|}{ Test for overall effect: $\mathrm{Z}=2.51(\mathrm{P}=0.01)$} \\
\hline \multicolumn{8}{|l|}{ 13.11.3 Other } \\
\hline Wang 2013a & 18 & $1.6(1.3)$ & 16 & $1.6(1.1)$ & - & $6.37 \%$ & $0.02[-0.66,0.69]$ \\
\hline Wang 2013b & 48 & $1.7(0.9)$ & 39 & $1.8(0.9)$ & $\longrightarrow$ & $11.94 \%$ & $-0.11[-0.53,0.31]$ \\
\hline Subtotal ${ }^{\star \star \star}$ & 66 & & 55 & & & $18.32 \%$ & $-0.08[-0.43,0.28]$ \\
\hline \multicolumn{8}{|c|}{ Heterogeneity: Tau $^{2}=0 ; \mathrm{Chi}^{2}=0.1, \mathrm{df}=1(\mathrm{P}=0.75) ; \mathrm{I}^{2}=0 \%$} \\
\hline \multicolumn{8}{|c|}{ Test for overall effect: $Z=0.41(P=0.68)$} \\
\hline Total $\star \star \star ~$ & 658 & & 403 & & $>$ & $100 \%$ & $-0.36[-0.55,-0.16]$ \\
\hline \multicolumn{8}{|c|}{ Heterogeneity: $\mathrm{Tau}^{2}=0.04 ; \mathrm{Chi}^{2}=13.62, \mathrm{df}=7(\mathrm{P}=0.06) ; \mathrm{I}^{2}=48.62 \%$} \\
\hline \multicolumn{8}{|c|}{ Test for overall effect: $Z=3.6(P=0)$} \\
\hline Test for subgroup dif & $5, \mathrm{df}=1$ & $=0.29), I^{2}=19$ & & & & & \\
\hline
\end{tabular}

Analysis 13.12. Comparison 13 Subgroup analysis: type of humanitarian crisis - adults, Outcome 12 Quality of life at endpoint.

\begin{tabular}{|c|c|c|c|c|c|c|c|}
\hline \multirow[t]{2}{*}{ Study or subgroup } & \multicolumn{2}{|c|}{ Psychotherapy } & \multicolumn{2}{|c|}{ Control } & \multirow{2}{*}{$\begin{array}{c}\text { Std. Mean Difference } \\
\text { Random, } 95 \% \mathrm{Cl}\end{array}$} & \multirow[t]{2}{*}{ Weight } & \multirow{2}{*}{$\begin{array}{c}\text { Std. Mean Difference } \\
\text { Random, } 95 \% \mathrm{Cl}\end{array}$} \\
\hline & $\mathbf{N}$ & Mean(SD) & $\mathbf{N}$ & Mean(SD) & & & \\
\hline \multicolumn{8}{|c|}{ 13.12.1 War/armed conflict } \\
\hline Ahmadizadeh 2013 & 75 & $-51.4(65.2)$ & 25 & $-45.7(3.2)$ & $\longrightarrow$ & $28.25 \%$ & $-0.1[-0.55,0.35]$ \\
\hline Azad Marzabadi 2014 & 14 & $-69.9(7.2)$ & 14 & $-55(12.8)$ & & $17.76 \%$ & $-1.39[-2.23,-0.55]$ \\
\hline Knaevelsrud 2015 & 79 & $-3(1)$ & 80 & $-2.3(0.7)$ & & $32.05 \%$ & $-0.83[-1.16,-0.51]$ \\
\hline Subtotal $* \star \star$ & 168 & & 119 & & & $78.06 \%$ & $-0.71[-1.35,-0.07]$ \\
\hline \multicolumn{8}{|c|}{ Heterogeneity: $\mathrm{Tau}^{2}=0.24 ; \mathrm{Chi}^{2}=9.86, \mathrm{df}=2(\mathrm{P}=0.01) ; \mathrm{I}^{2}=79.72 \%$} \\
\hline \multicolumn{8}{|c|}{ Test for overall effect: $\mathrm{Z}=2.18(\mathrm{P}=0.03)$} \\
\hline \multicolumn{8}{|c|}{ 13.12.2 Natural hazards (geophysical: earthquake; volcanic eruptions) } \\
\hline Jiang 2014 & 19 & $-19.9(6.1)$ & 19 & $-15.1(4.6)$ & 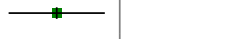 & $21.94 \%$ & $-0.88[-1.55,-0.21]$ \\
\hline Subtotal *** & 19 & & 19 & & & $21.94 \%$ & $-0.88[-1.55,-0.21]$ \\
\hline \multicolumn{8}{|c|}{ Heterogeneity: Not applicable } \\
\hline \multicolumn{8}{|c|}{ Test for overall effect: $Z=2.57(P=0.01)$} \\
\hline 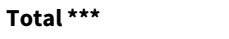 & 187 & & 138 & & & $100 \%$ & $-0.73[-1.22,-0.25]$ \\
\hline \multicolumn{8}{|c|}{ Heterogeneity: $\mathrm{Tau}^{2}=0.17 ; \mathrm{Chi}^{2}=10.23, \mathrm{df}=3(\mathrm{P}=0.02) ; \mathrm{I}^{2}=70.67 \%$} \\
\hline \multicolumn{8}{|c|}{ Test for overall effect: $Z=2.95(P=0)$} \\
\hline \multicolumn{8}{|c|}{ Test for subgroup differences: $\mathrm{Chi}^{2}=0.13, \mathrm{df}=1(\mathrm{P}=0.72), \mathrm{I}^{2}=0 \%$} \\
\hline
\end{tabular}

Psychological therapies for the treatment of mental disorders in low- and middle-income countries affected by humanitarian crises 
Analysis 13.13. Comparison 13 Subgroup analysis: type of humanitarian crisis - adults, Outcome 13 Diagnosis of PTSD.

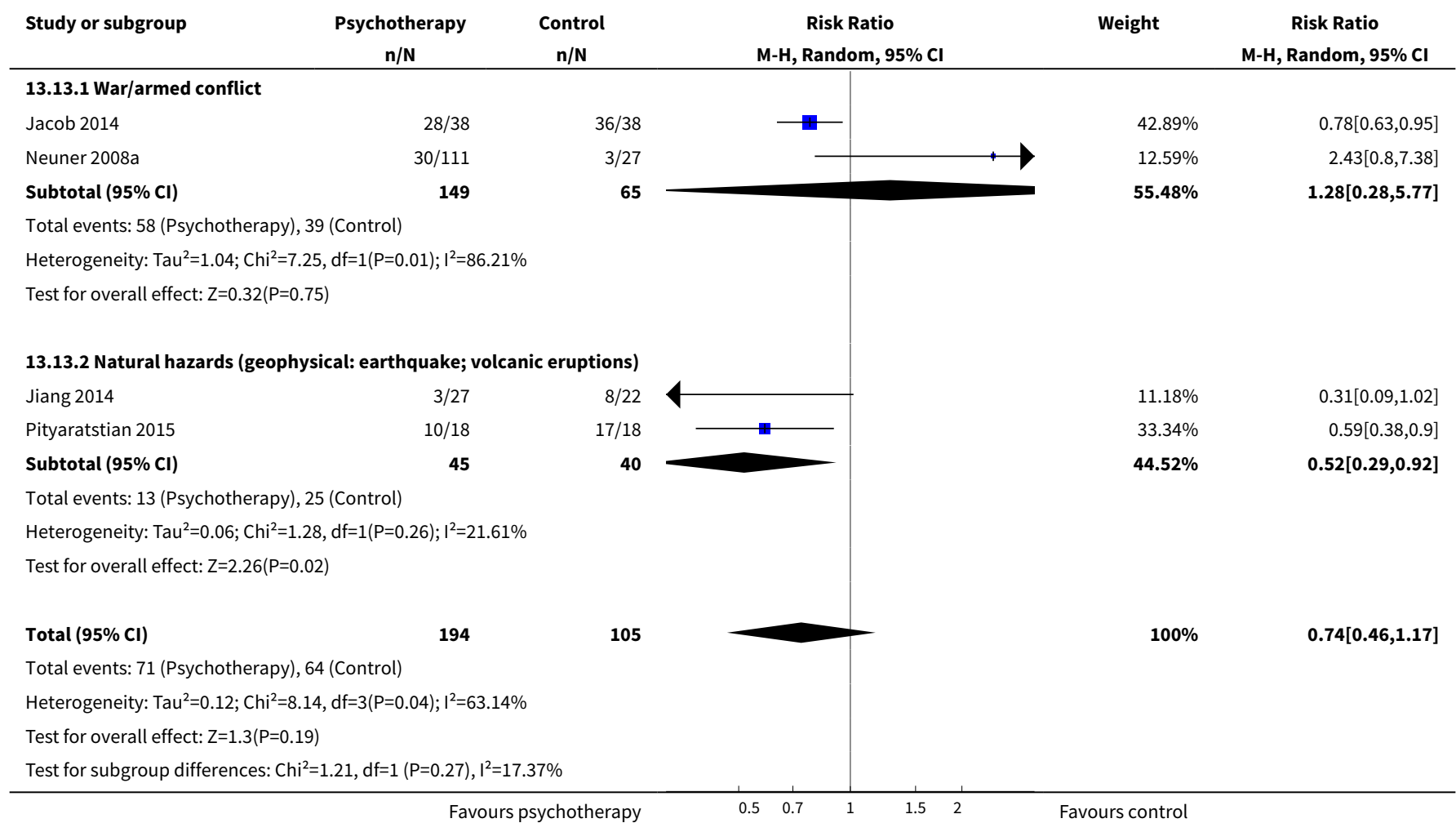

\section{Comparison 14. Subgroup analysis: type of interventionists - adults}

\begin{tabular}{|c|c|c|c|c|}
\hline $\begin{array}{l}\text { Outcome or subgroup } \\
\text { title }\end{array}$ & No. of studies & $\begin{array}{l}\text { No. of partici- } \\
\text { pants }\end{array}$ & Statistical method & Effect size \\
\hline $\begin{array}{l}1 \text { PTSD symptoms at end- } \\
\text { point }\end{array}$ & 16 & 1298 & $\begin{array}{l}\text { Std. Mean Difference (IV, Random, 95\% } \\
\mathrm{CI})\end{array}$ & $-1.21[-1.52,-0.90]$ \\
\hline 1.1 Professionals & 8 & 400 & $\begin{array}{l}\text { Std. Mean Difference (IV, Random, 95\% } \\
\text { CI) }\end{array}$ & $-1.51[-2.13,-0.90]$ \\
\hline 1.2 Paraprofessionals & 8 & 898 & $\begin{array}{l}\text { Std. Mean Difference (IV, Random, 95\% } \\
\text { CI) }\end{array}$ & $-0.99[-1.33,-0.65]$ \\
\hline $\begin{array}{l}2 \text { PTSD symptoms at } 1 \text { to } \\
4 \text { months }\end{array}$ & 16 & 1568 & $\begin{array}{l}\text { Std. Mean Difference (IV, Random, 95\% } \\
\mathrm{CI})\end{array}$ & $-0.47[-0.66,-0.29]$ \\
\hline 2.1 Professionals & 9 & 476 & $\begin{array}{l}\text { Std. Mean Difference (IV, Random, 95\% } \\
\mathrm{CI})\end{array}$ & $-0.57[-0.96,-0.19]$ \\
\hline 2.2 Paraprofessionals & 7 & 1092 & $\begin{array}{l}\text { Std. Mean Difference (IV, Random, 95\% } \\
\mathrm{CI} \text { ) }\end{array}$ & $-0.43[-0.61,-0.26]$ \\
\hline $\begin{array}{l}3 \text { PTSD symptoms } \geq 6 \\
\text { months }\end{array}$ & 5 & 400 & $\begin{array}{l}\text { Std. Mean Difference (IV, Random, 95\% } \\
\mathrm{Cl} \text { ) }\end{array}$ & $-0.37[-0.61,-0.14]$ \\
\hline
\end{tabular}

Psychological therapies for the treatment of mental disorders in low- and middle-income countries affected by humanitarian crises 


\begin{tabular}{|c|c|c|c|c|}
\hline $\begin{array}{l}\text { Outcome or subgroup } \\
\text { title }\end{array}$ & No. of studies & $\begin{array}{l}\text { No. of partici- } \\
\text { pants }\end{array}$ & Statistical method & Effect size \\
\hline 3.1 Professionals & 1 & 30 & $\begin{array}{l}\text { Std. Mean Difference (IV, Random, 95\% } \\
\mathrm{CI} \text { ) }\end{array}$ & $-0.72[-1.46,0.03]$ \\
\hline 3.2 Paraprofessionals & 4 & 370 & $\begin{array}{l}\text { Std. Mean Difference (IV, Random, 95\% } \\
\mathrm{CI} \text { ) }\end{array}$ & $-0.34[-0.58,-0.09]$ \\
\hline $\begin{array}{l}4 \text { Anxiety symptoms at } \\
\text { endpoint }\end{array}$ & 4 & 535 & $\begin{array}{l}\text { Std. Mean Difference (IV, Random, 95\% } \\
\mathrm{CI} \text { ) }\end{array}$ & $-0.76[-1.10,-0.42]$ \\
\hline 4.1 Professionals & 2 & 52 & $\begin{array}{l}\text { Std. Mean Difference (IV, Random, 95\% } \\
\mathrm{CI} \text { ) }\end{array}$ & $-1.30[-1.92,-0.67]$ \\
\hline 4.2 Paraprofessionals & 2 & 483 & $\begin{array}{l}\text { Std. Mean Difference (IV, Random, 95\% } \\
\mathrm{CI} \text { ) }\end{array}$ & $-0.60[-0.85,-0.35]$ \\
\hline $\begin{array}{l}5 \text { Anxiety symptoms at } 1 \\
\text { to } 4 \text { months }\end{array}$ & 6 & 969 & $\begin{array}{l}\text { Std. Mean Difference (IV, Random, 95\% } \\
\mathrm{CI} \text { ) }\end{array}$ & $-0.43[-0.71,-0.15]$ \\
\hline 5.1 Professionals & 2 & 52 & $\begin{array}{l}\text { Std. Mean Difference (IV, Random, 95\% } \\
\mathrm{CI} \text { ) }\end{array}$ & $0.06[-0.50,0.63]$ \\
\hline 5.2 Paraprofessionals & 4 & 917 & $\begin{array}{l}\text { Std. Mean Difference (IV, Random, 95\% } \\
\text { CI) }\end{array}$ & $-0.52[-0.81,-0.23]$ \\
\hline $\begin{array}{l}6 \text { Depressive symptoms } \\
\text { at endpoint }\end{array}$ & 13 & 1232 & $\begin{array}{l}\text { Std. Mean Difference (IV, Random, 95\% } \\
\mathrm{CI} \text { ) }\end{array}$ & $-0.90[-1.09,-0.71]$ \\
\hline 6.1 Professionals & 7 & 370 & $\begin{array}{l}\text { Std. Mean Difference (IV, Random, 95\% } \\
\mathrm{CI} \text { ) }\end{array}$ & $-1.13[-1.35,-0.90]$ \\
\hline 6.2 Paraprofessionals & 6 & 862 & $\begin{array}{l}\text { Std. Mean Difference (IV, Random, 95\% } \\
\text { CI) }\end{array}$ & $-0.74[-0.98,-0.50]$ \\
\hline $\begin{array}{l}7 \text { Depressive symptoms } \\
\text { at } 1 \text { to } 4 \text { months }\end{array}$ & 14 & 1364 & $\begin{array}{l}\text { Std. Mean Difference (IV, Random, 95\% } \\
\text { Cl) }\end{array}$ & $-0.40[-0.61,-0.18]$ \\
\hline 7.1 Professionals & 7 & 272 & $\begin{array}{l}\text { Std. Mean Difference (IV, Random, 95\% } \\
\mathrm{CI} \text { ) }\end{array}$ & $-0.38[-0.80,0.05]$ \\
\hline 7.2 Paraprofessionals & 7 & 1092 & $\begin{array}{l}\text { Std. Mean Difference (IV, Random, 95\% } \\
\text { CI) }\end{array}$ & $-0.40[-0.66,-0.15]$ \\
\hline $\begin{array}{l}8 \text { Depressive symptoms } \geq \\
6 \text { months }\end{array}$ & 2 & & $\begin{array}{l}\text { Std. Mean Difference (IV, Random, 95\% } \\
\mathrm{CI} \text { ) }\end{array}$ & Subtotals only \\
\hline 8.1 Paraprofessionals & 2 & 242 & $\begin{array}{l}\text { Std. Mean Difference (IV, Random, 95\% } \\
\mathrm{CI} \text { ) }\end{array}$ & $-0.20[-0.49,0.09]$ \\
\hline 9 Dropout & 24 & 2791 & Risk Ratio (M-H, Random, 95\% Cl) & $0.97[0.80,1.16]$ \\
\hline 9.1 Professionals & 9 & 394 & Risk Ratio (M-H, Random, 95\% Cl) & $1.38[0.56,3.38]$ \\
\hline 9.2 Paraprofessionals & 15 & 2397 & Risk Ratio (M-H, Random, 95\% Cl) & $0.95[0.78,1.15]$ \\
\hline
\end{tabular}




\begin{tabular}{|c|c|c|c|c|}
\hline $\begin{array}{l}\text { Outcome or subgroup } \\
\text { title }\end{array}$ & No. of studies & $\begin{array}{l}\text { No. of partici- } \\
\text { pants }\end{array}$ & Statistical method & Effect size \\
\hline $\begin{array}{l}10 \text { Functional impair- } \\
\text { ment at endpoint }\end{array}$ & 5 & 686 & $\begin{array}{l}\text { Std. Mean Difference (IV, Random, 95\% } \\
\mathrm{CI} \text { ) }\end{array}$ & $-0.54[-0.79,-0.29]$ \\
\hline 10.1 Paraprofessionals & 5 & 686 & $\begin{array}{l}\text { Std. Mean Difference (IV, Random, 95\% } \\
\mathrm{CI} \text { ) }\end{array}$ & $-0.54[-0.79,-0.29]$ \\
\hline $\begin{array}{l}11 \text { Functional impair- } \\
\text { ment at } 1 \text { to } 4 \text { months }\end{array}$ & 7 & 1061 & $\begin{array}{l}\text { Std. Mean Difference (IV, Random, 95\% } \\
\mathrm{CI} \text { ) }\end{array}$ & $-0.36[-0.55,-0.16]$ \\
\hline 11.1 Paraprofessionals & 7 & 1061 & $\begin{array}{l}\text { Std. Mean Difference (IV, Random, 95\% } \\
\text { CI) }\end{array}$ & $-0.36[-0.55,-0.16]$ \\
\hline $\begin{array}{l}12 \text { Quality of life at end- } \\
\text { point }\end{array}$ & 3 & 297 & $\begin{array}{l}\text { Std. Mean Difference (IV, Random, 95\% } \\
\text { CI) }\end{array}$ & $-0.59[-1.10,-0.08]$ \\
\hline 12.1 Professionals & 3 & 297 & $\begin{array}{l}\text { Std. Mean Difference (IV, Random, 95\% } \\
\text { CI) }\end{array}$ & $-0.59[-1.10,-0.08]$ \\
\hline 13 Diagnosis of PTSD & 4 & 402 & Risk Ratio (M-H, Random, 95\% Cl) & $1.07[0.43,2.68]$ \\
\hline 13.1 Professionals & 2 & 125 & Risk Ratio (M-H, Random, 95\% Cl) & $0.56[0.20,1.59]$ \\
\hline 13.2 Paraprofessionals & 2 & 277 & Risk Ratio (M-H, Random, 95\% Cl) & $2.34[1.14,4.80]$ \\
\hline
\end{tabular}

\begin{tabular}{|c|c|c|c|c|c|c|c|c|}
\hline \multirow{3}{*}{$\begin{array}{l}\text { Study or subgroup } \\
\text { 14.1.1 Professionals }\end{array}$} & \multicolumn{2}{|c|}{ Psychotherapy } & \multicolumn{2}{|c|}{ Control } & \multirow{2}{*}{\multicolumn{2}{|c|}{$\begin{array}{c}\text { Std. Mean Difference } \\
\text { Random, } 95 \% \mathrm{Cl}\end{array}$}} & \multirow[t]{2}{*}{ Weight } & \multirow{2}{*}{$\begin{array}{c}\text { Std. Mean Difference } \\
\text { Random, } 95 \% \mathrm{Cl}\end{array}$} \\
\hline & \multirow[t]{2}{*}{$\mathbf{N}$} & \multirow[t]{2}{*}{ Mean(SD) } & \multirow[t]{2}{*}{$\mathbf{N}$} & \multirow[t]{2}{*}{ Mean(SD) } & & & & \\
\hline & & & & & & & & \\
\hline Acarturk 2015 & 15 & $22.9(20.3)$ & 14 & $54.2(16.3)$ & - & & $5.23 \%$ & $-1.65[-2.51,-0.79]$ \\
\hline Acaturk 2016 & 37 & $1.4(0.4)$ & 33 & $2.4(0.5)$ & & & $6.58 \%$ & $-2.19[-2.78,-1.59]$ \\
\hline Basoglu 2007 & 16 & $38.7(18.7)$ & 16 & $54.5(16.9)$ & $\longrightarrow$ & & $5.89 \%$ & $-0.86[-1.59,-0.14]$ \\
\hline Bryant 2011 & 16 & $4.1(8)$ & 12 & $12.3(8.4)$ & + & & $5.54 \%$ & $-0.97[-1.77,-0.18]$ \\
\hline Hermenau 2013 & 15 & $11.5(5.2)$ & 15 & $13.7(5.8)$ & 1 & - & $5.92 \%$ & $-0.4[-1.12,0.32]$ \\
\hline Knaevelsrud 2015 & 79 & $20.3(12.5)$ & 80 & $30.2(8.7)$ & $\rightarrow$ & & $7.93 \%$ & $-0.92[-1.24,-0.59]$ \\
\hline Zang 2013 & 11 & $25.8(13.8)$ & 11 & $47.2(10.9)$ & - & & $4.61 \%$ & $-1.65[-2.64,-0.66]$ \\
\hline Zang 2014 & 20 & $17(5.8)$ & 10 & $54.7(10.8)$ & & & $2.91 \%$ & $-4.72[-6.21,-3.23]$ \\
\hline 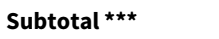 & 209 & & 191 & & 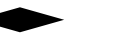 & & $44.6 \%$ & $-1.51[-2.13,-0.9]$ \\
\hline \multicolumn{9}{|c|}{ Heterogeneity: $\mathrm{Tau}^{2}=0.61 ; \mathrm{Chi}^{2}=42.37, \mathrm{df}=7(\mathrm{P}<0.0001) ; \mathrm{I}^{2}=83.48 \%$} \\
\hline \multicolumn{9}{|c|}{ Test for overall effect: $Z=4.82(P<0.0001)$} \\
\hline \multicolumn{9}{|c|}{ 14.1.2 Paraprofessionals } \\
\hline Bolton 2014b & 148 & $0.3(0.4)$ & 126 & $0.6(0.6)$ & $\rightarrow$ & & $8.25 \%$ & $-0.79[-1.04,-0.54]$ \\
\hline Connolly 2011 & 71 & $58.7(6.3)$ & 74 & $66.9(6.6)$ & $一$ & & $7.8 \%$ & $-1.27[-1.63,-0.91]$ \\
\hline McMullen 2013 & 24 & $10.6(4.5)$ & 24 & $34.8(11.6)$ & & & $5.53 \%$ & $-2.71[-3.51,-1.91]$ \\
\hline Meffert 2014 & 11 & $1.8(0.5)$ & 9 & $2.6(0.6)$ & + & & $4.53 \%$ & $-1.45[-2.46,-0.43]$ \\
\hline Rahman 2016a & 114 & $12.9(10.7)$ & 95 & $18.7(11.1)$ & $\rightarrow$ & & $8.14 \%$ & $-0.54[-0.81,-0.26]$ \\
\hline Rahman 2016b & 25 & $9.8(9.1)$ & 26 & $19.5(18.5)$ & $\longrightarrow$ & & $6.76 \%$ & $-0.65[-1.22,-0.09]$ \\
\hline
\end{tabular}




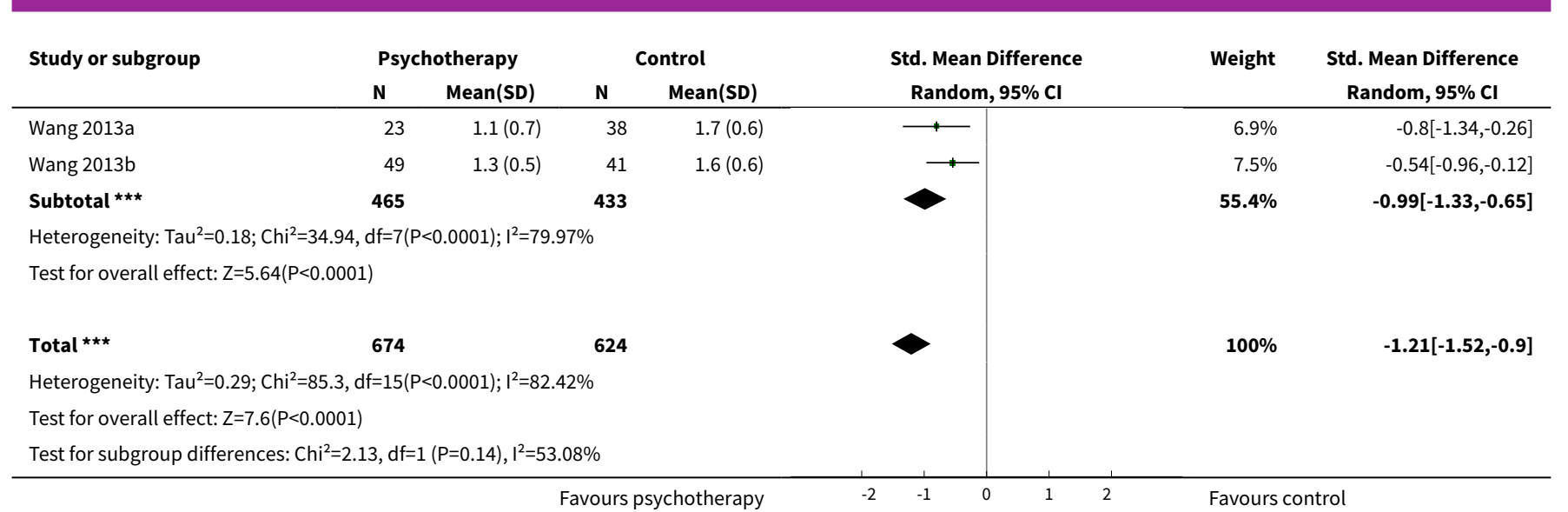

Analysis 14.2. Comparison 14 Subgroup analysis: type of interventionists - adults, Outcome 2 PTSD symptoms at 1 to 4 months.

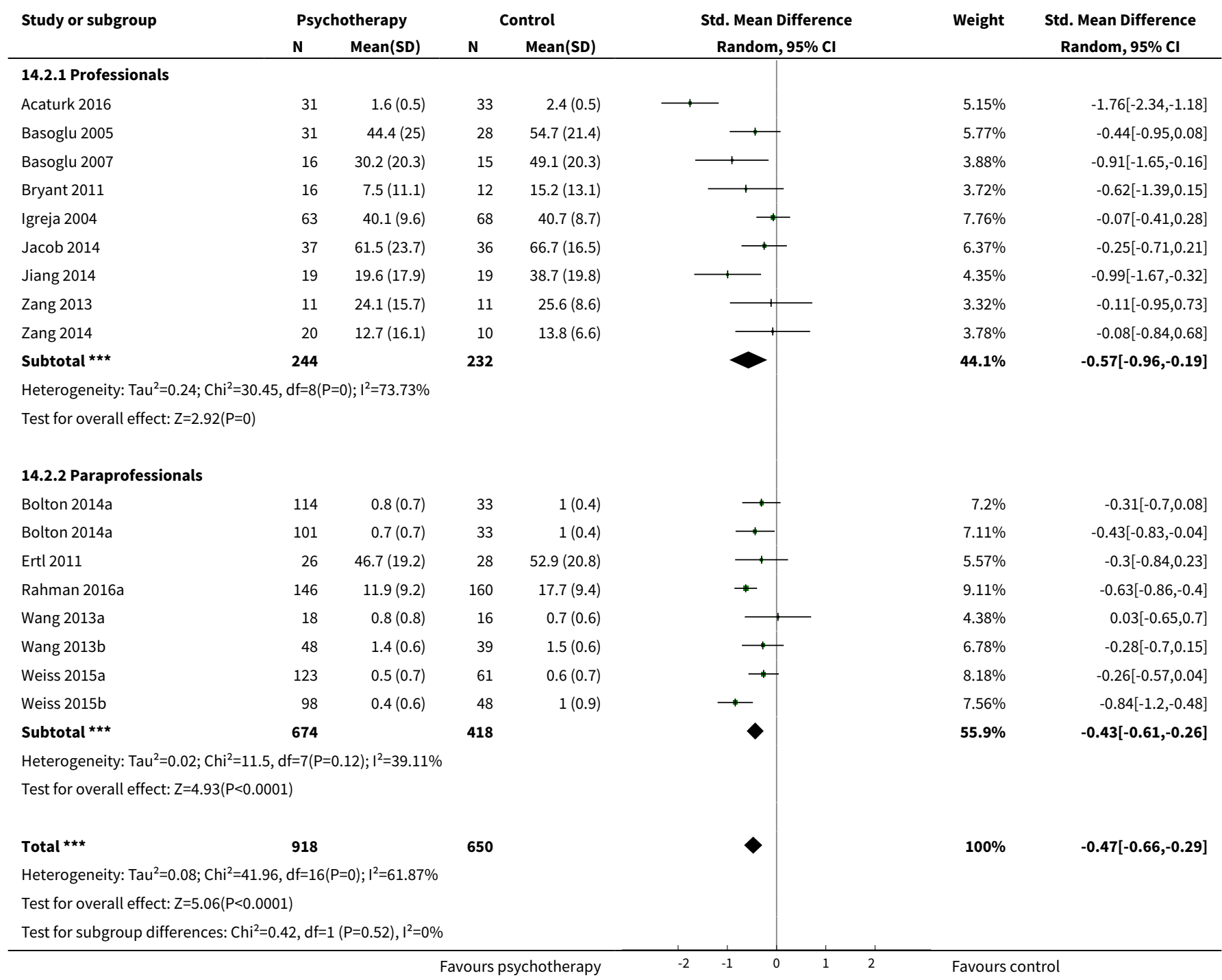

Psychological therapies for the treatment of mental disorders in low- and middle-income countries affected by humanitarian crises 
Analysis 14.3. Comparison 14 Subgroup analysis: type of interventionists - adults, Outcome 3 PTSD symptoms $\geq 6$ months.

\begin{tabular}{|c|c|c|c|c|c|c|c|}
\hline \multirow{3}{*}{$\begin{array}{l}\text { Study or subgroup } \\
\text { 14.3.1 Professionals }\end{array}$} & \multicolumn{2}{|c|}{ Psychotherapy } & \multicolumn{2}{|c|}{ Control } & \multirow{2}{*}{$\begin{array}{c}\text { Std. Mean Difference } \\
\text { Random, } 95 \% \mathrm{Cl}\end{array}$} & \multirow[t]{2}{*}{ Weight } & \multirow{2}{*}{$\begin{array}{c}\text { Std. Mean Difference } \\
\text { Random, } 95 \% \mathrm{Cl}\end{array}$} \\
\hline & $\mathbf{N}$ & Mean(SD) & $\mathbf{N}$ & Mean(SD) & & & \\
\hline & & & & & & & \\
\hline Hermenau 2013 & 15 & $8.9(7.6)$ & 15 & $16.5(12.5)$ & $\longrightarrow$ & $10.11 \%$ & $-0.72[-1.46,0.03]$ \\
\hline Subtotal $\star \star \star$ & 15 & & 15 & & & $10.11 \%$ & $-0.72[-1.46,0.03]$ \\
\hline \multicolumn{8}{|c|}{ Heterogeneity: Not applicable } \\
\hline \multicolumn{8}{|c|}{ Test for overall effect: $Z=1.89(P=0.06)$} \\
\hline \multicolumn{8}{|c|}{ 14.3.2 Paraprofessionals } \\
\hline Bass 2016 & 145 & $0.7(0.6)$ & 43 & $0.9(0.6)$ & 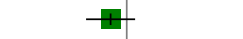 & $47.78 \%$ & $-0.22[-0.57,0.12]$ \\
\hline Ertl 2011 & 26 & $43(21.5)$ & 28 & $48.6(23.7)$ & $\because-$ & $19.35 \%$ & $-0.24[-0.78,0.29]$ \\
\hline Neuner 2008a & 56 & $6.1(6.8)$ & 10 & $10.1(8.1)$ & & $12.02 \%$ & $-0.56[-1.25,0.12]$ \\
\hline Neuner 2008b & 53 & $5(6.6)$ & 9 & $10.1(8.1)$ & & $10.75 \%$ & $-0.74[-1.46,-0.02]$ \\
\hline Subtotal $\star \star \star$ & 280 & & 90 & & & $89.89 \%$ & $-0.34[-0.58,-0.09]$ \\
\hline \multicolumn{8}{|c|}{ Test for overall effect: $Z=2.64(P=0.01)$} \\
\hline Total $\star \star \star$ & 295 & & 105 & & & $100 \%$ & $-0.37[-0.61,-0.14]$ \\
\hline \multicolumn{8}{|c|}{ Heterogeneity: $\operatorname{Tau}^{2}=0 ; \mathrm{Chi}^{2}=3.07, \mathrm{df}=4(\mathrm{P}=0.55) ; \mathrm{I}^{2}=0 \%$} \\
\hline \multicolumn{8}{|c|}{ Test for overall effect: $\mathrm{Z}=3.11(\mathrm{P}=0)$} \\
\hline Test for subgroup dif & $91, \mathrm{df}=$ & $P=0.34), I^{2}=0 \%$ & & & & & \\
\hline
\end{tabular}

Analysis 14.4. Comparison 14 Subgroup analysis: type of interventionists - adults, Outcome 4 Anxiety symptoms at endpoint.

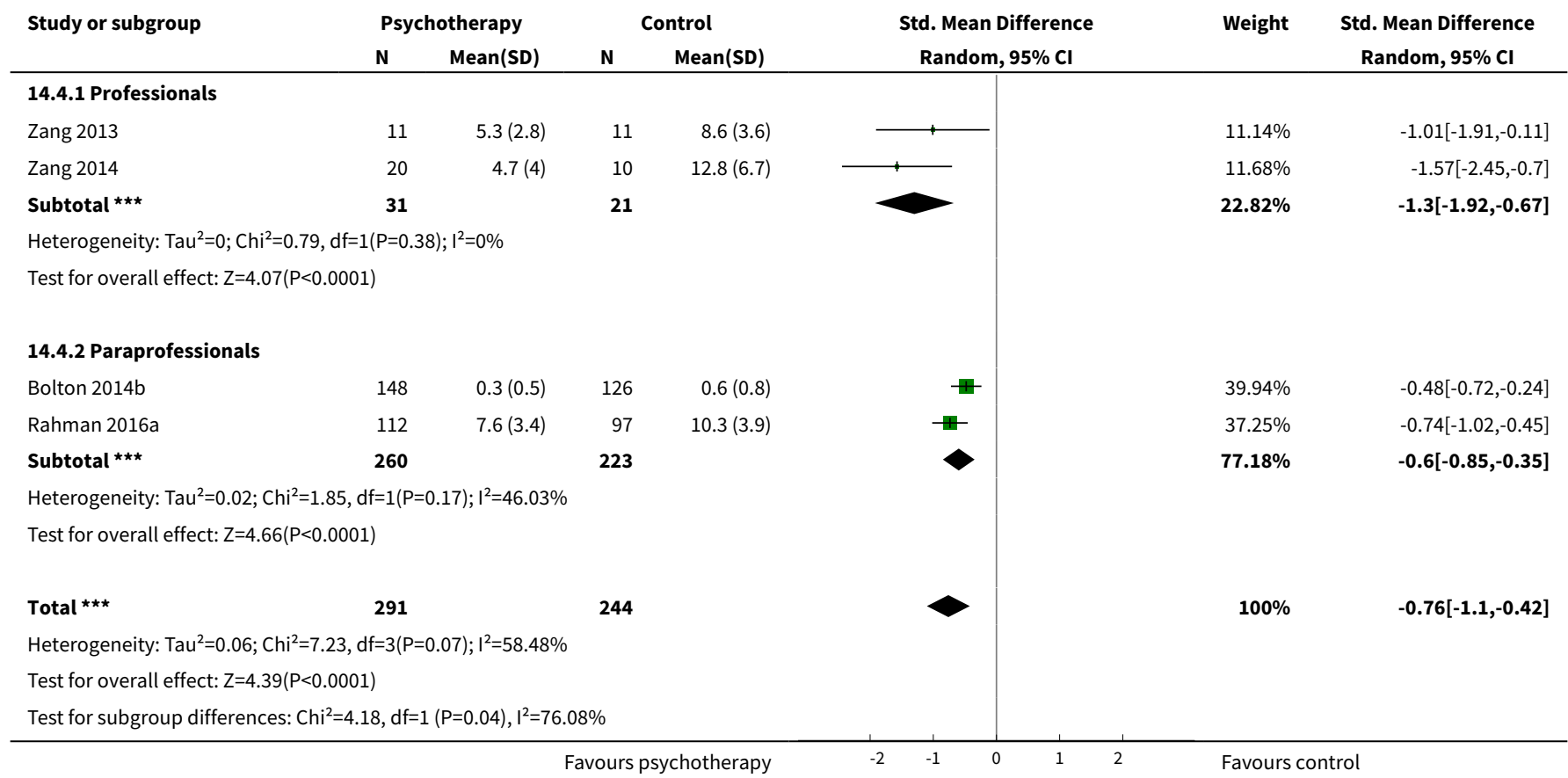

Psychological therapies for the treatment of mental disorders in low- and middle-income countries affected by humanitarian crises 
Analysis 14.5. Comparison 14 Subgroup analysis: type of interventionists - adults, Outcome 5 Anxiety symptoms at 1 to 4 months.

\begin{tabular}{|c|c|c|c|c|c|c|c|}
\hline \multirow[t]{2}{*}{ Study or subgroup } & \multicolumn{2}{|c|}{ Psychotherapy } & \multicolumn{2}{|c|}{ Control } & \multirow{2}{*}{$\begin{array}{c}\text { Std. Mean Difference } \\
\text { Random, } 95 \% \mathrm{Cl}\end{array}$} & \multirow[t]{2}{*}{ Weight } & \multirow{2}{*}{$\begin{array}{c}\text { Std. Mean Difference } \\
\text { Random, } 95 \% \mathrm{Cl}\end{array}$} \\
\hline & $\mathbf{N}$ & Mean(SD) & $\mathbf{N}$ & Mean(SD) & & & \\
\hline \multicolumn{8}{|l|}{ 14.5.1 Professionals } \\
\hline Zang 2013 & 11 & $5.5(3)$ & 11 & $4.8(2.5)$ & + & $7.39 \%$ & $0.22[-0.62,1.06]$ \\
\hline Zang 2014 & 20 & $3.4(3.2)$ & 10 & $3.6(2.2)$ & & $8.41 \%$ & $-0.07[-0.83,0.69]$ \\
\hline Subtotal $\star \star \star$ & 31 & & 21 & & & $15.81 \%$ & $0.06[-0.5,0.63]$ \\
\hline \multicolumn{8}{|c|}{ Heterogeneity: $\mathrm{Tau}^{2}=0 ; \mathrm{Chi}^{2}=0.24, \mathrm{df}=1(\mathrm{P}=0.62) ; \mathrm{I}^{2}=0 \%$} \\
\hline \multicolumn{8}{|c|}{ 14.5.2 Paraprofessionals } \\
\hline Bolton 2014a & 101 & $0.8(1)$ & 33 & $1(0.6)$ & $\longrightarrow$ & $15.47 \%$ & $-0.24[-0.63,0.15]$ \\
\hline Bolton 2014a & 114 & $0.8(1.1)$ & 33 & $0.9(0.5)$ & $\because$ & $15.61 \%$ & $-0.2[-0.59,0.19]$ \\
\hline Rahman 2016a & 146 & $7.3(3.6)$ & 160 & $10(3.9)$ & $\rightarrow$ & $19.38 \%$ & $-0.74[-0.97,-0.51]$ \\
\hline Weiss 2015a & 123 & $0.5(0.5)$ & 61 & $0.7(0.7)$ & $\rightarrow$ & $17.55 \%$ & $-0.36[-0.67,-0.05]$ \\
\hline Subtotal $\star \star \star$ & 582 & & 335 & & & $84.19 \%$ & $-0.52[-0.81,-0.23]$ \\
\hline \multicolumn{8}{|c|}{ Heterogeneity: Tau $^{2}=0.08 ; \mathrm{Chi}^{2}=15.68, \mathrm{df}=4(\mathrm{P}=0) ; \mathrm{I}^{2}=74.49 \%$} \\
\hline \multicolumn{8}{|c|}{ Test for overall effect: $\mathrm{Z}=3.5(\mathrm{P}=0)$} \\
\hline Total $\star \star \star ~$ & 613 & & 356 & & & $100 \%$ & $-0.43[-0.71,-0.15]$ \\
\hline \multicolumn{8}{|c|}{ Heterogeneity: $\mathrm{Tau}^{2}=0.09 ; \mathrm{Chi}^{2}=20.37, \mathrm{df}=6(\mathrm{P}=0) ; \mathrm{I}^{2}=70.54 \%$} \\
\hline \multicolumn{8}{|c|}{ Test for overall effect: $Z=3(P=0)$} \\
\hline Test for subgroup dif & $24, \mathrm{df}=$ & $P=0.07), I^{2}=6$ & & & & & \\
\hline
\end{tabular}

Analysis 14.6. Comparison 14 Subgroup analysis: type of interventionists - adults, Outcome 6 Depressive symptoms at endpoint.

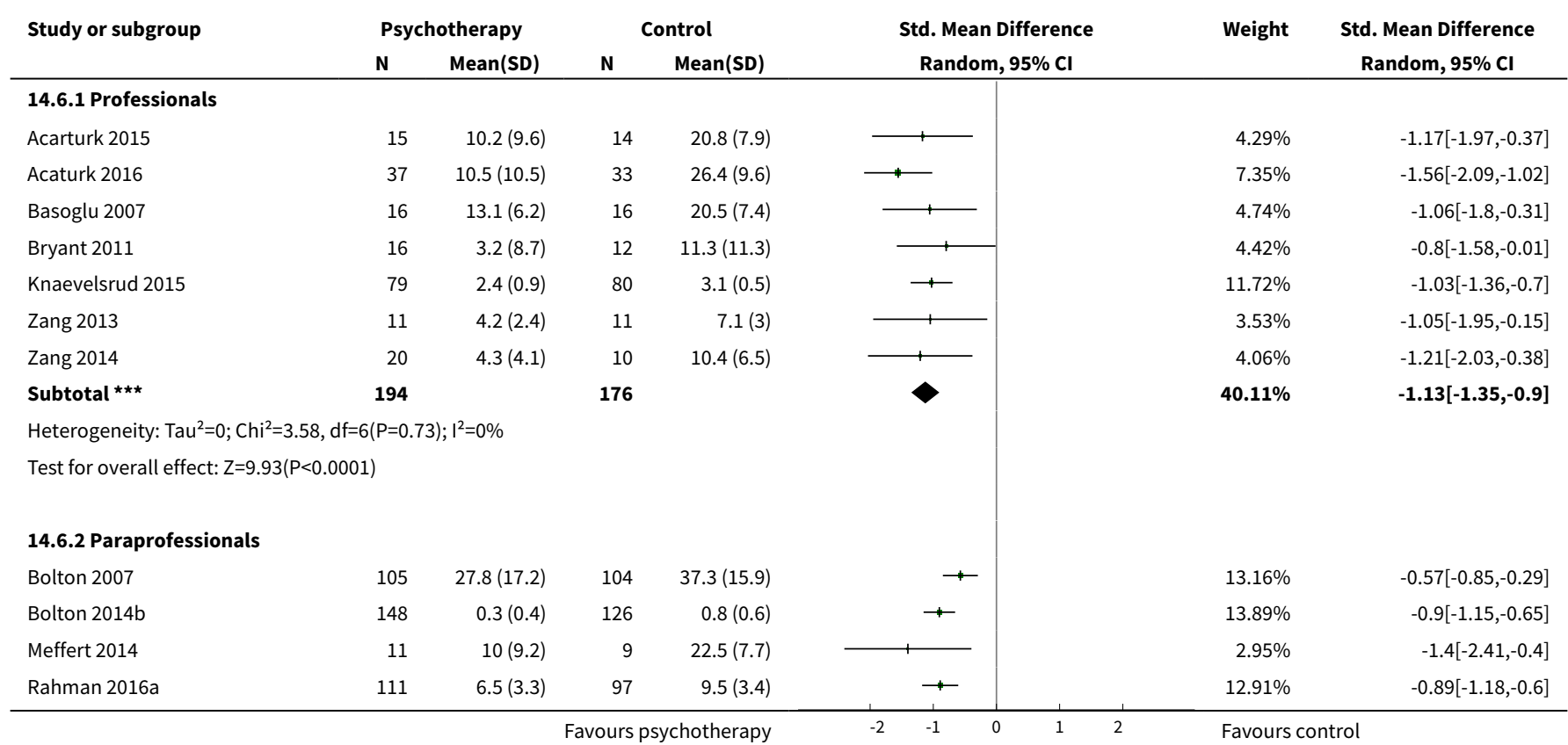

Psychological therapies for the treatment of mental disorders in low- and middle-income countries affected by humanitarian crises 


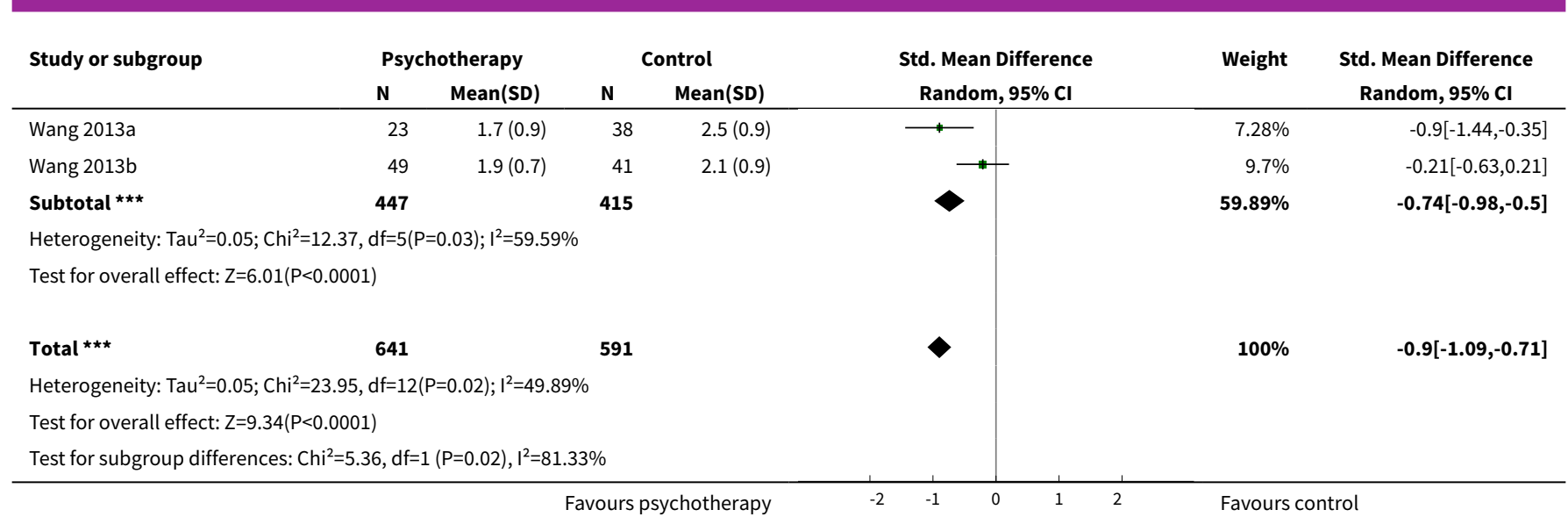

\section{Analysis 14.7. Comparison 14 Subgroup analysis: type of interventionists} - adults, Outcome 7 Depressive symptoms at 1 to 4 months.

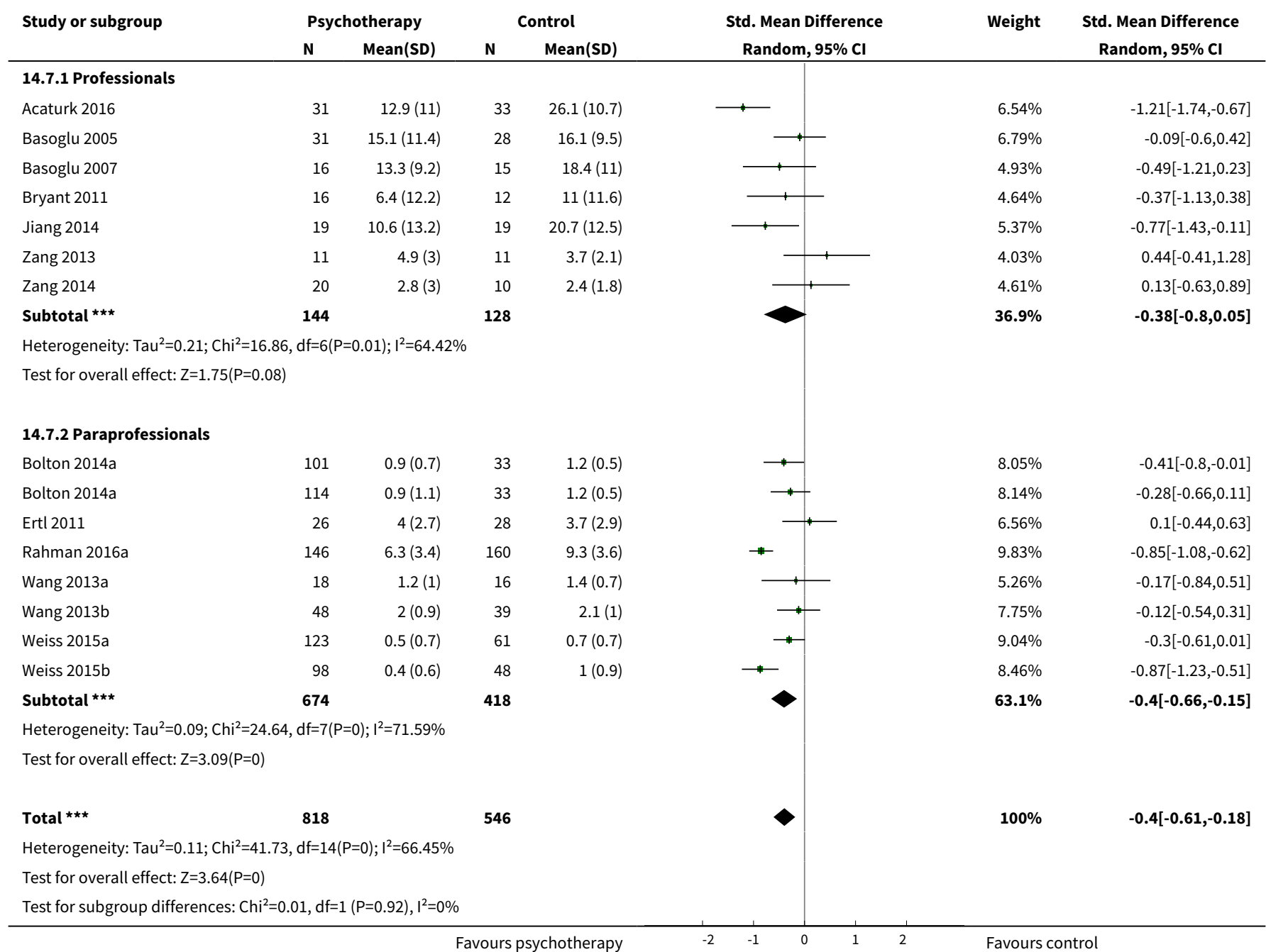


Analysis 14.8. Comparison 14 Subgroup analysis: type of interventionists - adults, Outcome 8 Depressive symptoms $\geq 6$ months.

\begin{tabular}{|c|c|c|c|c|c|c|c|}
\hline \multirow[t]{2}{*}{ Study or subgroup } & \multicolumn{2}{|c|}{ Psychotherapy } & \multicolumn{2}{|c|}{ Control } & \multirow{2}{*}{$\begin{array}{c}\text { Std. Mean Difference } \\
\text { Random, } 95 \% \mathrm{Cl}\end{array}$} & \multirow[t]{2}{*}{ Weight } & \multirow{2}{*}{$\begin{array}{c}\text { Std. Mean Difference } \\
\text { Random, } 95 \% \mathrm{Cl}\end{array}$} \\
\hline & $\mathbf{N}$ & Mean(SD) & $\mathbf{N}$ & Mean(SD) & & & \\
\hline \multicolumn{8}{|c|}{ 14.8.1 Paraprofessionals } \\
\hline Bass 2016 & 145 & $0.8(0.7)$ & 43 & $1(0.8)$ & & $70.97 \%$ & $-0.26[-0.61,0.08]$ \\
\hline Ertl 2011 & 26 & $3.1(3)$ & 28 & $3.2(3)$ & 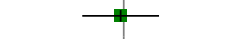 & $29.03 \%$ & $-0.04[-0.58,0.49]$ \\
\hline Subtotal $* \star \star$ & 171 & & 71 & & & $100 \%$ & $-0.2[-0.49,0.09]$ \\
\hline \multicolumn{8}{|c|}{ Heterogeneity: Tau $^{2}=0 ; \mathrm{Chi}^{2}=0.47, \mathrm{df}=1(\mathrm{P}=0.49) ; \mathrm{I}^{2}=0 \%$} \\
\hline
\end{tabular}

Analysis 14.9. Comparison 14 Subgroup analysis: type of interventionists - adults, Outcome 9 Dropout.

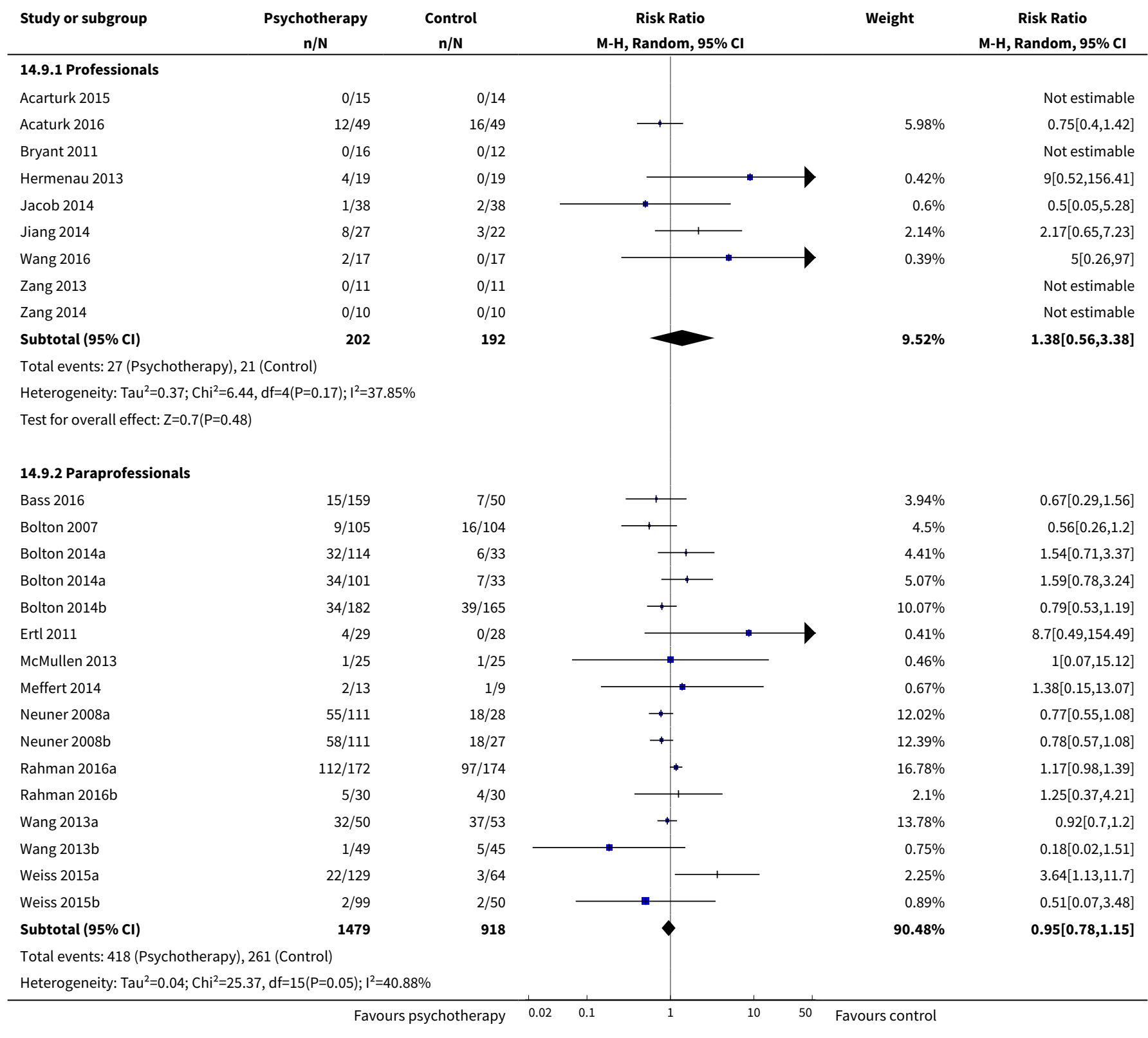

Psychological therapies for the treatment of mental disorders in low- and middle-income countries affected by humanitarian crises 


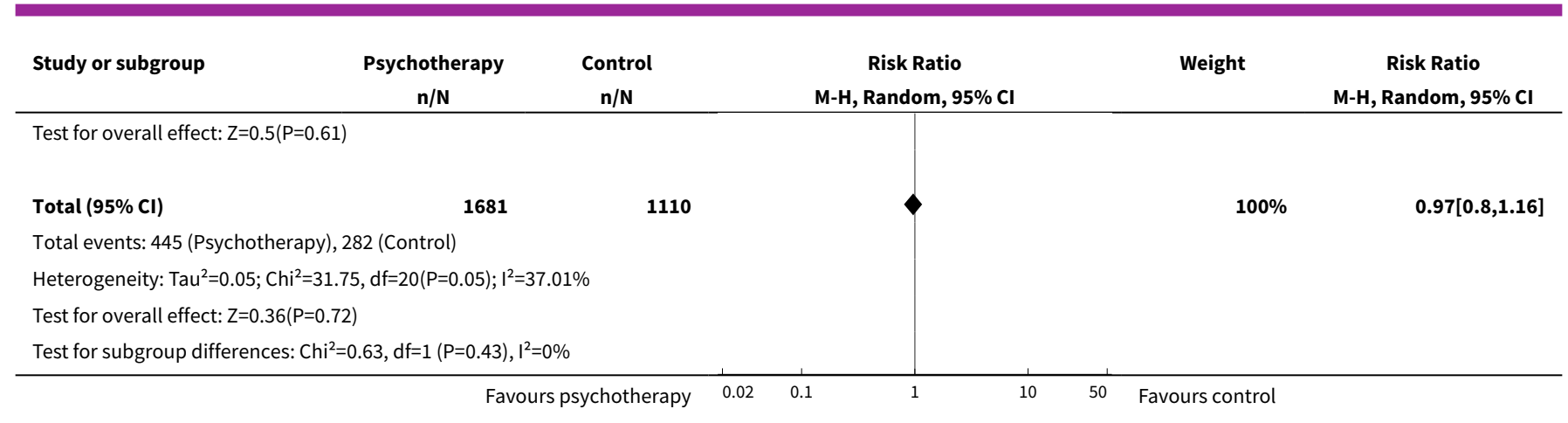

Analysis 14.10. Comparison 14 Subgroup analysis: type of interventionists - adults, Outcome 10 Functional impairment at endpoint.

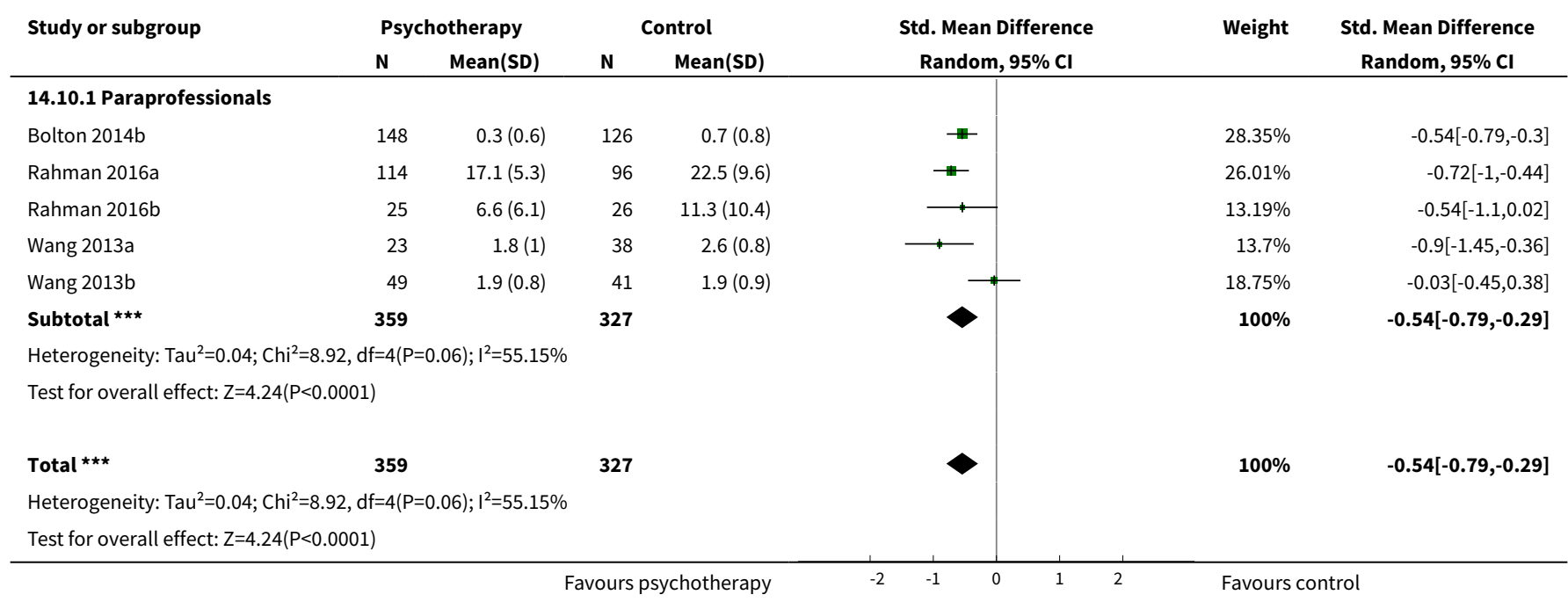

\section{Analysis 14.11. Comparison 14 Subgroup analysis: type of interventionists - adults, Outcome 11 Functional impairment at 1 to 4 months.}

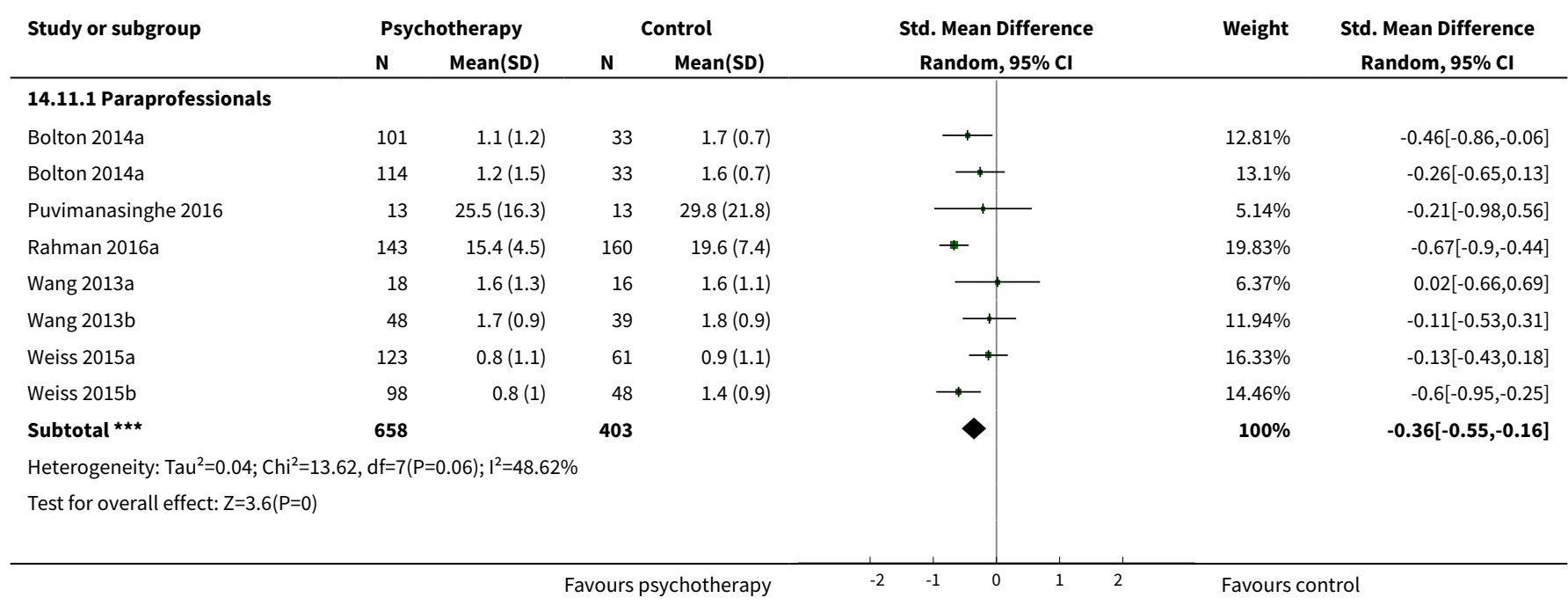




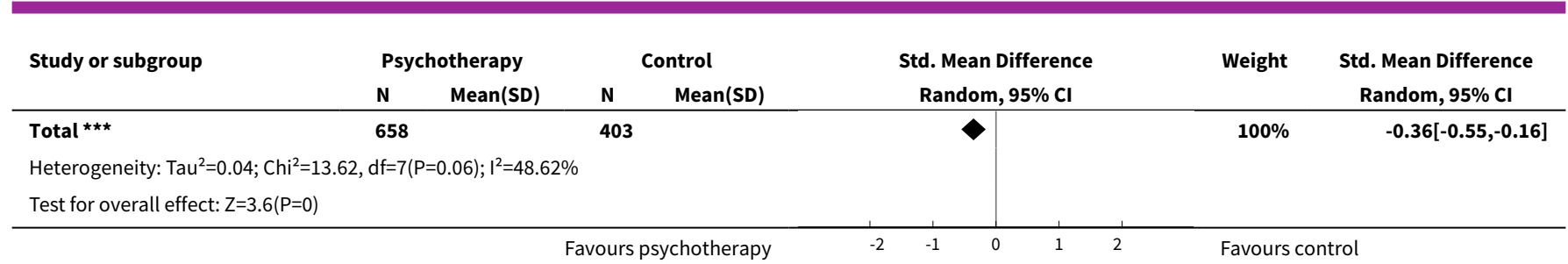

Analysis 14.12. Comparison 14 Subgroup analysis: type of interventionists - adults, Outcome 12 Quality of life at endpoint.

\begin{tabular}{|c|c|c|c|c|c|c|c|}
\hline \multirow[t]{2}{*}{ Study or subgroup } & \multicolumn{2}{|c|}{ Psychotherapy } & \multicolumn{2}{|c|}{ Control } & \multirow{2}{*}{$\begin{array}{c}\text { Std. Mean Difference } \\
\text { Random, } 95 \% \mathrm{Cl}\end{array}$} & \multirow[t]{2}{*}{ Weight } & \multirow{2}{*}{$\begin{array}{c}\text { Std. Mean Difference } \\
\text { Random, } 95 \% \mathrm{Cl}\end{array}$} \\
\hline & $\mathbf{N}$ & Mean(SD) & $\mathbf{N}$ & Mean(SD) & & & \\
\hline \multicolumn{8}{|c|}{ 14.12.1 Professionals } \\
\hline Ahmadizadeh 2013 & 75 & $-51.4(65.2)$ & 25 & $-45.7(3.2)$ & - & $34.37 \%$ & $-0.1[-0.55,0.35]$ \\
\hline Jiang 2014 & 19 & $-19.9(6.1)$ & 19 & $-15.1(4.6)$ & $\longrightarrow$ & $26.06 \%$ & $-0.88[-1.55,-0.21]$ \\
\hline Knaevelsrud 2015 & 79 & $-3(1)$ & 80 & $-2.3(0.7)$ & $\mp$ & $39.56 \%$ & $-0.83[-1.16,-0.51]$ \\
\hline 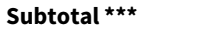 & 173 & & 124 & & & $100 \%$ & $-0.59[-1.1,-0.08]$ \\
\hline \multicolumn{8}{|c|}{ Test for overall effect: $Z=2.27(P=0.02)$} \\
\hline Total $\star \star \star$ & 173 & & 124 & & & $100 \%$ & $-0.59[-1.1,-0.08]$ \\
\hline \multicolumn{8}{|c|}{ Heterogeneity: $\mathrm{Tau}^{2}=0.14 ; \mathrm{Chi}^{2}=7.25, \mathrm{df}=2(\mathrm{P}=0.03) ; \mathrm{I}^{2}=72.42 \%$} \\
\hline \multicolumn{8}{|c|}{ Test for overall effect: $Z=2.27(P=0.02)$} \\
\hline & & & fours & chotherapy & -1 & Favours & trol \\
\hline
\end{tabular}

Analysis 14.13. Comparison 14 Subgroup analysis: type of interventionists - adults, Outcome 13 Diagnosis of PTSD.

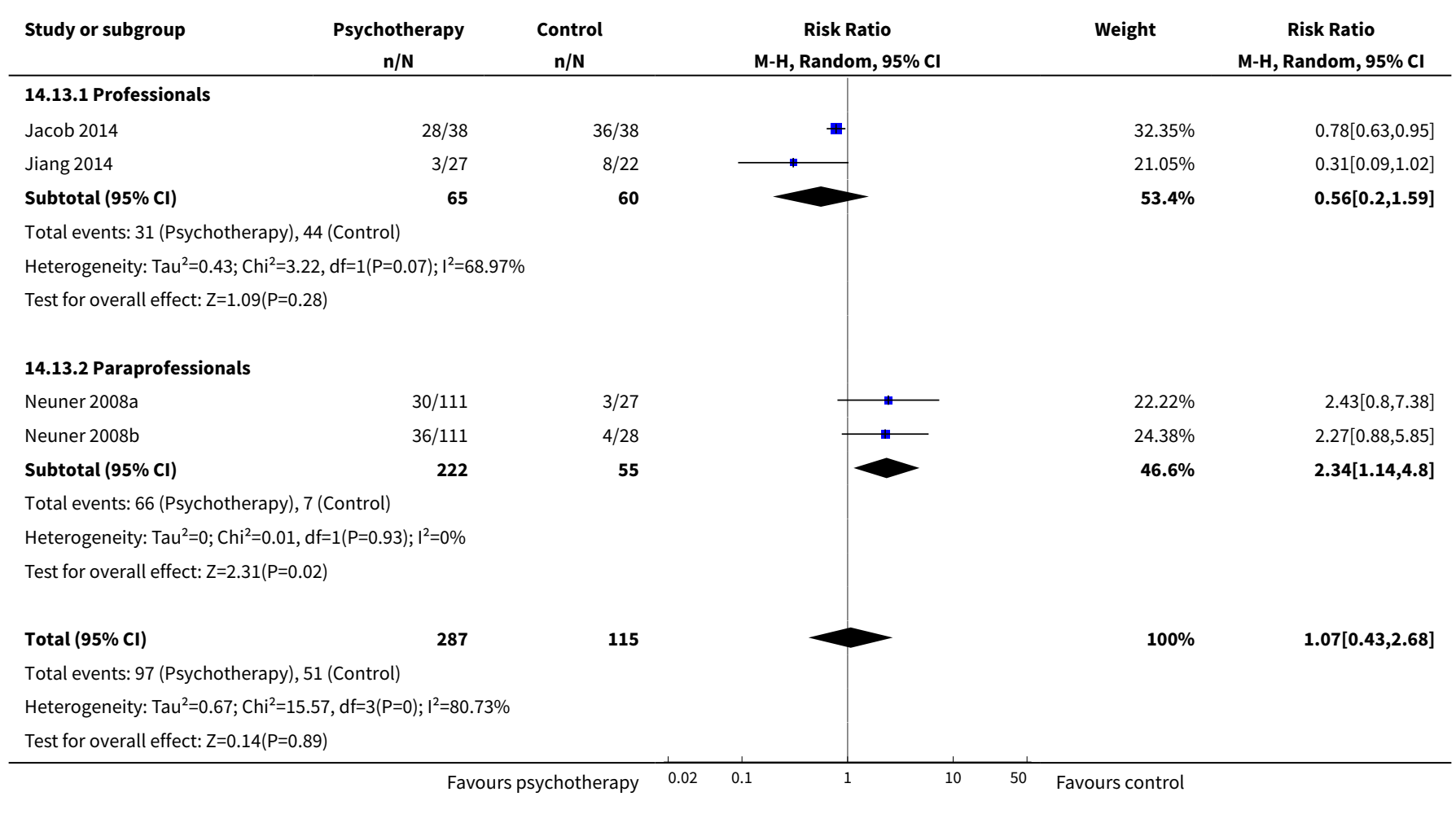




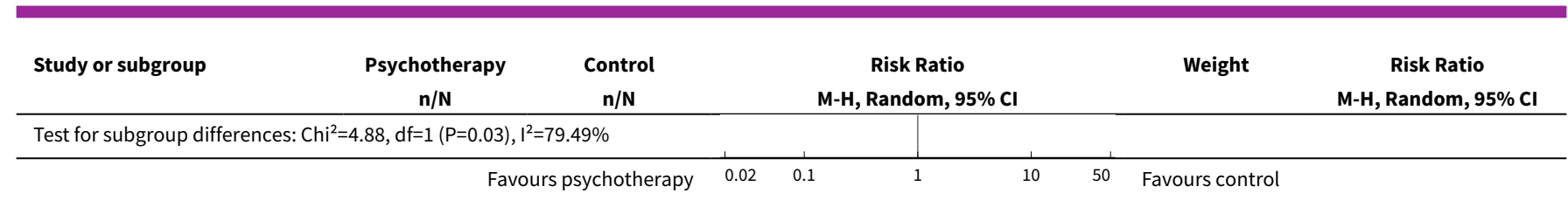

\section{Comparison 15. Subgroup analysis: type of control - adults}

\begin{tabular}{|c|c|c|c|c|}
\hline $\begin{array}{l}\text { Outcome or subgroup ti- } \\
\text { tle }\end{array}$ & No. of studies & $\begin{array}{l}\text { No. of partici- } \\
\text { pants }\end{array}$ & Statistical method & Effect size \\
\hline $\begin{array}{l}1 \text { PTSD symptoms at end- } \\
\text { point }\end{array}$ & 16 & 1272 & $\begin{array}{l}\text { Std. Mean Difference (IV, Random, 95\% } \\
\text { Cl) }\end{array}$ & $-1.07[-1.34,-0.79]$ \\
\hline 1.1 EMDR vs wait list & 2 & 99 & $\begin{array}{l}\text { Std. Mean Difference (IV, Random, 95\% } \\
\text { Cl) }\end{array}$ & $-2.01[-2.50,-1.52]$ \\
\hline 1.2 IPT vs wait list & 1 & 20 & $\begin{array}{l}\text { Std. Mean Difference (IV, Random, 95\% } \\
\text { CI) }\end{array}$ & $-1.45[-2.46,-0.43]$ \\
\hline 1.3 CBT vs wait list & 8 & 696 & $\begin{array}{l}\text { Std. Mean Difference (IV, Random, 95\% } \\
\text { CI) }\end{array}$ & $-1.08[-1.47,-0.68]$ \\
\hline 1.4 CBT vs no treatment & 2 & 52 & $\begin{array}{l}\text { Std. Mean Difference (IV, Random, 95\% } \\
\text { CI) }\end{array}$ & $-0.43[-0.98,0.12]$ \\
\hline 1.5 TFT vs wait list & 1 & 145 & $\begin{array}{l}\text { Std. Mean Difference (IV, Random, 95\% } \\
\text { CI) }\end{array}$ & $-1.27[-1.63,-0.91]$ \\
\hline $\begin{array}{l}1.6 \text { CBT vs TAU/enhanced } \\
\text { usual care }\end{array}$ & 2 & 260 & $\begin{array}{l}\text { Std. Mean Difference (IV, Random, 95\% } \\
\text { Cl) }\end{array}$ & $-0.56[-0.81,-0.31]$ \\
\hline $\begin{array}{l}2 \text { PTSD symptoms at } 1 \text { to } 4 \\
\text { months }\end{array}$ & 17 & 1590 & $\begin{array}{l}\text { Std. Mean Difference (IV, Random, 95\% } \\
\text { Cl) }\end{array}$ & $-0.49[-0.68,-0.31]$ \\
\hline 2.1 EMDR vs wait list & 1 & 64 & $\begin{array}{l}\text { Std. Mean Difference (IV, Random, 95\% } \\
\text { CI) }\end{array}$ & $-1.76[-2.34,-1.18]$ \\
\hline 2.2 CBT vs wait list & 13 & 1160 & $\begin{array}{l}\text { Std. Mean Difference (IV, Random, 95\% } \\
\text { CI) }\end{array}$ & $-0.35[-0.49,-0.21]$ \\
\hline 2.3 IPT vs TAU & 1 & 38 & $\begin{array}{l}\text { Std. Mean Difference (IV, Random, 95\% } \\
\text { Cl) }\end{array}$ & $-0.99[-1.67,-0.32]$ \\
\hline 2.4 CBT vs no treatment & 1 & 22 & $\begin{array}{l}\text { Std. Mean Difference (IV, Random, 95\% } \\
\text { CI) }\end{array}$ & $-1.12[-2.04,-0.21]$ \\
\hline $\begin{array}{l}2.5 \text { CBT vs TAU/enhanced } \\
\text { usual care }\end{array}$ & 1 & 306 & $\begin{array}{l}\text { Std. Mean Difference (IV, Random, 95\% } \\
\text { CI) }\end{array}$ & $-0.63[-0.86,-0.40]$ \\
\hline $\begin{array}{l}3 \text { PTSD symptoms } \geq 6 \\
\text { months }\end{array}$ & 5 & 400 & $\begin{array}{l}\text { Std. Mean Difference (IV, Random, 95\% } \\
\text { CI) }\end{array}$ & $-0.37[-0.61,-0.14]$ \\
\hline $\begin{array}{l}\text { 3.1 Trauma/Supportive } \\
\text { counselling vs wait list }\end{array}$ & 1 & 188 & $\begin{array}{l}\text { Std. Mean Difference (IV, Random, 95\% } \\
\text { CI) }\end{array}$ & $-0.22[-0.57,0.12]$ \\
\hline
\end{tabular}




\begin{tabular}{|c|c|c|c|c|}
\hline $\begin{array}{l}\text { Outcome or subgroup ti- } \\
\text { tle }\end{array}$ & No. of studies & $\begin{array}{l}\text { No. of partici- } \\
\text { pants }\end{array}$ & Statistical method & Effect size \\
\hline $\begin{array}{l}3.2 \text { Trauma/Supportive } \\
\text { counselling vs no treat- } \\
\text { ment }\end{array}$ & 1 & 62 & $\begin{array}{l}\text { Std. Mean Difference (IV, Random, 95\% } \\
\mathrm{CI} \text { ) }\end{array}$ & $-0.74[-1.46,-0.02]$ \\
\hline 3.3 CBT vs no treatment & 3 & 150 & $\begin{array}{l}\text { Std. Mean Difference (IV, Random, 95\% } \\
\mathrm{CI} \text { ) }\end{array}$ & $-0.45[-0.82,-0.09]$ \\
\hline $\begin{array}{l}4 \text { Anxiety symptoms at } \\
\text { endpoint }\end{array}$ & 4 & 535 & $\begin{array}{l}\text { Std. Mean Difference (IV, Random, 95\% } \\
\mathrm{CI} \text { ) }\end{array}$ & $-0.76[-1.10,-0.42]$ \\
\hline 4.1 CBT vs wait list & 3 & 326 & $\begin{array}{l}\text { Std. Mean Difference (IV, Random, 95\% } \\
\mathrm{CI} \text { ) }\end{array}$ & $-0.92[-1.61,-0.23]$ \\
\hline $\begin{array}{l}\text { 4.2 CBT vs TAU/enhanced } \\
\text { usual care }\end{array}$ & 1 & 209 & $\begin{array}{l}\text { Std. Mean Difference (IV, Random, 95\% } \\
\mathrm{Cl} \text { ) }\end{array}$ & $-0.74[-1.02,-0.45]$ \\
\hline $\begin{array}{l}5 \text { Anxiety symptoms at } 1 \text { to } \\
4 \text { months }\end{array}$ & 6 & 969 & $\begin{array}{l}\text { Std. Mean Difference (IV, Random, 95\% } \\
\mathrm{Cl} \text { ) }\end{array}$ & $-0.43[-0.71,-0.15]$ \\
\hline 5.1 CBT vs wait list & 5 & 663 & $\begin{array}{l}\text { Std. Mean Difference (IV, Random, 95\% } \\
\mathrm{Cl} \text { ) }\end{array}$ & $-0.35[-0.67,-0.03]$ \\
\hline $\begin{array}{l}5.2 \text { CBT vs TAU/enhanced } \\
\text { usual care }\end{array}$ & 1 & 306 & $\begin{array}{l}\text { Std. Mean Difference (IV, Random, 95\% } \\
\mathrm{CI} \text { ) }\end{array}$ & $-0.74[-0.97,-0.51]$ \\
\hline $\begin{array}{l}6 \text { Anxiety symptoms } \geq 6 \\
\text { months }\end{array}$ & 1 & 188 & $\begin{array}{l}\text { Std. Mean Difference (IV, Random, 95\% } \\
\mathrm{CI} \text { ) }\end{array}$ & $-0.18[-0.52,0.16]$ \\
\hline $\begin{array}{l}6.1 \text { Trauma/Supportive } \\
\text { counselling vs wait list }\end{array}$ & 1 & 188 & $\begin{array}{l}\text { Std. Mean Difference (IV, Random, 95\% } \\
\mathrm{CI} \text { ) }\end{array}$ & $-0.18[-0.52,0.16]$ \\
\hline $\begin{array}{l}7 \text { Depressive symptoms at } \\
\text { endpoint }\end{array}$ & 14 & 1254 & $\begin{array}{l}\text { Std. Mean Difference (IV, Random, 95\% } \\
\mathrm{CI} \text { ) }\end{array}$ & $-0.86[-1.06,-0.67]$ \\
\hline 7.1 EMDR vs wait list & 2 & 99 & $\begin{array}{l}\text { Std. Mean Difference (IV, Random, 95\% } \\
\mathrm{CI} \text { ) }\end{array}$ & $-1.43[-1.88,-0.99]$ \\
\hline 7.2 CBT vs wait list & 8 & 696 & $\begin{array}{l}\text { Std. Mean Difference (IV, Random, 95\% } \\
\mathrm{CI} \text { ) }\end{array}$ & $-0.85[-1.08,-0.61]$ \\
\hline 7.3 IPT vs wait list & 2 & 229 & $\begin{array}{l}\text { Std. Mean Difference (IV, Random, 95\% } \\
\mathrm{CI} \text { ) }\end{array}$ & $-0.84[-1.60,-0.08]$ \\
\hline 7.4 CBT vs no treatment & 1 & 22 & $\begin{array}{l}\text { Std. Mean Difference (IV, Random, 95\% } \\
\mathrm{CI} \text { ) }\end{array}$ & $0.11[-0.73,0.95]$ \\
\hline $\begin{array}{l}7.5 \text { CBT vs TAU/enhanced } \\
\text { usual care }\end{array}$ & 1 & 208 & $\begin{array}{l}\text { Std. Mean Difference (IV, Random, 95\% } \\
\mathrm{CI} \text { ) }\end{array}$ & $-0.89[-1.18,-0.60]$ \\
\hline $\begin{array}{l}8 \text { Depressive symptoms at } \\
1 \text { to } 4 \text { months }\end{array}$ & 15 & 1386 & $\begin{array}{l}\text { Std. Mean Difference (IV, Random, 95\% } \\
\mathrm{CI} \text { ) }\end{array}$ & $-0.42[-0.63,-0.21]$ \\
\hline 8.1 EMDR vs wait list & 1 & 64 & $\begin{array}{l}\text { Std. Mean Difference (IV, Random, 95\% } \\
\mathrm{CI} \text { ) }\end{array}$ & $-1.21[-1.74,-0.67]$ \\
\hline
\end{tabular}




\begin{tabular}{|c|c|c|c|c|}
\hline $\begin{array}{l}\text { Outcome or subgroup ti- } \\
\text { tle }\end{array}$ & No. of studies & $\begin{array}{l}\text { No. of partici- } \\
\text { pants }\end{array}$ & Statistical method & Effect size \\
\hline 8.2 CBT vs wait list & 11 & 956 & $\begin{array}{l}\text { Std. Mean Difference (IV, Random, 95\% } \\
\mathrm{Cl} \text { ) }\end{array}$ & $-0.27[-0.45,-0.08]$ \\
\hline 8.3 IPT vs TAU & 1 & 38 & $\begin{array}{l}\text { Std. Mean Difference (IV, Random, 95\% } \\
\mathrm{Cl} \text { ) }\end{array}$ & $-0.77[-1.43,-0.11]$ \\
\hline 8.4 CBT vs no treatment & 1 & 22 & $\begin{array}{l}\text { Std. Mean Difference (IV, Random, 95\% } \\
\mathrm{CI})\end{array}$ & $-1.07[-1.98,-0.16]$ \\
\hline $\begin{array}{l}\text { 8.5 CBT vs TAU/enhanced } \\
\text { usual care }\end{array}$ & 1 & 306 & $\begin{array}{l}\text { Std. Mean Difference (IV, Random, 95\% } \\
\mathrm{CI} \text { ) }\end{array}$ & $-0.85[-1.08,-0.62]$ \\
\hline $\begin{array}{l}9 \text { Depressive symptoms } \geq \\
6 \text { months }\end{array}$ & 2 & 242 & $\begin{array}{l}\text { Std. Mean Difference (IV, Random, 95\% } \\
\mathrm{CI} \text { ) }\end{array}$ & $-0.20[-0.49,0.09]$ \\
\hline $\begin{array}{l}\text { 9.1 Trauma/Supportive } \\
\text { counselling vs wait list }\end{array}$ & 1 & 188 & $\begin{array}{l}\text { Std. Mean Difference (IV, Random, 95\% } \\
\mathrm{Cl} \text { ) }\end{array}$ & $-0.26[-0.61,0.08]$ \\
\hline 9.2 CBT vs no treatment & 1 & 54 & $\begin{array}{l}\text { Std. Mean Difference (IV, Random, 95\% } \\
\mathrm{CI} \text { ) }\end{array}$ & $-0.04[-0.58,0.49]$ \\
\hline 10 Dropout & 25 & 2801 & Risk Ratio (M-H, Random, 95\% Cl) & $0.98[0.81,1.19]$ \\
\hline 10.1 EMDR vs wait list & 2 & 127 & Risk Ratio (M-H, Random, 95\% Cl) & $0.75[0.40,1.42]$ \\
\hline 10.2 CBT vs wait list & 14 & 1575 & Risk Ratio (M-H, Random, 95\% Cl) & $1.02[0.76,1.38]$ \\
\hline $\begin{array}{l}10.3 \text { CBT vs TAU/enhanced } \\
\text { usual care }\end{array}$ & 2 & 406 & Risk Ratio $(\mathrm{M}-\mathrm{H}$, Random, 95\% Cl) & $1.17[0.99,1.39]$ \\
\hline 10.4 IPT vs wait list & 2 & 231 & Risk Ratio $(\mathrm{M}-\mathrm{H}$, Random, 95\% Cl) & $0.61[0.30,1.27]$ \\
\hline 10.5 CBT vs no treatment & 2 & 66 & Risk Ratio (M-H, Random, 95\% Cl) & $9.47[1.29,69.52]$ \\
\hline $\begin{array}{l}10.6 \text { Trauma/Supportive } \\
\text { counselling vs no treat- } \\
\text { ment }\end{array}$ & 1 & 138 & Risk Ratio $(\mathrm{M}-\mathrm{H}$, Random, 95\% Cl) & $0.78[0.57,1.08]$ \\
\hline 10.7 IPT vs TAU & 1 & 49 & Risk Ratio (M-H, Random, 95\% Cl) & $2.17[0.65,7.23]$ \\
\hline $\begin{array}{l}\text { 10.8 Trauma/Supportive } \\
\text { counselling vs wait list }\end{array}$ & 1 & 209 & Risk Ratio (M-H, Random, 95\% Cl) & $0.67[0.29,1.56]$ \\
\hline $\begin{array}{l}11 \text { Functional impairment } \\
\text { at endpoint }\end{array}$ & 5 & 686 & $\begin{array}{l}\text { Std. Mean Difference (IV, Random, 95\% } \\
\mathrm{Cl} \text { ) }\end{array}$ & $-0.55[-0.80,-0.30]$ \\
\hline 11.1 CBT vs wait list & 3 & 425 & $\begin{array}{l}\text { Std. Mean Difference (IV, Random, 95\% } \\
\mathrm{Cl} \text { ) }\end{array}$ & $-0.48[-0.91,-0.06]$ \\
\hline $\begin{array}{l}11.2 \text { CBT vs TAU/enhanced } \\
\text { usual care }\end{array}$ & 2 & 261 & $\begin{array}{l}\text { Std. Mean Difference (IV, Random, 95\% } \\
\mathrm{CI} \text { ) }\end{array}$ & $-0.68[-0.93,-0.43]$ \\
\hline $\begin{array}{l}12 \text { Functional impairment } \\
\text { at } 1 \text { to } 4 \text { months }\end{array}$ & 7 & 1061 & $\begin{array}{l}\text { Std. Mean Difference (IV, Random, 95\% } \\
\mathrm{Cl} \text { ) }\end{array}$ & $-0.36[-0.55,-0.16]$ \\
\hline
\end{tabular}




\begin{tabular}{|c|c|c|c|c|}
\hline $\begin{array}{l}\text { Outcome or subgroup ti- } \\
\text { tle }\end{array}$ & No. of studies & $\begin{array}{l}\text { No. of partici- } \\
\text { pants }\end{array}$ & Statistical method & Effect size \\
\hline 12.1 CBT vs wait list & 6 & 758 & $\begin{array}{l}\text { Std. Mean Difference (IV, Random, 95\% } \\
\text { Cl) }\end{array}$ & $-0.29[-0.45,-0.12]$ \\
\hline $\begin{array}{l}12.2 \text { CBT vs TAU/enhanced } \\
\text { usual care }\end{array}$ & 1 & 303 & $\begin{array}{l}\text { Std. Mean Difference (IV, Random, 95\% } \\
\mathrm{CI} \text { ) }\end{array}$ & $-0.67[-0.90,-0.44]$ \\
\hline $\begin{array}{l}13 \text { Functional impairment } \\
\geq 6 \text { months }\end{array}$ & 1 & 188 & $\begin{array}{l}\text { Std. Mean Difference (IV, Random, 95\% } \\
\mathrm{Cl} \text { ) }\end{array}$ & $-0.25[-0.59,0.09]$ \\
\hline $\begin{array}{l}\text { 13.1 Trauma/Supportive } \\
\text { counselling vs wait list }\end{array}$ & 1 & 188 & $\begin{array}{l}\text { Std. Mean Difference (IV, Random, 95\% } \\
\mathrm{CI} \text { ) }\end{array}$ & $-0.25[-0.59,0.09]$ \\
\hline $\begin{array}{l}14 \text { Quality of life at end- } \\
\text { point }\end{array}$ & 4 & 325 & $\begin{array}{l}\text { Std. Mean Difference (IV, Random, 95\% } \\
\mathrm{CI} \text { ) }\end{array}$ & $-0.73[-1.22,-0.25]$ \\
\hline $\begin{array}{l}\text { 14.1 CBT vs wait list - } \\
\text { Adults }\end{array}$ & 3 & 287 & $\begin{array}{l}\text { Std. Mean Difference (IV, Random, 95\% } \\
\mathrm{CI} \text { ) }\end{array}$ & $-0.71[-1.35,-0.07]$ \\
\hline 14.2 IPT vs wait list - Adults & 1 & 38 & $\begin{array}{l}\text { Std. Mean Difference (IV, Random, 95\% } \\
\mathrm{CI} \text { ) }\end{array}$ & $-0.88[-1.55,-0.21]$ \\
\hline 15 Diagnosis of PTSD & 4 & 402 & Risk Ratio (M-H, Random, 95\% Cl) & $1.07[0.43,2.68]$ \\
\hline $\begin{array}{l}\text { 15.1 Trauma/Supportive } \\
\text { counselling vs wait list }\end{array}$ & 1 & 139 & Risk Ratio (M-H, Random, 95\% Cl) & $2.27[0.88,5.85]$ \\
\hline 15.2 IPT vs TAU & 1 & 49 & Risk Ratio (M-H, Random, 95\% Cl) & $0.31[0.09,1.02]$ \\
\hline 15.3 CBT vs wait list & 2 & 214 & Risk Ratio (M-H, Random, 95\% Cl) & $1.28[0.28,5.77]$ \\
\hline 16 Diagnosis of depression & 1 & 49 & Risk Ratio (M-H, Random, 95\% Cl) & $0.30[0.11,0.80]$ \\
\hline 16.1 IPT vs wait list & 1 & 49 & Risk Ratio (M-H, Random, 95\% Cl) & $0.30[0.11,0.80]$ \\
\hline 17 Coping at endpoint & 2 & 151 & $\begin{array}{l}\text { Std. Mean Difference (IV, Random, 95\% } \\
\mathrm{CI} \text { ) }\end{array}$ & $-0.27[-0.60,0.05]$ \\
\hline 17.1 CBT vs wait list & 2 & 151 & $\begin{array}{l}\text { Std. Mean Difference (IV, Random, 95\% } \\
\text { CI) }\end{array}$ & $-0.27[-0.60,0.05]$ \\
\hline 18 Coping at 1 to 4 months & 2 & 121 & $\begin{array}{l}\text { Std. Mean Difference (IV, Random, 95\% } \\
\mathrm{CI} \text { ) }\end{array}$ & $0.07[-0.49,0.64]$ \\
\hline 18.1 CBT vs wait list & 2 & 121 & $\begin{array}{l}\text { Std. Mean Difference (IV, Random, 95\% } \\
\mathrm{CI} \text { ) }\end{array}$ & $0.07[-0.49,0.64]$ \\
\hline
\end{tabular}

Analysis 15.1. Comparison 15 Subgroup analysis: type of control - adults, Outcome 1 PTSD symptoms at endpoint.

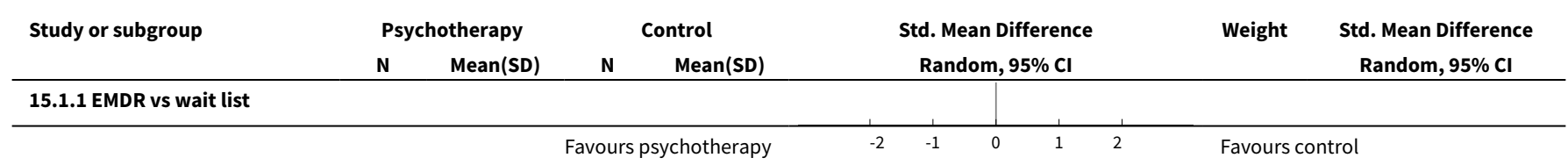




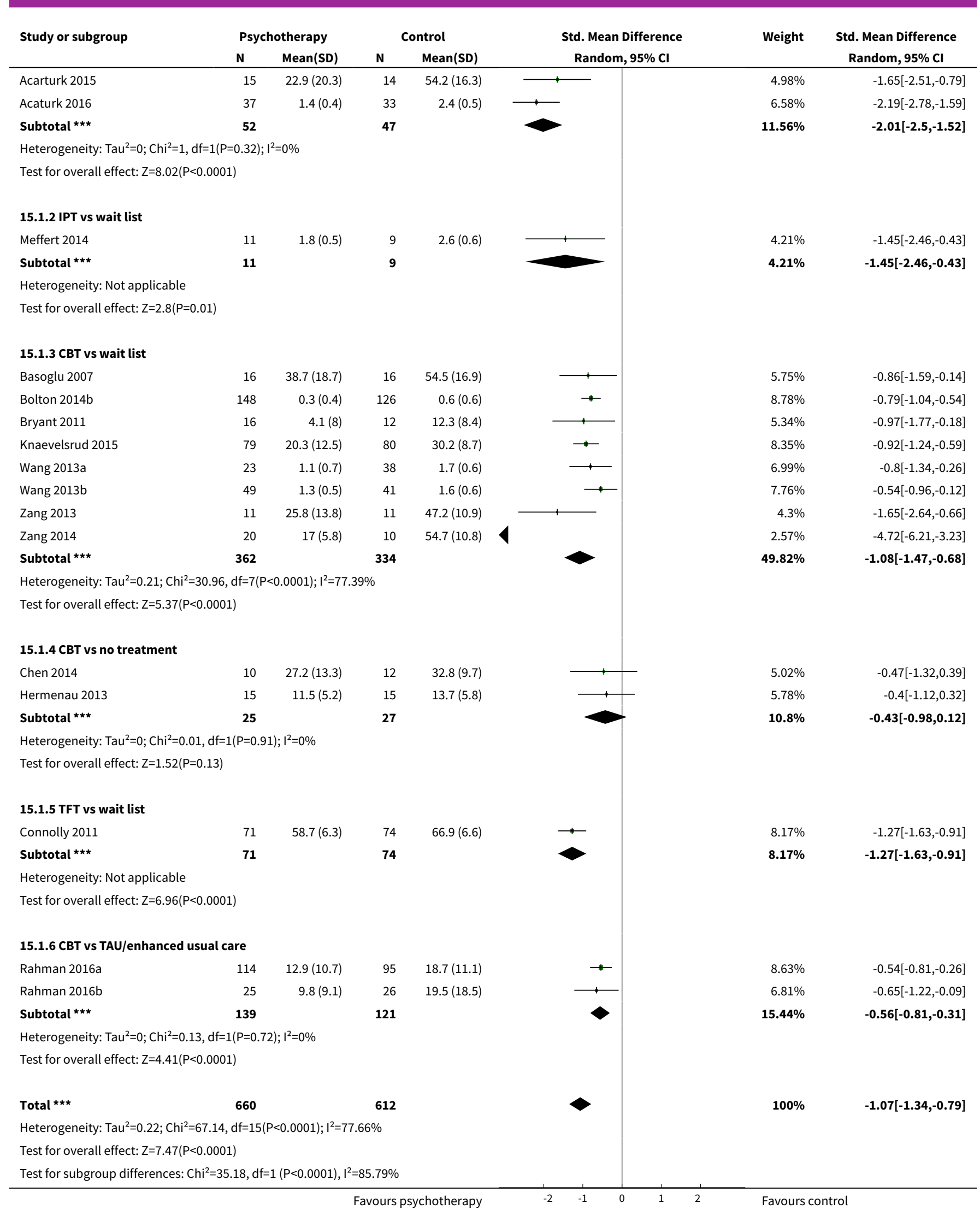

Psychological therapies for the treatment of mental disorders in low- and middle-income countries affected by humanitarian crises 
Analysis 15.2. Comparison 15 Subgroup analysis: type of control - adults, Outcome 2 PTSD symptoms at 1 to 4 months.

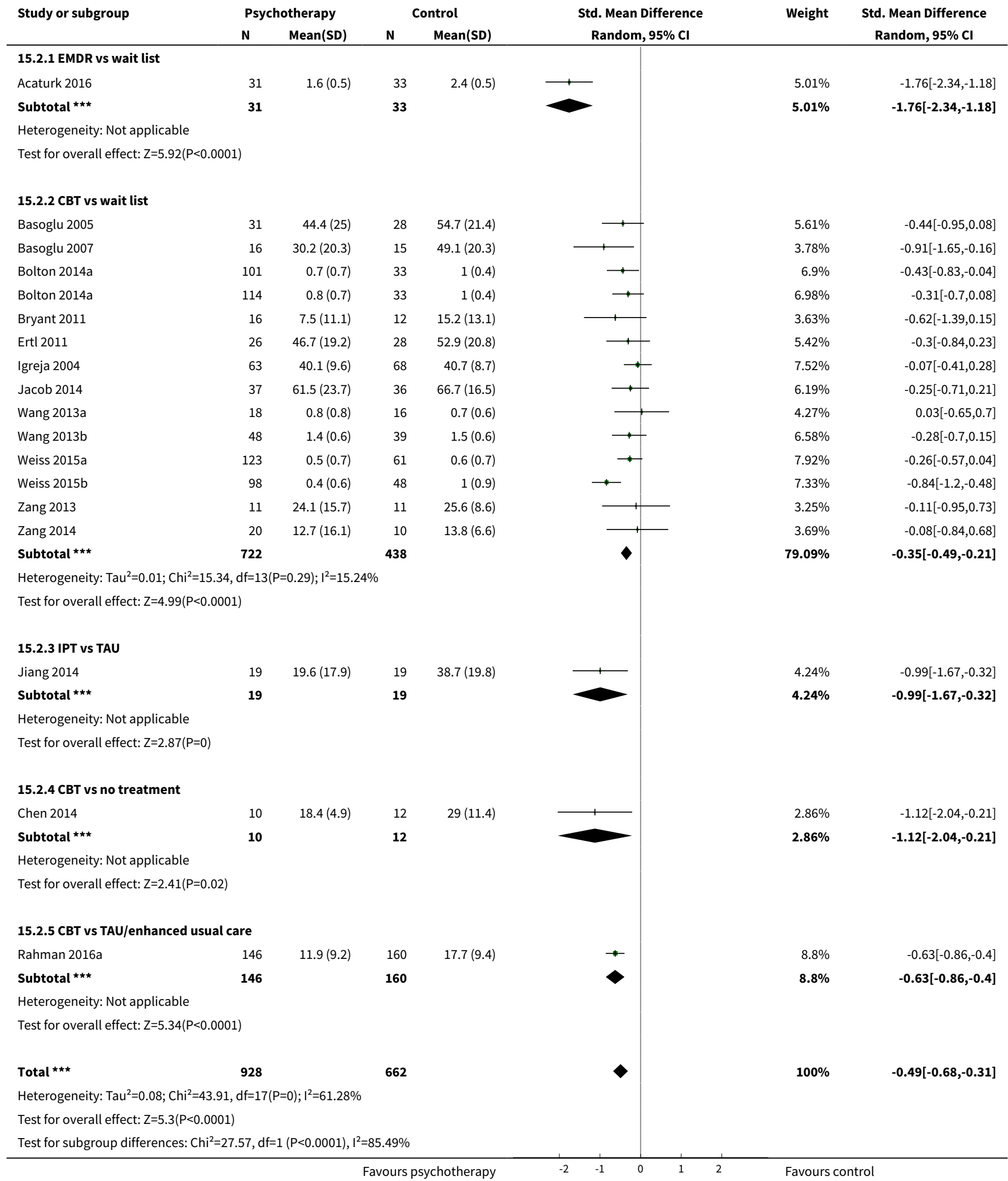

Psychological therapies for the treatment of mental disorders in low- and middle-income countries affected by humanitarian crises 
Analysis 15.3. Comparison 15 Subgroup analysis: type of control - adults, Outcome 3 PTSD symptoms $\geq 6$ months.

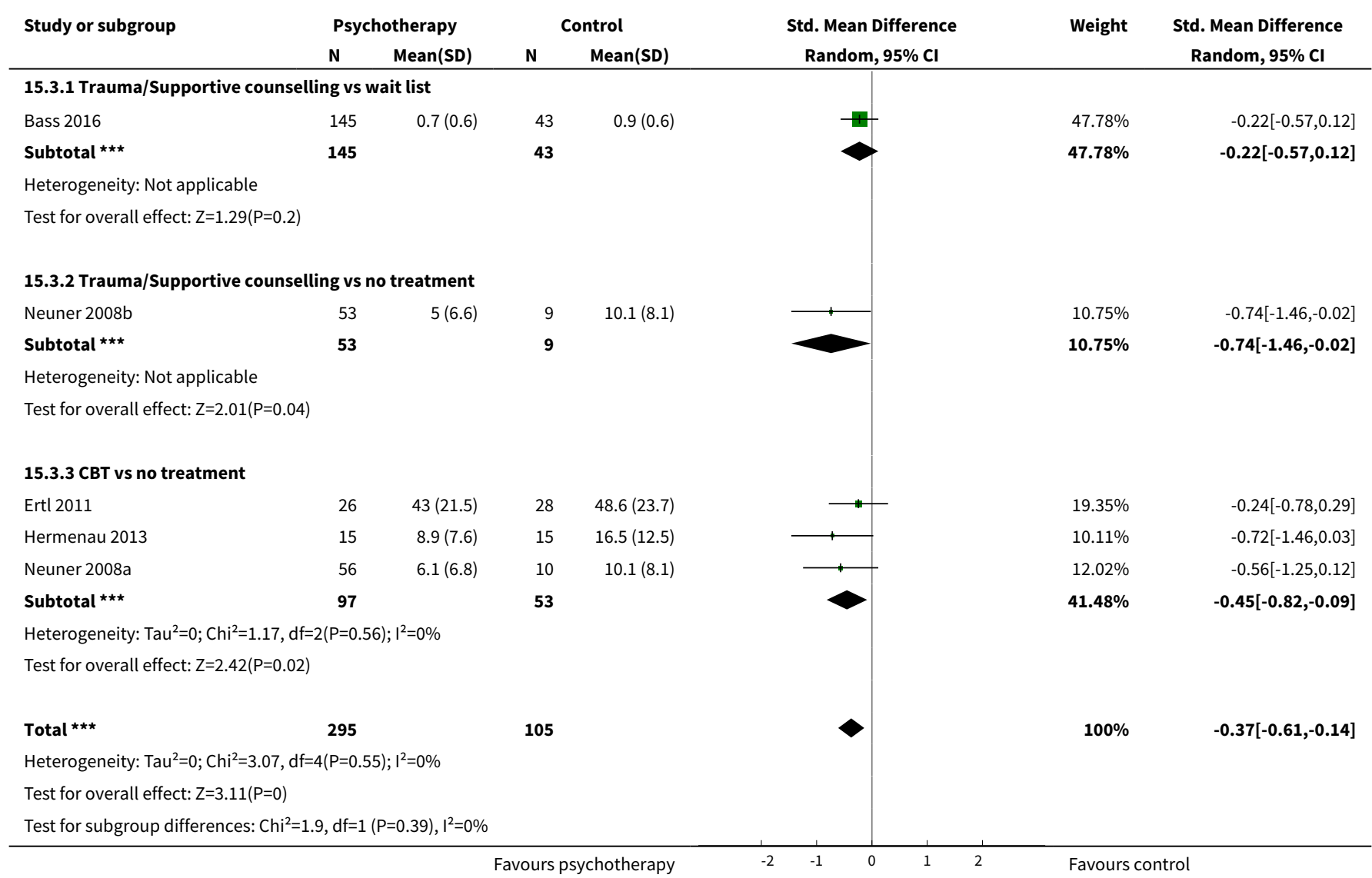

Analysis 15.4. Comparison 15 Subgroup analysis: type of control - adults, Outcome 4 Anxiety symptoms at endpoint.

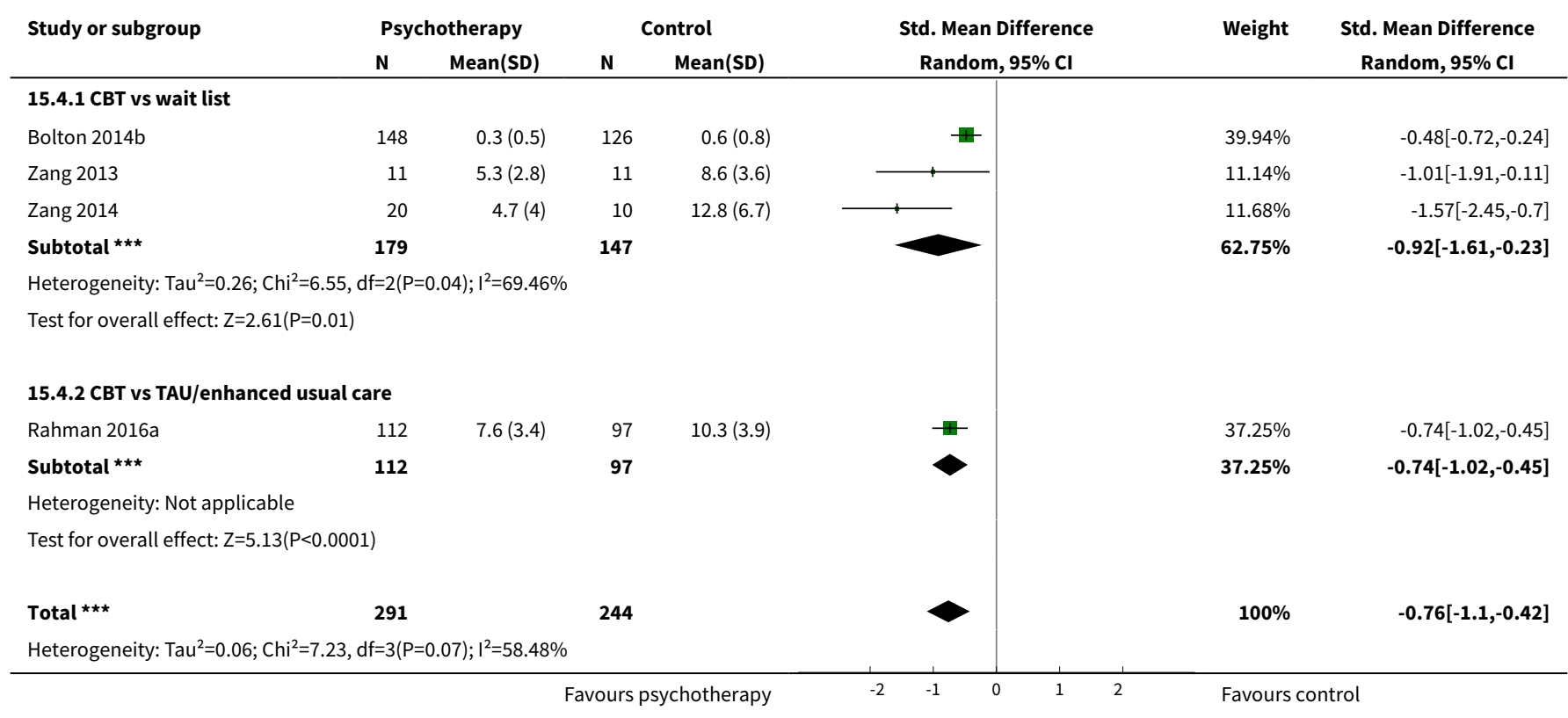

Psychological therapies for the treatment of mental disorders in low- and middle-income countries affected by humanitarian crises 


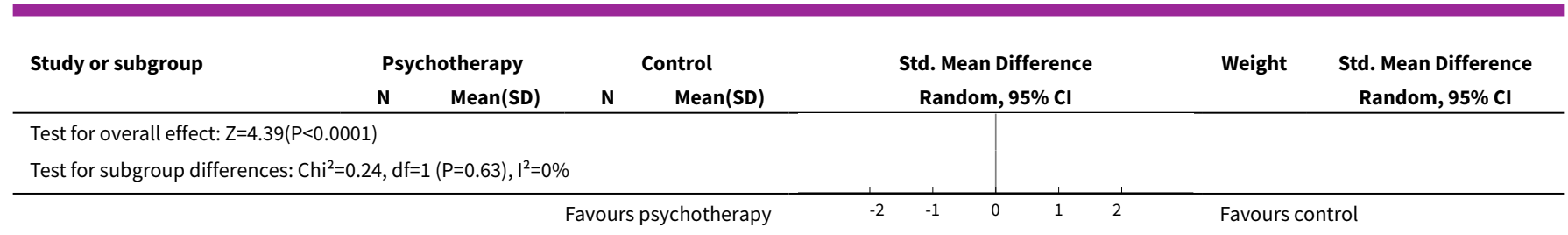

Analysis 15.5. Comparison 15 Subgroup analysis: type of control - adults, Outcome 5 Anxiety symptoms at 1 to 4 months.

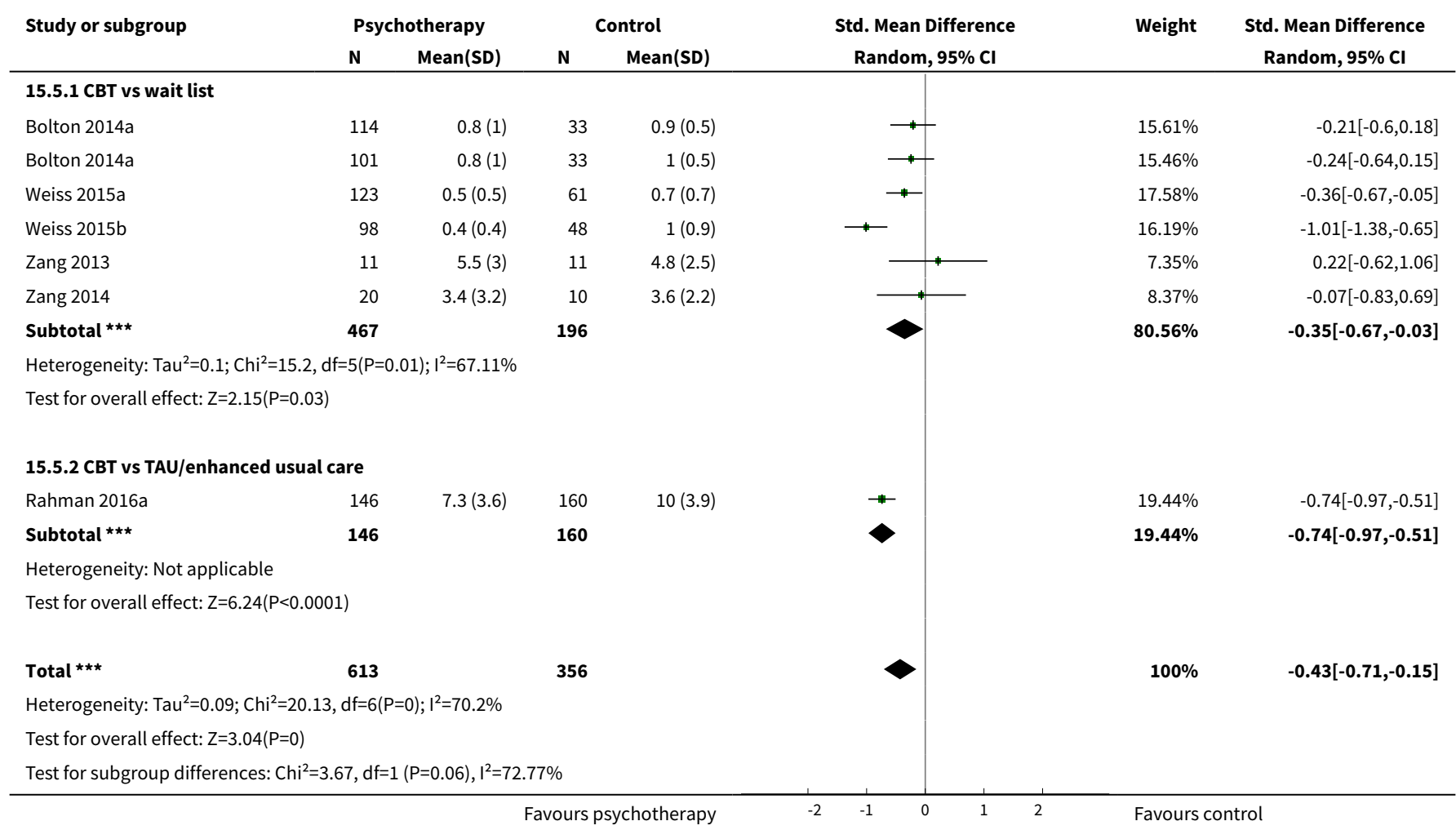

Analysis 15.6. Comparison 15 Subgroup analysis: type of control - adults, Outcome 6 Anxiety symptoms $\geq 6$ months.

\begin{tabular}{|c|c|c|c|c|c|c|c|}
\hline \multirow[t]{2}{*}{ Study or subgroup } & \multicolumn{2}{|c|}{ Psychotherapy } & \multicolumn{2}{|c|}{ Control } & \multirow{2}{*}{$\begin{array}{c}\text { Std. Mean Difference } \\
\text { Random, } 95 \% \mathrm{Cl}\end{array}$} & \multirow[t]{2}{*}{ Weight } & \multirow{2}{*}{$\begin{array}{c}\text { Std. Mean Difference } \\
\text { Random, } 95 \% \mathrm{Cl}\end{array}$} \\
\hline & $\mathbf{N}$ & $\operatorname{Mean}(S D)$ & $\mathbf{N}$ & Mean(SD) & & & \\
\hline \multicolumn{8}{|c|}{ 15.6.1 Trauma/Supportive counselling vs wait list } \\
\hline Bass 2016 & 145 & $0.7(0.8)$ & 43 & $0.8(0.8)$ & & $100 \%$ & $-0.18[-0.52,0.16]$ \\
\hline Subtotal ${ }^{\star \star \star}$ & 145 & & 43 & & & $100 \%$ & $-0.18[-0.52,0.16]$ \\
\hline \multicolumn{8}{|c|}{ Heterogeneity: Not applicable } \\
\hline \multicolumn{8}{|c|}{ Test for overall effect: $Z=1.05(P=0.29)$} \\
\hline Total $\star \star \star$ & 145 & & 43 & & & $100 \%$ & $-0.18[-0.52,0.16]$ \\
\hline \multicolumn{8}{|c|}{ Heterogeneity: Not applicable } \\
\hline \multicolumn{8}{|c|}{ Test for overall effect: $Z=1.05(P=0.29)$} \\
\hline & & & 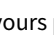 & hotherapy & -1 & Favours & \\
\hline
\end{tabular}


Analysis 15.7. Comparison 15 Subgroup analysis: type of control - adults, Outcome 7 Depressive symptoms at endpoint.

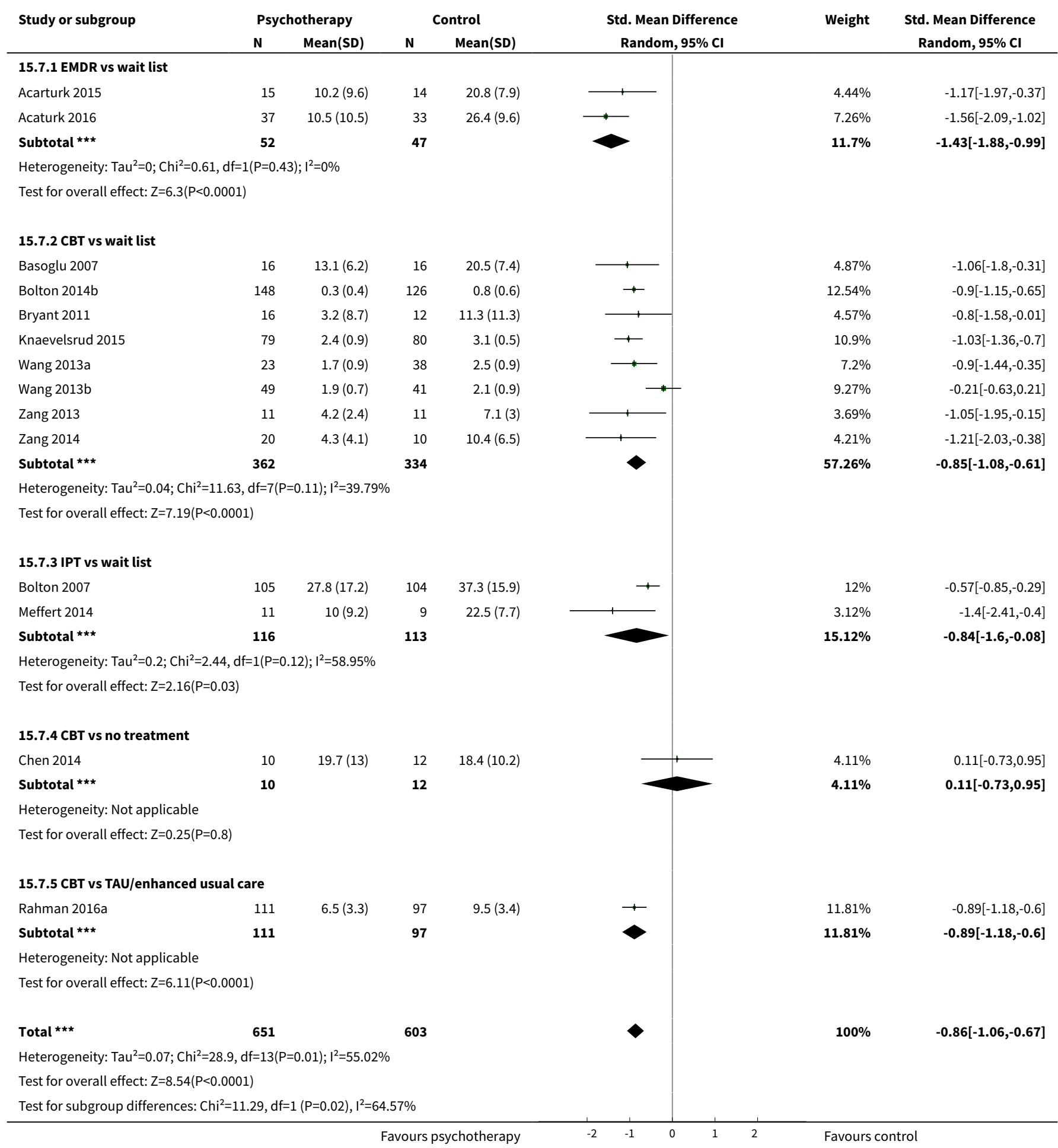

Psychological therapies for the treatment of mental disorders in low- and middle-income countries affected by humanitarian crises 


\section{Analysis 15.8. Comparison 15 Subgroup analysis: type of control} - adults, Outcome 8 Depressive symptoms at 1 to 4 months.

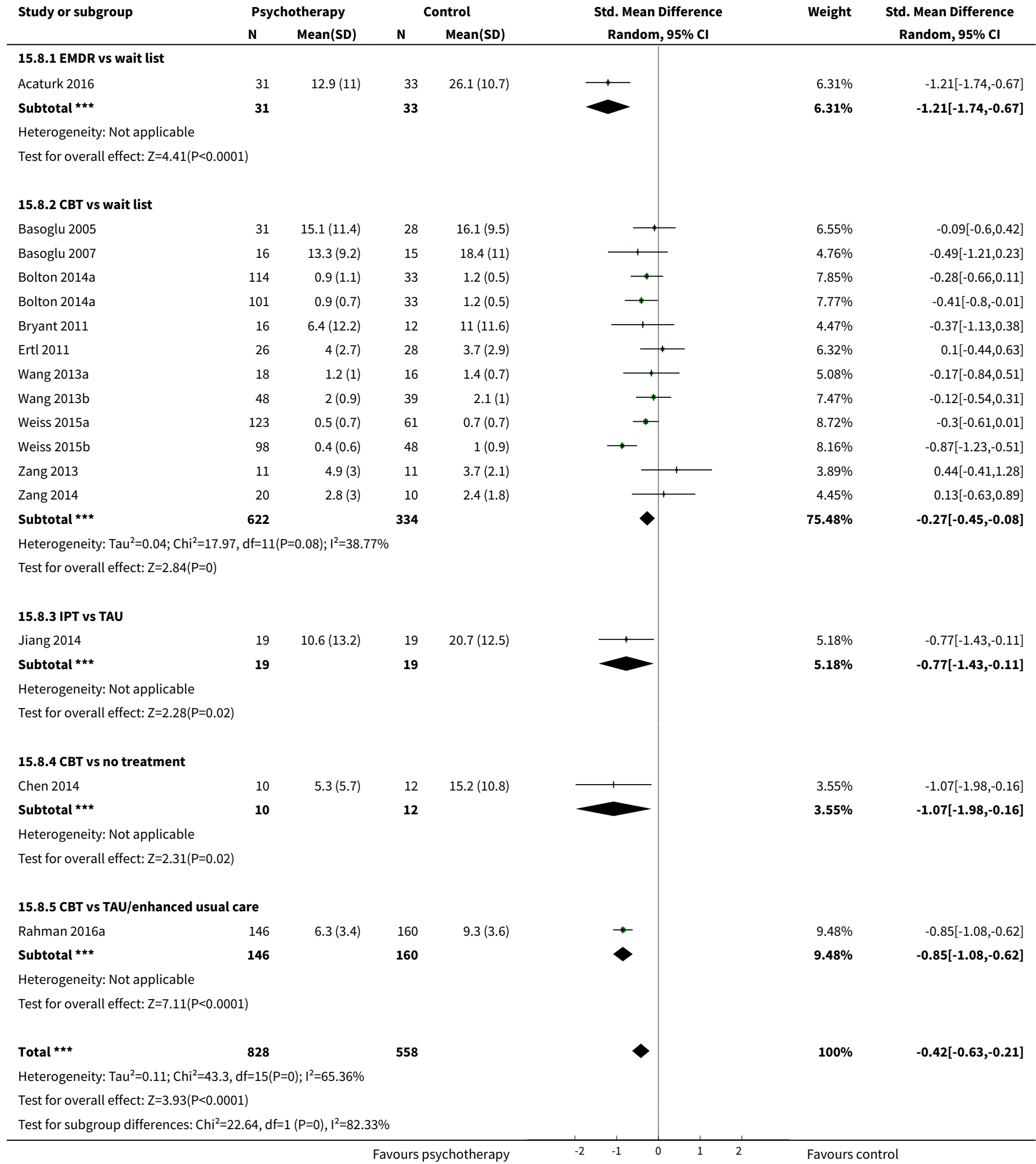

Psychological therapies for the treatment of mental disorders in low- and middle-income countries affected by humanitarian crises 
Analysis 15.9. Comparison 15 Subgroup analysis: type of control - adults, Outcome 9 Depressive symptoms $\geq 6$ months.

\begin{tabular}{|c|c|c|c|c|c|c|c|}
\hline \multirow[t]{2}{*}{ Study or subgroup } & \multicolumn{2}{|c|}{ Psychotherapy } & \multicolumn{2}{|c|}{ Control } & \multirow{2}{*}{$\begin{array}{c}\text { Std. Mean Difference } \\
\text { Random, } 95 \% \mathrm{Cl}\end{array}$} & \multirow[t]{2}{*}{ Weight } & \multirow{2}{*}{$\begin{array}{c}\text { Std. Mean Difference } \\
\text { Random, } 95 \% \mathrm{Cl}\end{array}$} \\
\hline & $\mathbf{N}$ & Mean(SD) & $\mathbf{N}$ & Mean(SD) & & & \\
\hline \multicolumn{8}{|c|}{ 15.9.1 Trauma/Supportive counselling vs wait list } \\
\hline Bass 2016 & 145 & $0.8(0.7)$ & 43 & $1(0.8)$ & & $70.97 \%$ & $-0.26[-0.61,0.08]$ \\
\hline 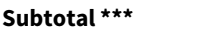 & 145 & & 43 & & & $70.97 \%$ & $-0.26[-0.61,0.08]$ \\
\hline \multicolumn{8}{|c|}{ Heterogeneity: Not applicable } \\
\hline \multicolumn{8}{|c|}{ Test for overall effect: $Z=1.52(P=0.13)$} \\
\hline \multicolumn{8}{|c|}{ 15.9.2 CBT vs no treatment } \\
\hline Ertl 2011 & 26 & $3.1(3)$ & 28 & $3.2(3)$ & & $29.03 \%$ & $-0.04[-0.58,0.49]$ \\
\hline Subtotal $\star \star \star$ & 26 & & 28 & & & $29.03 \%$ & $-0.04[-0.58,0.49]$ \\
\hline \multicolumn{8}{|c|}{ Heterogeneity: Not applicable } \\
\hline \multicolumn{8}{|c|}{ Test for overall effect: $Z=0.16(P=0.87)$} \\
\hline \multicolumn{8}{|c|}{ Heterogeneity: $\mathrm{Tau}^{2}=0 ; \mathrm{Chi}^{2}=0.47, \mathrm{df}=1(\mathrm{P}=0.49) ; \mathrm{I}^{2}=0 \%$} \\
\hline \multicolumn{8}{|c|}{ Test for overall effect: $Z=1.36(P=0.17)$} \\
\hline \multicolumn{8}{|c|}{ Test for subgroup differences: $\mathrm{Chi}^{2}=0.47, \mathrm{df}=1(\mathrm{P}=0.49), \mathrm{I}^{2}=0 \%$} \\
\hline
\end{tabular}

Analysis 15.10. Comparison 15 Subgroup analysis: type of control - adults, Outcome 10 Dropout.

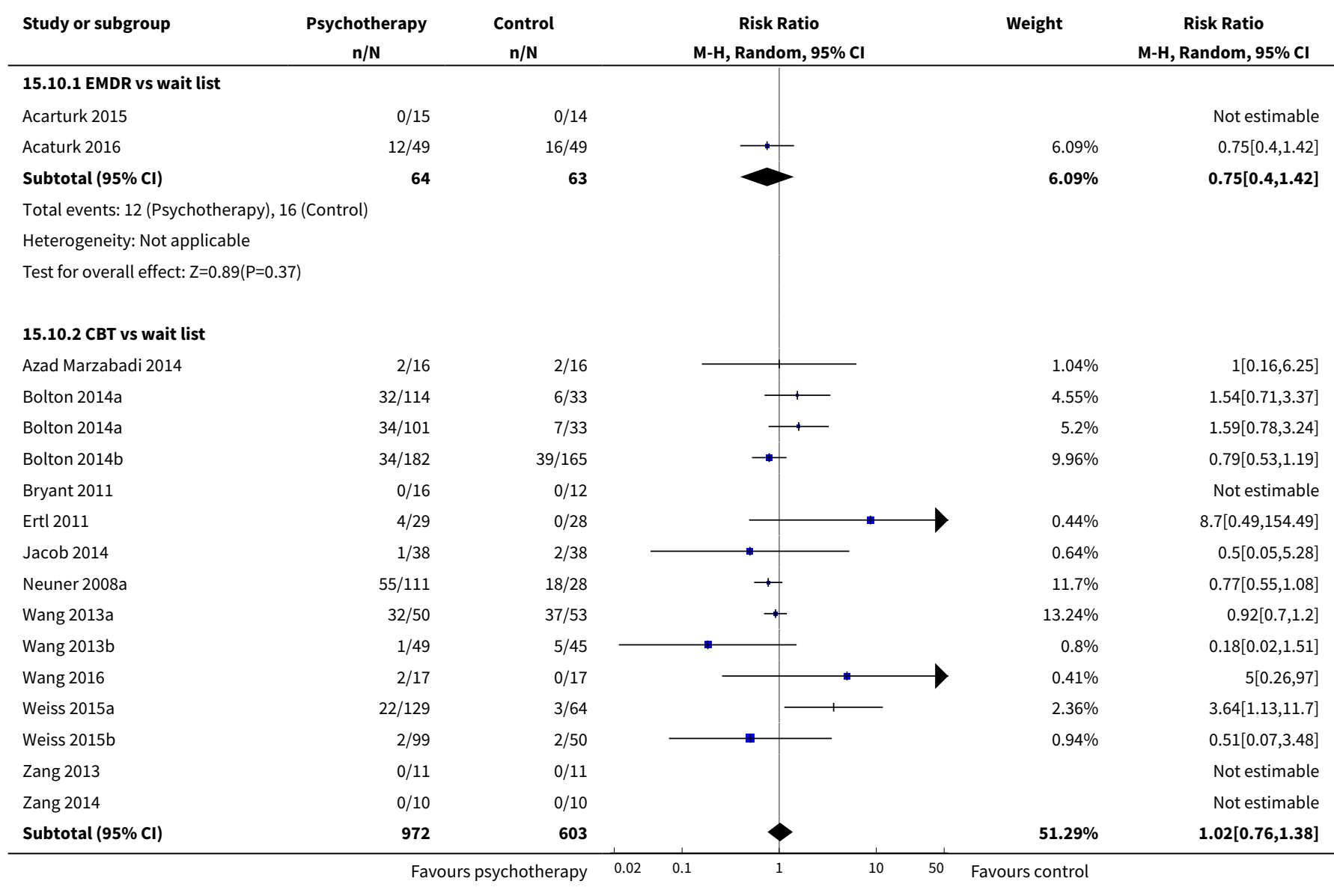

Psychological therapies for the treatment of mental disorders in low- and middle-income countries affected by humanitarian crises 148 (Review)

Copyright (c) 2018 The Cochrane Collaboration. Published by John Wiley \& Sons, Ltd. 


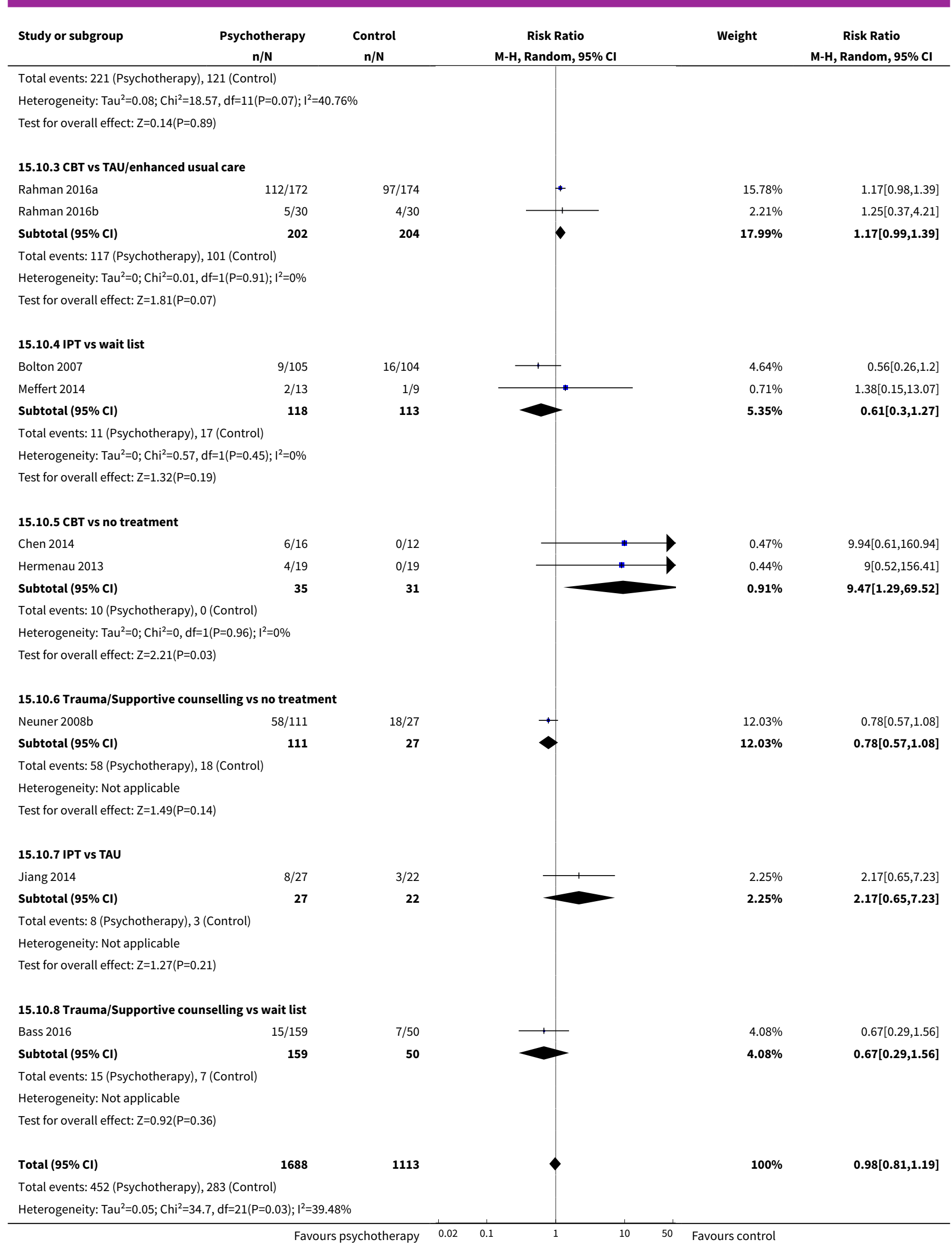

Psychological therapies for the treatment of mental disorders in low- and middle-income countries affected by humanitarian crises 


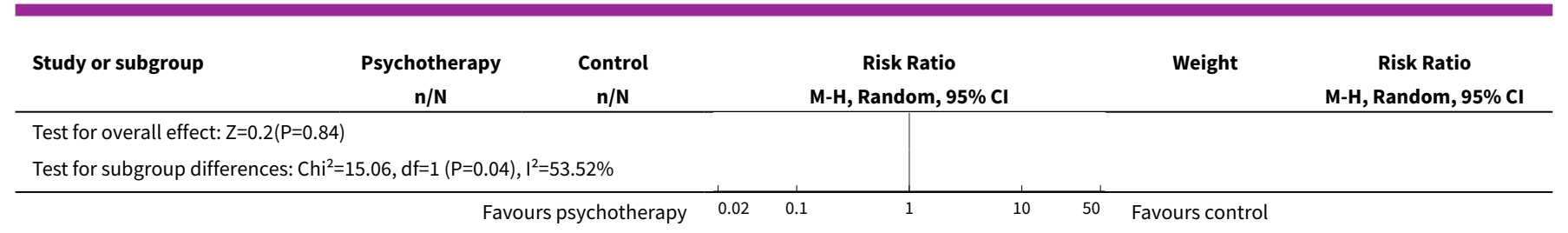

Analysis 15.11. Comparison 15 Subgroup analysis: type of control - adults, Outcome 11 Functional impairment at endpoint.

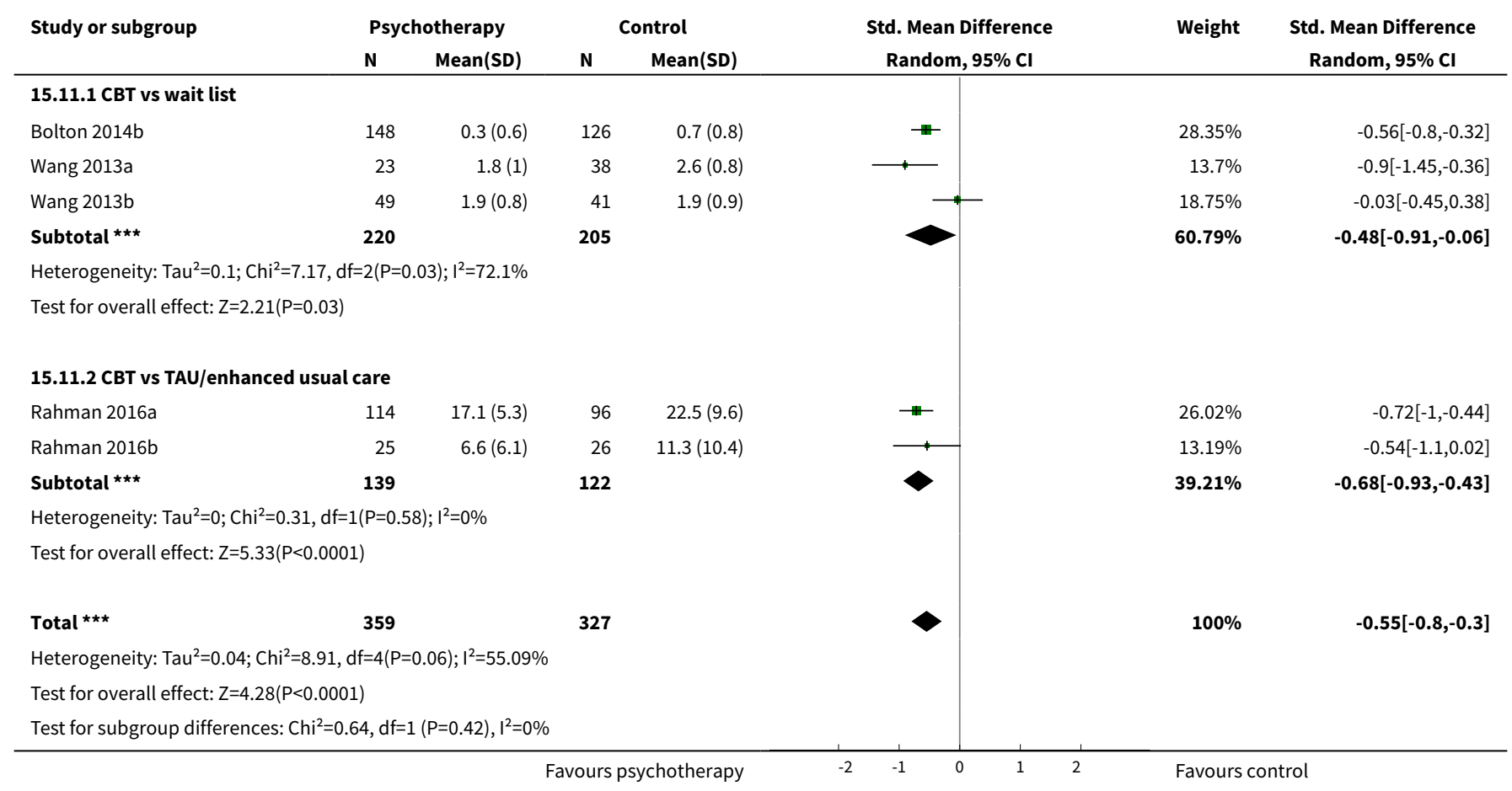

\section{Analysis 15.12. Comparison 15 Subgroup analysis: type of control - adults, Outcome 12 Functional impairment at 1 to 4 months.}

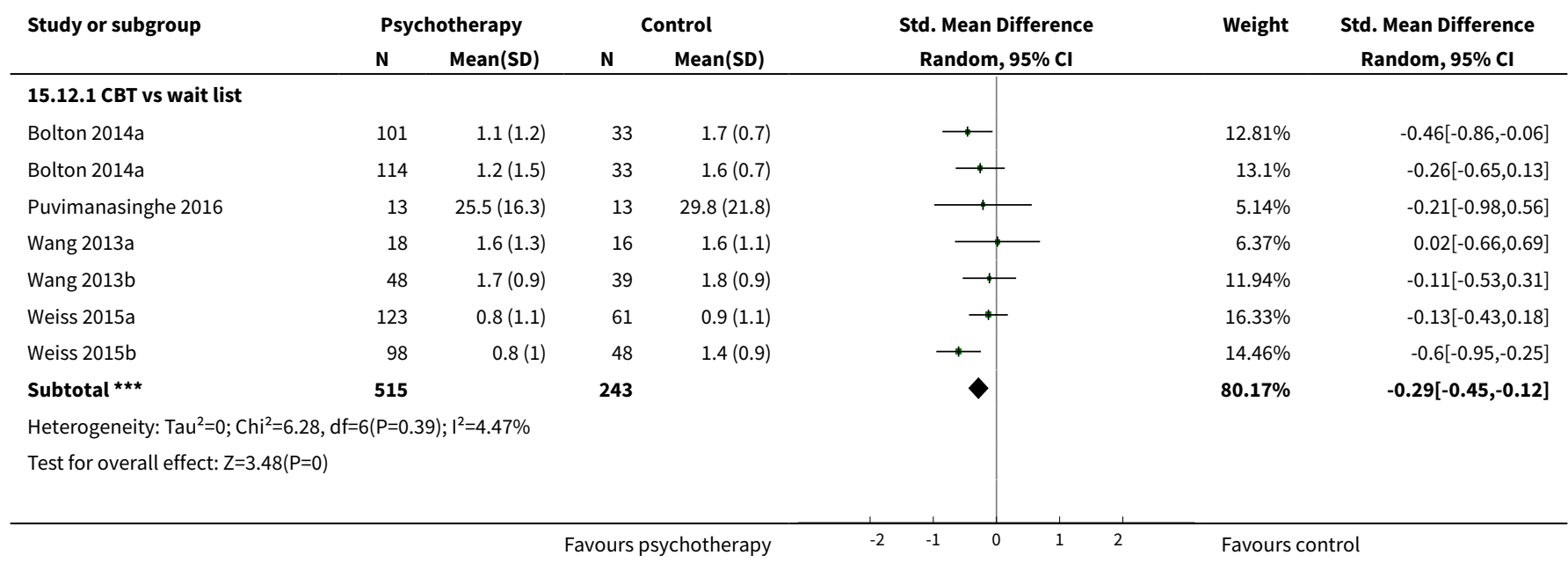




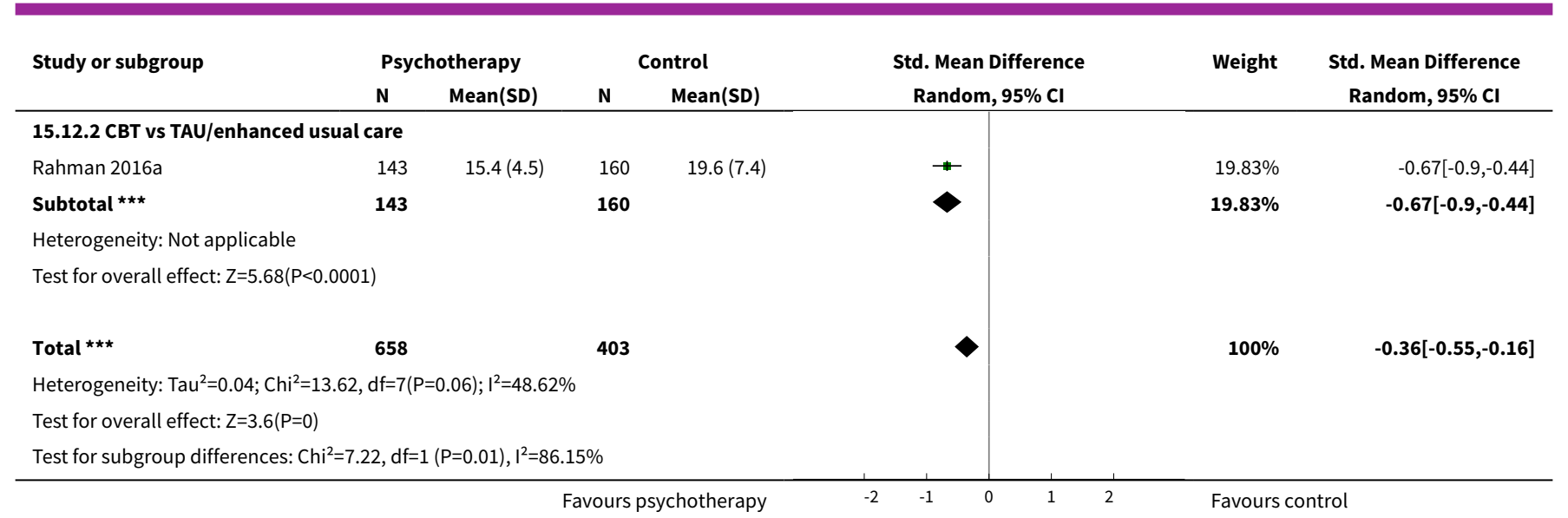

Analysis 15.13. Comparison 15 Subgroup analysis: type of control - adults, Outcome 13 Functional impairment $\geq 6$ months.

\begin{tabular}{|c|c|c|c|c|c|c|c|}
\hline \multirow[t]{2}{*}{ Study or subgroup } & \multicolumn{2}{|c|}{ Psychotherapy } & \multicolumn{2}{|c|}{ Control } & \multirow{2}{*}{$\begin{array}{c}\text { Std. Mean Difference } \\
\text { Random, } 95 \% \mathrm{Cl}\end{array}$} & \multirow[t]{2}{*}{ Weight } & \multirow{2}{*}{$\begin{array}{c}\text { Std. Mean Difference } \\
\text { Random, } 95 \% \mathrm{Cl}\end{array}$} \\
\hline & $\mathbf{N}$ & $\operatorname{Mean}(S D)$ & $\mathbf{N}$ & $\operatorname{Mean}(S D)$ & & & \\
\hline \multicolumn{8}{|c|}{ 15.13.1 Trauma/Supportive counselling vs wait list } \\
\hline Bass 2016 & 145 & $1.2(1.3)$ & 43 & $1.5(1.2)$ & & $100 \%$ & $-0.25[-0.59,0.09]$ \\
\hline Subtotal $\star \star \star ~$ & 145 & & 43 & & & $100 \%$ & $-0.25[-0.59,0.09]$ \\
\hline \multicolumn{8}{|c|}{ Heterogeneity: Not applicable } \\
\hline \multicolumn{8}{|c|}{ Test for overall effect: $Z=1.44(P=0.15)$} \\
\hline 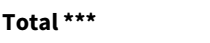 & 145 & & 43 & & & $100 \%$ & $-0.25[-0.59,0.09]$ \\
\hline \multicolumn{8}{|c|}{ Heterogeneity: Not applicable } \\
\hline \multicolumn{8}{|c|}{ Test for overall effect: $Z=1.44(P=0.15)$} \\
\hline
\end{tabular}

Analysis 15.14. Comparison 15 Subgroup analysis: type of control - adults, Outcome 14 Quality of life at endpoint.

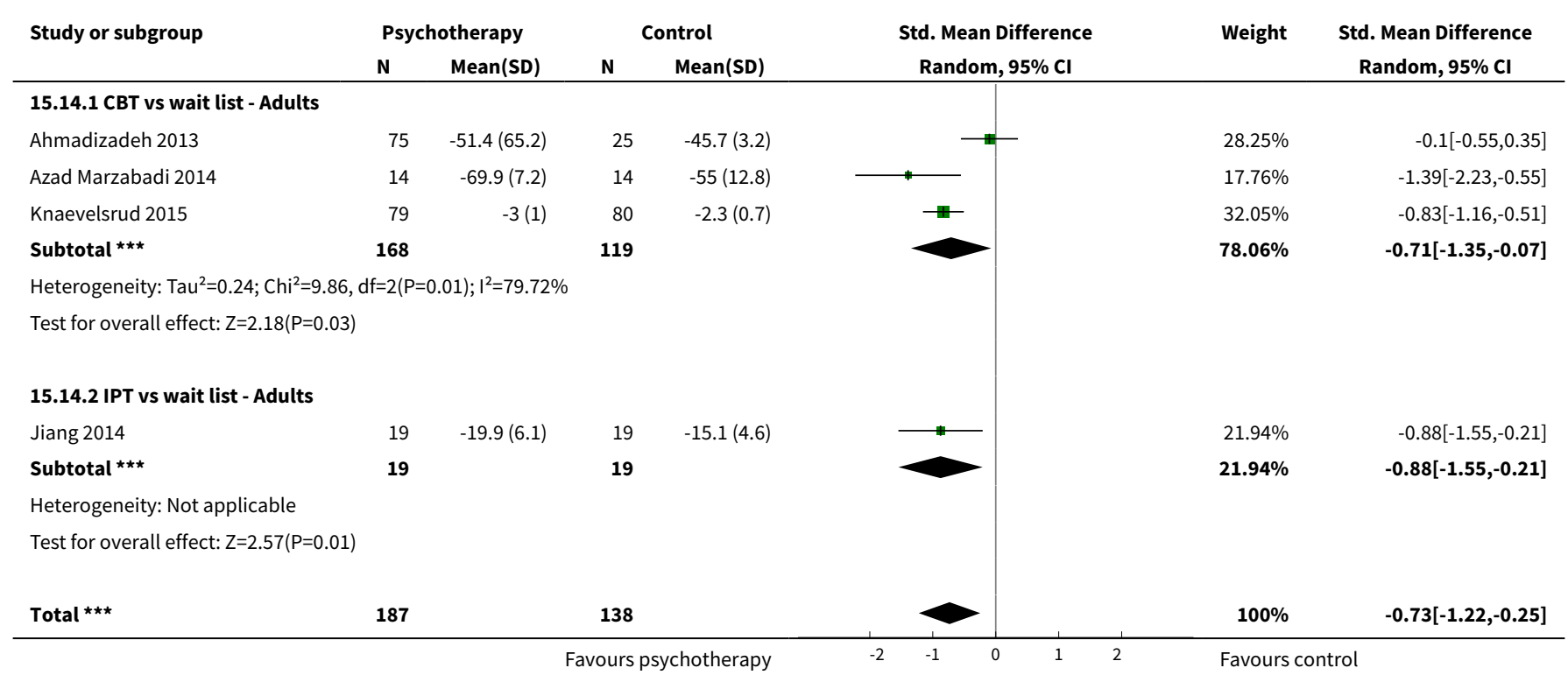




\begin{tabular}{|c|c|c|c|c|c|c|c|}
\hline \multirow[t]{2}{*}{ Study or subgroup } & Psychotherapy & \multicolumn{2}{|c|}{ Control } & \multirow{2}{*}{\multicolumn{2}{|c|}{$\begin{array}{c}\text { Std. Mean Difference } \\
\text { Random, } 95 \% \mathrm{Cl}\end{array}$}} & \multirow[t]{2}{*}{ Weight } & \multirow{2}{*}{$\begin{array}{c}\text { Std. Mean Difference } \\
\text { Random, } 95 \% \mathrm{Cl}\end{array}$} \\
\hline & N $\quad \operatorname{Mean}(\mathrm{SD})$ & $\mathbf{N}$ & $\operatorname{Mean}(S D)$ & & & & \\
\hline \multicolumn{8}{|c|}{ Heterogeneity: $\operatorname{Tau}^{2}=0.17 ; \mathrm{Chi}^{2}=10.23, \mathrm{df}=3(\mathrm{P}=0.02) ; \mathrm{I}^{2}=70.67 \%$} \\
\hline \multicolumn{8}{|c|}{ Test for overall effect: $Z=2.95(P=0)$} \\
\hline \multicolumn{8}{|c|}{ Test for subgroup differences: $\mathrm{Chi}^{2}=0.13, \mathrm{df}=1(\mathrm{P}=0.72), \mathrm{I}^{2}=0 \%$} \\
\hline
\end{tabular}

\section{Analysis 15.15. Comparison 15 Subgroup analysis: type of control - adults, Outcome 15 Diagnosis of PTSD.}

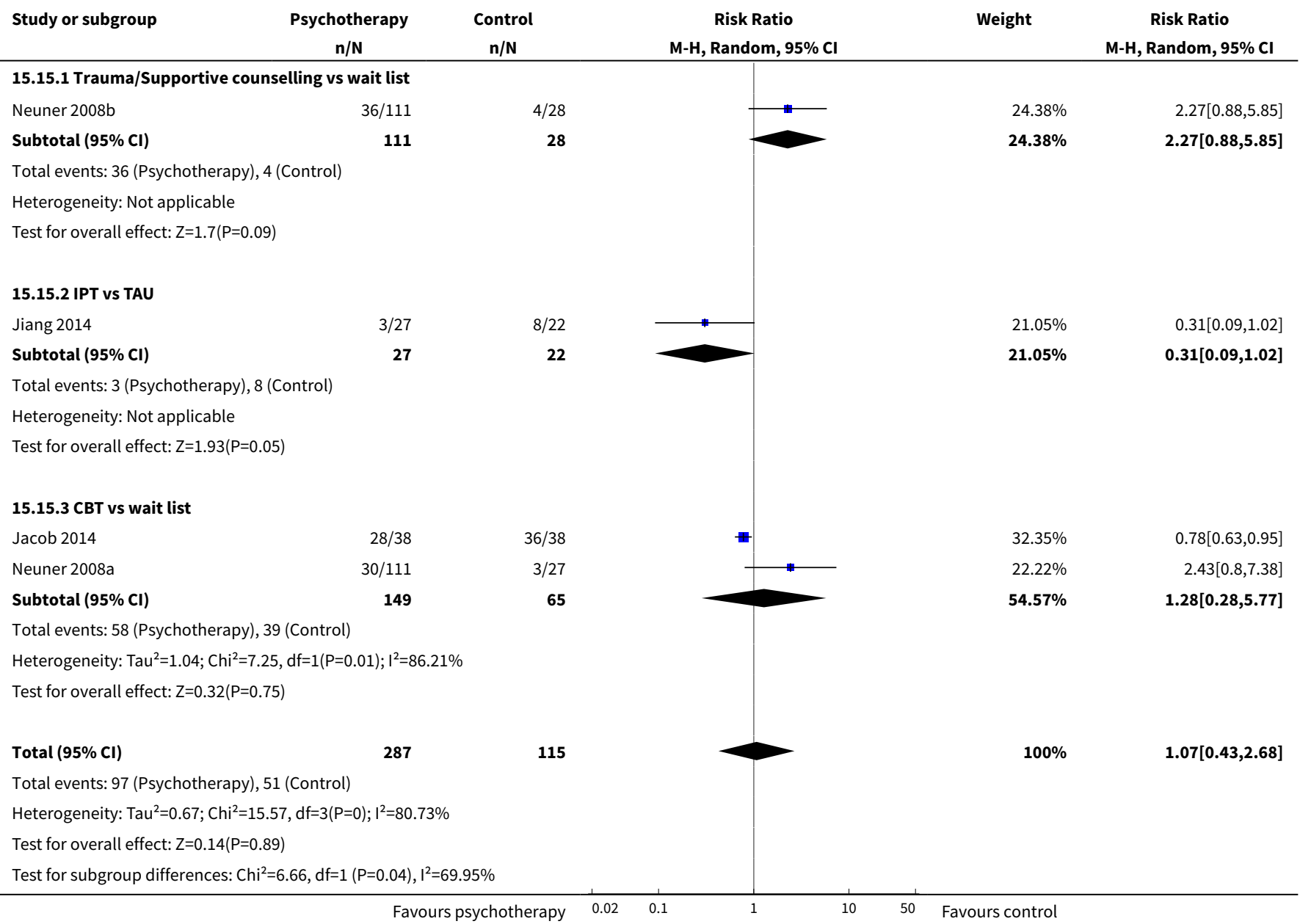

Analysis 15.16. Comparison 15 Subgroup analysis: type of control - adults, Outcome 16 Diagnosis of depression.

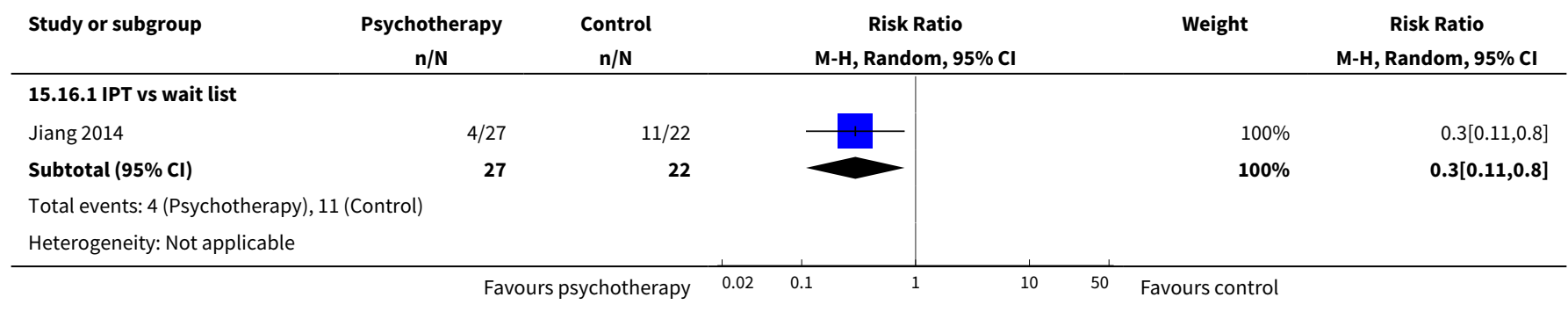

Psychological therapies for the treatment of mental disorders in low- and middle-income countries affected by humanitarian crises 


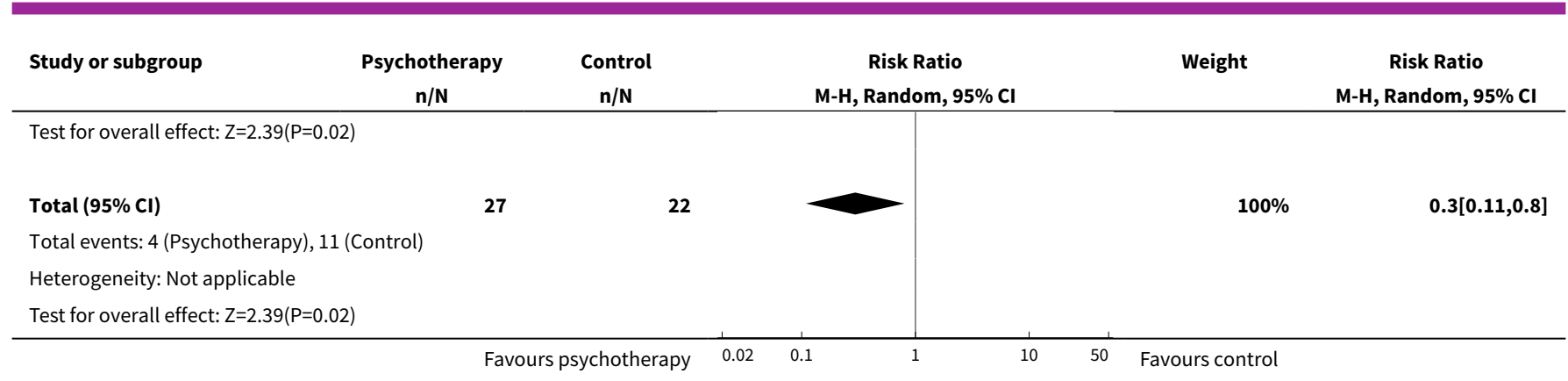

Analysis 15.17. Comparison 15 Subgroup analysis: type of control - adults, Outcome 17 Coping at endpoint.

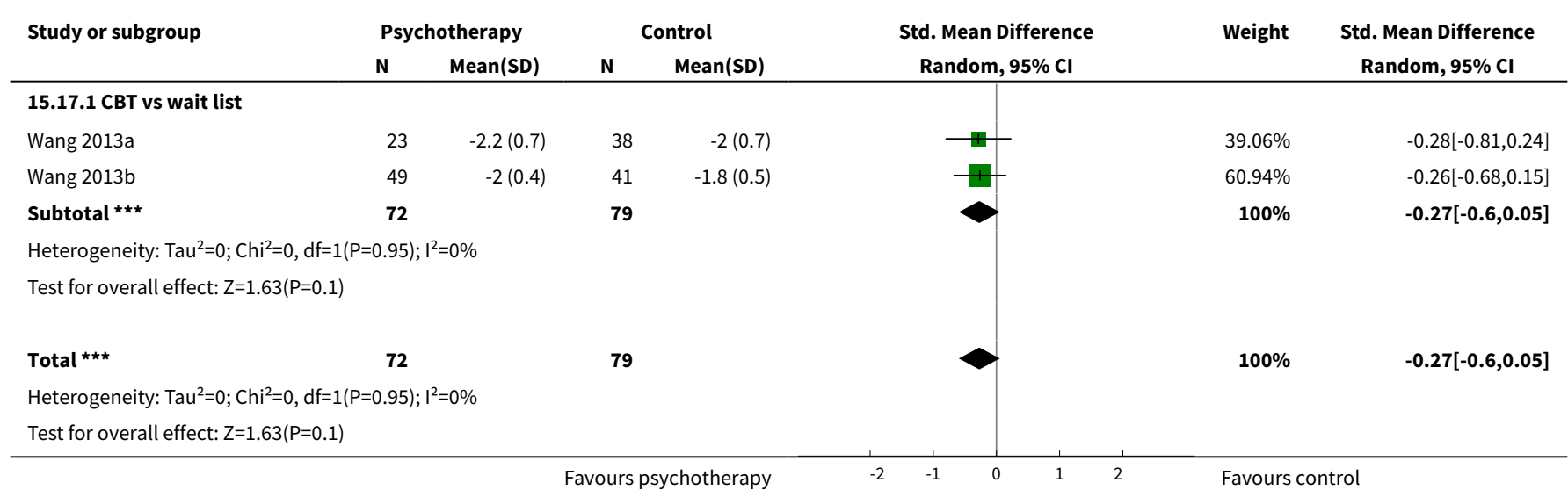

Analysis 15.18. Comparison 15 Subgroup analysis: type of control - adults, Outcome 18 Coping at 1 to 4 months.

\begin{tabular}{|c|c|c|c|c|c|c|c|}
\hline \multirow[t]{2}{*}{ Study or subgroup } & \multicolumn{2}{|c|}{ Psychotherapy } & \multicolumn{2}{|c|}{ Control } & \multirow{2}{*}{$\begin{array}{c}\text { Std. Mean Difference } \\
\text { Random, } 95 \% \mathrm{Cl}\end{array}$} & \multirow[t]{2}{*}{ Weight } & \multirow{2}{*}{$\begin{array}{c}\text { Std. Mean Difference } \\
\text { Random, } 95 \% \mathrm{Cl}\end{array}$} \\
\hline & $\mathbf{N}$ & Mean(SD) & $\mathbf{N}$ & $\operatorname{Mean}(S D)$ & & & \\
\hline \multicolumn{8}{|c|}{ 15.18.1 CBT vs wait list } \\
\hline Wang 2013a & 18 & $-2.3(1.2)$ & 16 & $-2.7(0.5)$ & + & $39.36 \%$ & $0.43[-0.25,1.11]$ \\
\hline Wang 2013b & 48 & $-2(0.5)$ & 39 & $-1.9(0.7)$ & & $60.64 \%$ & $-0.16[-0.58,0.26]$ \\
\hline 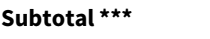 & 66 & & 55 & & & $100 \%$ & $0.07[-0.49,0.64]$ \\
\hline \multicolumn{8}{|c|}{ Heterogeneity: $\mathrm{Tau}^{2}=0.09 ; \mathrm{Chi}^{2}=2.09, \mathrm{df}=1(\mathrm{P}=0.15) ; \mathrm{I}^{2}=52.1 \%$} \\
\hline \multicolumn{8}{|c|}{ Test for overall effect: $Z=0.25(P=0.8)$} \\
\hline Total $\star \star \star ~$ & 66 & & 55 & & & $100 \%$ & $0.07[-0.49,0.64]$ \\
\hline \multicolumn{8}{|c|}{ Heterogeneity: $\mathrm{Tau}^{2}=0.09 ; \mathrm{Chi}^{2}=2.09, \mathrm{df}=1(\mathrm{P}=0.15) ; \mathrm{I}^{2}=52.1 \%$} \\
\hline Test for overall effect & & & & & & & \\
\hline
\end{tabular}


Comparison 16. Subgroup analysis: phase of humanitarian crisis - adults

\begin{tabular}{|c|c|c|c|c|}
\hline $\begin{array}{l}\text { Outcome or subgroup } \\
\text { title }\end{array}$ & No. of studies & $\begin{array}{l}\text { No. of partici- } \\
\text { pants }\end{array}$ & Statistical method & Effect size \\
\hline $\begin{array}{l}1 \text { PTSD symptoms at end- } \\
\text { point }\end{array}$ & 15 & 1250 & $\begin{array}{l}\text { Std. Mean Difference (IV, Random, 95\% } \\
\text { CI) }\end{array}$ & $-1.10[-1.39,-0.81]$ \\
\hline $\begin{array}{l}\text { 1.1 During the acute cri- } \\
\text { sis }\end{array}$ & 7 & 566 & $\begin{array}{l}\text { Std. Mean Difference (IV, Random, 95\% } \\
\text { Cl) }\end{array}$ & $-1.14[-1.58,-0.69]$ \\
\hline 1.2 After the acute crisis & 6 & 533 & $\begin{array}{l}\text { Std. Mean Difference (IV, Random, 95\% } \\
\text { CI) }\end{array}$ & $-1.33[-1.92,-0.73]$ \\
\hline 1.3 Other & 2 & 151 & $\begin{array}{l}\text { Std. Mean Difference (IV, Random, 95\% } \\
\text { Cl) }\end{array}$ & $-0.64[-0.97,-0.31]$ \\
\hline $\begin{array}{l}2 \text { PTSD symptoms at } 1 \text { to } \\
4 \text { months }\end{array}$ & 16 & 1568 & $\begin{array}{l}\text { Std. Mean Difference (IV, Random, 95\% } \\
\text { Cl) }\end{array}$ & $-0.47[-0.66,-0.29]$ \\
\hline $\begin{array}{l}\text { 2.1 During the acute cri- } \\
\text { sis }\end{array}$ & 5 & 728 & $\begin{array}{l}\text { Std. Mean Difference (IV, Random, 95\% } \\
\text { CI) }\end{array}$ & $-0.78[-1.18,-0.38]$ \\
\hline 2.2 After the acute crisis & 9 & 719 & $\begin{array}{l}\text { Std. Mean Difference (IV, Random, 95\% } \\
\text { CI) }\end{array}$ & $-0.33[-0.50,-0.17]$ \\
\hline 2.3 Other & 2 & 121 & $\begin{array}{l}\text { Std. Mean Difference (IV, Random, 95\% } \\
\text { Cl) }\end{array}$ & $-0.19[-0.55,0.17]$ \\
\hline $\begin{array}{l}3 \text { PTSD symptoms } \geq 6 \\
\text { months }\end{array}$ & 5 & 400 & $\begin{array}{l}\text { Std. Mean Difference (IV, Random, 95\% } \\
\text { CI) }\end{array}$ & $-0.37[-0.61,-0.14]$ \\
\hline $\begin{array}{l}\text { 3.1 During the acute cri- } \\
\text { sis }\end{array}$ & 1 & 188 & $\begin{array}{l}\text { Std. Mean Difference (IV, Random, 95\% } \\
\text { CI) }\end{array}$ & $-0.22[-0.57,0.12]$ \\
\hline 3.2 After the acute crisis & 4 & 212 & $\begin{array}{l}\text { Std. Mean Difference (IV, Random, 95\% } \\
\text { Cl) }\end{array}$ & $-0.51[-0.84,-0.18]$ \\
\hline $\begin{array}{l}4 \text { Anxiety symptoms at } \\
\text { endpoint }\end{array}$ & 4 & 535 & $\begin{array}{l}\text { Std. Mean Difference (IV, Random, 95\% } \\
\mathrm{CI} \text { ) }\end{array}$ & $-0.76[-1.10,-0.42]$ \\
\hline 4.1 After the acute crisis & 3 & 326 & $\begin{array}{l}\text { Std. Mean Difference (IV, Random, 95\% } \\
\mathrm{CI} \text { ) }\end{array}$ & $-0.92[-1.61,-0.23]$ \\
\hline $\begin{array}{l}\text { 4.2 During the acute cri- } \\
\text { sis }\end{array}$ & 1 & 209 & $\begin{array}{l}\text { Std. Mean Difference (IV, Random, 95\% } \\
\text { Cl) }\end{array}$ & $-0.74[-1.02,-0.45]$ \\
\hline $\begin{array}{l}5 \text { Anxiety symptoms at } 1 \\
\text { to } 4 \text { months }\end{array}$ & 6 & 969 & $\begin{array}{l}\text { Std. Mean Difference (IV, Random, 95\% } \\
\text { Cl) }\end{array}$ & $-0.43[-0.71,-0.15]$ \\
\hline $\begin{array}{l}\text { 5.1 During the acute cri- } \\
\text { sis }\end{array}$ & 3 & 636 & $\begin{array}{l}\text { Std. Mean Difference (IV, Random, 95\% } \\
\text { Cl) }\end{array}$ & $-0.69[-1.03,-0.36]$ \\
\hline 5.2 After the acute crisis & 3 & 333 & $\begin{array}{l}\text { Std. Mean Difference (IV, Random, 95\% } \\
\text { Cl) }\end{array}$ & $-0.16[-0.41,0.08]$ \\
\hline $\begin{array}{l}6 \text { Depressive symptoms } \\
\text { at endpoint }\end{array}$ & 13 & 1232 & $\begin{array}{l}\text { Std. Mean Difference (IV, Random, 95\% } \\
\text { CI) }\end{array}$ & $-0.90[-1.09,-0.71]$ \\
\hline
\end{tabular}

Psychological therapies for the treatment of mental disorders in low- and middle-income countries affected by humanitarian crises 


\begin{tabular}{|c|c|c|c|c|}
\hline $\begin{array}{l}\text { Outcome or subgroup } \\
\text { title }\end{array}$ & No. of studies & $\begin{array}{l}\text { No. of partici- } \\
\text { pants }\end{array}$ & Statistical method & Effect size \\
\hline $\begin{array}{l}\text { 6.1 During the acute cri- } \\
\text { sis }\end{array}$ & 6 & 514 & $\begin{array}{l}\text { Std. Mean Difference (IV, Random, 95\% } \\
\mathrm{CI} \text { ) }\end{array}$ & $-1.05[-1.26,-0.85]$ \\
\hline 6.2 After the acute crisis & 5 & 567 & $\begin{array}{l}\text { Std. Mean Difference (IV, Random, 95\% } \\
\text { CI) }\end{array}$ & $-0.82[-1.03,-0.60]$ \\
\hline 6.3 Other & 2 & 151 & $\begin{array}{l}\text { Std. Mean Difference (IV, Random, 95\% } \\
\mathrm{CI} \text { ) }\end{array}$ & $-0.53[-1.20,0.14]$ \\
\hline $\begin{array}{l}7 \text { Depressive symptoms } \\
\text { at } 1 \text { to } 4 \text { months }\end{array}$ & 14 & 1670 & $\begin{array}{l}\text { Std. Mean Difference (IV, Random, 95\% } \\
\mathrm{CI} \text { ) }\end{array}$ & $-0.42[-0.61,-0.23]$ \\
\hline $\begin{array}{l}\text { 7.1 During the acute cri- } \\
\text { sis }\end{array}$ & 5 & 1034 & $\begin{array}{l}\text { Std. Mean Difference (IV, Random, 95\% } \\
\mathrm{CI} \text { ) }\end{array}$ & $-0.70[-0.93,-0.47]$ \\
\hline 7.2 After the acute crisis & 7 & 515 & $\begin{array}{l}\text { Std. Mean Difference (IV, Random, 95\% } \\
\mathrm{CI} \text { ) }\end{array}$ & $-0.22[-0.44,0.00]$ \\
\hline 7.3 Other & 2 & 121 & $\begin{array}{l}\text { Std. Mean Difference (IV, Random, 95\% } \\
\mathrm{CI} \text { ) }\end{array}$ & $-0.13[-0.49,0.23]$ \\
\hline $\begin{array}{l}8 \text { Depressive symptoms } \geq \\
6 \text { months }\end{array}$ & 2 & 242 & $\begin{array}{l}\text { Std. Mean Difference (IV, Random, 95\% } \\
\mathrm{Cl} \text { ) }\end{array}$ & $-0.20[-0.49,0.09]$ \\
\hline $\begin{array}{l}\text { 8.1 During the acute cri- } \\
\text { sis }\end{array}$ & 1 & 188 & $\begin{array}{l}\text { Std. Mean Difference (IV, Random, 95\% } \\
\mathrm{CI} \text { ) }\end{array}$ & $-0.26[-0.61,0.08]$ \\
\hline 8.2 After the acute crisis & 1 & 54 & $\begin{array}{l}\text { Std. Mean Difference (IV, Random, 95\% } \\
\mathrm{CI} \text { ) }\end{array}$ & $-0.04[-0.58,0.49]$ \\
\hline 9 Dropout & 24 & 2773 & Risk Ratio (M-H, Random, 95\% Cl) & $0.97[0.80,1.16]$ \\
\hline $\begin{array}{l}9.1 \text { During the acute cri- } \\
\text { sis }\end{array}$ & 9 & 1134 & Risk Ratio $(\mathrm{M}-\mathrm{H}$, Random, 95\% Cl) & $1.07[0.78,1.48]$ \\
\hline 9.2 After the acute crisis & 13 & 1442 & Risk Ratio (M-H, Random, 95\% Cl) & $0.96[0.73,1.28]$ \\
\hline 9.3 Other & 2 & 197 & Risk Ratio $(\mathrm{M}-\mathrm{H}$, Random, 95\% Cl) & $0.56[0.12,2.67]$ \\
\hline $\begin{array}{l}10 \text { Functional impair- } \\
\text { ment at endpoint }\end{array}$ & 5 & 686 & $\begin{array}{l}\text { Std. Mean Difference (IV, Random, 95\% } \\
\mathrm{CI} \text { ) }\end{array}$ & $-0.54[-0.79,-0.29]$ \\
\hline 10.1 After the acute crisis & 1 & 274 & $\begin{array}{l}\text { Std. Mean Difference (IV, Random, 95\% } \\
\mathrm{CI} \text { ) }\end{array}$ & $-0.54[-0.79,-0.30]$ \\
\hline 10.2 Other & 2 & 151 & $\begin{array}{l}\text { Std. Mean Difference (IV, Random, 95\% } \\
\mathrm{CI} \text { ) }\end{array}$ & $-0.45[-1.30,0.40]$ \\
\hline $\begin{array}{l}10.3 \text { During the acute cri- } \\
\text { sis }\end{array}$ & 2 & 261 & $\begin{array}{l}\text { Std. Mean Difference (IV, Random, 95\% } \\
\mathrm{CI} \text { ) }\end{array}$ & $-0.68[-0.93,-0.43]$ \\
\hline $\begin{array}{l}11 \text { Functional impair- } \\
\text { ment at } 1 \text { to } 4 \text { months }\end{array}$ & 7 & 1061 & $\begin{array}{l}\text { Std. Mean Difference (IV, Random, 95\% } \\
\mathrm{Cl} \text { ) }\end{array}$ & $-0.36[-0.55,-0.16]$ \\
\hline
\end{tabular}




\begin{tabular}{|c|c|c|c|c|}
\hline $\begin{array}{l}\text { Outcome or subgroup } \\
\text { title }\end{array}$ & No. of studies & $\begin{array}{l}\text { No. of partici- } \\
\text { pants }\end{array}$ & Statistical method & Effect size \\
\hline $\begin{array}{l}11.1 \text { During the acute cri- } \\
\text { sis }\end{array}$ & 3 & 633 & $\begin{array}{l}\text { Std. Mean Difference (IV, Random, 95\% } \\
\mathrm{CI} \text { ) }\end{array}$ & $-0.47[-0.81,-0.13]$ \\
\hline 11.2 After the acute crisis & 2 & 307 & $\begin{array}{l}\text { Std. Mean Difference (IV, Random, 95\% } \\
\mathrm{CI} \text { ) }\end{array}$ & $-0.34[-0.60,-0.08]$ \\
\hline 11.3 Other & 2 & 121 & $\begin{array}{l}\text { Std. Mean Difference (IV, Random, 95\% } \\
\mathrm{CI} \text { ) }\end{array}$ & $-0.08[-0.43,0.28]$ \\
\hline $\begin{array}{l}12 \text { Quality of life at end- } \\
\text { point }\end{array}$ & 4 & 325 & $\begin{array}{l}\text { Std. Mean Difference (IV, Random, 95\% } \\
\mathrm{CI} \text { ) }\end{array}$ & $-0.73[-1.22,-0.25]$ \\
\hline $\begin{array}{l}12.1 \text { During the acute cri- } \\
\text { sis }\end{array}$ & 1 & 159 & $\begin{array}{l}\text { Std. Mean Difference (IV, Random, 95\% } \\
\mathrm{Cl} \text { ) }\end{array}$ & $-0.83[-1.16,-0.51]$ \\
\hline 12.2 After the acute crisis & 3 & 166 & $\begin{array}{l}\text { Std. Mean Difference (IV, Random, 95\% } \\
\mathrm{CI} \text { ) }\end{array}$ & $-0.73[-1.49,0.04]$ \\
\hline 13 Diagnosis of PTSD & 4 & 402 & Risk Ratio $(\mathrm{M}-\mathrm{H}$, Random, 95\% Cl) & $1.07[0.43,2.68]$ \\
\hline 13.1 After the acute crisis & 4 & 402 & Risk Ratio $(\mathrm{M}-\mathrm{H}$, Random, 95\% Cl) & $1.07[0.43,2.68]$ \\
\hline
\end{tabular}

Analysis 16.1. Comparison 16 Subgroup analysis: phase of humanitarian crisis - adults, Outcome 1 PTSD symptoms at endpoint.

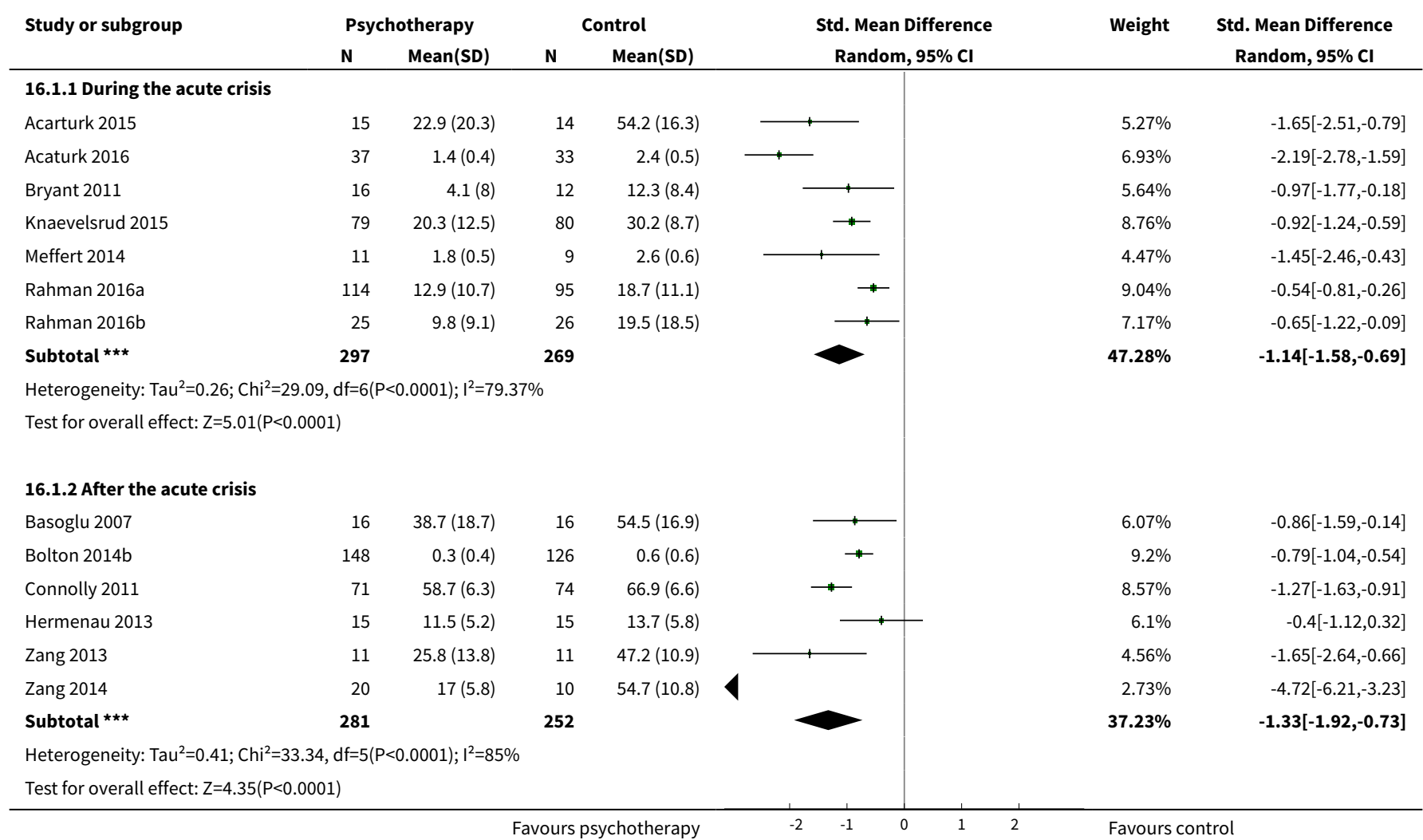




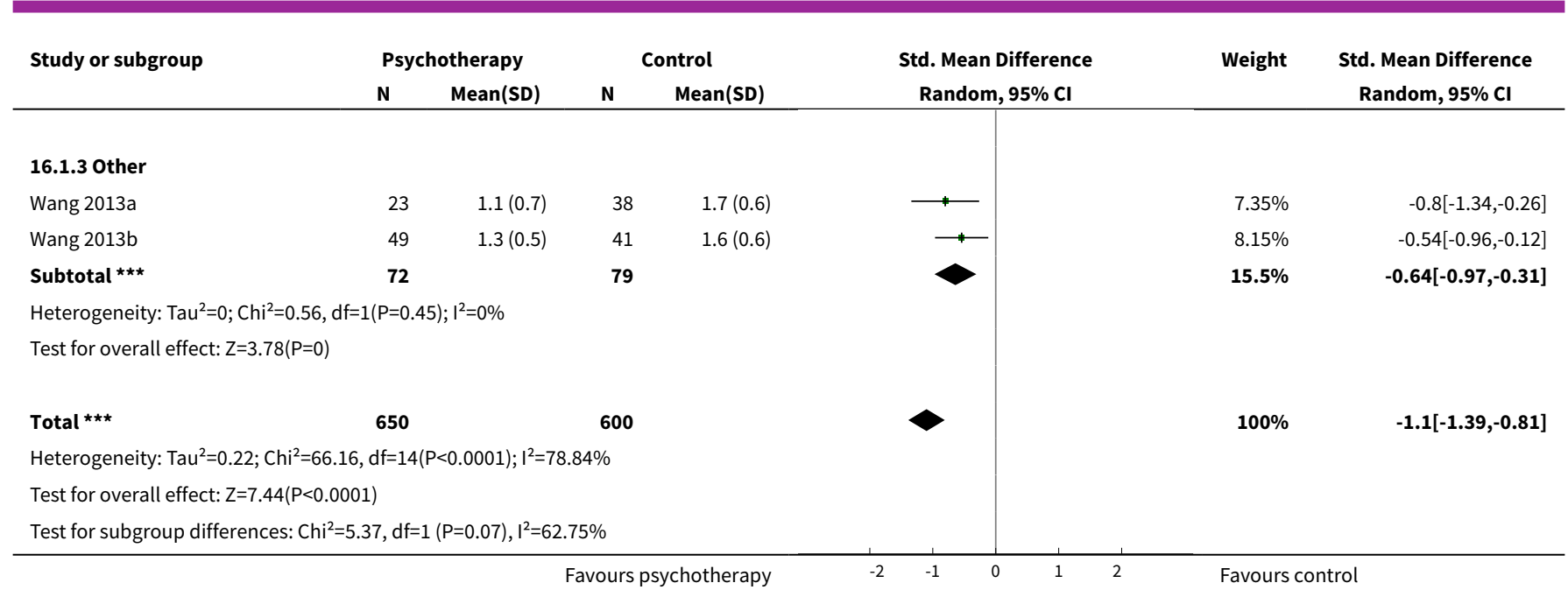

\section{Analysis 16.2. Comparison 16 Subgroup analysis: phase of humanitarian crisis - adults, Outcome 2 PTSD symptoms at 1 to 4 months.}

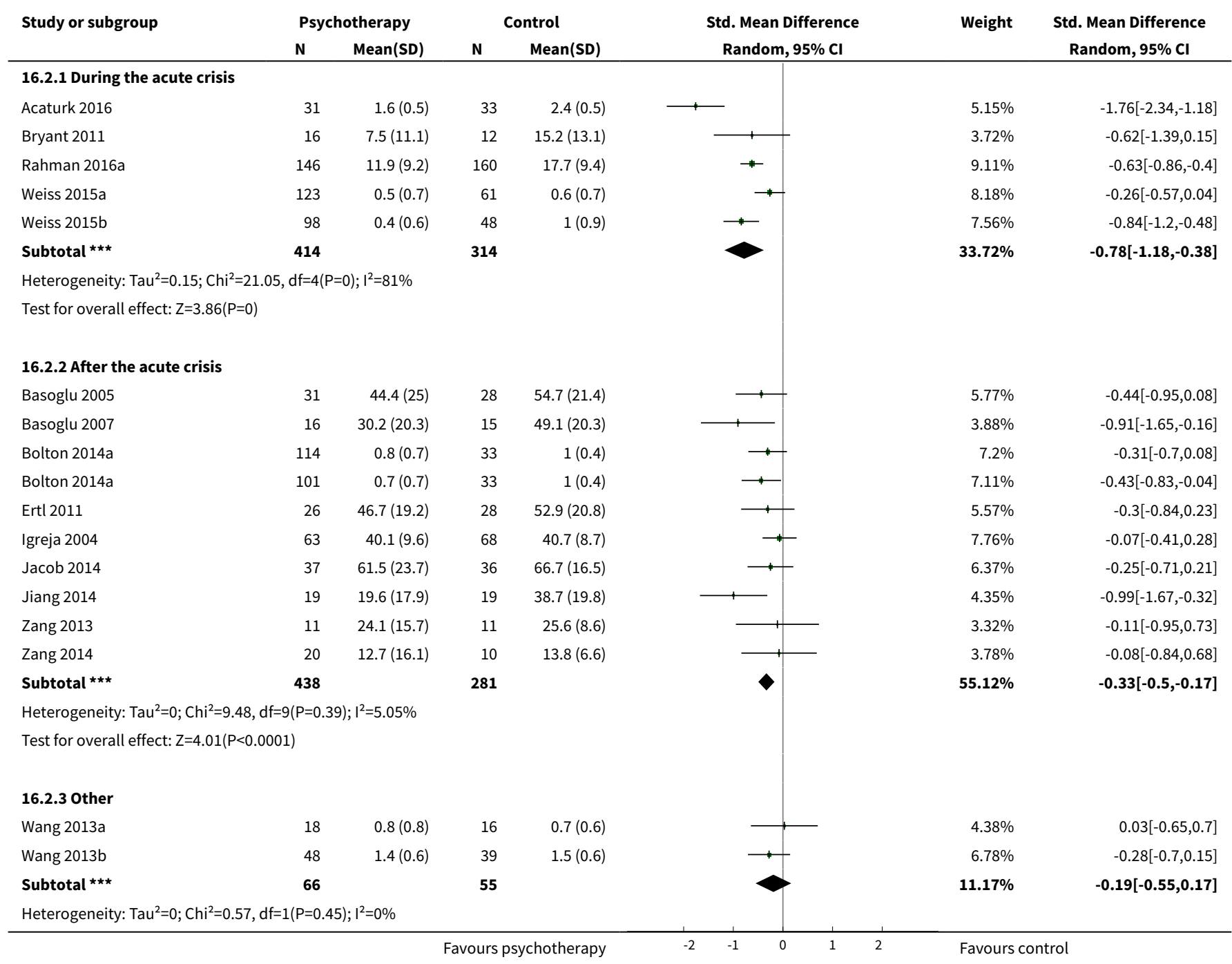

Psychological therapies for the treatment of mental disorders in low- and middle-income countries affected by humanitarian crises 


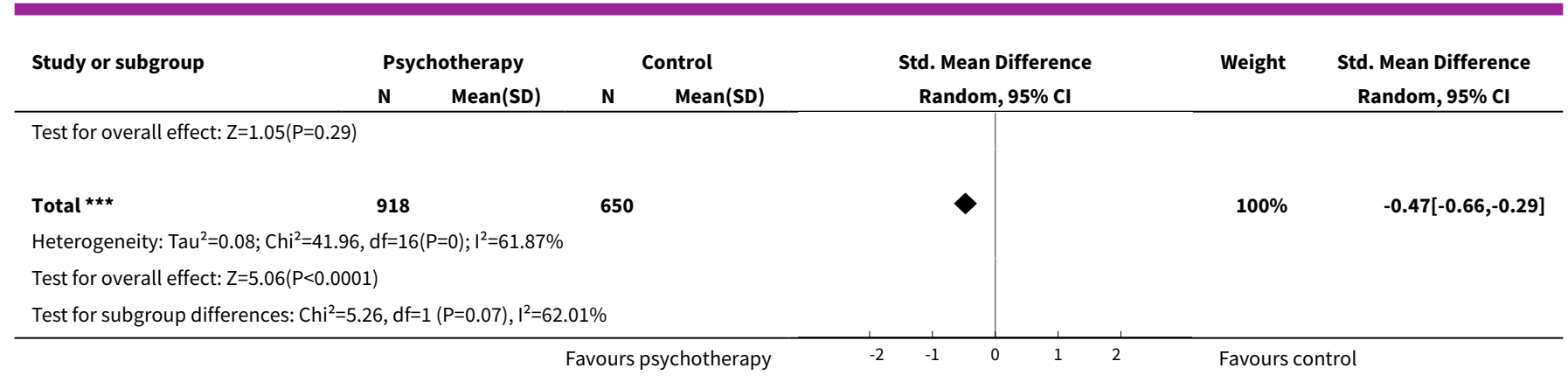

Analysis 16.3. Comparison 16 Subgroup analysis: phase of humanitarian crisis - adults, Outcome 3 PTSD symptoms $\geq 6$ months.

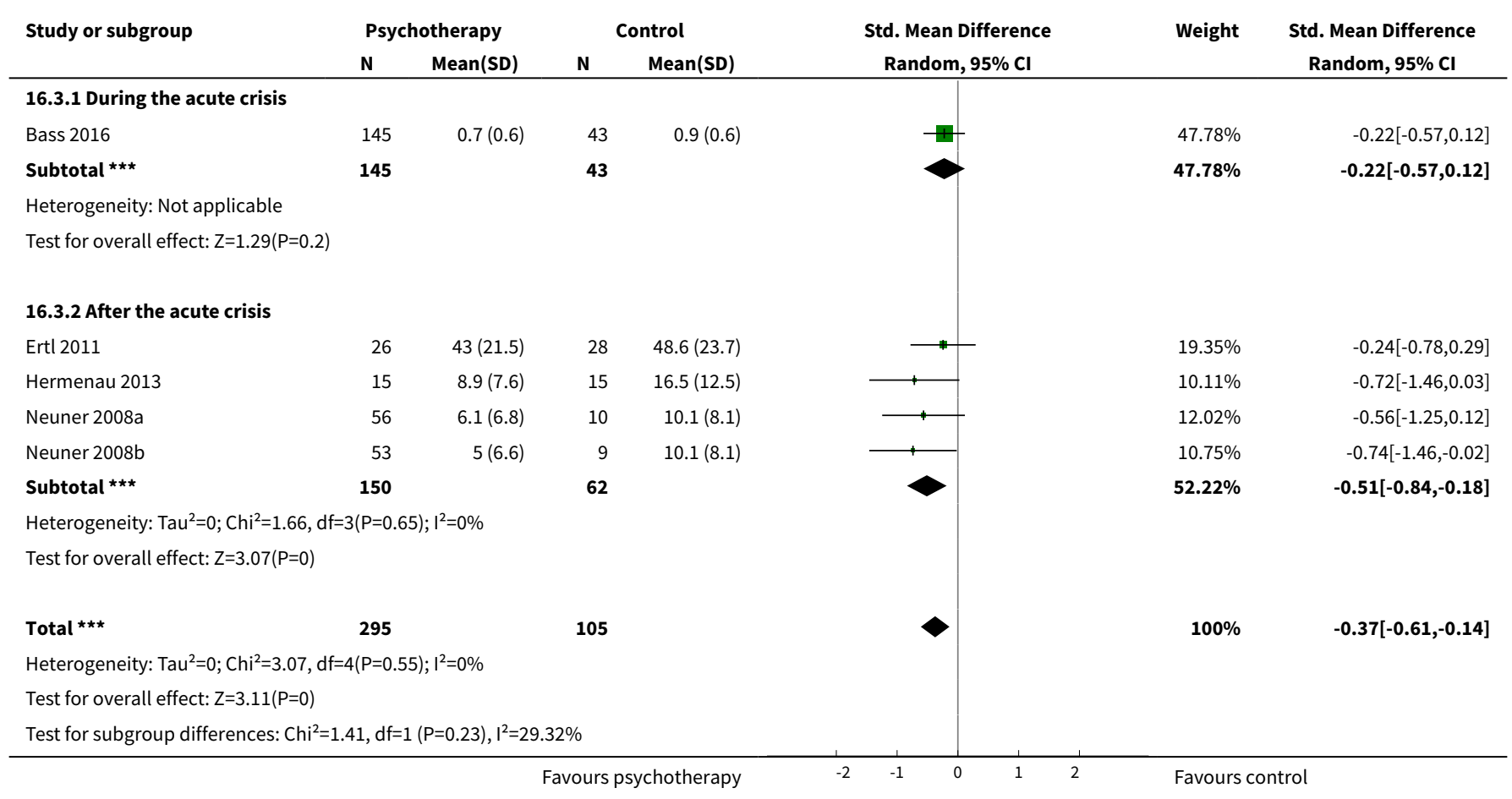

Analysis 16.4. Comparison 16 Subgroup analysis: phase of humanitarian crisis - adults, Outcome 4 Anxiety symptoms at endpoint.

\begin{tabular}{|c|c|c|c|c|c|c|c|c|}
\hline \multirow[t]{2}{*}{ Study or subgroup } & \multicolumn{2}{|c|}{ Psychotherapy } & \multicolumn{2}{|c|}{ Control } & \multirow{2}{*}{\multicolumn{2}{|c|}{$\begin{array}{c}\text { Std. Mean Difference } \\
\text { Random, } 95 \% \mathrm{Cl} \\
\end{array}$}} & \multirow[t]{2}{*}{ Weight } & \multirow{2}{*}{$\begin{array}{c}\text { Std. Mean Difference } \\
\text { Random, } 95 \% \mathrm{CI}\end{array}$} \\
\hline & $\mathbf{N}$ & Mean(SD) & $\mathbf{N}$ & $\operatorname{Mean}(\mathrm{SD})$ & & & & \\
\hline \multicolumn{9}{|c|}{ 16.4.1 After the acute crisis } \\
\hline Bolton 2014b & 148 & $0.3(0.5)$ & 126 & $0.6(0.8)$ & 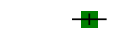 & & $39.94 \%$ & $-0.48[-0.72,-0.24]$ \\
\hline Zang 2013 & 11 & $5.3(2.8)$ & 11 & $8.6(3.6)$ & & & $11.14 \%$ & $-1.01[-1.91,-0.11]$ \\
\hline Zang 2014 & 20 & $4.7(4)$ & 10 & $12.8(6.7)$ & 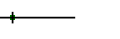 & & $11.68 \%$ & $-1.57[-2.45,-0.7]$ \\
\hline Subtotal $* \star \star$ & 179 & & 147 & & & & $62.75 \%$ & $-0.92[-1.61,-0.23]$ \\
\hline \multicolumn{9}{|c|}{ Heterogeneity: $\mathrm{Tau}^{2}=0.26 ; \mathrm{Chi}^{2}=6.55, \mathrm{df}=2(\mathrm{P}=0.04) ; \mathrm{I}^{2}=69.46 \%$} \\
\hline \multicolumn{9}{|c|}{ Test for overall effect: $Z=2.61(P=0.01)$} \\
\hline & & & & otherapy & -1 & 1 & Favour & \\
\hline
\end{tabular}

Psychological therapies for the treatment of mental disorders in low- and middle-income countries affected by humanitarian crises 


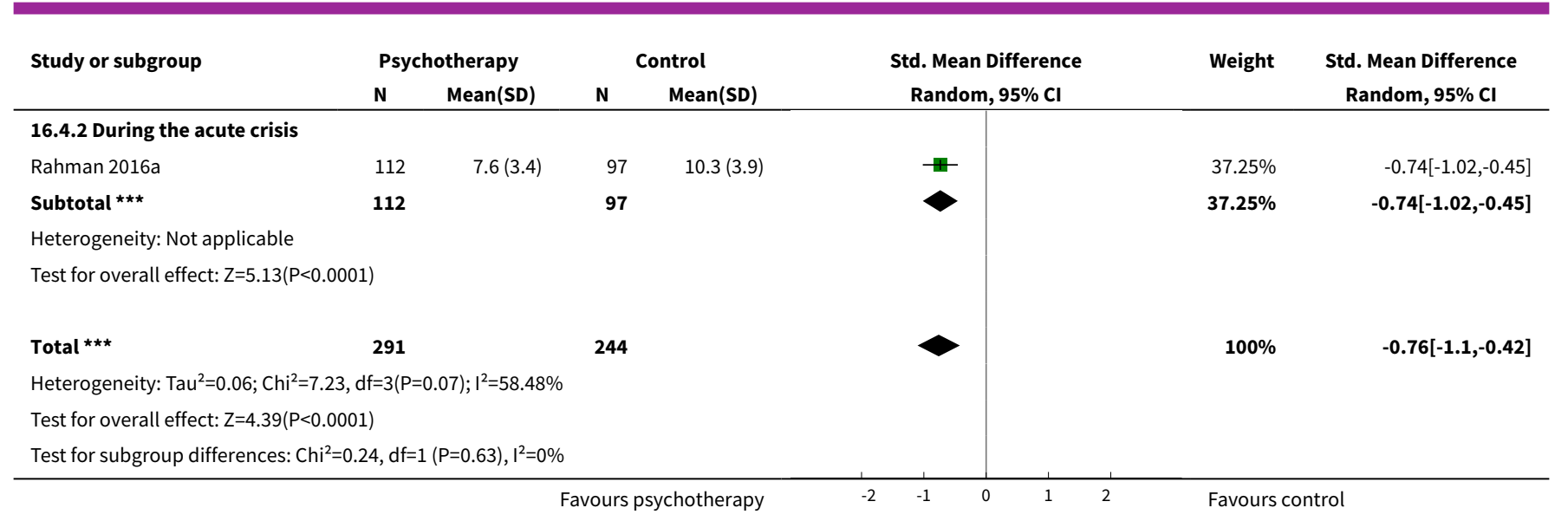

\section{Analysis 16.5. Comparison 16 Subgroup analysis: phase of humanitarian} crisis - adults, Outcome 5 Anxiety symptoms at 1 to 4 months.

\begin{tabular}{|c|c|c|c|c|c|c|c|}
\hline \multirow[t]{2}{*}{ Study or subgroup } & \multicolumn{2}{|c|}{ Psychotherapy } & \multicolumn{2}{|c|}{ Control } & \multirow{2}{*}{$\begin{array}{c}\text { Std. Mean Difference } \\
\text { Random, } 95 \% \mathrm{Cl}\end{array}$} & \multirow[t]{2}{*}{ Weight } & \multirow{2}{*}{$\begin{array}{c}\text { Std. Mean Difference } \\
\text { Random, } 95 \% \mathrm{Cl}\end{array}$} \\
\hline & $\mathbf{N}$ & Mean(SD) & $\mathbf{N}$ & Mean(SD) & & & \\
\hline \multicolumn{8}{|c|}{ 16.5.1 During the acute crisis } \\
\hline Rahman 2016a & 146 & $7.3(3.6)$ & 160 & $10(3.9)$ & + & $19.39 \%$ & $-0.74[-0.97,-0.51]$ \\
\hline Weiss 2015a & 123 & $0.5(0.5)$ & 61 & $0.7(0.7)$ & $\rightarrow$ & $17.56 \%$ & $-0.36[-0.67,-0.05]$ \\
\hline Weiss 2015b & 98 & $0.4(0.4)$ & 48 & $1(0.9)$ & $\rightarrow$ & $16.18 \%$ & $-1.01[-1.38,-0.65]$ \\
\hline 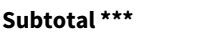 & 367 & & 269 & & & $53.14 \%$ & $-0.69[-1.03,-0.36]$ \\
\hline \multicolumn{8}{|c|}{ Heterogeneity: $\mathrm{Tau}^{2}=0.06 ; \mathrm{Chi}^{2}=7.59, \mathrm{df}=2(\mathrm{P}=0.02) ; \mathrm{I}^{2}=73.63 \%$} \\
\hline \multicolumn{8}{|c|}{ Test for overall effect: $Z=4.05(P<0.0001)$} \\
\hline \multicolumn{8}{|c|}{ 16.5.2 After the acute crisis } \\
\hline Bolton 2014a & 101 & $0.8(1)$ & 33 & $1(0.5)$ & $\rightarrow$ & $15.46 \%$ & $-0.24[-0.64,0.15]$ \\
\hline Bolton 2014a & 114 & $0.8(1.1)$ & 33 & $0.9(0.5)$ & 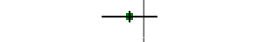 & $15.61 \%$ & $-0.2[-0.59,0.19]$ \\
\hline Zang 2013 & 11 & $5.5(3)$ & 11 & $4.8(2.5)$ & & $7.39 \%$ & $0.22[-0.62,1.06]$ \\
\hline Subtotal $\star \star \star$ & 246 & & 87 & & & $46.86 \%$ & $-0.16[-0.41,0.08]$ \\
\hline \multicolumn{8}{|c|}{ Heterogeneity: $\operatorname{Tau}^{2}=0 ; \mathrm{Chi}^{2}=1.05, \mathrm{df}=3(\mathrm{P}=0.79) ; \mathrm{I}^{2}=0 \%$} \\
\hline \multicolumn{8}{|c|}{ Test for overall effect: $Z=1.3(P=0.19)$} \\
\hline Total $\star \star \star ~$ & 613 & & 356 & & & $100 \%$ & $-0.43[-0.71,-0.15]$ \\
\hline \multicolumn{8}{|c|}{ Heterogeneity: $\mathrm{Tau}^{2}=0.09 ; \mathrm{Chi}^{2}=20.32, \mathrm{df}=6(\mathrm{P}=0) ; \mathrm{I}^{2}=70.47 \%$} \\
\hline \multicolumn{8}{|c|}{ Test for overall effect: $Z=3.01(P=0)$} \\
\hline \multicolumn{8}{|c|}{ Test for subgroup differences: $\mathrm{Chi}^{2}=6.15, \mathrm{df}=1(\mathrm{P}=0.01), \mathrm{I}^{2}=83.75 \%$} \\
\hline
\end{tabular}

Analysis 16.6. Comparison 16 Subgroup analysis: phase of humanitarian crisis - adults, Outcome 6 Depressive symptoms at endpoint.

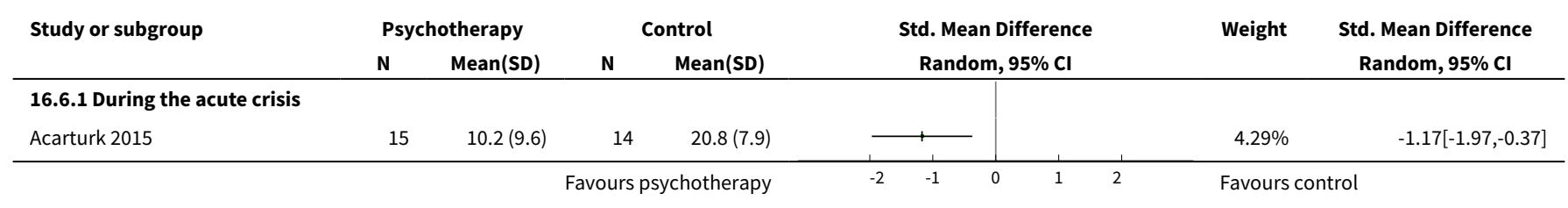

Psychological therapies for the treatment of mental disorders in low- and middle-income countries affected by humanitarian crises 


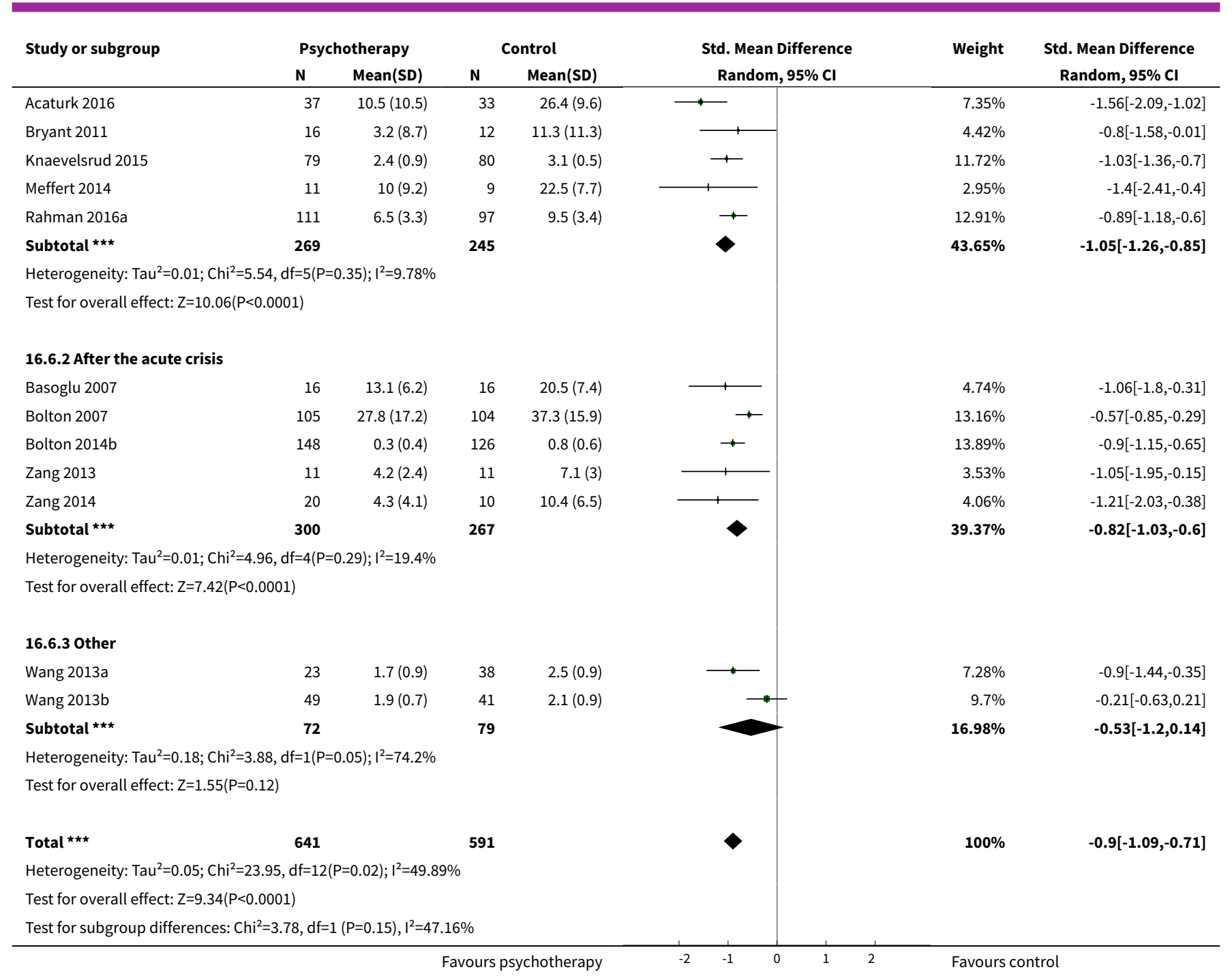

Analysis 16.7. Comparison 16 Subgroup analysis: phase of humanitarian crisis - adults, Outcome 7 Depressive symptoms at 1 to 4 months.

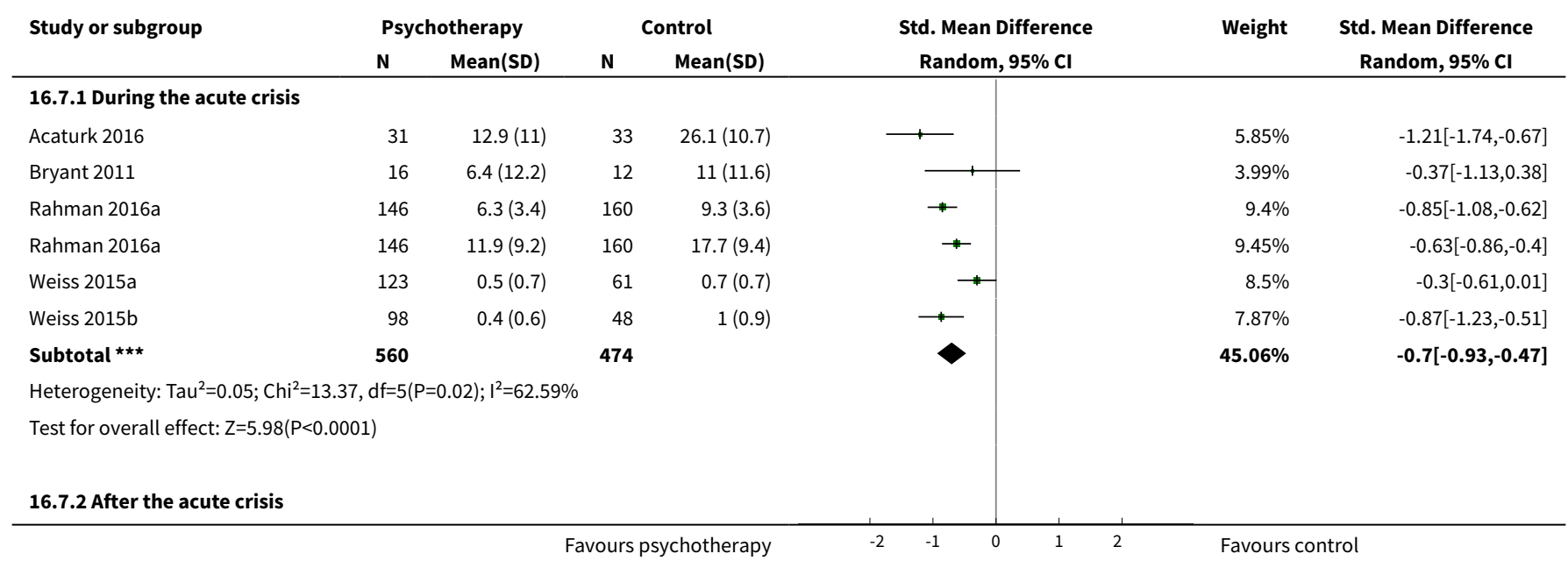

Psychological therapies for the treatment of mental disorders in low- and middle-income countries affected by humanitarian crises 


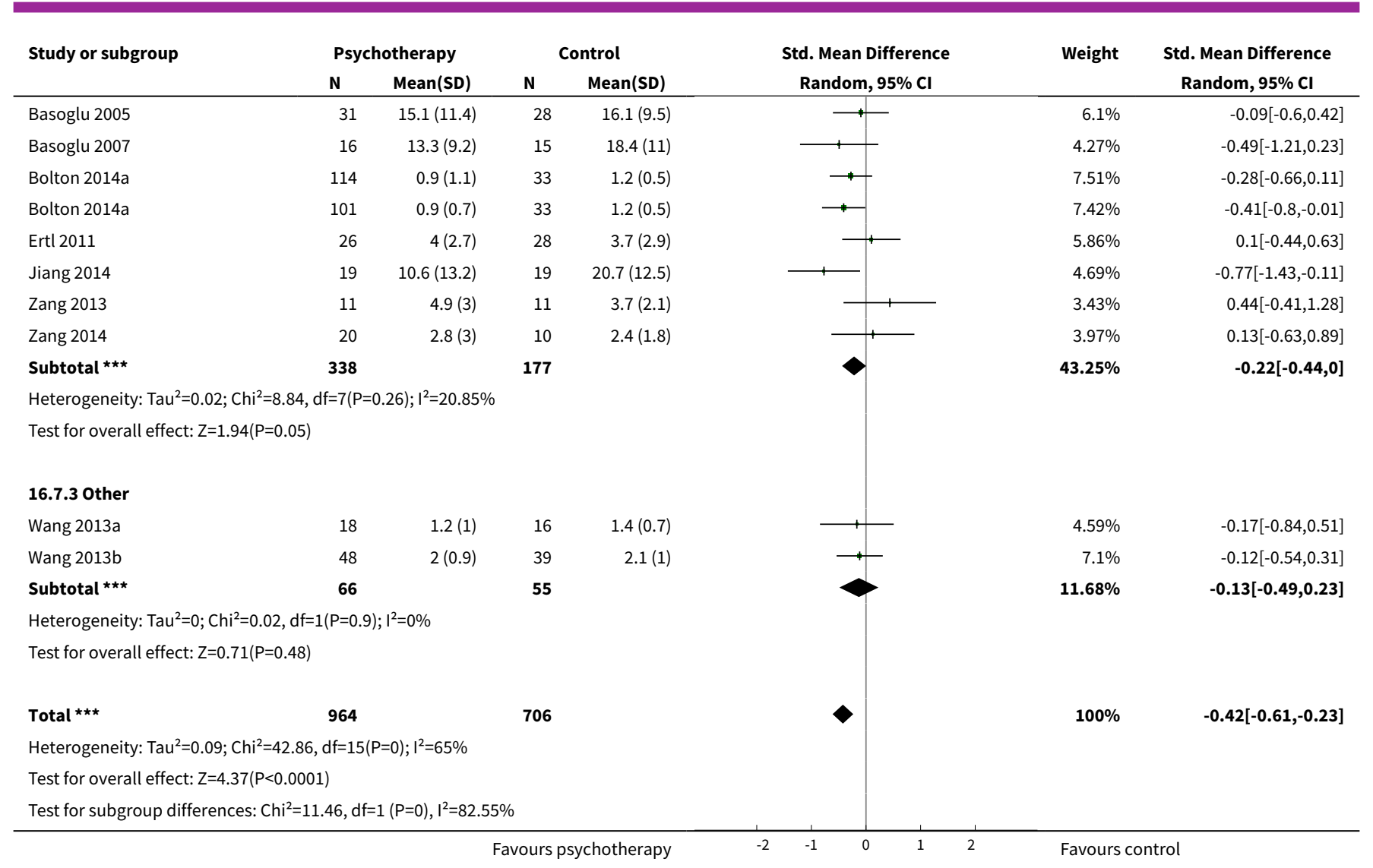

Analysis 16.8. Comparison 16 Subgroup analysis: phase of humanitarian crisis - adults, Outcome 8 Depressive symptoms $\geq 6$ months.

\begin{tabular}{|c|c|c|c|c|c|c|c|}
\hline \multirow[t]{2}{*}{ Study or subgroup } & \multicolumn{2}{|c|}{ Psychotherapy } & \multicolumn{2}{|c|}{ Control } & \multirow{2}{*}{$\begin{array}{c}\text { Std. Mean Difference } \\
\text { Random, } 95 \% \mathrm{Cl}\end{array}$} & \multirow[t]{2}{*}{ Weight } & \multirow{2}{*}{$\begin{array}{c}\text { Std. Mean Difference } \\
\text { Random, } 95 \% \mathrm{Cl}\end{array}$} \\
\hline & $\mathbf{N}$ & Mean(SD) & $\mathbf{N}$ & $\operatorname{Mean}(S D)$ & & & \\
\hline \multicolumn{8}{|c|}{ 16.8.1 During the acute crisis } \\
\hline Bass 2016 & 145 & $0.8(0.7)$ & 43 & $1(0.8)$ & & $70.97 \%$ & $-0.26[-0.61,0.08]$ \\
\hline 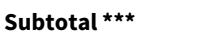 & 145 & & 43 & & & $70.97 \%$ & $-0.26[-0.61,0.08]$ \\
\hline \multicolumn{8}{|c|}{ Heterogeneity: Not applicable } \\
\hline \multicolumn{8}{|c|}{ Test for overall effect: $Z=1.52(P=0.13)$} \\
\hline \multicolumn{8}{|c|}{ 16.8.2 After the acute crisis } \\
\hline Ertl 2011 & 26 & $3.1(3)$ & 28 & $3.2(3)$ & — & $29.03 \%$ & $-0.04[-0.58,0.49]$ \\
\hline Subtotal $\star \star \star ~$ & 26 & & 28 & & & $29.03 \%$ & $-0.04[-0.58,0.49]$ \\
\hline \multicolumn{8}{|c|}{ Heterogeneity: Not applicable } \\
\hline \multicolumn{8}{|c|}{ Test for overall effect: $Z=0.16(P=0.87)$} \\
\hline \multicolumn{8}{|c|}{ Heterogeneity: $\mathrm{Tau}^{2}=0 ; \mathrm{Chi}^{2}=0.47, \mathrm{df}=1(\mathrm{P}=0.49) ; \mathrm{I}^{2}=0 \%$} \\
\hline \multicolumn{8}{|c|}{ Test for overall effect: $Z=1.36(P=0.17)$} \\
\hline \multicolumn{8}{|c|}{ Test for subgroup differences: $\mathrm{Chi}^{2}=0.47, \mathrm{df}=1(\mathrm{P}=0.49), \mathrm{I}^{2}=0 \%$} \\
\hline
\end{tabular}


Analysis 16.9. Comparison 16 Subgroup analysis: phase of humanitarian crisis - adults, Outcome 9 Dropout.

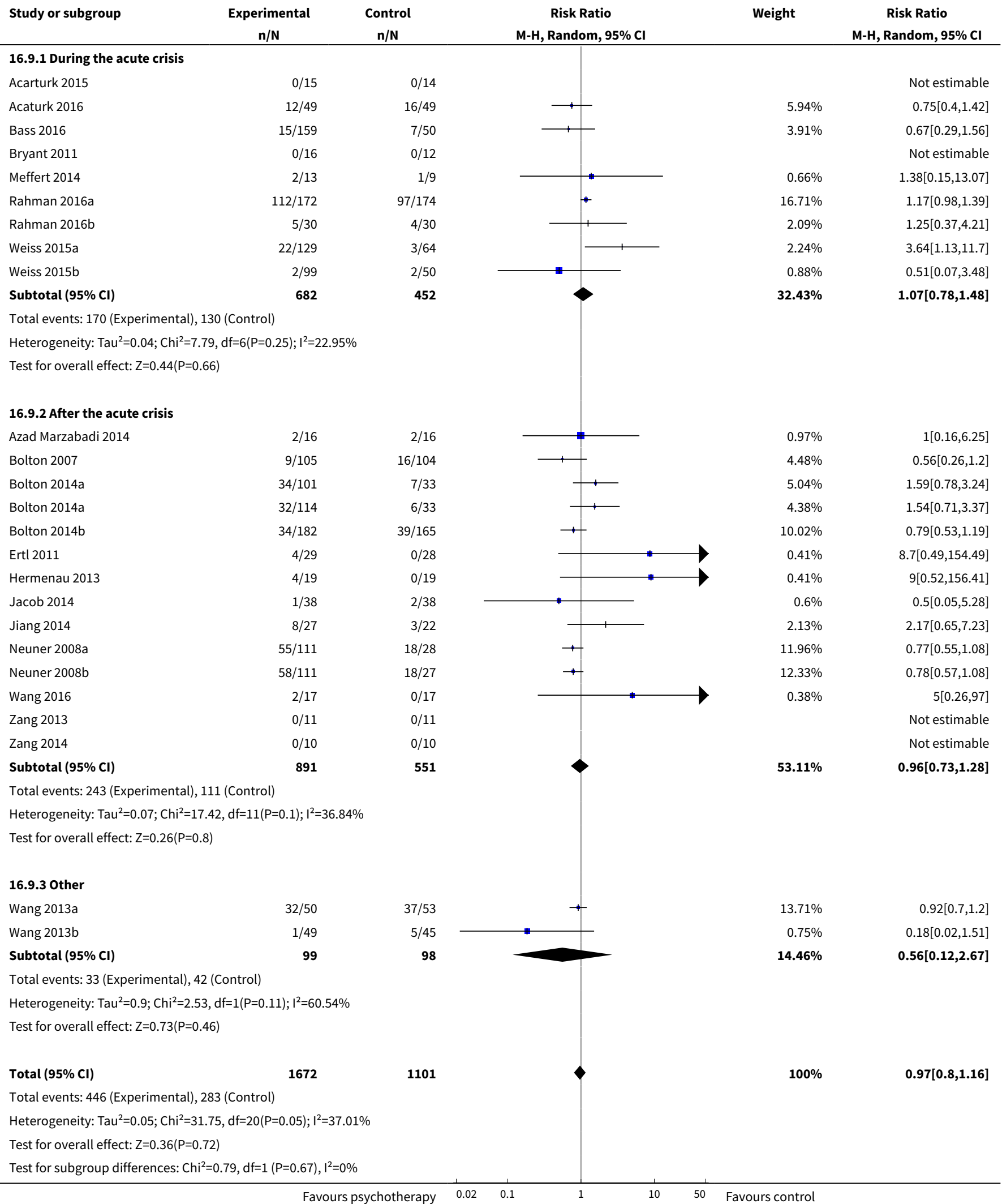


Analysis 16.10. Comparison 16 Subgroup analysis: phase of humanitarian crisis - adults, Outcome 10 Functional impairment at endpoint.

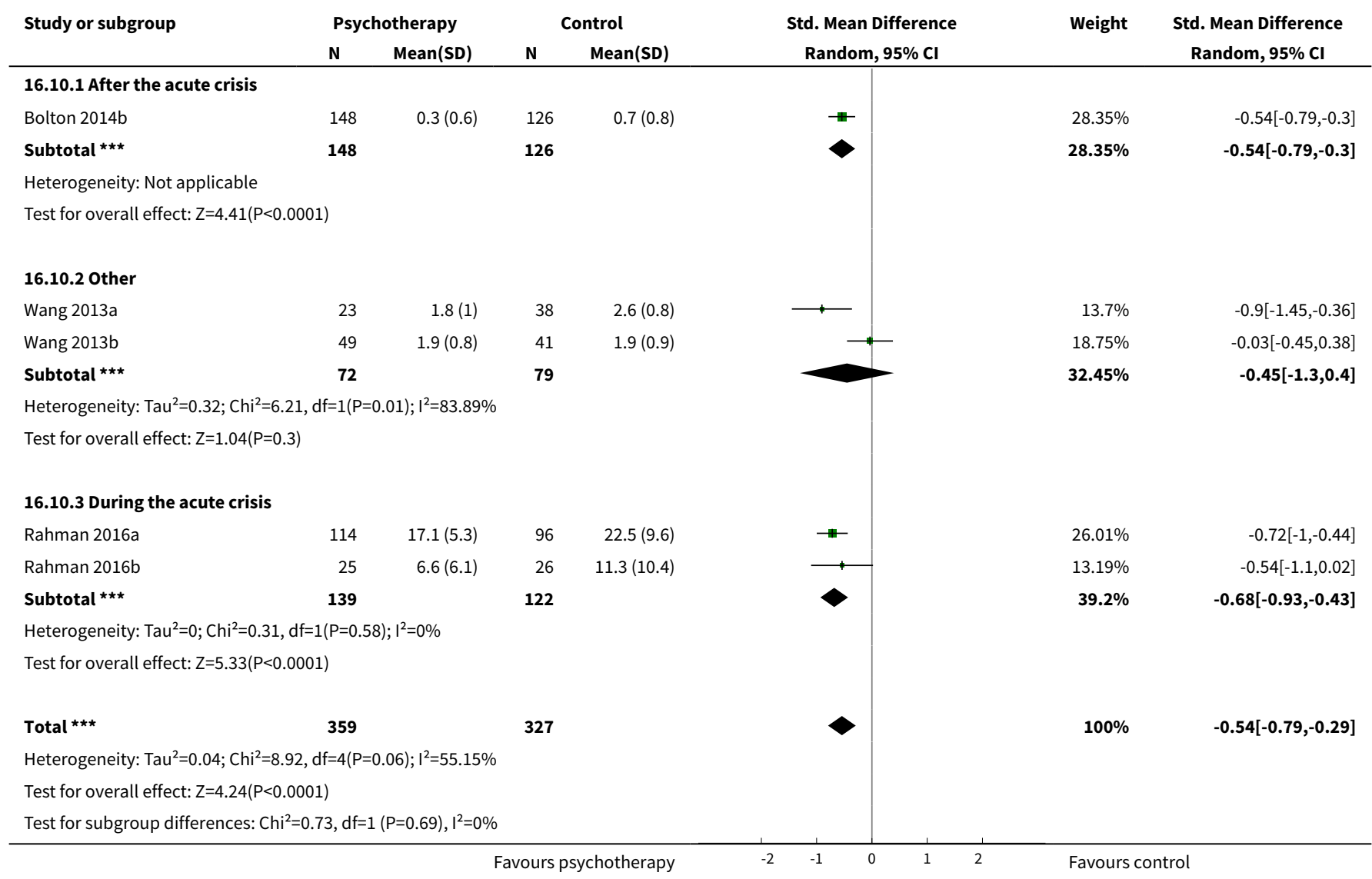

Analysis 16.11. Comparison 16 Subgroup analysis: phase of humanitarian crisis - adults, Outcome 11 Functional impairment at 1 to 4 months.

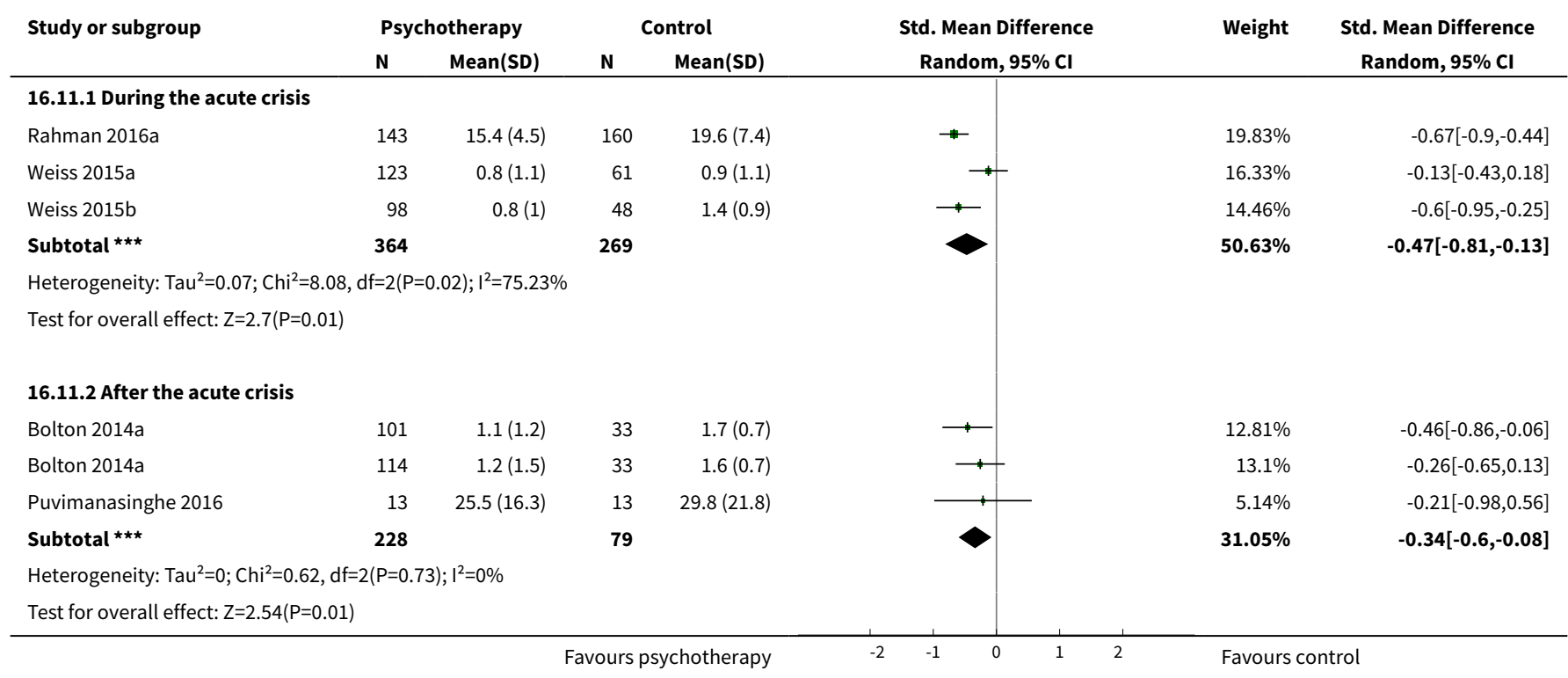

Psychological therapies for the treatment of mental disorders in low- and middle-income countries affected by humanitarian crises 


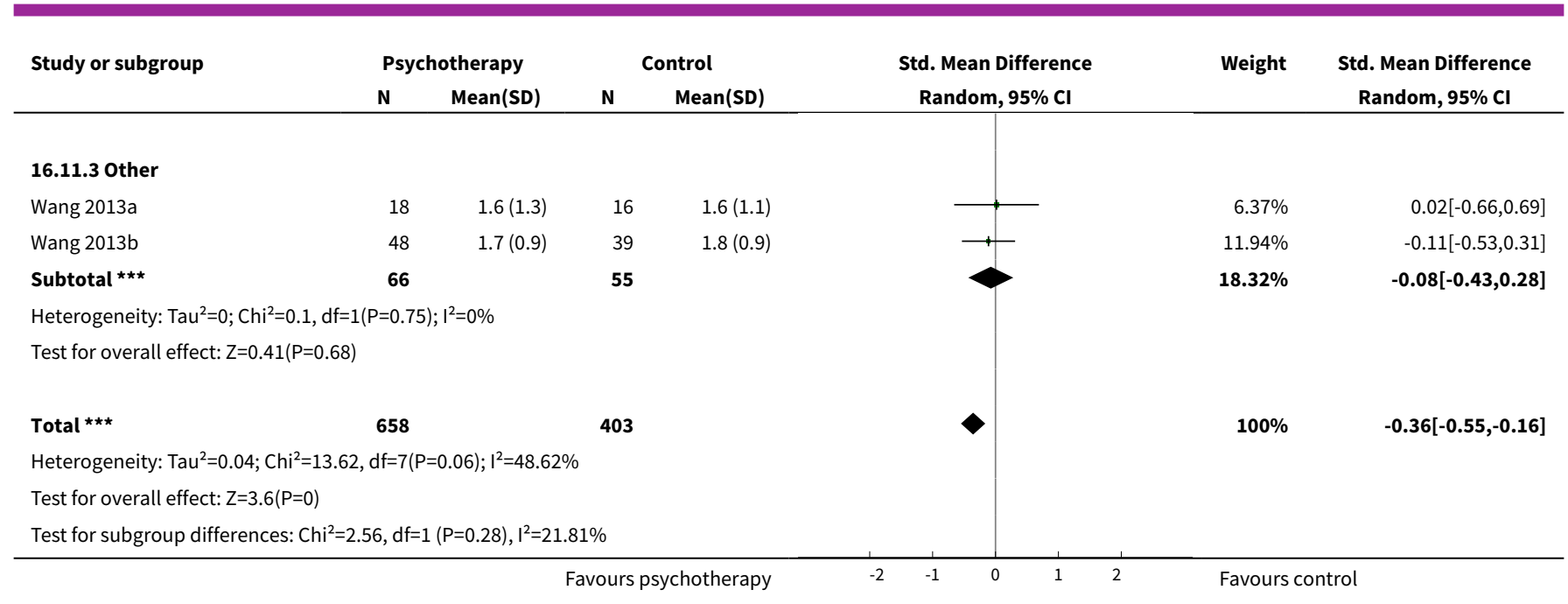

Analysis 16.12. Comparison 16 Subgroup analysis: phase of humanitarian crisis - adults, Outcome 12 Quality of life at endpoint.

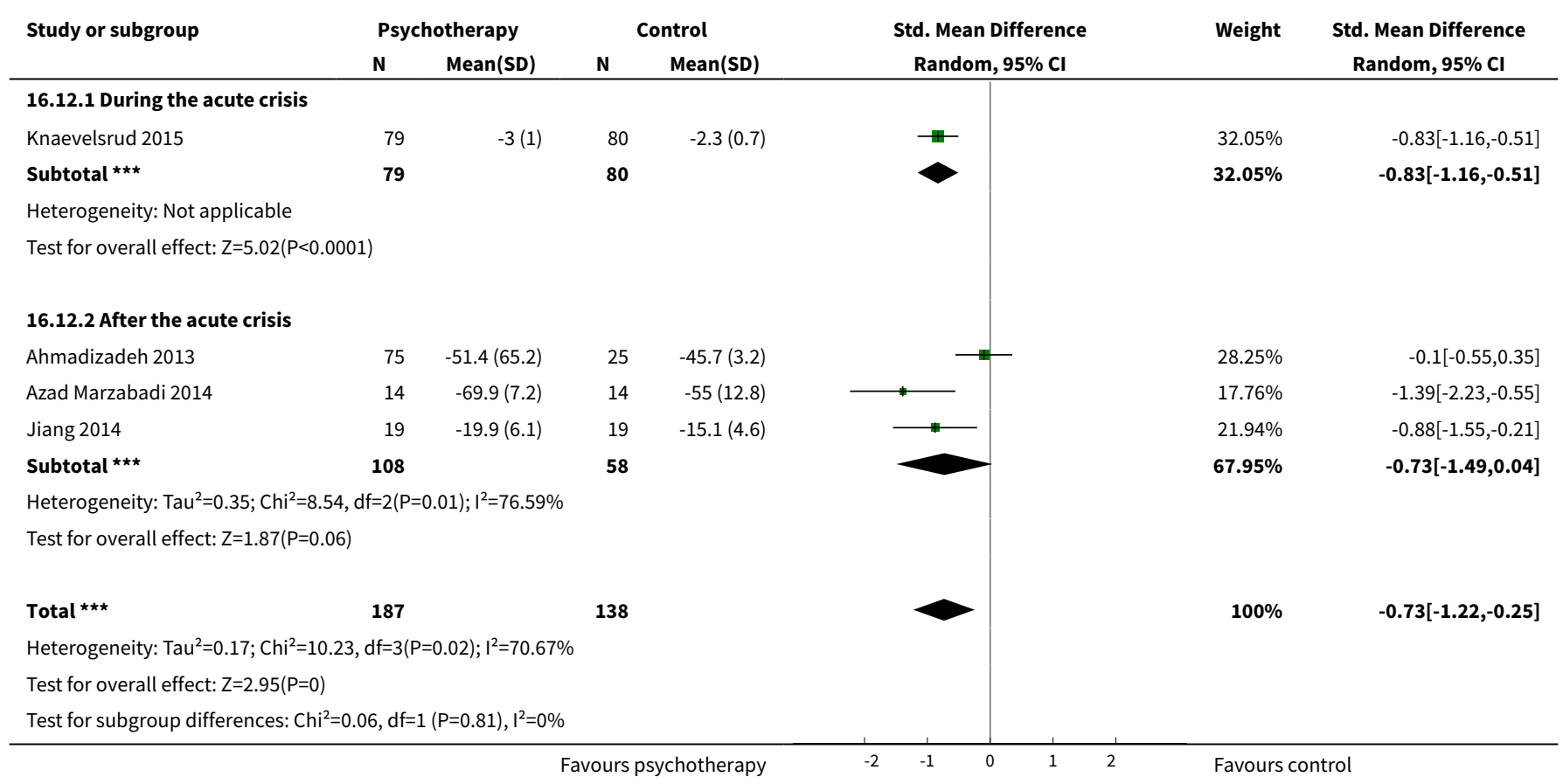

Analysis 16.13. Comparison 16 Subgroup analysis: phase of humanitarian crisis - adults, Outcome 13 Diagnosis of PTSD.

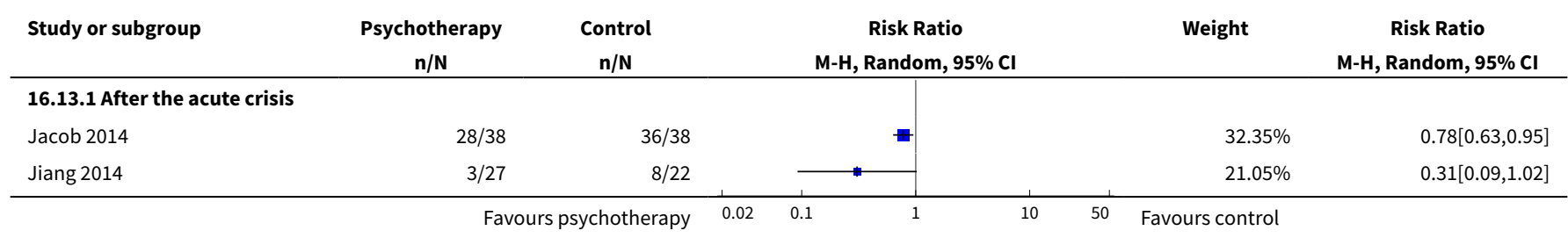




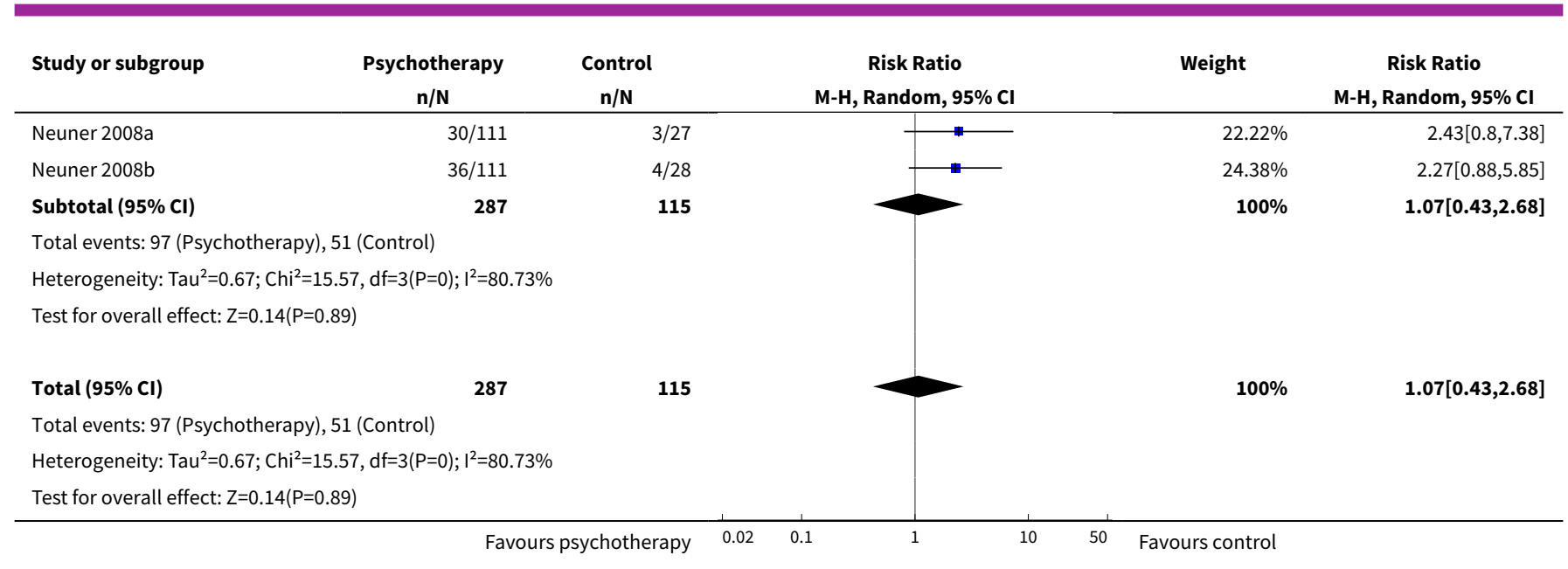

\section{Comparison 17. Sensitivity analysis: incomplete outcome data - adults}

\begin{tabular}{|c|c|c|c|c|}
\hline $\begin{array}{l}\text { Outcome or subgroup } \\
\text { title }\end{array}$ & No. of studies & $\begin{array}{l}\text { No. of partici- } \\
\text { pants }\end{array}$ & Statistical method & Effect size \\
\hline $\begin{array}{l}1 \text { PTSD symptoms at end- } \\
\text { point }\end{array}$ & 9 & 401 & $\begin{array}{l}\text { Std. Mean Difference (IV, Random, 95\% } \\
\text { CI) }\end{array}$ & $-1.27[-1.76,-0.78]$ \\
\hline 1.1 EMDR vs control & 1 & 29 & $\begin{array}{l}\text { Std. Mean Difference (IV, Random, 95\% } \\
\text { CI) }\end{array}$ & $-1.65[-2.51,-0.79]$ \\
\hline 1.2 CBT vs control & 7 & 352 & $\begin{array}{l}\text { Std. Mean Difference (IV, Random, 95\% } \\
\text { CI) }\end{array}$ & $-1.21[-1.80,-0.63]$ \\
\hline 1.3 IPT vs control & 1 & 20 & $\begin{array}{l}\text { Std. Mean Difference (IV, Random, 95\% } \\
\text { CI) }\end{array}$ & $-1.45[-2.46,-0.43]$ \\
\hline $\begin{array}{l}2 \text { PTSD symptoms at } 1 \text { to } \\
4 \text { months }\end{array}$ & 12 & 943 & $\begin{array}{l}\text { Std. Mean Difference (IV, Random, 95\% } \\
\text { CI) }\end{array}$ & $-0.40[-0.58,-0.22]$ \\
\hline 2.1 CBT vs control & 11 & 905 & $\begin{array}{l}\text { Std. Mean Difference (IV, Random, 95\% } \\
\text { CI) }\end{array}$ & $-0.37[-0.54,-0.20]$ \\
\hline 2.2 IPT vs control & 1 & 38 & $\begin{array}{l}\text { Std. Mean Difference (IV, Random, 95\% } \\
\text { CI) }\end{array}$ & $-0.99[-1.67,-0.32]$ \\
\hline $\begin{array}{l}3 \text { PTSD symptoms } \geq 6 \\
\text { months }\end{array}$ & 3 & 272 & $\begin{array}{l}\text { Std. Mean Difference (IV, Random, 95\% } \\
\text { CI) }\end{array}$ & $-0.29[-0.56,-0.03]$ \\
\hline $\begin{array}{l}3.1 \text { Trauma/Supportive } \\
\text { counselling vs control - } \\
\text { Adults }\end{array}$ & 1 & 188 & $\begin{array}{l}\text { Std. Mean Difference (IV, Random, 95\% } \\
\text { CI) }\end{array}$ & $-0.22[-0.57,0.12]$ \\
\hline 3.2 CBT vs control & 2 & 84 & $\begin{array}{l}\text { Std. Mean Difference (IV, Random, 95\% } \\
\text { CI) }\end{array}$ & $-0.41[-0.85,0.03]$ \\
\hline $\begin{array}{l}4 \text { Anxiety symptoms at } \\
\text { endpoint }\end{array}$ & 3 & 211 & $\begin{array}{l}\text { Std. Mean Difference (IV, Random, 95\% } \\
\text { CI) }\end{array}$ & $-0.98[-1.42,-0.54]$ \\
\hline
\end{tabular}




\begin{tabular}{|c|c|c|c|c|}
\hline $\begin{array}{l}\text { Outcome or subgroup } \\
\text { title }\end{array}$ & No. of studies & $\begin{array}{l}\text { No. of partici- } \\
\text { pants }\end{array}$ & Statistical method & Effect size \\
\hline 4.1 CBT vs control & 3 & 211 & $\begin{array}{l}\text { Std. Mean Difference (IV, Random, 95\% } \\
\mathrm{CI} \text { ) }\end{array}$ & $-0.98[-1.42,-0.54]$ \\
\hline $\begin{array}{l}5 \text { Anxiety symptoms at } 1 \\
\text { to } 4 \text { months }\end{array}$ & 5 & 663 & $\begin{array}{l}\text { Std. Mean Difference (IV, Random, 95\% } \\
\text { CI) }\end{array}$ & $-0.35[-0.67,-0.03]$ \\
\hline 5.1 CBT vs control & 5 & 663 & $\begin{array}{l}\text { Std. Mean Difference (IV, Random, 95\% } \\
\mathrm{CI} \text { ) }\end{array}$ & $-0.35[-0.67,-0.03]$ \\
\hline $\begin{array}{l}6 \text { Depressive symptoms } \geq \\
6 \text { months }\end{array}$ & 2 & 242 & $\begin{array}{l}\text { Std. Mean Difference (IV, Random, 95\% } \\
\mathrm{CI} \text { ) }\end{array}$ & $-0.20[-0.49,0.09]$ \\
\hline $\begin{array}{l}\text { 6.1 Trauma/Supportive } \\
\text { counselling vs control }\end{array}$ & 1 & 188 & $\begin{array}{l}\text { Std. Mean Difference (IV, Random, 95\% } \\
\mathrm{CI} \text { ) }\end{array}$ & $-0.26[-0.61,0.08]$ \\
\hline 6.2 CBT vs control & 1 & 54 & $\begin{array}{l}\text { Std. Mean Difference (IV, Random, 95\% } \\
\mathrm{CI} \text { ) }\end{array}$ & $-0.04[-0.58,0.49]$ \\
\hline $\begin{array}{l}7 \text { Depressive symptoms } \\
\text { at endpoint }\end{array}$ & 8 & 529 & $\begin{array}{l}\text { Std. Mean Difference (IV, Random, 95\% } \\
\mathrm{CI} \text { ) }\end{array}$ & $-0.89[-1.09,-0.68]$ \\
\hline 7.1 EMDR vs control & 1 & 29 & $\begin{array}{l}\text { Std. Mean Difference (IV, Random, 95\% } \\
\mathrm{CI} \text { ) }\end{array}$ & $-1.17[-1.97,-0.37]$ \\
\hline 7.2 CBT vs control & 5 & 271 & $\begin{array}{l}\text { Std. Mean Difference (IV, Random, 95\% } \\
\mathrm{CI} \text { ) }\end{array}$ & $-1.02[-1.28,-0.77]$ \\
\hline 7.3 IPT vs control & 2 & 229 & $\begin{array}{l}\text { Std. Mean Difference (IV, Random, 95\% } \\
\mathrm{CI} \text { ) }\end{array}$ & $-0.84[-1.60,-0.08]$ \\
\hline $\begin{array}{l}8 \text { Depressive symptoms } \\
\text { at } 1 \text { to } 4 \text { months }\end{array}$ & 10 & 739 & $\begin{array}{l}\text { Std. Mean Difference (IV, Random, 95\% } \\
\text { CI) }\end{array}$ & $-0.52[-1.07,0.03]$ \\
\hline 8.1 IPT vs control & 1 & 38 & $\begin{array}{l}\text { Std. Mean Difference (IV, Random, 95\% } \\
\text { CI) }\end{array}$ & $-0.77[-1.43,-0.11]$ \\
\hline 8.2 CBT vs control & 9 & 701 & $\begin{array}{l}\text { Std. Mean Difference (IV, Random, 95\% } \\
\mathrm{CI} \text { ) }\end{array}$ & $-0.49[-1.09,0.11]$ \\
\hline 9 Dropout & 17 & 1535 & Risk Ratio (M-H, Random, 95\% Cl) & $1.22[0.83,1.79]$ \\
\hline 9.1 EMDR vs control & 1 & 29 & Risk Ratio (M-H, Random, 95\% Cl) & $0.0[0.0,0.0]$ \\
\hline 9.2 CBT vs control & 12 & 1017 & Risk Ratio (M-H, Random, 95\% Cl) & $1.53[0.92,2.54]$ \\
\hline 9.3 IPT vs control & 2 & 231 & Risk Ratio (M-H, Random, 95\% Cl) & $0.61[0.30,1.27]$ \\
\hline 9.4 IPT vs control & 1 & 49 & Risk Ratio (M-H, Random, 95\% Cl) & $2.17[0.65,7.23]$ \\
\hline $\begin{array}{l}\text { 9.5 Trauma/Supportive } \\
\text { counselling vs control }\end{array}$ & 1 & 209 & Risk Ratio (M-H, Random, 95\% Cl) & $0.67[0.29,1.56]$ \\
\hline $\begin{array}{l}10 \text { Functional impair- } \\
\text { ment at endpoint }\end{array}$ & 2 & 141 & $\begin{array}{l}\text { Std. Mean Difference (IV, Random, 95\% } \\
\mathrm{CI} \text { ) }\end{array}$ & $-0.25[-0.74,0.24]$ \\
\hline
\end{tabular}




\begin{tabular}{|c|c|c|c|c|}
\hline $\begin{array}{l}\text { Outcome or subgroup } \\
\text { title }\end{array}$ & No. of studies & $\begin{array}{l}\text { No. of partici- } \\
\text { pants }\end{array}$ & Statistical method & Effect size \\
\hline 10.1 CBT vs control & 2 & 141 & $\begin{array}{l}\text { Std. Mean Difference (IV, Random, 95\% } \\
\mathrm{CI} \text { ) }\end{array}$ & $-0.25[-0.74,0.24]$ \\
\hline $\begin{array}{l}11 \text { Functional impair- } \\
\text { ment at } 1 \text { to } 4 \text { months }\end{array}$ & 4 & 637 & $\begin{array}{l}\text { Std. Mean Difference (IV, Random, 95\% } \\
\mathrm{CI} \text { ) }\end{array}$ & $-0.32[-0.50,-0.14]$ \\
\hline 11.1 CBT vs control & 4 & 637 & $\begin{array}{l}\text { Std. Mean Difference (IV, Random, 95\% } \\
\mathrm{CI} \text { ) }\end{array}$ & $-0.32[-0.50,-0.14]$ \\
\hline $\begin{array}{l}12 \text { Quality of life at end- } \\
\text { point }\end{array}$ & 3 & 297 & $\begin{array}{l}\text { Std. Mean Difference (IV, Random, 95\% } \\
\text { CI) }\end{array}$ & $-0.59[-1.10,-0.08]$ \\
\hline 12.1 IPT vs control & 1 & 38 & $\begin{array}{l}\text { Std. Mean Difference (IV, Random, 95\% } \\
\text { CI) }\end{array}$ & $-0.88[-1.55,-0.21]$ \\
\hline 12.2 CBT vs control & 2 & 259 & $\begin{array}{l}\text { Std. Mean Difference (IV, Random, 95\% } \\
\text { CI) }\end{array}$ & $-0.48[-1.20,0.23]$ \\
\hline 13 Diagnosis of PTSD & 2 & 125 & Risk Ratio (M-H, Random, 95\% Cl) & $0.56[0.20,1.59]$ \\
\hline 13.1 IPT vs control & 1 & 49 & Risk Ratio (M-H, Random, 95\% Cl) & $0.31[0.09,1.02]$ \\
\hline 13.2 CBT vs control & 1 & 76 & Risk Ratio (M-H, Random, 95\% Cl) & $0.78[0.63,0.95]$ \\
\hline
\end{tabular}

\section{Analysis 17.1. Comparison 17 Sensitivity analysis: incomplete} outcome data - adults, Outcome 1 PTSD symptoms at endpoint.

\begin{tabular}{|c|c|c|c|c|c|c|c|c|}
\hline \multirow[t]{2}{*}{ Study or subgroup } & \multicolumn{2}{|c|}{ Psychotherapy } & \multicolumn{2}{|c|}{ Control } & \multirow{2}{*}{\multicolumn{2}{|c|}{$\begin{array}{c}\text { Std. Mean Difference } \\
\text { Random, } 95 \% \mathrm{Cl}\end{array}$}} & \multirow[t]{2}{*}{ Weight } & \multirow{2}{*}{$\begin{array}{c}\text { Std. Mean Difference } \\
\text { Random, } 95 \% \mathrm{Cl}\end{array}$} \\
\hline & $\mathbf{N}$ & $\operatorname{Mean}(S D)$ & $\mathbf{N}$ & Mean(SD) & & & & \\
\hline \multicolumn{9}{|c|}{ 17.1.1 EMDR vs control } \\
\hline Acarturk 2015 & 15 & $22.9(20.3)$ & 14 & $54.2(16.3)$ & & & $10.79 \%$ & $-1.65[-2.51,-0.79]$ \\
\hline 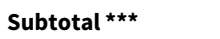 & 15 & & 14 & & & & $10.79 \%$ & $-1.65[-2.51,-0.79]$ \\
\hline \multicolumn{9}{|c|}{ Heterogeneity: $\operatorname{Tau}^{2}=0 ; \mathrm{Chi}^{2}=0, \mathrm{df}=0(\mathrm{P}<0.0001) ; \mathrm{I}^{2}=100 \%$} \\
\hline \multicolumn{9}{|c|}{ Test for overall effect: $Z=3.76(P=0)$} \\
\hline \multicolumn{9}{|l|}{ 17.1.2 CBT vs control } \\
\hline Basoglu 2007 & 16 & $38.7(18.7)$ & 16 & $54.5(16.9)$ & & & $11.89 \%$ & $-0.86[-1.59,-0.14]$ \\
\hline Bryant 2011 & 16 & $4.1(8)$ & 12 & $12.3(8.4)$ & & & $11.31 \%$ & $-0.97[-1.77,-0.18]$ \\
\hline Hermenau 2013 & 15 & $11.5(5.2)$ & 15 & $13.7(5.8)$ & 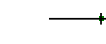 & - & $11.93 \%$ & $-0.4[-1.12,0.32]$ \\
\hline Knaevelsrud 2015 & 79 & $20.3(12.5)$ & 80 & $30.2(8.7)$ & $\rightarrow$ & & $15 \%$ & $-0.92[-1.24,-0.59]$ \\
\hline Rahman 2016b & 25 & $9.8(9.1)$ & 26 & $19.5(18.5)$ & $\longrightarrow$ & & $13.27 \%$ & $-0.65[-1.22,-0.09]$ \\
\hline Zang 2014 & 20 & $17(5.8)$ & 10 & $54.7(10.8)$ & & & $6.52 \%$ & $-4.72[-6.21,-3.23]$ \\
\hline Subtotal $\star \star \star ~$ & 182 & & 170 & & & & $79.63 \%$ & $-1.21[-1.8,-0.63]$ \\
\hline \multicolumn{9}{|c|}{ Heterogeneity: $\operatorname{Tau}^{2}=0.45 ; \mathrm{Chi}^{2}=29.9, \mathrm{df}=6(\mathrm{P}<0.0001) ; \mathrm{I}^{2}=79.93 \%$} \\
\hline \multicolumn{9}{|c|}{ Test for overall effect: $Z=4.08(P<0.0001)$} \\
\hline \multicolumn{9}{|l|}{ 17.1.3 IPT vs control } \\
\hline Meffert 2014 & 11 & $1.8(0.5)$ & 9 & $2.6(0.6)$ & & & $9.57 \%$ & $-1.45[-2.46,-0.43]$ \\
\hline & & & onts & chotherapy & -1 & 1 & Favours & \\
\hline
\end{tabular}




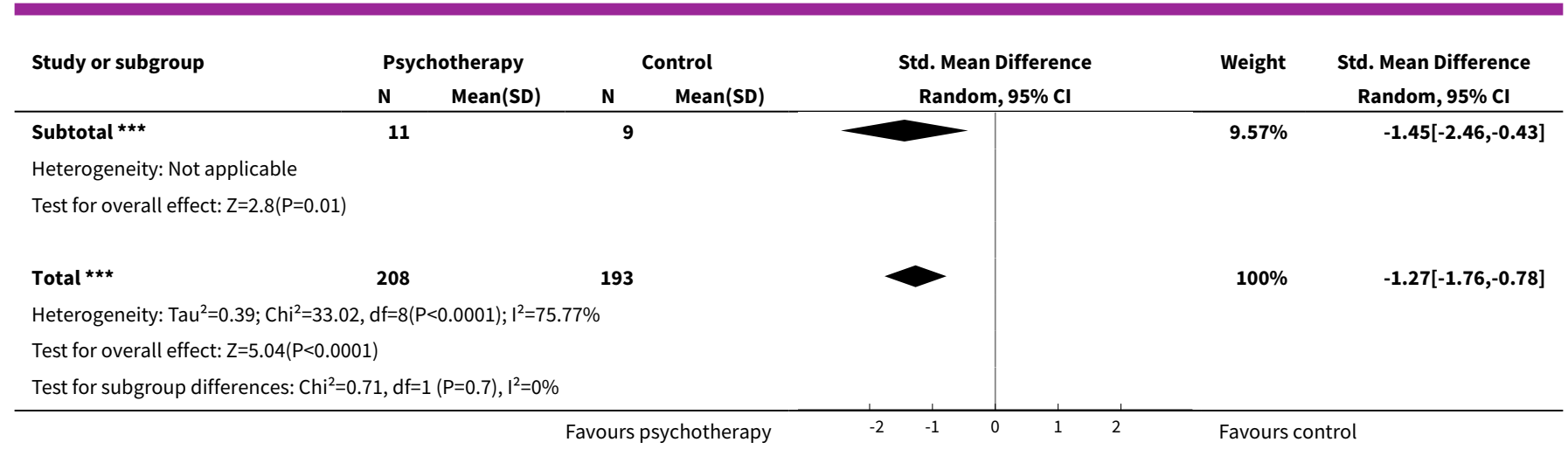

Analysis 17.2. Comparison 17 Sensitivity analysis: incomplete outcome data - adults, Outcome 2 PTSD symptoms at 1 to 4 months.

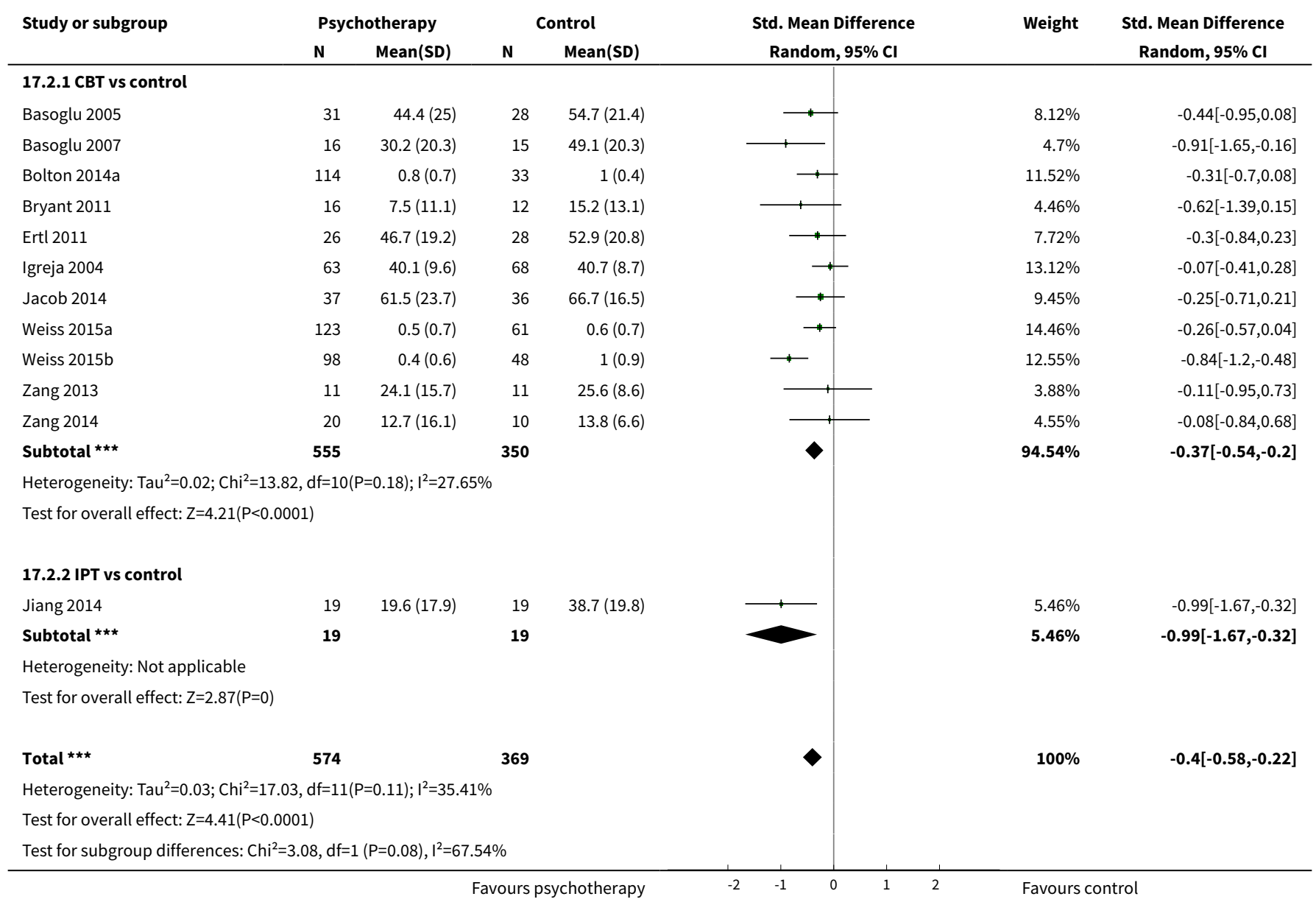


Analysis 17.3. Comparison 17 Sensitivity analysis: incomplete outcome data - adults, Outcome 3 PTSD symptoms $\geq 6$ months.

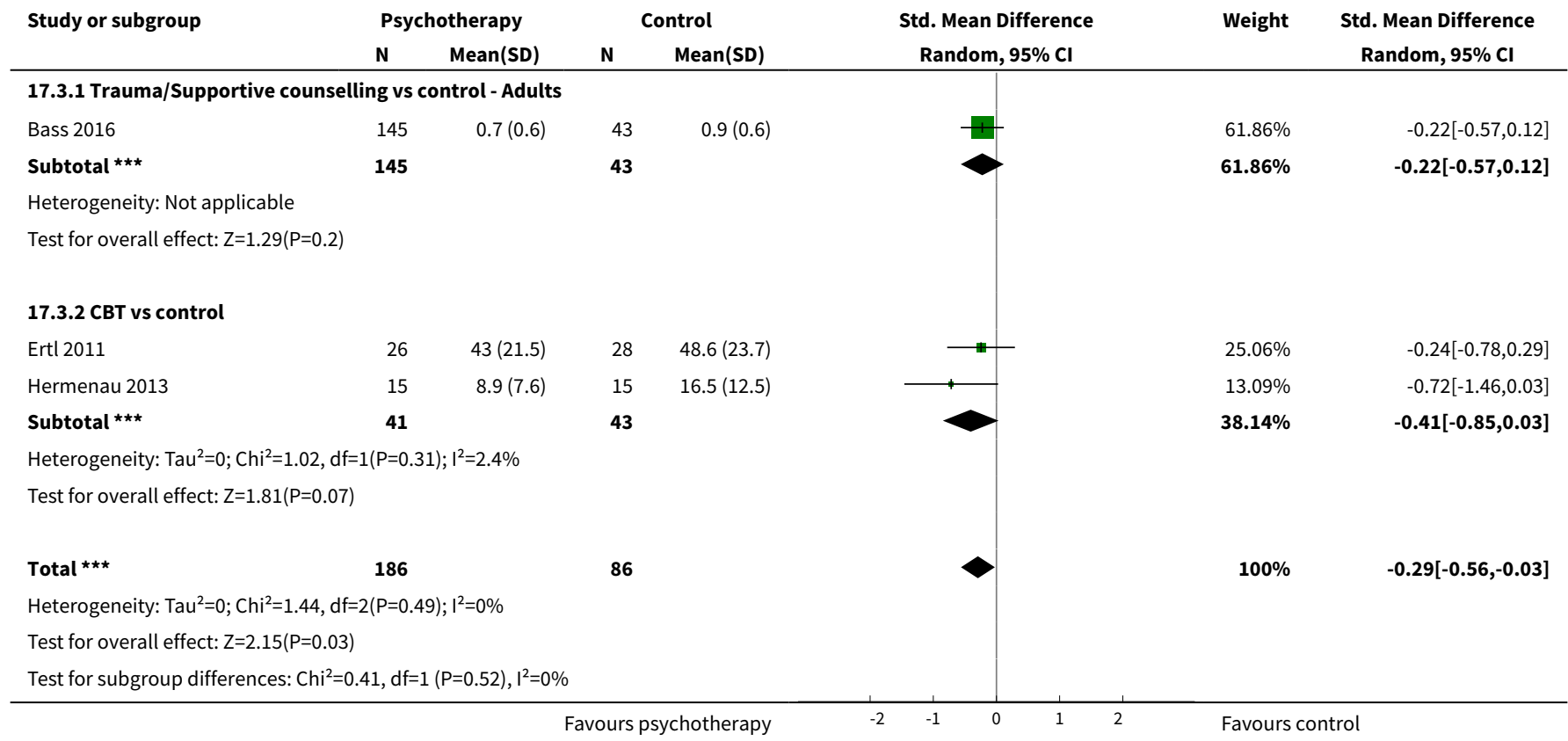

Analysis 17.4. Comparison 17 Sensitivity analysis: incomplete outcome data - adults, Outcome 4 Anxiety symptoms at endpoint.

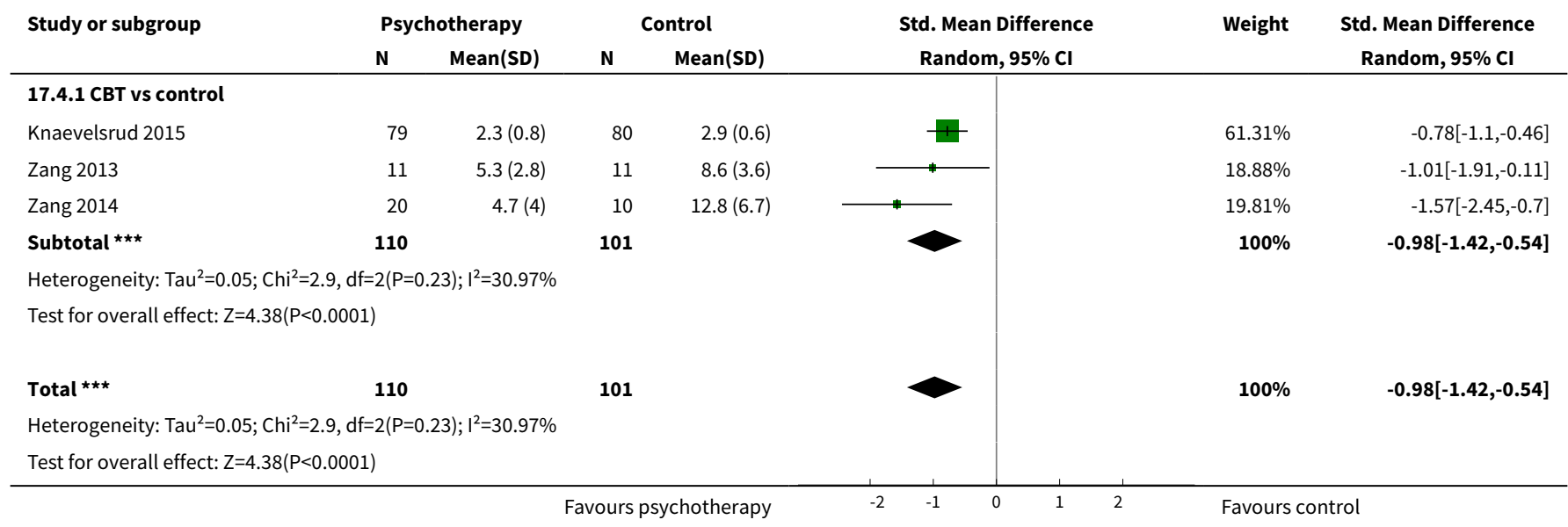

Analysis 17.5. Comparison 17 Sensitivity analysis: incomplete outcome data - adults, Outcome 5 Anxiety symptoms at 1 to 4 months.

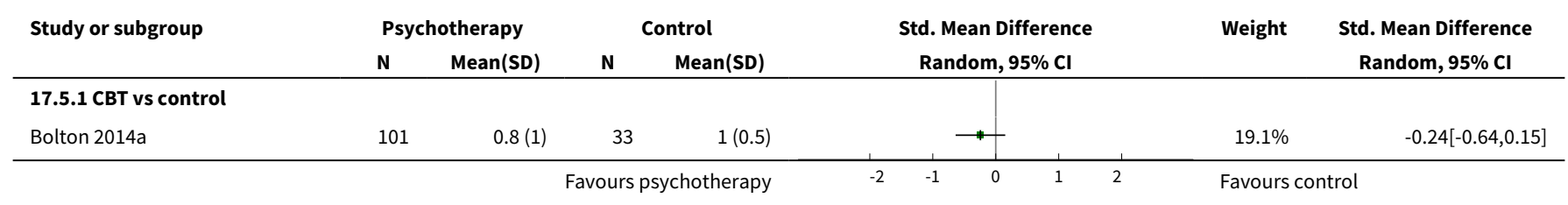




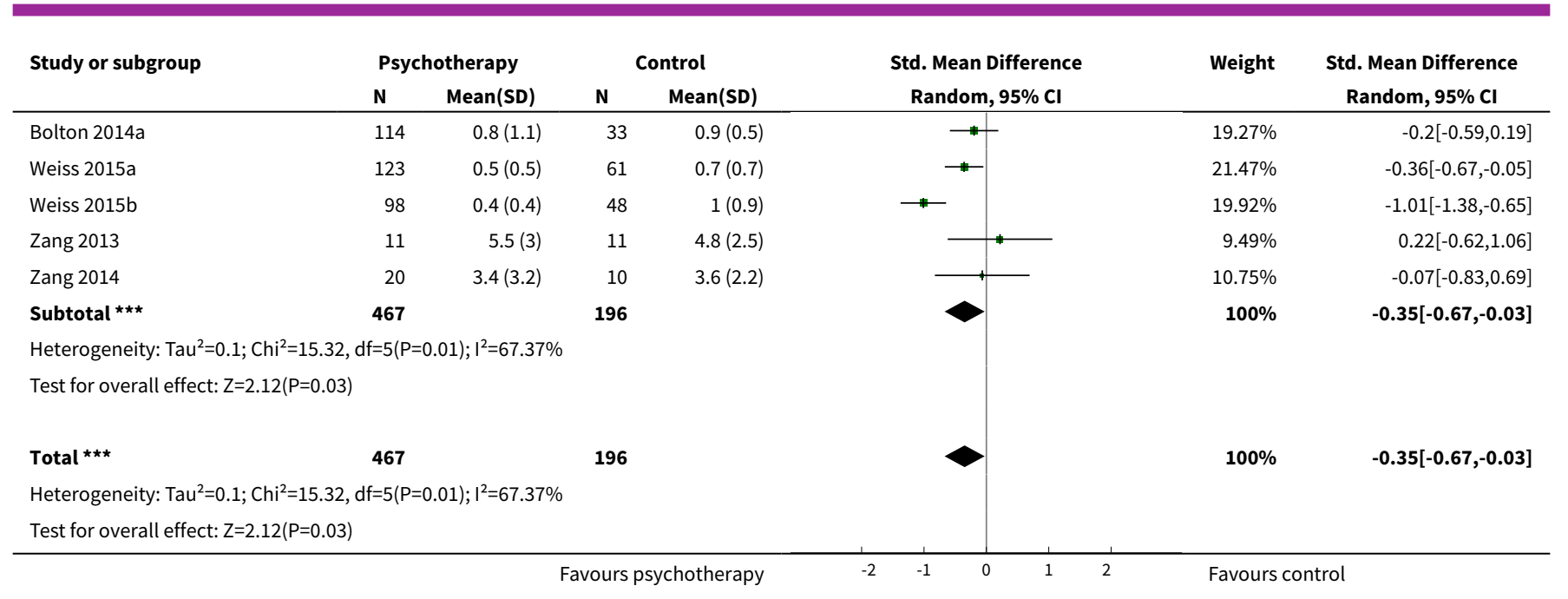

Analysis 17.6. Comparison 17 Sensitivity analysis: incomplete outcome data - adults, Outcome 6 Depressive symptoms $\geq 6$ months.

\begin{tabular}{|c|c|c|c|c|c|c|c|}
\hline \multirow[t]{2}{*}{ Study or subgroup } & \multicolumn{2}{|c|}{ Psychotherapy } & \multicolumn{2}{|c|}{ Control } & \multirow{2}{*}{$\begin{array}{c}\text { Std. Mean Difference } \\
\text { Random, } 95 \% \mathrm{Cl}\end{array}$} & \multirow[t]{2}{*}{ Weight } & \multirow{2}{*}{$\begin{array}{c}\text { Std. Mean Difference } \\
\text { Random, } 95 \% \mathrm{CI}\end{array}$} \\
\hline & $\mathbf{N}$ & Mean(SD) & $\mathbf{N}$ & Mean(SD) & & & \\
\hline \multicolumn{8}{|c|}{ 17.6.1 Trauma/Supportive counselling vs control } \\
\hline Bass 2016 & 145 & $0.8(0.7)$ & 43 & $1(0.8)$ & & $70.97 \%$ & $-0.26[-0.61,0.08]$ \\
\hline Subtotal $\star \star \star$ & 145 & & 43 & & & $70.97 \%$ & $-0.26[-0.61,0.08]$ \\
\hline \multicolumn{8}{|c|}{ Heterogeneity: Not applicable } \\
\hline \multicolumn{8}{|c|}{ Test for overall effect: $Z=1.52(P=0.13)$} \\
\hline \multicolumn{8}{|l|}{ 17.6.2 CBT vs control } \\
\hline Ertl 2011 & 26 & $3.1(3)$ & 28 & $3.2(3)$ & - & $29.03 \%$ & $-0.04[-0.58,0.49]$ \\
\hline Subtotal $\star \star \star$ & 26 & & 28 & & & $29.03 \%$ & $-0.04[-0.58,0.49]$ \\
\hline \multicolumn{8}{|c|}{ Heterogeneity: Not applicable } \\
\hline \multicolumn{8}{|c|}{ Test for overall effect: $Z=0.16(P=0.87)$} \\
\hline 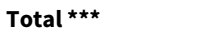 & 171 & & 71 & & & $100 \%$ & $-0.2[-0.49,0.09]$ \\
\hline \multicolumn{8}{|c|}{ Heterogeneity: $\operatorname{Tau}^{2}=0 ; \mathrm{Chi}^{2}=0.47, \mathrm{df}=1(\mathrm{P}=0.49) ; \mathrm{I}^{2}=0 \%$} \\
\hline \multicolumn{8}{|c|}{ Test for overall effect: $Z=1.36(P=0.17)$} \\
\hline \multicolumn{8}{|c|}{ Test for subgroup differences: $\mathrm{Chi}^{2}=0.47, \mathrm{df}=1(\mathrm{P}=0.49), \mathrm{I}^{2}=0 \%$} \\
\hline
\end{tabular}

Analysis 17.7. Comparison 17 Sensitivity analysis: incomplete outcome data - adults, Outcome 7 Depressive symptoms at endpoint.

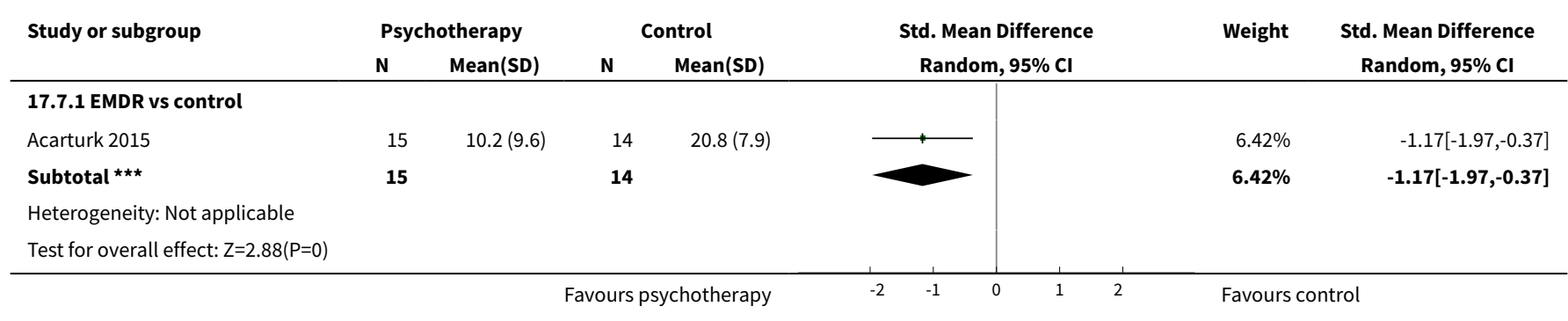

Psychological therapies for the treatment of mental disorders in low- and middle-income countries affected by humanitarian crises 


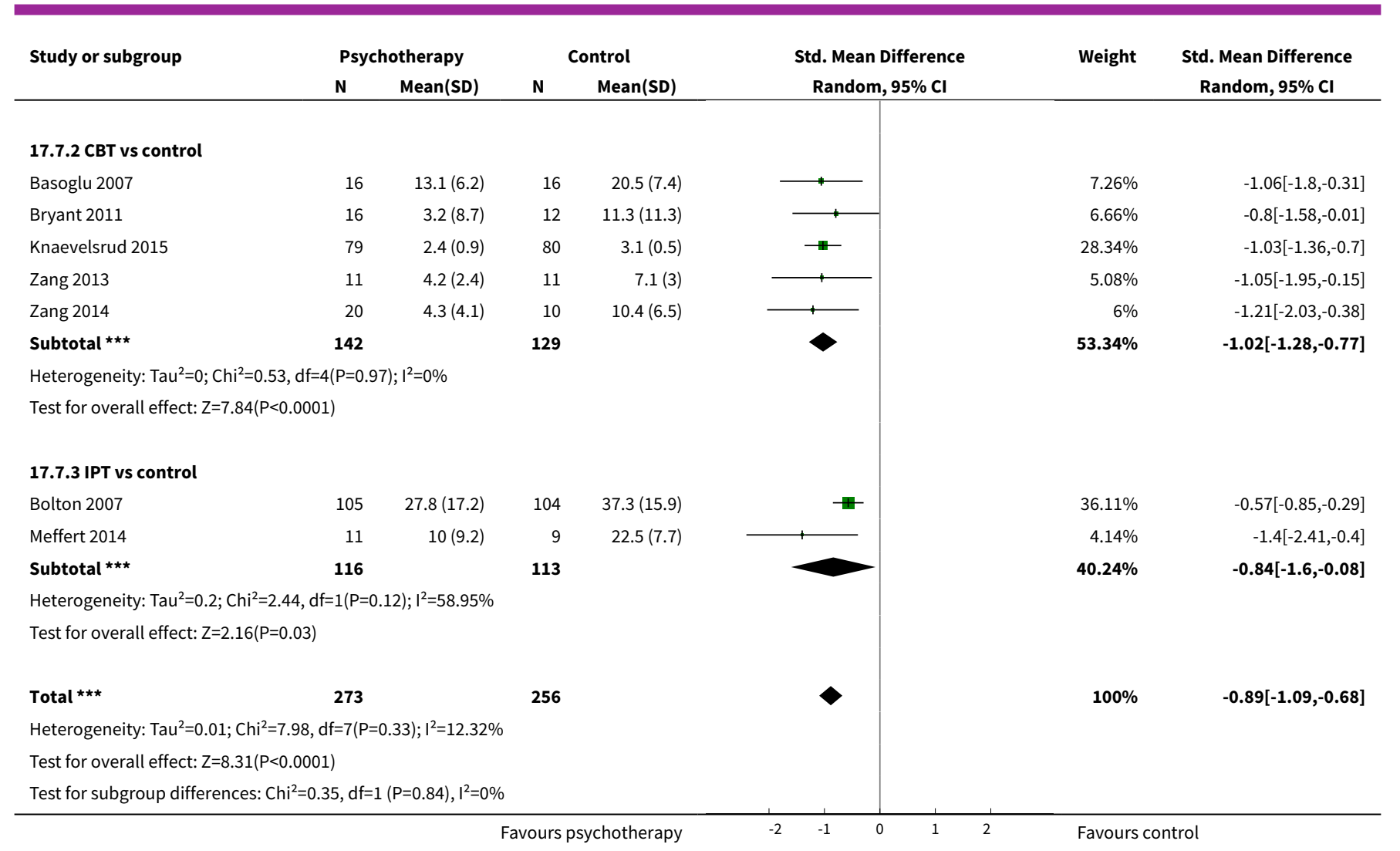

\section{Analysis 17.8. Comparison 17 Sensitivity analysis: incomplete outcome data - adults, Outcome 8 Depressive symptoms at 1 to 4 months.}

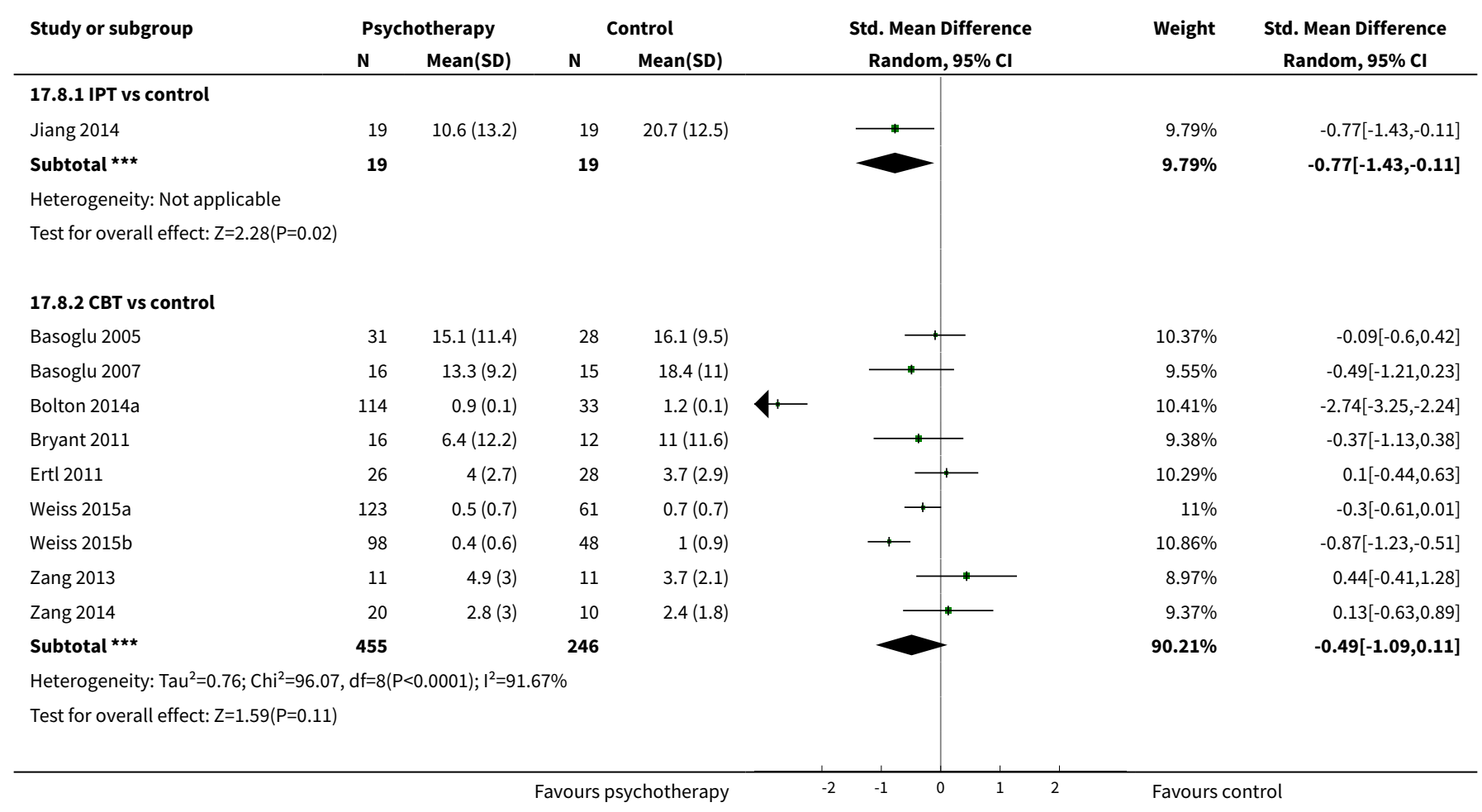

Psychological therapies for the treatment of mental disorders in low- and middle-income countries affected by humanitarian crises 171 (Review)

Copyright (c) 2018 The Cochrane Collaboration. Published by John Wiley \& Sons, Ltd. 


\begin{tabular}{|c|c|c|c|c|c|c|}
\hline \multirow[t]{2}{*}{ Study or subgroup } & \multicolumn{2}{|c|}{ Psychotherapy } & Control & \multirow{2}{*}{$\begin{array}{c}\text { Std. Mean Difference } \\
\text { Random, } 95 \% \mathrm{CI}\end{array}$} & \multirow[t]{2}{*}{ Weight } & \multirow{2}{*}{$\begin{array}{c}\text { Std. Mean Difference } \\
\text { Random, } 95 \% \mathrm{Cl}\end{array}$} \\
\hline & $\mathbf{N}$ & Mean(SD) & Mean(SD) & & & \\
\hline 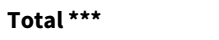 & 474 & & 265 & & $100 \%$ & $-0.52[-1.07,0.03]$ \\
\hline \multicolumn{7}{|c|}{ Heterogeneity: $\mathrm{Tau}^{2}=0.7 ; \mathrm{Chi}^{2}=96.32, \mathrm{df}=9(\mathrm{P}<0.0001) ; \mathrm{I}^{2}=90.66 \%$} \\
\hline \multicolumn{7}{|c|}{ Test for overall effect: $Z=1.84(P=0.07)$} \\
\hline \multicolumn{7}{|c|}{ Test for subgroup differences: $\mathrm{Chi}^{2}=0.37, \mathrm{df}=1(\mathrm{P}=0.54), \mathrm{I}^{2}=0 \%$} \\
\hline
\end{tabular}

Analysis 17.9. Comparison 17 Sensitivity analysis: incomplete outcome data - adults, Outcome 9 Dropout.

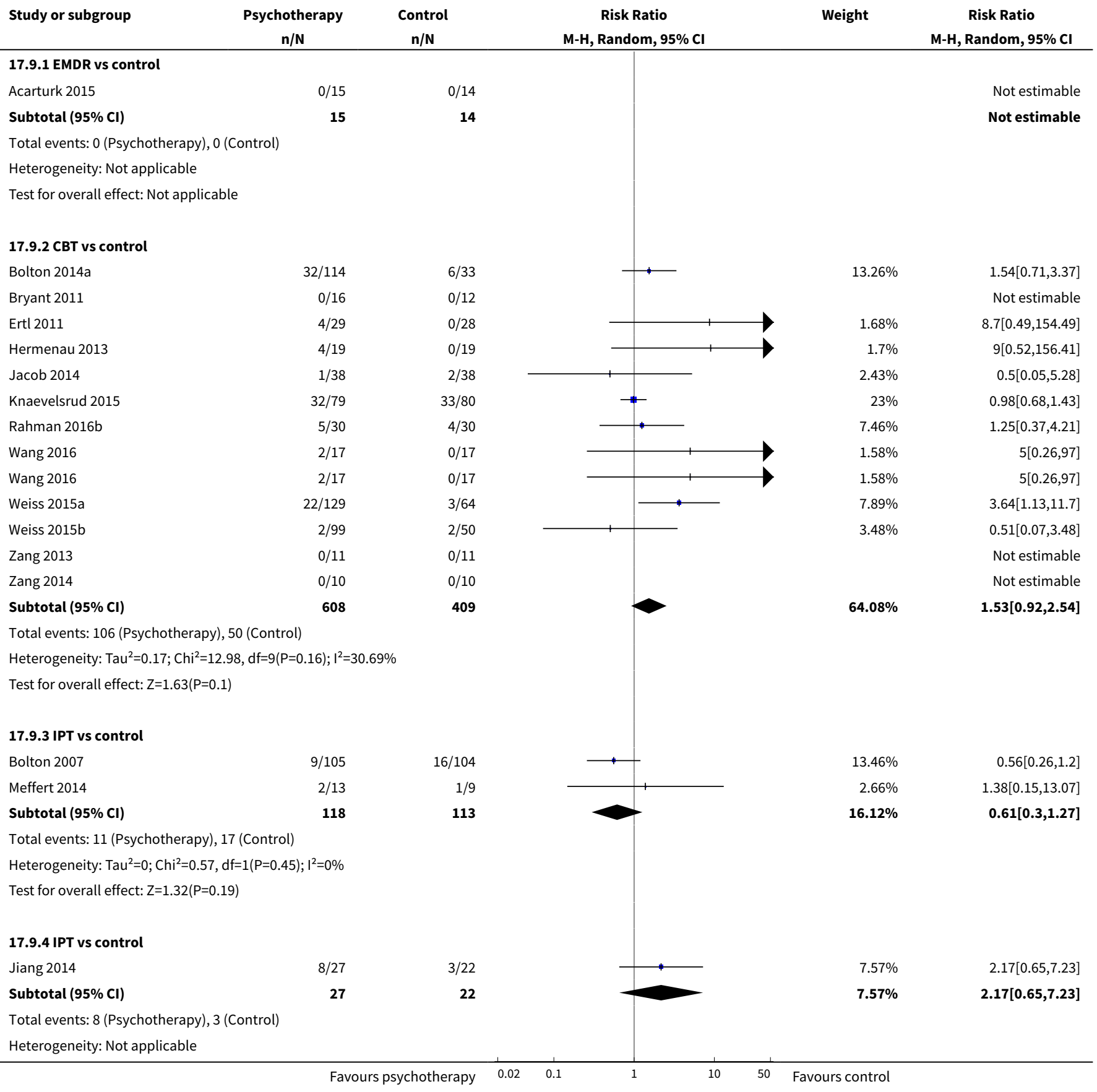

Psychological therapies for the treatment of mental disorders in low- and middle-income countries affected by humanitarian crises 


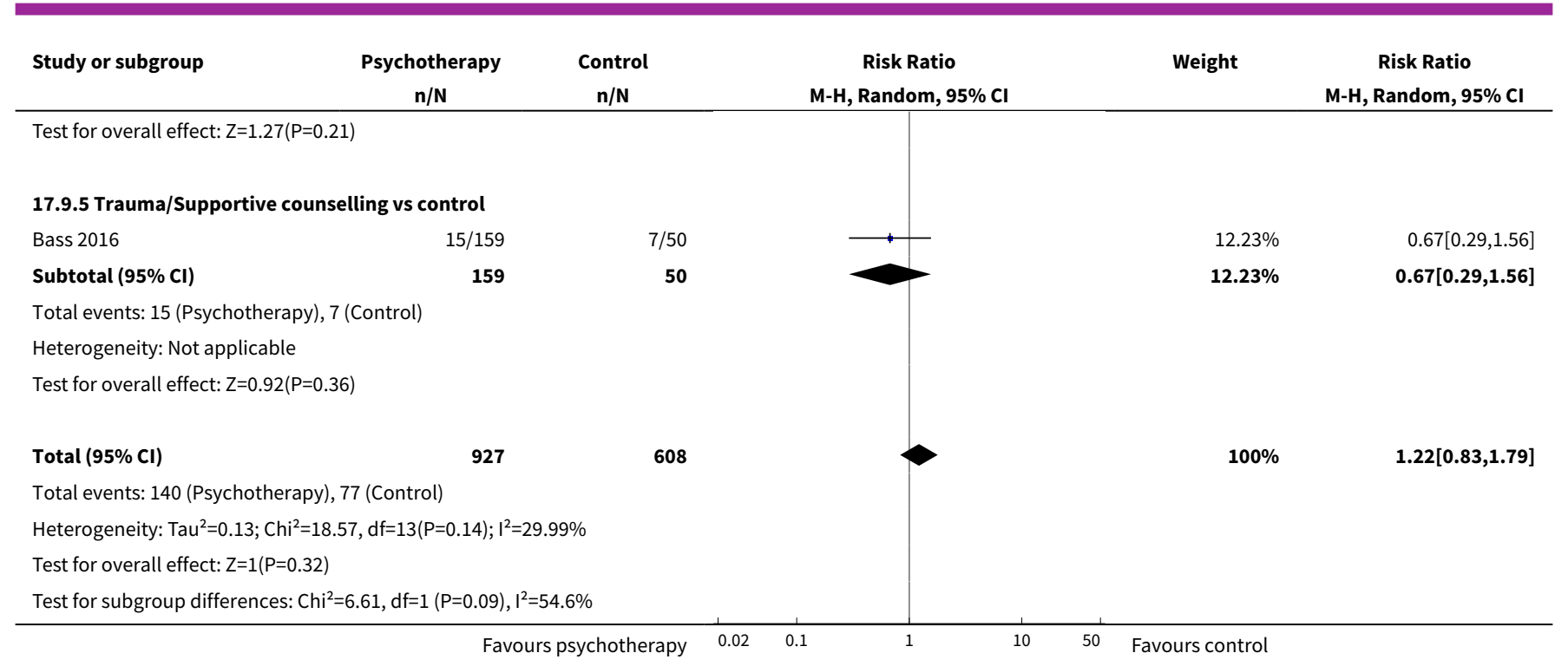

Analysis 17.10. Comparison 17 Sensitivity analysis: incomplete outcome data - adults, Outcome 10 Functional impairment at endpoint.

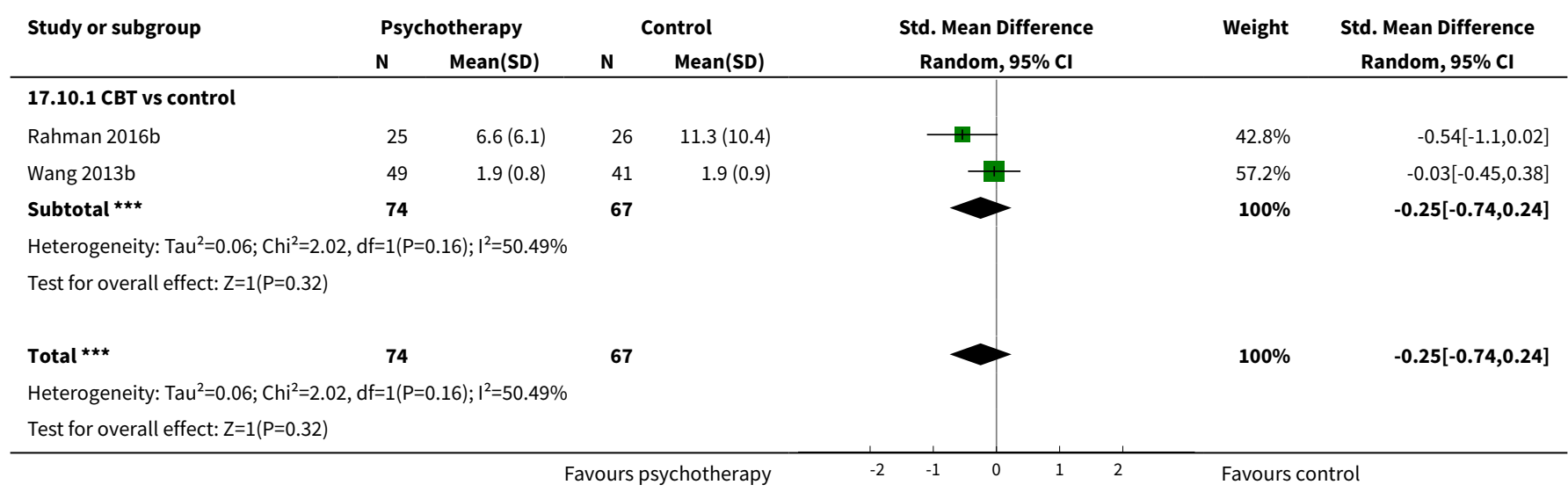

Analysis 17.11. Comparison 17 Sensitivity analysis: incomplete outcome data - adults, Outcome 11 Functional impairment at 1 to 4 months.

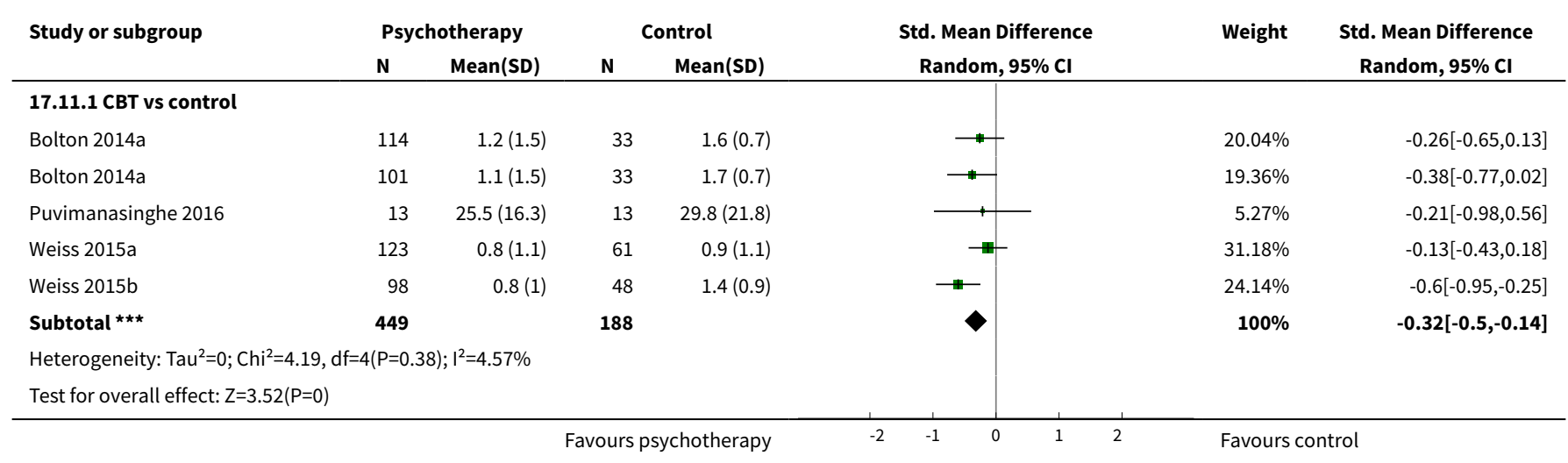

Psychological therapies for the treatment of mental disorders in low- and middle-income countries affected by humanitarian crises 


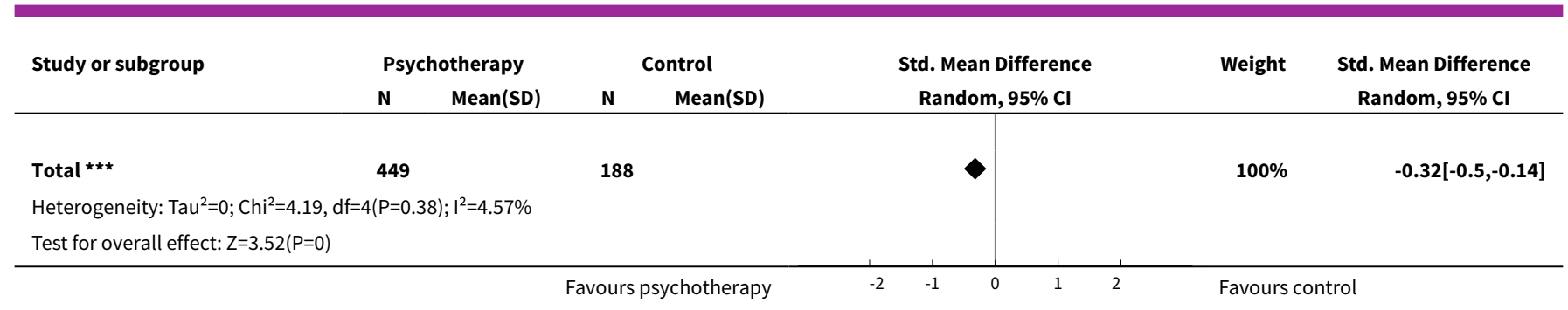

\section{Analysis 17.12. Comparison 17 Sensitivity analysis: incomplete} outcome data - adults, Outcome 12 Quality of life at endpoint.

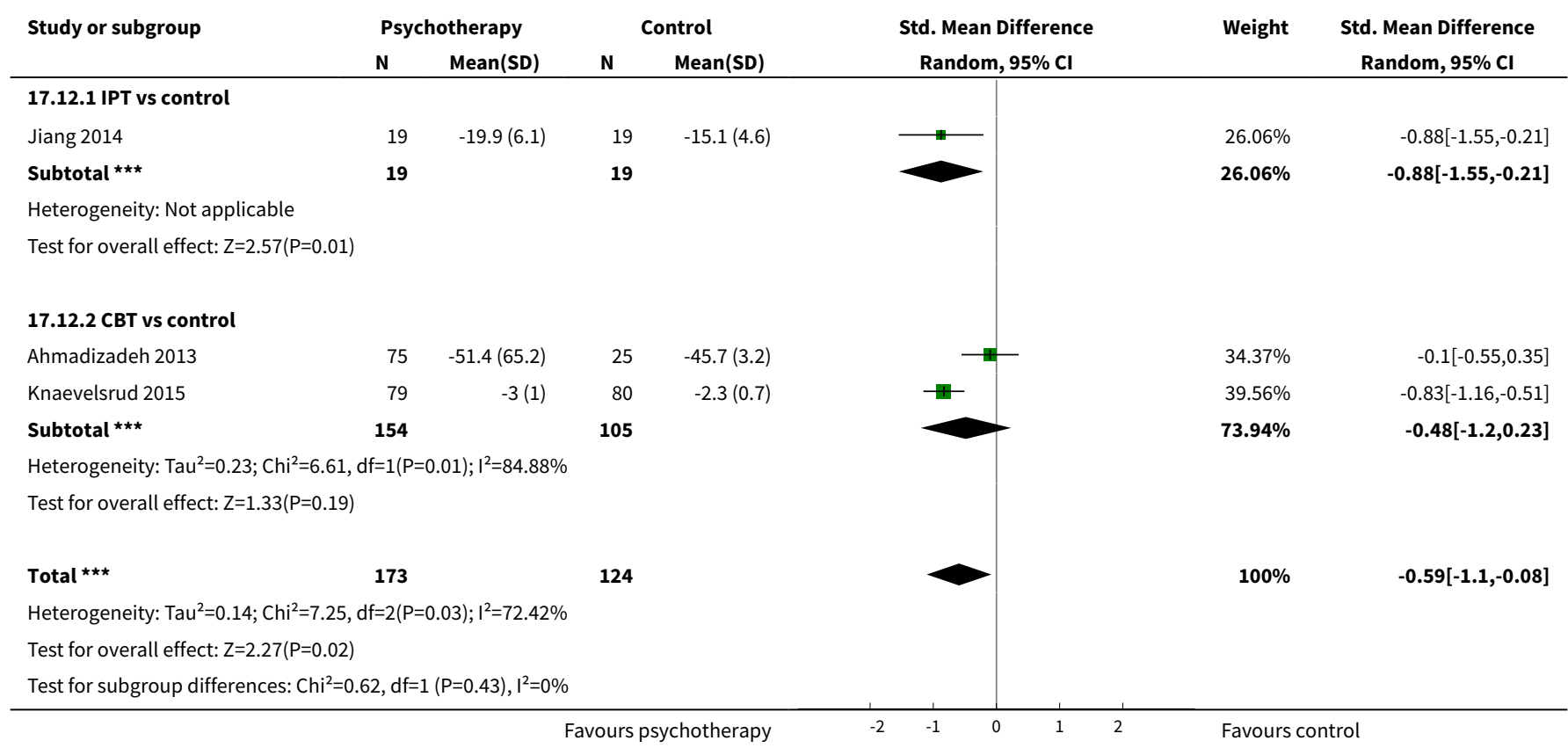

Analysis 17.13. Comparison 17 Sensitivity analysis: incomplete outcome data - adults, Outcome 13 Diagnosis of PTSD.

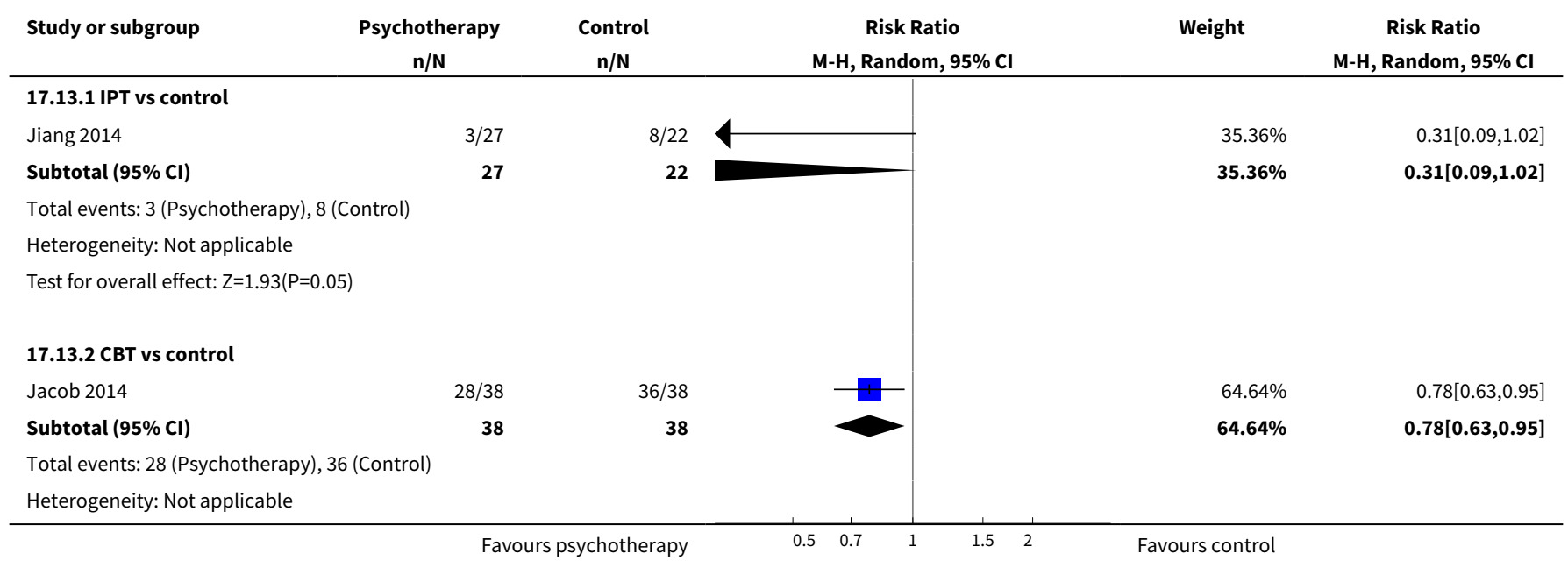

Psychological therapies for the treatment of mental disorders in low- and middle-income countries affected by humanitarian crises 


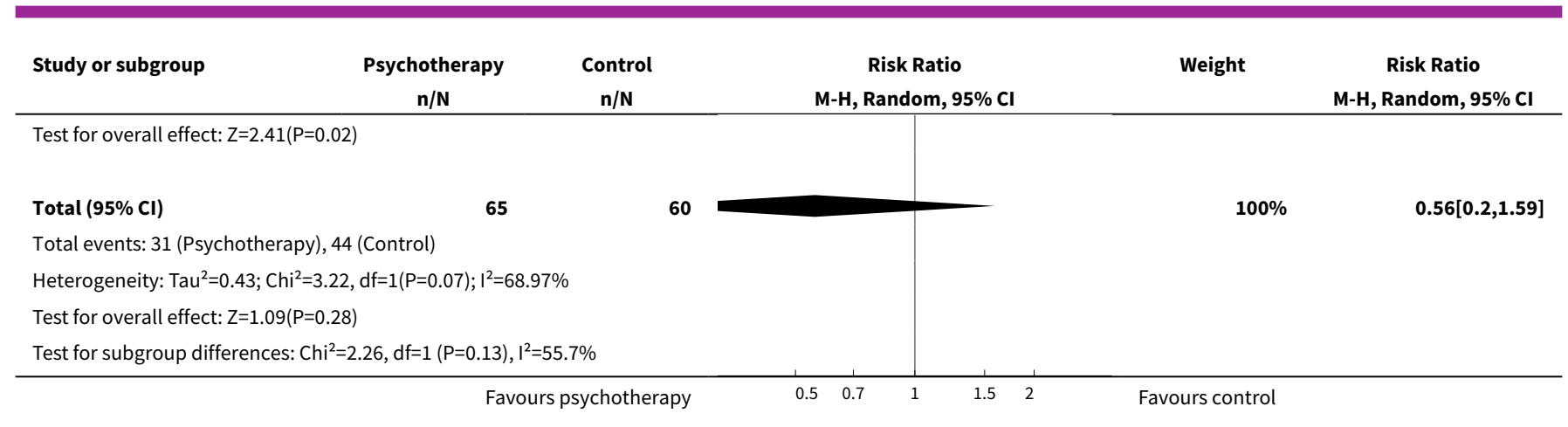

\section{Comparison 18. Sensitivity analysis: selective reporting - adults}

\begin{tabular}{|c|c|c|c|c|}
\hline $\begin{array}{l}\text { Outcome or subgroup } \\
\text { title }\end{array}$ & No. of studies & $\begin{array}{l}\text { No. of partici- } \\
\text { pants }\end{array}$ & Statistical method & Effect size \\
\hline $\begin{array}{l}1 \text { PTSD symptoms at } \\
\text { endpoint }\end{array}$ & 15 & 1127 & $\begin{array}{l}\text { Std. Mean Difference (IV, Random, 95\% } \\
\text { Cl) }\end{array}$ & $-1.05[-1.35,-0.75]$ \\
\hline 1.1 EMDR vs control & 2 & 99 & $\begin{array}{l}\text { Std. Mean Difference (IV, Random, 95\% } \\
\text { Cl) }\end{array}$ & $-2.01[-2.50,-1.52]$ \\
\hline $1.2 \mathrm{CBT}$ vs control & 12 & 1008 & $\begin{array}{l}\text { Std. Mean Difference (IV, Random, 95\% } \\
\text { Cl) }\end{array}$ & $-0.85[-1.13,-0.58]$ \\
\hline 1.3 IPT vs control & 1 & 20 & $\begin{array}{l}\text { Std. Mean Difference (IV, Random, 95\% } \\
\text { Cl) }\end{array}$ & $-1.45[-2.46,-0.43]$ \\
\hline $\begin{array}{l}2 \text { PTSD symptoms at } 1 \\
\text { to } 4 \text { months }\end{array}$ & 16 & 1459 & $\begin{array}{l}\text { Std. Mean Difference (IV, Random, 95\% } \\
\text { Cl) }\end{array}$ & $-0.53[-0.71,-0.34]$ \\
\hline 2.1 EMDR vs control & 1 & 64 & $\begin{array}{l}\text { Std. Mean Difference (IV, Random, 95\% } \\
\text { Cl) }\end{array}$ & $-1.76[-2.34,-1.18]$ \\
\hline 2.2 CBT vs control & 14 & 1357 & $\begin{array}{l}\text { Std. Mean Difference (IV, Random, 95\% } \\
\text { Cl) }\end{array}$ & $-0.44[-0.58,-0.31]$ \\
\hline 2.3 IPT vs control & 1 & 38 & $\begin{array}{l}\text { Std. Mean Difference (IV, Random, 95\% } \\
\text { Cl) }\end{array}$ & $-0.99[-1.67,-0.32]$ \\
\hline 2.4 BATD vs control & 0 & 0 & $\begin{array}{l}\text { Std. Mean Difference (IV, Random, 95\% } \\
\text { Cl) }\end{array}$ & $0.0[0.0,0.0]$ \\
\hline 2.5 NET vs control & 0 & 0 & $\begin{array}{l}\text { Std. Mean Difference (IV, Random, 95\% } \\
\text { Cl) }\end{array}$ & $0.0[0.0,0.0]$ \\
\hline 2.6 CETA vs control & 0 & 0 & $\begin{array}{l}\text { Std. Mean Difference (IV, Random, 95\% } \\
\text { Cl) }\end{array}$ & $0.0[0.0,0.0]$ \\
\hline 3 Dropout & 24 & 2753 & Risk Ratio (M-H, Random, 95\% Cl) & $1.01[0.84,1.21]$ \\
\hline 3.1 EMDR vs control & 2 & 127 & Risk Ratio (M-H, Random, 95\% Cl) & $0.75[0.40,1.42]$ \\
\hline 3.2 CBT vs control & 18 & 2208 & Risk Ratio (M-H, Random, 95\% Cl) & $1.07[0.86,1.33]$ \\
\hline
\end{tabular}

Psychological therapies for the treatment of mental disorders in low- and middle-income countries affected by humanitarian crises 


\begin{tabular}{|c|c|c|c|c|}
\hline $\begin{array}{l}\text { Outcome or subgroup } \\
\text { title }\end{array}$ & No. of studies & $\begin{array}{l}\text { No. of partici- } \\
\text { pants }\end{array}$ & Statistical method & Effect size \\
\hline 3.3 IPT vs control & 2 & 71 & Risk Ratio (M-H, Random, 95\% Cl) & $1.97[0.68,5.67]$ \\
\hline $\begin{array}{l}\text { 3.4 Trauma/Supportive } \\
\text { counselling vs control }\end{array}$ & 2 & 347 & Risk Ratio (M-H, Random, 95\% Cl) & $0.77[0.57,1.04]$ \\
\hline $\begin{array}{l}4 \text { Functional impair- } \\
\text { ment at endpoint }\end{array}$ & 5 & 686 & $\begin{array}{l}\text { Std. Mean Difference (IV, Random, 95\% } \\
\mathrm{CI} \text { ) }\end{array}$ & $-0.55[-0.80,-0.30]$ \\
\hline 4.1 CBT vs control & 5 & 686 & $\begin{array}{l}\text { Std. Mean Difference (IV, Random, 95\% } \\
\mathrm{CI} \text { ) }\end{array}$ & $-0.55[-0.80,-0.30]$ \\
\hline $\begin{array}{l}5 \text { Quality of life at end- } \\
\text { point }\end{array}$ & 1 & 38 & $\begin{array}{l}\text { Std. Mean Difference (IV, Random, 95\% } \\
\mathrm{CI} \text { ) }\end{array}$ & $-0.88[-1.55,-0.21]$ \\
\hline 5.1 IPT vs control & 1 & 38 & $\begin{array}{l}\text { Std. Mean Difference (IV, Random, 95\% } \\
\mathrm{CI} \text { ) }\end{array}$ & $-0.88[-1.55,-0.21]$ \\
\hline
\end{tabular}

\section{Analysis 18.1. Comparison 18 Sensitivity analysis: selective reporting - adults, Outcome 1 PTSD symptoms at endpoint.}

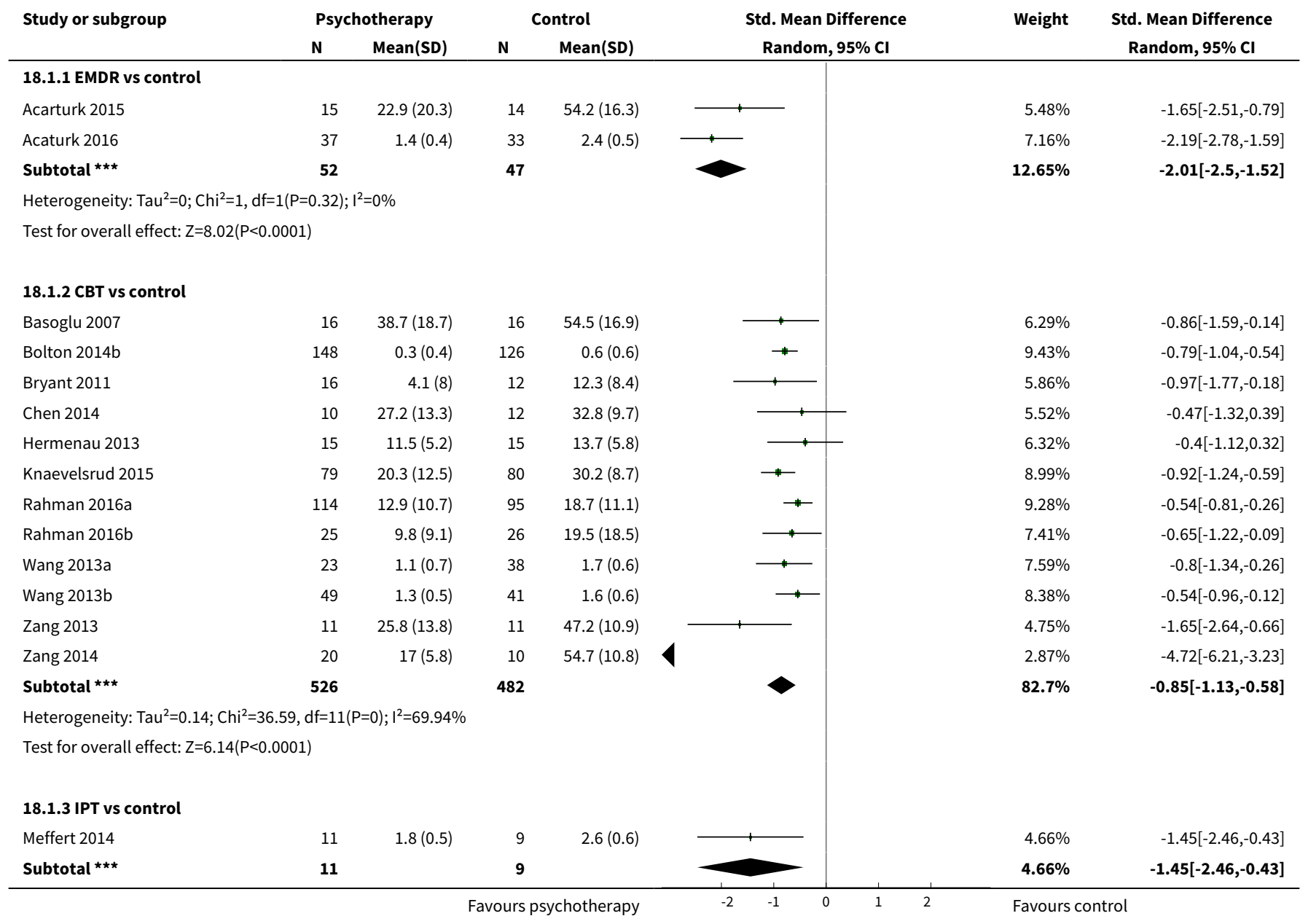




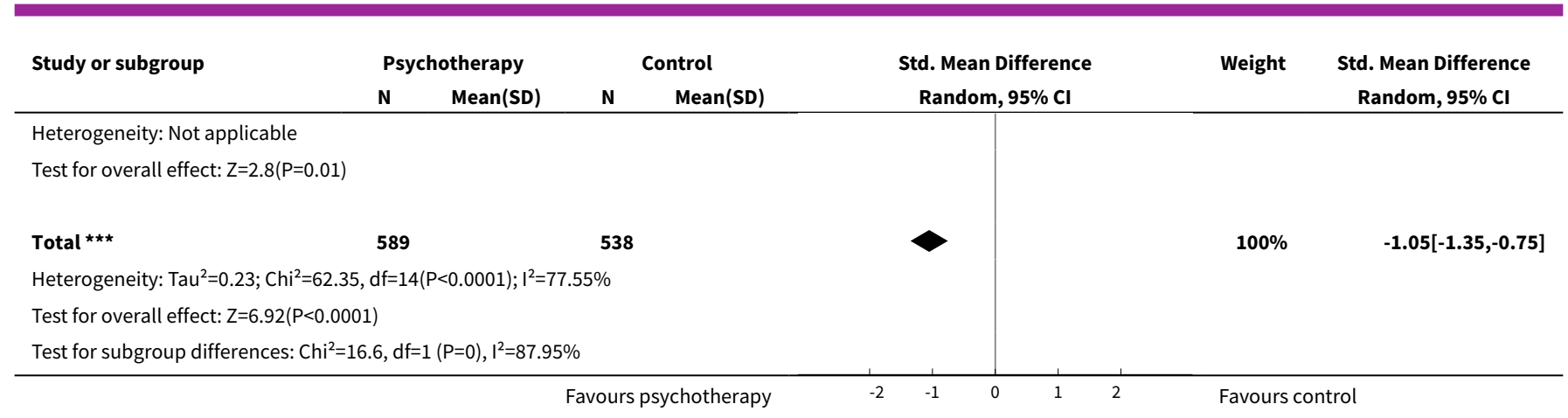

Analysis 18.2. Comparison 18 Sensitivity analysis: selective reporting - adults, Outcome 2 PTSD symptoms at 1 to 4 months.

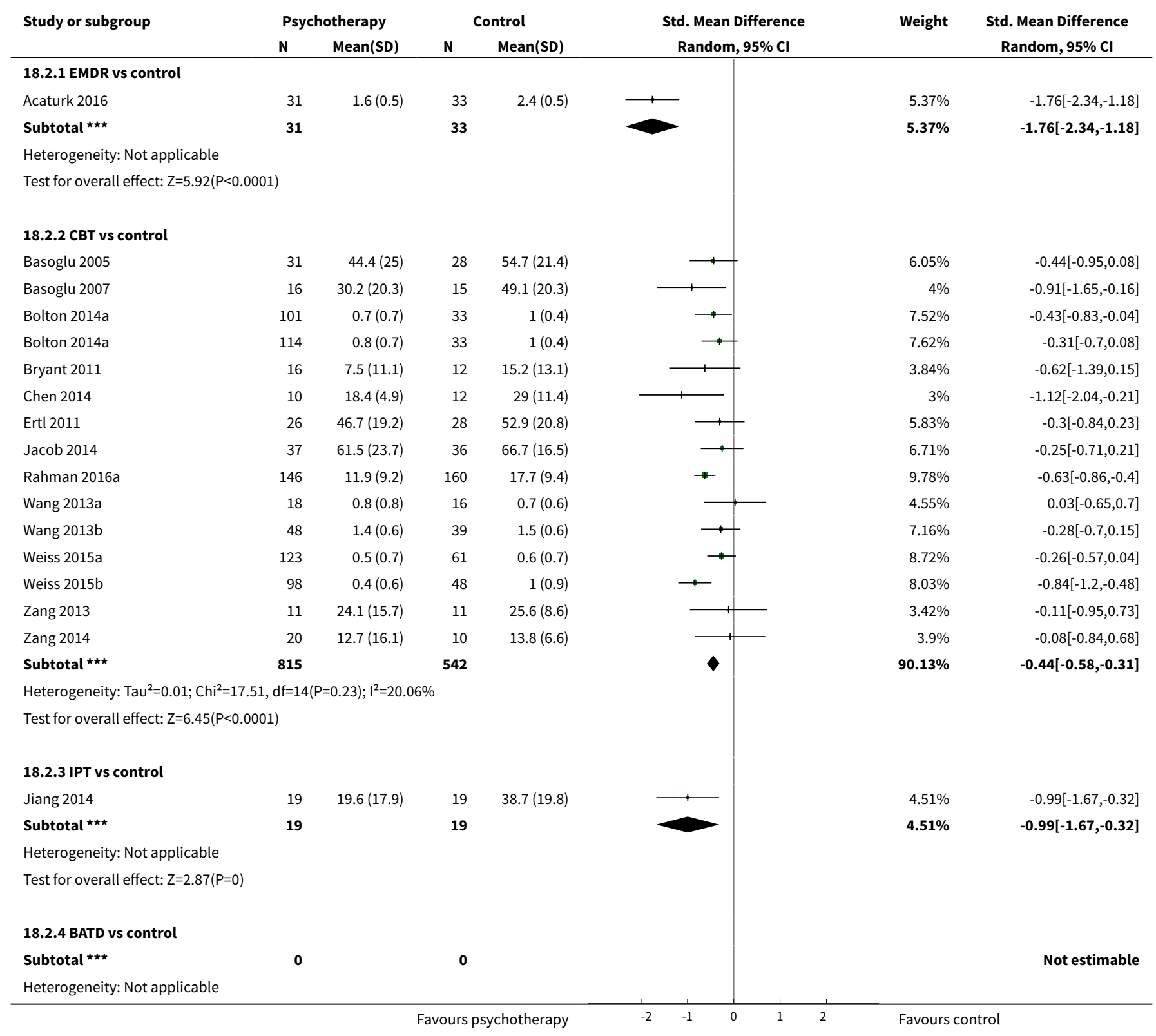

Psychological therapies for the treatment of mental disorders in low- and middle-income countries affected by humanitarian crises 


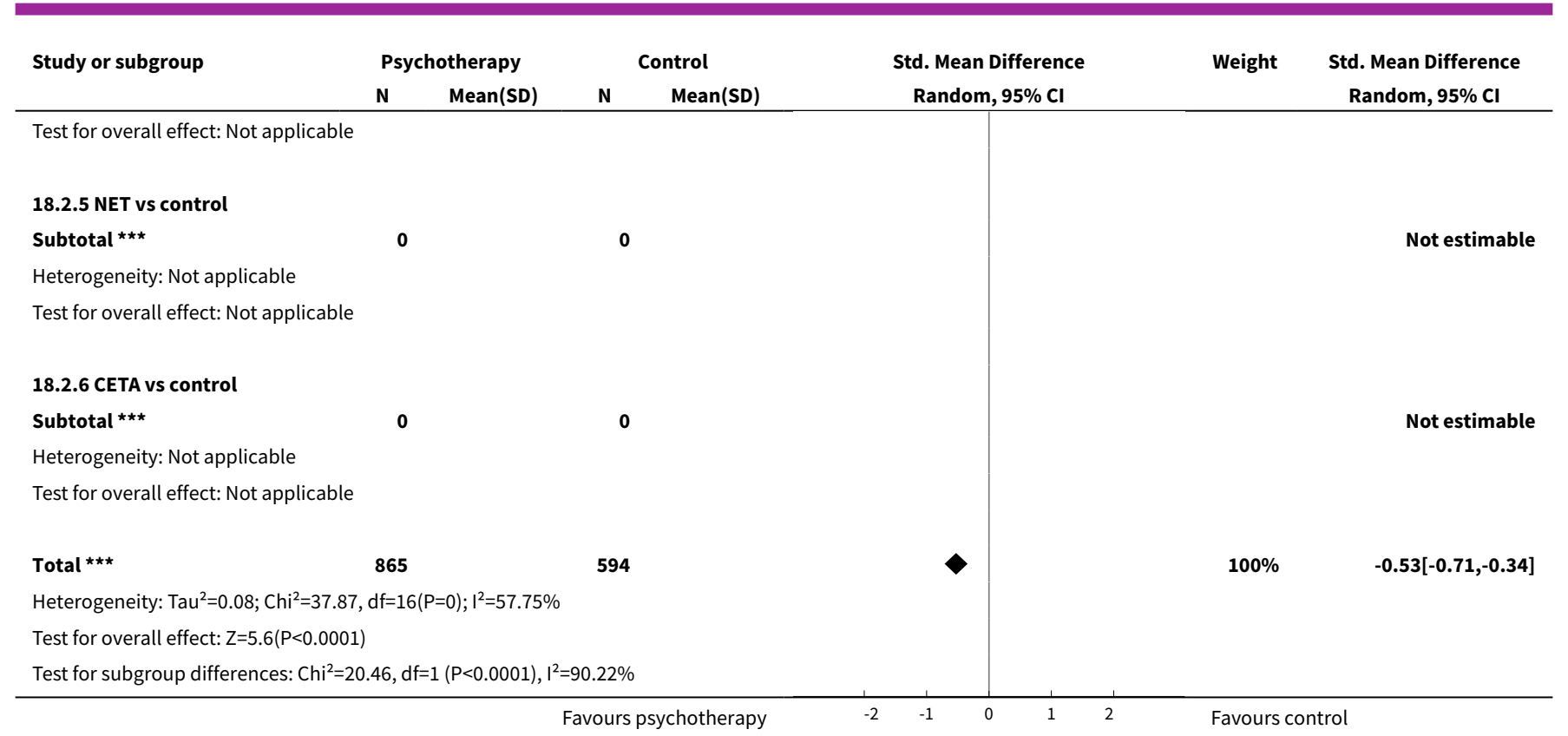

Analysis 18.3. Comparison 18 Sensitivity analysis: selective reporting - adults, Outcome 3 Dropout.

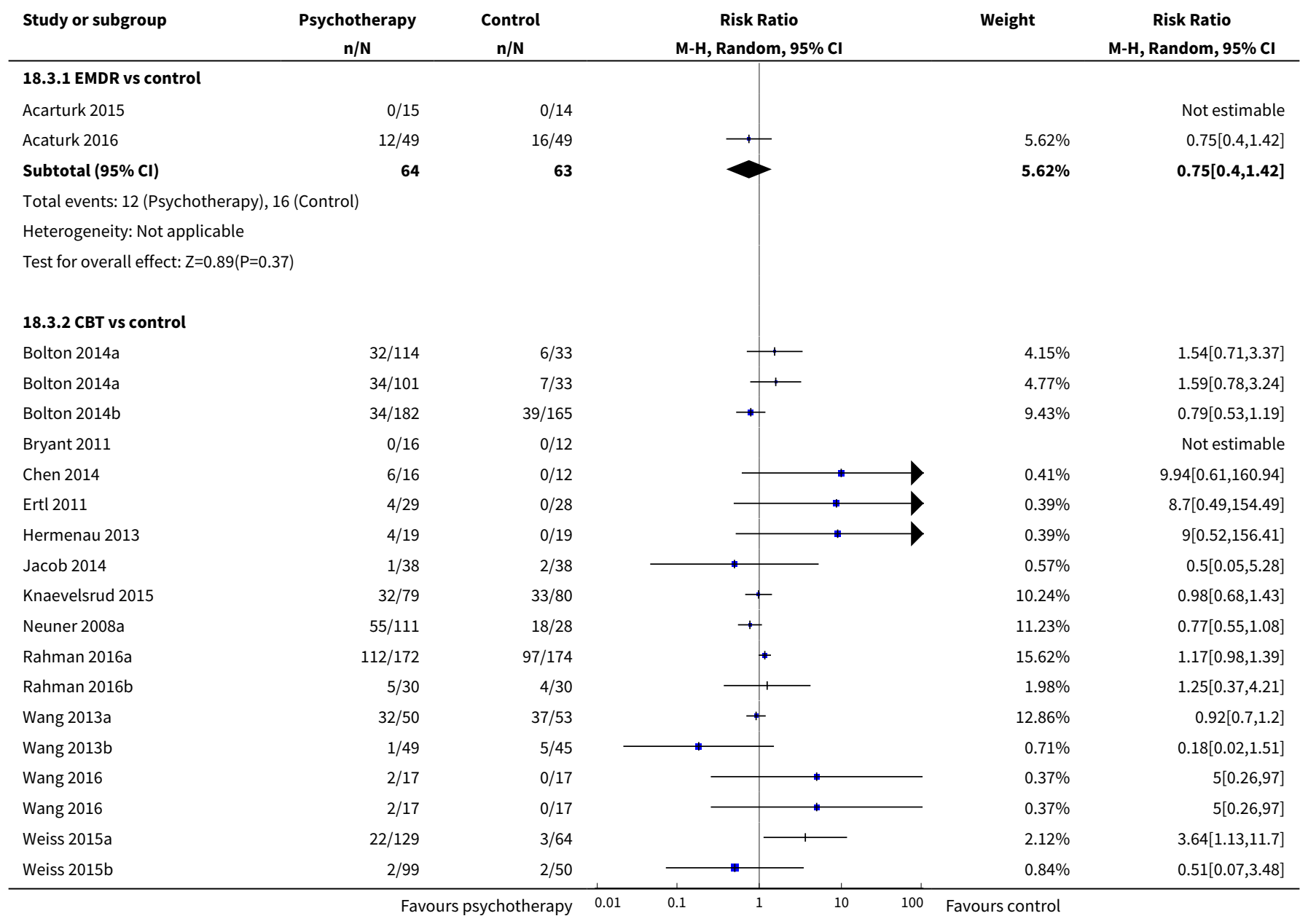

Psychological therapies for the treatment of mental disorders in low- and middle-income countries affected by humanitarian crises 178 (Review)

Copyright (c) 2018 The Cochrane Collaboration. Published by John Wiley \& Sons, Ltd. 


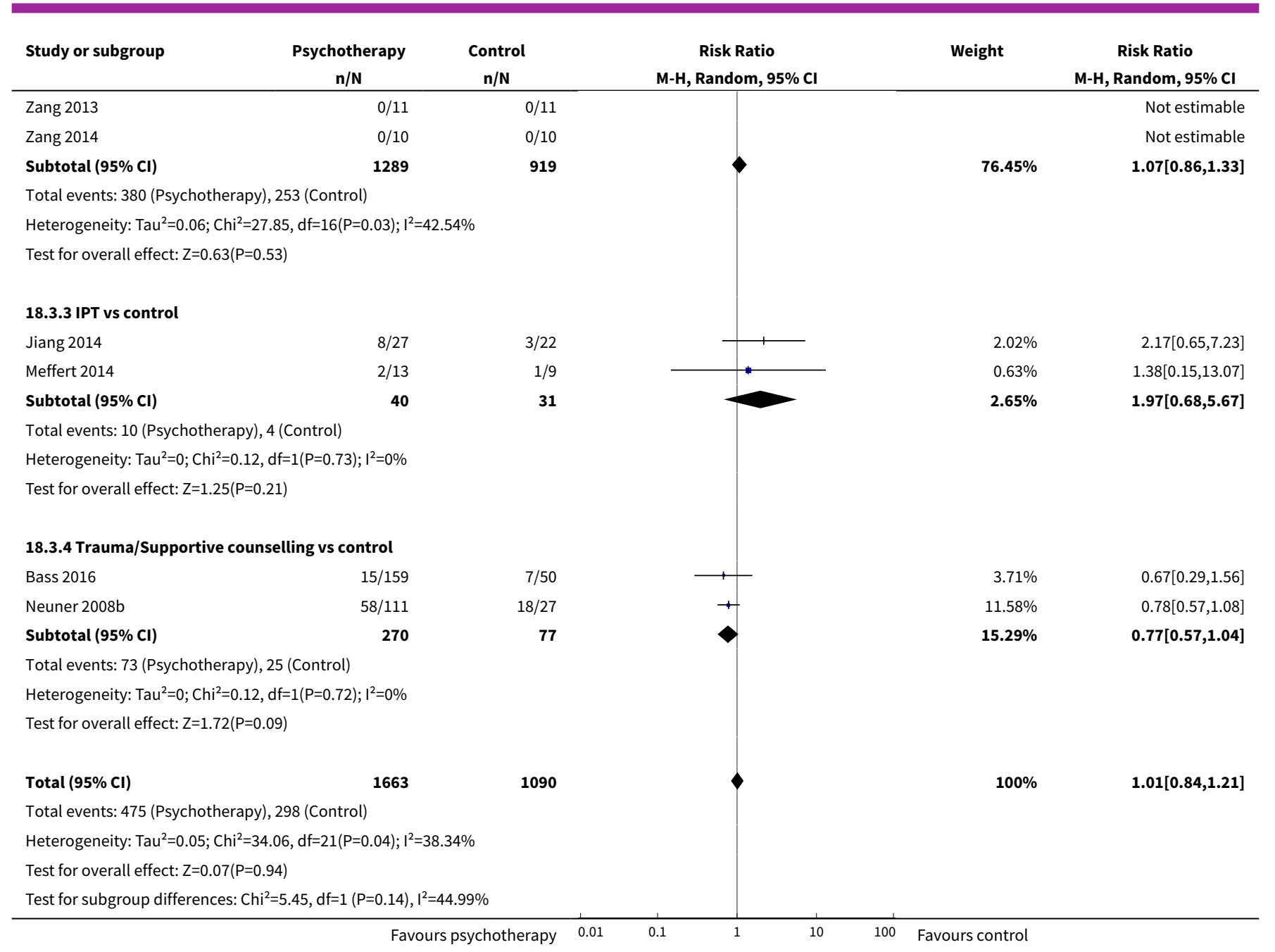

Analysis 18.4. Comparison 18 Sensitivity analysis: selective reporting - adults, Outcome 4 Functional impairment at endpoint.

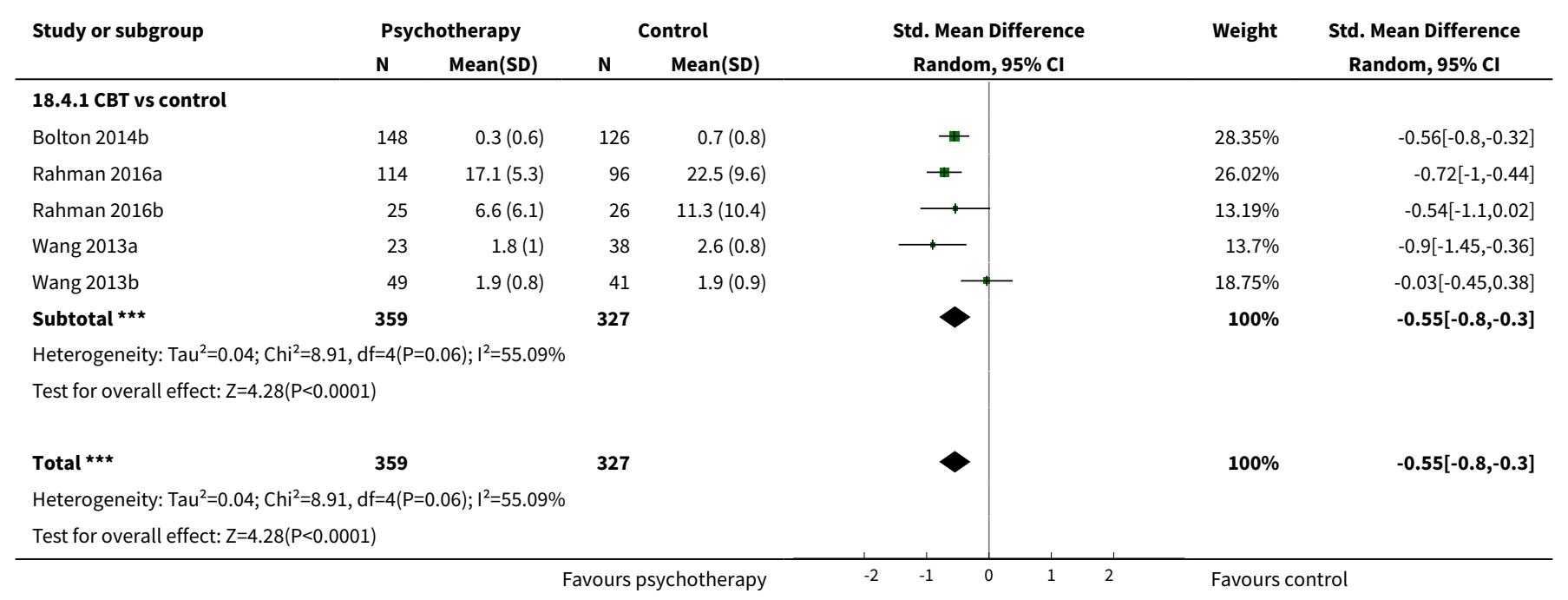

Psychological therapies for the treatment of mental disorders in low- and middle-income countries affected by humanitarian crises 179 (Review)

Copyright (c) 2018 The Cochrane Collaboration. Published by John Wiley \& Sons, Ltd. 


\section{Analysis 18.5. Comparison 18 Sensitivity analysis: selective reporting - adults, Outcome 5 Quality of life at endpoint.}

\begin{tabular}{|c|c|c|c|c|c|c|c|}
\hline \multirow{3}{*}{$\begin{array}{l}\text { Study or subgroup } \\
\text { 18.5.1 IPT vs control }\end{array}$} & \multicolumn{2}{|c|}{ Psychotherapy } & \multicolumn{2}{|c|}{ Control } & \multirow{2}{*}{$\begin{array}{c}\text { Std. Mean Difference } \\
\text { Random, } 95 \% \mathrm{Cl}\end{array}$} & \multirow[t]{2}{*}{ Weight } & \multirow{2}{*}{$\begin{array}{c}\text { Std. Mean Difference } \\
\text { Random, } 95 \% \mathrm{Cl}\end{array}$} \\
\hline & \multirow[t]{2}{*}{$\mathbf{N}$} & \multirow[t]{2}{*}{ Mean(SD) } & \multirow[t]{2}{*}{$\mathbf{N}$} & \multirow[t]{2}{*}{ Mean(SD) } & & & \\
\hline & & & & & & & \\
\hline Jiang 2014 & 19 & $-19.9(6.1)$ & 19 & $-15.1(4.6)$ & - & $100 \%$ & $-0.88[-1.55,-0.21]$ \\
\hline Subtotal $\star \star \star$ & 19 & & 19 & & & $100 \%$ & $-0.88[-1.55,-0.21]$ \\
\hline \multicolumn{8}{|l|}{ Heterogeneity: Not applicable } \\
\hline \multicolumn{8}{|l|}{ Test for overall effect: $Z=2.57(P=0.01)$} \\
\hline Total $\star \star \star ~$ & 19 & & 19 & & & $100 \%$ & $-0.88[-1.55,-0.21]$ \\
\hline \multicolumn{8}{|l|}{ Heterogeneity: Not applicable } \\
\hline \multicolumn{8}{|l|}{ Test for overall effect: $Z=2.57(P=0.01)$} \\
\hline & & & ours & chotherapy & -1 & Favours & trol \\
\hline
\end{tabular}

\section{APPENDICES}

\section{Appendix 1. CCMDCTR - core MEDLINE search}

\section{Core search strategy used to inform the Cochrane Common Mental Disorders Group's specialised register: OVID MEDLINE}

A weekly search alert based on condition + RCT filter only

1. [MeSH Headings]:

eating disorders/ or anorexia nervosa/ or binge-eating disorder/ or bulimia nervosa/ or female athlete triad syndrome/ or pica/ or hyperphagia/ or bulimia/ or self-injurious behavior/ or self mutilation/ or suicide/ or suicidal ideation/ or suicide, attempted/ or mood disorders/ or affective disorders, psychotic/ or bipolar disorder/ or cyclothymic disorder/ or depressive disorder/ or depression, postpartum/ or depressive disorder, major/ or depressive disorder, treatment-resistant/ or dysthymic disorder/ or seasonal affective disorder/ or neurotic disorders/ or depression/ or adjustment disorders/ or exp antidepressive agents/ or anxiety disorders/ or agoraphobia/ or neurocirculatory asthenia/ or obsessive-compulsive disorder/ or obsessive hoarding/ or panic disorder/ or phobic disorders/ or stress disorders, traumatic/ or combat disorders/ or stress disorders, post-traumatic/ or stress disorders, traumatic, acute/ or anxiety/ or anxiety, castration/ or koro/ or anxiety, separation/ or panic/ or exp anti-anxiety agents/or somatoform disorders/ or body dysmorphic disorders/ or conversion disorder/ or hypochondriasis/or neurasthenia/ or hysteria/ or munchausen syndrome by proxy/ or munchausen syndrome/ or fatigue syndrome, chronic/ or obsessive behavior/ or compulsive behavior/ or behavior, addictive/ or impulse control disorders/ or firesetting behavior/ or gambling/ or trichotillomania/ or stress, psychological/ or burnout, professional/ or sexual dysfunctions, psychological/ or vaginismus/ or Anhedonia/ or Affective Symptoms/ or *Mental Disorders/

\section{2. [Title/Author Keywords]:}

(eating disorder* or anorexia nervosa or bulimi ${ }^{\star}$ or binge eat ${ }^{\star}$ or (self adj (injur ${ }^{\star}$ or mutilat ${ }^{\star}$ )) or suicide ${ }^{\star}$ or suicidal or parasuicid ${ }^{\star}$ or mood disorder ${ }^{\star}$ or affective disorder ${ }^{\star}$ or bipolar i or bipolar ii or (bipolar and (affective or disorder $\left.{ }^{\star}\right)$ ) or mania or manic or cyclothymic ${ }^{\star}$ or depression or depressive or dysthymi ${ }^{\star}$ or neurotic or neurosis or adjustment disorder ${ }^{\star}$ or antidepress* or anxiety disorder ${ }^{\star}$ or agoraphobia or obsess ${ }^{\star}$ or compulsi ${ }^{\star}$ or panic or phobi* or ptsd or posttrauma* or post trauma* or combat or somatoform or somati\#ation or medical ${ }^{\star}$ unexplained or body dysmorphi* or conversion disorder or hypochondria* or neurastheni* or hysteria or munchausen or chronic fatigue* or gambling or trichotillomania or vaginismus or anhedoni* or affective symptoms or mental disorder* or mental health).ti,kf.

\section{3. [RCT filter]:}

(controlled clinical trial.pt. or randomized controlled trial.pt. or (randomi\#ed or randomi\#ation).ab,ti. or randomly.ab. or (random ${ }^{\star}$ adj3

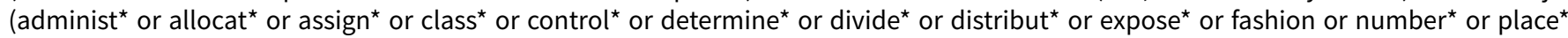
or recruit ${ }^{\star}$ or subsitut ${ }^{\star}$ or treat $\left.{ }^{\star}\right)$ ).ab. or placebo ${ }^{\star}$.ab,ti. or drug therapy.fs. or trial.ab,ti. or groups.ab. or (control* adj3 (trial* or study or studies)).ab,ti. or ((singl ${ }^{\star}$ or doubl* or tripl* or trebl*) adj3 (blind ${ }^{\star}$ or mask ${ }^{*}$ or dummy $\left.\left.{ }^{\star}\right)\right) . m p$. or clinical trial, phase ii/ or clinical trial, phase iii/ or clinical trial, phase iv/ or randomized controlled trial/ or pragmatic clinical trial/ or (quasi adj (experimental or random*)).ti,ab. or

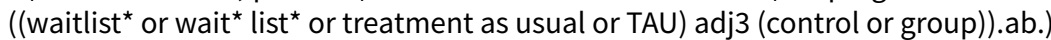

\section{4. (1 and 2 and 3 )}

Records are screened for reports of RCTs within the scope of the Cochrane Common Mental Disorders Group. Secondary reports of RCTs are tagged to the appropriate study record.

Psychological therapies for the treatment of mental disorders in low- and middle-income countries affected by humanitarian crises 
Similar weekly search alerts are also conducted on OVID Embase and PsycINFO, using relevant subject headings (controlled vocabularies) and search syntax, appropriate to each resource.

\section{Appendix 2. LMIC search filter}

The LMIC filter was created by the Norwegian Satellite of the Cochrane Effective Practice and Organisation of Care Group (2012). It is based on the World Bank list of countries (2009), classified as low-income, lower-middle-income or upper-middle-income economies:data.worldbank.org/about/country-classifications. (The search syntax has been adapted forthe Cochrance Register of Studies (CRS)).

\#1 (Africa or Asia or Caribbean or "West Indies" or "South America" or "Latin America" or "Central America"):ti,ab,kw,ky,emt,mh,mc

\#2 (Afghanistan or Albania or Algeria or Angola or Antigua or Barbuda or Argentina or Armenia or Armenian or Aruba or Azerbaijan or Bahrain or Bangladesh or Barbados or Benin or Byelarus or Byelorussian or Belarus or Belorussian or Belorussia or Belize or Bhutan or Bolivia or Bosnia or Herzegovina or Hercegovina or Botswana or Brasil or Brazil or Bulgaria or "Burkina Faso" or "Burkina Fasso" or "Upper Volta" or Burundi or Urundi or Cambodia or "Khmer Republic" or Kampuchea or Cameroon or Cameroons or Cameron or Camerons or "Cape Verde" or "Central African Republic" or Chad or Chile or China or Colombia or Comoros or "Comoro Islands" or Comores or Mayotte or Congo or Zaire or "Costa Rica" or "Cote d'Ivoire" or "Ivory Coast" or Croatia or Cuba or Cyprus or Czechoslovakia or "Czech Republic" or Slovakia or "Slovak Republic"):ti,ab,kw,ky,emt,mh,mc

\#3 (Djibouti or "French Somaliland" or Dominica or "Dominican Republic" or "East Timor" or "East Timur" or "Timor Leste" or Ecuador or Egypt or "United Arab Republic" or "El Salvador" or Eritrea or Estonia or Ethiopia or Fiji or Gabon or "Gabonese Republic" or Gambia or Gaza or Georgia or Georgian or Ghana or "Gold Coast" or Greece or Grenada or Guatemala or Guinea or Guam or Guiana or Guyana or Haiti or Honduras or Hungary or India or Maldives or Indonesia or Iran or Iraq or "Isle of Man" or Jamaica or Jordan or Kazakhstan or Kazakh or Kenya or Kiribati or Korea or Kosovo or Kyrgyzstan or Kirghizia or "Kyrgyz Republic" or Kirghiz or Kirgizstan or "Lao PDR" or Laos or Latvia or Lebanon or Lesotho or Basutoland or Liberia or Libya or Lithuania):ti,ab,kw,ky,emt,mh,mc

\#4 (Macedonia or Madagascar or "Malagasy Republic" or Malaysia or Malaya or Malay or Sabah or Sarawak or Malawi or Nyasaland or Mali or Malta or "Marshall Islands" or Mauritania or Mauritius or "Agalega Islands" or Mexico or Micronesia or "Middle East" or Moldova or Moldovia or Moldovian or Mongolia or Montenegro or Morocco or Ifni or Mozambique or Myanmar or Myanma or Burma or Namibia or Nepal or "Netherlands Antilles" or "New Caledonia" or Nicaragua or Niger or Nigeria or "Northern Mariana Islands" or Oman or Muscat or Pakistan or Palau or Palestine or Panama or Paraguay or Peru or Philippines or Philipines or Phillipines or Phillippines or Poland or Portugal or "Puerto Rico"):ti,ab,kw,ky,emt,mh,mc

\#5 (Romania or Rumania or Roumania or Russia or Russian or Rwanda or Ruanda or "Saint Kitts" or "St Kitts" or Nevis or "Saint Lucia" or "St Lucia" or "Saint Vincent" or "St Vincent" or Grenadines or Samoa or "Samoan Islands" or "Navigator Island" or "Navigator Islands" or "Sao Tome" or "Saudi Arabia" or Senegal or Serbia or Montenegro or Seychelles or "Sierra Leone" or Slovenia or "Sri Lanka" or Ceylon or "Solomon Islands" or Somalia or Sudan or Suriname or Surinam or Swaziland or Syria or Tajikistan or Tadzhikistan or Tadjikistan or Tadzhik or Tanzania or Thailand or Togo or "Togolese Republic" or Tonga or Trinidad or Tobago or Tunisia or Turkey or Turkmenistan or Turkmen or Uganda or Ukraine or Uruguay or USSR or "Soviet Union" or "Union of Soviet Socialist Republics" or Uzbekistan or Uzbek or Vanuatu or "New Hebrides" or Venezuela or Vietnam or "Viet Nam" or "West Bank" or Yemen or Yugoslavia or Zambia or Zimbabwe or Rhodesia):ti,ab,kw,ky,emt,mh,mc

\#6 ((developing or "less* developed" or "under developed" or underdeveloped or "middle income" or "low* income" or underserved or "under served" or deprived or poor ${ }^{\star}$ NEAR (countr* or nation* or population* or world)):ti,ab,kw,ky,emt,mh,mc

\#7 ((developing or "less* developed" or "under developed" or underdeveloped or "middle income" or "low* income") NEXT (economy or economies)):ti,ab,kw,ky,emt,mh,mc

\#8 (low* NEXT (GDP or GNP or "gross domestic" or "gross national")):ti,ab,kw,ky,emt,mh,mc

\#9 (low NEAR3 middle NEAR3 countr $\left.{ }^{\star}\right): t i, a b, k w, k y, e m t, m h, m c$

\#10 (LMIC or LMICs or "third world" or "LAMI country" or "LAMI countries"):ti,ab,kw,ky,emt,mh,mc

\#11 ("transitional country" or "transitional countries"):ti,ab,kw,ky,emt,mh,mc

\#12 (\#1 OR \#2 OR \#3 OR \#4 OR \#5 OR \#6 OR \#7 OR \#8 OR \#9 OR \#10 OR \#11)

\section{Appendix 3. Additional database searches}

\section{OVID PsycINFO}

We searched PsycINFO (all years to 1 September 2017) using terms for humanitarian crises in LMIC and RCTs:

[Humanitarian Crises]

Psychological therapies for the treatment of mental disorders in low- and middle-income countries affected by humanitarian crises 


\author{
1. CONFLICT/ \\ 2. CRISES/ \\ 3. CRISIS INTERVENTION/ \\ 4. exp DISASTERS/ \\ 5. REFUGEES/ \\ 6. "RESILIENCE (Psychological)" \\ 7. $\exp$ TERRORISM/ \\ 8. WAR/ \\ 9. TORTURE/
}

10. VICTIMIZATION/

11. (humanitarian adj3 (aid or affair ${ }^{\star}$ or agenc ${ }^{\star}$ or assistance or catastrophe* or crisis or crises or disaster* or effort ${ }^{\star}$ or emergenc ${ }^{\star}$ or evacuation ${ }^{\star}$ or integration or reintegration or mission or organization ${ }^{\star}$ or organisation ${ }^{\star}$ or program ${ }^{\star}$ or relief or setting ${ }^{\star}$ or support $^{\star}$ or task $^{*}$ force or work*)).ti,ab,id.

12. (genocide or armed conflict* or mass execution* or mass violence).ti,ab,id.

13. (cataclysmic or catastroph* or devastation or disaster ${ }^{\star}$ or drought ${ }^{\star}$ or earthquake ${ }^{\star}$ or evacuation ${ }^{\star}$ or famine ${ }^{\star}$ or flood or floods or

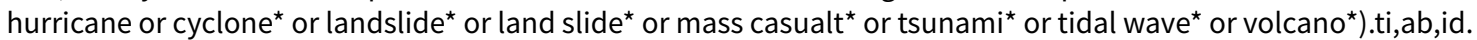

14. (refugee ${ }^{\star}$ or forced migration or (displac* adj2 (internal or forced or mass or person ${ }^{\star}$ or people* or population $\left.\left.{ }^{\star}\right)\right)$ ).ti,ab,id.

15. (torture* or (politic ${ }^{\star}$ adj2 (persecut* or prison $^{\star}$ or imprison ${ }^{\star}$ or violen $\left.{ }^{\star}\right)$ )).ti,ab,id.

16. (war and (abuse* or crime* or rape $^{\star}$ or survivor ${ }^{\star}$ or victim*)).ti, ab,id.

17. (bereav ${ }^{\star}$ or orphan ${ }^{\star}$ or widow*).ti,ab,id.

18. or $/ 1-17$

[Location - country where study was conducted]

19. (Africa or Asia or Caribbean or West Indies or South America or Latin America or Central America or Afghanistan or Albania or Algeria or Angola or Antigua or Barbuda or Argentina or Armenia or Armenian or Aruba or Azerbaijan or Bahrain or Bangladesh or Barbados or Benin or Byelarus or Byelorussian or Belarus or Belorussian or Belorussia or Belize or Bhutan or Bolivia or Bosnia or Herzegovina or Hercegovina or Botswana or Brasil or Brazil or Bulgaria or Burkina Faso or Burkina Fasso or Upper Volta or Burundi or Urundi or Cambodia or Khmer Republic or Kampuchea or Cameroon or Cameroons or Cameron or Camerons or Cape Verde or Central African Republic or Chad or Chile or China or Colombia or Comoros or Comoro Islands or Comores or Mayotte or Congo or Zaire or Costa Rica or Cote d'Ivoire or Ivory Coast or Croatia or Cuba or Cyprus or Czechoslovakia or Czech Republic or Slovakia or Slovak Republic or Djibouti or French Somaliland or Dominica or Dominican Republic or East Timor or East Timur or Timor Leste or Ecuador or Egypt or United Arab Republic or El Salvador or Eritrea or Estonia or Ethiopia or Fiji or Gabon or Gabonese Republic or Gambia or Gaza or Georgia or Georgian or Ghana or Gold Coast or Greece or Grenada or Guatemala or Guinea or Guam or Guiana or Guyana or Haiti or Honduras or Hungary or India or Maldives or Indonesia or Iran or Iraq or Isle of Man or Jamaica or Jordan or Kazakhstan or Kazakh or Kenya or Kiribati or Korea or Kosovo or Kyrgyzstan or Kirghizia or Kyrgyz Republic or Kirghiz or Kirgizstan or Lao PDR or Laos or Latvia or Lebanon or Lesotho or Basutoland or Liberia or Libya or Lithuania or Macedonia or Madagascar or Malagasy Republic or Malaysia or Malaya or Malay or Sabah or Sarawak or Malawi or Nyasaland or Mali or Malta or Marshall Islands or Mauritania or Mauritius or Agalega Islands or Mexico or Micronesia or Middle East or Moldova or Moldovia or Moldovian or Mongolia or Montenegro or Morocco or Ifni or Mozambique or Myanmar or Myanma or Burma or Namibia or Nepal or Netherlands Antilles or New Caledonia or Nicaragua or Niger or Nigeria or Northern Mariana Islands or Oman or Muscat or Pakistan or Palau or Palestine or Panama or Paraguay or Peru or Philippines or Philipines or Phillipines or Phillippines or Poland or Portugal or Puerto Rico or Romania or Rumania or Roumania or Russia or Russian or Rwanda or Ruanda or Saint Kitts or St Kitts or Nevis or Saint Lucia or St Lucia or Saint Vincent or St Vincent or Grenadines or Samoa or Samoan Islands or Navigator Island or Navigator Islands or Sao Tome or Saudi Arabia or Senegal or Serbia or Montenegro or Seychelles or Sierra Leone or Slovenia or Sri Lanka or Ceylon or Solomon Islands or Somalia or Sudan or Suriname or Surinam or Swaziland or Syria or Tajikistan or Tadzhikistan or Tadjikistan or Tadzhik or Tanzania or Thailand or Togo or Togolese Republic or Tonga or Trinidad or Tobago or Tunisia or Turkey or Turkmenistan or Turkmen or Uganda or Ukraine or Uruguay or USSR or Soviet Union or Union of Soviet Socialist Republics or Uzbekistan or Uzbek or Vanuatu or New Hebrides or Venezuela or Vietnam or Viet Nam or West Bank or Yemen or Yugoslavia or Zambia or Zimbabwe or Rhodesia).ti,ab.id,lo.

20. ((developing or less* developed or under developed or underdeveloped or middle income or low* income or underserved or under served or deprived or poor ${ }^{\star}$ ) adj3 (countr* or nation* or population* or world)).ti,ab,id.

21. ((developing or less ${ }^{\star}$ developed or under developed or underdeveloped or middle income or low* income) adj1 (economy or economies)).ti,ab,id.

22. (low* adj1 (GDP or GNP or gross domestic or gross national)).ti,ab,id.

23. (low adj3 middle adj3 countr ${ }^{\star}$ ).ti,ab,id.

24. (LMIC or LMICs or third world or LAMI country or LAMI countries).ti,ab,id.

25. (transitional country or transitional countries).ti,ab,id.

26. or/19-25

[RCT filter]

27. treatment effectiveness evaluation.sh.

28. clinical trials.sh.

29. mental health program evaluation.sh.

30. randomly.ab.

31. randomi\#ed.ti,ab,id.

Psychological therapies for the treatment of mental disorders in low- and middle-income countries affected by humanitarian crises 
32. (random* adj3 (administ ${ }^{\star}$ or allocat ${ }^{\star}$ or assign* or class ${ }^{\star}$ or control* or determine* or divide ${ }^{\star}$ or distribut ${ }^{\star}$ or expose ${ }^{\star}$ or fashion or number $^{\star}$ or place ${ }^{\star}$ or recruit ${ }^{\star}$ or subsitut ${ }^{\star}$ or treat $\left.{ }^{\star}\right)$ ).ab.

33. trial.ti,ab.

34. (control* adj3 (trial or study or group $\left.\left.{ }^{\star}\right)\right) . t i, a b$.

35. "2000".md.

36. (quasi adj (experimental or random*)).mp.

37. ((waitlist* or wait ${ }^{\star}$ list $^{\star}$ or treatment as usual or usual treatment or TAU or no treatment or care as usual or usual care or standard care) and (control or group)).ab.

38. or $/ 20-30$

39. (18 and 26 and 38 )

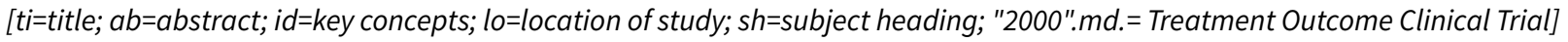

\section{ProQuest PILOTS}

This database covers post-traumatic stress disorder (PTSD) and other mental-health sequelae of traumatic events. We searched PILOTS (all years to 3 February 2016) using terms for: (humanitarian crises or LMIC) and RCTs

[Humanitarian Crises]

S1 SU.EXACT("Humanitarian Intervention")

S2 (SU.EXACT.EXPLODE("Accidents" OR "Agent Orange" OR "Air Traffic Accidents" OR "Avalanches" OR "Blizzards" OR "Building Collapse" OR "Disasters" OR "Drought" OR "Earthquakes" OR "Epidemics" OR "Epizootics" OR "Explosions" OR "Famine" OR "Fires" OR "Floods" OR "Home Accidents" OR "Hurricanes" OR "Industrial Accidents" OR "Landmines" OR "Landslides" OR "Lightning" OR "Motor Traffic Accidents" OR "Natural Disasters" OR "Nuclear Accidents" OR "Nuclear Testing" OR "Oil Spills" OR "Pedestrian Accidents" OR "Railroad Accidents" OR "Ship Accidents" OR "Technological Disasters" OR "Tornadoes" OR "Toxic Contamination" OR "Tsunamis" OR "Volcanoes")) S3 (altruis* or humanitarian or "human right*")

S4 (cataclysmic or catastroph* or devastation or disaster ${ }^{\star}$ or drought ${ }^{\star}$ or earthquake* or evacuation* or famine* or flood or floods or

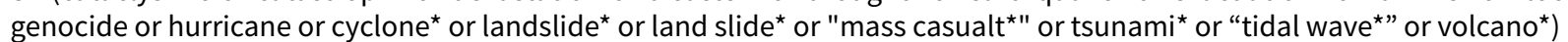

S5 ((war or conflict) near/2 (affect ${ }^{\star}$ or effect* or expos* or related or victim ${ }^{\star}$ or survivor $\left.{ }^{\star}\right)$ )

S6 (refugee* or "forced migration") or (displac* NEAR/2 (internal or forced or mass or person* or people* or population*))

S7 (politic ${ }^{\star}$ near $/ 2$ (persecut ${ }^{\star}$ or prison ${ }^{\star}$ or imprison* or violen $\left.{ }^{\star}\right)$ )

S8 SU.EXACT("Developing Countries")

[Location]

S9 (Africa or Asia or Caribbean or West Indies or South America or Latin America or Central America or Afghanistan or Albania or Algeria or Angola or Antigua or Barbuda or Argentina or Armenia or Armenian or Aruba or Azerbaijan or Bahrain or Bangladesh or Barbados or Benin or Byelarus or Byelorussian or Belarus or Belorussian or Belorussia or Belize or Bhutan or Bolivia or Bosnia or Herzegovina or Hercegovina or Botswana or Brasil or Brazil or Bulgaria or Burkina Faso or Burkina Fasso or Upper Volta or Burundi or Urundi or Cambodia or Khmer Republic or Kampuchea or Cameroon or Cameroons or Cameron or Camerons or Cape Verde or Central African Republic or Chad or Chile or China or Colombia or Comoros or Comoro Islands or Comores or Mayotte or Congo or Zaire or Costa Rica or Cote d'Ivoire or Ivory Coast or Croatia or Cuba or Cyprus or Czechoslovakia or Czech Republic or Slovakia or Slovak Republic or Djibouti or French Somaliland or Dominica or Dominican Republic or East Timor or East Timur or Timor Leste or Ecuador or Egypt or United Arab Republic or El Salvador or Eritrea or Estonia or Ethiopia or Fiji or Gabon or Gabonese Republic or Gambia or Gaza or Georgia or Georgian or Ghana or Gold Coast or Greece or Grenada or Guatemala or Guinea or Guam or Guiana or Guyana or Haiti or Honduras or Hungary or India or Maldives or Indonesia or Iran or Iraq or Isle of Man or Jamaica or Jordan or Kazakhstan or Kazakh or Kenya or Kiribati or Korea or Kosovo or Kyrgyzstan or Kirghizia or Kyrgyz Republic or Kirghiz or Kirgizstan or Lao PDR or Laos or Latvia or Lebanon or Lesotho or Basutoland or Liberia or Libya or Lithuania or Macedonia or Madagascar or Malagasy Republic or Malaysia or Malaya or Malay or Sabah or Sarawak or Malawi or Nyasaland or Mali or Malta or Marshall Islands or Mauritania or Mauritius or Agalega Islands or Mexico or Micronesia or Middle East or Moldova or Moldovia or Moldovian or Mongolia or Montenegro or Morocco or Ifni or Mozambique or Myanmar or Myanma or Burma or Namibia or Nepal or Netherlands Antilles or New Caledonia or Nicaragua or Niger or Nigeria or Northern Mariana Islands or Oman or Muscat or Pakistan or Palau or Palestine or Panama or Paraguay or Peru or Philippines or Philipines or Phillipines or Phillippines or Poland or Portugal or Puerto Rico or Romania or Rumania or Roumania or Russia or Russian or Rwanda or Ruanda or Saint Kitts or St Kitts or Nevis or Saint Lucia or St Lucia or Saint Vincent or St Vincent or Grenadines or Samoa or Samoan Islands or Navigator Island or Navigator Islands or Sao Tome or Saudi Arabia or Senegal or Serbia or Montenegro or Seychelles or Sierra Leone or Slovenia or Sri Lanka or Ceylon or Solomon Islands or Somalia or Sudan or Suriname or Surinam or Swaziland or Syria or Tajikistan or Tadzhikistan or Tadjikistan or Tadzhik or Tanzania or Thailand or Togo or Togolese Republic or Tonga or Trinidad or Tobago or Tunisia or Turkey or Turkmenistan or Turkmen or Uganda or Ukraine or Uruguay or USSR or Soviet Union or Union of Soviet Socialist Republics or Uzbekistan or Uzbek or Vanuatu or New Hebrides or Venezuela or Vietnam or Viet Nam or West Bank or Yemen or Yugoslavia or Zambia or Zimbabwe or Rhodesia)

[RCT filter-precision maximizing]

$\mathrm{S} 10$ (randomiz* OR randomis*)

S11 (waitlist* OR "wait list*" OR "waiting list*" OR "treatment as usual" OR TAU) NEAR/3 (control* OR group)

S12 "no intervention"

S13 (random* NEAR/3 (administer* OR allocat* OR assign* OR class* OR control* OR determine* OR divide* OR division OR distribute* OR expose ${ }^{\star}$ OR fashion OR number ${ }^{\star}$ OR place ${ }^{\star}$ OR recruit* OR substitute* OR treat $\left.^{\star}\right)$ )

Psychological therapies for the treatment of mental disorders in low- and middle-income countries affected by humanitarian crises 
[((Humanitarian Crises OR Location) AND RCT filter)]

$\mathrm{S} 14$ (S1 or S2 or S3 or S4 or S5 or S6 or S7 or S8 or S9) and (S10 or S11 or S12 or S13)

We did not repeat the PILOTS search in 2017 , as it did not retrieve any unique studies in any of the previous searches (to February 2016).

\section{Cochrane CENTRAL search}

We searched the Cochrane Central Register of Controlled Trials (CENTRAL) (all years to issue 8, 2017) using terms for: (humanitarian crises and LMIC and mental health)

\#1. MeSH descriptor: [Crisis Intervention] explode all trees

\#2. MeSH descriptor: [Disasters] explode all trees

\#3. MeSH descriptor: [Refugees] this term only

\#4. MeSH descriptor: [Adaptation, Psychological] explode all trees

\#5. MeSH descriptor: [Resilience, Psychological] this term only

\#6. MeSH descriptor: [Terrorism] explode all trees

\#7. MeSH descriptor: [War] explode all trees

\#8. MeSH descriptor: [Torture] this term only

\#9. (humanitarian and (aid or affair* or agenc ${ }^{\star}$ or assistance or catastrophe* or crisis or crises or disaster ${ }^{\star}$ or effort $^{\star}$ or emergenc $^{\star}$ or evacuation* or integration or reintegration or mission or organization* or organisation* or program ${ }^{\star}$ or relief or setting or support $^{\star}$ or task force or work $\left.{ }^{*}\right)$ )

\#10. (genocide or "armed conflict*" or "mass execution*" or "mass violence")

\#11. (cataclysmic or catastroph* or devastation or disaster ${ }^{\star}$ or drought* or earthquake* or evacuation* or famine* or flood or floods or hurricane or cyclone* or landslide* or "land slide " or landslide or "mass casualt " or tsunami* or "tidal wave*" or volcano*)

\#12. (refugee* or forced migration or (displac* near/2 (internal or forced or mass or person* or people* or population $\left.\left.{ }^{\star}\right)\right)$ )

\#13. (torture* or (politic ${ }^{\star}$ near/2 (persecut ${ }^{\star}$ or prison $^{\star}$ or imprison ${ }^{\star}$ or violen $\left.\left.{ }^{\star}\right)\right)$ )

\#14. (war and (abuse* or crime* or rape $^{\star}$ or survivor ${ }^{\star}$ or victim $\left.{ }^{\star}\right)$ )

\#15. (bereav* or orphan ${ }^{\star}$ or widow*)

$\# 16$. (\#1 or \#2 or \#3 or \#4 or \#5 or \#6 or \#7 or \#8 or \#9 or \#10 or \#11 or \#12 or \#13 or \#14 or \#15)

\#17. (Africa or Asia or Caribbean or West Indies or South America or Latin America or Central America or Afghanistan or Albania or Algeria or Angola or Antigua or Barbuda or Argentina or Armenia or Armenian or Aruba or Azerbaijan or Bahrain or Bangladesh or Barbados or Benin or Byelarus or Byelorussian or Belarus or Belorussian or Belorussia or Belize or Bhutan or Bolivia or Bosnia or Herzegovina or Hercegovina or Botswana or Brasil or Brazil or Bulgaria or Burkina Faso or Burkina Fasso or Upper Volta or Burundi or Urundi or Cambodia or Khmer Republic or Kampuchea or Cameroon or Cameroons or Cameron or Camerons or Cape Verde or Central African Republic or Chad or Chile or China or Colombia or Comoros or Comoro Islands or Comores or Mayotte or Congo or Zaire or Costa Rica or Cote d'Ivoire or Ivory Coast or Croatia or Cuba or Cyprus or Czechoslovakia or Czech Republic or Slovakia or Slovak Republic or Djibouti or French Somaliland or Dominica or Dominican Republic or East Timor or East Timur or Timor Leste or Ecuador or Egypt or United Arab Republic or El Salvador or Eritrea or Estonia or Ethiopia or Fiji or Gabon or Gabonese Republic or Gambia or Gaza or Georgia or Georgian or Ghana or Gold Coast or Greece or Grenada or Guatemala or Guinea or Guam or Guiana or Guyana or Haiti or Honduras or Hungary or India or Maldives or Indonesia or Iran or Iraq or Isle of Man or Jamaica or Jordan or Kazakhstan or Kazakh or Kenya or Kiribati or Korea or Kosovo or Kyrgyzstan or Kirghizia or Kyrgyz Republic or Kirghiz or Kirgizstan or Lao PDR or Laos or Latvia or Lebanon or Lesotho or Basutoland or Liberia or Libya or Lithuania or Macedonia or Madagascar or Malagasy Republic or Malaysia or Malaya or Malay or Sabah or Sarawak or Malawi or Nyasaland or Mali or Malta or Marshall Islands or Mauritania or Mauritius or Agalega Islands or Mexico or Micronesia or Middle East or Moldova or Moldovia or Moldovian or Mongolia or Montenegro or Morocco or Ifni or Mozambique or Myanmar or Myanma or Burma or Namibia or Nepal or Netherlands Antilles or New Caledonia or Nicaragua or Niger or Nigeria or Northern Mariana Islands or Oman or Muscat or Pakistan or Palau or Palestine or Panama or Paraguay or Peru or Philippines or Philipines or Phillipines or Phillippines or Poland or Portugal or Puerto Rico or Romania or Rumania or Roumania or Russia or Russian or Rwanda or Ruanda or Saint Kitts or St Kitts or Nevis or Saint Lucia or St Lucia or Saint Vincent or St Vincent or Grenadines or Samoa or Samoan Islands or Navigator Island or Navigator Islands or Sao Tome or Saudi Arabia or Senegal or Serbia or Montenegro or Seychelles or Sierra Leone or Slovenia or Sri Lanka or Ceylon or Solomon Islands or Somalia or Sudan or Suriname or Surinam or Swaziland or Syria or Tajikistan or Tadzhikistan or Tadjikistan or Tadzhik or Tanzania or Thailand or Togo or Togolese Republic or Tonga or Trinidad or Tobago or Tunisia or Turkey or Turkmenistan or Turkmen or Uganda or Ukraine or Uruguay or USSR or Soviet Union or Union of Soviet Socialist Republics or Uzbekistan or Uzbek or Vanuatu or New Hebrides or Venezuela or Vietnam or Viet Nam or West Bank or Yemen or Yugoslavia or Zambia or Zimbabwe or Rhodesia)

\#18. MeSH descriptor: [Developing Countries] this term only

\#19. ((developing or less ${ }^{\star}$ developed or under developed or underdeveloped or middle income or low* income or underserved or under served or deprived or poor ${ }^{\star}$ ) near (countr ${ }^{\star}$ or nation* or population ${ }^{\star}$ or world))

\#20. ((developing or less ${ }^{\star}$ developed or under developed or underdeveloped or middle income or low* income) next (economy or economies))

\#21. (low* next (GDP or GNP or "gross domestic" or "gross national"))

\#22. (low near middle near countr ${ }^{\star}$ )

\#23. (LMIC or LMICs or third world or LAMI country or LAMI countries)

\#24. (transitional country or transitional countries)

\#25. (\#17 or \#18 or \#19 or \#20 or \#21 or \#22 or \#23 or \#24)

Psychological therapies for the treatment of mental disorders in low- and middle-income countries affected by humanitarian crises 
\#26. MeSH descriptor: [Mental Health] this term only

\#27. MeSH descriptor: [Health Promotion] this term only

\#28. MeSH descriptor: [Mental Disorders] explode all trees

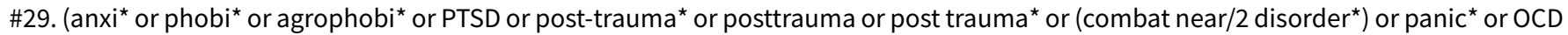
or obsess ${ }^{\star}$ or compulsi* or GAD or stress disorder ${ }^{\star}$ or stress reaction* or acute stress or neurosis or neuroses or neurotic or psychoneuro*) \#30. ("substance use " $^{\star}$ or "substance abuse*" or SUD or addict*)

\#31. (somatiz* or somatis* or hysteri* or briquet or multisomat ${ }^{\star}$ or multi somat* or MUPs or "medically unexplained")

\#32. ((dissociative near/3 (disorder* or reaction*)) or dissociation)

\#33. (mental or psychiatri ${ }^{\star}$ or psycho* or "affective disorder*" or "affective symptom ${ }^{\star}$ " or mood or depressi^ or depressed or MDD)

\#34. (\#26 or \#27 or \#28 or \#29 or \#30 or \#31 or \#32 or \#33)

\#35. (\#16 and \#25 and \#34)

\section{OVID MEDLINE search}

We searched MEDLINE (all years to 1 September 2017) using terms for: (humanitarian crises and LMIC and mental health and RCTs)

[Humanitarian Crises]

1. CRISIS INTERVENTION/

2. exp DISASTERS/

3. REFUGEES/

4. ADAPTATION, PSYCHOLOGICAL/ Or RESILIENCE, PSYCHOLOGICAL/

5. $\exp$ TERRORISM/

6. $\exp$ WAR/

7. TORTURE/

8. (humanitarian adj3 (aid or affair ${ }^{\star}$ or agenc ${ }^{\star}$ or assistance or catastrophe ${ }^{\star}$ or crisis or crises or disaster ${ }^{\star}$ or effort ${ }^{\star}$ or emergenc ${ }^{\star}$ or evacuation* or integration or reintegration or mission or organization* or organisation* or program ${ }^{\star}$ or relief or setting* or support ${ }^{\star}$ or task force or work $\left.{ }^{\star}\right)$ ).mp.

9. (genocide or armed conflict* ${ }^{\star}$ or mass execution ${ }^{\star}$ or mass violence).mp.

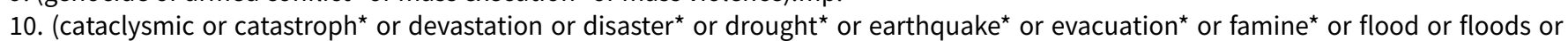
hurricane or cyclone* or landslide* or land slide* or mass casualt* or tsunami* or tidal wave* or volcano*).mp.

11. (refugee* or forced migration or (displac ${ }^{\star}$ adj2 (internal or forced or mass or person* or people* or population*))).mp.

12. (torture ${ }^{\star}$ or (politic ${ }^{\star}$ adj2 (persecut ${ }^{\star}$ or prison ${ }^{\star}$ or imprison ${ }^{\star}$ or violen $\left.\left.{ }^{\star}\right)\right)$ ).mp.

13. (war and (abuse or crime $^{\star}$ or rape ${ }^{\star}$ or survivor ${ }^{\star}$ or victim $\left.^{\star}\right)$ ).mp.

14. (bereav* or orphan* or widow*).mp.

15. or/1-14

[Location]

16. (Africa or Asia or Caribbean or West Indies or South America or Latin America or Central America or Afghanistan or Albania or Algeria or Angola or Antigua or Barbuda or Argentina or Armenia or Armenian or Aruba or Azerbaijan or Bahrain or Bangladesh or Barbados or Benin or Byelarus or Byelorussian or Belarus or Belorussian or Belorussia or Belize or Bhutan or Bolivia or Bosnia or Herzegovina or Hercegovina or Botswana or Brasil or Brazil or Bulgaria or Burkina Faso or Burkina Fasso or Upper Volta or Burundi or Urundi or Cambodia or Khmer Republic or Kampuchea or Cameroon or Cameroons or Cameron or Camerons or Cape Verde or Central African Republic or Chad or Chile or China or Colombia or Comoros or Comoro Islands or Comores or Mayotte or Congo or Zaire or Costa Rica or Cote d'Ivoire or Ivory Coast or Croatia or Cuba or Cyprus or Czechoslovakia or Czech Republic or Slovakia or Slovak Republic or Djibouti or French Somaliland or Dominica or Dominican Republic or East Timor or East Timur or Timor Leste or Ecuador or Egypt or United Arab Republic or El Salvador or Eritrea or Estonia or Ethiopia or Fiji or Gabon or Gabonese Republic or Gambia or Gaza or Georgia or Georgian or Ghana or Gold Coast or Greece or Grenada or Guatemala or Guinea or Guam or Guiana or Guyana or Haiti or Honduras or Hungary or India or Maldives or Indonesia or Iran or Iraq or Isle of Man or Jamaica or Jordan or Kazakhstan or Kazakh or Kenya or Kiribati or Korea or Kosovo or Kyrgyzstan or Kirghizia or Kyrgyz Republic or Kirghiz or Kirgizstan or Lao PDR or Laos or Latvia or Lebanon or Lesotho or Basutoland or Liberia or Libya or Lithuania or Macedonia or Madagascar or Malagasy Republic or Malaysia or Malaya or Malay or Sabah or Sarawak or Malawi or Nyasaland or Mali or Malta or Marshall Islands or Mauritania or Mauritius or Agalega Islands or Mexico or Micronesia or Middle East or Moldova or Moldovia or Moldovian or Mongolia or Montenegro or Morocco or Ifni or Mozambique or Myanmar or Myanma or Burma or Namibia or Nepal or Netherlands Antilles or New Caledonia or Nicaragua or Niger or Nigeria or Northern Mariana Islands or Oman or Muscat or Pakistan or Palau or Palestine or Panama or Paraguay or Peru or Philippines or Philipines or Phillipines or Phillippines or Poland or Portugal or Puerto Rico or Romania or Rumania or Roumania or Russia or Russian or Rwanda or Ruanda or Saint Kitts or St Kitts or Nevis or Saint Lucia or St Lucia or Saint Vincent or St Vincent or Grenadines or Samoa or Samoan Islands or Navigator Island or Navigator Islands or Sao Tome or Saudi Arabia or Senegal or Serbia or Montenegro or Seychelles or Sierra Leone or Slovenia or Sri Lanka or Ceylon or Solomon Islands or Somalia or Sudan or Suriname or Surinam or Swaziland or Syria or Tajikistan or Tadzhikistan or Tadjikistan or Tadzhik or Tanzania or Thailand or Togo or Togolese Republic or Tonga or Trinidad or Tobago or Tunisia or Turkey or Turkmenistan or Turkmen or Uganda or Ukraine or Uruguay or USSR or Soviet Union or Union of Soviet Socialist Republics or Uzbekistan or Uzbek or Vanuatu or New Hebrides or Venezuela or Vietnam or Viet Nam or West Bank or Yemen or Yugoslavia or Zambia or Zimbabwe or Rhodesia).mp.

17. DEVELOPING COUNTRIES/

18. ((developing or less* developed or under developed or underdeveloped or middle income or low ${ }^{\star}$ income or underserved or under served or deprived or poor ${ }^{\star}$ ) adj3 (countr ${ }^{\star}$ or nation ${ }^{\star}$ or population ${ }^{\star}$ or world)).mp.

Psychological therapies for the treatment of mental disorders in low- and middle-income countries affected by humanitarian crises 
19. ((developing or less ${ }^{\star}$ developed or under developed or underdeveloped or middle income or low* income) adj1 (economy or economies)).mp.

20. (low* adj1 (GDP or GNP or gross domestic or gross national)).mp.

21. (low adj3 middle adj3 countr ${ }^{\star}$ ).mp.

22. (LMIC or LMICs or third world or LAMI country or LAMI countries).mp.

23. (transitional country or transitional countries).mp.

24. or/16-23

[Mental disorders]

25. HEALTH PROMOTION/

26. MENTAL HEALTH/

27. exp MENTAL DISORDERS/

28. (anxi ${ }^{\star}$ or phobi ${ }^{\star}$ or agrophobi ${ }^{\star}$ or PTSD or post-trauma* or posttrauma or post trauma* or (combat adj3 disorder ${ }^{\star}$ ) or panic ${ }^{\star}$ or OCD or obsess $^{\star}$ or compulsi ${ }^{\star}$ or GAD or stress disorder* or stress reaction* or acute stress or neurosis or neuroses or neurotic or psychoneuro*).mp.

29. (substance use ${ }^{\star}$ or substance abuse* or SUD or addict*).mp.

30. (somatiz ${ }^{\star}$ or somatis ${ }^{\star}$ or hysteri ${ }^{\star}$ or briquet or multisomat ${ }^{\star}$ or multi somat ${ }^{\star}$ or MUPs or medically unexplained).mp.

31. ((dissociative adj3 (disorder* or reaction $\left.^{\star}\right)$ ) or dissociation).mp.

32. (mental or psychiatr ${ }^{\star}$ or psycho* or affective disorder* or affective symptom* or mood or depressi* or depressed or MDD).mp.

33. or $/ 25-32$

[RCT filter-precision maximizing]

34. randomised controlled trial.pt.

35. (randomi\#ted or randomi\#ation).ab,ti.

36. (random ${ }^{\star}$ adj3 (administ ${ }^{\star}$ or allocat ${ }^{\star}$ or assign ${ }^{\star}$ or class $^{\star}$ or control ${ }^{\star}$ or determine ${ }^{\star}$ or divide ${ }^{\star}$ or division or distribut ${ }^{\star}$ or expose* or fashion or number ${ }^{\star}$ or place ${ }^{\star}$ or recruit ${ }^{\star}$ or subsitut ${ }^{\star}$ or treat $\left.{ }^{\star}\right)$ ).ab.

37. ((waitlist* or wait* $^{\star}$ list $^{\star}$ or treatment as usual or TAU) adj3 (control or group)).ab.

38. intervention as usual.ab.

39. or/33-37

40. (15 and 24 and 33 and 39)

\section{OVID Embase search}

We searched Embase (all years to 1 September) using terms for: (humanitarian crises and LMIC and mental health and RCTs)

[Humanitarian Crises]

1. (crisis or crises).mp.

2. ADAPTATIVE BEHAVIOR/

3. ALTRUISM/

4. $\exp$ COPING BEHAVIOR/

5. $\exp$ DISASTER/

6. $\exp$ EMOTIONAL DEPRIVATION/

7. $\exp$ MILITARY PHENOMENA/

8. $\exp$ REFUGEE/

9. $\exp$ VIOLENCE/

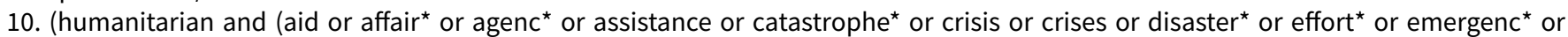
evacuation* or integration or reintegration or mission or organization* or organisation* or program* or relief or setting* or support* or task force or work $\left.\left.{ }^{\star}\right)\right) . t i, a b, k w$.

11. (genocide or armed conflict* or mass execution* or mass violence).ti,ab,kw.

12. (refugee ${ }^{\star}$ or forced migration or (displac ${ }^{\star}$ adj2 (internal or forced or mass or person* or people* or population*))).ti,ab,kw.

13. (torture* or (politic $^{\star}$ adj2 (persecut* or prison $^{\star}$ or imprison ${ }^{\star}$ or violen $\left.\left.{ }^{\star}\right)\right)$ ).ti,ab,kw.

14. (war and (abuse or crime $^{\star}$ or rape or survivor $^{\star}$ or victim $\left.{ }^{\star}\right)$ ).ti,ab,kw.

15. $\left(\right.$ bereav $^{\star}$ or orphan ${ }^{\star}$ or widow* ${ }^{\star}$.ti,ab,kw.

16. (cataclysmic or catastroph* or devastation or disaster ${ }^{\star}$ or drought* or earthquake* or evacuation* or famine ${ }^{\star}$ or flood or floods or hurricane or cyclone $e^{\star}$ or landslide* or "land slide*" or landslide or "mass casualt*" or tsunami* or "tidal wave*" or volcano*).mp.

17. or/1-16

[Location]

18. DEVELOPING COUNTRY/

19. LOWEST INCOME GROUP/

20. MIDDLE INCOME GROUP/

21. RED CROSS/

22. UNITED NATIONS/

23. WORLD HEALTH ORGANIZATION/

24. ((developing or less ${ }^{\star}$ developed or under developed or underdeveloped or middle income or low income or underserved or under served or deprived or poor ${ }^{\star}$ ) adj3 (countr* or nation* or population* or world)).ti,ab,kw.

Psychological therapies for the treatment of mental disorders in low- and middle-income countries affected by humanitarian crises 
25. ((developing or less ${ }^{\star}$ developed or under developed or underdeveloped or middle income or low* income) adj1 (economy or economies)).ti,ab,kw.

26. (low* adj1 (GDP or GNP or "gross domestic" or "gross national")).ti,ab,kw.

27. (low adj3 middle adj3 countr ${ }^{\star}$ ).ti,ab,kw.

28. (LMIC or LMICs or third world or LAMI country or LAMI countries).ti,ab,kw.

29. (transitional country or transitional countries).ti,ab,kw.

30. (Africa or Asia or Caribbean or West Indies or South America or Latin America or Central America or Afghanistan or Albania or Algeria or Angola or Antigua or Barbuda or Argentina or Armenia or Armenian or Aruba or Azerbaijan or Bahrain or Bangladesh or Barbados or Benin or Byelarus or Byelorussian or Belarus or Belorussian or Belorussia or Belize or Bhutan or Bolivia or Bosnia or Herzegovina or Hercegovina or Botswana or Brasil or Brazil or Bulgaria or Burkina Faso or Burkina Fasso or Upper Volta or Burundi or Urundi or Cambodia or Khmer Republic or Kampuchea or Cameroon or Cameroons or Cameron or Camerons or Cape Verde or Central African Republic or Chad or Chile or China or Colombia or Comoros or Comoro Islands or Comores or Mayotte or Congo or Zaire or Costa Rica or Cote d'Ivoire or Ivory Coast or Croatia or Cuba or Cyprus or Czechoslovakia or Czech Republic or Slovakia or Slovak Republic or Djibouti or French Somaliland or Dominica or Dominican Republic or East Timor or East Timur or Timor Leste or Ecuador or Egypt or United Arab Republic or El Salvador or Eritrea or Estonia or Ethiopia or Fiji or Gabon or Gabonese Republic or Gambia or Gaza or Georgia or Georgian or Ghana or Gold Coast or Greece or Grenada or Guatemala or Guinea or Guam or Guiana or Guyana or Haiti or Honduras or Hungary or India or Maldives or Indonesia or Iran or Iraq or Isle of Man or Jamaica or Jordan or Kazakhstan or Kazakh or Kenya or Kiribati or Korea or Kosovo or Kyrgyzstan or Kirghizia or Kyrgyz Republic or Kirghiz or Kirgizstan or Lao PDR or Laos or Latvia or Lebanon or Lesotho or Basutoland or Liberia or Libya or Lithuania or Macedonia or Madagascar or Malagasy Republic or Malaysia or Malaya or Malay or Sabah or Sarawak or Malawi or Nyasaland or Mali or Malta or Marshall Islands or Mauritania or Mauritius or Agalega Islands or Mexico or Micronesia or Middle East or Moldova or Moldovia or Moldovian or Mongolia or Montenegro or Morocco or Ifni or Mozambique or Myanmar or Myanma or Burma or Namibia or Nepal or Netherlands Antilles or New Caledonia or Nicaragua or Niger or Nigeria or Northern Mariana Islands or Oman or Muscat or Pakistan or Palau or Palestine or Panama or Paraguay or Peru or Philippines or Philipines or Phillipines or Phillippines or Poland or Portugal or Puerto Rico or Romania or Rumania or Roumania or Russia or Russian or Rwanda or Ruanda or Saint Kitts or St Kitts or Nevis or Saint Lucia or St Lucia or Saint Vincent or St Vincent or Grenadines or Samoa or Samoan Islands or Navigator Island or Navigator Islands or Sao Tome or Saudi Arabia or Senegal or Serbia or Montenegro or Seychelles or Sierra Leone or Slovenia or Sri Lanka or Ceylon or Solomon Islands or Somalia or Sudan or Suriname or Surinam or Swaziland or Syria or Tajikistan or Tadzhikistan or Tadjikistan or Tadzhik or Tanzania or Thailand or Togo or Togolese Republic or Tonga or Trinidad or Tobago or Tunisia or Turkey or Turkmenistan or Turkmen or Uganda or Ukraine or Uruguay or USSR or Soviet Union or Union of Soviet Socialist Republics or Uzbekistan or Uzbek or Vanuatu or New Hebrides or Venezuela or Vietnam or Viet Nam or West Bank or Yemen or Yugoslavia or Zambia or Zimbabwe or Rhodesia).mp.

31. exp AFRICAN/ or exp ASIAN/ or exp "CARIBBEAN (person)"/ or exp CENTRAL AMERICAN/ or exp EASTERN EUROPEAN/ or exp MELANESIAN/ or exp MICRONESIAN/ or exp POLYNESIAN/ or exp SOUTH AMERICAN/

32. or $/ 18-31$

[Mental disorders]

33. exp MENTAL DISEASE/

34. exp "PSYCHOLOGICAL and PSYCHIATRIC PROCEDURES"/

35. PSYCHOSOCIAL CARE/

36. $\left(\mathrm{anxi}^{\star}\right.$ or phobi* or agrophobi ${ }^{\star}$ or PTSD or post-trauma* or posttrauma or post trauma* or (combat adj2 disorder ${ }^{\star}$ ) or panic ${ }^{\star}$ or OCD or obsess ${ }^{\star}$ or compulsi* or GAD or stress disorder ${ }^{\star}$ or stress reaction ${ }^{\star}$ or acute stress or neurosis or neuroses or neurotic or psychoneuro*).ti,ab,kw.

37. (substance use ${ }^{\star}$ or substance abuse ${ }^{\star}$ or SUD or addict*).ti,ab,kw.

38. (somatiz ${ }^{\star}$ or somatis ${ }^{\star}$ or hysteri* ${ }^{\star}$ or briquet or multisomat ${ }^{\star}$ or multi somat ${ }^{\star}$ or MUPs or medically unexplained).ti,ab,kw.

39. ((dissociative adj3 (disorder ${ }^{\star}$ or reaction $\left.{ }^{\star}\right)$ ) or dissociation).ti,ab,kw.

40. (mental or psychiatri* or psycho* or affective disorder ${ }^{\star}$ or affective symptom or mood or depressi* or depressed or MDD).ti,ab,kw.

41. or $/ 33-40$

[RCT filter - precision maximizing]

42. randomiz ${ }^{*}$.de.

43. (randomi\#ed or randomi\#ation).ab,ti,kw.

44. (random* adj3 (administ* or allocat ${ }^{\star}$ or assign* or class ${ }^{\star}$ or control ${ }^{\star}$ or determine ${ }^{\star}$ or divide* or division or distribut ${ }^{\star}$ or expose $^{\star}$ or fashion or number ${ }^{\star}$ or place* or recruit* or subsitut ${ }^{\star}$ or treat $\left.\left.{ }^{\star}\right)\right)$.ab.

45. ((waitlist* or wait $^{\star}$ list $^{\star}$ or treatment as usual or TAU) adj3 (control or group)).ab.

46. intervention as usual.ab.

47. or $/ 42-46$

48. (17 and 32 and 41 and 47 )

In 2017 we appended the following terms to the searches:

(i) Demonyms:

((Africa? or Asia? or Arab ${ }^{*}$ or Caribbean or West Indi ${ }^{\star}$ or South America? or Latin America? or Central America? or Afghan ${ }^{\star}$ or Albania? or Algeria? or Angola? or Antigu* or Barbuda? or Argentin* or Armenia? or Aruba? or Azerbaijan? or Bahrain* or Bangladesh? or Barbados or Barbadian? or

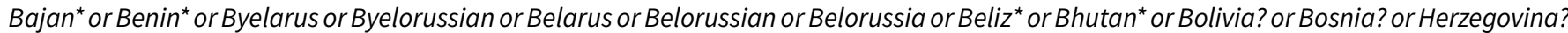
or Hercegovin* or Botswana? or Brasil* or Brazil* or Bulgaria? or Burkina Faso or Burkina Fasso or Upper Volta or Burundi* or Urundi* or

Psychological therapies for the treatment of mental disorders in low- and middle-income countries affected by humanitarian crises 
Cambodia? or Khmer Republic* or Kampuchea? or Cameroon* or Cameroons or Cameron* or Camerons or Cape Verde* or Central Africa* or Chad $^{*}$ or Chile* or China or Chinese or Colombia? or Comoros or Comoro Island* or Comores or Comoran or Mayotte* or Congo* or Zaire* or Costa Rica? or Cote d'Ivoire or Ivory Coast or Croatia? or Cuba? or Cyprus or Cyprian or Czechoslovakia? or Czech Republic* or Slovakia? or Slovak Republic or Djibouti* or French Somaliland or Dominica? or Dominican Republic or East Timor ${ }^{\star}$ or East Timur ${ }^{\star}$ or Timor Leste* or Timorese or Ecuador* or Egypt* or United Arab Republic or El Salvador ${ }^{\star}$ or Eritrea? or Estonia? or Ethiopia? or Fiji* or Gabon or Gabonese or Gambia? or Gaza? or Georgia? or Ghana or Ghanaian or Gold Coast or Greece or Greek or Grenada or Grenadian or Guatemala? or Guinea? or Guam* or Guiana or Guyana? or Haiti* or Hondura? or Hungary or Hungarian or India? or Maldives or Maldivian? or Indonesia? or Iran* or Iraq? or Isle of Man or Jamaica? or Jordan* or Kazakhstan or Kazakh or Kenya? or Kiribati* or Korea? or Kosov* or Kyrgyzstan or Kirghizia or Kyrgyz or Kirghiz or Kirgizstan or Lao PDR or Lao? or Latvia? or Lebanon or Lebanese or Lesotho* or Basutoland or Liberia? or Libya? or Lithuania? or Macedonia? or Madagasca? or Malagasy Republic or Malaysia or Malay? or Sabah* or Sarawak* or Malawi* or Nyasaland or Mali or Malta or Maltese or Marshall Island* or Mauritania? or Mauritius or Mauritian or Agalega Islands* or Mexico or Mexican or Micronesia or Middle East* or Moldova or Moldovia or Moldovian or Mongolia? or Montenegro or Morocc* or Ifni or Mozambique or Myanmar or Myanma or Burma or Burmese or Namibia? or Nepal* or Netherlands Antilles or New Caledonia? or Nicaragua? or Niger or Nigeria? or Northern Mariana Island* or Oman ${ }^{\star}$ or Muscat or Pakistan? or Palau or Palestin* or Panama or Paraguay or Peru or Peruvian or Philippin* or Philipin* or Phillipin* or Phillippin* or Poland or Polish or Portugal or Portuguese or Puerto Ric* or Romania? or Rumania? or Roumania? or Russia or Russian or Rwanda? or Ruanda? or Saint Kitts or St Kitts or Nevis or Saint Lucia? or St Lucia? or Saint Vincent or St Vincent or Grenadines or Samoa? or Samoan Island* or Navigator Island* or Sao Tom* or Saudi Arabia? or Senegal* or Serbia? or Montenegr ${ }^{\star}$ or Seychell* or Sierra Leon* or Slovenia? or Slovak* or Sri Lanka? or Ceylon or Solomon Island ${ }^{*}$ or Somali* or Sudan* or Surinam* or Swaziland* or Syria? or Tajikistan or Tadzhikistan or Tadjikistan or Tadzhik or Tanzania? or Thailand or Thai or Togo or Togolese or Tonga? or Trinidad* or Tobag* or Tunisia? or Turkey or Turkish or Turkmenistan? or Turkmen or Uganda? or Ukrain* or Uruguay* or USSR? or Soviet Union? or Union of Soviet Socialist Republics or Uzbekistan? or Uzbek? or Vanuat ${ }^{\star}$ or New Hebride* or Venezuel* or Vietnam ${ }^{\star}$ or Viet Nam* or West Bank or Yemen? or Yugoslavia? or Zambia? or Zimbabwe* or Rhodesia?) adj3 (combatant? or ex-combatant? or soldier? or ((conflict or terroris ${ }^{*}$ or war) adj2 (affected or afflicted or trauma $\left.{ }^{\star}\right)$ or refugee? or survivor? or victim? or orphan* or widow*)) [Title, Abstract, Keywords (PsycINFO, CENTRAL, MEDLINE, Embase)]

(ii) Additional terms for warfare:

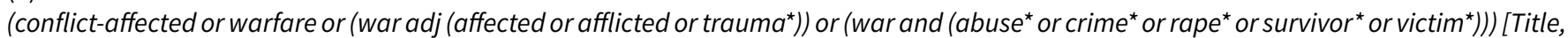
Abstract, Keywords (PsycINFO, CENTRAL, MEDLINE, Embase)]

(iii) Additional terms for mental health in low or poor resource settings:

(((low or poor) adj resource setting?) and (anxi ${ }^{\star}$ or phobi* or agrophobi* or PTSD or post-trauma* or posttrauma* or post trauma* or (combat adj3 disorder ${ }^{\star}$ ) or panic ${ }^{\star}$ or OCD or obsess ${ }^{\star}$ or compulsi ${ }^{\star}$ or GAD or stress disorder ${ }^{\star}$ or stress reaction ${ }^{\star}$ or acute stress or neurosis or neuroses or neurotic or psychoneuro* or mental or psychiatr ${ }^{\star}$ or psycho* or affective disorder* or affective symptom* or mood or depressi ${ }^{\star}$ or depressed

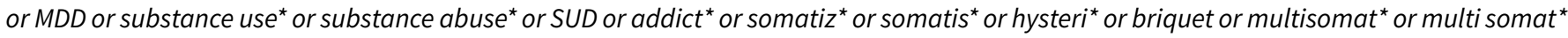
or MUPs or medically unexplained or (dissociative adj3 (disorder ${ }^{\star}$ or reaction $\left.{ }^{\star}\right)$ ) or dissociation)).ti,ab,id, hw. [PsycINFO only]

\section{International trial registries}

ClinicalTrials.gov: (all years to 1-September 2017): Title Search - Interventional Studies | earthquake OR earthquakes OR tsunami OR tsunamis OR floods OR hurricane OR hurricanes OR refugee OR refugees OR war OR warfare OR postconflict OR post-conflict OR genocide OR sexual violence OR torture OR terrorism OR terrorists

\section{CONTRIBUTIONS OF AUTHORS}

MP, WT, MvO, and CB designed the review structure. MP, CG, and DP collected data; MP and CB ran the analyses; MP, WT, and CB drafted and critically revised the manuscript.

MvO critically revised the manuscript.

All review authors contributed actively to development of the review, participated in discussions, helped clarify questions, and provided suggestions for overall preparation.

The review authors alone are responsible for the views expressed in this article, which do not necessarily represent the views, decisions, or policies of the institutions with which they are affiliated.

\section{DECLARATIONSOF INTEREST}

None known.

\section{SOURCES OF SUPPORT}

\section{Internal sources}

- WHO Collaborating Centre for Research and Training in Mental Health and Service Evaluation, Department of Neuroscience, Biomedicine, and Movement Sciences, University of Verona, Italy.

- Department of Mental Health, Johns Hopkins Bloomberg School of Public Health, Johns Hopkins University, Baltimore, MD, USA.

Psychological therapies for the treatment of mental disorders in low- and middle-income countries affected by humanitarian crises 


\section{External sources}

- None, Other

\section{DIFFERENCES BETWEEN PROTOCOLANDREVIEW}

1. MUPS were added to the somatoform disorders category, and "depressive disorders" were changed into "major depressive disorders." These changes have been made, as they reflect the definitions used in the current scientific literature focused on psychosocial interventions in LMICs.

2. Behavioural therapy has been removed from and thought field therapy has been added to the list of interventions.

3. We added a combined subgroups analysis according to types of psychological therapies, as we believe it was more informative and appropriate to explore this issue rather than following the original plan of conducting separate comparisons for each type of therapy (as stated in the original protocol).

\section{N DEX TERMS}

\section{Medical Subject Headings (MeSH)}

*Developing Countries; Age Factors; Anxiety Disorders [psychology] [ ${ }^{\star}$ therapy]; Armed Conflicts [psychology]; Behavior Therapy; Depressive Disorder, Major [psychology] [*therapy]; Disasters; Eye Movement Desensitization Reprocessing [methods]; Narrative Therapy; Patient Dropouts [statistics \& numerical data]; Psychotherapy [ ${ }^{\star}$ methods]; Randomized Controlled Trials as Topic; Somatoform Disorders [therapy]; Stress Disorders, Post-Traumatic [psychology] [*therapy]; Stress, Psychological [complications]; Violence [psychology]; Waiting Lists

\section{MeSH check words}

Adolescent; Adult; Aged; Child; Child, Preschool; Humans; Middle Aged 